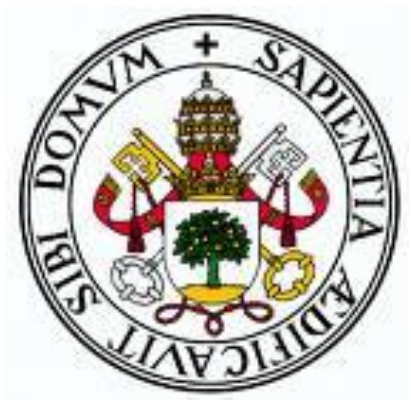

UNIVERSIDAD DE VALLADOLID

Departamento de Didáctica de las CCSS y de las CCEE

\title{
ESTUDIO DE CONTRASTE SOBRE LA PREFERENCIA Y SIGNIFICACIÓN DE PRUEBAS FORMALES Y PREFORMALES
}

TESIS DOCTORAL

Juan Carlos González Vara

Valladolid, marzo de 2012 


\begin{abstract}
Memoria presentada para optar al grado de Doctor por la Universidad de Valladolid por el licenciado en la Universidad de Valladolid, D. Juan Carlos González Vara, en el Programa de Doctorado: Investigación en Didáctica de las Ciencias Sociales, Experimentales, y de la Matemática.
\end{abstract}

Director de la Tesis: Dr. Tomás Ortega del Rincón. Departamento de Didáctica de las CCSS y de las CCEE. Universidad de Valladolid. 
Esta tesis ha sido desarrollada en el marco del proyecto I+D+i, EDU2009-12063, del Ministerio de Ciencia e Innovación. 
TOMÁS ORTEGA DEL RINCÓN, CAUN de Didáctica de la Matemática de la Universidad de Valladolid,

\section{CERTIFICA:}

Que la presente memoria, Estudio de contraste sobre la preferencia y significación de pruebas formales y preformales, ha sido realizada por Don Juan Carlos González Vara bajo mi dirección en la Universidad de Valladolid.

Valladolid, marzo de 2012.

Fdo.: Tomás Ortega del Rincón 

Me siento especialmente obligado a manifestar mi más sincero agradecimiento en primer lugar al director de esta tesis, Dr. Tomás Ortega del Rincón, por sus continuas y sabias orientaciones, por la generosa y amable atención que me ha dedicado en todo momento, por la paciencia que siempre ha mostrado conmigo y, sobre todo, por su cercanía y amistad que convierte en un honor y un regalo el trabajo que se realiza bajo su acertada y responsable dirección.

Mi gratitud también a los familiares que tanto me han ayudado con su aliento y comprensión.

Expreso asimismo mi reconocimiento a las personas que desde el ámbito universitario han colaborado desinteresadamente en la elaboración de esta tesis respondiendo a cuestionarios, ofreciendo ayuda, presencia, espacios de trabajo y cuantos materiales didácticos han sido necesarios para su realización. 

A mi familia 




\section{ÍNDICE}

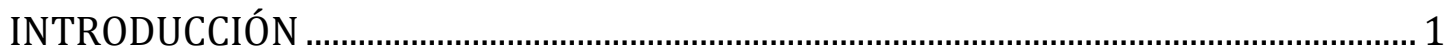

CAPÍTULO 1

ANTECEDENTES Y MARCO TEÓRICO................................................................................ 9

CAPÍTULO 2

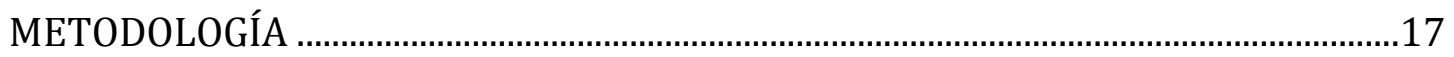

2.1. Introducción....................................................................................................17

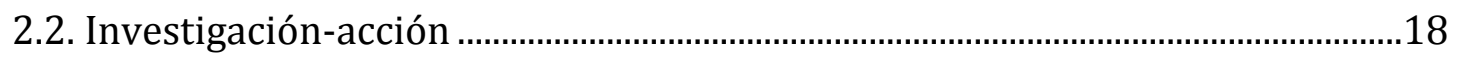

2.3. Investigación de diseño, experimentos de enseñanza..........................................24

2.4. Evolución de los experimentos de diseño ………………………………………...27

2.5. Experimentos de enseñanza transformativos dirigidos por una conjetura...30

2.6. Metodología cuantitativa ………………………………………………………….....33

CAPÍTULO 3

EXPERIENCIA PREVIA E HIPÓTESIS DE INVESTIGACIÓN ……………………….........37

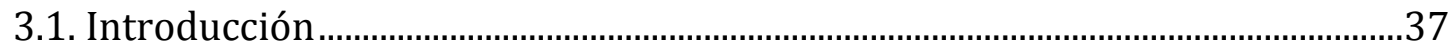

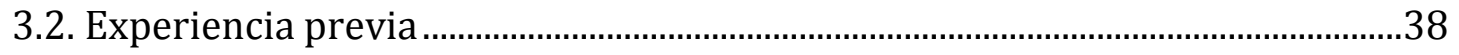

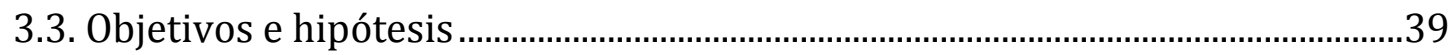

CAPÍTULO 4

CICLOS DE INVESTIGACIÓN-ACCIÓN ...............................................................................41

PLANIFICACIÓN GENERAL .......................................................................................... 41

4.1. Ciclo I (ciclo de exploración) ………………………………………………………..43

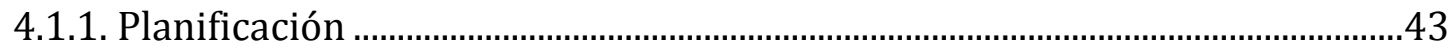

4.1.2. Desarrollo del primer ciclo (acción) ……………………………………………....4

4.1.3. Observación de la acción .................................................................................45

4.1.4. Recogida de datos a través del cuestionario ....................................................46

4.1.5. Análisis de los datos ..............................................................................................

4.1.6. Análisis de las entrevistas ..................................................................................73

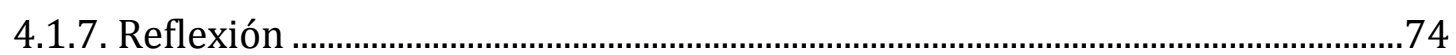

4.2. Ciclo II (ciclo de confirmación) …………………………....................................78 
4.2.2. Desarrollo del segundo ciclo (acción) ………………......................................79

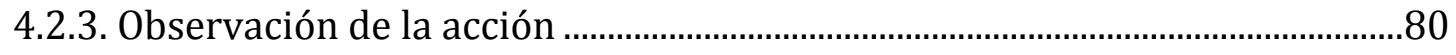

4.2.4. Recogida de datos a través del cuestionario .........................................................89

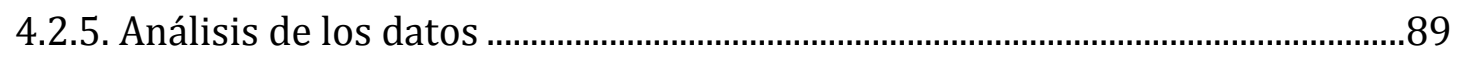

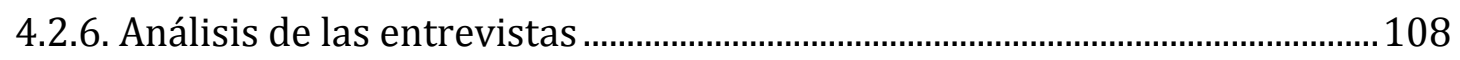

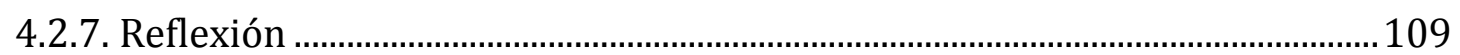

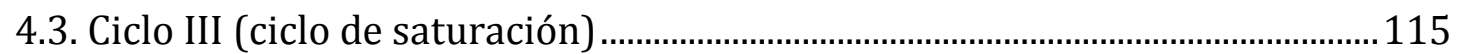

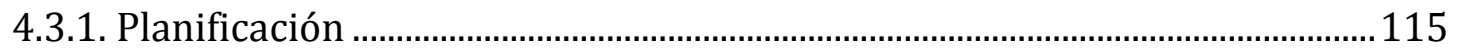

4.3.2. Desarrollo del tercer ciclo (acción) ……………………………………............116

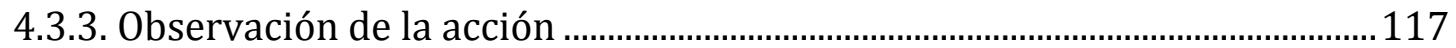

4.3.4. Recogida de datos a través del cuestionario ................................................... 120

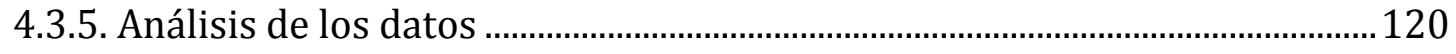

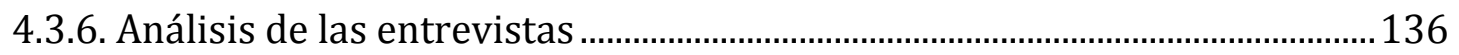

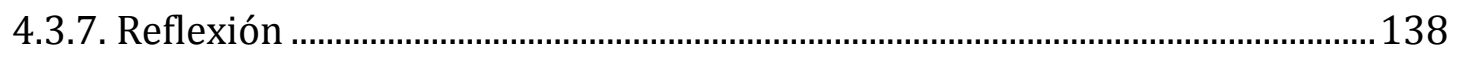

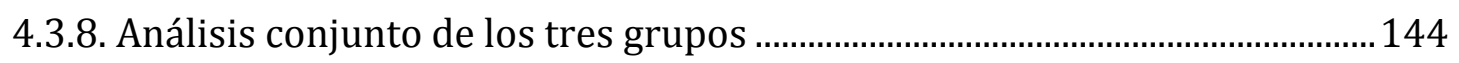

CAPÍTULO 5

ANÁLISIS ESTADÍSTICO DE LOS DATOS DEL TERCER CICLO (2009) ....................147

5.1. Comparaciones de los resultados de la facilidad (F) de cada una de las demostraciones

5.1.1. Comparaciones de los resultados de la facilidad (F) para la primera demostración.

5.1.2. Comparaciones de los resultados de la facilidad (F) para la segunda demostración

5.2. Comparaciones de los resultados del gusto (G) de cada una de las demostraciones

5.2.1. Comparaciones de los resultados de gusto $(G)$ para la primera demostración

5.2.2. Comparaciones de los resultados de gusto (G) para la segunda demostración

5.3. Comparaciones de los resultados de claridad (C) de cada una de las demostraciones

5.3.1. Comparaciones de los resultados de claridad (C) para la primera demostración

5.3.2. Comparaciones de los resultados de claridad (C) para la segunda demostración

5.4. Comparaciones de los resultados de aprendizaje (A) de cada una de las demostraciones 
5.4.1. Comparaciones de los resultados de aprendizaje (A) para la primera demostración

5.4.2. Comparaciones de los resultados de aprendizaje (A) para la segunda demostración .

5.5. Comparaciones de los resultados medios para el conjunto de las demostraciones

5.5.1. Comparaciones de los resultados medios para la primera demostración.

5.5.2. Comparaciones de los resultados medios para la segunda demostración .

CAPÍTULO 6

ANÁLISIS ESTADÍSTICO GLOBAL

6.1. Comparaciones sobre la facilidad (F) de las pruebas .........................................226

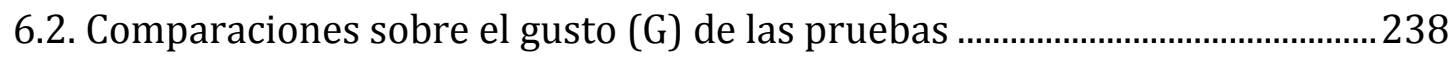

6.3. Comparaciones sobre claridad (C) de las pruebas..............................................249

6.4. Comparaciones sobre el aprendizaje (A) de las pruebas....................................261

6.5. Comparaciones sobre la valoración global de las pruebas ................................ 278

6.6. Reflexiones que se desprenden de este análisis................................................... 287

\section{CAPÍTULO 7}

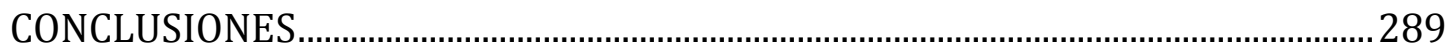

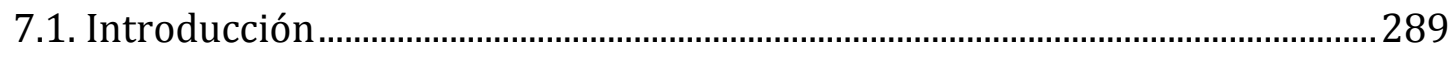

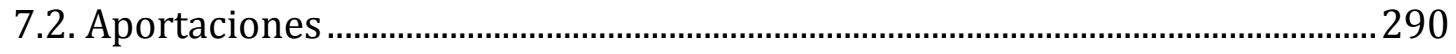

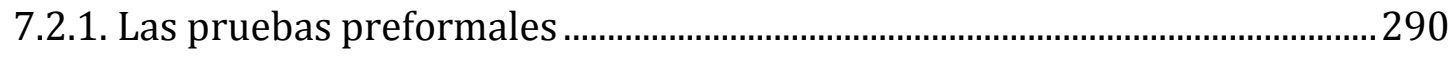

7.2.2. La integración de las metodologías cualitativa y cuantitativa ....................... 291

7.2.3. El protocolo de observación del OE..................................................................292

7.2.4. El cuestionario y protocolo de entrevistas ........................................................ 292

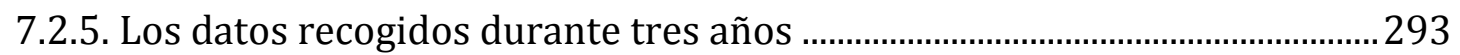

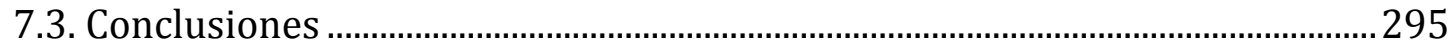

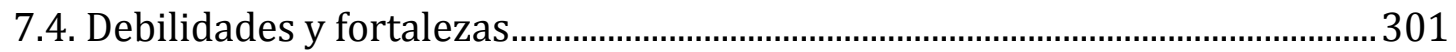

7.4.1. La falta de bibliografía al respecto....................................................................... 301

7.4.2. Las deficiencias matemáticas del alumnado ........................................................ 302

7.4.3. La experimentación. Combinación de metodologías ..........................................304

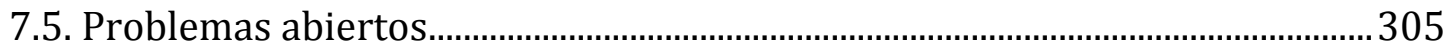




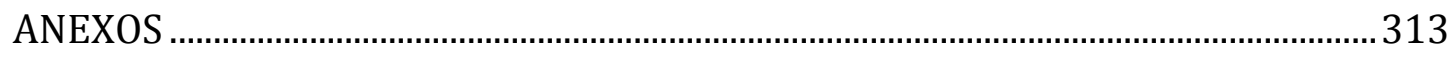

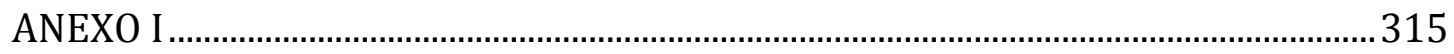

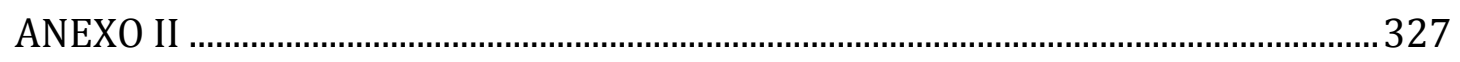

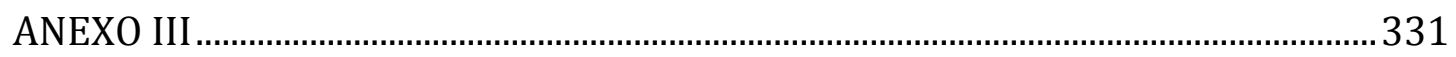

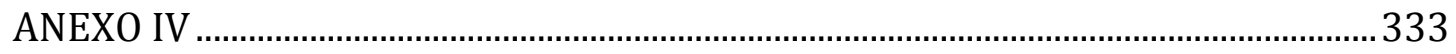

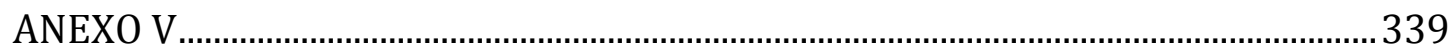

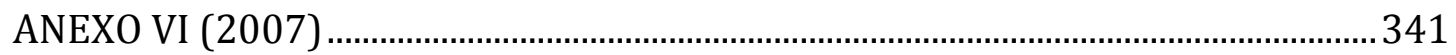

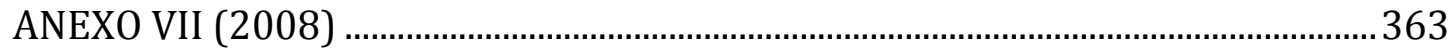

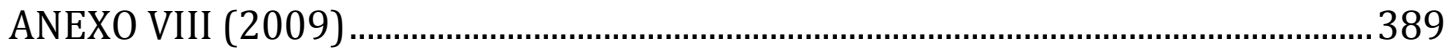




\section{INTRODUCCIÓN}

En los últimos años se están llevando a cabo investigaciones sobre la enseñanza y aprendizaje de las matemáticas realizadas con alumnos en el aula. Estos estudios se centran fundamentalmente en la educación básica (enseñanza infantil, primaria y secundaria) y son menos frecuentes en el ámbito universitario. En concreto no se han realizado investigaciones sobre el aprendizaje de la demostración matemática en los primeros cursos de carreras universitarias.

Nos encontramos hoy con nuevos factores que han cambiado la realidad del alumnado y de los centros docentes universitarios: mayor diversidad y heterogeneidad en los estudiantes, la motivación, la disciplina, el clima del aula. Esto requiere nuevos planteamientos en la acción docente dirigida a todo el alumnado, en la que se contemplen todos los niveles de avance en el aprendizaje, procurando un modo diferente de trabajo que afronte la nueva problemática con el fin de darle la solución más adecuada.

Desde mi experiencia docente en bachillerato y primer curso de carreras universitarias he podido observar que, con la profunda transformación de la sociedad, los alumnos de nuestros centros docentes quieren participar en vivo en su proceso de aprendizaje. Quieren vivir mientras aprenden y aprender mientras viven y rechazan la idea de una forma pasiva de aprender: quieren ser protagonistas de su propio aprendizaje. Se hace necesario emplear una metodología apropiada para detectar cómo aprenden nuestros alumnos y desarrollar una enseñanza adecuada a sus características de aprendizaje. Los profesores somos quienes mejor conocemos a 
los alumnos y, desde ese conocimiento, siguiendo el temario establecido, debemos elaborar un producto didáctico adaptado a su realidad y a su contexto.

Asimismo, en lo que se refiere a la enseñanza-aprendizaje de la demostración matemática he experimentado que los alumnos en la mayoría de los casos no son conscientes de lo que supone haber demostrado un teorema, es decir no captan su verdadero significado, pues no saben extraer consecuencias ni aplicar el resultado cuando procede. Cuando se les pide que expongan una demostración, en muchas ocasiones se limitan a reproducir mecánicamente de forma aislada algunos pasos del razonamiento, ignorando las conexiones lógicas existentes a lo largo del proceso deductivo. Por otra parte, no suelen distinguir la demostración matemática de ejemplificaciones. Ante esta situación, la experiencia nos lleva a rechazar una metodología esencialmente receptiva, en la que el profesor se limita a presentar a los alumnos el producto final.

Para optimizar el aprendizaje del alumno en general y en lo que se refiere a la demostración matemática en particular, y facilitar el trabajo del profesor, éste debe conectar con el alumno, captar su curiosidad, conseguir su interés y motivación, fines que en la tarea educativa se pretenden alcanzar por medio del aprendizaje significativo. Este aprendizaje se apoya en el constructivismo, según la investigación reciente en psicología educativa, iniciado por el psicólogo Lev Semionovich Vygotski (1896-1934). Ausubel, Novak y Hanesian (1983), psicólogos educativos, han desarrollado apoyados en Vygotski, la teoría del aprendizaje significativo. Según dicha teoría, este tipo de aprendizaje es apropiación de conocimiento encajando unas piezas con otras en un todo coherente.

Todos tenemos esta experiencia: al pretender enseñar una palabra o un concepto nuevo a otra persona tratamos de apoyarnos en las palabras o conceptos conocidos por esa persona que sean afines, sinónimos, semejantes, cercanos al que pretendemos comunicarle para conectar lo nuevo con lo que la persona ya sabe, relacionándolo entre sí, haciendo surgir, crear, “construir" en ella el nuevo concepto o significado que le queremos transmitir. 
De modo semejante, y a diferencia del proceso en el aprendizaje por "repetición" en el que los estudiantes memorizan información sin relacionarla con su conocimiento previo anterior o con sus experiencias actuales, en este aprendizaje "significativo" los estudiantes se esfuerzan por conectar el nuevo conocimiento con el que ya poseían y con lo que acontece en su actual entorno de aprendizaje. Se facilita así la expansión del gran potencial humano de aprendizaje, lo que aumenta la autoestima, potencia el enriquecimiento personal, ayuda a pensar y a retener, mantiene alta una motivación interna (más eficaz) por aprender.

Los resultados cognitivos y afectivos del aprendizaje significativo son muy positivos para profesores y alumnos. Para el profesor siempre resultará gratificante saber cómo aprenden sus alumnos y contemplar sus conocimientos previos para poder enlazarlos con los nuevos y obtener así una amplia eficacia en su labor docente.

Hoy día, después de las múltiples pruebas e investigaciones realizadas en esta dirección, no hay duda sobre la eficacia del aprendizaje significativo en orden a conseguir elevados niveles de calidad y positivos resultados en la enseñanza en las aulas.

Un elemento siempre importante del aprendizaje en matemáticas son las demostraciones o pruebas de los enunciados. En esta tesis doctoral vamos a referirnos a las llamadas pruebas "preformales” en su relación con el aprendizaje significativo.

La experiencia y múltiples investigaciones realizadas en el área de Didáctica de la Matemática ponen de relieve que los alumnos tienen grandes dificultades para percibir la necesidad de una demostración, así como para comprender las demostraciones formales, rigurosas. Los problemas que los alumnos tienen con las demostraciones no pueden atribuirse solamente a un lento desarrollo cognitivo o a su limitada capacidad de razonamiento y puede ser interesante buscar las relaciones que pueda haber entre las dificultades de comprensión de las demostraciones y el grado de significación, las funciones y la utilidad que tienen las demostraciones para ellos. 
Creemos que las pruebas preformales, que constituyen el núcleo de esta tesis, apuntan en la dirección adecuada que permite amortiguar o incluso resolver estas dificultades. Nuestro objetivo es establecer si este tipo de pruebas resultan más cercanas, más comprensibles y, por tanto, más significativas para los alumnos.

La larga experiencia docente del Dr. Ortega coincide con estas apreciaciones y, además, su experiencia como investigador en este campo avala lo anterior, y todo ello impulsa a continuar en esta línea de investigación.

Vamos a fijar nuestra atención, dentro del marco de la demostración matemática, en la prueba preformal y sus características. Recordaremos la noción y elementos del aprendizaje significativo. Expondremos la metodología investigación-acción como instrumento de ayuda al profesor de matemáticas para investigar los conocimientos de sus alumnos y facilitar su labor docente.

Hacia los años setenta del siglo pasado comienza a cambiar la habitual concepción de demostración formal en matemáticas. La demostración pretende ser más activa, social, práctica, conectada con la realidad. Las pruebas preformales en las funciones y características propias de la demostración se presentan como más favorables a esta línea de significación. Esta investigación se presenta en la memoria de tesis, que se estructura en siete capítulos bien diferenciados, amén de la introducción. A continuación se hace una breve descripción de los contenidos de los mismos.

En el capítulo 1 se habla del aprendizaje significativo y se introduce el concepto de prueba preformal. El aprendizaje significativo no es memorizar conocimientos, sino comprenderlos y "encajarlos" en la estructura mental y bagaje de los conocimientos ya adquiridos por el sujeto aprendiz. Esta integración en la estructura cognitiva personal requiere intencionalidad, actividad (el discípulo no es ni acepta ser pasivo) y consonancia entre la información que adviene y los esquemas mentales adquiridos.

En el capítulo 2 se abordan diversas metodologías, distinguiendo la perspectiva positivista, tradicional, más propia de las ciencias naturales, análisis estadísticos, 
metodología cuantitativa, y la fenomenológica, más propia de las ciencias sociales, que usa más la metodología cualitativa y se fija en el entorno y situación de las personas. En esta perspectiva se describe la metodología investigación-acción, que une teoría y práctica; sus ciclos, las etapas de éstos, sus rasgos característicos vinculados a la "acción", la "investigación”... Asimismo, se describe la investigación de diseño y se expone una breve evolución de su aplicación en el ámbito de Didáctica de la Matemática. Se contrasta con la investigación psicológica aportando una tabla muy clarificadora de sus diferencias. Esta evolución tiene su origen en la búsqueda del por qué se cree algo y se fija en la estructura que resulta del proceso, al aprender, entre el conocedor, el ambiente y la actividad de quienes participan en ese proceso, frente al interés por la búsqueda de resultados (la rapidez, el rendimiento...) del aprendizaje. El capítulo termina con una breve descripción de las características de las metodologías cuantitativa y cualitativa, inclinándose por la conjunción de ambas (así se hace en este trabajo) que se complementan y corrigen los posibles riesgos y limitaciones de una y otra.

En el capítulo 3 se relata el origen de esta investigación: el curso de doctorado impartido por el Dr. Ortega, La demostración en Matemáticas, y el trabajo tutelado bajo su dirección, Contribución de las pruebas preformales al aprendizaje significativo (septiembre de 2005). Se hace referencia a la experiencia previa en el aula con alumnos de la asignatura Cálculo del curso 2005-2006, mediante presentación y contacto con pruebas preformales. A partir de los resultados de esta actividad se señalaron las hipótesis para el trabajo posterior: aunque complementarias (pruebas formales y preformales), los alumnos prefieren las preformales por su facilidad, sus características, aplicabilidad, grado de influencia, se aprenden mejor y su aprendizaje es más significativo.

El capítulo 4 describe con detalle el proceso de tres ciclos de investigación-acción con sus fases, desarrollados en esta investigación: planificación, acción, recogida de datos, análisis y reflexión sobre los mismos. Los alumnos responden a un cuestionario manifestando cómo han entendido los teoremas (del valor medio de Lagrange y de la unicidad del límite) con cada prueba y valorando categorías y significa- 
ción de las mismas. Se hacen dos entrevistas en cada ciclo. El esquema se repite en los tres, refinándose con las aportaciones del anterior. Los datos se recogen en tablas y gráficos.

En el capítulo 5 se realiza el análisis estadístico de las respuestas de los alumnos del tercer ciclo en relación con las categorías de las pruebas. En cada una de las categorías investigadas (Facilidad-Gusto-Claridad-Aprendizaje), se comparan las respuestas aportadas por los alumnos en relación con uno y otro teorema, $1^{\underline{a}}$ y $2^{\underline{a}}$ demostración, analizando estadísticamente correlaciones, comparación de muestras, de medias, de desviaciones típicas, contrastes, terminando con el análisis también de las comparaciones de los resultados medios para el conjunto de las demostraciones.

En el capítulo 6 se compara y analiza estadísticamente el conjunto de todas las respuestas de los alumnos de los tres ciclos sobre las categorías de las dos pruebas en cada uno de los dos teoremas. Se presentan los resultados que se obtienen con varios test estadísticos: comparación de medias, de varianzas y KolmogorovSmirnov.

Finalmente, en el capítulo 7 resumimos las aportaciones que se han producido en el desarrollo de todo el proceso de investigación. Asimismo, presentamos y enumeramos las conclusiones del mismo. Señalamos también sus fortalezas y algunas de sus limitaciones. Sugerimos posibles cuestiones abiertas o perspectivas de posteriores investigaciones. Hacemos referencia a una propuesta de desarrollo curricular sobre las demostraciones, citando el ANEXO I, en el que se incluyen diversas pruebas preformales de teoremas propios del Análisis Matemático.

La estructura general de la memoria de investigación se presenta en el gráfico de la figura 1 con el fin de apreciar la globalidad de la investigación desarrollada desde el inicio de la memoria. 


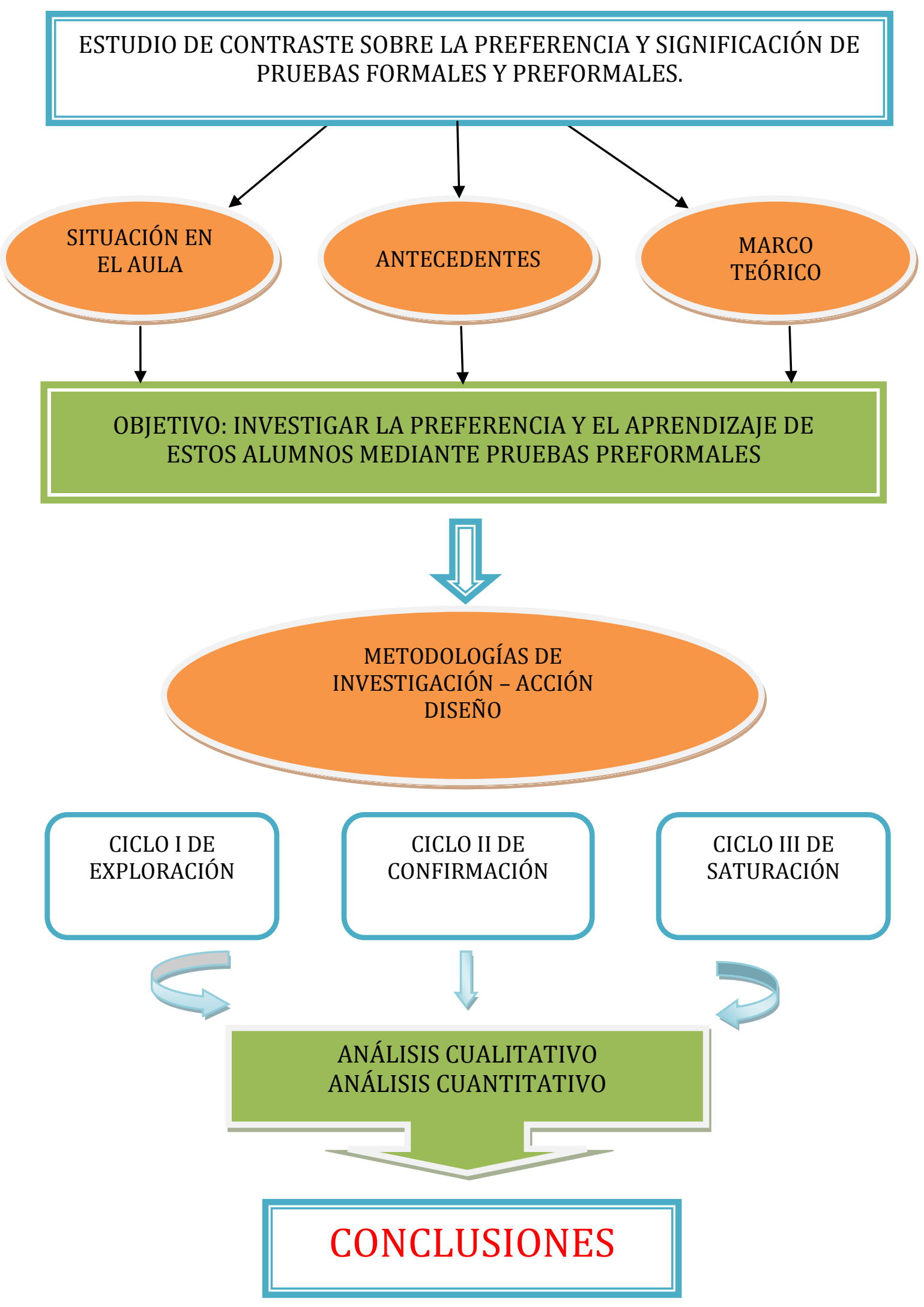

Figura 1. Esquema de la investigación 



\section{CAPÍTULO 1}

\section{ANTECEDENTES Y MARCO TEÓRICO}

En este capítulo se describe el "aprendizaje significativo" y se presenta la noción de "prueba preformal". Tiene asociado el ANEXO I en el que se presentan diversas pruebas preformales.

Sobre la importancia del aprendizaje significativo, Ausubel afirma que

“...es el mecanismo humano por excelencia para adquirir y almacenar la vasta cantidad de ideas e información representadas por cualquier campo de conocimiento" (Ausubel, 1976, p. 78).

El aprendizaje significativo es el aprendizaje que conduce a la generalización de lo aprendido. Para aclarar esta afirmación, fijémonos brevemente en los conceptos de aprendizaje y de transferencia.

Según Mayer (2004, p. 3), el aprendizaje es "un cambio relativamente estable en el conocimiento de alguien como consecuencia de la experiencia de esa persona". Debe ser permanente, es decir, a largo plazo (un cambio que desaparece al cabo de un breve tiempo no refleja un aprendizaje). Debe operarse un cambio cognitivo que se refleja en un cambio de conducta (como de no saber a sí saber algo). Si no hay tal cambio no hay aprendizaje. Finalmente debe estar basado sobre la experiencia del aprendiz. Más aún, no depende sólo de lo que haya hecho el aprendiz sino de cómo 
ha interpretado lo ocurrido, es decir, depende de la experiencia personal del aprendiz.

Es decir, el aprendizaje es un cambio en el conocimiento debido a la experiencia. Esta perspectiva nos parece especialmente sensible a la temática de cómo promover el aprendizaje significativo, al centrarse en el aprendizaje como búsqueda de comprensión. Entender lo que se aprende capacita mucho mejor para transferir el aprendizaje a nuevas situaciones.

La transferencia "es el efecto del aprendizaje previo (A) sobre uno nuevo o sobre la resolución de un problema (B)" (ibid. p. 5). Será positiva, negativa o neutra cuando el conocimiento previo (A) facilita, perjudica o no influye para nada sobre un nuevo aprendizaje o resolución de problemas (B), respectivamente.

La transferencia puede ser específica, general o mixta. Es específica cuando los contenidos de (A) son idénticos a los que aparecen en (B). Es general cuando, aunque los contenidos no sean idénticos, el aprendizaje de (A) mejora la propia capacidad para enfrentarse al nuevo problema (B). Finalmente, la transferencia mixta se refiere a la transferencia específica de un principio o estrategia general; se produce cuando un principio o estrategia general aprendida en (A) también se puede utilizar para resolver el problema (B).

Edward Lee Thorndike (1874-1949), reconocido como el primer psicólogo educativo, y sus colegas contrastaron a principios del siglo XX la eficacia de la transferencia general, llegando a rechazar la doctrina de la disciplina formal (con el énfasis en la transferencia general) y sustituyéndola por su teoría de la transferencia por elementos idénticos (con el énfasis sobre la transferencia específica). La importante tarea investigadora desarrollada por Thorndike ha supuesto un considerable descrédito para la transferencia general.

Existe una importante alternativa a las dos anteriores teorías de la transferencia: la transferencia específica de principios o estrategias generales. De acuerdo con esta teoría, es posible aprender ciertos principios o estrategias generales especialmente 
aplicables a nuevas tareas. Esta idea forma parte de las modernas teorías de transferencia y de que la transferencia se produce cuando los estudiantes llevan a cabo una "abstracción consciente", es decir, reflexionan sobre el principio general que han aprendido.

Los psicólogos de la Gestalt (Katona, 1940; Wertheimer, 1959) compararon dos métodos de enseñanza: el aprendizaje por memorización (basado en la transferencia específica de conductas específicas) y el aprendizaje por comprensión (basado en la transferencia específica de principios generales). Las investigaciones de Judd (1908) y Katona (1940) demostraron que el aprendizaje por comprensión produce la transferencia, mientras que el aprendizaje por memorización no la produce.

Volviendo a Ausubel, el aprendizaje significativo es un proceso de "relación" del contenido o material a aprender con el bagaje o almacenamiento de conocimientos ya poseído por el alumno que aprende y la estructura mental de ese bagaje. Dicha "relación” se realiza de modo "sustancial" (Ausubel, 1976), no mecánico, arbitrario, al pie de la letra. Presupone:

- Una disposición para relacionar sustancialmente, para encajar el contenido nuevo con la estructura cognitiva ya existente, y

- Que este nuevo material tenga capacidad de ser "relacionado", de encajarse, que sea potencialmente significativo para la estructura de conocimiento del alumno; lo cual depende tanto de la naturaleza del saber o contenido a aprender (aprehender) como de la estructura cognitiva de la persona concreta que lo va a adquirir. El resultado, pues, variará según los antecedentes educativos, la edad, los diversos factores afectivos y socioculturales de esa persona.

Las dos características específicas, intencionalidad y sustancialidad de este aprendizaje son enormemente eficaces como procesamiento de información (para entender, fijar, incorporar y emplear un gran volumen de nuevas ideas) y como me- 
canismo de almacenamiento de toda esa información (que supera muy ampliamente el periodo de mero recuerdo mecánico).

Ausubel distingue dos modelos de enseñanza-aprendizaje:

- Por recepción, si el contenido a integrar, a aprender, se presenta al alumno explícitamente en su forma final, definitiva; el contenido es "recibido".

- Por descubrimiento, si ese contenido lo va descubriendo el alumno por sí mismo generando soluciones a problemas planteados o pasos sucesivos para resolverlos; aquí el contenido es "descubierto". Este segundo modelo puede ser guiado, libre, programado, etc.

Ambos modelos serán significativos si el alumno, por su voluntad, integra los contenidos en su personal estructura cognitiva acomodándolos a sus saberes anteriores.

Fischbein (1982) afirma que todo maestro conoce que el discípulo no es un pasivo receptor de información y de soluciones de trámite. El concepto de Piaget de asimilación es esencial para la descripción y la explicación del proceso de aprendizaje en relación con el desarrollo. Aprendemos, entendemos y usamos información procesándola e integrándola en consonancia con nuestros propios esquemas mentales jerárquicamente organizados. Una prueba no está basada en una multitud de muchos resultados prácticos muy bien controlados. La validez del teorema se garantiza únicamente por la validez de la inferencia formal lógica utilizada. La totalidad de la verdad expresada por el teorema está garantizada por la validez universal de las reglas lógicas utilizadas por la prueba (Fischbein, 1982).

En el aprendizaje de las matemáticas ocupa un lugar importante la demostración. Entre las investigaciones que resaltan esta importancia destaca la realizada por Hanna (1989), quien, partiendo de que la demostración "formal" ha sido la característica más importante de las matemáticas modernas de la primera mitad del siglo XX, de acuerdo con el logicismo, formalismo e intuicionismo, afirma que estas creencias se discuten a partir de los años setenta con Lakatos, Davis, Kitcher 
y otros, que conciben la demostración no como puro formalismo, sino como proceso social, práctica.

Distingue Hanna diferentes percepciones de demostración:

- Demostración formal: La demostración como un concepto teórico de la lógica (o metalógica) formal, completamente rigurosa, a la que solamente se aproxima la actual práctica matemática.

- Demostración aceptable: La demostración como un concepto normativo que define lo que es aceptable para los matemáticos cualificados. Influyen procesos sociales. Los matemáticos valoran más una demostración que tenga implicaciones interesantes para la rama de las matemáticas en la que está inmersa y proporcione intuiciones hacia campos relacionados. Una demostración es valorada cuando saca a relucir relaciones matemáticas esenciales más que cuando simplemente demuestra la exactitud de un resultado.

- La enseñanza de la demostración: La demostración como una actividad en la educación matemática que sirve para aclarar ideas que merece la pena transmitir al estudiante. Los educadores conceden mayor énfasis al concepto de demostración como "argumento convincente". Deben presentarse a los estudiantes, siempre que sea posible, demostraciones que expliquen en lugar de aquellas que solamente prueben, demostraciones que expliquen, que muestren el "por qué" es cierto un teorema (rationes essendi) en lugar de las que solamente prueban "que" un teorema es cierto (rationes cognoscendi). El objetivo primordial es que los estudiantes entiendan las matemáticas. La comprensión es mucho más que la confirmación de que los enlaces en una cadena de deducciones sean correctos. Por ello son las demostraciones explicativas las que representan un papel decisivo en la comprensión matemática.

Analiza Hanna los desafíos actuales a la demostración: el ordenador, la influencia de valores sociales, teorías que fomentan la pasividad del profesor en el aula o suscitan la idea de la verdad infalible de las matemáticas o las matemáticas como cien- 
cia "a priori", y señala las funciones de la demostración: en matemáticas, verificación (el fin considerado tradicionalmente), ayuda a comprender el significado del teorema; y en la educación matemática, principalmente la explicación.

Bell (1976) señala tres significados de la demostración matemática: verificación o justificación respecto a la verdad de una proposición (verdad); iluminación, lo que se espera de una buena prueba es que transmita una perspectiva de por qué es verdadera la proposición (por qué), esto no afecta a la validez de una prueba, pero su presencia en una prueba es estéticamente complementario; y sistematización, el significado matemático más característico, i. e. la organización de resultados dentro de un sistema deductivo de axiomas en un todo coherente.

Balacheff (1987) muestra la necesidad de una aproximación a la vez situacional y cognitiva, especialmente en referencia al funcionamiento de los conocimientos en el aprendizaje de las matemáticas. Distingue diferentes fases en la evolución de los alumnos desde pruebas pragmáticas a pruebas intelectuales y demostraciones.

De Villiers (1993) critica duramente la posición tradicional y distingue las siguientes funciones de la demostración, que comenta detalladamente: verificación, explicación, sistematización, descubrimiento (nuevos resultados) y comunicación (transmisión del conocimiento matemático). Insiste en que la convicción no se obtiene únicamente mediante la demostración y resalta el valor de la función de "explicación".

Nos fijamos en van Asch (1993), quien (después de considerar argumentos a favor y en contra de presentar u omitir la demostración para justificar un teorema) distingue tres maneras diferentes por su grado de abstracción para justificar un teorema: prueba formal (las "rigurosas" de los textos universitarios), prueba preformal y prueba por medio de un dibujo o un ejemplo (pruebas sin palabras). El término prueba preformal (término original de Semadeni, 1984) se define así:

"By a preformal proof we understand a line of reasoning which can be formalized to a formal proof, but in which the essential idea is already present" (van Asch, 1993, p. $310)$. 
Sus características: se adaptan mejor a la capacidad intelectual de los estudiantes, son argumentos matemáticos correctos, no es una verificación experimental de una proposición, no están basadas íntegramente en argumentos visuales, a menudo suponen acciones concretas, no es una simple comprobación en casos particulares.

Concluye advirtiendo que los matemáticos deberían estar dispuestos a demostrar teoremas de forma menos abstracta a la que ellos probablemente preferirían. Una demostración debe presentarse de tal manera que contribuya realmente a la enseñanza.

Blum y Kirsch (1991) distinguen también tres niveles de demostración: pruebas, pruebas preformales y pruebas formales. Definen las preformales:

"a chain of correct, but not formally represented conclusions which refer to valid, non-formal premises".

Consideran varias clases de estas pruebas: pruebas acción (acciones concretas que corresponden a argumentos correctos); intuitivo-geométricas (concepciones geométricas básicas y hechos intuitivamente evidentes); orientadas por la realidad (ideas de significado claro y muy accesible).

Ortega (2004), en la línea de Hanna (en la aceptación de un teorema es más importante su significado global que una demostración rigurosa) va más allá al afirmar que en los teoremas los procesos constructivos son muchísimo más importantes que las demostraciones y que es la interpretación de los mismos al conexionarlos fuertemente con la realidad lo que les da verdadero significado.

Otros han investigado sobre las funciones de la demostración: Renz (1981), Hersh (1993), Thurston (1995), Reid (1996), Hanna y Jahnke (1996), Harel y Sowder (1998), etc. 
Ibañes y Ortega (2005), por fin, al tratar de su dimensión histórica hacen estas reflexiones muy interesantes, mesuradas e ilustrativas sobre la demostración matemática:

- Las exigencias de rigor en la argumentación matemática han ido variando a lo largo de la historia debido a los distintos conocimientos, necesidades y sensibilidades.

- Aun en las épocas en que se ha trabajado con mayor rigor no se ha podido conseguir una certeza total en los resultados obtenidos. El rigor absoluto es inalcanzable.

- No existe un modelo de demostración independiente de la época ni de las personas que han construido el conocimiento matemático. Distintas argumentaciones, comprobaciones, justificaciones o pruebas se han utilizado para verificar o explicar los teoremas.

- No debemos pensar que la idea actual de demostración será la última palabra. Siempre es posible introducir alguna mejora, proponer otro enfoque, o, incluso que surjan dificultades inesperadas.

- No ha habido siempre un total acuerdo entre los matemáticos en cuanto a la conveniencia de los conceptos y procedimientos utilizados, produciéndose a veces fuertes y prolongadas controversias.

- Resulta enriquecedora la búsqueda de distintas fundamentaciones para una teoría, o diferentes explicaciones para un resultado, o distintos estilos y métodos para una demostración.

- La evolución del lenguaje y el simbolismo han tenido mucho que ver con el estilo y el modo de las demostraciones, y su adaptación ha resultado esencial para el desarrollo de las matemáticas y, en particular, para el perfeccionamiento de las demostraciones. 


\section{CAPÍTULO 2}

\section{METODOLOGíA}

Se analiza la metodología cuantitativa: datos, estadística, validez de resultados, y la metodología cualitativa: investigación de diseño y su evolución hacia experimentos de enseñanza y aprendizaje de las matemáticas. Se presenta la metodología de investigación-acción: sus ciclos y etapas de los mismos.

\subsection{Introducción}

Fijar una metodología de investigación adecuada es crucial en el desarrollo de la misma. En nuestro caso, por las características específicas del tema tratado consideramos que es más apropiado utilizar una metodología de integración cualitativacuantitativa, ya que ambas aportan apreciaciones diferentes: datos estadísticos de los hechos, validaciones de los alumnos... y atención a la situación subjetiva de los protagonistas de toda la experiencia.

La metodología cualitativa, a su vez, integra dos marcos metodológicos: la investigación-acción y la metodología de diseño. En la primera integramos docencia, praxis e investigación en una acción planificada y observada a través de un proceso de ciclos en espiral unidos por la reflexión del precedente con la planificación del siguiente; y en la segunda, situamos la experiencia en la vida real del contexto del aula dando importante protagonismo a todos sus agentes, con cuyas aportaciones, 
analizadas y reflexionadas, se irá refinando y enriqueciendo el propio proceso y la hipótesis de partida.

Por otra parte la metodología cuantitativa aporta la validez de las pruebas estadísticas y, en definitiva, puede establecer la validez de los resultados.

\subsection{Investigación-acción}

La metodología de trabajo elegida ha sido investigación-acción, enmarcada en la metodología cualitativa, de las dos grandes perspectivas teóricas prevalentes en la investigación en ciencias sociales: positivismo, tradicional, que busca las causas de los hechos sociales sin atender la situación de los sujetos; y fenomenología, que ve en la subjetiva situación del individuo, y en especial en las ciencias del aprendizaje, una fuente de conocimiento, objeto de la misma ciencia y en consecuencia presupuesto metodológico.

El método cualitativo, según Pérez Serrano (1994, p. 18) se inserta dentro de la corriente teórica fenomenológica en las ciencias sociales, cuyas características principales enumeramos: a) la teoría constituye una reflexión en y desde la praxis; b) intenta comprender la realidad; c) describe el hecho en el que se desarrolla el acontecimiento; d) profundiza en los diferentes motivos de los hechos; e) el individuo es un sujeto interactivo, comunicativo, que comparte significados. Distingue así Pérez Serrano estas dos perspectivas o modelos de investigación: el positivista toma el modelo del método cuantitativo propio de las ciencias naturales y busca las causas mediante análisis estadísticos; el fenomenológico busca comprender la realidad mediante métodos cualitativos que proporcionan mayor comprensión personal de motivos y creencias que influyen en las acciones de las personas (citado por García, 2008, p. 80). La práctica educativa, afirma Pérez Serrano, posee una lógica muy distinta de la racional y científica postulada por la investigación positivista, y unos contenidos que no se reducen a habilidades para la gestión eficaz de la enseñanza. Estos dos métodos, cuantitativo y cualitativo, no son contrapuestos o excluyentes. 
Dentro del modelo cualitativo, el psicólogo norteamericano Kurt Lewin inició, a mediados del siglo XX, la metodología investigación-acción, aplicándola al estudio de las relaciones humanas. Lewin define la investigación-acción como un proceso de peldaños en espiral, cada uno de los cuales se compone de planificación, acción y evaluación del resultado de la acción; describe los aspectos más relevantes de esta línea de investigación: planificación de la acción, recogida de datos, análisis, reflexión y carácter cíclico del proceso.

A partir de entonces el método ha recibido aportaciones de otros investigadores, y se ha aplicado a otros ámbitos, en concreto al de la educación. Kemmis y McTaggart (1988) lo definen así:

"La investigación-acción es una forma de indagación introspectiva colectiva, emprendida por participantes en situaciones sociales con objeto de mejorar la racionalidad y la justicia de sus prácticas sociales y educativas, así como la comprensión de esas prácticas y de las situaciones en que éstas tienen lugar" (p. 9).

Y, resaltando la unión de ambos términos, afirman: “La vinculación de los términos 'acción' e 'investigación' pone de relieve el rasgo esencial del enfoque: el sometimiento a la prueba de la práctica de las ideas como medio de mejorar y de lograr un aumento del conocimiento acerca de los planes de estudio, la enseñanza y el aprendizaje. El resultado es una mejora de aquello que ocurre en la clase y la escuela, y una articulación y justificación mejores de la argumentación educacional aplicada a aquello que sucede. La investigación-acción proporciona un medio para trabajar que vincula la teoría y la práctica en un todo único: ideas en acción".

El proceso comienza, en la práctica, con la idea de mejorar o cambiar un hecho, una preocupación temática que se establece con las cuatro etapas fundamentales del método que deben entrar en juego dinámicamente con esa preocupación:

a) Planificación: elaboración de un plan que anticipa y prevé la actividad y las formas de observación, con flexibilidad para poder adaptarse a situaciones imprevistas. 
b) Acción diseñada por el plan: guiada y controlada por la planificación, decidida de forma que permita a los profesionales actuar eficazmente y les convierta en educadores más efectivos; al desarrollarse en una realidad viva, dinámica y sujeta a limitaciones, debe estar abierta a cambios posibles, a limitaciones reales de la situación, dispuesta a la negociación y al compromiso; la acción debe ser examinada en su contexto para ser evaluada posteriormente; se trata de una acción observada.

c) Observación: recogida de datos durante la acción, que la documentan y proporcionan la base para la reflexión; observación abierta y flexible para registrar lo inesperado; los autores recomiendan registrar siempre en un diario observaciones adicionales que encajan en las planificadas; los investigadores deben observar el proceso, sus efectos, sus circunstancias, sus limitaciones para obtener una base fiable para la introspección crítica.

d) Reflexión sobre lo observado: hallar el sentido de los procesos, los problemas y las restricciones que se han manifestado; esta etapa tiene un aspecto valorativo y otro aspecto descriptivo a través de un retrato más vigoroso de la vida y el trabajo en la situación dada y de las limitaciones de la acción. La reflexión sirve de base para una nueva planificación, con lo que se pone en marcha otro ciclo.

Lo mostramos en el siguiente esquema:

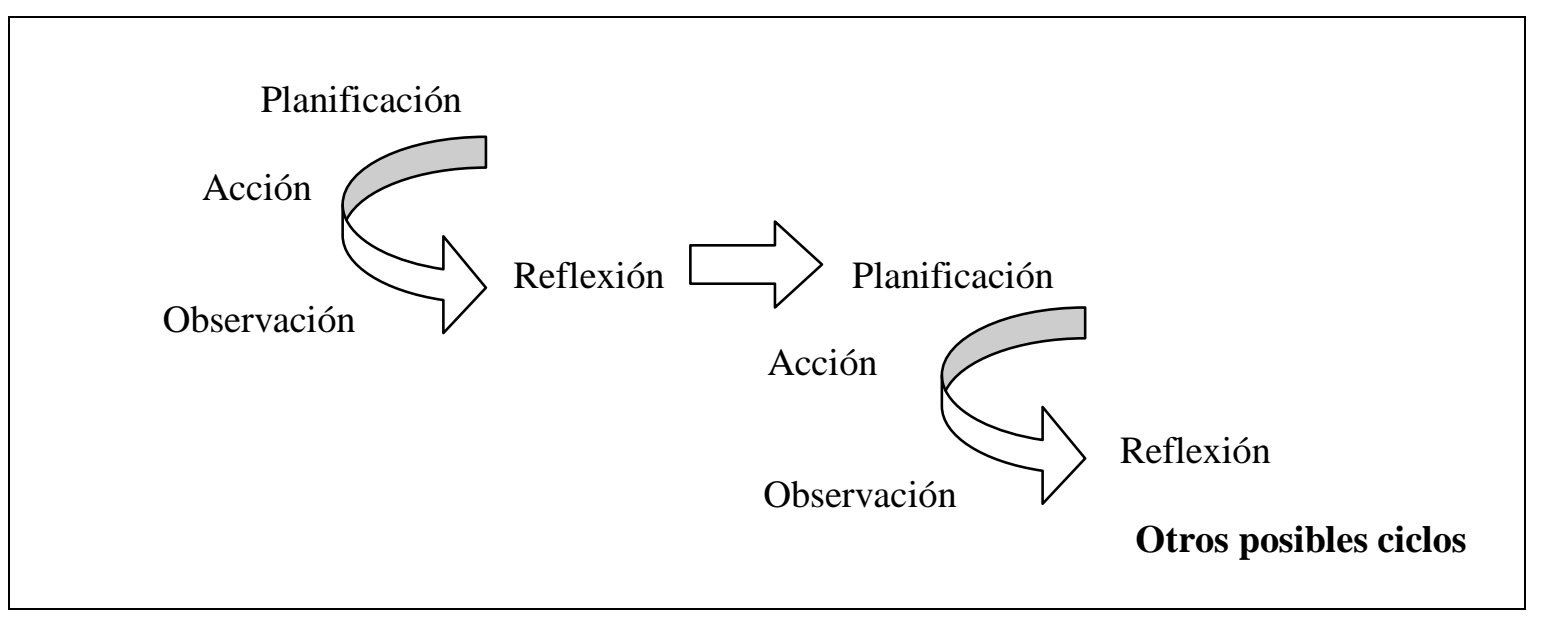

\subsection{Esquema de ciclo de investigación-acción}


Todos estos pasos no son momentos estáticos, sino pasos en espiral: tomar una decisión sobre una cuestión o preocupación temática lleva consigo un juicio acerca de cómo podría mejorarse. La planificación que en consecuencia se decide, puede detectar cambios en el decurso del proceso, a medida que se va precisando el problema y la estrategia planificada va dando frutos. Las conclusiones finales estarán precedidas de varias reflexiones que inciden en ellas.

Los autores consideran que la investigación-acción no es el quehacer normal del profesor (proceso de reflexión); ni la resolución de un problema concreto; ni investigación acerca de otras personas; ni un método científico aplicado a la enseñanza. Pérez Serrano (1994, pp. 158-173) describe unos rasgos que la caracterizan, así se resume en Ibañes y Ortega (2005, pp. 30-32):

1. Rasgos vinculados a la acción.

- Unión de teoría y praxis. La teoría orienta la práctica y ésta supone una reflexión para la elaboración de teorías. En el ámbito de la educación, supone incorporar la investigación educativa a la práctica escolar.

- Orientada a la mejora de la acción. Desde sus inicios, la investigación-acción ha tratado de actuar sobre el medio para mejorarlo. En el plano educativo se orienta a mejorar la acción, contribuyendo a la resolución de problemas con una visión dinámica de la realidad.

- Parte de problemas prácticos. El objeto de estudio son los problemas prácticos tal y como acontecen en su propio contexto. En consecuencia, los problemas y preocupaciones concretas de los profesores pueden ser un tema de investigación.

- Protagonismo del práctico. Los profesores se convierten en protagonistas de sus propias investigaciones y la investigación-acción implica activamente a los docentes. 
2. Rasgos vinculados a la investigación.

- Un nuevo tipo de investigador. La investigación-acción reconoce la posibilidad de construir el conocimiento científico con los implicados en la acción social y educativa, creando una figura de investigador -por ejemplo, la del profesor- más próxima a los problemas cotidianos.

- Investigación amplia y flexible. El diseño no se moldea a priori, sino que se va negociando con los que están implicados en la acción educativa. La flexibilidad se manifiesta también en la posibilidad de volver las veces que sea preciso sobre los datos, así como reinterpretar y contrastar con nuevas fuentes.

- Perspectiva ecológica. Estudia los problemas en su propio contexto, dentro de unidades locales.

- Interés por la clarificación de los valores y las características del profesor. Al ser un proceso autorreflexivo y crítico, hace salir a la luz de la reflexión las creencias y valores que actúan de motor en el profesor.

- Rigor metodológico. Pretende un rigor metodológico, aunque tiene una amplia visión del control ya que no sacrifica su relevancia al rigor del método.

3. Rasgos vinculados al cambio de actitudes.

- Dimensión de colaboración. Es necesario contar con un grupo de personas para llevar a cabo una tarea de transformación, de cambio y de mejora, por lo que la investigación-acción es colaboradora y participativa.

- Democratización del proceso de la investigación. En el campo educativo esto implica la igualdad del docente frente al investigador y la consiguiente coparticipación.

- Función crítica. La investigación-acción se orienta hacia la creación de comunidades autocríticas de personas que se implican en el proceso de transformación en todo el período de investigación. 
- Función de comunicación. La investigación-acción hace énfasis en los procesos de comunicación que se generan en los grupos. Por otra parte el lenguaje empleado suele ser sencillo, claro y asequible.

- Acción como cambio social. Se pretende, a través de la acción, una adaptación o transformación social, que no tiene por qué ser muy amplia, sino que puede limitarse a mejorar una situación concreta.

- Finalidad de formación. La investigación-acción tiene también una dimensión educativa o formativa, en virtud de la cual se potencia el desarrollo personal y la formación profesional de las personas implicadas.

La misma autora, Pérez Serrano, señala las características que debe cumplir un trabajo para que se pueda considerar que sigue este método de investigación:

1. Que el proyecto surja de problemas y preocupaciones educativas de carácter práctico que sientan como propios los educadores.

2. Que el proyecto implique a todos los responsables del mismo, formando un equipo preocupado por las cuestiones prácticas vinculadas con la acción que se desea mejorar.

3. Que se eviten estas formas de actuación: depender exclusivamente de pruebas, dirigirse estrictamente a la determinación del logro, ignorar las perspectivas de otros profesores, servir a quienes toman las decisiones, o romper el ciclo necesario de acción y reflexión.

4. Que se mantenga la redacción de un diario del proyecto en el que se registren las reflexiones críticas relativas al tema a investigar.

5. Que el grupo siga una espiral de ciclos acción-reflexión.

6. Es preciso: partir de abajo arriba; integrar docencia, praxis e investigación; relacionar la investigación documental y la formación investigadora; y aplicar los resultados a la práctica educativa. 


\subsection{Investigación de diseño, experimentos de enseñanza}

La investigación de diseño es una metodología muy presente hoy en el ámbito de la Didáctica de la Matemática y las Ciencias. Brown (1992) y Collins (1992) fueron los primeros en emplear este término que otros denominan estudios de diseño o experimentos de diseño o investigación basada en diseño. Se trata de un intento de elaborar nuevas teorías, artificios y prácticas que expliquen e impacten el aprendizaje y enseñanza de ámbitos naturalistas; vinculan dos cosas: ingeniería de formas particulares de aprendizaje y estudio de esas formas desde el contexto definidas por los medios que las apoyan. Este contexto está sometido a prueba, revisión y sucesivas iteraciones, papel similar al de variación sistemática de experimento. Collins y otros proponen estas siete diferencias entre métodos tradicionales psicológicos y esta metodología: (citado por Barab y Squire, 2004, p. 4):

\begin{tabular}{|c|c|c|}
\hline Categoría & $\begin{array}{l}\text { Experimentación } \\
\text { psicológica }\end{array}$ & Investigación basada en diseño \\
\hline Lugar & Escenarios de laboratorio & $\begin{array}{l}\text { En la ruidosa y floreciente confusión } \\
\text { de la vida real es donde acontece la } \\
\text { mayor parte del aprendizaje }\end{array}$ \\
\hline $\begin{array}{l}\text { Complejidad de va- } \\
\text { riables }\end{array}$ & $\begin{array}{l}\text { Una o dos variables de- } \\
\text { pendientes }\end{array}$ & $\begin{array}{l}\text { Implica múltiples variables depen- } \\
\text { dientes incluyendo de ambiente, de } \\
\text { resultado y de sistema }\end{array}$ \\
\hline $\begin{array}{l}\text { Foco de investiga- } \\
\text { ción }\end{array}$ & $\begin{array}{l}\text { Identificación y control de } \\
\text { unas pocas variables }\end{array}$ & $\begin{array}{c}\text { Caracterizar la situación en su comple- } \\
\text { jidad, que no lo está a priori en gran } \\
\text { parte }\end{array}$ \\
\hline $\begin{array}{l}\text { Despliegue de pro- } \\
\text { cedimientos }\end{array}$ & Procedimientos fijados & $\begin{array}{l}\text { Revisión flexible del conjunto inicial } \\
\text { dependiendo de su éxito en la práctica }\end{array}$ \\
\hline $\begin{array}{l}\text { Carga de interac- } \\
\text { ción social }\end{array}$ & $\begin{array}{l}\text { Aprendices aislados para } \\
\text { control de interacción }\end{array}$ & $\begin{array}{l}\text { Complejas interacciones sociales, } \\
\text { compartir ideas, confrontaciones de } \\
\text { unos con otros }\end{array}$ \\
\hline $\begin{array}{l}\text { Caracterización de } \\
\text { hallazgos }\end{array}$ & Sobre prueba de hipótesis & $\begin{array}{l}\text { Múltiples aspectos y desarrollo de per- } \\
\text { fil que caracterice el diseño en la } \\
\text { práctica }\end{array}$ \\
\hline $\begin{array}{l}\text { Papel de los parti- } \\
\text { cipantes }\end{array}$ & $\begin{array}{l}\text { Los participantes como su- } \\
\text { jetos }\end{array}$ & $\begin{array}{c}\text { Participantes diferentes en el diseño, } \\
\text { en aportaciones de destrezas, en el } \\
\text { análisis }\end{array}$ \\
\hline
\end{tabular}

Tabla 2.2. Comparación de métodos de experimentación psicológica y la basada en diseño 
Lo central de esta distinción es que la investigación de diseño se enfoca y centra en la ruidosa y creativa confusión de la vida real, es práctica con su contexto, y es parte importante y no una variable extraña para ser trivializada. Además implica revisión flexible del diseño, dependencia múltiple de variables dependientes (ambiente: colaboraciones, recursos disponibles...; resultado: aprendizaje de contenido, cambio, transmisión...; y de sistema: difusión, continuidad...), compleja interacción social.

El diseño se revisa constantemente a partir de la experiencia en un "refinamiento progresivo" a través de ciclos, puesta en práctica, análisis y rediseño. Se hacen, testan y refinan conjeturas sobre la trayectoria de aprendizaje, basándose en las evidencias obtenidas en el transcurso de la investigación, colaborando o actuando como docentes y recogiendo extensos registros sobre lo que los alumnos, los docentes y los investigadores aprenden a lo largo del proceso (Molina, 2006, p. 267). Los participantes no son sujetos asignados para el experimento, sino coparticipantes en el diseño e incluso en los análisis; por último se interesan en caracterizar situaciones (opuesto a controlar variables), en desarrollar tal vez un perfil o teoría que caracterice el diseño en la práctica (opuesto a simple prueba de hipótesis).

Un reto de la investigación basada en diseño es mostrar en otros contextos la relevancia de las deducciones realizadas a partir del contexto de intervención. Stake (1995) se refiere a este tipo de generalización con la expresión "petite generalization". El antropólogo Clifford Geertz discutió la importancia de trabajar teniendo tanto el significado de la "experiencia próxima" como la relevancia de la "experiencia distante": "Confinement to experience-near concepts leaves the ethnographer awash in immediacies, as well as entangled in vernacular. Confinement to experience-distant ones leaves him stranded in abstractions and smothered in jargon." (Geertz, 1983, p. 57). La investigación de diseño se esfuerza por generar y avanzar un conjunto de explicaciones teóricas que transcienden las particularidades ambientales de sus contextos, lo que la sitúa aparte de experimentos de laboratorio o investigación de evaluación. 
Shavelson y Towne (2002, citado por Confrey, 2006, cap. 9) definen así estos estudios: "Enfoques analíticos para examinar mecanismos que comienzan con ideas teóricas que son testadas a lo largo del diseño, implementación y estudio sistemático de herramientas educativas (currículo, métodos de enseñanza, applets informáticos) que dan cuerpo al mecanismo conjeturado inicialmente". Enmarcan estas investigaciones en la pretensión de responder al interrogante de cómo y por qué está pasando algo.

En contraste con otros métodos creadores de teoría, el cambio radical de esta investigación basada en diseño es reconocer la importancia del contexto local y tratar los cambios en ese contexto como evidencia necesaria para la viabilidad de una teoría: cambios demostrables en el nivel local; si no se demuestra el valor del diseño en la creación de un impacto sobre el aprendizaje en el contexto local, no se ha justificado adecuadamente el valor de la teoría. Esta investigación supone, pues, un apuntalamiento filosófico pragmático en el valor de una teoría: que sea apoyada por su habilidad para producir cambios en el mundo. Así un sistema de investigación se inspiraría menos en la ciencia positivista tradicional o tradiciones etnográficas y más en líneas pragmáticas de investigación, donde las teorías son juzgadas no por sus pretensiones de exactitud, sino por su habilidad de trabajo en el mundo (Dewey, 1938).

La complejidad de estos experimentos supone una dificultad para garantizar sus argumentos y resultados, pero son numerosos los que reconocen su importancia y potencial como metodología de investigación educativa, en función de los criterios de fiabilidad: análisis sistemáticos con descripción detallada de las diversas fases y con fundamento de las inferencias realizadas; replicabilidad: aspectos potencialmente repetibles en otros contextos; capacidad de generación: no se persiguen leyes universales, sino obtener "modelos" de aprendizaje que permitan a los docentes guiar la enseñanza en la práctica; y utilidad: resultados en la práctica eliminando la desconexión entre investigación y praxis y promoviendo comunidades de profesionales de la enseñanza. 


\subsection{Evolución de los experimentos de diseño}

Surgen estos estudios a raíz de una reflexión sobre el proceso del aprendizaje y de las entrevistas clínicas, a lo que se suman los experimentos rusos de enseñanza y las aportaciones de Piaget, Vygotsky y Dewey. Frente a quienes miden el aprendizaje por los resultados computables del alumno (rapidez, rendimiento..., criterios insuficientes al no ofrecer "estructura" del aprendizaje), hay que afirmar que la cognición no es algo situado en el individuo pensante, sino un proceso entre el conocedor, el ambiente en el que tiene lugar y la actividad en la que participan los alumnos, lo que exige un desarrollo de teorías que ayuden a comprender y predecir sistemáticamente cómo ocurre el aprendizaje. En este contexto surge la investigación de diseño. En estas ciencias la clave característica incluye por qué se cree algo, cómo ha sucedido, cómo se relaciona con otras ideas, qué permite ejecutarlo, lo que implica una amplia serie de resultados medibles que capten el proceso así como la situación final del aprendiz.

Dewey, basado en la filosofía y epistemología así como en la psicología, influido por el pragmatismo (James) y la evolución (Darwin) considera que una teoría corresponde a los hechos cuando conduce a sus consecuencias a través de la experiencia, pero están sujetas a ser corregidas, han de pasar el test pragmático de utilidad en múltiples situaciones a lo largo del tiempo. El pragmatismo no sitúa la teoría en una estantería, sino que la encaja en la complejidad de la experiencia. La verdad absoluta es un ideal irrealizable. Existe la posibilidad de errar en la observación de algunos hechos o de obviar futuras consecuencias. Mediante la consideración de variadas circunstancias, dichas consecuencias se van haciendo más estables y seguras, siempre dentro de una provisionalidad.

Esta perspectiva conduce a Dewey a enfatizar la investigación, definida como "la transformación controlada de una situación indeterminada a una determinada en un todo unificado, que va evolucionando hacia una hipótesis y la hipótesis conduce a un conjunto de afirmaciones de conocimiento que deberían contrastarse a lo largo del 
tiempo, comprobando su utilidad para interpretar otras situaciones" (Dewey, 1981, pp. 49-50).

Así la erudición de Piaget, Vygotsky y Dewey proporciona la razón teórica fundamental para seleccionar los estudios de diseño como metodología para producir teorías sobre dirección educativa. El aula es compleja, no determinista. En estos escenarios se puede influir en la probabilidad de ciertos resultados, realizaciones diferentes y múltiples que ofrecen "tendencias" que pueden ser un marco paramétrico y dirigir la toma de una decisión. En base a estas premisas, puede dirigirse (no prescribir) la práctica mediante sistemas explicativos, acompañados de datos, evidencia y argumento. Un sistema de éstos es: hacia el mejor modelo de resultados verosímiles, conectados con sus teorías, con relaciones de interacción dentro de escenarios ecológicos, con rigurosa documentación y análisis y validez de utilidad para otros contextos similares. La teoría de los estudios de diseño incorpora esta visión epistemológica de la praxis del aula.

La metodología de diseño ha seguido evolucionando. Así Collins et al. (2004) desarrollaron la idea de refinamiento progresivo, en referencia a la industria japonesa del automóvil de revisiones frecuentes de mejora de la calidad. Cobb et al. (2003) se fijan en cinco rasgos transversales metodológicos: diseños fundados, intervencionistas más que naturalistas, generadores de teoría, iterativos, ecológicamente válidos y orientados a la práctica. Confrey (2006) habla de la articulación de dos conceptos relacionados: un corredor o pasillo conceptual y una trayectoria conceptual. El corredor es una construcción teórica del posible espacio a recorrer para aprender un contenido conceptual y durante el experimento de diseño los alumnos recorrerán una particular trayectoria conceptual a través del corredor.

Con el desarrollo de las teorías constructivistas, la metodología fue evolucionando hacia los experimentos de enseñanza procedentes de las investigaciones pedagógicas en la Unión Soviética (Kelly, 2003; Confrey, 2006), orientados a descubrir los procesos del aprendizaje de las asignaturas escolares: estudio longitudinal, intervención del investigador en el proceso, constante iteración de datos recogidos y planificación de actividades, datos más cualitativos que cuantitativos. 
Investigadores de EE.UU. aplicaron esta metodología al aprendizaje y desarrollo en el conocimiento de alumnos en aulas de comunicación matemática. Existía un abismo entre la práctica de la investigación y la práctica de la enseñanza. El paradigma experimental clásico no atendía a variables ajenas al experimento. Entonces el objetivo era la búsqueda de relaciones causales entre el comportamiento de los alumnos y su ambiente. Estos experimentos marcaron cambios en los modos de concebir el aprendizaje y enseñanza de las matemáticas. Molina (2006), citando a Steffe y otros, resume así la novedad de estos experimentos: "Los experimentos de enseñanza estudian las maneras y los modos de influenciar el conocimiento de los alumnos, yendo más allá de los objetivos de las entrevistas clínicas, intentando formular un modelo de aprendizaje de los contenidos particulares involucrados... persiguen entender el progreso que los alumnos hacen a lo largo de periodos en el tiempo. El investigador toma a la vez el papel de entrevistador y de docente; ...bajo la perspectiva constructivista... los investigadores investigaron las matemáticas de los niños, localizándolas en situaciones de negociación y comunicación interactiva, centrándose en la evolución de sus actividades más que en los productos, con la intención de examinar las modificaciones que se producen en sus esquemas en relación con actividades dirigidas a un objetivo" (Steffe y Thompson, 2000).

En la actualidad, los experimentos de enseñanza, característicos en Educación Matemática y Educación de las Ciencias, son considerados como investigación de diseño. El intento de observar directamente el aprendizaje y razonamiento, hace que profesor e investigador se coordinen estrechamente hasta coincidir, si el docente se involucra plenamente en la investigación. Alumnos, profesor e investigador colaboran y cambian a veces sus roles en complejas interacciones en una secuencia de episodios de enseñanza en el aula donde se recoge información, notas, grabaciones, cuanto ocurre en el aula.

Al principio, al final y durante los distintos episodios del experimento van generándose hipótesis a validar, corregir o abandonar, analizando, incluso en nuevos análisis retrospectivos, todos los datos recogidos. Así va desarrollándose un refinamiento progresivo de hipótesis hacia un modelo firme de la actividad y evolución 
mental de los investigados; el modelo va surgiendo de la actividad de los alumnos entrelazada con la teoría previamente establecida para analizar esa actividad, lo que puede conducir a modificar esos constructos teóricos empleados.

La evolución de estos estudios, según Steffe y Thompson (2000) está en función de los resultados, justificados con evidencias de apoyos, de la replicabilidad no estricta, sino sustitución del modelo por otro más avanzado, y capacidad de generalización aplicable a más contextos en el sentido de poder ser adaptados con otros alumnos.

\subsection{Experimentos de enseñanza transformativos dirigidos por una conjetura}

Son experimentos de enseñanza de diseño, algo diferentes de los experimentos de enseñanza, de importancia actualmente y con especial eficacia en ámbitos de enseñanza de las Matemáticas y las Ciencias. Marta Molina los describe como una intervención en el aula "dirigidos a investigar nuevas estrategias de enseñanza o a analizar diferentes enfoques para el contenido y la pedagogía de un conjunto de conceptos matemáticos, siendo su característica fundamental la conjetura que lo define y actúa de guía en el proceso de investigación... enfocado al trabajo en clases normales, no en clases donde se suponen las 'mejores prácticas'”' (Molina, 2006, p. 285).

Resumiendo a Confrey y Lachance (2000) detallamos más estos experimentos. Su base es una conjetura: inferencia basada en pruebas incompletas o no concluyentes, que se revisa, evoluciona y va refinándose y emergiendo durante el proceso. Estos autores unieron la conjetura a la obra de Lakatos, que reconoce que la teoría se establece "mediante la incesante mejora de las conjeturas, gracias a la especulación y a la crítica" (Lakatos, 1978, p. 20). La conjetura debe situarse en una teoría que la relacione con otros aspectos educativos; dicha teoría, con la ideología del investigador, influirán ambas en los elementos y desarrollo de la propia conjetura; tiene ésta dos dimensiones: de contenido, qué enseñar, y pedagógica, cómo enseñarlo. De acuerdo con esta dimensión se elaboran cuatro elementos principales de la enseñanza: 
- Currículo: áreas de "contenido" a tener en cuenta; contenido flexible en función del trabajo y peculiaridades de los alumnos.

- Interacción en el aula de las dos dimensiones de la conjetura; interacción determinante de métodos de recogida de datos. Datos abundantes sobre alumnos (incluso individualmente) y su trabajo; interacciones en el aula (recomendable grabación en vídeo); reflexiones, notas, decisiones y pensamiento de investigadores (incluso entrevistados) en el decurso del experimento. Los datos se analizan en dos momentos: después de cada intervención, análisis preliminar; y al final de todo el proceso, análisis final de éste con todos los datos recogidos, lo que conduce a construir una historia, con su evolución justificada, de todo el experimento realizado.

- Papel del Profesor: decidir el docente en función de la Conjetura y actividades a desarrollar.

- Evaluación: informada y congruente con el contenido, pedagogía, marco teórico de la conjetura y otros integrantes de la intervención; evaluación del impacto de la intervención y su proceso. Confrey y Lachance recomiendan diversas evaluaciones en el transcurso del experimento.

La peculiaridad de la conjetura reguladora de este experimento induce a Confrey y Lachance a proponer como factores reguladores de su evaluación la calidad de los procesos internos de la investigación y su impacto potencial en la práctica, en lugar de los factores estándares aplicados habitualmente. En relación con la calidad de los procesos internos, proponen tres medidas: poder explicativo de la conjetura, racionalidad de la historia del proceso (evaluar la coherencia entre conjetura y hechos sucedidos en esa historia) y fidelidad a la posición ideológica (juicio externo sobre si convencen y son consecuentes los datos aportados de los alumnos). En relación con el impacto potencial en la práctica, evaluación del grado posible de catarsis, para el cambio, de los resultados obtenidos en función de estos criterios: su viabilidad (en otras aulas), sostenibilidad (en el tiempo), convicción (del cambio a realizar), adaptabilidad (a otros contextos) y capacidad generativa (aporte de un modo de volver a conceptualizar sucesos, relaciones y prácticas). 
La investigación-acción puede considerarse como complementaria de la investigación de diseño. Ambas identifican problemas del mundo real para mejorar las situaciones concretas llevando a cabo, previa reflexión, las acciones adecuadas. En el ámbito educativo, ambas se centran en problemas prácticos con el objetivo de mejorar el aprendizaje. En ambas metodologías, el profesor está muy involucrado en la investigación y en nuestro caso es el investigador. Uno de los principales objetivos de la investigación basada en diseño es establecer nueva teoría para afrontar los problemas prácticos. La investigación-acción, por su parte, pretende tratar de manera directa, concreta, operativa, esos problemas prácticos.

Ante estas opciones metodológicas estrechamente relacionadas, el trabajo que presentamos sigue la línea de la investigación basada en diseño, concretándose en el método de investigación-acción, con el objetivo de presentar las pruebas preformales como adecuadas para facilitar el aprendizaje de las demostraciones matemáticas.

Consideramos más apropiada la línea fenomenológica, ya que investigamos aspectos cualitativos en la preferencia y valoración de unas pruebas, las pruebas preformales, por medio de instrucciones, cuestionarios, entrevistas (dentro de las limitaciones que tenemos). Además, algunas sesiones se desarrollaron en presencia de una profesora, que ha aportado sus observaciones. Realizamos también tablas y estudios estadísticos que nos prestan datos clarificadores a la hora de contabilizar los resultados obtenidos en las respuestas y actitudes de los alumnos. Hemos analizado y comentado estos resultados.

En Pecharromán y Ortega (2009), finalmente, se crea un marco de investigación que integra el marco teórico que proporciona el Enfoque Lógico Semiótico (ELOS), (marco descrito por Socas, 2007) con un marco metodológico de investigación cualitativa, basado en la investigación-acción (I-A) y la investigación de diseño. En este artículo, se describe cómo las dos metodologías cualitativas citadas se integran para formar un marco de investigación más robusto. En nuestra investigación seguimos el modelo creado por estos autores. 


\subsection{Metodología cuantitativa}

En los años ochenta del siglo pasado, la revista Educational Researcher recogía el juicio de diversos autores sobre la aportación de esta metodología al ámbito de la educación. La reticencia que alguno manifestó entonces sobre la validez de sus resultados ha quedado atenuada, y actualmente es aceptada la coordinación de ambos enfoques, cualitativo y cuantitativo en análisis, métodos e investigación. La conjunción de ambos paradigmas está logrando resultados óptimos en experiencias y trabajos relacionados con el aprendizaje y la enseñanza.

Los enfoques cuantitativo y cualitativo cumplen diversos roles en el progreso del conocimiento y ambos, con sus diferencias estructurales, resultan esenciales y complementarios, ya que en este campo de la investigación es preciso cuantificar datos estadísticamente y también comprender la realidad en la situación concreta en que intervienen los proyectos y programas, para mejorarla y estudiar y conocer los procesos y resultados desde una reflexión crítica y sistemática.

Se señalan como diferencias entre ambas metodologías:

- El análisis cuantitativo examina y valora los datos especialmente en el campo de la estadística. Utiliza variables, relación entre ellas, unidad de observación. El abordaje de estos datos es numérico, busca la certeza objetiva, por lo que utiliza la medición exhaustiva y controlada, distingue elementos de su todo y realiza inferencias. El riesgo puede estar en deformar el enfoque cualitativo en cantidades calculadas artificialmente, tomar una parte como variable independiente, propensión a "servirse de" más que a "comunicarse con" los sujetos analizados: la información es procesada sin devolución al origen de los datos; es una concepción lineal de lo analizado en el marco de una teoría y a través de una estrategia deductiva; estudia la asociación o relación de variables cuantificadas numéricamente; trata de determinar la fuerza de correlación entre variables y la objetivación y la generalización de los resultados a través de la información obtenida de una 
muestra para hacer inferencia, con un determinado nivel de confianza y error, a la población de la que procede, y explicar por qué las cosas suceden o no de una forma determinada. El análisis cuantitativo se realiza sobre elementos medibles y cuantificables. Los análisis cuantitativos se hacen sobre casos concretos para llegar a descripciones más generales, comprobar hipótesis o causas.

- El análisis cualitativo es, generalmente, inductivo. Analiza contextos estructurales, hace registros narrativos de los fenómenos estudiados mediante técnicas de observación participante y entrevistas o cuestionarios; trata de identificar la naturaleza profunda de las realidades, su estructura dinámica. El análisis cualitativo analiza los objetos investigados generadores de determinadas conductas en los individuos considerados dentro de su concreta situación. No se basa, de suyo, en medidas numéricas, sino en entrevistas, cuestionarios, materiales históricos. Intenta analizar de forma global un acontecimiento, situación. El análisis cualitativo permite una comunicación más horizontal entre investigador e investigados, aunque también hay que considerar con detalle las condiciones en que se realiza esa comunicación.

El empleo de ambas metodologías y análisis puede ayudar a corregir los riesgos de cada una de ellas. La cuantificación, hoy tan utilizada, facilita la comprensión del universo que nos rodea. Ya decía Galileo: "Mide lo que sea medible y haz medible lo que no lo sea".

Los dos enfoques son importantes: en el cualitativo se pone mayor atención a lo "profundo" de los resultados y no a su generalización; en el cuantitativo lo importante es la generalización de los resultados. Ambos son complementarios como enfoques de una misma investigación: los resultados cualitativos pueden estar mejor "validados", pero no tener tanta "fiabilidad"; los cuantitativos serán más "fiables", pero tendrán una menor "validez". 
Utilizamos Statgraphics, un programa suficientemente acreditado después de treinta años utilizándose en cálculos estadísticos para obtener resultados cuantitativos. Los contrastes de medias, varianzas, el test de Kolmogorov-Smirnov (K-S) son muy apropiados para detectar y establecer diferencias entre variables. En nuestro caso, las diferencias señaladas por los alumnos entre las "características y funciones" de las dos modalidades que se estudian y comparan en este trabajo: el empleo de pruebas formales y el empleo de pruebas preformales en las demostraciones matemáticas.

El test de Kolmogorov-Smirnov, el más extendido en la práctica para cuantificar de un modo más exacto las desviaciones de la distribución normal, se basa en la idea de comparar la función de distribución acumulada de los datos observados con la de una distribución normal, midiendo la máxima distancia entre ambas curvas. Como en cualquier contraste de hipótesis, la hipótesis nula se rechaza cuando el valor del estadístico supera un cierto valor crítico que se obtiene de una tabla de probabilidad. No obstante, este procedimiento debe ser utilizado con precaución. Cuando se dispone de un número suficiente de datos, cualquier test será capaz de detectar diferencias pequeñas aún cuando estas no sean relevantes para la mayor parte de los propósitos. El test de Kolmogorov-Smirnov, en este sentido, otorga un peso menor a las observaciones extremas y, por tanto, es menos sensible a las desviaciones que normalmente se producen en estos tramos. 



\section{CAPÍTULO 3}

\section{EXPERIENCIA PREVIA}

\section{E HIPÓTESIS DE INVESTIGACIÓN}

\subsection{Introducción}

En este capítulo se va a describir el origen de esta investigación. La investigación surge a partir del curso de doctorado La demostración en matemáticas realizado en la Universidad de Valladolid e impartido por el Dr. Ortega y del Trabajo de Investigación Tutelado Contribución de las pruebas preformales al aprendizaje significativo llevado a cabo por el Profesor Investigador (en adelante PI) bajo la dirección del referido doctor.

En el Trabajo Tutelado se reflexiona sobre la facilidad que las pruebas preformales aportan al aprendizaje. Al comienzo del Trabajo se alude a los cambios operados en las aulas y los centros académicos, a la transformación de la sociedad y al rechazo de los alumnos a un aprendizaje pasivo. Todo ello induce al profesor a conectar más personalmente con el alumno y su entorno. Esta aproximación se persigue por medio del aprendizaje significativo que construye el conocimiento encajando las piezas nuevas con las anteriores en un todo coherente. 
Este Trabajo, estructurado en tres capítulos, es de índole práctica: tras realizar una fundamentación del mismo en los dos primeros capítulos (I. Antecedentes. El marco teórico; II. Metodología investigación-acción), en un tercer capítulo, se ofrecen las pruebas preformales con diversos ejemplos de este tipo de pruebas.

\subsection{Experiencia previa}

La experimentación comenzó en el curso 2005-2006 en el desarrollo de la asignatura Cálculo, con una experiencia previa en la que se trató de establecer una primera aproximación sobre la conveniencia de utilizar pruebas preformales en lugar de demostraciones formales. Se experimentó con un grupo de alumnos de primero de Ingeniería Técnica en Informática de Gestión, de la Universidad Europea Miguel de Cervantes (Valladolid).

El PI realizó una serie de pruebas preformales de Análisis Matemático para alcanzar una formación y destreza en el uso de este tipo de pruebas (Desigualdad triangular, Propiedad arquimediana, Unicidad del límite, Teorema de Weierstrass, Teorema de Bolzano, Derivabilidad implica continuidad, Teorema de singularidad, Teorema de Rolle, Teorema del valor medio de Lagrange, Teorema fundamental del cálculo integral). En suma, se puede decir que remodeló sus materiales de cálculo diferencial utilizando pruebas preformales. Estas pruebas constituyen el ANEXO I y, por sí solas, constituyen una aportación de la tesis.

El PI desarrolló una docencia utilizando algunas de estas pruebas preformales en el grupo de alumnos. En concreto se les mostraron varias pruebas preformales de las citadas antes (Desigualdad triangular, Propiedad arquimediana, Derivabilidad implica continuidad, Teorema de Weierstrass, Teorema de la unicidad del límite y Teorema del valor medio de Lagrange) para analizar el comportamiento de los alumnos ante esta nueva forma de establecer los teoremas de matemáticas.

La observación directa del PI sobre esta docencia y las respuestas que dieron los alumnos a preguntas relacionadas con la comprensión y preferencias, permitieron conjeturar que este tipo de pruebas son más beneficiosas que las demostraciones 
formales para estos alumnos por varias razones: a los alumnos les parecen más atractivas, más fáciles, y las comprenden mejor. Este trabajo nos permite enunciar los objetivos de la investigación.

\subsection{Objetivos e hipótesis}

La conjetura del párrafo anterior, junto con la hipótesis formulada en el citado Trabajo de Investigación Tutelado (TIT), determinó el problema que pretendemos estudiar en esta tesis. Al final de las consideraciones realizadas en el TIT avanzábamos como hipótesis general de investigación que las pruebas preformales son más significativas para los alumnos que las pruebas formales.

Este objetivo general da pie a formular otros objetivos específicos de la investigación. Establecemos los siguientes:

01. Indagar qué tipo de aprendizaje en relación con la demostración matemática es más activo, más participativo y los aprendizajes.

02. Iniciar a los alumnos en el uso de la prueba preformal con precisión en el concepto y su distinción de la prueba formal.

03. Comparar ambos tipos de demostración, preformal y formal, para optar por el que sea preferido para los alumnos, sopesando debidamente las características y funciones de cada una de las dos modalidades.

04. Descubrir la posible complementariedad de ambos tipos de demostración.

05. Analizar cómo valoran los alumnos la influencia de cada una de las dos modalidades de prueba para un mismo teorema al aplicar dicho teorema a algún ejercicio práctico.

06. Investigar el proceso de enseñanza-aprendizaje de las demostraciones matemáticas mediante pruebas preformales y formales, y detectar cuál de estos dos tipos de prueba resulta más significativo para los alumnos. 
Como indican en una de sus conclusiones Ibañes y Ortega (2002, p. 11) "sólo la tercera parte de los alumnos muestra disposición a aplicar el teorema recién demostrado; el resto repite la demostración en un caso concreto o argumentan en su caso particular con independencia del teorema expuesto". Ésta y otras conclusiones de estos autores, la dificultad del aprendizaje, enraizamiento, evolución, distinción y reconocimiento de las demostraciones nos hacen pensar que las pruebas preformales contribuyen eficazmente a facilitar el aprendizaje significativo de los alumnos.

Aunque no es necesario fijar hipótesis de investigación, ya que los objetivos señalados antes orientan por sí solos la finalidad de la misma, para fijar nuestra posición respecto de las pruebas preformales sí que vamos a establecer las siguientes hipótesis:

H1. El aprendizaje en relación con la demostración matemática es más activo, más participativo y los aprendizajes son más significativos a través de las pruebas preformales.

H2. Las pruebas preformales son un complemento de las pruebas formales y como tales cumplen una función de explicación.

H3. No es difícil que los alumnos aprendan a usar pruebas preformales con precisión y que las distingan de las pruebas formales.

H4. El proceso de aprendizaje de las demostraciones matemáticas mediante pruebas preformales resulta más significativo para los alumnos, son más motivadoras (gusto) y consideran que las entienden mejor, que son más fáciles, más claras.

H5. Los alumnos prefieren este tipo de pruebas, las comprenden mejor y perduran más en la memoria.

H6. Las pruebas preformales son más convincentes para estos alumnos y son más fáciles de aplicar. 


\section{CAPÍTULO 4}

\section{CICLOS DE INVESTIGACIÓN-ACCIÓN}

En este capítulo se describe la actividad de tres ciclos de investigación, I (2007), II (2008) y III (2009), con sus etapas de planificación, acción, observación de la acción, recogida de datos (cuestionario del ANEXO II), análisis de los datos (respuesta a las preguntas 1, 2 y 3 del cuestionario) y, por último, reflexión. Se adjunta al final una tabla y un gráfico resumen de la respuesta de todos los alumnos de los tres ciclos a la pregunta 3 del cuestionario.

\section{PLANIFICACIÓN GENERAL}

Con el objetivo general de descubrir las diferentes valoraciones de los alumnos con respecto a demostraciones matemáticas en las modalidades de prueba formal y prueba preformal se han diseñado tres ciclos de investigación-acción, cada uno con sus cuatro fases (planificación, acción, recogida y análisis de datos, y reflexión) y retroalimentados, es decir, en la planificación de los nuevos ciclos se utilizan las reflexiones de los anteriores. El primer ciclo se ha desarrollado en el curso 20062007 (ciclo de exploración) y los dos siguientes (ciclos de confirmación y de saturación) se han desarrollado en los cursos 2007-2008 y 2008-2009. En cada uno de estos ciclos se han tenido en cuenta las orientaciones de la metodología de diseño, concretándose en el método de investigación-acción (sección 2.2). 
La acción ha sido similar en los tres ciclos y las observaciones de la misma también, pero por tratarse de alumnos y cursos diferentes se describe en cada uno de ellos, aunque los contenidos que se han considerado en los tres ciclos hayan sido los mismos. En el desarrollo de los mismos es cuando los alumnos han tenido contacto por primera vez con las pruebas preformales siguiendo el modelo de van Asch, resaltando la peculiaridad de este tipo de prueba distinguiéndola de la formal, la habitualmente conocida por el alumno. Esta acción ha sido observada, en los ciclos II y III, por un Observador Externo (en adelante OE) y, lógicamente, por el PI, quien ha anotado todas sus apreciaciones en el cuaderno de aula.

Para la recogida de datos se elaboró un cuestionario a partir de las observaciones realizadas en la experiencia previa del curso 2005-2006, cuestionario que ha resultado eficaz y que no ha mostrado variación en los ciclos de investigación-acción. Los ítems del cuestionario trataban de averiguar los comportamientos de los alumnos frente a las pruebas preformal y formal del teorema del valor medio de Lagrange y del teorema de la unicidad del límite, y las ventajas de un tipo de prueba frente al otro a la hora de aplicar el teorema del valor medio. Este cuestionario figura en el ANEXO II.

También se planificaron entrevistas en todos los ciclos a dos parejas de alumnos para esclarecer o profundizar en sus respuestas algunos de los ítems del cuestionario, entrevistas que serían transcritas y analizadas con las anotaciones correspondientes.

La primera cuestión planteada pretendía conocer la impresión general sobre cómo habían comprendido los alumnos uno y otro teorema con cada modalidad de prueba (formal y preformal).

En segundo lugar, dentro de la comprensión, el PI consideró oportuno detectar la preferencia de los alumnos por una u otra de las modalidades de prueba en relación con cuatro importantes categorías: su facilidad, su claridad, el gusto por ellas y su influjo en el aprendizaje del alumno. 
En tercer lugar, respecto de las funciones de la demostración (Hanna, 1989) se proyectó que comparasen los dos procedimientos en relación con la capacidad de convicción, la ayuda a la comprensión, memorización y utilización de los teoremas.

Finalmente se consideró muy conveniente completar el cuestionario con una aplicación de uno de los dos teoremas, el del valor medio, para valorar el grado de influencia de cada una de las dos modalidades de prueba.

Una vez recopiladas las respuestas al cuestionario, el PI analizaría los datos aportados y reflexionando sobre ellos concluiría un ciclo dando paso al siguiente.

Pasamos a describir las cuatro fases del desarrollo de cada uno de los ciclos de investigación acción.

\subsection{Ciclo I (ciclo de exploración)}

\subsubsection{Planificación}

Este primer ciclo se realizó en marzo y abril de 2007 con el grupo de 28 alumnos de primer curso en la asignatura Cálculo de la titulación Ingeniería Técnica en Informática de Gestión, integrada en la Escuela Politécnica Superior de la Universidad Europea Miguel de Cervantes de Valladolid.

El PI proyectó este ciclo de la manera siguiente. Primeramente se realizaría una preparación de los alumnos en una serie de clases con el objetivo de iniciarlos y familiarizarlos con la noción de prueba preformal según van Asch. Cumplido este objetivo, tendría lugar la sesión principal del ciclo, consistente en una breve explicación sobre la tarea a realizar, la exposición por parte del PI de las demostraciones formales y preformales de los teoremas elegidos, la entrega a los alumnos del cuestionario señalado en la planificación general y la recogida del mismo una vez cumplimentado por ellos. Seguidamente se procedería al análisis de la información 
proporcionada por los alumnos sobre la cual se realizaría la reflexión correspondiente. Finalmente, tendrían lugar sendas entrevistas con dos parejas de estos alumnos.

\subsubsection{Desarrollo del primer ciclo (acción)}

La fase de acción se llevó a cabo con arreglo al siguiente calendario:

\begin{tabular}{|c|c|}
\hline Fechas & Contenidos \\
\hline $20 / 03 / 2007$ & $\begin{array}{l}\text { Repaso de axiomática de los números reales } \\
\text { Concepto de prueba preformal según van Asch. } \\
\text { Demostraciones preformales de la desigualdad triangular y de la } \\
\text { propiedad arquimediana }\end{array}$ \\
\hline $22 / 03 / 2007$ & Teoría de límites funcionales \\
\hline $29 / 03 / 2007$ & $\begin{array}{l}\text { Continuidad } \\
\text { Demostraciones preformales de los teoremas de Bolzano y } \\
\text { Weierstrass }\end{array}$ \\
\hline $17 / 04 / 2007$ & Primeras definiciones de cálculo diferencial \\
\hline $19 / 04 / 2007$ & $\begin{array}{l}\text { Teoremas de cálculo diferencial } \\
\text { Demostración preformal del teorema "derivabilidad implica } \\
\text { continuidad" y del teorema de singularidad }\end{array}$ \\
\hline $26 / 04 / 2007$ & $\begin{array}{l}\text { Sesión principal } \\
\text { Demostraciones formales y preformales de los teoremas del valor me- } \\
\text { dio de Lagrange y de la unicidad del límite. } \\
\text { Cuestionario }\end{array}$ \\
\hline $30 / 04 / 2007$ & Entrevistas \\
\hline
\end{tabular}

Tabla 4.1. Calendario del primer ciclo

Como ya se ha indicado antes, las pruebas preformales que se han utilizado en esta docencia y otras más aparecen en el ANEXO I.

En la sesión principal, reunidos los 28 alumnos del grupo, el PI comenzó recordando en qué consiste una prueba preformal y su diferencia con la formal. Seguida- 
mente, después de solicitar a los alumnos una actitud atenta y activa (hacer preguntas, pedir aclaraciones, tomar notas), el PI expuso en la pizarra ante ellos las demostraciones de los teoremas del valor medio de Lagrange y de la unicidad del límite. Para el teorema del valor medio (teorema 1) hizo en primer lugar una prueba preformal y, a continuación, una prueba formal. En cambio, con el teorema de la unicidad del límite (teorema 2), primero hizo una prueba formal y posteriormente una prueba preformal.

\subsubsection{Observación de la acción}

Los alumnos de este grupo no conocían la modalidad de demostración preformal, por lo que, desde el principio de la experiencia, su actitud fue de expectación y curiosidad. El PI pudo observar y anotar en su cuaderno de aula, cómo los alumnos acogían con agrado las explicaciones cada vez que introducía una prueba preformal en el transcurso de las clases. En el cuaderno de aula han quedado recogidas cuestiones como éstas: en la prueba preformal, si cambiamos la función o el intervalo ¿sigue siendo válido el razonamiento?; la modalidad preformal ¿no es simplemente un ejemplo?; ¿se puede realizar una prueba preformal para demostrar cualquier teorema matemático? Estas preguntas fueron respondidas por el PI en las clases preparatorias previas a la sesión principal e, invariablemente, se producía el asentimiento de los alumnos. Durante la sesión principal, los alumnos mantuvieron en todo momento un buen nivel de atención e interés, solicitando numerosas aclaraciones sobre diversos pasos y características de las demostraciones y, también, sobre el cuestionario cuando lo estaban cumplimentando. Concretamente, varios alumnos solicitaron alguna explicación adicional respecto de la forma de cumplimentar la columna preferencia de la tabla de la cuestión 2 (cf. ANEXO II). También quedó reflejado en el cuaderno de aula que en el ejercicio de aplicación del teorema del valor medio a la función seno (cf. ANEXO II) no se trataba de realizar una demostración de este teorema, sino simplemente de aplicarlo a una situación concreta, valorando el grado de influencia de cada una de las modalidades de prueba en la citada aplicación. Esta cuestión fue planteada por varios alumnos. Finalmente, es destacable la actitud de disponibilidad, espontaneidad y sincera cola- 
boración mostrada por los alumnos en las entrevistas que se hicieron posteriormente.

\subsubsection{Recogida de datos a través del cuestionario}

Terminadas las explicaciones, el PI entregó a los alumnos el cuestionario establecido, para ser cumplimentado personalmente por cada uno de ellos. Al cabo de dos horas, se procedió a la recogida de los cuestionarios finalizando así la sesión. La recogida la realizó el PI en dos fases: en primer lugar las cuestiones 1, 2 y 3, y después el ejercicio de aplicación del teorema del valor medio. Las respuestas de los alumnos a las cuestiones 1 y 3 se han transcrito y se presentan íntegramente en el ANEXO VI.

\subsubsection{Análisis de los datos}

El PI realizó un análisis cualitativo y cuantitativo de los datos aportados por los alumnos. En cuanto al análisis cuantitativo, con el fin de tener una muestra más significativa, el PI optó por integrar estos datos junto con los correspondientes a los ciclos II y III que habrían de realizarse en los dos años siguientes.

\section{Análisis cualitativo}

Cuestión 1: Explica cómo has entendido cada uno de los teoremas con cada procedimiento. Indica en cada caso claramente el porqué.

El objetivo de esta cuestión es analizar la comprensión de los alumnos de las demostraciones matemáticas sobre las pruebas preformales en contraste con las pruebas formales.

Responden los 28 alumnos matriculados en el curso y a continuación se presenta una síntesis de las respuestas emitidas por cada uno de ellos en relación con el teorema 1 (teorema del valor medio). Identificamos la respuesta del alumno n con la notación An: 
A1. Este alumno ve la prueba preformal como una mera aplicación del teorema; y en la formal, que entiende mejor, ve más claramente los pasos de la prueba. En rigor no analiza las pruebas "en sí", sino alguno de sus aspectos.

A2. Este alumno sí juzga las pruebas en sí mismas: más clara e intuitiva la preformal, pero menos complicada (enrevesada) la formal. Se da una cierta contradicción entre los términos "más clara" y "menos complicada": si es más clara, parece que no debe ser "más" complicada o enrevesada, como él dice.

A3. Entiende la preformal como una aplicación a un caso concreto, que carece de la generalización propia de todo teorema. Pero una prueba no es aún el teorema. La formal, dice, es una demostración de libro; supone, pues, que las pruebas presentadas en los libros suelen ser sólo las formales.

A4. A este alumno le "caen mal" las letras, prefiere valores concretos y luego dar el paso desde ellos a la generalización.

A5. Sin aportación apreciable.

A6. El alumno establece un orden entre las dos pruebas: primero la preformal (ejemplo concreto) y luego "generalizar" con la formal, que le parece "confusa" (quizá ha querido decir "más difícil”). Piensa bien en relación al orden de las dos pruebas y en relación también a su dificultad.

A7. Se fija también en la dificultad de las pruebas: mayor dificultad la formal y menor la preformal por partir, afirma, de una función concreta, la logarítmica.

A8. Analiza también la dificultad de las pruebas y el ámbito de su extensión en aplicaciones. Acierta al juzgar más fácil e intuitiva la preformal y más completa como teoría, pero más compleja para entender la formal.

A9. Apunta un posible peligro, según él, de la preformal: creer que sólo es aplicable a un solo caso determinado previamente, mientras la formal, que él prefiere, abarca a todos los casos posibles. 
A10. A este alumno no le gusta la función logarítmica (probablemente aún no la ha entendido), por ello le resulta más sencilla la formal por más general, aunque le cuesta imaginarse la función. Se contradice en cierto sentido al juzgar más sencilla la formal, pero confiesa que le cuesta imaginarse la función.

A11. Manifiesta, sin profundizar en el análisis, que la prueba formal es "general" y la preformal no es "general" y que ambas se apoyan en el teorema de Rolle. (Su análisis también es muy "general”).

A12. La prueba preformal no le agrada "por no especificar el enunciado con claridad lo que persigo"; la prueba formal es mejor "sé hasta dónde tengo que llegar en el análisis del problema”. No es coherente, ya que el enunciado es el mismo en las dos pruebas.

A13. La prueba preformal, afirma este alumno, clarifica mucho, ayuda mejor a la comprensión; con la formal se ve menos claro, pero tiene un mayor ámbito de aplicación.

A14. No ha entendido las pruebas ni, en consecuencia, sus características.

A15. Incide, como otros, en lo "concreto" de la prueba preformal y en la mayor "generalidad" de la formal, en la que este alumno dice ver más clara la resolución.

A16. Parece contradecirse al afirmar que con la prueba formal comprende el teorema y, a la vez, que la preformal resulta clarificadora, ayuda a verlo (el teorema) mejor.

A17. Un poco complicada la prueba preformal y más claro (el teorema) por la formal.

A18. Más fácil entenderlo con la preformal; más precisa y aplicable la formal.

A19. Le gusta más la prueba preformal con la que entiende mejor cómo llegar a la conclusión. 
A20. Éste no tiene preferencia por ninguna de las dos pruebas: ve la preformal como un ejemplo; un poco más claro con la formal.

A21. Éste, por el contrario, lo entiende mejor con la prueba preformal.

A22. También para éste resulta más sencilla la comprensión con la prueba preformal por verla como una comprobación en un caso particular.

A23. Para éste, en la prueba preformal se muestra más (mejor) el funcionamiento (se referirá al proceso de la prueba).

A24. Este alumno se contradice, en cierto modo: al referirse a la prueba preformal la califica de "liosa y complicada de ver", mientras que la formal, dice, que es más sencilla aunque la función es "difícil" de obtener. La contradicción está en los términos que utiliza, "liosa” para la más difícil y "difícil” para la más sencilla.

A25. Dice entenderlo mejor con la prueba formal; en la preformal "usa" el teorema, no su demostración. (Una prueba no es "uso", sino más bien explicación, justificación del teorema).

A26. Para éste son más claras las explicaciones en la prueba preformal.

A27. También éste lo entiende mejor con la prueba preformal, pues se comprende mejor el funcionamiento con casos concretos.

A28. La prueba preformal, dice, es la que más le ha ayudado a entenderlo. Establece, según su criterio, este orden para exponer el teorema: primero la prueba formal y luego la preformal, para concluir con un ejemplo.

Para el teorema 2 (teorema de la unicidad del límite) también responden los 28 alumnos y a continuación se presenta una síntesis de sus respuestas:

A1. Este alumno afirma que la prueba preformal le ha sido más fácil de entender. La formal más difícil por ser más estricta y utilizar muchas variables. 
A2. El ejemplo de la prueba preformal es más claro. Cree que el camino más fácil para resolver teoremas sobre unicidad es la reducción al absurdo. Afirma también, contradiciéndose en cierto modo, que esta reducción al absurdo es una dificultad "añadida".

A3. Le parece más fácil de entender que el primer teorema. En la prueba preformal ve matices que aportan mayor precisión a la formal.

A4. Se decanta por la prueba preformal porque se hace la aplicación de forma muy visual y luego se da el paso a conceptos generales, mejor que pasar de lo abstracto a lo más sencillo. Analiza las pruebas: más sencilla la preformal y muestra preferencia por el orden de ellas: de lo más sencillo, concreto, a lo abstracto.

A5. Ha entendido el teorema con la prueba preformal. No aporta nada en relación con la formal.

A6. Ha recibido más ayuda con la prueba preformal: ejemplos concretos, funciones conocidas, pasos y operaciones más fáciles. La formal se le hace confusa.

A7. Lo he entendido algo mejor con la prueba preformal por dar valores concretos, ha sido bastante clara, me decanto por la preformal; también me ha resultado clara la formal.

A8. Más fácil de intuir el teorema en la prueba preformal, más difícil en la formal.

A9. Es más ilustrativa la prueba preformal: dados los intervalos $(0,2)$ y $(2,4)$ se ve que la imagen de la función no puede estar a la vez en los dos, pues no tienen ningún punto común.

A10. Es más sencilla la prueba preformal, el teorema se entiende mejor con esta prueba. La formal no es mal método al ser "general". 
A11. La prueba preformal sólo demuestra que la función no puede tener límites 1 y 3 en el punto 4; la formal se extiende a más casos, me satisface más.

A12. La única forma de entender este teorema, más difícil que el anterior, es con la prueba preformal; con la formal me quedaría sin palabras ante mis múltiples dudas. (En rigor se trata de "entenderlo" más que de "hablarlo"). Manifiesta que le es difícil la formal.

A13. Prefiero la prueba preformal: ver el teorema en forma más concreta me ayudó a comprenderlo mejor. La formal, dice, es la forma genérica que ha de memorizarse. 0 sea: para entenderlo, la prueba preformal; para memorizarlo, la formal.

A14. No he entendido nada de la prueba formal; en la preformal no entiendo por qué utiliza el $\varepsilon$. Alguna preferencia, pues, por la preformal.

A15. La prueba preformal me ayudó en la última parte de la demostración formal, se ve más claro con los intervalos concretos que no tienen puntos comunes. No parece muy clara la mayor facilidad de la prueba formal. En todo caso este alumno ve más sencilla y útil la prueba preformal.

A16. Me ayudó más la prueba preformal: la representación gráfica ayuda bastante a ver que es imposible que, en un punto tengamos dos límites distintos. Una vez vista la prueba preformal, al volver a la formal sí pude entenderlo y generalizarlo. Este alumno aporta dos facilidades a favor de la prueba preformal: la representación gráfica la hace más fácil en sí misma; y, entendida la preformal, facilita el entendimiento de la formal.

A17. Más clara la prueba preformal; menos la formal por las letras.

A18. A este alumno le quedó bastante claro el teorema con la prueba formal. 
A19. Más fácil de entender con la prueba preformal porque tomamos valores concretos.

A20. Lo he entendido mejor con la prueba preformal.

A21. Este alumno se fija en dos aspectos de interés: 1) que una prueba preformal puede ayudar siempre a entender cualquier teorema; 2) pero no sería suficiente para entender de dónde salen los resultados.

A22. Para este alumno es la prueba formal la que demuestra el teorema; la preformal le aplica a un caso concreto; así se ve mejor si no se ha entendido algún paso de la prueba formal. (No es así, también la prueba preformal demuestra a través, si se quiere, de una aplicación concreta; el mismo alumno afirma que la prueba preformal ayuda a comprobar los pasos dados en la formal).

A23. La prueba preformal ayuda bastante a seguir el razonamiento. Lo entiendo mejor con la prueba formal. (Pero el ayudar a seguir el razonamiento ¿no es aportar un mejor entendimiento?).

A24. Para este alumno la prueba preformal es algo más liosa y complicada. Con la formal ve mejor lo que está realizado.

A25. Este alumno lo entiende con las dos pruebas, pero mejor con la preformal.

A26. Más sencilla la prueba preformal y ofrece una visión de conjunto bastante mejor.

A27. Este prefiere la prueba formal por más técnica; la reducción al absurdo parece lo más adecuado. 
A28. La prueba preformal es la que más me ha ayudado a entenderlo. Para exponerlo, sugiere el orden siguiente: primero la prueba formal y luego la preformal para terminar con un ejemplo.

Sonia M. G. (21), que prefiere la prueba preformal en el teorema 1 y la prueba formal en el teorema 2, concluye así su respuesta:

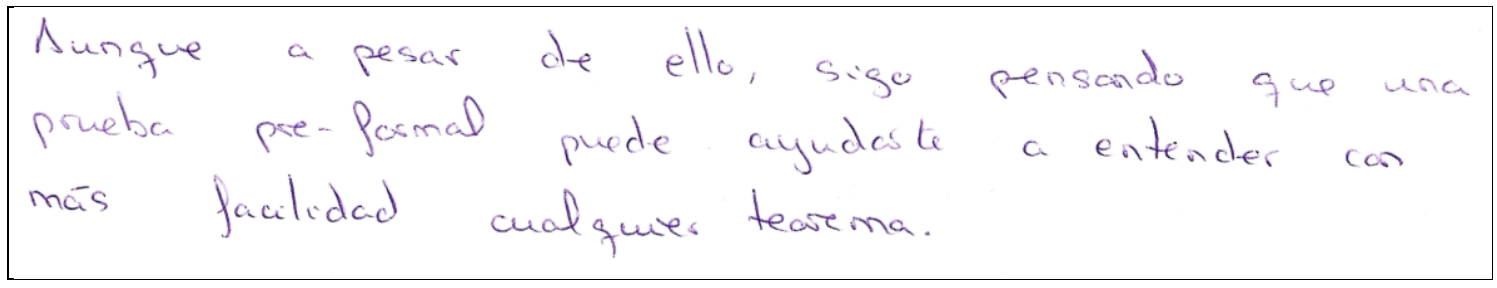

Los escaneos siguientes corresponden a las respuestas de Jesús B. G. (4) y Pablo G. S. (12). El primer alumno prefiere la prueba preformal, por su sencillez, y el orden preformal-formal en la sucesión de ambas pruebas; el segundo prefiere la prueba formal para el teorema 1, por ser más general, pero prefiere la preformal en el teorema 2, por su claridad, como se deduce de sus explicaciones.

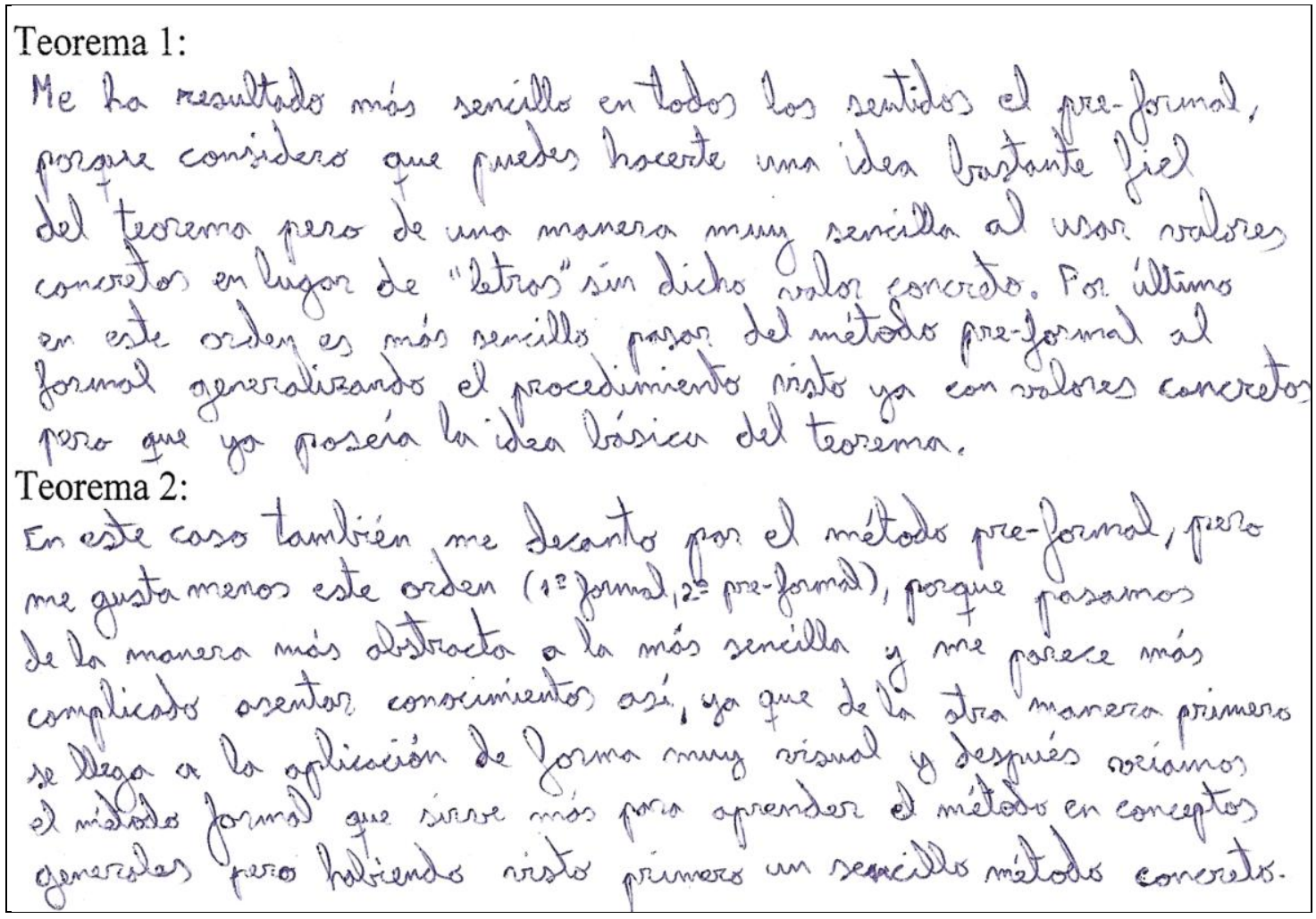


- Teorema 1: Para el Teorena del vaior redio (Lagange) la rejo opción esla formal, sin waber mucha diterencia entre las dos. Prefiero una explicación mas geveralizada, no con un ejemplo. porque as se hasta dende tengo que legar en el anavisis del problema. La Pre-formal no ne agrada, creo yo quees por gje el ennciado no re especifica can claridad 10 que persigo y no se si los puntos $(\sqrt{2}, \ln \sqrt{2})$ y $(6, \ln 6)$ vieren dados o son anteriomente. calcula dos.

- Teorema 2: Partiendo de que este segur do tecrema es más canplejo que el anterior, aqui por muy bien que se expliquen todos los pasos de una forma precisa lo que conseguina seria liarme, más todaria de 10 que estoy tras leer delevidarente el teorema mas cuantas veces. Por cansiguiente. la úrca foma de poder entender 10 que la unicidad del limite nos permite ver, es can un ejemplo. Deesta forma pued delendeime ante una exposición de este problema isituese car la formal ive quedaria sin palabras, ante mis nutiples dudos.

En las dos tablas siguientes se muestran las categorías que prefieren los alumnos:

\begin{tabular}{|c|c|c|c|}
\hline \multicolumn{4}{|c|}{ TEOREMA 1} \\
\hline \multicolumn{2}{|l|}{ Prueba preformal } & \multicolumn{2}{|l|}{ Prueba formal } \\
\hline Categoría & $\mathbf{n}_{\mathbf{i}}$ & Categoría & $\mathbf{n}_{\mathbf{i}}$ \\
\hline $\begin{array}{l}\text { La preformal convence más que } \\
\text { la formal }\end{array}$ & 1 & $\begin{array}{l}\text { La formal convence más que } \\
\text { la preformal }\end{array}$ & 1 \\
\hline Se entiende mejor & 10 & Se entiende mejor & 2 \\
\hline Más difícil, compleja & 0 & Más difícil, compleja & 3 \\
\hline Más general & 0 & Más general & 3 \\
\hline Orden: preformal-formal & 3 & Orden: formal-preformal & 1 \\
\hline Aplicación caso concreto & 3 & Aplicación caso concreto & 0 \\
\hline $\begin{array}{l}\text { Concisa, clara, fácil, intuitiva, } \\
\text { tangible, muestra más el } \\
\text { funcionamiento }\end{array}$ & 8 & $\begin{array}{l}\text { Fácil de intuir, ilustrativa, } \\
\text { ayuda bastante a seguir el } \\
\text { razonamiento }\end{array}$ & 3 \\
\hline
\end{tabular}

Tabla 4.2. Categorías preferidas en el teorema 1, ciclo I 


\begin{tabular}{|c|c|c|c|}
\hline \multicolumn{3}{|c|}{} & $\mathbf{n}_{\mathbf{i}}$ \\
\hline Prueba preformal & $\mathbf{n}_{\mathbf{i}}$ & Categoría & 1 \\
\hline Categoría & 10 & Se entiende mejor & 1 \\
\hline $\begin{array}{c}\text { Se entiende mejor } \\
\text { contenidos }\end{array}$ & 5 & $\begin{array}{c}\text { Ayuda más a entender } \\
\text { contenidos }\end{array}$ & 8 \\
\hline Más difícil, compleja & 1 & Más difícil, compleja & 2 \\
\hline Más general & 0 & Más general & 2 \\
\hline Orden: preformal-formal & 2 & Orden: formal-preformal & 0 \\
\hline Aplicación caso concreto & 1 & Aplicación caso concreto & 1 \\
\hline Convence más & 0 & Convence más & 1 \\
\hline
\end{tabular}

Tabla 4.3. Categorías preferidas en el teorema 2, ciclo I

Para el análisis de las respuestas de los alumnos a esta primera cuestión se han tenido en cuenta las dos tablas anteriores de categorías sobre ambas modalidades de prueba (preformal y formal) realizadas directamente a partir de la información proporcionada por los alumnos.

Dentro de la variedad de respuestas de estos alumnos, se perfilan dos corrientes en relación con las características detectadas por ellos en cada una de las dos modalidades de prueba, más favorable una, en su conjunto, a la prueba preformal y menos favorable la otra a la formal.

Algunos alumnos, cuatro en concreto, consideran la prueba preformal como una aplicación a un caso particular. Puede ser así, y tratarse, a la vez, de una prueba auténtica a través de una aplicación concreta. No sería apropiado entender esta característica como la mera aportación de un ejemplo. 
Frente a este juicio sobre la prueba preformal, otros cinco alumnos aplican a la formal la condición de generalizadora a todos los casos, lo que en verdad corresponde a toda prueba verdadera utilizada en matemáticas, también a la prueba preformal, pero es cierto que en la prueba formal puede aparecer esta condición con mayor claridad.

En las cualidades que los alumnos señalan como motivos para establecer sus valoraciones sobre las pruebas inciden, en relación con la prueba formal, en su dificultad, complejidad, el uso de letras en vez de números, y en considerarla más técnica, más completa, "de libro". Por otra parte, aplican a la prueba preformal las siguientes cualidades: concisión, claridad, ayuda a entender el funcionamiento. Respuestas que, al analizarlas, parecen responder a sentimientos personales o impresiones un tanto espontáneas, sin un discernimiento más profundo.

Una categoría importante de valoración de una prueba matemática ha de estar en su fuerza de convencimiento y su ayuda a entender el teorema o los contenidos. Estos alumnos se decantan, en estos aspectos, por la prueba preformal (26 vs. 6), más aún en el teorema 2, lo que se refleja también al considerar a la prueba formal más compleja y difícil (11 vs. 1).

Varios alumnos afirman la necesidad de utilizar ambas pruebas, y establecen el orden de las mismas que le parece a cada uno más eficaz y conveniente: el orden preformal-formal (cinco alumnos) y formal-preformal (tres alumnos). Puede influir en esto el esquema de aprendizaje personal de cada uno y el orden seguido en la explicación de los teoremas.

Las cuestiones $2^{\underline{a}}$ y $3^{\underline{a}}$ se centran en las categorías de facilidad (F), gusto $(G)$, claridad (C), aprendizaje (A), comprensión, preferencia (la $2^{\underline{a}}$ ), y convencimiento, entendimiento, memorización y aplicabilidad (la $3^{a}$ ).

La condición de facilidad se refiere al grado de esfuerzo necesario para la comprensión de la prueba, el gusto a la atracción que motiva su aprendizaje y utilización, la claridad a la percepción del razonamiento libre de oscuridad y confusión, y 
el aprendizaje al grado de integración en el esquema conceptual del alumno. Aspectos de gran interés a considerar en la asimilación de las demostraciones matemáticas.

Cuestión 2: Puntúa de 1 a 10 las cuatro pruebas que hemos realizado atendiendo a las siguientes categorías: facilidad (F), gusto (G), claridad (C), aprendizaje (A). Entiende que:

> 1 es muy difícil y 10 es muy fácil.

> 1 no me gusta y 10 me gusta mucho.

1 no es nada clara y 10 es muy clara.

1 no se aprende nada y 10 se aprende mucho.

- Preformal del teorema 1: $F_{ـ}, G_{\_}, C_{\_}, A_{-}$.

- Formal del teorema 1: $F_{\_}, G_{\_}, C_{\_}, A_{-}$.

- Preformal del teorema 2: $F_{\_}, G_{\_}, C_{\_}, A_{-}$.

- Formal del teorema 2: $F_{\_}, G_{\_}, C_{\_}, A_{-}$.

En la columna COMPRENSIÓN: se reescriben las puntuaciones de la cuestión 2. En la columna PREFERENCIA: para cada teorema, comparando las puntuaciones de cada modalidad de prueba en cada una de las cuatro categorías, se indica el número de veces en que "gana” (supera) la prueba preformal a la formal, y viceversa. 


\begin{tabular}{|c|c|c|c|}
\hline TEOREMA & MODALIDAD & COMPRENSIÓN & PREFERENCIA \\
\hline \multirow{2}{*}{ Teorema 1} & Preformal & F_, G_, C, _, A _ & \\
\hline & Formal & $F_{-}, G_{-}, C_{-}, A_{-}$ & \\
\hline \multirow{2}{*}{ Teorema 2} & Preformal & $F_{-}, G_{-}, C_{-}, A_{-}$ & \\
\hline & Formal & F__, G_, $C_{-}, A_{-}$ & \\
\hline \multirow{2}{*}{ Total } & Preformal & & \\
\hline & Formal & & \\
\hline
\end{tabular}

Tabla 4.4. Esquema de la pregunta $2^{\mathrm{a}}$ del cuestionario

El objetivo de esta cuestión es, dentro de la comprensión, conocer la preferencia de los alumnos por una u otra de las modalidades de prueba en relación con cuatro importantes categorías: su facilidad, su claridad, el gusto por ellas y su influjo en el aprendizaje del alumno.

\subsubsection{PREFERENCIA}

\section{(F: facilidad; G: gusto; C: claridad; A: aprendizaje)}

En la tabla siguiente (4.5) aparecen los resultados de las respuestas de los alumnos sobre la significación de las pruebas según las categorías asignadas. En las columnas destinadas a preferencia aparecen reflejadas con sus iniciales las categorías preferidas por los alumnos en cada modalidad de prueba y para cada teorema. En las columnas destinadas a puntuación se consigna en cada casilla el número de categorías preferidas en cada modalidad de prueba y para cada teorema. 


\begin{tabular}{|c|c|c|c|c|c|c|c|c|}
\hline $\begin{array}{l}\mathbf{A} \\
\mathbf{L}\end{array}$ & \multicolumn{4}{|c|}{ TEOREMA 1} & \multicolumn{4}{|c|}{ TEOREMA 2} \\
\hline $\mathbf{M}$ & \multicolumn{2}{|c|}{ PREFORMAL } & \multicolumn{2}{|c|}{ FORMAL } & \multicolumn{2}{|c|}{ PREFORMAL } & \multicolumn{2}{|c|}{ FORMAL } \\
\hline $\begin{array}{l}\mathbf{N} \\
\mathbf{0}\end{array}$ & $\begin{array}{l}\text { Prefe- } \\
\text { rencia }\end{array}$ & $\begin{array}{c}\text { Pun- } \\
\text { tuación }\end{array}$ & $\begin{array}{l}\text { Prefe- } \\
\text { rencia }\end{array}$ & $\begin{array}{c}\text { Pun- } \\
\text { tuación }\end{array}$ & $\begin{array}{l}\text { Prefe- } \\
\text { rencia }\end{array}$ & $\begin{array}{c}\text { Pun- } \\
\text { tuación }\end{array}$ & $\begin{array}{l}\text { Prefe- } \\
\text { rencia }\end{array}$ & $\begin{array}{c}\text { Pun- } \\
\text { tuación }\end{array}$ \\
\hline 1 & - & - & $\mathrm{G}, \mathrm{C}, \mathrm{A}$ & 3 & $\mathrm{~A}$ & 1 & - & - \\
\hline 2 & $\mathrm{~F}, \mathrm{C}, \mathrm{A}$ & 3 & G & 1 & $\mathrm{~F}, \mathrm{G}, \mathrm{C}, \mathrm{A}$ & 4 & - & - \\
\hline 3 & $\mathrm{~F}, \mathrm{~A}$ & 2 & - & - & $\mathrm{A}$ & 1 & - & - \\
\hline 4 & $\mathrm{~F}, \mathrm{G}, \mathrm{C}, \mathrm{A}$ & 4 & - & - & $\mathrm{F}, \mathrm{G}, \mathrm{C}, \mathrm{A}$ & 4 & - & - \\
\hline 5 & - & - & $\mathrm{G}, \mathrm{C}, \mathrm{A}$ & 3 & $\mathrm{~F}, \mathrm{C}$ & 2 & G & 1 \\
\hline 6 & $\mathrm{~F}, \mathrm{G}, \mathrm{C}$ & 3 & - & - & $\mathrm{F}, \mathrm{G}, \mathrm{C}, \mathrm{A}$ & 4 & - & - \\
\hline 7 & $\mathrm{~F}, \mathrm{G}, \mathrm{C}, \mathrm{A}$ & 4 & - & - & $\mathrm{F}, \mathrm{G}, \mathrm{A}$ & 3 & $\mathrm{C}$ & 1 \\
\hline 8 & $\mathrm{~F}, \mathrm{G}, \mathrm{C}, \mathrm{A}$ & 4 & - & - & $\mathrm{F}, \mathrm{G}, \mathrm{C}, \mathrm{A}$ & 4 & - & - \\
\hline 9 & - & - & $\mathrm{F}, \mathrm{G}, \mathrm{C}, \mathrm{A}$ & 4 & $\mathrm{~F}, \mathrm{G}, \mathrm{C}, \mathrm{A}$ & 4 & - & - \\
\hline 10 & - & - & $\mathrm{F}, \mathrm{G}, \mathrm{C}, \mathrm{A}$ & 4 & $\mathrm{~F}, \mathrm{G}, \mathrm{C}, \mathrm{A}$ & 4 & - & - \\
\hline 11 & - & - & $\mathrm{F}, \mathrm{G}, \mathrm{C}, \mathrm{A}$ & 4 & - & - & $\mathrm{G}, \mathrm{C}, \mathrm{A}$ & 3 \\
\hline 12 & - & - & $\mathrm{G}, \mathrm{C}, \mathrm{A}$ & 3 & $\mathrm{~F}, \mathrm{C}, \mathrm{A}$ & 3 & - & - \\
\hline 13 & $\mathrm{~F}, \mathrm{G}, \mathrm{C}$ & 3 & $\mathrm{~A}$ & 1 & $\mathrm{~F}, \mathrm{G}, \mathrm{C}, \mathrm{A}$ & 4 & - & - \\
\hline 14 & $\mathrm{~F}$ & 1 & - & - & - & - & $\mathrm{F}$ & 1 \\
\hline 15 & - & - & $\mathrm{F}, \mathrm{G}, \mathrm{C}$ & 3 & - & - & $\mathrm{F}, \mathrm{C}$ & 2 \\
\hline 16 & $\mathrm{~F}, \mathrm{C}$ & 2 & $\mathrm{G}, \mathrm{A}$ & 2 & $\mathrm{~F}, \mathrm{G}, \mathrm{C}$ & 3 & - & - \\
\hline 17 & - & - & $\mathrm{F}, \mathrm{C}, \mathrm{A}$ & 3 & $\mathrm{~F}, \mathrm{G}, \mathrm{C}, \mathrm{A}$ & 4 & - & - \\
\hline 18 & $\mathrm{~F}, \mathrm{G}$ & 2 & $\mathrm{C}, \mathrm{A}$ & 2 & - & - & $\mathrm{F}, \mathrm{G}, \mathrm{C}, \mathrm{A}$ & 4 \\
\hline 19 & $\mathrm{~F}, \mathrm{G}, \mathrm{C}, \mathrm{A}$ & 4 & - & - & $\mathrm{F}, \mathrm{G}, \mathrm{C}, \mathrm{A}$ & 4 & - & - \\
\hline 20 & - & - & $\mathrm{G}, \mathrm{C}, \mathrm{A}$ & 3 & $\mathrm{~F}, \mathrm{C}, \mathrm{A}$ & 3 & $\mathrm{G}$ & 1 \\
\hline 21 & $\mathrm{G}, \mathrm{C}$ & 2 & - & - & $\mathrm{F}$ & 1 & $\mathrm{C}$ & 1 \\
\hline 22 & $\mathrm{~F}, \mathrm{G}, \mathrm{C}$ & 3 & - & - & $\mathrm{F}, \mathrm{G}, \mathrm{C}, \mathrm{A}$ & 4 & - & - \\
\hline 23 & $\mathrm{G}, \mathrm{A}$ & 2 & $\mathrm{~F}, \mathrm{C}$ & 2 & - & - & $\mathrm{F}, \mathrm{C}$ & 2 \\
\hline 24 & - & - & $\mathrm{F}, \mathrm{G}, \mathrm{C}, \mathrm{A}$ & 4 & - & - & $\mathrm{F}, \mathrm{G}, \mathrm{C}, \mathrm{A}$ & 4 \\
\hline 25 & - & - & $\mathrm{F}, \mathrm{G}, \mathrm{C}, \mathrm{A}$ & 4 & $\mathrm{~F}, \mathrm{G}, \mathrm{C}$ & 3 & A & 1 \\
\hline 26 & $\mathrm{~F}, \mathrm{G}, \mathrm{C}$ & 3 & - & - & $\mathrm{F}, \mathrm{C}$ & 2 & - & - \\
\hline 27 & $\mathrm{~F}, \mathrm{G}, \mathrm{C}, \mathrm{A}$ & 4 & - & - & - & - & $\mathrm{F}, \mathrm{G}, \mathrm{C}, \mathrm{A}$ & 4 \\
\hline 28 & $\mathrm{C}, \mathrm{A}$ & 2 & - & - & - & - & - & - \\
\hline $\begin{array}{l}\text { To- } \\
\text { tal }\end{array}$ & & 48 & & 46 & & 62 & & 25 \\
\hline
\end{tabular}

Tabla 4.5. Preferencias y puntuaciones del primer ciclo según categorías de las pruebas preformal y formal en ambos teoremas

El gráfico de la figura siguiente muestra la frecuencia de las preferencias de las pruebas preformal y formal para los dos teoremas, y la suma total de ambas frecuencias. 


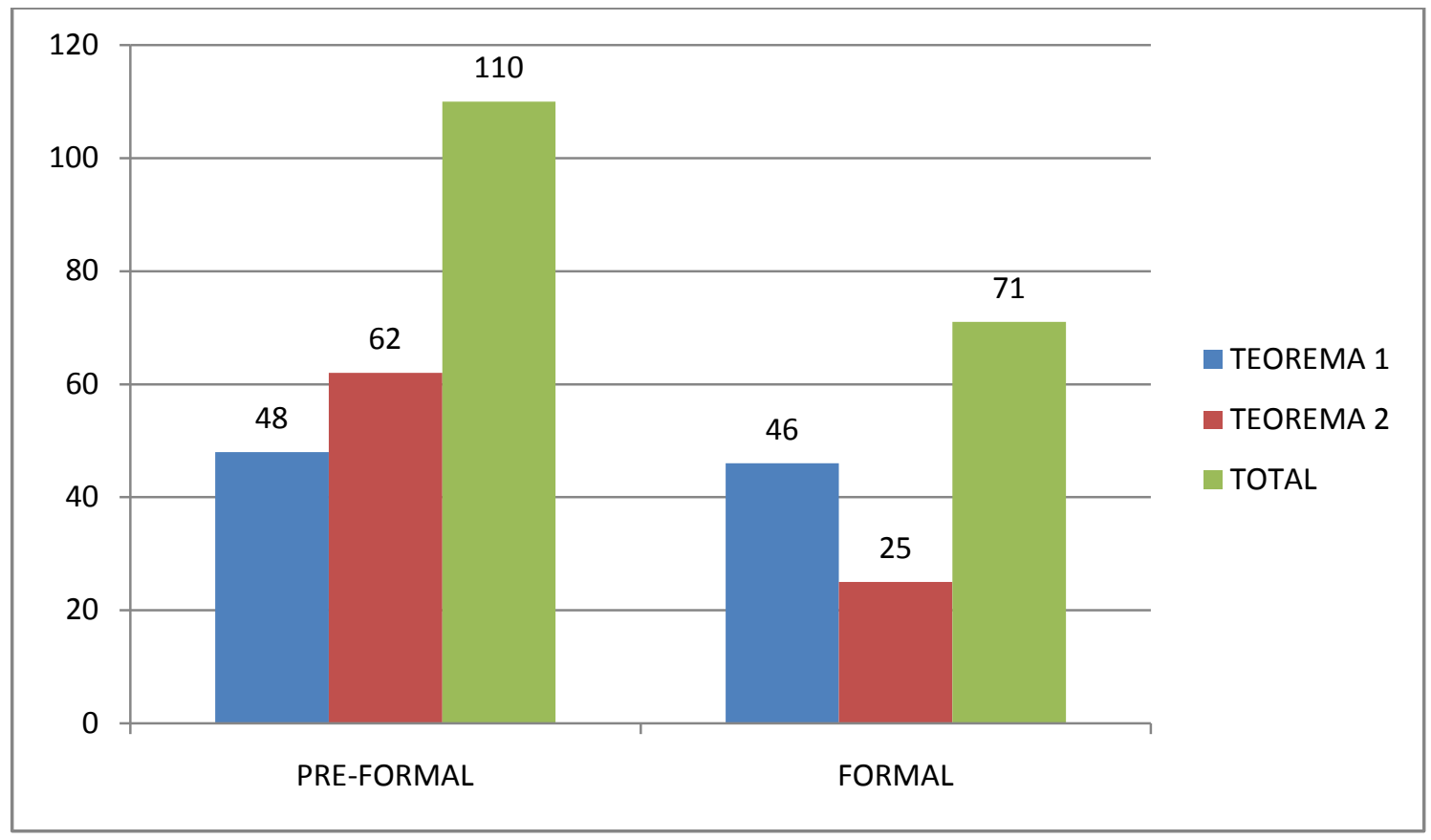

\section{Gráfico 4.1. Preferencia por las categorías de las pruebas, ciclo I}

Los veintiocho alumnos piensan que son diferentes los procedimientos en alguna de las cuatro categorías. Sólo hay uno (el n⿳0 14, alumno que resulta muy poco significativo dentro de este conjunto) que diferencia una sola categoría, y tres más (3, $21,28)$ que señalan dos diferencias.

Tomados en conjunto ambos teoremas, destaca la preferencia de la prueba preformal $(48+62=110)$ sobre la formal $(46+25=71)$. En porcentaje, $60,8 \%$ frente a $39,2 \%$.

Destaca asimismo la diferencia de preferencia en el caso del teorema del valor medio (Lagrange), 48 vs. 46, y la diferencia notable 62 vs. 25 (71,3\% frente a 28,7\%) en el caso del teorema de la unicidad del límite. El orden de aplicación de las pruebas fue preformal-formal en el primer teorema y formal-preformal en el segundo teorema. El orden de la aplicación de las pruebas influye en la valoración de los alumnos. 


\subsubsection{SIGNIFICACIÓN}

Nos centramos ahora en las categorías que hacen referencia a la significación de ambos tipos de prueba (convencimiento, entendimiento, memorización y aplicabilidad de las dos modalidades de prueba). Es decir, su grado de persuasión y seguridad, su contribución a aprehender significativamente, a retener y no olvidar los teoremas y a la aplicación de los mismos en diferentes situaciones, respectivamente.

Cuestión 3: Responde a las siguientes preguntas, señalando en cada caso claramente el porqué:

- ¿Cuál de las dos formas te convence más de la veracidad del teorema?

- ¿Cuál de las dos pruebas puede ayudar más a entender el significado del teorema?

- ¿Cuál de las dos pruebas puede ayudar más a memorizar el teorema?

- ¿Cuál de las dos pruebas es más útil para ilustrar cómo se aplica el teorema?

El objetivo de esta cuestión es, detectar la contribución de la demostración respecto a la significación de los teoremas (Hanna, 1989). Para ello, los alumnos comparan los dos procedimientos en relación con la capacidad de convicción, la ayuda a la comprensión, memorización y utilización de los teoremas.

La tabla siguiente resume las respuestas de los 28 alumnos a la pregunta 3 del cuestionario. Cada alumno (en este ciclo, así como en los ciclos II y III) viene designado por un número con el fin de facilitar las referencias posteriores a dicho alumno. Los totales se muestran en el gráfico posterior a la tabla.

En adelante PF designa prueba formal y PPF prueba preformal. 


\begin{tabular}{|c|c|c|c|c|}
\hline Alumno & $\begin{array}{c}\text { Convence } \\
\text { más }\end{array}$ & $\begin{array}{c}\text { Ayuda más a } \\
\text { entender }\end{array}$ & $\begin{array}{l}\text { Ayuda más a } \\
\text { memorizar }\end{array}$ & $\begin{array}{c}\text { Más útil para } \\
\text { aplicar el teorema }\end{array}$ \\
\hline 1 & $\mathrm{PF}$ & PPF & PPF PF & PPF \\
\hline 2 & PPF & PPF & PPF PF & PPF \\
\hline 3 & $\mathrm{PF}$ & PF & PPF & PPF \\
\hline 4 & PPF & PPF & $\mathrm{PF}$ & PPF \\
\hline 5 & $\mathrm{PF}$ & PPF & $\mathrm{PF}$ & PPF \\
\hline 6 & $\mathrm{PF}$ & PPF & $\mathrm{PF}$ & PPF \\
\hline 7 & PPF & PPF & $\mathrm{PF}$ & PPF \\
\hline 8 & $\mathrm{PF}$ & PPF & PPF & PPF \\
\hline 9 & $\mathrm{PF}$ & PPF PF & PF & PPF \\
\hline 10 & $\mathrm{PF}$ & PPF & PPF & PPF \\
\hline 11 & $\mathrm{PF}$ & PPF PF & $\mathrm{PF}$ & PPF \\
\hline 12 & $\mathrm{PF}$ & PPF & $\mathrm{PF}$ & PPF \\
\hline 13 & PPF & $\mathrm{PF}$ & PF & PPF \\
\hline 14 & - & - & - & - \\
\hline 15 & $\mathrm{PF}$ & PPF & $\mathrm{PF}$ & PPF PF \\
\hline 16 & $\mathrm{PF}$ & $\mathrm{PF}$ & PPF PF & PPF \\
\hline 17 & - & - & - & - \\
\hline 18 & $\mathrm{PF}$ & PPF & PF & PPF \\
\hline 19 & PPF & PPF & $\mathrm{PF}$ & PPF \\
\hline 20 & $\mathrm{PF}$ & $\mathrm{PF}$ & $\mathrm{PF}$ & PPF \\
\hline 21 & $\mathrm{PF}$ & PPF & $\mathrm{PF}$ & PPF \\
\hline 22 & $\mathrm{PF}$ & PPF & PPF PF & PPF \\
\hline 23 & PPF & $\mathrm{PF}$ & $\mathrm{PF}$ & PPF \\
\hline 24 & PF & $\mathrm{PF}$ & $\mathrm{PF}$ & PF \\
\hline 25 & $\mathrm{PF}$ & $\mathrm{PF}$ & PF & PPF \\
\hline 26 & PPF & $\mathrm{PF}$ & $\mathrm{PF}$ & PPF \\
\hline 27 & PPF & PPF & $\mathrm{PF}$ & PPF \\
\hline 28 & PPF & PPF & PPF & PPF \\
\hline PF/PPF & $17 / 9$ 2nc & $\mathbf{1 0 / 1 8} 2 \mathrm{nc}$ & $22 / 82 n c$ & $2 / 252 n c$ \\
\hline PF /PPF (\%) & $65,4 / 34,6$ & $35,7 / 64,3$ & $73,3 / 26,7$ & $7,4 / 92,6$ \\
\hline
\end{tabular}

Tabla 4.6. Preferencias sobre significación: convencimiento, entendimiento, memorización y utilidad de las PPF y PF, ciclo I 


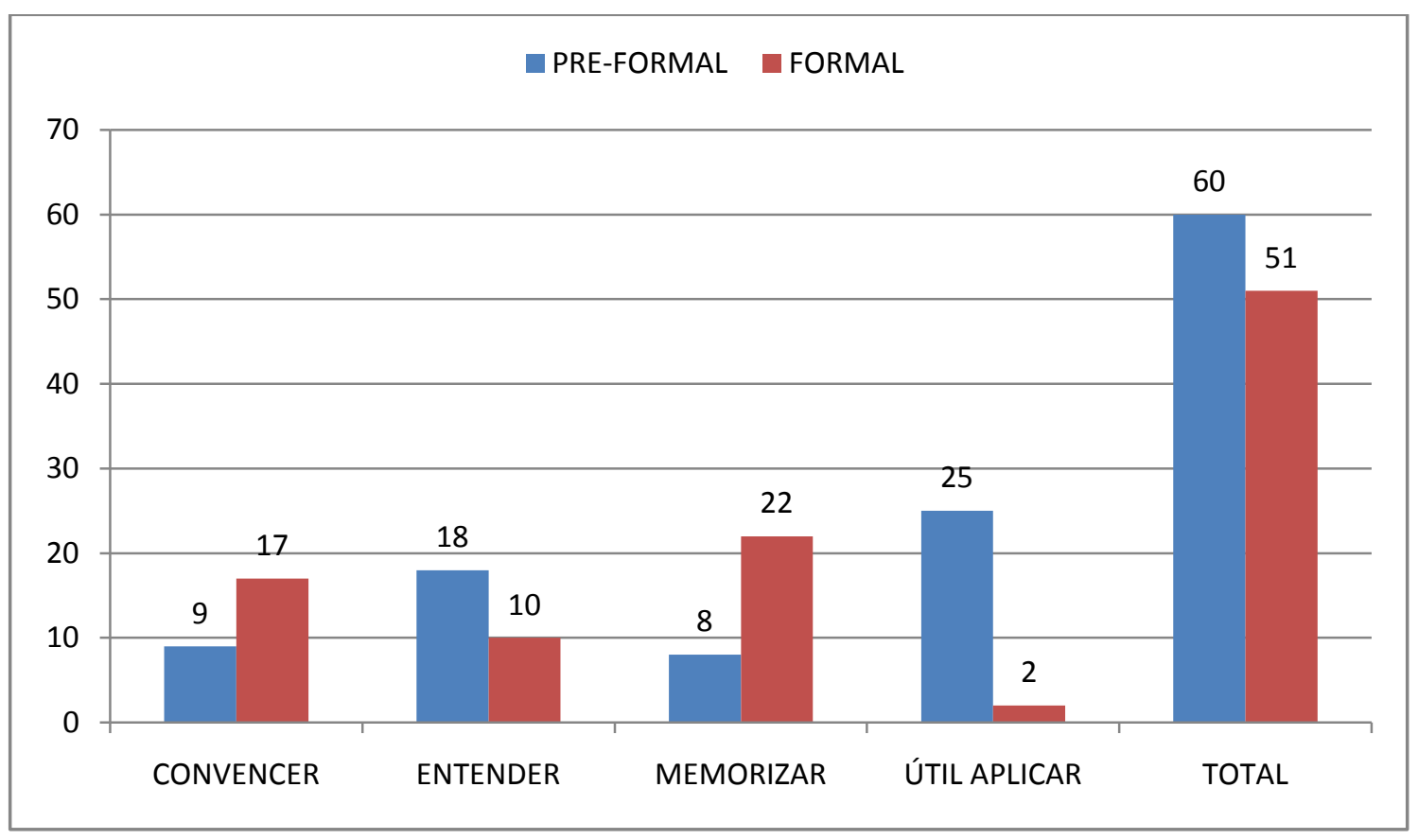

Gráfico 4.2. Preferencias por la significación de las pruebas PPF y PF, ciclo I

1) Destaca la casi unanimidad de las respuestas (92,6\%) sobre la utilidad de la prueba PPF a la hora de aplicar el teorema. La aplicación es importante para estos alumnos que cursan una carrera técnica. Supone un valor añadido a favor de la prueba PPF.

2) Prescindiendo de los dos alumnos que no contestan, la experiencia queda reducida a veintiséis respuestas. Han sido recogidas y cuantificadas las respuestas dobles, i. e. que señalan las dos pruebas PF y PPF, es decir la preferencia se decanta a favor de las dos pruebas, no de una sobre la otra.

3) Destaca también el alto porcentaje (64,3\%) dado a la prueba PPF en la respuesta a la pregunta sobre la ayuda que las pruebas ofrecen para el aprendizaje (entender) del teorema. Indica que la prueba PPF es de más fácil comprensión que la prueba PF, y por ello facilita más el aprendizaje del teorema a los alumnos. 
4) Relacionando los porcentajes de las tres respuestas (convence más, ayuda más a entender, ayuda más a memorizar), destaca cierta contradicción entre el 64,3\% a favor de la prueba PPF en la respuesta a la mayor ayuda para el aprendizaje y el porcentaje a favor de la prueba PF en las otras dos respuestas, convence más y ayuda más a memorizar, 65,4\% y 73,3\%, respectivamente. ¿Cómo convence más si se entiende menos? y también ¿cómo se memoriza mejor si no es la prueba con la que mejor se ha comprendido?

5) Tres alumnos optan por la misma prueba en todas las respuestas a las cuatro cuestiones planteadas: el no 28 opta por la prueba PPF; el no 2 también, aunque en cuanto a la ayuda para memorizar se decanta asimismo por la PF; el no 24 opta por la PF y éste es el único que prefiere la prueba PF, solamente, como más útil para aplicar el teorema.

6) Cinco alumnos $(2,4,7,19$ y 27) eligen la prueba PPF como respuesta a las dos primeras cuestiones, convence más y ayuda más a entender el teorema. Otros cinco $(3,9$ (que señala ambas pruebas como la mejor ayuda para entender), 16, 20 y 25) están a favor de la prueba PF en la respuesta a estas dos cuestiones. Los cinco primeros, que prefieren la prueba PPF como idéntica respuesta a las dos cuestiones indicadas, son más coherentes que los otros cinco que prefieren la PF, pues ciertamente lo que se entiende mejor es lo que convence más. Los cinco alumnos, que optan por la prueba PF como respuesta a estas dos cuestiones, incurren en la contradicción que antes apuntábamos en 4) ¿cómo convence más la prueba PF si se entiende menos?

7) Un grupo de cinco más ocho alumnos, en las respuestas a la segunda y tercera cuestión (ayuda más a entender-ayuda más a memorizar), señalan a la misma prueba como respuesta en ambas cuestiones: los alumnos 1, 2, 8, 10 y 22 la prueba PPF; los alumnos 9, 11, 13, 16, 20, 23, 25 y 26 eligen, por el contrario, la prueba PF. En este caso, todos estos trece alumnos que responden con la misma prueba PPF o PF a las dos cuestiones citadas, son todos coherentes, pues es cierto que siempre contribuirá mejor a la memorización lo que se ha entendido mejor (los alumnos 9 y 11 señalan ambas 
pruebas PPF y PF como respuesta a la cuestión sobre la ayuda a entender, y los alumnos 1, 2, 16 y 22 señalan ambas pruebas PPF y PF en la respuesta a la cuestión sobre la ayuda a memorizar).

8) Finalmente, existe un grupo formado por diez alumnos $(1,5,6,9,11,12,15$, 18, 21 y 22) que responden al conjunto de las tres cuestiones (convence más-ayuda más a entender-ayuda más a memorizar) señalando todos a la prueba PPF como la que más ayuda a entender y la prueba PF en las respuestas a las otras dos cuestiones (convence más-ayuda más a memorizar). Es de notar la elección por todos de la prueba PPF como la que más les ayuda a entender el teorema. Pero incurren en dos incoherencias ya apuntadas anteriormente en 4): que les convence más lo que entienden peor y memorizan mejor lo que peor han comprendido. Si es la prueba PPF la que comprenden mejor ¿cómo convence más la prueba PF que se entiende menos? y, por lo mismo, ¿cómo lo que menos entienden les ayuda más a memorizar?

Veamos con más detalle los motivos en que se apoyan estas respuestas.

\subsubsection{CUÁL DE LAS DOS FORMAS TE CONVENCE MÁS DE LA VERACIDAD DEL TEOREMA}

\section{RESUMEN}

Las respuestas a esta cuestión se reparten en nueve alumnos que prefieren la PPF, diecisiete que se inclinan a favor de la PF y dos $(14,17)$ contestaciones irrelevantes.

\begin{tabular}{|c|c|c|c|c|c|c|c|}
\hline & \multirow{2}{*}{$\begin{array}{c}\text { Ambas } \\
\text { pruebas }\end{array}$} & \multicolumn{2}{|c|}{ Teorema 1} & \multicolumn{2}{|c|}{ Teorema 2} & \multirow{2}{*}{ PPF } & \multirow{2}{*}{ PF } \\
\hline & & PPF & PF & PPF & PF & & \\
\hline $\begin{array}{c}\text { № de } \\
\text { Alumnos }\end{array}$ & 0 & 0 & 0 & 0 & 0 & 9 & 17 \\
\hline Identif. & & & & & & $\begin{array}{l}2,4,7,13,19 \\
23,26,27,28\end{array}$ & $\begin{array}{c}1,3,5,6,8,9,10,11,12,15 \\
16,18,20,21,22,24,25\end{array}$ \\
\hline
\end{tabular}

Tabla 4.7. Respuesta a la cuestión convence más, ciclo I 


\section{MOTIVOS}

Estos son los motivos aportados por los que prefieren la PPF:

- Las cosas en la vida se entienden mejor con casos prácticos, aunque no sirve el caso aislado como demostración, no tiene la misma validez matemáticamente que la PF (2).

- Por demostrarse cierto en un caso, lo que es imposible con la PF sin valores concretos no puedo comprobar su veracidad con constantes (4).

- Con un ejemplo se ve todo mejor $(7,13)$.

- Al realizarse con números, la idea ya está presente en la forma que más me convence (23).

- Se demuestra a base de números (26).

- Es más concisa (7).

- $\quad$ Es más clara $(19,26)$.

En síntesis: concreción, sobre todo; también claridad, concisión.

Los que optan por la PF aducen estos motivos:

- Es general, para todos los casos. Por lógica al abarcar todos los casos convence más, se puede aplicar a cualquier caso, a cualquier función que cumpla las condiciones, para cualquier valor de las variables $(6,8,10,11,15,18$, $21,22)$.

- La PF es más concreta, pero la PPF puede ayudar a hacerse una idea rápida, sin ser muy específico (9).

- Enuncia el teorema de forma más técnica y precisa, aunque la PPF demuestra la veracidad con un ejemplo (12).

- Es más factible (18).

- La PPF no sería suficiente, tendríamos que probar todos los casos (3). En la PPF puede quedar la duda de si se cumplirá para cualquier función o solo la del ejemplo (6). No da la sensación de ser general por tratarse de un caso concreto (16).

En síntesis, la PF es: general, más técnica y precisa, más concreta, más factible. 
Parecen muy acordes con las características de la PPF los motivos señalados. Ciertamente esta prueba se apoya en datos concretos, lo que para estos alumnos les ha de proporcionar más claridad e incluso sencillez, que quizá es lo que pretenden señalar al caracterizarla como más concisa.

También es acorde con la PF ver esa "generalización" que, con diversas expresiones, aplican a esta prueba la mayoría de los que optan por ella; no están tan acertados en lo que niegan a la PPF en sus reflexiones un tanto precipitadas.

\subsubsection{CUÁl DE LAS DOS PRUEBAS PUEDE AYUDAR MÁS A ENTENDER EL SIGNIFICADO DEL TEOREMA}

\section{RESUMEN}

Las respuestas de los alumnos a este tema se reparten entre dieciséis que prefieren la PPF, ocho la PF, dos que optan por ambas pruebas y dos $(14,17)$ que no responden a la cuestión propuesta.

\begin{tabular}{|c|c|c|c|c|c|c|c|}
\hline & \multirow{2}{*}{$\begin{array}{c}\text { Ambas } \\
\text { pruebas }\end{array}$} & \multicolumn{2}{|c|}{$\begin{array}{c}\text { Teorema } \\
\mathbf{1}\end{array}$} & \multicolumn{2}{c|}{$\begin{array}{c}\text { Teorema } \\
\mathbf{2}\end{array}$} & PPF & PF \\
\cline { 3 - 6 } & PPF & PF & PPF & PF & & 10 \\
\hline $\begin{array}{c}\text { No de } \\
\text { Alumnos }\end{array}$ & 2 & 0 & 0 & 0 & 0 & 18 & 10 \\
\hline Identif. & 9,11 & & & & & $\begin{array}{c}1,2,4,5,6,7,8,9,10,11,12, \\
15,18,19,21,22,27,28\end{array}$ & $\begin{array}{c}3,9,11,13,16,20, \\
23,24,25,26\end{array}$ \\
\hline
\end{tabular}

Tabla 4.8. Respuesta a la cuestión ayuda más a entender el significado, ciclo I

\section{MOTIVOS}

Los dieciséis alumnos que prefieren sola la PPF, apoyan su opción en estos motivos:

- Más fácil asimilarlo al verlo en un caso práctico (1). 
- Los ejemplos numéricos clarifican; las letras griegas y múltiples variables complican bastante la comprensión (2).

- Más fácil, se ve mejor al ser un caso determinado $(5,6,28)$.

- Un ejemplo es más fácil de comprender, la mejor manera, usa valores reales $(10,12,15,19,21,22)$.

- Más intuitiva (8).

- Es directa y visual, posee la idea central del teorema (4).

- Me sirve para verlo antes, la PF también es necesaria (18).

El alumno 7 no aduce motivo alguno, el 27 tampoco y sólo dice "sobre todo en el teorema de Lagrange".

Los motivos señalados se concentran en su mayoría en la facilidad y claridad de la PPF por utilizar valores concretos, reales, un caso práctico, un ejemplo y resultar así más intuitiva, visual, directa y que anticipa verlo (entenderlo) antes.

En resumen, la PPF aparece en esta cuestión para estos alumnos, más fácil, clara e intuitiva.

Para dos alumnos, 9 y 11, la preferencia es en esta cuestión para las dos pruebas, PF y PPF. Sus motivos:

- La PPF es bastante intuitiva, la PF clara por extrapolable a todos los casos (9); ambas pruebas se complementan (11).

Ocho alumnos prefieren sola la PF en sus respuestas apoyados en que:

- La PPF tendría que formalizarla. Me ayudan las dos, PF como demostración y PPF como aplicación (3).

- $\quad$ La PF es más técnica, la PPF más visual (20).

- Me da una visión más amplia del teorema (16).

- Lo veo mejor en la forma generalizada del teorema (23).

- Es más estructurada y tiene más que ver con el teorema (26). 
Los alumnos 13, 24, 25 no manifiestan ningún motivo de su preferencia; el 3 y el 20 afirman ventajas con ambas pruebas; los motivos de los alumnos 16, 23, 26 se resumen en considerar esta prueba, $\mathrm{PF}$, más estructurada y generalizada.

Al tratarse de la ayuda para entender el significado del teorema, estos alumnos optan, de hecho en un 64,3\%, a favor de la prueba que realmente les supone mayor ayuda, la PPF. Esto muestra el alto grado de significación que esta prueba tiene para ellos en una cuestión tan decisiva en el aprendizaje de las matemáticas: entender el significado de los teoremas.

\subsubsection{CUÁL DE LAS DOS PRUEBAS PUEDE AYUDAR MÁS A MEMORIZAR EL TEOREMA}

\section{RESUMEN}

En esta cuestión de nuevo se han respetado las expresiones literales de los alumnos. De ellos, dos o no contestan o su respuesta es irrelevante; cuatro prefieren las dos pruebas PF y PPF; otros cuatro optan por la PPF; y el resto, trece señalando motivo y cinco sin señalarlo, prefieren la PF. Un alumno (2) especificó que le ayudaba más a memorizar la PF en el teorema 1 y la PPF en el teorema 2.

\begin{tabular}{|c|c|c|c|c|c|c|c|}
\hline & \multirow{2}{*}{$\begin{array}{c}\text { Ambas } \\
\text { pruebas }\end{array}$} & \multicolumn{2}{|c|}{ Teorema1 } & \multicolumn{2}{|c|}{ Teorema2 } & \multirow{2}{*}{ PPF } & \multirow{2}{*}{ PF } \\
\hline $\begin{array}{c}\text { No de } \\
\text { Alumnos }\end{array}$ & 4 & 0 & 1 & 1 & 0 & 8 & 22 \\
\hline & & & PPF & PPF & PF & \\
Identif. & $\begin{array}{c}1,2,16, \\
22\end{array}$ & & 2 & 2 & & $\begin{array}{c}1,2,3,8, \\
10,16, \\
22,28\end{array}$ & $\begin{array}{c}1,2,4,5,6,7,9,11,12,13,18,19,20,21,22, \\
23,24,25,26,27\end{array}$ \\
\hline
\end{tabular}

Tabla 4.9. Respuesta a la cuestión ayuda más a memorizar el teorema, ciclo I 


\section{MOTIVOS}

Los que prefieren ambas pruebas, lo motivan así:

- Es necesario prueba estricta y ejemplo práctico (1).

- Se memoriza mejor lo que se entiende y lo más corto: en el teorema 1 la PF y en el teorema 2 la PPF (2).

- $\quad$ La PF es más impactante, la PPF ayuda por su claridad (16).

Afirmaciones espontáneas, un tanto subjetivas, de estos alumnos.

Los que prefieren la PPF lo razonan así:

- Se memoriza mejor por ser una aplicación directa (3).

- Por ser mucho más intuitiva y entenderla antes (8).

- $\quad$ Los números te ayudan (10).

Aparece, una vez más, lo concreto (así lo captan estos alumnos) de esta prueba: números, aplicación, prueba más intuitiva.

La preferencia de los alumnos por la PF se basa en los motivos siguientes:

- $\quad$ Es general $(4,6,7,9,13,15,21,23,26)$.

- Es más clara, se ve mejor el desarrollo teórico $(11,27)$.

- Explicación técnica que asegura mejor evitar errores (12).

- Es más corta que la PPF (19).

Cinco alumnos $(5,18,20,22,25)$ no aducen razones.

El motivo más repetido es considerar a la prueba PF general, como una "fórmula" válida para muchos casos, en contraste con la PPF que la entienden como un caso concreto, un ejemplo. Otros motivos menos repetidos: es más clara, se ve mejor el desarrollo teórico, más corta, con mayor garantía de evitar errores. 


\subsubsection{CUÁL DE LAS DOS PRUEBAS ES MÁS ÚTIL PARA ILUSTRAR CÓMO SE APLICA EL TEOREMA}

\section{RESUMEN}

Son veinticuatro, los alumnos que en esta cuestión eligen sola la PPF como más útil para ilustrar cómo se aplica el teorema. De estos, tres no indican motivo alguno. Un alumno, el 15, señala a las dos pruebas en su respuesta afirmando que "la PF lo explica de un modo general", pero cree que "es necesaria la PPF para demostrarlo a modo de ejemplo". Y sólo un alumno, el 24, sin manifestar motivo alguno, opta por la PF.

\begin{tabular}{|c|c|c|c|c|c|c|c|}
\hline & \multirow{2}{*}{$\begin{array}{c}\text { Ambas } \\
\text { pruebas }\end{array}$} & \multicolumn{2}{|c|}{ Teorema 1} & \multicolumn{2}{|c|}{ Teorema 2} & \multirow{2}{*}{ PPF } & \multirow{2}{*}{$\mathbf{P F}$} \\
\hline & & PPF & $\mathbf{P F}$ & PPF & PF & & \\
\hline $\begin{array}{c}\text { No de } \\
\text { Alumnos }\end{array}$ & 1 & 0 & 0 & 0 & 0 & 25 & 2 \\
\hline Identif. & 15 & & & & & $\begin{array}{c}1,2,3,4,5,6,7,8,9,10,11 \\
12,13,15,16,18,19,20 \\
21,22,23,25,26,27,28\end{array}$ & 15,24 \\
\hline
\end{tabular}

Tabla 4.10. Respuesta a la cuestión útil para ilustrar la aplicación del teorema, ciclo I

\section{MOTIVOS}

Los motivos de los que están a favor de la PPF son los siguientes:

- Con algunas expresiones como sin duda, evidentemente, sin duda alguna, sin lugar a dudas $(8,11,12,13,27)$ la mayoría atribuye a la PPF ser la mejor en esta cuestión por realizarse con valores definidos, números concretos, caso concreto, ejemplo aplicado, caso práctico, ejemplo numérico, datos reales, situaciones concretas $(2,3,7,9,10,18,19,22,23)$.

- El alumno 4 manifiesta que "eso es su función (de la PPF), ilustrar una aplicación concreta del teorema".

- Otros destacan los motivos de la mayor claridad de esta prueba (5), más sencilla gráficamente (26), ayuda más a entender el teorema y su aplicación $(20,21)$. 
En definitiva, siguen estos alumnos valorando ese matiz de mayor concreción que caracteriza a la PPF en relación con la PF que es más general, más abstracta. Esto hace que la PPF se les ofrezca más sencilla, más atractiva (un alumno, el 28, afirma espontáneamente "la PPF es más de mi gusto"), y en consecuencia, podemos afirmar, para estos alumnos también más significativa.

\begin{tabular}{|c|c|c|c|c|c|c|c|}
\hline \multicolumn{8}{|c|}{ RESUMEN DE LAS CUATRO PREGUNTAS (28 alumnos) } \\
\hline \multirow[t]{2}{*}{ Qué prueba ayuda más a... } & \multirow[t]{2}{*}{ PPF } & \multirow[t]{2}{*}{$\mathbf{P F}$} & \multicolumn{2}{|c|}{$\begin{array}{c}\text { Teorema } \\
\quad 1\end{array}$} & \multicolumn{2}{|c|}{$\begin{array}{l}\text { Teorema } \\
\quad 2\end{array}$} & \multirow[t]{2}{*}{ Ambas } \\
\hline & & & PPF & $\mathbf{P F}$ & PPF & $\mathbf{P F}$ & \\
\hline $\begin{array}{c}\text {...convencer de la veracidad del } \\
\text { teorema }\end{array}$ & 9 & 17 & 0 & 0 & 0 & 0 & 0 \\
\hline $\begin{array}{c}\text {...entender el significado del } \\
\text { teorema }\end{array}$ & 18 & 10 & 0 & 0 & 0 & 0 & 2 \\
\hline ...memorizar el teorema & 8 & 22 & 0 & 1 & 1 & 0 & 4 \\
\hline ...ilustrar la aplicación del teorema & 25 & 2 & 0 & 0 & 0 & 0 & 1 \\
\hline Tot a l & 60 & 51 & $\mathbf{0}$ & 1 & 1 & $\mathbf{0}$ & 7 \\
\hline
\end{tabular}

Tabla 4.11. Resumen de las cuatro respuestas anteriores, ciclo I

\section{CONSIDERACIÓN GENERAL}

Las preferencias de estos alumnos en las cuatro cuestiones que se les han presentado están, en términos generales, en torno al 54\% a favor de la PPF. Los alumnos prefieren la PF en las cuestiones relativas al convencimiento de la veracidad del teorema y a la memorización. Se inclinan por la conjunción de ambas pruebas: en la cuestión segunda, dos alumnos (9 y 11); en la cuestión tercera, cuatro alumnos (1, 2 (PF en el teorema 1, PPF en el teorema 2), 16 y 22); y, finalmente, en la cuarta cuestión, únicamente el alumno no 15 .

Los motivos a favor de la PPF pueden resumirse así: utiliza valores o números concretos lo que ilustra mejor cómo se aplica el teorema, es un caso práctico con lo que se comprende más fácilmente, es directa y visual. 
A favor de la PF se aporta lo siguiente: es general, abarca todos los casos, es más corta, técnica y mecánica.

\subsubsection{Análisis de las entrevistas}

En cada ciclo se realizaron dos entrevistas. Se hicieron entre el PI y dos parejas de alumnos. En cada una de las entrevistas, los alumnos habían manifestado su preferencia uno por la prueba formal y el otro por la prueba preformal. El clima en el que se desarrollaron fue de gran confianza, responsabilidad y espontaneidad por parte del PI y los alumnos.

Las cuestiones o preguntas se iniciaban por el PI y cada uno de los alumnos de la pareja respondía de forma animada y distendida (cf. ANEXOS VI, VII y VIII).

La entrevista comenzaba manifestando cada alumno su conocimiento y convicción previa a la experiencia de los teoremas demostrados, y la verdad y comprensión para él de los mismos después de la demostración realizada en esta experiencia. A continuación se conversaba sobre la ayuda o facilidad de aplicación de estas pruebas en trabajos matemáticos. Seguidamente confesaban los alumnos el esfuerzo aportado por ellos en cada una de ambas demostraciones y exponían cuál de los dos procedimientos aporta a cada uno mayor ayuda al comunicar contenidos matemáticos. Por último, se les preguntaba sobre la mejor explicación y comprensión de los pasos en cada una de las pruebas.

En las entrevistas, los alumnos no son muy precisos en sus respuestas. Tienen la idea pero no aciertan a expresarla con propiedad. Responden con cierta impulsividad a las cuestiones, sin una reflexión más pausada.

El conocimiento previo de estos teoremas por parte de los alumnos de este primer ciclo es muy superficial: alguna noción, sobre todo del teorema del valor medio, pero sin demostraciones. Después de esta experiencia, manifiestan su total convicción de la veracidad de los teoremas demostrados. Cada uno apoya esta convicción 
más en la prueba de su preferencia, pero todos conceden también valor probatorio a la no preferida. Incluso alguno que, a la prueba preformal, comienza calificándola como un caso concreto o un ejemplo.

Sobre la ayuda de estos procedimientos de prueba en trabajos de matemáticas, tres de los cuatro entrevistados estiman el procedimiento preformal como el que ayuda más a la hora de los ejercicios y prácticas (afirmación en consonancia con la respuesta, mayoritaria en este grupo (tabla 4.10, p. 71) favorable a la prueba preformal, a la cuestión sobre cuál de las dos pruebas aporta mayor utilidad para ilustrar cómo se aplica el teorema). A los mismos alumnos que prefieren la prueba preformal en trabajos y ejercicios matemáticos les ha supuesto más esfuerzo, por su abstracción, el procedimiento formal.

Para comunicar contenidos matemáticos, estos alumnos se muestran integradores y se decantan por el uso de ambas demostraciones con algún matiz preferente hacia la formal "por su rigor", afirma uno de los entrevistados partidario de dicha prueba.

En cuanto a la explicación y entendimiento de la sucesión y conexión de los pasos de las demostraciones, también se inclinan a la conjunción de ambas pruebas con tendencia a la prueba formal en relación con la "explicación", por su mayor generalización, y a la prueba preformal, por ser más manejable, fácil y cercana, en relación con el mejor "entendimiento" de dichos pasos.

\subsubsection{Reflexión}

De los datos correspondientes a este primer ciclo, que aparecen en el ANEXO VI, se desprende con claridad la preferencia de estos 28 alumnos por la PPF, mayor aún en el caso del teorema de la unicidad del límite. El orden de aplicación de ambas pruebas ha podido influir en las valoraciones de los alumnos, por lo que el PI determina el cambio de este orden para el ciclo II. 
Las entrevistas son muy interesantes por el alto grado de espontaneidad con que se expresan los alumnos. En cada pareja entrevistada se escogió uno de los alumnos con preferencia por la prueba formal y el otro con preferencia por la preformal, lo que ha resultado interactivo y enriquecedor en las respuestas y comentarios.

Todos constatan lo positivo de esta experiencia por la claridad, profundidad y aprendizaje de los teoremas. Alguno ha evolucionado en su concepto de prueba preformal desde una consideración como mero ejemplo hasta aceptarla como una auténtica demostración.

Aunque cada miembro de la pareja se muestra consecuente en las respuestas con su prueba preferida, ambos estiman y valoran también la prueba preferida por el otro compañero.

En las entrevistas, así como en las demás actividades desarrolladas en el ciclo, se observa en los alumnos una tendencia a favor de la facilidad y utilidad de aplicación y comunicación de la prueba preformal y a favor de la generalización de la prueba formal.

Como consecuencia del debate surgido en la segunda de las entrevistas sobre el orden más conveniente de presentación de las pruebas, el PI decidió incluir una nueva pregunta sobre este aspecto de cara a las entrevistas a realizar en el siguiente ciclo.

Como apreciación general, atendiendo a las motivaciones de la valoración de los alumnos, la PPF se muestra para ellos como la prueba de la concreción y la PF como la prueba de la generalización.

\section{REFLEXIÓN SOBRE LAS ENTREVISTAS}

Los números entre paréntesis hacen referencia a las preguntas del protocolo utilizado en las entrevistas (cf. ANEXO V). 


\section{ENTREVISTA I: José Luis/Eduardo}

\section{I $(1,2,3)$. CERTEZA DE LOS TEOREMAS.}

Poca o nula convicción anterior a esta experiencia. La demostración ofrecida ahora les parece un razonamiento completo. Equivocadamente uno de ellos valora la PPF como un ejemplo. Ambos quedan convencidos de la veracidad de los teoremas para todos los casos.

Al ver las demostraciones te convences que no puede aparecer ningún caso extraño que lo incumpla. (Eduardo).

\section{II (4). FACILIDAD DE APLICACIÓN DE LAS PRUEBAS.}

Cada uno es fiel a su preferencia: José Luis a la PF por su generalización, Eduardo a la PPF por ser más intuitiva. Ambos reconocen valor a la prueba que no es de su preferencia.

\section{III (5). ESFUERZO EMPLEADO. IMPRESIÓN GENERAL SOBRE LA ACTIVIDAD.}

A cada uno le ha supuesto menos esfuerzo la prueba de su preferencia. A ambos la actividad les ha ayudado a un conocimiento más profundo de los teoremas.

Más esfuerzo en el procedimiento formal, por la abstracción. (Eduardo).

\section{IV (6). AYUDA AL COMUNICAR CONTENIDOS MATEMÁTICOS.}

Consecuentes también en este punto, a cada uno le ayuda más su prueba preferida. Utilizaría el procedimiento preformal, aunque, naturalmente, ofrecería también el formal. (Eduardo).

V $(7,8)$. PASOS DE AMBOS PROCEDIMIENTOS: EXPLICACIÓN Y COMPRENSIÓN.

Finalmente, coherentes, cada cual señala su prueba preferida en ambos aspectos. El procedimiento formal. Cualquier situación queda contemplada en el razonamiento. (José Luis). 


\section{ENTREVISTA II: Roberto/Fernando}

\section{I $(1,2,3)$. CERTEZA DE LOS TEOREMAS.}

Tienen algún conocimiento previo de ambos teoremas, algo mayor respecto del teorema del valor medio. Después de la demostración ven la prueba favorita como definitiva o como la justificación propiamente dicha del teorema.

Ambas pruebas clarifican, especialmente la prueba preformal por su concreción. (Fernando).

\section{II (4). FACILIDAD DE APLICACIÓN DE LAS PRUEBAS.}

Ambos señalan la prueba preformal como mejor ayuda.

\section{III (5). ESFUERZO EMPLEADO. IMPRESIÓN GENERAL SOBRE LA ACTIVIDAD.}

Mayor esfuerzo la prueba formal, por su abstracción. Muy positiva la impresión general.

Son los teoremas que mejor he asimilado porque la actividad me ha impulsado a trabajarlos con mayor profundidad. (Fernando).

\section{IV (6). AYUDA AL COMUNICAR CONTENIDOS MATEMÁTICOS.}

Los dos procedimientos ayudan: para el teorema del valor medio primero la PF y luego la PPF; para el teorema de la unicidad del límite

Yo haría en primer lugar la prueba preformal y después la prueba formal. (Fernando).

\section{V $(7,8)$. PASOS DE AMBOS PROCEDIMIENTOS. EXPLICACIÓN Y COMPRENSIÓN.}

Mejor en el procedimiento preformal aunque ambos se decantan por la conjunción de los dos tipos de prueba. 


\subsection{Ciclo II (ciclo de confirmación)}

El objetivo de este segundo ciclo, que denominamos ciclo de confirmación, es fundamentalmente ampliar el campo de nuestra investigación, no a un ámbito distinto del analizado en el ciclo I, sino a nuevos alumnos protagonistas en las mismas cuestiones planteadas.

\subsubsection{Planificación}

El segundo ciclo fue planificado por el PI para ser realizado en los meses de marzo y abril de 2008 con el grupo de los 19 alumnos que en el curso 2008-2009 se matricularon en la misma asignatura de la titulación y escuela indicadas en el ciclo I.

También en este ciclo se llevaría a cabo la adecuada preparación de los alumnos instruyéndolos en la noción de prueba preformal según van Asch. Tendría lugar también una sesión principal de exposición de las pruebas y la entrega posterior a los alumnos del mismo cuestionario preparado para el primer ciclo. Para esta sesión se elegiría de nuevo el teorema del valor medio de Lagrange y el teorema de la unicidad del límite. En este segundo ciclo se invertiría el orden de realización de las demostraciones en cada uno de los teoremas: para el teorema del valor medio, en primer lugar una prueba formal y a continuación una preformal; con el teorema de la unicidad del límite, por el contrario, se expondría primeramente una prueba preformal y posteriormente una prueba formal. Si los resultados fueran los mismos o similares que en el ciclo anterior, avalarían con mayor fuerza la hipótesis propuesta: se hacen pruebas preformales porque son más significativas para los alumnos.

Durante la sesión principal asistiría una profesora de matemáticas de la Universidad Europea Miguel de Cervantes con el fin de observar el desarrollo de la actividad, tomar notas y responder a un protocolo de observación que consta de seis apartados y trata de cubrir todos los aspectos del desarrollo de la docencia en el aula. En primer lugar se trata de recabar información sobre el diseño metodológico, su viabilidad y la adecuación del tiempo. A continuación se contempla la participación y calidad de las intervenciones del profesor en el proceso docente. El ter- 
cer apartado atiende al comportamiento, actitudes y participación de los alumnos. El cuarto aspecto se refiere a la frecuencia y modo de las interacciones profesoralumnos. A la idoneidad y posibles inconvenientes de los recursos se atiene el quinto apartado. Y por último se tienen en cuenta posibles incidencias, disfunciones o inconvenientes producidos (cf. ANEXO III).

Recogidas las respuestas de los alumnos al cuestionario y analizados los datos aportados, se procedería a una reflexión sobre los mismos en orden a proyectar un tercer ciclo de la investigación. También se efectuarían dos entrevistas por parte del PI a sendas parejas escogidas entre los alumnos de este grupo.

\subsubsection{Desarrollo del segundo ciclo (acción)}

La fase de acción se llevó a cabo con arreglo al siguiente calendario:

\begin{tabular}{|c|c|}
\hline Fechas & Contenidos \\
\hline $4 / 03 / 2008$ & $\begin{array}{l}\text { Repaso de axiomática de los números reales } \\
\text { Concepto de prueba preformal según van Asch. } \\
\text { Demostraciones preformales de la desigualdad triangular y de la } \\
\text { propiedad arquimediana }\end{array}$ \\
\hline $6 / 03 / 2008$ & Teoría de límites funcionales \\
\hline $13 / 03 / 2008$ & $\begin{array}{l}\text { Continuidad } \\
\text { Demostraciones preformales de los teoremas de Bolzano y } \\
\text { Weierstrass }\end{array}$ \\
\hline $1 / 04 / 2008$ & Primeras definiciones de cálculo diferencial \\
\hline $3 / 04 / 2008$ & $\begin{array}{l}\text { Teoremas del cálculo diferencial } \\
\text { Demostración preformal del teorema "derivabilidad implica } \\
\text { continuidad" y del teorema de singularidad }\end{array}$ \\
\hline $10 / 04 / 2008$ & $\begin{array}{l}\text { Sesión principal } \\
\text { Demostraciones formales y preformales de los teoremas del valor me- } \\
\text { dio de Lagrange y de la unicidad del límite. } \\
\text { Cuestionario }\end{array}$ \\
\hline $15 / 04 / 2008$ & Entrevistas \\
\hline
\end{tabular}

Tabla 4.12. Calendario del segundo ciclo 
En la sesión principal, se reunieron los 19 alumnos de este ciclo con el PI y la profesora de observación. De nuevo el PI inició la actividad solicitando a los alumnos una actitud receptiva y colaboradora, pasando a continuación a la exposición en la pizarra de las demostraciones de los teoremas elegidos cambiando el orden de las pruebas según se había planificado.

\subsubsection{Observación de la acción}

Al no variar las actividades y el cuestionario a responder por los 19 alumnos de este ciclo, la acción se desarrolló de forma similar a la descrita en el ciclo I, salvo la variación referente al orden de las pruebas. Terminada la exposición de las pruebas en la sesión principal, se entregó a los alumnos el cuestionario preparado para toda la experiencia. Los 19 alumnos mostraron una actitud positiva a lo largo de las dos horas de duración de la sesión y al final de la misma, todos entregaron el cuestionario respondido. Todo se desarrolló bajo la atenta observación de la profesora de matemáticas antes aludida, que proporcionó las siguientes respuestas al protocolo de observación establecido en la planificación de este ciclo.

\subsubsection{Observaciones recogidas en el protocolo del $\mathrm{OE}$}

A continuación se reproducen íntegramente las anotaciones realizadas por el OE en su cuaderno de observación respondiendo al protocolo del ANEXO III.

\section{a) Tratamiento y metodología}

1. Finalidad/grado de adecuación del diseño:

- Objetivos

Analizar la enseñanza-aprendizaje de las demostraciones con pruebas formales y preformales, así como las preferencias de los alumnos sobre dichas pruebas.

- Contenidos

Teorema del valor medio.

Demostración formal del teorema del valor medio.

Demostración preformal del teorema del valor medio. 
Teorema de unicidad del límite.

Demostración preformal del teorema de unicidad del límite.

Demostración formal del teorema de unicidad del límite.

- Metodología

Exposición por parte del profesor de los teoremas y sus respectivas demostraciones preformal y formal, en el orden cronológico que se indica en los contenidos. Además, explica cómo deben cumplimentarse las tareas que deben realizar los alumnos.

A continuación los alumnos realizan las tres tareas solicitadas por el profesor, relativas a los contenidos que se acaban de exponer.

Tanto en el periodo de exposición por parte del profesor como en el de realización de tareas, el profesor pregunta a los alumnos si existen dudas e informa que se pueden preguntar dudas en cualquier momento.

2. ¿Se especifican adecuadamente los diferentes contenidos?

Sí, la exposición y especificación de cada uno de los contenidos se realiza de forma clara y detallada.

3. Viabilidad/utilidad práctica del tratamiento.

Enseñanza y aprendizaje de algunos teoremas de análisis utilizando pruebas preformales.

4. Tiempo: ¿Se ajusta el desarrollo al tiempo previsto? ¿Qué modificaciones son necesarias en el tiempo?

A pesar de que la tarea se ha realizado demasiado deprisa, se ha utilizado más tiempo del previsto.

Sería necesario ampliar el tiempo o realizar alguna de las tareas en otro momento.

\section{b) Profesor}

1. Comportamiento y papel durante el desarrollo. (Actitud, interés, atención,...). 
El profesor muestra interés por el aprendizaje de los alumnos. Está pendiente en todo momento de las dudas del alumno, resolviendo éstas de forma individual y prestando especial interés por aquellos alumnos que tienen más dificultades. Además, realiza algunas preguntas para motivar la participación de los alumnos y observar la atención prestada. Muestra en todo momento una actitud positiva.

2. Tipo de participación en el proceso docente.

Durante la primera hora del proceso el profesor recuerda qué es una demostración preformal, a continuación expone los teoremas y sus demostraciones y explica la forma de cumplimentar los cuestionarios, deteniéndose en algunos momentos para resolver las dudas de los alumnos o preguntar él si existen dudas.

En la segunda parte de la sesión, cuando los alumnos responden a las preguntas que se realizan en los cuestionarios, resuelve de forma individual las dudas que surgen a cada uno de los alumnos.

3. Calidad y claridad de las intervenciones.

Las intervenciones del profesor son claras, rigurosas y concisas en todo momento.

4. Tiempo de intervención.

Durante la primera hora interviene casi de forma continua, y en los 45 minutos que los alumnos dedican a realizar las tareas, de forma puntual resolviendo sus dudas.

\section{c) Alumnos}

1. Actividad central durante el desarrollo del tratamiento. ¿Qué hacen?

Durante el periodo de exposición del profesor, la gran mayoría de los alumnos prestan atención a la explicación y toman notas como apoyo en la resolución de las tareas propuestas.

Están expectantes ya que se encuentran en una clase distinta y especial. 
2. Tipo de participación (Iniciativa, pasividad, silencio, hacen las tareas, siguen las explicaciones).

Siguen las explicaciones del profesor en silencio y tomando notas. En esta fase la participación es escasa, con una actitud de pasividad en bastantes alumnos.

Durante la realización de tareas los alumnos muestran bastante interés en su desarrollo, preguntando al profesor cuando surgen dudas.

3. Tipo de preguntas que realizan.

Los alumnos preguntan sobre la posibilidad de tomar notas en el transcurso de la exposición.

Muy pocas preguntas sobre los teoremas expuestos y sus demostraciones, hacen referencia a la forma de cumplimentar adecuadamente las tareas expuestas.

4. Atención/asimilación.

a) ¿Atienden a las explicaciones?

En el periodo de exposición los alumnos han estado muy atentos en la parte correspondiente al teorema del valor medio, aunque se observa menor atención durante el desarrollo correspondiente al teorema de unicidad del límite.

b) ¿Parece que entienden las explicaciones?

Se aprecia un entendimiento bastante claro de la exposición y demostración formal del teorema del valor medio, pero en menor medida cuando se realiza la demostración preformal al utilizar la función logaritmo neperiano.

El teorema de unicidad del límite se aprecia de comprensión más difícil.

c) ¿Qué síntomas se detectan?

En el periodo relativo al teorema del valor medio se percibe interés por parte de la mayoría de los alumnos, pero en el teorema de unicidad del límite algunos alumnos muestran síntomas de cansancio con movimientos y bostezos. 
Desconexión y miradas hacia diversos sitios de cuatro alumnos que parecen perdidos, dejan de tomar notas. Otro alumno juega con la calculadora.

5. Actitud general: ¿Positiva, neutra, negativa,...?

Positiva.

\section{d) Interacciones profesor-alumnos}

1. Frecuencia: Nunca, a menudo, constantemente. Duración de las intervenciones.

Las intervenciones son pocas y de duración breve.

2. Tipo:

- Atención fluida con diálogo profesor-alumno.

(No responde).

- A instancias del alumno.

Los alumnos intervienen principalmente para que el profesor aclare dudas sobre la forma de cumplimentar adecuadamente las tareas.

- El profesor crea situaciones de interacción.

(No responde).

- El profesor muestra una actitud que favorece el diálogo.

(No responde).

\section{e) Recursos y condiciones materiales}

1. Idoneidad de los recursos empleados en relación con el tratamiento.

Se ha desarrollado en la misma aula teórica que se imparten las clases de la asignatura, cuyo tamaño es adecuado al número de alumnos, y se ha utilizado pizarra y tizas de diversos colores, considerando estos recursos suficientes para el tratamiento realizado. 
2. Inconvenientes materiales observados.

En el aula no funcionan las luces más cercanas a la pizarra.

3. Otras observaciones.

(No responde).

\section{f) Incidencias especiales}

1. Retrasos dignos de mención, puntualidad: alumnos y profesor.

Puntualidad tanto por parte del profesor como de los alumnos, sólo uno de los alumnos llegó tarde, pero terminó las pruebas antes de que acabara el tiempo permitido.

2. Disfunciones o inconvenientes producidos. Posibles motivos. No se detectan.

3. Otras observaciones. (Orden en la clase, alboroto, atención, participación, estudio, trabajo).

(No responde).

\subsubsection{Observaciones del cuaderno de aula del PI}

Al igual que los alumnos del ciclo I, los de este grupo recibieron con interés por lo novedoso que era para la mayoría, las explicaciones basadas en pruebas preformales en las clases de preparación anteriores a la sesión principal, solicitando aclaraciones al PI sobre estas cuestiones: la prueba preformal, ¿es una verdadera demostración?; ¿por qué en los libros de texto habituales no aparecen pruebas preformales?; a partir de una prueba preformal, ¿es posible realizar una prueba formal del mismo teorema?, y recíprocamente. Preguntas que fueron recibiendo adecuada respuesta por parte del PI. En la sesión principal, la presencia de la observadora externa inicialmente causó sorpresa a los alumnos pero rápidamente se adaptaron a la nueva situación. El nivel de atención de los alumnos fue aceptable, especialmente durante la exposición del teorema del valor medio. En la demostración pre- 
formal de este teorema, el PI tuvo que recordar a los alumnos la derivada de la función logaritmo neperiano, al manifestar alguno de ellos que no la tenía "actualizada". En el tiempo dedicado a cumplimentar el cuestionario, el PI atendió inmediatamente las dudas planteadas por diversos alumnos. Las entrevistas se desarrollaron en un buen clima de confianza, participación e interés. En la segunda entrevista (cf. ANEXO VII), iniciada con un diálogo espontáneo entre profesor y alumnos, estos hicieron varias sugerencias en relación con la prueba preformal del teorema del valor medio, alguna de las cuales decidió tomar en consideración el PI a la hora de planificar el tercer ciclo de esta investigación.

\subsubsection{Reflexiones del protocolo de observación del OE y del cuaderno de aula del PI}

Han resultado especialmente relevantes las notas aportadas por la profesora que acudió en calidad de observadora a la sesión principal de este ciclo y que, en gran medida, coinciden con las recogidas por el PI en el cuaderno de aula. Es motivo de reflexión para el PI la disminución de la participación de los alumnos durante la exposición del segundo teorema, circunstancia que responde posiblemente a la menor familiaridad de estos alumnos con dicho teorema. Asimismo el PI toma en consideración procurar motivar a los alumnos para que superen el natural cansancio que siempre se acumula en el transcurso de una sesión intensa y que precisa concentración continuada.

Se hace a continuación una breve reflexión de cada uno de los bloques del protocolo de observación.

\section{a) Tratamiento y metodología:}

El OE no hace ninguna valoración, se limita a transcribir los títulos de los contenidos que se desarrollan en el aula, contenidos que se corresponden con los de la programación de la asignatura y, por tanto, manifiestan que el uso de las PPF no entorpece el desarrollo del programa. 
Sobre metodología, el OE Indica que es adecuada. Es clara y detallada la exposición del PI, abierto en todo momento a las dudas y preguntas de los alumnos.

Se sugiere la necesidad de ampliar el tiempo o dividir tareas en tiempos distintos.

En el cuaderno de aula (en adelante CA) del PI está apreciada también esta circunstancia en relación con el tiempo para tenerlo en consideración en el ciclo siguiente. Se señala como una cuestión abierta que invita a una mayor atención la pregunta de algún alumno sobre la ausencia de pruebas preformales en los libros de texto al uso.

\section{b) Profesor:}

El OE describe la actuación del PI y valora su actitud como "positiva" destacando su interés en resolver las dudas que surgen y motivar la participación de los alumnos.

Sus intervenciones se califican como claras, rigurosas y concisas, con mayor frecuencia en la primera parte de la sesión principal y menor en el tiempo en que los alumnos responden al cuestionario.

\section{c) Alumnos:}

El OE valora su actividad como positiva. Están expectantes ante la novedad de la experiencia que realizan, participan preguntando y tomando notas. Ponen bastante interés en cumplimentar adecuadamente el cuestionario, sobre el que hacen la mayoría de sus preguntas.

Se dan situaciones distintas en cuanto a la atención prestada, por la diversa dificultad de los propios contenidos y la duración de la sesión, con alguna expresión externa de cansancio en los momentos finales de la exposición.

El PI en su CA hace referencia a la aceptable atención general de los alumnos. Las actitudes de unos pocos pueden explicarse por la duración de la sesión principal, que precisa concentración continuada y por darse en ellos una menor familiaridad con conocimientos importantes para la realización de esta experiencia (derivada 
de la función logaritmo neperiano). Se anota como dentro de la normalidad la actitud de estos alumnos que en nada entorpece el proceso de la investigación que estamos realizando.

\section{d) Interacciones profesor-alumnos:}

El OE se muestra muy parco en este punto: pocas intervenciones, la mayoría para responder a dudas sobre la forma de cumplimentar sus tareas los alumnos. Ya ha señalado anteriormente el interés del PI en hacer preguntas con el fin de motivar la participación.

En el CA se consigna el clima de confianza en que se han desarrollado las entrevistas llevadas a cabo en este ciclo con las dos parejas de alumnos. Asimismo, aparece la interacción que estas entrevistas suponen entre los mismos alumnos, al desarrollarse entre personas seleccionadas partidarias de cada una de las modalidades de prueba. Incluso se acoge la sugerencia que se hace al PI de algunos cambios en la prueba preformal del teorema del valor medio.

Por otra parte, consideramos como muy positivo que el mismo profesor sea también "investigador" en estas experiencias, ya que el contacto y el contexto habitual "profesor-alumnos" crea sin dificultad, esta es mi experiencia personal, una gran confianza que propicia la franca interacción entre ambos.

\section{e) Recursos y condiciones materiales:}

El OE, en su informe, considera específicamente que son suficientes.

En el CA no se hace ninguna observación sobre este extremo. Las posibilidades y medios ordinarios del Centro Académico han estado en todo momento a disposición de esta experiencia.

\section{f) Incidencias especiales:}

Ni el OE en sus respuestas a este Protocolo ni el PI en el CA consideran incidencia especial alguna. 


\subsubsection{Recogida de datos a través del cuestionario}

Los datos aportados en este segundo ciclo son las respuestas de los alumnos al cuestionario que se les entregó después de la explicación en la sesión principal y cumplimentado en un intervalo de tiempo similar al del ciclo anterior (algunos alumnos no respondieron al ejercicio relativo al grado de influencia de las pruebas en una aplicación del teorema del valor medio, quizá por falta de tiempo, según manifiesta la profesora observadora).

\subsubsection{Análisis de los datos}

Fue llevado a cabo por el PI, quien en relación con el análisis cuantitativo de la primera cuestión, optó por integrar los datos con los del ciclo I, ya realizado, y los del ciclo III, previsto para el siguiente curso.

\section{Análisis cualitativo}

Cuestión 1: Explica cómo has entendido cada uno de los teoremas con cada procedimiento. Indica en cada caso claramente el porqué.

El objetivo de esta cuestión es analizar la comprensión de los alumnos de las demostraciones matemáticas sobre las pruebas preformales en contraste con las pruebas formales.

Para el análisis de las respuestas de los alumnos a esta primera cuestión se han tenido en cuenta las dos tablas siguientes de categorías sobre ambas modalidades de prueba (preformal y formal) realizadas directamente a partir de la información proporcionada por los alumnos. 


\begin{tabular}{|c|c|c|c|}
\hline \multicolumn{4}{|c|}{ TEOREMA 1} \\
\hline \multicolumn{2}{|l|}{ Prueba preformal } & \multicolumn{2}{|l|}{ Prueba formal } \\
\hline Categoría & $\mathbf{n}_{\mathbf{i}}$ & Categoría & $\mathbf{n}_{\mathbf{i}}$ \\
\hline Preferencia por ambas pruebas & 6 & Preferencia por ambas pruebas & 6 \\
\hline Convence más & 5 & Convence más & 8 \\
\hline Más útil y fáciles operaciones & 0 & Más útil y fáciles operaciones & 6 \\
\hline Más general & 0 & Más general & 5 \\
\hline Orden: preformal-formal & 0 & Orden: formal-preformal & 1 \\
\hline $\begin{array}{l}\text { Aplicación caso concreto, } \\
\text { números }\end{array}$ & 4 & $\begin{array}{c}\text { Aplicación caso concreto, } \\
\text { números }\end{array}$ & 0 \\
\hline Alcanza comprensión completa & 3 & Alcanza comprensión completa & 0 \\
\hline
\end{tabular}

Tabla 4.13. Categorías preferidas en el teorema 1, ciclo II

\begin{tabular}{|c|c|c|c|}
\hline \multicolumn{4}{|c|}{ TEOREMA 2} \\
\hline \multicolumn{2}{|l|}{ Prueba preformal } & \multicolumn{2}{|l|}{ Prueba formal } \\
\hline Categoría & $\mathbf{n}_{\mathbf{i}}$ & Categoría & $\mathbf{n}_{\mathbf{i}}$ \\
\hline Convence más & 15 & Convence más & 1 \\
\hline Aplicación caso concreto & 5 & Aplicación caso concreto & 0 \\
\hline $\begin{array}{c}\text { Preferencia por ambas } \\
\text { pruebas }\end{array}$ & 3 & $\begin{array}{c}\text { Preferencia por ambas } \\
\text { pruebas }\end{array}$ & 3 \\
\hline Clara/fácil/sencilla & $4 / 4 / 3$ & Teórica/completa/abstracta & $1 / 1 / 1$ \\
\hline Orden: preformal-formal & 1 & Orden: formal-preformal & 1 \\
\hline Más general & 0 & Más general & 1 \\
\hline
\end{tabular}

Tabla 4.14. Categorías preferidas en el teorema 2, ciclo II 


\section{Observaciones del PI en torno a estas respuestas}

\section{TEOREMA 1}

En este teorema (del valor medio) la tendencia más acusada de estos alumnos es a favor de la PF. No obstante, tres alumnos que prefieren la PF, señalan matices restrictivos en su elección: fácil, excepto la fórmula de la función diferencia (alumno no 3); ideal las dos pruebas $1^{\circ}$ la PF y $2^{\circ}$ la PPF porque primero se capta la idea general y después se concreta (alumno no 4); es verdad que ha sido (la PF) la primera explicada y siempre te parece más sencillo y estás más atento (alumno no 14), observación objetiva y muy acertada, de lo cual todos tenemos larga experiencia.

\section{MOTIVOS}

Los aportados a favor de la PF son los siguientes: fáciles las operaciones $(3,7,14$, $15)$; fácil de entender el teorema $(15,19)$; útil, al ser general, para aplicar a cualquier caso $(1,4)$. La mayoría se fija en la facilidad de la prueba por las operaciones que se realizan para entender el teorema; dos alumnos aluden a la utilidad de esta prueba para su aplicación al ser general. El alumno no 14 ofrece una posible explicación de la facilidad señalada por seis de los ocho alumnos que la indican como motivo de su opción por esta prueba: "ha sido la primera explicada y siempre te parece más sencillo y estás más atento".

Los aportados a favor de la PPF: al manejar números y no letras, se capta mejor la idea (5); menos abstracta al ser un ejemplo concreto (12); con valores concretos se ve mejor lo que se quiere demostrar (16); me ha gustado más (10). El alumno no 10 alude al gusto; los otros tres que optan por esta prueba coinciden en que se entiende mejor al usar números concretos y no letras y ser menos abstracta.

Vuelven a aparecer una vez más los criterios tan frecuentes en estos alumnos sobre la concreción de la PPF y la generalización de la PF.

Seis alumnos optan en este teorema a favor de ambas pruebas por estos motivos: lo he entendido perfectamente tanto con la PF como con la PPF, en ésta algo com- 
plejo los logaritmos (2); las dos, primero la PF visión amplia teórica y luego la PPF refuerza los conceptos adquiridos (6); con la PF consigo saber las variables a combinar y colocar, y con la PPF averiguar errores posibles al sustituir con la PF (9); la PF es general, la PPF más concreta y en el teorema de Rolle me complico con los cálculos (11); lo he entendido bien con las dos pruebas, un poco mejor con la PF (13); la PF es básica para entender el teorema, pero con la PPF se alcanza la comprensión completa (17). Tres alumnos de esta opción abogan por las dos pruebas porque la PF es general, básica, y la PPF refuerza los conceptos adquiridos, ofrece la comprensión completa. Dos alumnos lo han entendido perfectamente con ambas pruebas, uno dice que algo complejo los logaritmos, otro que un poco mejor con la PF porque se ve de dónde sale cada dato. Uno ve la ventaja en la PF al combinar y colocar variables, y la ventaja en la PPF de evitar posibles errores con la PF. Consideran, pues, las dos pruebas como complementarias en cuanto a ventajas y utilidad e iguales en cuanto a su comprensión.

\section{TEOREMA 2}

En este segundo teorema (unicidad del límite) la opción a favor de la PPF es muy mayoritaria. A favor de la PF solamente se decanta un alumno (5), y otros tres (6, 12 y 17) optan por ambas pruebas.

\section{MOTIVOS}

a) A favor de la PF: la PF parece ser (no muestra suficiente convicción) la que me ha dejado entender el objetivo del teorema (5).

b) A favor de la PPF: lo entiendo mejor, mucho más fácil de ver y de aplicar (1); más clara la PPF debido a la aplicación de números concretos (2); más fácil de comprender al visualizar el problema (3); menos complicada e influye el orden de las pruebas (4); bastante más fácil, más clara y concisa la solución, incluso antes de terminar la demostración (7); se ve más claro, por anticipado (9); se ha explicado mejor y en cuanto al orden, 1ํ PPF y $2^{\circ}$ PF (10); más claro y sencillo con números concretos (11); más fácil de entender 
(13); las operaciones más sencillas (14); más sencilla, utilizamos números concretos (15); por ser más concreta, he entendido mejor la PPF (16); se hace necesaria la PPF, la PF es más abstracta (17); más entendible (18); más concreta y conclusa, la gráfica es imprescindible para ambas (19). La práctica totalidad de los quince alumnos que optan por esta prueba en este teorema se apoya en que es más fácil, más clara, más concreta, se entiende y comprende mejor y son más sencillas las operaciones. Se utilizan números concretos. Se la considera para este teorema más necesaria. Solamente el alumno no 10 manifiesta que se ha explicado mejor y el no 4 que el orden de las pruebas influye. En este teorema se presentó primeramente la PPF.

c) A favor de ambas pruebas. Para el alumno no 6, la PPF es más fácil de entender y la PF más completa. Coincide con su opinión respecto del teorema 1. Concibe la PF, por ser general y más teórica, como más completa también al poder aplicarse a más casos y la PPF, que juzga más fácil, como la que ratifica y refuerza a la PF. El orden de las pruebas, para este alumno, es primero la PF y en segundo lugar la PPF. El alumno no 12 viene a afirmar, en una frase general, que ambas pruebas son positivas para la finalidad que se pretende. El alumno no 17 juzga necesaria la preformal dada la mayor abstracción de la formal.

En resumen y atendiendo a la totalidad de los juicios de alumnos en las dos pruebas, PF y PPF, y en ambos teoremas, es la PPF la preferida por ellos.

En las tablas (4.13 y 4.14, p. 90) se detalla y concentra a la vez esta variada y rica dispersión de respuestas. La casilla convence más refleja la preferencia por cada modalidad de prueba (preformal vs. formal) por separado, en ambos teoremas, 5 vs. 8 en el $1^{\circ}$, 15 vs. 1 en el $2^{\circ}$. Es seis el dígito de preferencia por ambas pruebas unidas en el teorema $1^{\circ}$ y tres en el $2^{\circ}$. En el resto de casillas aparecen los detalles más repetidos de los motivos aludidos por los alumnos en sus estimaciones de las pruebas. Respecto de la prueba formal: más general, útil por esto mismo, abstracta, teórica, completa, operaciones fáciles. Y en relación con la prueba preformal: apli- 
cación más concreta, uso de números en lugar de letras, clara, fácil, sencilla y, a continuación de la formal, refuerza conceptos y alcanza la completa comprensión.

Para el PI se trata de valoraciones en general acertadas, que han sabido detectar los matices propios y más específicos de cada una de estas dos modalidades de prueba, y las realizan alumnos que, al menos en su mayoría, desconocían antes de empezar el ciclo la demostración preformal, su concepto, posibilidades, uso, aplicaciones.

Escaneamos algunas respuestas de este segundo grupo de alumnos.

Cristina G. P. (2) es partidaria de ambas pruebas en los dos teoremas y en el escaneo siguiente aparece su justificación:

- Teorema 1:

El teorema del vator medio lo he entendido perfectamente tanto en la prueba formal como en la pre-formal.

He ha resultado un poca más compleja la pre-formal debido a la aplicación de los logaritmos que me lían un poro. pero en ambas pruebas he enterdudo lo que se obtiene, es decir. el proceso a realizar para obtener el resultado.

- Teorema 2:

Tanto la prueba pre-formal como la formal son sencillas a la hora de entender. Minguna me ha resultado complicada, sólo a la hora de la representación gráfica me resulta má dara la prueba pre-formal debido a la aplicación de uno números concretos.

Antonio B. M. (14) hace referencia en su preferencia al orden en que respondió a las pruebas: 


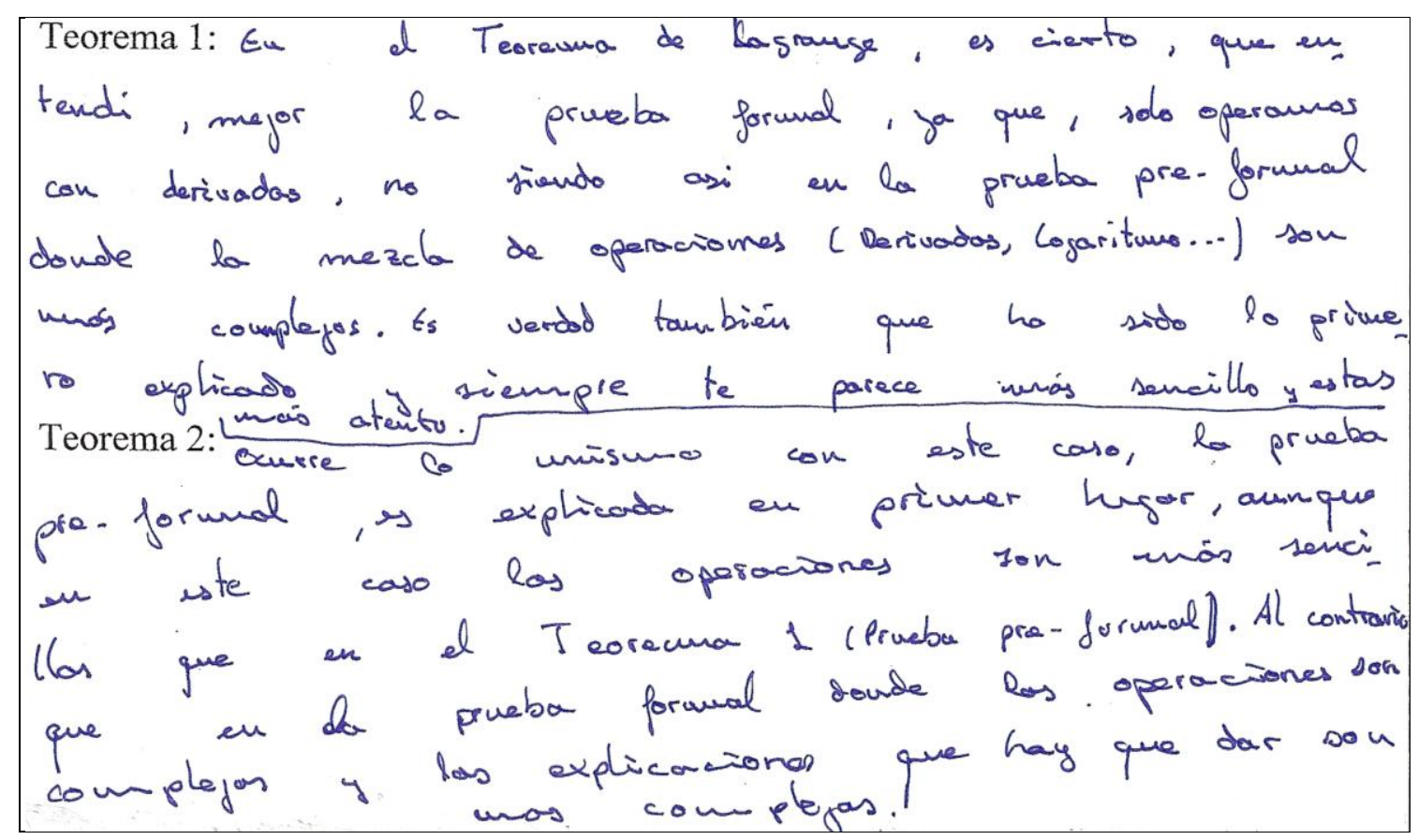

Por último, Antonio B. V. (13) afirma que es la prueba preformal, más sencilla, la que le hace comprender plenamente el $2^{\circ}$ de los teoremas. Estas respuestas se muestran en el escaneo siguiente:

- Teorema 1:

El teorecuia 1 lo he entendido bien cou las dos prvebas; quizá lo he entendido un soro urejor con la formal porque se H urejor de doude sale cada dato

- Teorema 2:

El teorema 2 no lo he ectendido hasta que hemos llogado al frial de la explicacion pre-formal, considero que en este trorena es mas facil de entender on la provba pre-formal 
La cuestión $2^{\text {a }}$ se centra en las categorías de facilidad (F), gusto (G), claridad (C), aprendizaje (A).

Cuestión 2: Puntúa de 1 a 10 las cuatro pruebas que hemos realizado atendiendo a las siguientes categorías: facilidad (F), gusto (G), claridad (C), aprendizaje (A). Entiende que:

1 es muy difícil y 10 es muy fácil.

1 no me gusta y 10 me gusta mucho.

1 no es nada clara y 10 es muy clara.

1 no se aprende nada y 10 se aprende mucho.

- Preformal del teorema 1: $F_{\_}, G_{\_}, C_{\_}, A_{-}$.

- Formal del teorema 1: $F_{\_}, G_{\_}, C_{\_}, A_{-}$.

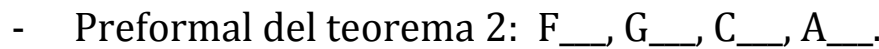

- Formal del teorema 2: $\mathrm{F}_{\_}, \mathrm{G}_{\_}, \mathrm{C}_{\_}, \mathrm{A}_{-}$.

En la columna COMPRENSIÓN: se reescriben las puntuaciones de la cuestión 2. En la columna PREFERENCIA: para cada teorema, comparando las puntuaciones de cada modalidad de prueba en cada una de las cuatro categorías, se indica el número de veces en que "gana" (supera) la prueba preformal a la formal, y viceversa. 


\begin{tabular}{|c|c|c|c|}
\hline TEOREMA & MODALIDAD & COMPRENSIÓN & PREFERENCIA \\
\hline \multirow{2}{*}{ Teorema 1} & Preformal & F_, G_, $, C_{2}, A_{-}$ & \\
\hline & Formal & $F_{-}, G_{-}, C_{-}, A_{-}$ & \\
\hline \multirow{2}{*}{ Teorema 2} & Preformal & $F_{-}, G_{-}, C_{2}, A_{-}$ & \\
\hline & Formal & $F_{-}, G_{-}, C_{2}, A_{-}$ & \\
\hline \multirow{2}{*}{ Total } & Preformal & & \\
\hline & Formal & & \\
\hline
\end{tabular}

Tabla 4.15. Esquema de la pregunta $2^{\mathrm{a}}$ del cuestionario

El objetivo de esta cuestión es, dentro de la comprensión, conocer la preferencia de los alumnos por una u otra de las modalidades de prueba en relación con cuatro importantes categorías: su facilidad, su claridad, el gusto por ellas y su influjo en el aprendizaje del alumno.

\subsubsection{PREFERENCIA}

\section{(F: facilidad; G: gusto; C: claridad; A: aprendizaje)}

En la tabla siguiente se muestran los resultados de las respuestas de los alumnos sobre la significación de las pruebas según las categorías asignadas. En las columnas destinadas a preferencia aparecen reflejadas con sus iniciales las categorías preferidas por los alumnos en cada modalidad de prueba y para cada teorema. En las columnas destinadas a puntuación se consigna en cada casilla el número de categorías preferidas en cada modalidad de prueba y para cada teorema. 


\begin{tabular}{|c|c|c|c|c|c|c|c|c|}
\hline $\begin{array}{l}\mathbf{A} \\
\mathbf{L}\end{array}$ & \multicolumn{4}{|c|}{ TEOREMA 1} & \multicolumn{4}{|c|}{ TEOREMA 2} \\
\hline $\mathbf{M}$ & PREF & RMAL & FOI & MAL & PREF & RMAL & FO & MAL \\
\hline $\begin{array}{l}\mathbf{N} \\
\mathbf{O}\end{array}$ & $\begin{array}{l}\text { Prefe- } \\
\text { rencia }\end{array}$ & $\begin{array}{c}\text { Pun- } \\
\text { tuación }\end{array}$ & $\begin{array}{l}\text { Prefe- } \\
\text { rencia }\end{array}$ & $\begin{array}{c}\text { Pun- } \\
\text { tuación }\end{array}$ & $\begin{array}{l}\text { Prefe- } \\
\text { rencia }\end{array}$ & $\begin{array}{c}\text { Pun- } \\
\text { tuación }\end{array}$ & $\begin{array}{l}\text { Prefe- } \\
\text { rencia }\end{array}$ & $\begin{array}{c}\text { Pun- } \\
\text { tuación }\end{array}$ \\
\hline 1 & $\mathrm{C}$ & 1 & $\mathrm{~F}, \mathrm{~A}$ & 2 & $\mathrm{G}, \mathrm{C}, \mathrm{A}$ & 3 & - & - \\
\hline 2 & - & - & $\mathrm{G}, \mathrm{C}$ & 2 & $\mathrm{G}, \mathrm{C}, \mathrm{A}$ & 3 & - & - \\
\hline 3 & - & - & $\mathrm{F}, \mathrm{G}, \mathrm{C}$ & 3 & $\mathrm{~F}, \mathrm{G}, \mathrm{C}$ & 3 & - & - \\
\hline 4 & - & - & $\mathrm{G}, \mathrm{C}, \mathrm{A}$ & 3 & $\mathrm{~F}, \mathrm{G}, \mathrm{C}, \mathrm{A}$ & 4 & - & - \\
\hline 5 & $\mathrm{G}, \mathrm{C}, \mathrm{A}$ & 3 & - & - & $\mathrm{A}$ & 1 & $\mathrm{~F}, \mathrm{G}, \mathrm{C}$ & 3 \\
\hline 6 & $F$ & 1 & $\mathrm{G}, \mathrm{C}, \mathrm{A}$ & 3 & - & - & $\mathrm{F}, \mathrm{C}, \mathrm{A}$ & 3 \\
\hline 7 & - & - & $\mathrm{F}$ & 1 & $\mathrm{~F}, \mathrm{C}$ & 2 & - & - \\
\hline 8 & - & - & $\mathrm{F}, \mathrm{G}$ & 2 & $\mathrm{~F}$ & 1 & $G$ & 1 \\
\hline 9 & $\mathrm{~F}$ & 1 & $\mathrm{C}$ & 1 & $\mathrm{~F}$ & 1 & $\mathrm{~A}$ & 1 \\
\hline 10 & $\mathrm{~F}, \mathrm{G}$ & 2 & $\mathrm{C}$ & 1 & $\mathrm{~F}, \mathrm{G}, \mathrm{C}, \mathrm{A}$ & 4 & - & - \\
\hline 11 & $\mathrm{~F}, \mathrm{G}, \mathrm{C}, \mathrm{A}$ & 4 & - & - & $\mathrm{C}, \mathrm{A}$ & 2 & - & - \\
\hline 12 & - & - & $\mathrm{G}, \mathrm{C}, \mathrm{A}$ & 3 & - & - & - & - \\
\hline 13 & - & - & $\mathrm{F}, \mathrm{C}$ & 2 & $\mathrm{~F}, \mathrm{C}$ & 2 & - & - \\
\hline 14 & $\mathrm{C}, \mathrm{A}$ & 2 & $\mathrm{~F}, \mathrm{G}$ & 2 & $\mathrm{~F}, \mathrm{C}, \mathrm{A}$ & 3 & - & - \\
\hline 15 & - & - & $\mathrm{F}, \mathrm{G}$ & 2 & $\mathrm{~F}, \mathrm{G}, \mathrm{C}$ & 3 & - & - \\
\hline 16 & $\mathrm{~F}$ & 1 & - & - & $\mathrm{F}$ & 1 & - & - \\
\hline 17 & $\mathrm{~F}, \mathrm{C}, \mathrm{A}$ & 3 & - & - & $\mathrm{F}, \mathrm{C}, \mathrm{A}$ & 3 & - & - \\
\hline 18 & - & - & $\mathrm{F}, \mathrm{G}, \mathrm{C}, \mathrm{A}$ & 4 & $\mathrm{~F}, \mathrm{G}, \mathrm{C}, \mathrm{A}$ & 4 & - & - \\
\hline 19 & - & - & $\mathrm{F}, \mathrm{G}, \mathrm{C}, \mathrm{A}$ & 4 & $\mathrm{G}, \mathrm{C}, \mathrm{A}$ & 3 & $\mathrm{~F}$ & 1 \\
\hline $\begin{array}{l}\text { To- } \\
\text { tal }\end{array}$ & & 18 & & 35 & & 43 & & 9 \\
\hline
\end{tabular}

Tabla 4.16. Preferencias y puntuaciones según categorías de las pruebas PPF y PF en ambos teoremas, ciclo II

El gráfico siguiente muestra la frecuencia de las preferencias de las pruebas preformal y formal para los dos teoremas, y la suma total de ambas frecuencias. 


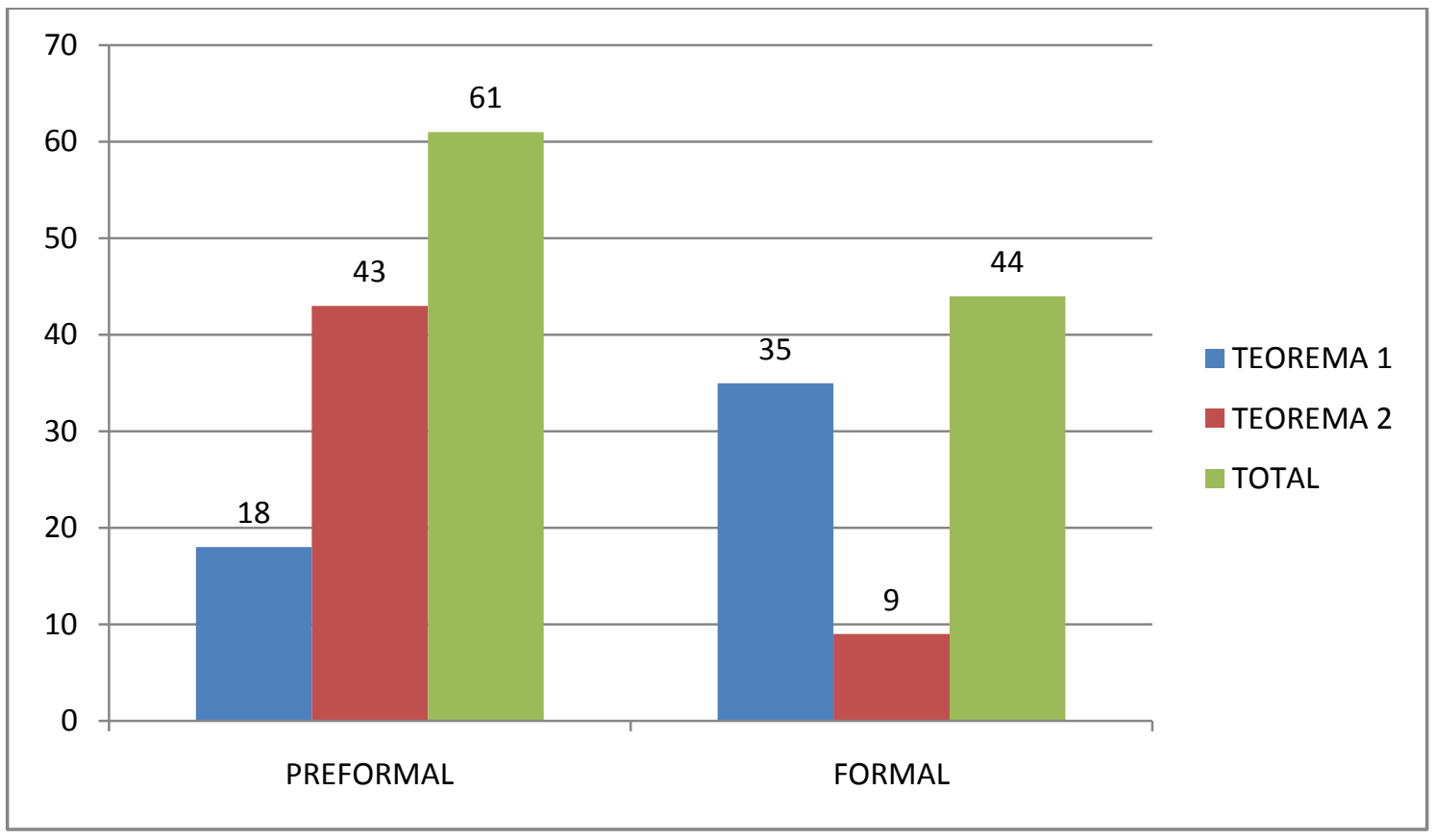

Gráfico 4.3. Preferencia por las categorías de las pruebas, ciclo II

Es distinta la preferencia de estos alumnos en cada uno de los dos teoremas. En el teorema 1 (teorema del valor medio) su preferencia se inclina a favor de la PF: dos alumnos $(18,19)$ en las cuatro categorías; cuatro alumnos $(3,4,6,12)$ en tres de ellas; seis alumnos $(1,2,8,13,14,15)$ en dos de ellas; y tres alumnos $(7,9,10)$ en una de ellas. Representan estas preferencias el 66\% (35 frente a 18 en la última fila de la tabla anterior).

Por el contrario, en el teorema 2 (teorema de la unicidad del límite) y con mayor porcentaje a su favor, el $82,7 \%$ (43 frente a 9 en la tabla citada), los alumnos se inclinan a favor de la PPF: tres alumnos $(4,10,18)$ en las cuatro categorías; siete alumnos $(1,2,3,14,15,17,19)$ en tres categorías; tres alumnos $(7,11,13)$ en dos categorías; y cuatro alumnos $(5,8,9,16)$ en una categoría. Cabe destacar que la categoría más considerada en estas preferencias es la claridad, en trece ocasiones.

El orden de las pruebas ha influido, sin duda, en los alumnos al establecer sus preferencias. Aun así la diferencia a favor de la PPF en el teorema de la unicidad del 
límite es notablemente mayor que la diferencia a favor de la PF en el teorema del valor medio, que fue estudiado en primer lugar (en términos de porcentajes, $82,7 \%$ frente a $66 \%)$.

Tomados los resultados de ambos teoremas en conjunto, destaca la preferencia de la $P P F(18+43=61)$ sobre la $P F(35+9=44)$. En porcentaje, 58,1\% frente a 41,9\%.

\subsubsection{SIGNIFICACIÓN}

$\mathrm{Al}$ igual que en el ciclo anterior, ahora, nos centramos en las categorías que hacen referencia a la significación de ambos tipos de prueba (convencimiento, entendimiento, memorización y aplicabilidad de las dos modalidades de prueba). El objetivo de esta cuestión, igual que en el ciclo anterior, es analizar la contribución de la demostración sobre la significación de los teoremas (Hanna, 1989).

Cuestión 3: Responde a las siguientes preguntas, señalando en cada caso claramente el porqué:

- ¿Cuál de las dos formas te convence más de la veracidad del teorema?

- ¿Cuál de las dos pruebas puede ayudar más a entender el significado del teorema?

- ¿Cuál de las dos pruebas puede ayudar más a memorizar el teorema?

- ¿Cuál de las dos pruebas es más útil para ilustrar cómo se aplica el teorema?

La siguiente tabla se elabora utilizando las siglas PPF o PF, según que un alumno concreto crea que para él tiene más significación la prueba preformal o formal. Esto se repite en cada una de las cuatro características señaladas en la cuestión 3. 


\begin{tabular}{|c|c|c|c|c|}
\hline Alumno & $\begin{array}{c}\text { Convence } \\
\text { más }\end{array}$ & $\begin{array}{c}\text { Ayuda más a } \\
\text { entender }\end{array}$ & $\begin{array}{c}\text { Ayuda más a } \\
\text { memorizar }\end{array}$ & $\begin{array}{c}\text { Más útil para } \\
\text { aplicar el teorema }\end{array}$ \\
\hline 1 & PPF PF & PPF & PPF PF & PPF \\
\hline 2 & PPF & PPF & $\mathrm{PF}$ & PPF \\
\hline 3 & $\mathrm{PF}$ & PPF & PPF PF & PPF \\
\hline 4 & PPF & $\mathrm{PF}$ & $\mathrm{PF}$ & PPF \\
\hline 5 & PPF & PPF PF & $\mathrm{PF}$ & PPF \\
\hline 6 & PPF PF & PPF & $\mathrm{PF}$ & PPF \\
\hline 7 & PPF & $\mathrm{PF}$ & PPF & PPF \\
\hline 8 & PPF PF & PPF PF & PPF PF & PPF PF \\
\hline 9 & PPF & PPF & PPF & PPF \\
\hline 10 & PPF & PPF & $\mathrm{PF}$ & PPF \\
\hline 11 & PPF PF & PPF PF & $\mathrm{PF}$ & PPF \\
\hline 12 & $\mathrm{PF}$ & PPF & PPF & $\mathrm{PF}$ \\
\hline 13 & PPF & $\mathrm{PF}$ & PPF & PPF \\
\hline 14 & PPF PF & $\mathrm{PF}$ & $\mathrm{PF}$ & PPF \\
\hline 15 & PPF & PPF & - & PPF \\
\hline 16 & PPF & PPF & PPF & PPF \\
\hline 17 & $\mathrm{PF}$ & PPF & $\mathrm{PF}$ & PPF PF \\
\hline 18 & $\mathrm{PF}$ & $\mathrm{PF}$ & $\mathrm{PF}$ & $\mathrm{PF}$ \\
\hline 19 & PPF PF & PPF & $\mathrm{PF}$ & PPF \\
\hline PF/PPF & $10 / 15$ & $8 / 14$ & $13 / 8$ & $4 / 17$ \\
\hline PF/PPF (\%) & $40 / 60$ & $36,4 / 63,6$ & $61,9 / 38,1$ & $19 / 81$ \\
\hline
\end{tabular}

Tabla 4.17. Preferencias sobre significación: convencimiento, entendimiento, memorización y utilidad de las PPF y PF, ciclo II

Siguiendo la misma línea que en el gráfico 4.2 (p. 63), el gráfico siguiente muestra la frecuencia de las categorías de significación de las pruebas preformal y formal para los dos teoremas, y la suma total de ambas frecuencias. 


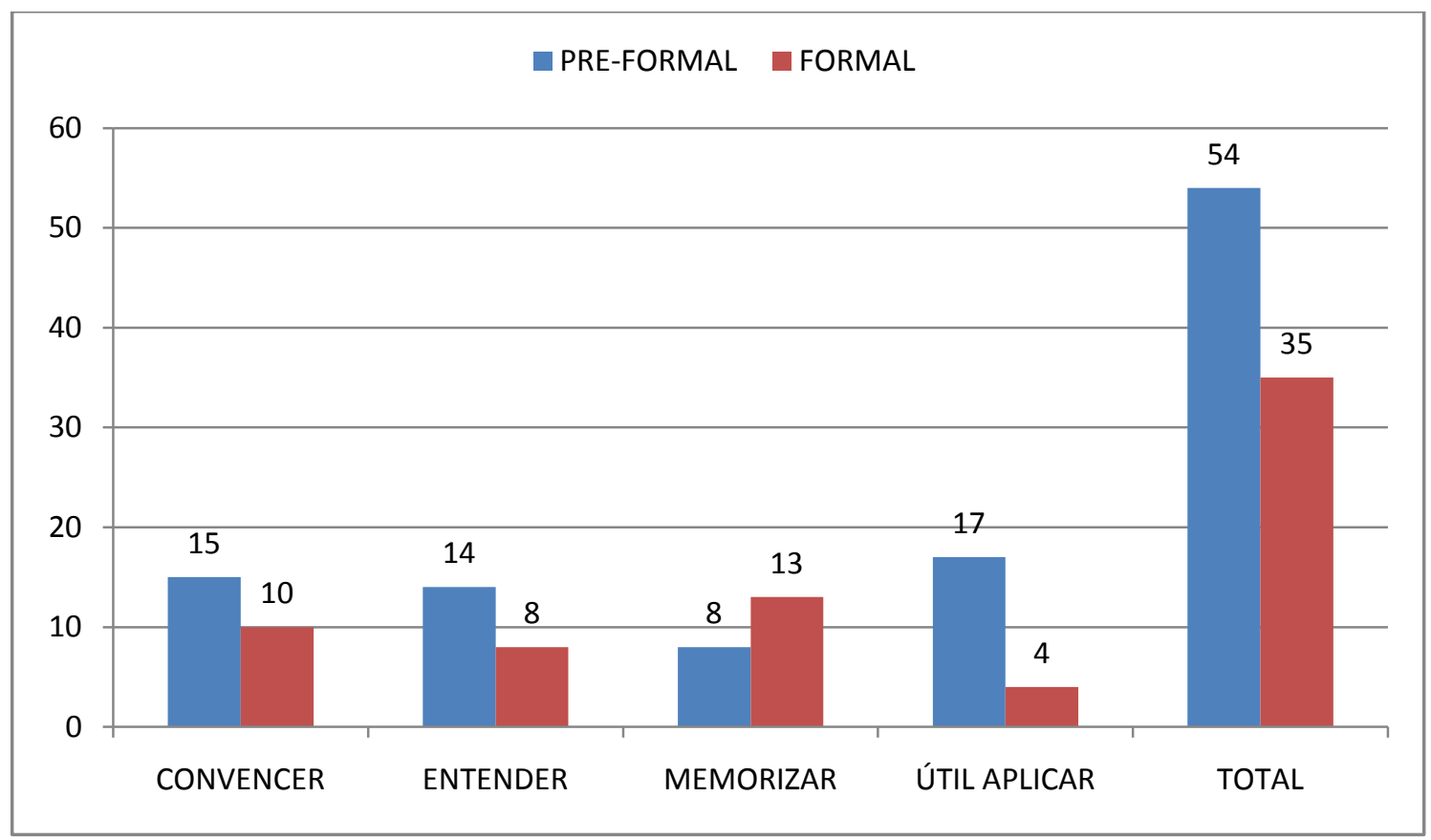

Gráfico 4.4. Preferencias por la significación de las pruebas, convencimiento, entendimiento, memorización y utilidad de las pruebas PPF y PF, ciclo II

El profesor cree que algunos alumnos señalan la PF en "ayuda a memorizar" porque es más corta y la PPF alarga el proceso porque se obtiene explícitamente la derivada de la función y el cálculo del punto.

Veamos con más detalle los motivos en que se apoyan estas respuestas.

\subsubsection{CUÁL DE LAS DOS FORMAS TE CONVENCE MÁS DE LA VERACIDAD DEL TEOREMA}

\section{RESUMEN}

\begin{tabular}{|c|c|c|c|c|c|c|c|}
\hline & \multirow{2}{*}{$\begin{array}{c}\text { Ambas } \\
\text { pruebas }\end{array}$} & \multicolumn{2}{|c|}{ Teorema 1 } & \multicolumn{2}{c|}{ Teorema 2 } & \multirow{2}{*}{ PPF } & \multirow{2}{*}{ PF } \\
\cline { 3 - 6 } & PPF & PF & PPF & PF & 15 & 10 \\
\hline $\begin{array}{c}\text { No de } \\
\text { Alumnos }\end{array}$ & 4 & 1 & 1 & 1 & 1 & 15 & $1,3,6,8,11,12$, \\
Identif. & $1,6,14,19$ & 11 & 8 & 8 & 11 & $\begin{array}{c}1,2,4,5,6,7,8,9,10,14,14,18,19 \\
11,13,14,16,19\end{array}$ \\
\hline
\end{tabular}

Tabla 4.18. Respuesta a la cuestión convence más, ciclo II 


\section{MOTIVOS}

PPF: Es más concreta (4); usa valores conocidos (5); se ve de modo más visible (7); más fácil (8); asequible para nosotros (15).

PF: Es más general (3); es aplicable a todos los casos (12); sirve de base para su aplicación (17); más sencilla (18).

Los motivos que señalan los cuatro alumnos que optan en esta cuestión por ambas pruebas son: el alumno no 6 alude a la conjunción de ambos procedimientos como ideal y a que la PF da una visión general de todos los casos; los restantes tres alumnos de esta opción $(1,14,19)$ no aportan motivos.

La mayoría se decanta a favor de la PPF: nueve alumnos en ambos teoremas, un alumno (11) en el teorema 1 y otro alumno (8) en el teorema 2. Los motivos señalados son: la PPF utiliza valores concretos conocidos, en vez de letras; se la considera más fácil, asequible, más apropiada para teoremas complicados; se ve más claro.

Los motivos de los alumnos que prefieren la PF, cuatro en ambos teoremas, uno (el alumno $n$ o 8) en el teorema 1 y uno (el no 11 ) en el teorema 2 , se reducen a considerar esta prueba general, aplicable y válida para todos los casos.

Cinco alumnos $(1,13,14,16,19)$ no aportan motivo alguno apreciable.

\section{ESTIMACIÓN:}

Parecen, en general, motivos apropiados que sí corresponden a las características de cada una de las pruebas. La preferencia de estos alumnos a favor de la PPF, en esta cuestión, se sitúa en torno al 60\%. Por una parte, a favor de la PPF destaca la concreción, el uso de valores conocidos, la accesibilidad; por otra, los alumnos que creen lo contrario y son favorables a la PF, destacan la universalidad. Sin embargo, la sencillez es una característica señalada por ambos colectivos, lo que sin duda es 
un síntoma consecuente con sus aprendizajes o al menos con el entendimiento de las mismas. Es decir, para un alumno una prueba es más fácil que otra si la ha aprendido o entendido mejor. Precisamente en la tabla 4.19 se resumen las respuestas a esta apreciación y se estudiará la correlación entre ambas respuestas: "más fácil" y "entender mejor". Efectivamente, buscando las respuestas sobre cómo entender los significados, entre los alumnos que responden que es más fácil una prueba u otra, se obtiene que

- Los alumnos que responden que es más fácil la PPF $(10,15)$ también opinan que entienden mejor este tipo de prueba.

- Los alumnos que expresan que la facilidad del tipo de prueba depende del teorema, también responden que entienden mejor su significado con la misma prueba (para el alumno no 11 la PPF en el teorema 1 y la PF en el teorema 2, mientras que para el alumno no 8 ocurre lo contrario y elige PF en el teorema 1 y PPF en el teorema 2).

\subsubsection{CUÁL DE LAS DOS PRUEBAS PUEDE AYUDAR MÁS A ENTENDER EL SIGNIFICADO DEL TEOREMA}

\section{RESUMEN}

\begin{tabular}{|c|c|c|c|c|c|c|c|}
\hline & Ambas & \multicolumn{2}{|c|}{ Teorema 1 } & \multicolumn{2}{c|}{ Teorema 2 } & \multirow{2}{*}{ PPF } & \multirow{2}{*}{ PF } \\
\cline { 3 - 6 } & pruebas & PPF & PF & PPF & PF & & 8 \\
\hline $\begin{array}{c}\text { No de } \\
\text { Alumnos }\end{array}$ & 0 & 1 & 2 & 2 & 1 & 14 & 8 \\
\hline Identif. & & 5 & 8,11 & 8,11 & 5 & $\begin{array}{c}1,2,3,5,6,8,9,10, \\
11,12,15,16,17.19\end{array}$ & $\begin{array}{c}4,5,7,8,11, \\
13,14,18\end{array}$ \\
\hline
\end{tabular}

Tabla 4.19. Respuesta a la cuestión ayuda más a entender el significado, ciclo II

\section{MOTIVOS}

PPF: Aparece más clara (2); concreta (3, 6, 12, 15); acertada (10); menos abstracta (17). 
PF: Te centras más en el teorema al no usar valores concretos (4); más sencilla, si lo estudias (14).

En esta segunda pregunta, 14 alumnos (1 en el teorema 1 y 2 en el teorema 2 ) son favorables a la PPF. Estos son los motivos: se ve (se entiende) más claramente al ofrecer un caso concreto, un ejemplo claro y comprensible; la explicación es más sencilla, menos abstracta, más acertada.

Los 8 alumnos (2 en el teorema 1 y 1 en el teorema 2) que optan a favor de la PF, se apoyan en varios motivos que se reducen a: no utilizar valores, números concretos, así te distraes menos y te centras más en el teorema. El alumno no 14 dice "me parece más sencilla si lo estudias".

\section{ESTIMACIÓN:}

Parecen apropiados los motivos a favor de la PPF y algo menos los aportados a favor de la PF: valores, números concretos, no tienen por qué distraer en un estudio matemático. La preferencia a favor de la PPF es aún mayor, en torno al 64\%, que en la cuestión anterior.

\subsubsection{CUÁL DE LAS DOS PRUEBAS PUEDE AYUDAR MÁS A MEMORIZAR EL TEOREMA}

RESUMEN

\begin{tabular}{|c|c|c|c|c|c|c|c|}
\hline & \multirow{2}{*}{$\begin{array}{c}\text { Ambas } \\
\text { pruebas }\end{array}$} & \multicolumn{2}{|c|}{$\begin{array}{c}\text { Teorema } \\
\mathbf{1}\end{array}$} & \multicolumn{2}{c|}{$\begin{array}{c}\text { Teorema } \\
\mathbf{2}\end{array}$} & \multirow{2}{*}{ PPF } & \multirow{2}{*}{ PF } \\
\cline { 3 - 6 } & & PPF & PF & PPF & PF & & 13 \\
\hline $\begin{array}{c}\text { No de } \\
\text { Alumnos }\end{array}$ & 0 & 0 & 3 & 3 & 0 & 8 & 13 \\
\hline Identif. & & & $1,3,8$ & $1,3,8$ & & $1,3,7,8,9,12,13,16$ & $\begin{array}{c}1,2,3,4,5,6,8,10, \\
11,14,17,18,19\end{array}$ \\
\hline
\end{tabular}

Tabla 4.20. Respuesta a la cuestión ayuda más a memorizar el teorema, ciclo II 


\section{MOTIVOS}

PPF: Más fácil con aplicación numérica que con la formal (7); "es lo que miro al estudiar" (9); "se usa un caso concreto llamativo" (12).

PF: Se puede generalizar (2); no usa valores concretos (4); se basa en conceptos (6); por su gráfica y puntos obtenidos (14); es como un molde (5); menos mecánica (17).

En esta tercera pregunta la mayoría, 13 alumnos (3 en el teorema 1), se decantan a favor de la PF: más general, como un molde, sin considerar valores concretos. Por el contrario, los 8 alumnos (3 en el teorema 2) que eligen la PPF, justifican su elección principalmente en que les resulta más fácil de memorizar por mostrar una situación concreta.

ESTIMACIÓN:

Parecen más objetivos los motivos a favor de la PF y más subjetivos los aportados a favor de la PPF. En los apoyos a favor de cada prueba en los teoremas 1 (la PF) y 2 (la PPF), ha podido influir el orden en que fueron presentadas. El porcentaje de preferencia, en torno al 62\%, cambia únicamente en esta pregunta a favor de la PF.

\subsubsection{CUÁL DE LAS DOS PRUEBAS ES MÁS ÚTIL PARA ILUSTRAR CÓMO SE APLICA EL TEOREMA}

RESUMEN

\begin{tabular}{|c|c|c|c|c|c|c|c|}
\hline & \multirow{2}{*}{$\begin{array}{c}\text { Ambas } \\
\text { pruebas }\end{array}$} & \multicolumn{2}{|c|}{$\begin{array}{c}\text { Teorema } \\
\mathbf{1}\end{array}$} & \multicolumn{2}{c|}{$\begin{array}{c}\text { Teorema } \\
\mathbf{2}\end{array}$} & \multirow{2}{*}{ PPF } & PF \\
\cline { 3 - 6 } & PPF & PF & PPF & PF & & \\
\hline $\begin{array}{c}\text { No de } \\
\text { Alumnos }\end{array}$ & 1 & 0 & 1 & 2 & 0 & 17 & 4 \\
\hline Identif. & 17 & & 8 & 8,15 & & $\begin{array}{c}1,2,3,4,5,6,7,8,9,10, \\
11,13,14,15,16,17,19\end{array}$ & $8,12,17,18$ \\
\hline
\end{tabular}

Tabla 4.21. Respuesta a la cuestión útil para ilustrar la aplicación del teorema, ciclo II 


\section{MOTIVOS}

PPF: Usa números concretos (2,11); gráfica conocida (4); un ejemplo (10); aclara más dónde introducir los valores (3); se ve mejor "el molde" (5); ver un caso en el plano real y entendible (6); obtienes todos los datos e información para la resolución del ejercicio (14).

PF: Me parece más ilustrativa (18).

Es patente la preferencia a favor de la PPF: 17 alumnos (1 en ambas pruebas y 2 en el teorema 2): es concreta, aclara más, aporta todos los datos e información. A favor de la PF están 4 alumnos (1 en ambas pruebas y 1 en el teorema 1). Únicamente el alumno no 18 señala le parece más ilustrativa, sin decir por qué.

ESTIMACIÓN:

La preferencia es evidente por la PPF. No se dan motivos relevantes en las dos únicas opciones a favor de la PF. El porcentaje, en esta cuestión es del $81 \%$ a favor de la PPF.

\begin{tabular}{|c|c|c|c|c|c|c|c|}
\hline \multicolumn{8}{|c|}{ RESUMEN DE LAS CUATRO PREGUNTAS (19 alumnos) } \\
\hline \multirow[t]{2}{*}{ Qué prueba ayuda más a... } & \multirow[t]{2}{*}{ PPF } & \multirow[t]{2}{*}{ PF } & \multicolumn{2}{|c|}{$\begin{array}{c}\text { Teorema } \\
1\end{array}$} & \multicolumn{2}{|c|}{$\begin{array}{c}\text { Teorema } \\
2\end{array}$} & \multirow[t]{2}{*}{ Ambas } \\
\hline & & & PPF & PF & PPF & PF & \\
\hline $\begin{array}{c}\text {...convencer de la veracidad del } \\
\text { teorema }\end{array}$ & 15 & 10 & 1 & 1 & 1 & 1 & 4 \\
\hline $\begin{array}{c}\text {...entender el significado del teo- } \\
\text { rema }\end{array}$ & 14 & 8 & 1 & 2 & 2 & 1 & 0 \\
\hline ...memorizar el teorema & 8 & 13 & 0 & 3 & 3 & 0 & 0 \\
\hline ...ilustrar la aplicación del teorema & 17 & 4 & 0 & 1 & 2 & 0 & 1 \\
\hline Tot a l & 54 & 35 & 2 & 7 & 8 & 2 & 5 \\
\hline
\end{tabular}

Tabla 4.22. Resumen de las cuatro respuestas anteriores, ciclo II 


\section{CONSIDERACIÓN GENERAL}

Las preferencias de estos alumnos en las cuatro cuestiones que se les han presentado están en torno al 61\% a favor de la PPF (54 vs. 35, cf. tabla 4.22). Únicamente en la pregunta sobre la ayuda a memorizar el teorema, los alumnos prefieren la PF. Tres alumnos $(13,16,19)$ no señalan razón alguna de sus preferencias en ninguna de las cuatro cuestiones planteadas. El alumno no 1 en tres cuestiones, y seis alumnos más $(3,9,11,12,15,18)$ en una de ellas, tampoco aportan motivo alguno. Dos alumnos, el no 6 en la cuestión primera y el no 17 en la cuarta, se inclinan por la conjunción de ambas pruebas. El no 17 señala además el orden de ambas: la PF primero "como base" y PPF después "para ilustrar".

Los motivos a favor de la PPF pueden resumirse en que utiliza valores o números concretos, con lo que se ve o comprende más fácilmente, se hace más asequible y la explicación es más sencilla. A favor de la PF se aporta que generaliza más, es aplicable a todos los casos y se basa en conceptos.

\subsubsection{Análisis de las entrevistas}

Es muy interesante el inicio de las entrevistas de este grupo, sobre todo de la segunda. Manifiestan, con una espontánea y responsable naturalidad, la confianza entre profesor y alumnos, lo cual valora el acierto del profesor-investigador en este tipo de experiencias. En este marco confiado y espontáneo se consideran aspectos por los alumnos que el PI toma en consideración en el ciclo siguiente y hasta en la docencia de estos temas en el aula. Se respeta y valora la "autoridad" del profesor, incluso como argumento de certeza en la demostración del teorema. Se explican matices de ambas pruebas: la formal se ve más fácilmente como general para todos los casos, pero con mayor dificultad al entenderla, y la preformal como más fácil de entender, pero con cierta dificultad para su aplicación a todos los casos. Se aclara que ambos procedimientos probatorios, también el preformal aunque se fija en un caso concreto, son absolutamente rigurosos y válidos para todos los casos. Los alumnos, algunos con algún conocimiento previo, quedan plenamente convencidos y ven con mayor claridad los teoremas después de estas demostraciones. 
Los cuatro entrevistados de este segundo grupo encuentran en la prueba preformal mayor facilidad al realizar ejercicios y aplicaciones prácticas habituales en matemáticas, por la representación numérica frente a la simbólica, con la que "se lían más". La prueba preformal, afirma un alumno, "ya es en sí un ejemplo modelo". Y también los cuatro afirman que les ha costado más esfuerzo la prueba formal, tomarla con mayor atención y cuidado porque "hay que hacerse la imagen de cada valor, cada intervalo, cada variable".

Para comunicar contenidos matemáticos, estos alumnos, lógicamente, tendrían en cuenta la dificultad del contenido y la capacidad del receptor. Con el ya iniciado en matemáticas, "emplearía el procedimiento formal” afirma uno de ellos. En general, optan por emplear ambas pruebas, en primer lugar la preformal por su mayor facilidad y posteriormente, la formal.

En la explicación y entendimiento de los pasos de las pruebas todos prefieren, por su facilidad constantemente aludida, la demostración preformal. Alguno destaca esta preferencia especialmente en el teorema del valor medio (Lagrange).

\subsubsection{Reflexión}

En este grupo se da una diferencia en los teoremas en relación a la preferencia de las pruebas: en el teorema del valor medio los alumnos se inclinan a favor de la prueba formal, y en el teorema de la unicidad del límite a favor de la prueba preformal. Ha influido en ello el cambio del orden de las pruebas en el primero de los teoremas, así lo cree el PI y a esto alude también uno de los alumnos (el no la respuesta sobre la prueba formal del primero de los teoremas: "es verdad que ha sido la primera explicada (la prueba formal) y siempre parece más sencillo y estás más atento".

En relación con las funciones de las demostraciones, solamente en la función de ayudar a memorizar los alumnos se decantan más por la prueba formal, por ser más general. En todas las demás funciones la preferida es la prueba preformal por 
ser más concreta, mucho más fácil, más clara, menos abstracta, por entenderse mejor y ser más sencillas las operaciones.

Se tienen en cuenta las sugerencias de la profesora que actúa como observadora de cara al siguiente ciclo: ampliar el tiempo e intentar mantener mejor la atención de los alumnos en la demostración del segundo teorema. Como la misma profesora manifiesta, la actitud general ha sido positiva.

Los alumnos entrevistados estiman estas demostraciones. En ellas la representación numérica es preferida a la simbólica. También es valorada pedagógicamente la aportación de lo concreto (prueba preformal) a lo teórico (prueba formal).

Aprecian también, con respecto a sus exámenes, las explicaciones de clase. Señalan el poco interés que hay en gran número de alumnos por las disciplinas matemáticas y la dificultad de afrontar estas materias. Todos expresan su preferencia por la prueba preformal por la facilidad de su comprensión y del seguimiento de los pasos en el proceso de la demostración y su ventaja para comunicar contenidos matemáticos a otras personas.

El orden de las demostraciones sí influye en la comprensión de las mismas. Señalan como el más eficaz el siguiente: primero la PPF y luego la PF, con cierta flexibilidad.

Afirman la complementariedad de ambos procedimientos de demostración. Esta experiencia es considerada por todos muy positiva. Se sugiere al PI que utilice puntos más sencillos en la PPF del teorema del valor medio (y no los "números raros" $\sqrt{2}, \ln \sqrt{2})$.

Se va perfilando con nitidez un resultado concreto de esta experiencia investigadora: los alumnos prefieren la facilidad, convicción, concreción, claridad y utilidad de la prueba preformal en las demostraciones matemáticas. En cuanto a la prueba formal, estos alumnos valoran su ayuda para memorizar el teorema. A la hora de comunicar contenidos matemáticos, son muy altos los porcentajes a favor de la prueba preformal. No se infravalora la prueba formal pero la prueba preformal si- 
gue valorándose también por este grupo como más significativa en estas demostraciones.

\section{REFLEXIÓN SOBRE LAS ENTREVISTAS}

Los números entre paréntesis hacen referencia a las preguntas del protocolo utilizado en las entrevistas (cf. ANEXO V).

\section{ENTREVISTA I: Cristina/Luis}

\section{I $(1,2,3)$. CERTEZA DE LOS TEOREMAS.}

En relación con la certeza de los teoremas, ambos alumnos indican una convicción sustentada de forma muy subjetiva. Así, por ejemplo:

Yo creo que sí. Si realmente es un teorema y se puede demostrar, pues me lo creo (Cristina).

Yo creo que sí,... yo creo que sí que se cumple (Luis).

También afirman quedar convencidos del enunciado de los teoremas invocando la autoridad matemática y el valor de las pruebas:

Con demostraciones y viéndolo más en profundidad te queda más claro todo (Cristina).

Al ser teorema se tiene que cumplir siempre (Luis).

\section{II (4). FACILIDAD DE APLICACIÓN DE LAS PRUEBAS.}

Ambos prefieren la PPF, destacando la importancia de la representación numérica frente a la simbólica:

Yo con números me aclaro de lo que estoy viendo (Luis).

...sí que lo veo más claro con la PPF (Cristina).

III (5). ESFUERZO EMPLEADO. IMPRESIÓN GENERAL SOBRE LA ACTIVIDAD.

La PF les ha exigido más esfuerzo en ambos teoremas. A Luis en el teorema de la unicidad del límite (nuevo para él) más que en el teorema de Lagrange. 
La PPF se ve más clara (Cristina).

La impresión de la experiencia ha sido para ambos muy positiva. Cristina se fija en lo beneficioso de este tipo de explicaciones en clase de cara a los exámenes. Luis parece confundir la PPF con un mero ejemplo, aunque señala la ventaja pedagógica que aporta a lo teórico una situación concreta.

Me pones esto en el examen y si no lo hubiera visto en clase no... no sabría sacar nada (Cristina).

\section{IV (6). AYUDA AL COMUNICAR CONTENIDOS MATEMÁTICOS.}

Ambos conceden mayor ayuda a la PPF, pero optan por el empleo de las dos pruebas por su complementariedad. Difieren en el orden al usarlas que, en efecto, no debe ser estricto, sino flexible.

La PPF es la que yo creo entendería más la gente (Luis).

Me basaría más en la PPF porque resulta más fácil (Cristina).

Yo explicaría las dos, primero la PF y luego la PPF porque tienes que saber la base teórica para hacer el ejercicio con los números (Luis, que aún confunde "prueba" con “ejercicio").

\section{V $(7,8)$. PASOS DE AMBOS PROCEDIMIENTOS: EXPLICACIÓN Y COMPRENSIÓN.}

Mejor explicados los pasos en el proceso de la PPF y, en el teorema de Lagrange, también aprecian ambos mayor facilidad en la comprensión de los mismos.

Yo también. En Lagrange, a mí muchísimo más fácil la PPF, pero en unicidad del límite las dos he llegado a entender porque no hay mucho cambio de una a otra (Luis).

\section{VI (9). INFLUJO DEL ORDEN DE LAS PRUEBAS EN SU COMPRENSIÓN.}

Afirman los dos que sí influye el orden. Y al aplicarlas, coinciden ambos en utilizar primero la PPF y luego la PF. Luis, influenciado quizá por el desarrollo de la entrevista, matiza aquí con claridad sus respuestas anteriores, añadiendo incluso que se evitaría así algún posible desaliento: 
Hay gente que le va a entrar mucho mejor si directamente se lo cuentas con la PPF. A lo mejor con la PF no se empieza a enterar desde el principio y ya lo deja (Luis).

Yo la PPF, porque realmente yo lo comprendí mejor así (Cristina, apelando a su experiencia personal).

\section{ENTREVISTA II: Pablo/Álvaro}

\section{I $(1,2,3)$. CERTEZA DE LOS TEOREMAS.}

La entrevista comienza con un diálogo espontáneo y distendido entre el profesor y los dos alumnos. Sugieren éstos que, a su criterio, las pruebas se verían mejor con otros datos no irracionales y dedicando una sesión distinta a cada teorema. En diálogo posterior los dos alumnos reconocen que es muy positivo repasar estos teoremas que ellos ya conocen, con estos métodos. A Álvaro la demostración PPF le ha dado bastante más claridad y facilidad, "imagen mental” afirma, para recordar. Consideran ambos la PPF como una prueba rigurosa, no un simple ejemplo. Indican la facilidad de la PPF frente a la dificultad que entraña el simbolismo formal y llegan a señalar las características fundamentales de ambos procedimientos. Se expresan así:

Diría yo que la PF tiene unas ventajas, que puede ser que se ve fácilmente que es para todos los casos y tendrá la dificultad de entenderla. Y la PPF tiene la ventaja de que es más fácil entenderla, pero que tiene la dificultad de si quiero demostrarlo para todos los casos tendré que transformarla (Pablo).

La PF te asegura que eso se cumple en todos los casos, la PPF siempre se te puede escapar algún caso, pues por ejemplo los números negativos puede que no se cumpla, pero vamos, la PF es completa (Álvaro).

\section{II (4). FACILIDAD DE APLICACIÓN DE LAS PRUEBAS.}

Manifiestan ambos su convicción a favor de la PPF:

Yo también la PPF, ya tienes un ejemplo que es casi como un ejercicio modelo, y solo sería trasladarlo cambiando los valores (Álvaro). 


\section{III (5). ESFUERZO EMPLEADO. IMPRESIÓN GENERAL SOBRE LA ACTIVIDAD.}

Para ambos ha supuesto mayor esfuerzo la PF. Pablo, incluso, se atreve a generalizar:

...si fuera otro teorema, un tercer teorema que no conociera, la PF me sería mucho más difícil de asimilar (Pablo).

Impresión de la experiencia:

Yo creo que las dos pruebas se complementan. Puede llevar algo más de tiempo, pero los conceptos te quedan más claros y los teoremas se recuerdan mejor (Álvaro).

Las matemáticas a este nivel son algo difícil. La gente no sabe cómo afrontar esta asignatura. Hay gente que las matemáticas las toma... bueno, a ver si el día del examen hay suerte. Está claro que es más fácil de entender la PPF, pero siempre que se haya estudiado (Pablo).

\section{IV (6). AYUDA AL COMUNICAR CONTENIDOS MATEMÁTICOS.}

En este punto, inclinándose ambos por la facilidad de la PPF, consideran que, en general, habrá que tener en cuenta los lógicos criterios de sopesar la dificultad del teorema concreto y la capacidad del destinatario. Álvaro cree que el simbolismo supone un obstáculo para seguir el razonamiento en las pruebas formales.

Hay muchos símbolos que mucha gente no sabe lo que significan (Álvaro).

El teorema y la PPF sí que lo pondría; primero explicar el teorema y pasar a la PF puede ser un poco de golpe (Pablo, muy claro: primero la PPF; sería brusco comenzar por la PF).

\section{V $(7,8)$. PASOS DE AMBOS PROCEDIMIENTOS: EXPLICACIÓN Y COMPRENSIÓN.}

Los dos entrevistados se inclinan por la PPF.

Como en la PPF son operaciones de sustituir o tal, se puede entender más fácil el paso de una operación a otra (Pablo). En general, yo creo que con la PPF se entiende mejor (Álvaro).

\section{VI (9). INFLUJO DEL ORDEN DE LAS PRUEBAS EN SU COMPRENSIÓN.}

Se muestran coherentes con sus respuestas anteriores y consideran que el orden adecuado es la PPF en primer lugar y luego la PF. 
Yo creo que como es más fácil de entender la PPF, se debería hacer primero para tener una idea general del teorema. Luego, para abstraerla, pues llevarla ya a la PF y acabar de comprender el teorema (Álvaro). Yo pienso lo mismo (Pablo).

\subsection{Ciclo III (ciclo de saturación)}

Este tercer y último ciclo de nuestro trabajo lo denominamos de saturación porque se produce este fenómeno, no aparecen datos nuevos relevantes. Con la conclusión de este ciclo nuestra hipótesis quedaría razonablemente consolidada.

\subsubsection{Planificación}

Ampliaríamos el campo de la investigación, manteniendo las mismas cuestiones, con un nuevo grupo de alumnos que se incorporaría a la experiencia. El PI planificó este tercer ciclo para ser desarrollado en marzo y abril de 2009 con los 20 alumnos que cursaban ese año la misma asignatura de idéntica titulación que en los dos ciclos anteriores. También eligió los mismos teoremas (del valor medio y de la unicidad del límite), como continuación de la línea de investigación emprendida, teniendo en cuenta el refinamiento progresivo con que las sugerencias, aportaciones y reflexiones habían ido enriqueciendo en el transcurso de la experiencia la hipótesis inicial. Con los nuevos resultados, si fueran similares a los anteriores, nuestra hipótesis quedaría aún más reforzada.

En la sesión de exposición de las pruebas y posterior respuesta de los alumnos asistiría la misma profesora de matemáticas presente ya en el ciclo anterior en esta actividad, para responder al protocolo de observación que ya fue utilizado en el ciclo II. Por sugerencia suya, se prolongaría veinte minutos la duración de la actividad. Asimismo, como se sugirió en una de las entrevistas realizadas en el ciclo anterior, para la prueba preformal del teorema del valor medio, se utilizarían puntos más sencillos (números enteros) en el intervalo de definición de la función lo- 
garítmica. La prueba preformal del teorema del valor medio que aparece en el ANEXO I incorpora esta modificación.

Los datos recopilados en este grupo de alumnos se unirían a los obtenidos de los otros dos grupos para realizar un análisis cuantitativo conjunto de todos los alumnos que han participado en los tres ciclos de investigación.

Se efectuarían dos entrevistas por parte del PI a sendas parejas escogidas entre los alumnos de este grupo.

Finalmente, se llevaría a cabo la reflexión general de toda la experiencia investigadora, que daría paso a las correspondientes conclusiones.

\subsubsection{Desarrollo del tercer ciclo (acción)}

La fase de acción se llevó a cabo con arreglo al siguiente calendario:

\begin{tabular}{|c|c|}
\hline Fechas & Contenidos \\
\hline $23 / 03 / 2009$ & $\begin{array}{l}\text { Repaso de axiomática de los números reales } \\
\text { Concepto de prueba preformal según van Asch. } \\
\text { Demostraciones preformales de la desigualdad triangular y de la } \\
\text { propiedad arquimediana }\end{array}$ \\
\hline $25 / 03 / 2009$ & Teoría de límites funcionales \\
\hline $1 / 04 / 2009$ & $\begin{array}{l}\text { Continuidad } \\
\text { Demostraciones preformales de los teoremas de Bolzano y Weierstrass }\end{array}$ \\
\hline $20 / 04 / 2009$ & Primeras definiciones de cálculo diferencial \\
\hline $22 / 04 / 2009$ & $\begin{array}{l}\text { Teoremas del cálculo diferencial } \\
\text { Demostración preformal del teorema "derivabilidad implica } \\
\text { continuidad" y del teorema de singularidad }\end{array}$ \\
\hline $29 / 04 / 2009$ & $\begin{array}{l}\text { Sesión principal } \\
\text { Demostraciones formales y preformales de los teoremas del valor medio } \\
\text { de Lagrange y de la unicidad del límite. } \\
\text { Cuestionario }\end{array}$ \\
\hline $30 / 04 / 2009$ & Entrevistas \\
\hline
\end{tabular}

Tabla 4.23. Calendario del tercer ciclo 
Reunidos los 20 alumnos de este nuevo grupo en su clase habitual de la universidad, el PI explicó el concepto de prueba preformal según van Asch y expuso los mismos teoremas con las demostraciones en el mismo orden que en el ciclo anterior. De nuevo el PI inició la actividad solicitando a los alumnos una actitud receptiva y colaboradora. Al final entregó a los alumnos el mismo cuestionario que en los ciclos anteriores para que lo cumplimentaran.

\subsubsection{Observación de la acción}

Al no darse en este ciclo una variación sustancial en los contenidos de la investigación, el desarrollo de la tercera fase (conforme a la metodología investigaciónacción) ha seguido los pasos de los ciclos anteriores, con las lógicas actualizaciones, según aparece en el calendario de este período. Los alumnos aceptaron con interés toda la experiencia colaborando con empeño en las actividades que tuvieron que realizar. En la sesión principal asistió como observadora la misma persona que en el ciclo anterior.

\subsubsection{Observaciones recogidas en el protocolo del OE}

El protocolo de observación es el mismo del ciclo anterior y, para no ser repetitivos, las respuestas íntegras del OE se reproducen en el ANEXO IV. Aquí, en la memoria, más abajo (sección 4.3.3.3) sólo se muestran las reflexiones sobre el mismo.

\subsubsection{Observaciones del cuaderno de aula del PI}

También en este ciclo, los alumnos manifestaron su interés por la prueba preformal y sus características en las clases previas a la sesión principal. Sus preguntas incidieron en cuestiones semejantes a las de los ciclos I y II: si la prueba preformal puede aplicarse a todos los teoremas; sobre la diferencia fundamental de ambas pruebas, formal y preformal; si el uso de una sola de ellas es suficiente para la completa comprensión de un teorema; en qué sentido pueden ser complementarias ambas modalidades de prueba. De la sesión principal, queda registrado el mayor interés de los alumnos cuando la actividad es protagonizada principalmente por ellos mismos. También queda anotado el mayor uso que el PI hizo, para moti- 
var la atención y colaboración de los alumnos, de la pedagogía mayéutica, con profusión de preguntas en el transcurso de las explicaciones. Fueron numerosas las preguntas referidas al cumplimiento de las tareas encomendadas.

\subsubsection{Reflexiones del protocolo de observación del OE y del cuaderno de aula del PI}

Se ha mantenido, como en ciclos anteriores, el proceso de la metodología investigación-acción, con sus fases de planificación, acción, observación y reflexión. En estas fases se han implicado proporcionalmente los protagonistas principales de la investigación: PI, alumnos y observadora externa.

Destaca la actitud general de orden y compostura que ha primado en todas las actividades llevadas a cabo. No se detectan disfunciones y la observadora califica como positivas las actitudes tanto del profesor como de los alumnos.

En este ciclo III se han recogido los refinamientos obtenidos a partir de los ciclos anteriores. El PI ha tomado buena nota de la experiencia con la que se considera enriquecido en sus actitudes pedagógicas en relación a la enseñanza y a la mejora del aprendizaje matemático en el aula.

\section{a) Tratamiento y metodología:}

Al coincidir el Protocolo de este ciclo III con el del ciclo anterior y tratarse, en consecuencia, de los mismos objetivos y metodología, el OE transcribe nuevamente los títulos de los contenidos, describe las tareas, valora la del PI como clara y detallada e insiste en la ampliación del tiempo dado para responder al cuestionario.

En el CA: han tenido relevancia las sesiones del PI con los alumnos previas a la sesión principal, según se describe en el calendario seguido en la fase de acción del ciclo. Se ha pedido y se ha puesto el mayor interés en el desarrollo de la sesión principal. Su duración, debido a la gran ventaja de concentrar el núcleo de esta experiencia lo más posible, ha podido influir en una leve relajación de alguno de los alumnos al final de la parte expositiva de dicha sesión. El PI ha tratado de paliarlo 
con sus intervenciones y preguntas motivadoras y situando en la parte conclusiva la intervención más activa y personal de los alumnos. Éstos, por su parte, han redoblado su interés y colaboración preguntando y pidiendo todo tipo de aclaraciones.

\section{b) Profesor:}

El OE describe con brevedad la actividad del PI y señala sus actitudes: interés por ser comprendido en la exposición contestando y motivando preguntas. De hecho se trata de una clase especial bien planteada y realizada.

El CA destaca la frecuencia de intervenciones en el tiempo destinado a responder el cuestionario, que se ha prolongado en este ciclo III.

\section{c) Alumnos:}

La mayoría acoge bien la novedad de la clase, la presencia del OE, las explicaciones y avisos del PI. Preguntan con confianza, sobre todo en relación con el cuestionario a responder. La atención decae en la exposición del segundo teorema.

CA: detecta estas circunstancias. Hubo que recordar la fórmula punto-pendiente de la ecuación de una recta y explicar la función logaritmo neperiano. Señala la actitud positiva general.

\section{d) Interacciones profesor-alumnos:}

Con brevedad responde el $\mathrm{OE}$ que sí se crean, favoreciendo el diálogo. Las respuestas del PI tienen rigor, concisión y claridad.

En el CA se alude al interés en una economía en el empleo del tiempo, sin merma de la claridad en la exposición, para evitar cansancios de alumnos en la sesión principal, intensa de suyo y en la que se precisa una continuada atención. Se alude a la actitud mayéutica motivadora de respuestas a la que el PI ha recurrido en varios momentos de la sesión principal. Se constata que esta interacción es perfecta en las entrevistas realizadas a los alumnos (cf. ANEXO VIII, entrevista II, respuesta del alumno $\mathrm{A} 1$ a la pregunta $\mathrm{P}^{9}$ ). 


\section{e) Recursos y condiciones materiales:}

Repite el OE, en este apartado, la respuesta dada en el protocolo del ciclo anterior, ya que no ha habido ninguna alteración en este punto. Sigue considerando suficientes los recursos empleados.

\section{f) Incidencias especiales:}

El OE se muestra muy breve: sin incidencias ni disfunciones.

\subsubsection{Recogida de datos a través del cuestionario}

Tuvo lugar, como en los ciclos anteriores, al final de la sesión principal. Los datos corresponden a las respuestas de los alumnos al cuestionario entregado en dicha sesión después de la explicación, y cumplimentado por ellos en un intervalo de tiempo ampliado veinte minutos respecto al del ciclo anterior.

\subsubsection{Análisis de los datos}

Obtenidos los datos de este tercer ciclo se unieron a los de ciclos anteriores y se analizaron cuantitativamente de manera conjunta en un detallado estudio estadístico, que se presenta en el capítulo 6.

\section{Análisis cualitativo}

Cuestión 1: Explica cómo has entendido cada uno de los teoremas con cada procedimiento. Indica en cada caso claramente el porqué.

El objetivo de esta cuestión es analizar la comprensión de los alumnos de las demostraciones matemáticas sobre las pruebas preformales en contraste con las pruebas formales.

Para el análisis de las respuestas de los alumnos a esta primera cuestión se tienen en cuenta las siguientes tablas de categorías que los alumnos aprecian para justificar su elección en cada modalidad de prueba (preformal y formal), realizadas directamente a partir de la información proporcionada por los alumnos. 


\begin{tabular}{|c|c|c|c|}
\hline \multicolumn{3}{|c|}{ TEOREMA 1 } \\
\hline Prueba preformal & $\mathbf{n}_{\mathbf{i}}$ & Categoría & $\mathbf{n}_{\mathbf{i}}$ \\
\hline Categoría & 9 & Se entiende mejor & 5 \\
\hline Se entiende mejor & 6 & Ambas convencen igual & 6 \\
\hline Ambas convencen igual & 3 & Ayudan más las operaciones & 1 \\
\hline Ayudan más las operaciones & 5 & Más general & 3 \\
\hline Se basa en caso concreto & 5 & Más sencilla & 2 \\
\hline Más sencilla & 0 & Orden: formal-preformal & 1 \\
\hline Orden: preformal-formal & & & \\
\hline
\end{tabular}

Tabla 4.24. Categorías preferidas en el teorema 1, ciclo III

\begin{tabular}{|c|c|c|c|}
\hline \multicolumn{3}{|c|}{ TEREMA 2 } \\
\hline Prueba preformal & $\mathbf{n}_{\mathbf{i}}$ & Categoría & $\mathbf{n}_{\mathbf{i}}$ \\
\hline Categoría & 13 & Convence más & 2 \\
\hline Ambas convencen igual & 5 & Ambas convencen igual & 5 \\
\hline Aplicación caso concreto & 4 & General/práctica & $2 / 1$ \\
\hline Clara/sencilla & $4 / 3$ & Difícil & 1 \\
\hline $\begin{array}{c}\text { Fácil de recordar, ayuda a en- } \\
\text { tender pasos }\end{array}$ & 2 & Gráfica fácil & 2 \\
\hline
\end{tabular}

Tabla 4.25. Categorías preferidas en el teorema 2, ciclo III 


\section{Observaciones del PI en torno a estas respuestas}

\section{TEOREMA 1}

De los veinte alumnos de este grupo, nueve orientan su juicio a favor de la PPF, prueba realizada en segundo lugar después de la PF, que es preferida por cinco alumnos. Los seis alumnos restantes señalan cualidades positivas en ambas pruebas.

\section{MOTIVOS}

a) A favor de ambas pruebas: tres alumnos (2, 6 y 17) se fijan en lo positivo del orden en que se han realizado, primero la PF, segundo la PPF. Los otros tres $(4,8$ y 16) resaltan aspectos concretos de una y otra: lo ilustrativo de las gráficas, su forma abstracta, recordarla mejor al aplicarla, en la PF; utilización de la derivada, la utilidad del ejemplo, más claro el desarrollo, en relación con la PPF.

b) A favor de la PF: más clara (1); "genérica” (querrá decir general) (7); para cualquier valor (15); me ha parecido más sencilla (14); algo más sencilla (20).

c) A favor de la PPF: se explica ejemplificada (3); por la función logarítmica más clara y explícita (5); se basa en un caso concreto (9); la PF es muy abstracta (10); veo mejor la sucesión de pasos (11); más sencilla (13) al usar números (12); se toman partes concretas, más fácil de comprender (18) y (19).

\section{TEOREMA 2}

En el segundo de los teoremas trece alumnos optan por la PPF, tan sólo dos están a favor de la PF, y de los restantes cinco alumnos, tres señalan semejante dificultad 
en ambas pruebas (1, 2 y 19) y los otros dos (4 y 17) las equiparan en sencillez y ayuda para entender los conceptos.

\section{MOTIVOS}

a) A favor de la PF: el alumno no 5 ve esta prueba "mínimamente" más práctica, clara y dinámica; el no 18 lo ve y visualiza mejor con la PF.

b) A favor de la PPF: en general es considerada más fácil, clara y sencilla. Concretamente: diez alumnos $(3,6,7,9,10,12,13,14,15$ y 16) la juzgan más clara, intuitiva y sencilla; se ve mejor la utilidad (8); se ven mejor los pasos (11); más fácil de recordar (20).

En resumen, si nos fijamos en los juicios de estos alumnos sobre las dos pruebas para ambos teoremas, la PPF resulta ser la mejor valorada y preferida.

Las tablas 4.24 y 4.25 (p. 121) muestran, resumidas, las características más señaladas en esta primera cuestión de la experiencia, por estos alumnos del ciclo tercero. Como sucedió en los grupos de los ciclos anteriores, también en este grupo destacan en relación con la prueba preformal, las categorías de sencillez, claridad, facilidad, aplicación concreta y ayuda al mejor entendimiento de los teoremas, y en relación con la prueba formal, el aspecto de generalización.

Escaneamos algunas respuestas significativas. No están desorientados estos alumnos en sus apreciaciones, en ellas reflejan motivos y orden de sucesión del uso de ambas pruebas.

El alumno Alberto S. C. (20), que prefiere la prueba formal en el primer teorema, afirma de la preformal, en plena sintonía con van Asch, que en esta prueba está ya la idea esencial, considerándola como una verdadera prueba. 
Teorema 1: He entendido mejor la pruesa Forvell del T.V.Medis puquee considero que es algo mas senailla a la hora de exponer los conceptes. La prueba preformal tiene la ida esencial pero no me es suficiente par una comprension bastante buena.

Teorema 2: En este cas prefieo ela prueba preformal. He nisto la explicación muctó más clara y creo que es facil de comperder, s recordarto. La pruebs formel me werta also más

Álvaro C. C. (5) reparte su preferencia de las pruebas en cada uno de los dos teoremas.

- Teorema 1:

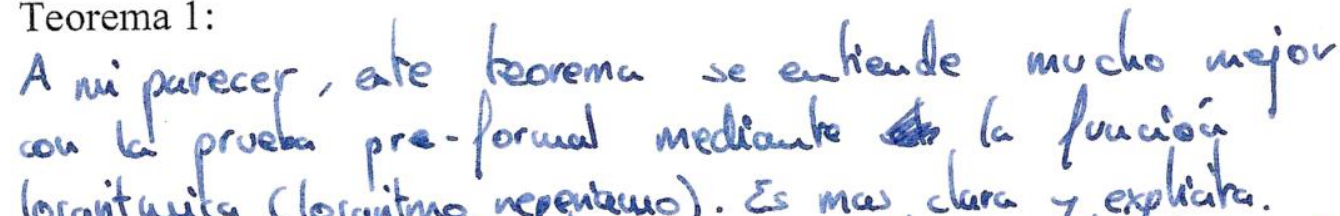
logantmica (loganitro neppriamo). Es mas clara 7 expliata. elentrow que (aventiunto.

- Teorema 2:

En ate teorema, medicumbe el sejundo procedimionto, la proveba foranal, Io he visto como más prachico. mas ligero para el

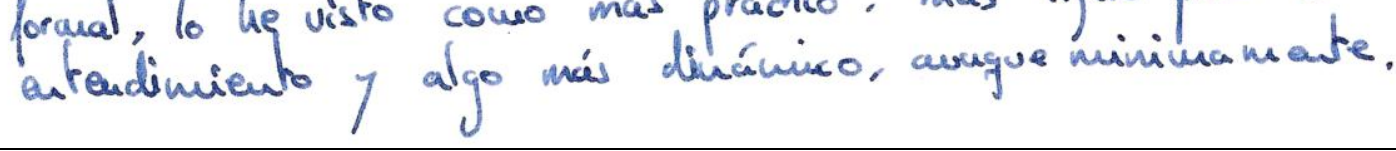

Javier F. M. (9) opta por la prueba preformal por ser más fácil, por basarse en un caso concreto y como paso previo, en caso de uso sucesivo, a la prueba formal. 


\section{Teorema 1:}

Con la proba formal, he euteridido las cavas de una fonua in poco abstracta, er decir, prefiero la formea pre-fonual ya que te bata eu un caso contreto, y a partir de ahi lleqausos a la forma foneral.

Teorema 2:

Con la forua pre-fonual, ocurre conno en el teasema auterior. AL exponer un caso concreto, es weás intivitivo que la preba fonual y más facil de ver. Por lo tauto me. "ha aclarado acás la procba pre-formal.

Cuestión 2: Puntúa de 1 a 10 las cuatro pruebas que hemos realizado atendiendo a las siguientes categorías: facilidad (F), gusto $(G)$, claridad (C), aprendizaje (A). Entiende que:

$>1$ es muy difícil y 10 es muy fácil.

$>1$ no me gusta y 10 me gusta mucho.

$>1$ no es nada clara y 10 es muy clara.

$>1$ no se aprende nada y 10 se aprende mucho.

- Preformal del teorema 1: F_, G_, C_, A_.

- Formal del teorema 1: F_, G_, C__, A_. 
- Preformal del teorema 2: $\mathrm{F}_{\_}, \mathrm{G}_{\_}, \mathrm{C}_{\longrightarrow}, \mathrm{A}$

- Formal del teorema 2: $\mathrm{F}_{\_}, \mathrm{G}_{\_}, \mathrm{C}_{\_}, \mathrm{A}_{-}$.

En la columna COMPRENSIÓN: se reescriben las puntuaciones de la cuestión 2. En la columna PREFERENCIA: para cada teorema, comparando las puntuaciones de cada modalidad de prueba en cada una de las cuatro categorías, se indica el número de veces en que "gana" (supera) la prueba preformal a la formal, y viceversa.

\begin{tabular}{|c|c|c|c|}
\hline TEOREMA & MODALIDAD & COMPRENSIÓN & PREFERENCIA \\
\hline \multirow{2}{*}{ Teorema 1} & Preformal & $\mathrm{F}_{-}, \mathrm{G}_{-}, \mathrm{C}_{\ldots}, \mathrm{A}_{-}$ & \\
\hline & Formal & F_, $G_{-}, C_{2}, A_{-}$ & \\
\hline \multirow{2}{*}{ Teorema 2} & Preformal & F_, $G_{-}, C_{2}, A_{-}$ & \\
\hline & Formal & $\mathrm{F}_{-}, \mathrm{G}_{-}, \mathrm{C}_{-}, \mathrm{A}_{-}$ & \\
\hline \multirow{2}{*}{ Total } & Preformal & & \\
\hline & Formal & & \\
\hline
\end{tabular}

Tabla 4.26. Esquema de la pregunta $2^{a}$ del cuestionario

El objetivo de esta cuestión es, dentro de la comprensión, conocer la preferencia de los alumnos por una u otra de las modalidades de prueba en relación con cuatro importantes categorías: su facilidad, su claridad, el gusto por ellas y su influjo en el aprendizaje del alumno. En la siguiente tabla se recopilan las respuestas correspondientes a la pregunta 2 del cuestionario entregado a los alumnos. 


\subsubsection{PREFERENCIA \\ (F: facilidad; G: gusto; C: claridad; A: aprendizaje)}

En la tabla 4.27 se muestran los resultados de las respuestas de los alumnos sobre la significación de las pruebas según las categorías asignadas. En las columnas destinadas a preferencia aparecen reflejadas con sus iniciales las categorías preferidas por los alumnos en cada modalidad de prueba y para cada teorema. En las columnas destinadas a puntuación se consigna en cada casilla el número de categorías preferidas en cada modalidad de prueba y para cada teorema.

\begin{tabular}{|c|c|c|c|c|c|c|c|c|}
\hline $\begin{array}{l}\text { A } \\
\mathbf{L}\end{array}$ & \multicolumn{4}{|c|}{ TEOREMA 1} & \multicolumn{4}{|c|}{ TEOREMA 2} \\
\hline $\mathbf{M}$ & PREFC & RMAL & FOI & MAL & PREF & RMAL & FOI & AAL \\
\hline $\begin{array}{l}\mathbf{N} \\
\mathbf{0}\end{array}$ & $\begin{array}{l}\text { Prefe- } \\
\text { rencia }\end{array}$ & $\begin{array}{c}\text { Pun- } \\
\text { tuación }\end{array}$ & $\begin{array}{l}\text { Prefe- } \\
\text { rencia }\end{array}$ & $\begin{array}{c}\text { Pun- } \\
\text { tuación }\end{array}$ & $\begin{array}{l}\text { Prefe- } \\
\text { rencia }\end{array}$ & $\begin{array}{c}\text { Pun- } \\
\text { tuación }\end{array}$ & $\begin{array}{l}\text { Prefe- } \\
\text { rencia }\end{array}$ & $\begin{array}{c}\text { Pun- } \\
\text { tuación }\end{array}$ \\
\hline 1 & - & - & $\mathrm{F}, \mathrm{G}, \mathrm{C}, \mathrm{A}$ & 4 & G,A & 2 & $\mathrm{C}$ & 1 \\
\hline 2 & $\mathrm{~F}$ & 1 & - & - & $\mathrm{F}, \mathrm{G}, \mathrm{C}$ & 3 & - & - \\
\hline 3 & $\mathrm{~F}, \mathrm{~A}$ & 2 & - & - & $\mathrm{F}, \mathrm{A}$ & 2 & - & - \\
\hline 4 & $\overline{F, G, A}$ & 3 & $\mathrm{C}$ & 1 & $\mathrm{~F}, \mathrm{G}, \mathrm{C}$ & 3 & $\bar{A}$ & 1 \\
\hline 5 & $\mathrm{~F}, \mathrm{G}, \mathrm{C}, \mathrm{A}$ & 4 & - & - & - & - & $\mathrm{G}, \mathrm{C}, \mathrm{A}$ & 3 \\
\hline 6 & - & - & $\mathrm{F}, \mathrm{G}, \mathrm{C}, \mathrm{A}$ & 4 & $\mathrm{~F}, \mathrm{G}, \mathrm{C}, \mathrm{A}$ & 4 & - & - \\
\hline 7 & - & - & $\mathrm{F}, \mathrm{G}, \mathrm{C}, \mathrm{A}$ & 4 & $\mathrm{~A}$ & 1 & $\mathrm{~F}, \mathrm{C}$ & 2 \\
\hline 8 & $\mathrm{~F}, \mathrm{C}$ & 2 & A & 1 & $\mathrm{C}$ & 1 & $\mathrm{~F}$ & 1 \\
\hline 9 & $\mathrm{~F}, \mathrm{G}, \mathrm{C}, \mathrm{A}$ & 4 & - & - & $\mathrm{F}, \mathrm{C}, \mathrm{A}$ & 3 & - & - \\
\hline 10 & $\mathrm{~F}, \mathrm{G}, \mathrm{C}, \mathrm{A}$ & 4 & - & - & $\mathrm{F}, \mathrm{G}, \mathrm{C}, \mathrm{A}$ & 4 & - & - \\
\hline 11 & G & 1 & $\mathrm{C}, \mathrm{A}$ & 2 & $\mathrm{~F}, \mathrm{C}$ & 2 & - & - \\
\hline 12 & $\mathrm{~F}, \mathrm{C}$ & 2 & - & - & $\mathrm{F}, \mathrm{G}, \mathrm{C}, \mathrm{A}$ & 4 & - & - \\
\hline 13 & $\overline{F, G}$ & 2 & - & - & G & 1 & $\mathrm{C}$ & 1 \\
\hline 14 & - & - & $\mathrm{F}, \mathrm{G}, \mathrm{C}, \mathrm{A}$ & 4 & $\mathrm{~F}, \mathrm{G}$ & 2 & A & 1 \\
\hline 15 & $\mathrm{~F}$ & 1 & $\mathrm{G}, \mathrm{C}$ & 2 & G & 1 & $\mathrm{C}$ & 1 \\
\hline 16 & $\overline{F, G, C}$ & 3 & A & 1 & $\mathrm{~F}, \mathrm{G}, \mathrm{C}, \mathrm{A}$ & 4 & - & - \\
\hline 17 & - & - & $\mathrm{F}, \mathrm{G}, \mathrm{C}, \mathrm{A}$ & 4 & $\mathrm{~F}$ & 1 & $\mathrm{C}$ & 1 \\
\hline 18 & $\mathrm{~F}, \mathrm{~A}$ & 2 & $\mathrm{C}$ & 1 & - & - & $\mathrm{F}, \mathrm{G}, \mathrm{C}, \mathrm{A}$ & 4 \\
\hline 19 & $\mathrm{~F}, \mathrm{G}, \mathrm{C}, \mathrm{A}$ & 4 & - & - & $\mathrm{F}, \mathrm{G}$ & 2 & - & - \\
\hline 20 & - & - & $\mathrm{F}, \mathrm{G}, \mathrm{C}, \mathrm{A}$ & 4 & $\mathrm{C}, \mathrm{A}$ & 2 & - & - \\
\hline $\begin{array}{l}\text { To- } \\
\text { tal }\end{array}$ & & 35 & & 32 & & 42 & & 16 \\
\hline
\end{tabular}

Tabla 4.27. Preferencias y puntuaciones según categorías de las pruebas PPF y PF en ambos teoremas, ciclo III 
El gráfico de la figura siguiente muestra la frecuencia de las preferencias de las pruebas preformal y formal para los dos teoremas, y la suma total de ambas frecuencias.

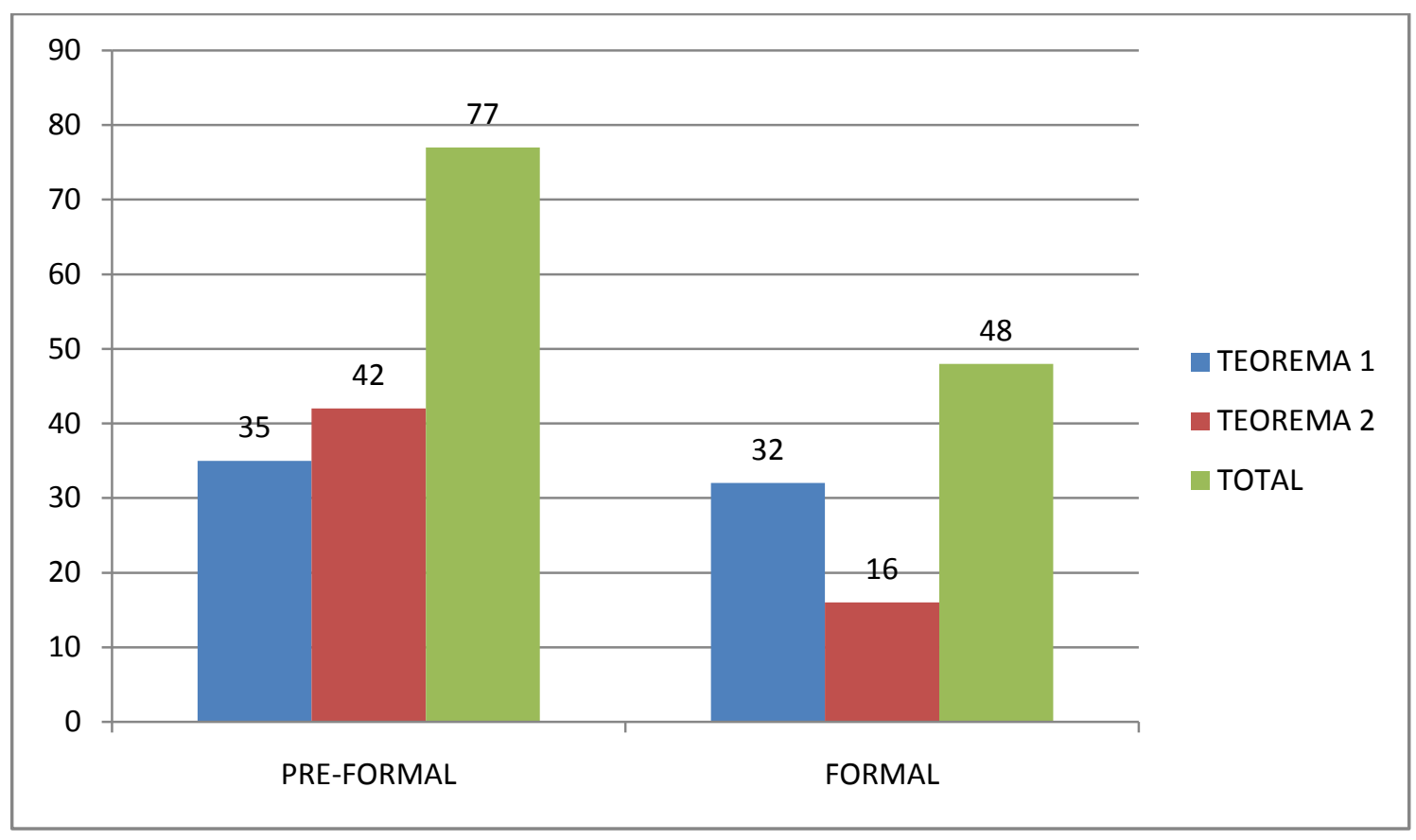

Gráfico 4.5. Preferencia por las categorías de las pruebas, ciclo III

Estos alumnos otorgan su preferencia a la PPF en los dos teoremas (teorema del valor medio y teorema de la unicidad del límite), siendo mayor la diferencia entre ambas pruebas en el 2o de ellos (42 frente a 16) que en el 1ํ (35 frente a 32 ).

Tomados los resultados de ambos teoremas en conjunto, destaca la preferencia de la PPF $(35+42=77)$ sobre la PF $(32+16=48)$. En porcentaje, $61,6 \%$ frente a $38,4 \%$.

Por el número de alumnos, los que se manifiestan a favor de la PPF se distribuyen de la siguiente manera: en las cuatro categorías, ocho alumnos (el no 10 coincide en los dos teoremas); en tres categorías, cinco alumnos; once alumnos, en dos de las categorías y en una sola categoría, ocho alumnos.

A favor de la PF, la distribución queda así: siete alumnos están a favor de esta prueba en las cuatro categorías; un alumno solamente $\left(\mathrm{n}^{\circ} \mathrm{o}\right.$, en el $2^{\mathrm{o}}$ de los teore- 
mas) en tres de ellas; en dos categorías, tres alumnos y en una sola categoría once alumnos.

Y la preferencia, según categorías, en el conjunto de ambos teoremas es la siguiente: la más "preferida" es la facilidad con 25 opciones en favor de la PPF frente a 9 en favor de la PF. En segundo lugar, el gusto: 19 veces a favor de la PPF frente a 9 a favor de la PF. En tercer lugar, la claridad: 17 veces igualmente preferida en la PF y en la PPF. Por último, en cuanto al aprendizaje: 16 veces en favor de la PPF y 13 en favor de la PF.

\subsubsection{SIGNIFICACIÓN}

En este tercer ciclo, que resultó ser de saturación, se vuelve a analizar la significación de las pruebas para los alumnos: convencimiento, entendimiento, memorización y aplicabilidad de las dos modalidades de prueba.

Cuestión 3: Responde a las siguientes preguntas, señalando en cada caso claramente el porqué:

- ¿Cuál de las dos formas te convence más de la veracidad del teorema?

- ¿Cuál de las dos pruebas puede ayudar más a entender el significado del teorema?

- ¿Cuál de las dos pruebas puede ayudar más a memorizar el teorema?

- ¿Cuál de las dos pruebas es más útil para ilustrar cómo se aplica el teorema?

El objetivo de esta cuestión es, respecto de las funciones de la demostración (Hanna, 1989), que los alumnos comparen los dos procedimientos en relación con la capacidad de convicción, la ayuda a la comprensión, memorización y utilización de los teoremas.

La siguiente tabla resume las respuestas de los 20 alumnos de este ciclo a la pregunta 3 del cuestionario y se elabora utilizando las siglas PPF o PF, según que un 
alumno concreto crea que para él tiene más significación la prueba preformal o formal. Esto se repite en cada una de las cuatro características señaladas en la cuestión 3.

\begin{tabular}{|c|c|c|c|c|}
\hline Alumno & $\begin{array}{l}\text { Convence } \\
\text { más }\end{array}$ & $\begin{array}{c}\text { Ayuda más a } \\
\text { entender }\end{array}$ & $\begin{array}{c}\text { Ayuda más a } \\
\text { memorizar }\end{array}$ & $\begin{array}{c}\text { Más útil para } \\
\text { aplicar el teorema }\end{array}$ \\
\hline 1 & PPF & PPF PF & PPF & PPF \\
\hline 2 & PPF & PPF PF & $\mathrm{PF}$ & PPF \\
\hline 3 & PPF & PPF & PPF & PPF \\
\hline 4 & PPF & PPF & PPF & PPF PF \\
\hline 5 & PPF PF & PPF PF & PPF & PPF \\
\hline 6 & PPF PF & PPF & $\mathrm{PF}$ & PPF PF \\
\hline 7 & $\mathrm{PF}$ & PPF & $\mathrm{PF}$ & PPF \\
\hline 8 & PPF & PPF & $\mathrm{PF}$ & PPF \\
\hline 9 & $\mathrm{PF}$ & PPF & PPF & $\mathrm{PPF}$ \\
\hline 10 & PPF & PPF & PPF & PPF \\
\hline 11 & PPF & PPF & PPF PF & PPF \\
\hline 12 & PPF & PPF & $\mathrm{PF}$ & PPF \\
\hline 13 & PPF & PPF & $\mathrm{PF}$ & PPF PF \\
\hline 14 & PPF PF & PPF PF & $\mathrm{PF}$ & $\mathrm{PPF}$ \\
\hline 15 & PF & - & $\mathrm{PF}$ & PPF \\
\hline 16 & PPF & PPF & PPF PF & PPF \\
\hline 17 & PPF PF & $\mathrm{PF}$ & $\mathrm{PF}$ & PPF \\
\hline 18 & $\mathrm{PF}$ & PPF & $\mathrm{PF}$ & $\mathrm{PF}$ \\
\hline 19 & PPF & $\mathrm{PF}$ & PPF & $\mathrm{PPF}$ \\
\hline 20 & $\mathrm{PF}$ & $\mathrm{PF}$ & PPF & PPF \\
\hline PF/PPF & $9 / 15$ & $7 / 16$ & $12 / 10$ & $4 / 19$ \\
\hline PF/PPF (\%) & $37,5 / 62,5$ & $30,4 / 69,6$ & $54,5 / 45,5$ & $17,4 / 82,6$ \\
\hline
\end{tabular}

Tabla 4.28. Preferencias sobre significación: convencimiento, entendimiento, memorización y utilidad de las PPF y PF, ciclo III

Siguiendo la misma línea que en los gráficos 4.2 y 4.4 (pp. 63 y 102), el 4.6 siguiente muestra la frecuencia de las categorías de significación de las pruebas preformal $\mathrm{y}$ formal para los dos teoremas, y la suma total de ambas frecuencias. 


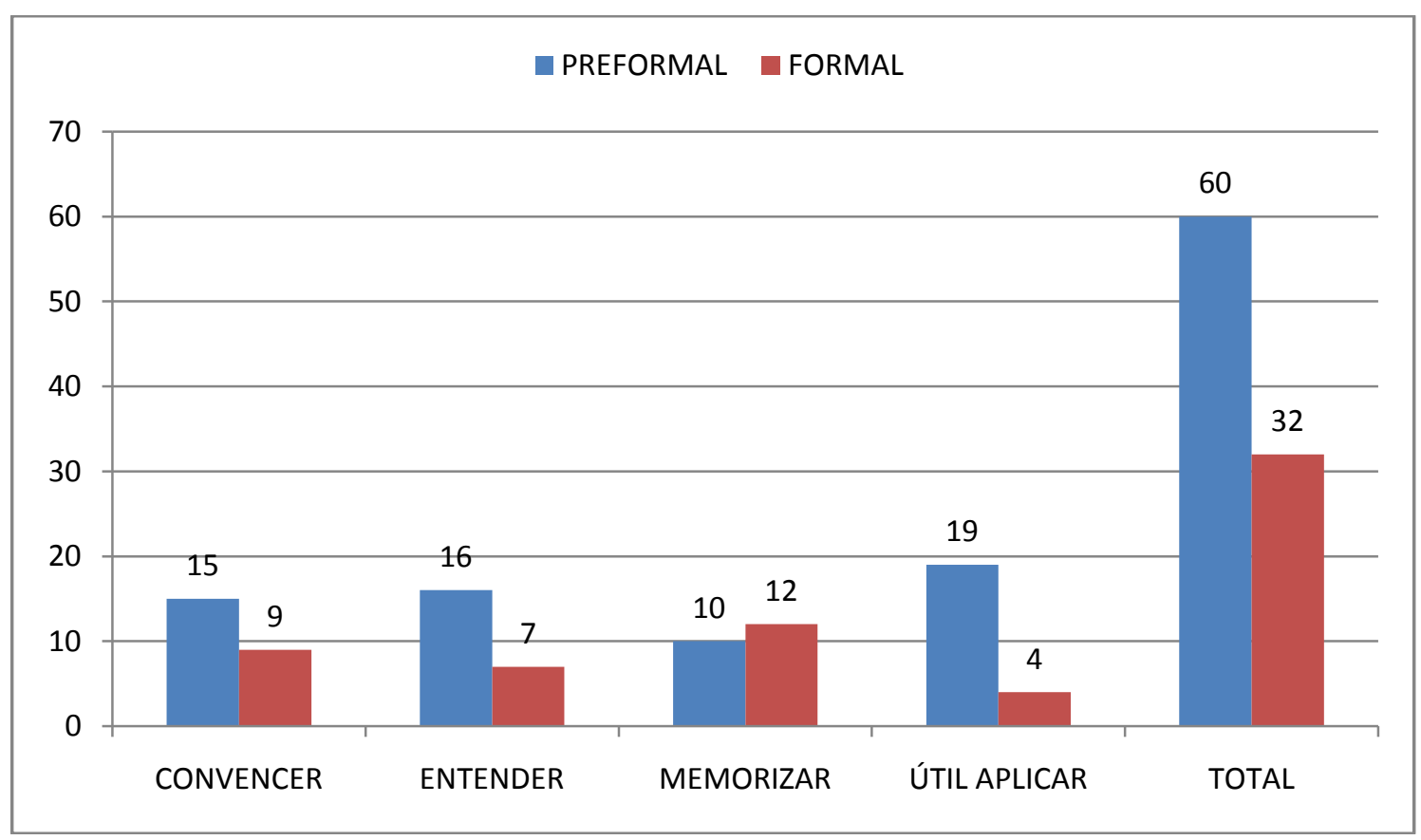

Gráfico 4.6. Preferencias por la significación de las pruebas, convencimiento, entendimiento, memorización y utilidad de las pruebas PPF y PF, ciclo III

El profesor cree que algunos alumnos señalan la PF en "ayuda a memorizar" porque es más corta y la PPF alarga el proceso porque se obtiene explícitamente la derivada de la función y el cálculo del punto.

Veamos con más detalle los motivos en que se apoyan estas respuestas.

\subsubsection{CUÁL DE LAS DOS FORMAS TE CONVENCE MÁS DE LA VERACIDAD DEL TEOREMA}

RESUMEN

\begin{tabular}{|c|c|c|c|c|c|c|c|}
\hline & \multirow{2}{*}{$\begin{array}{l}\text { Ambas } \\
\text { pruebas }\end{array}$} & \multicolumn{2}{|c|}{ Teorema 1} & \multicolumn{2}{|c|}{ Teorema 2} & \multirow{2}{*}{ PPF } & \multirow{2}{*}{ PF } \\
\hline & & PPF & $\mathbf{P F}$ & PPF & PF & & \\
\hline $\begin{array}{c}\text { № de } \\
\text { Alumnos }\end{array}$ & 0 & 1 & 3 & 3 & 1 & 15 & 9 \\
\hline Identif. & & 14 & $5,6,20$ & $5,6,17$ & 14 & $\begin{array}{c}1,2,3,4,5,6,8,10,11 \\
12,13,14,16,17,19\end{array}$ & $\begin{array}{c}5,6,7,9,14 \\
15,17,18,20\end{array}$ \\
\hline
\end{tabular}

Tabla 4.29. Respuesta a la cuestión convence más, ciclo III 


\section{MOTIVOS}

La mayoría, 15 alumnos ( 1 en el teorema 1 y 3 en el teorema 2), designan a la PPF como la que convence más de la veracidad de los teoremas. Motivos señalados: verlo aplicado $(2,10,11,12)$; se ve más claro $(8,16,19)$; se entiende mejor $(4,13)$. Nueve alumnos (3 en el teorema 1 y 1 en el teorema 2) indican a la PF como más convincente. Los motivos que alegan son: es una demostración seria para todos los casos (7); la PPF no es general (9); verlo gráficamente (18).

\section{ESTIMACIÓN:}

Los alumnos señalan, acertadamente, cualidades de la PPF: claridad, facilidad y confirmación. El alumno nº 18 al referirse a la PF afirma como motivo "verlo gráficamente, aunque sea fórmula sin números", alude a la PPF inclinándose por la PF por su expresión gráfica, circunstancia que también se da en la PPF; lo que afirma el alumno no 7 de la PF "demostración seria para todos los casos" es aplicable también a la PPF al ser auténtica demostración a través de una concreción, un cálculo concreto; el alumno no 9 atribuye, también erróneamente, la misma limitación a la PPF. Es notable la diferencia de apreciación favorable de la PPF, 15 vs. 9 en el conjunto de los dos teoremas (62,5\% frente a 37,5\%) en esta cualidad tan importante y decisiva en la docencia y tan estimada y valorada por profesores y alumnos.

\subsubsection{CUÁL DE LAS DOS PRUEBAS PUEDE AYUDAR MÁS A ENTENDER EL SIGNIFICADO DEL TEOREMA}

\section{RESUMEN}

\begin{tabular}{|c|c|c|c|c|c|c|c|}
\hline & \multirow{2}{*}{$\begin{array}{c}\text { Ambas } \\
\text { pruebas }\end{array}$} & \multicolumn{2}{|c|}{ Teorema 1 } & \multicolumn{2}{c|}{ Teorema 2 } & \multirow{2}{*}{ PPF } & \multirow{2}{*}{ PF } \\
\cline { 3 - 6 } & PPF & PF & PPF & PF & 16 & 7 \\
Alumnos & 2 & 1 & 1 & 1 & 1 & 16 & $7,2,3,4,5,6,7,8,9,10$, \\
\hline Identif. & 2,14 & 5 & 1 & 1 & 5 & $\begin{array}{c}1,2,5,14, \\
11,12,13,14,16,18\end{array}$ \\
\hline
\end{tabular}

Tabla 4.30. Respuesta a la cuestión ayuda más a entender el significado, ciclo III 


\section{MOTIVOS}

También en esta cuestión se da mayoría a favor de la PPF: 16 alumnos (1 en el teorema 1, 1 en el teorema 2 y 2 en ambos teoremas). Parece coherente que sea lo que más ayuda a entender el significado del teorema lo que también convence más de su veracidad, pues si convence es que también se entiende. Optan por la PF: 7 alumnos ( 1 en el teorema 1, 1 en el teorema 2 y 2 en ambos teoremas). Los motivos señalados por los alumnos son:

A favor de la PPF: utilidad (4); claridad $(6,16)$; facilidad $(7,8,9)$; verlo aplicado $(11,12,18)$.

A favor de la PF: es más amplia, general, integra todos los conceptos $(19,20)$.

ESTIMACIÓN:

Los motivos aportados son estimables y recurrentes en todos los grupos que han realizado esta experiencia, lo cual confirma con mayor fuerza las conclusiones anteriores. En la tabla 4.28 (p. 130) el porcentaje a favor de la PPF es de 69,6\%, frente a $30,4 \%$ favorable a la PF.

\subsubsection{CUÁL DE LAS DOS PRUEBAS PUEDE AYUDAR MÁS A MEMORIZAR EL TEOREMA}

RESUMEN

\begin{tabular}{|c|c|c|c|c|c|c|c|}
\hline & \multirow{2}{*}{$\begin{array}{c}\text { Ambas } \\
\text { pruebas }\end{array}$} & \multicolumn{2}{|c|}{ Teorema 1} & \multicolumn{2}{|c|}{ Teorema 2} & \multirow{2}{*}{ PPF } & \multirow{2}{*}{ PF } \\
\hline & & PPF & PF & PPF & PF & & \\
\hline $\begin{array}{c}\text { № de } \\
\text { Alumnos }\end{array}$ & 1 & 1 & 1 & 1 & 0 & 10 & 12 \\
\hline Identif. & 11 & 1 & 16 & 16 & & $\begin{array}{l}1,3,4,5,9,10 \\
11,16,19,20\end{array}$ & $\begin{array}{r}2,6,7,8,11,12,13 \\
14,15,16,17,18\end{array}$ \\
\hline
\end{tabular}

Tabla 4.31. Respuesta a la cuestión ayuda más a memorizar el teorema, ciclo III 


\section{MOTIVOS}

Para "memorizar", este grupo de veinte alumnos se decanta en mayoría a favor de la PF: 12 alumnos (1 en el teorema 1). Un alumno, el no 11 , opta por las dos pruebas: "la PPF me ayuda a memorizar los pasos a seguir, la PF a aprender las fórmulas utilizadas". Otro alumno, no 16, divide las pruebas según el teorema. Por la PPF se decantan 10 alumnos ( 1 en el teorema 1, 1 en el teorema 2 y 1 en ambos teoremas). Los motivos alegados para la PF son: es general, se puede aplicar a todos los casos, $(6,8,12,17,18)$; fácil (2); adecuada (14); fórmula con pasos metódicos (7). Para la PPF: el ejemplo ayuda a asimilar (9), y a recordar el teorema (19); se aprende el método (10); hay menor cantidad de conceptos (20).

ESTIMACIÓN:

El grupo coincide con los grupos de los ciclos anteriores en señalar en esta cuestión a la PF mayor porcentaje favorable. El alumno, al memorizar, suele tener la experiencia de que una fórmula general es más fácil de retener y se presta mejor a ser recordada. Los que optan por la PPF señalan motivos muy atinados que inciden muy positivamente en asimilar, entender y en consecuencia recordar y retener los teoremas. En la tabla 4.28 (p. 130) los porcentajes son 54,5\% favorable a la PF frente a $45,5 \%$ favorable a la PPF.

\subsubsection{CUÁL DE LAS DOS PRUEBAS ES MÁS ÚTIL PARA ILUSTRAR CÓMO SE APLICA EL TEOREMA}

\section{RESUMEN}

\begin{tabular}{|c|c|c|c|c|c|c|c|}
\hline & Ambas & \multicolumn{2}{|c|}{ Teorema 1 } & \multicolumn{2}{|c|}{ Teorema 2 } & \multirow{2}{*}{ PPF } & \multirow{2}{*}{ PF } \\
\cline { 3 - 6 } & pruebas & PPF & PF & PPF & PF & & 4 \\
\hline $\begin{array}{c}\text { No de } \\
\text { Alumnos }\end{array}$ & 1 & 2 & 1 & 1 & 1 & 19 & 4 \\
\hline Identif. & 13 & 1,4 & 4 & 1 & 6 & $\begin{array}{c}1,2,3,4,5,7,8,9,10,13,14,15,16, \\
17,18,19,20\end{array}$ & $4,6,13,18$ \\
\hline
\end{tabular}

Tabla 4.32. Respuesta a la cuestión útil para ilustrar la aplicación del teorema, ciclo III 


\section{MOTIVOS}

Se repite una vez más aquí la tónica de los grupos anteriores, es decir, una gran ventaja otorgada a la PPF: 19 alumnos (2 en el teorema 1, 1 en el teorema 2 y 1 en ambos teoremas). A favor de la PF: 4 alumnos (1 en el teorema 1, 1 en el teorema 2 y 1 en ambos teoremas). En los motivos, el alumno no 13 se inclina por ambas pruebas "ambas son igual de útiles", aunque matiza "en mi opinión la PPF es más útil”. Solamente un alumno, el nำ 18, señala la PF como más útil para ilustrar cómo se aplica el teorema por "el dibujo de la gráfica". Los motivos aducidos a favor de la PPF son: ilustra mejor la aplicación (5, 7, 9 (los gráficos más útiles)); nada mejor que un ejemplo, caso concreto $(10,19)$; más clara (8); más ordenada (14); más rápida (20); el alumno no 17 pone una condición previa "haber explicado antes la PF".

\section{ESTIMACIÓN:}

La coincidencia de los tres grupos otorgando un alto porcentaje a favor de la PPF avala y confirma la gran utilidad de esta prueba para ilustrar cómo se aplica el teorema. Los motivos son también recurrentes en todos los grupos. La tabla 4.28 (p. 130) muestra un porcentaje de $82,6 \%$ a favor de la PPF, frente a $17,4 \%$ favorable a la PF.

\begin{tabular}{|c|c|c|c|c|c|c|c|}
\hline \multicolumn{8}{|c|}{ RESUMEN DE LAS CUATRO PREGUNTAS (20 alumnos) } \\
\hline \multirow[t]{2}{*}{ Qué prueba ayuda más a... } & \multirow[t]{2}{*}{ PPF } & \multirow[t]{2}{*}{ PF } & \multicolumn{2}{|c|}{$\begin{array}{c}\text { Teorema } \\
1\end{array}$} & \multicolumn{2}{|c|}{$\begin{array}{c}\text { Teorema } \\
2\end{array}$} & \multirow[t]{2}{*}{ Ambas } \\
\hline & & & PPF & PF & PPF & $\mathbf{P F}$ & \\
\hline $\begin{array}{c}\text {...convencer de la veracidad del } \\
\text { teorema }\end{array}$ & 15 & 9 & 1 & 3 & 3 & 1 & 0 \\
\hline $\begin{array}{c}\text {...entender el significado del teo- } \\
\text { rema }\end{array}$ & 16 & 7 & 1 & 1 & 1 & 1 & 2 \\
\hline ...memorizar el teorema & 10 & 12 & 1 & 1 & 1 & 0 & 1 \\
\hline ...ilustrar la aplicación del teorema & 19 & 4 & 2 & 1 & 1 & 1 & 1 \\
\hline Tot a l & 60 & 32 & 5 & 6 & 6 & 3 & 4 \\
\hline
\end{tabular}

Tabla 4.33. Resumen de las cuatro respuestas anteriores, ciclo III 


\section{CONSIDERACIÓN GENERAL}

Las preferencias de estos alumnos en las cuatro cuestiones que se les han presentado, están, en términos generales, en torno al $65 \%$ a favor de la PPF (60 vs. 32, cf. tabla 4.33). Únicamente en la tercera pregunta (cuál te ayuda más a memorizar el teorema) los alumnos prefieren la PF. Cuatro alumnos se inclinan por la conjunción de ambas pruebas: dos alumnos ( 2 y 14), en la cuestión segunda (ayuda a entender el significado del teorema) sin señalar motivos; un alumno, el no 11, en la cuestión tercera (ayuda a memorizar el teorema) "con la PPF memoriza los pasos y con la PF las fórmulas"; y un alumno, el no 13, en la cuarta cuestión (útil para ilustrar la aplicación del teorema) sin aportar motivo. Los motivos más citados a favor de la PF son: se puede aplicar a todos los casos, es general, amplia, procede con pasos metódicos, la gráfica ayuda y lo hace ver. Y en favor de la PPF: gráficas más útiles, más clara, nada mejor que un ejemplo, lo concreto, más fácil, rápida para enseñar, más útil para ilustrar la aplicación del teorema, se entiende mejor, menor cantidad de conceptos.

En resumen: la PF es considerada más general; la PPF se entiende mejor, es sencilla, clara, útil para ilustrar aplicaciones. De nuevo los motivos giran alrededor de la generalización de la PF y de la concreción, sencillez, claridad y utilidad de la PPF. Excepto en la cuestión tercera (ayuda a memorizar el teorema), en la que es preferida con poca diferencia la $\mathrm{PF}$, en las otras tres cuestiones, y con mayor distancia, la preferida es la PPF.

\subsubsection{Análisis de las entrevistas}

En la primera cuestión sobre el conocimiento previo de estos teoremas, los entrevistados de este tercer ciclo manifiestan tener "alguna idea", pero sin convicción de ellos. Con esta experiencia han adquirido absoluta y rigurosa claridad de su verdad para todos los casos. Conocen perfectamente ambas pruebas y pueden así elegir la aplicación de la más adecuada. 
Al trabajar en ejercicios y prácticas en matemáticas, todos, excepto uno, se inclinan abiertamente por el procedimiento preformal, que, como prueba verdadera, pasa del caso concreto a la generalidad, tiene mayor "ejemplificación".

La prueba formal les ha supuesto a todos mayor esfuerzo de comprensión por la representación simbólica, "valores surrealistas" ("tanto simbolito griego", dice uno de los entrevistados). Hay que discurrir la aplicación de la fórmula, lo que exige conocer previamente bien el teorema, y esto se consigue más fácilmente con la prueba preformal.

La experiencia les ha parecido bastante positiva ("interactiva y demás" añade uno), claro el cuestionario y ordenada la distribución. Muestran con sinceridad su satisfacción.

A la hora de comunicar los teoremas, comentan que, "a su nivel", lo confiesan con sencillez, les ayuda más hacer uso de los procedimientos preformales por utilizar un caso concreto, por ser más sencillos, más asequibles de entender y de expresar. Les cuesta distinguir con claridad ejemplo de prueba con caso concreto.

En relación con los "pasos" de las pruebas, para entenderlos prefieren la prueba preformal, aduciendo que es más fácil, pero sin prescindir de la formal. Como explicación de su engarce, su nexo lógico, prefieren la prueba formal, pues la preformal, dicen, "lo da por supuesto", afirmación con que se reconoce valor de auténtica prueba a la preformal. También señalan, muy acertadamente, que la explicación de los pasos dependerá de la capacidad del receptor.

A modo de síntesis de los tres ciclos, podemos decir que las entrevistas han constituido para estos alumnos un ejercicio agradable y de interés. Todos manifiestan con sinceridad que han quedado satisfechos. Como aportación a toda la experiencia, sus manifestaciones confirman los resultados de otros análisis. Confiesan que parten de un conocimiento insuficiente de los contenidos, que se transforma, con el 
empleo de estas pruebas, en conocimiento preciso de las mismas y firme convicción de la verdad de los teoremas para todos los casos.

Dicen que es la prueba preformal la que más les va a ayudar en los trabajos y ejercicios en matemáticas por su claridad y el uso de notación numérica, y la prueba formal, por su mayor abstracción y representación simbólica, la que más esfuerzo les ha supuesto para su comprensión.

Para comunicar contenidos matemáticos a otros, piensan que en su nivel, que consideran modesto, además de discernir la medida entre el contenido a transmitir y la capacidad del receptor, lo mejor es utilizar las dos pruebas, primero la preformal, por su facilidad y sencillez y luego la formal, por su rigor y generalización. Consideran también que el uso de ambas pruebas les ha facilitado la explicación de los pasos probatorios, acudiendo a la formal para la explicación de su nexo lógico, y jugando un papel primordial la preformal, por ser manejable, fácil y cercana, para entender dichos pasos.

\subsubsection{Reflexión}

Con este tercer grupo se confirman y refuerzan los resultados de los dos anteriores a favor de la PPF, con dos matices: en las categorías, la claridad es considerada igualmente en ambos tipos de prueba y en las funciones de la demostración, una leve preferencia por la prueba formal en ayuda a memorizar, que responde a que el alumno suele experimentar las fórmulas generales como más fáciles de retener.

Los motivos de estas preferencias están señalados con bastante acierto. Es muy notable la diferencia a favor de la PPF en la función utilidad de aplicación del teorema, como ha ocurrido también en las dos experiencias anteriores, lo que avala con mayor firmeza la gran utilidad del procedimiento preformal en las demostraciones matemáticas.

En las entrevistas se concede mayor facilidad a la hora de las aplicaciones a la prueba preformal, que por alguno de ellos es calificada como prueba verdadera (ri- 
gurosa), afirmando que viene a ser lo mismo que la prueba formal y menos abstracta.

Los alumnos entrevistados coinciden en que la prueba formal es la que más esfuerzo les ha supuesto. Asimismo coinciden en que la experiencia de ver las demostraciones según los dos procedimientos resulta positiva. No dudan al afirmar que si se trata de comunicar contenidos matemáticos donde encuentran mayor ayuda es en la modalidad preformal.

En resumen el PI considera que a lo largo de todo el tercer ciclo queda patente que es la prueba preformal la más significativa para estos alumnos.

\section{REFLEXIÓN SOBRE LAS ENTREVISTAS}

Los números entre paréntesis hacen referencia a las preguntas del protocolo utilizado en las entrevistas (cf. ANEXO V).

\section{ENTREVISTA I: David/Javier}

\section{I $(1,2,3)$. CERTEZA DE LOS TEOREMAS.}

Parten ambos alumnos de algún conocimiento previo, muy poco asegurado y consistente, más bien olvidado.

Tengo mis conocimientos de matemáticas muy guardados (lejanos) dentro de la cabeza. Yo lo tengo muy olvidado. (Javier).

El de la unicidad del límite sabía lo que es, pero no sabía que había un teorema (David).

Rememorar lo ya olvidado y realizar nuevas experiencias, les da claridad y reafirmación en su veracidad.

...recordarlo de forma más clara (David).

...se te quedan mejor los conceptos (Javier). 


\section{II (4). FACILIDAD DE APLICACIÓN DE LAS PRUEBAS.}

Con cierta confusión ambos, David parece preferir como mejor ayuda del trabajo en matemáticas la PF en primer lugar y luego, para explicar el teorema, la PPF. Por su parte, Javier se fija en primer lugar en la PPF para "entender" el teorema y luego la PF como demostración definitiva.

La demostración formal está bien, pero para indicar cómo es la forma de hacerlo, y luego ya para explicarlo, pues el ejercicio (¿la PPF?) (David).

A mí personalmente el (procedimiento) preformal... primero prefiero ver un ejemplo (PPF) y luego que me digan la demostración (Javier).

\section{III (5). ESFUERZO EMPLEADO. IMPRESIÓN GENERAL SOBRE LA ACTIVIDAD.}

Javier afirma que en la PF hay que discurrir más. Para David el esfuerzo es más o menos igual en ambas pruebas si es algo que ya conoces; si no lo conoces, cree necesario más esfuerzo en la PF. También le cuesta más el uso de símbolos formales.

Discurres más en la formal (Javier).

...otra cosa que no sabes, pues a lo mejor sí que me cuesta más la formal (David).

Ambos afirman que "esta experiencia de ver una demostración de dos maneras diferentes siempre es positiva".

\section{IV (6). AYUDA AL COMUNICAR CONTENIDOS MATEMÁTICOS.}

Se inclinan los dos alumnos por la PPF.

Yo creo que el (procedimiento) preformal siempre que yo voy a explicar algo a una persona que conozco o me tienen que explicar algo que yo desconozco, con un ejemplo se ve más fácil. Entonces yo creo que la preformal es una manera más fácil de hacerlo (Javier).

Estoy de acuerdo (David).

\section{V $(7,8)$. PASOS DE AMBOS PROCEDIMIENTOS: EXPLICACIÓN Y COMPRENSIÓN.}

Con espontaneidad afirman que con la PPF es más fácil. Las explicaciones que dan reflejan que no se han liberado totalmente de esa tendencia a percibir esta prueba como un ejemplo por su peculiaridad de cálculo concreto. 
Una prueba preformal es más fácil (David).

Estoy de acuerdo (Javier).

\section{VI (9). INFLUJO DEL ORDEN DE LAS PRUEBAS EN SU COMPRENSIÓN.}

Expresan estos alumnos su deseo de "facilidad" en el lenguaje matemático, "que sea coloquial, normal, más cercano a nosotros". Desde este anhelo, señalan el procedimiento preformal como más indicado al comienzo en el orden de las dos pruebas. Pero quieren también lograr una total convicción que contemple y se extienda a todo el ámbito de aplicación del teorema. Por eso afirman que, después de la PPF, no debe prescindirse de la PF.

A mí me ayudó a comprenderla antes más la preformal (Javier).

Yo también pienso lo mismo: la preformal. Pero yo en mi elección me quedaría con una mezcla de las dos (David).

\section{ENTREVISTA II: Julio/Vicente}

\section{I $(1,2,3)$. CERTEZA DE LOS TEOREMAS.}

La situación de conocimiento de los teoremas propuestos de la que parten estos alumnos es muy semejante.

Yo la primera vez que les veía aquí contigo (Vicente).

Únicamente valor medio, y un poco por encima, pasando rápidamente (Julio).

Reconocen lo positivo de la experiencia y haberla realizado con estas dos pruebas, que así pueden ser comparadas.

Queda bastante claro cómo está una demostración, cómo está otra y se ve de dónde sale. Entonces creo que no hay ningún problema (Julio).

Con las dos demostraciones quedaron bastante más claras las cosas de cómo se aplicaban a los ejemplos los dos teoremas (Vicente). 


\section{II (4). FACILIDAD DE APLICACIÓN DE LAS PRUEBAS.}

Estiman ambos que el procedimiento que más les ayuda es el preformal, y al indicar el motivo, señalan la característica de esta prueba, ser rigurosa: "viene a ser lo mismo que la formal", pero es preferible "aplicándolo con un ejemplo".

Yo la preformal, ya que aplicándolo con un ejemplo... de mi forma de ser me ayuda más la preformal (Vicente).

Yo también me tiro por la preformal. Aparte de que tiene la misma base que la formal, puesto que viene a ser lo mismo, pero una tiene más ejemplificación (Julio).

\section{III (5). ESFUERZO EMPLEADO. IMPRESIÓN GENERAL SOBRE LA ACTIVIDAD.}

También en relación con el trabajo aportado, los dos están de acuerdo en que ha sido la PF la que ha supuesto mayor esfuerzo.

A mí el formal me ha parecido bastante más abstracto... con valores un poco surrealistas. Entonces tienes que estar más pendiente de dónde salen los valores, las fórmulas y demás. Me ha parecido un poco más complicada (Julio). Yo también porque la formal tienes que estar un poco más atento de dónde van saliendo las letras (Vicente).

Impresión sobre toda la actividad:

Sí, estuvo muy bien. Sí, estaba claro y ordenado (el cuestionario, la distribución) (Vicente).

Ha sido productiva. Bastante positivo, interactivo y demás. Se podía ver por dónde ibas (Julio).

\section{IV (6). AYUDA AL COMUNICAR CONTENIDOS MATEMÁTICOS.}

Desde su nivel consideran en la PPF mayor ayuda a la hora de comunicar contenidos matemáticos. Son un tanto pudorosos en esta estimación, pero ciertamente acertados en que la PPF resulta más asequible y práctica para comunicar estos contenidos.

Yo creo que estando en nuestro nivel, así entre nosotros, un (procedimiento) preformal sería más sencillo, más práctico, más asequible para entender (Julio). 
A la hora de explicárselo a un compañero, hacer una preformal estaría mejor. Seríamos más capaces de explicárselo mejor que con una prueba formal (Vicente).

\section{V $(7,8)$. PASOS DE AMBOS PROCEDIMIENTOS: EXPLICACIÓN Y COMPRENSIÓN.}

Para los dos, los pasos de la demostración quedan mejor explicados en la PF, aunque Vicente al añadir que "depende de la gente" parece advertir que es decisiva la mayor o menor claridad del que recibe la exposición de la prueba y, lógicamente, de quien la presenta. Los dos coinciden en que la PPF sí es la más adecuada para entender el enlace de unos pasos con otros en la demostración. Se inclinan, pues, por una complementariedad de ambos procedimientos para explicar y entender sus diversos pasos.

Para mí, para explicar los pasos de cómo se tiene que ir realizando yo creo que la formal a secas, puesto que la preformal ya lo da un poco por supuesto (Julio).

Sí, yo también estoy de acuerdo..., aunque, bueno, depende de la gente, más claro o menos claro (Vicente).

La preformal para entender, sí (Julio).

Yo también la preformal, pero también luego no está de más ver la formal para ver de dónde han salido esas demostraciones preformales (Vicente).

\section{VI (9). INFLUJO DEL ORDEN DE LAS PRUEBAS EN SU COMPRENSIÓN.}

Las respuestas a esta cuestión parecen volver a adolecer de esa difusa impresión que considera a veces a la PPF como un "ejemplo" más que como una verdadera demostración, a pesar de que en las anteriores intervenciones estos alumnos mostraban haberla superado. Desde esta impresión, quizá aún latente en ellos, afirman que si no se conoce un teorema, el orden adecuado de las pruebas será la PF en primer lugar, seguida de la PPF, y al revés, si ya se tiene algún conocimiento previo del teorema.

En los teoremas que no conoces de antes, quizá hacer una demostración formal para ver en conjunto todo el teorema y luego ejemplificarlo con una de- 
mostración preformal. Pero en otros teoremas que tengas conocimiento previo de ellos, con una preformal bastaría (Vicente).

En el primer teorema, bueno, yo tenía un poco de idea de cómo iba, entonces... primero la formal y luego la preformal; no tuve problemas. En el siguiente no tenía conocimiento previo de él. Entonces primero la preformal y luego la formal. Me costó más entenderle así, de primeras, con una preformal, sin saber de dónde salían previamente las cosas, pero... (Julio).

\subsubsection{Análisis conjunto de los tres grupos}

Aportamos como muestra de la tendencia constante manifestada a favor de la preferencia por la prueba preformal como más significativa en los tres ciclos desarrollados con los 67 alumnos, las respuestas dadas a la 3로 ellos.

\begin{tabular}{|c|c|c|c|c|c|c|c|}
\hline \multicolumn{8}{|c|}{ RESUMEN DE LAS CUATRO PREGUNTAS (67 alumnos) } \\
\hline \multirow{2}{*}{ Qué prueba ayuda más a... } & \multirow{2}{*}{ PPF } & \multirow{2}{*}{ PF } & \multicolumn{2}{|c|}{ Teorema1 } & \multicolumn{2}{|c|}{ Teorema2 } & \multirow{2}{*}{ Ambas } \\
\hline & & & PPF & PF & PPF & PF & \\
\hline $\begin{array}{c}\text {...convencer de la veracidad del } \\
\text { teorema }\end{array}$ & 39 & 36 & 2 & 4 & 4 & 2 & 4 \\
\hline $\begin{array}{l}\text {...entender el significado del teo- } \\
\text { rema }\end{array}$ & 48 & 25 & 2 & 3 & 3 & 2 & 4 \\
\hline ...memorizar el teorema & 26 & 47 & 1 & 5 & 5 & 0 & 5 \\
\hline $\begin{array}{l}\text {...ilustrar la aplicación del teore- } \\
\text { ma }\end{array}$ & 61 & 10 & 2 & 2 & 3 & 1 & 3 \\
\hline Total & 174 & 118 & 6 & 14 & 15 & 5 & 16 \\
\hline
\end{tabular}

Tabla 4.34. Resumen de las cuatro respuestas anteriores, ciclos I, II y III

Refleja las características señaladas en los tres grupos. En la tercera pregunta el porcentaje es $64,4 \%$ a favor de la PF, pero en la cuarta, el porcentaje a favor de la PPF es 85,9\%. En términos absolutos, la tendencia es a favor de la PPF en un porcentaje de 59,6\% (174 vs. 118). 
El gráfico siguiente refleja las frecuencias de la tabla 4.34 .

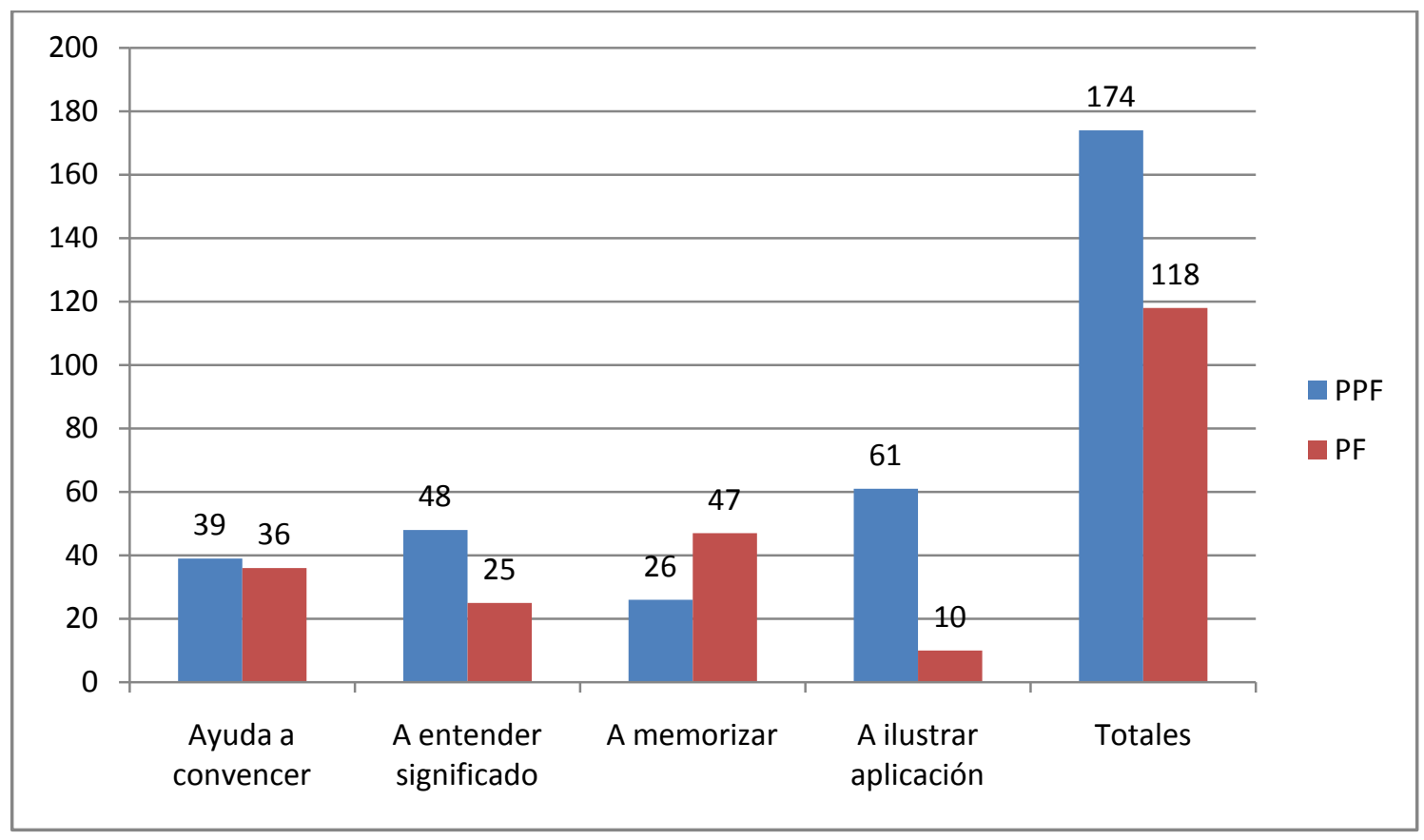

\section{Gráfico 4.7. Resumen de los tres grupos de alumnos}

Finalmente, en marzo de 2010, realizamos una última experiencia con unos alumnos del Máster de Profesor de Matemáticas en Secundaria de la Universidad de Valladolid, que consistió en preparar una prueba preformal del teorema de Weierstrass: toda función continua en un intervalo cerrado y acotado admite un máximo absoluto y un mínimo absoluto en dicho intervalo.

El objetivo de esta actividad era experimentar con estos profesores la dificultad para preparar pruebas preformales en su labor docente. El PI sólo les había demostrado con una prueba preformal el teorema del valor medio de Lagrange y les pidió como tarea que hicieran una prueba preformal del citado teorema de Weierstrass. Respondieron siete de los nueve alumnos del máster. Las calificaciones han sido muy positivas $(8,10,10,5,10,10,10)$. Por tanto, se ve que son capaces de transformar sin dificultad una prueba formal en prueba preformal. Así pues, a los profe- 
sores de este máster no les resultaría difícil elaborar pruebas preformales para su labor docente. 


\section{CAPÍTULO 5}

\section{ANÁLISIS ESTADÍSTICO DE LOS DATOS}

\section{DEL TERCER CICLO (2009)}

Vamos a tratar a continuación de analizar pormenorizadamente para cada una de las características estudiadas, si existe o no igualdad de unas demostraciones a otras, tratando de ver si efectivamente los alumnos prefieren las pruebas preformales a las formales. Se hace este análisis como un complemento del cualitativo que se ha realizado en los tres ciclos de investigación-acción.

Sólo se analiza el tercer ciclo en este capítulo; en el siguiente se hace el análisis global de los tres ciclos. Es un análisis sencillo que no es pretencioso y que se limita a tratar de descubrir posibles diferencias estadísticamente significativas en las respuestas de los alumnos.

En cada una de las cuatro categorías investigadas facilidad, gusto, claridad, aprendizaje, se comparan las respuestas aportadas por los alumnos en relación con uno y otro teorema, $1^{\mathrm{a}}$ y $2^{\mathrm{a}}$ demostración, analizando estadísticamente: correlaciones, comparación de muestras, de medias, de desviaciones típicas, contrastes; terminando con el análisis también de las comparaciones de los resultados medios para el conjunto de las demostraciones. Los signos representan: p1, p2 en 1er. y $2^{\text {o }}$ lugar, demostración del teorema 1, demostración del teorema 2; p, f en 3er. lugar, 
prueba preformal (p), prueba formal (f); F, G, C, A, Me en 4ํㅡ lugar, Facilidad, Gusto, Claridad, Aprendizaje, Media. En el texto, PPF representa la pueba preformal; PF la formal. El capítulo se completa con una breve reflexión derivada de este análisis.

\subsection{Comparaciones de los resultados de la facilidad (F) de cada una de las demostraciones}

La tabla 5.1 recoge las frecuencias de cada una de las calificaciones asignadas por los alumnos en la categoría facilidad en cada una de las cuatro demostraciones.

\begin{tabular}{rrrrrr}
\hline & $\mathbf{p 1 p F}$ & $\mathbf{p 1 f F}$ & $\mathbf{p 2 p F}$ & \multicolumn{2}{c}{$\mathbf{p 2 f F}$} \\
\hline $\mathbf{1}$ & 0 & 0 & 0 & 0 \\
$\mathbf{2}$ & 0 & 0 & 0 & 0 \\
$\mathbf{3}$ & 0 & 2 & 3 & 6 \\
$\mathbf{4}$ & 3 & 4 & 3 & 5 \\
$\mathbf{5}$ & 2 & 5 & 6 & 4 \\
$\mathbf{6}$ & 8 & 6 & 4 & 4 \\
$\mathbf{7}$ & 4 & 2 & 4 & 1 \\
$\mathbf{8}$ & 3 & 1 & 0 & 0 \\
$\mathbf{9}$ & 0 & 0 & 0 & 0 \\
$\mathbf{1 0}$ & 0 & 0 & 0 & 0 \\
\hline Total & 20 & 20 & 20 & 20
\end{tabular}

Tabla 5.1. Frecuencias en la categoría facilidad en cada una de las pruebas y de los teoremas

Asimismo, estos datos quedan reflejados en los gráficos 5.1 y 5.1 bis siguientes. 

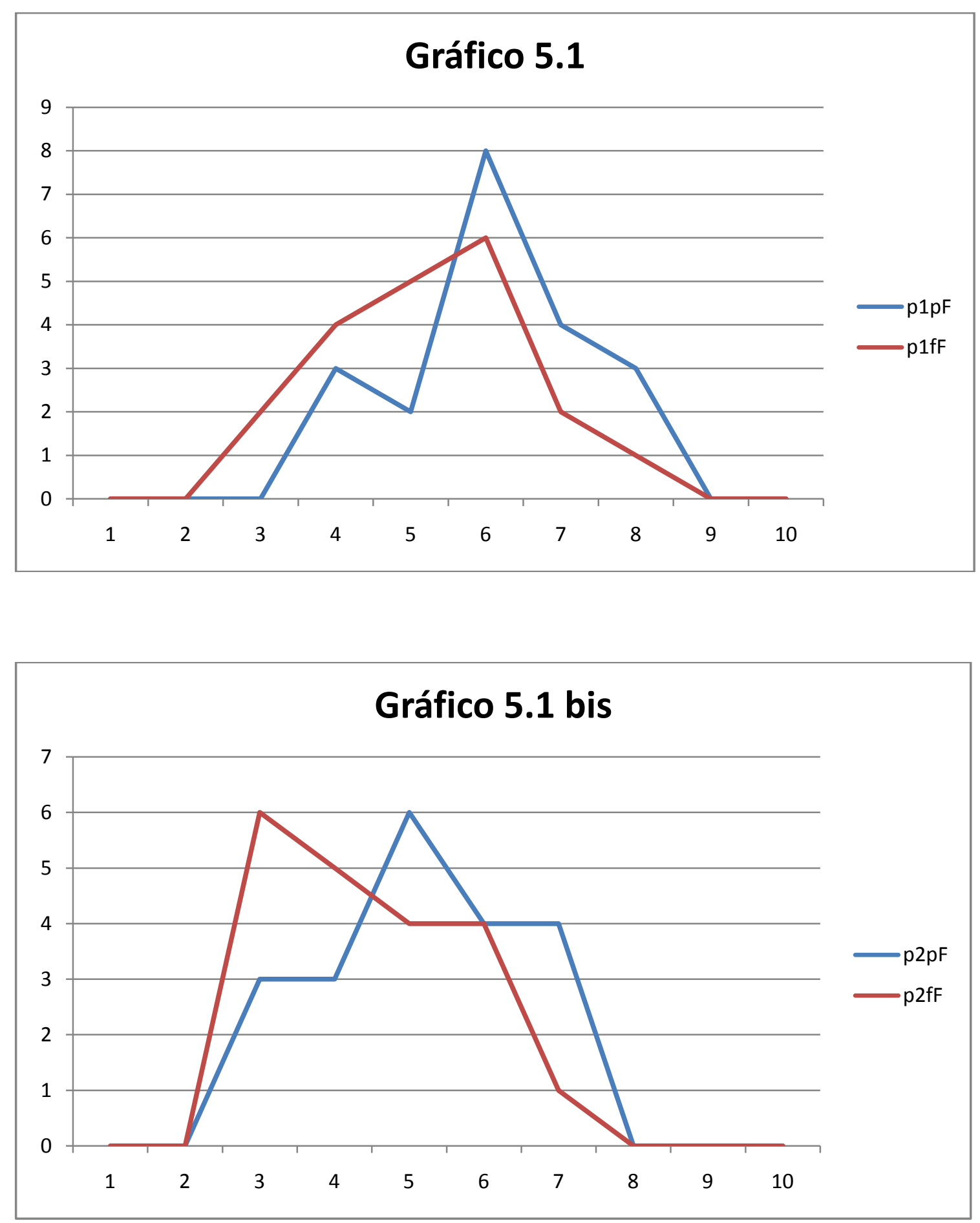

Dichas distribuciones ponen ya de manifiesto lo que queremos mostrar, que las pruebas PPF son mejor valoradas que las PF. Tanto para el primer teorema como para el segundo, la PPF (en azul) está más a la derecha que la PF (en rojo). Esto 
significa que obtienen mayores puntuaciones ambas PPF sobre su respectiva PF. También se observa que el primer teorema tiene mejores calificaciones que el segundo, comparando las dos PPF o las dos PF.

Las tablas 5.2 y 5.3 recogen las comparaciones de las características estadísticas de estas cuatro variables, que vienen a confirmarnos la preferencia por la PPF. Así en la tabla 5.2, observamos que las características de tendencia central son superiores en las PPF ( $1^{\underline{a}}$ y $3^{\underline{a}}$ columna) respecto a las PF $\left(2^{\underline{a}}\right.$ y $4^{\underline{a}}$ columna) en media, mediana y moda en la primera y segunda prueba, con unas diferencias en los resultados de aproximadamente un punto a favor de las PPF, llegando a caer por debajo del 5 sólo en la PF de la segunda prueba. Se observa lo mismo para mínimo, máximo y cuartiles.

Por lo que se refiere a la dispersión, tomando el coeficiente de variación se aprecia cómo va creciendo de PPF a PF y de $1^{\text {a }}$ a $2^{\text {a }}$ demostración, lo que significaría una mayor unanimidad en las votaciones para aquellas pruebas con un coeficiente de variación menor.

\begin{tabular}{|c|c|c|c|c|}
\hline \multicolumn{5}{|l|}{ Análisis Multivariable } \\
\hline \multicolumn{5}{|l|}{ Resumen Estadístico } \\
\hline & $\mathrm{plpF}$ & $\mathrm{p} 1 \mathrm{fF}$ & $\mathrm{p} 2 \mathrm{pF}$ & $\mathrm{p} 2 \mathrm{fF}$ \\
\hline Frecuencia & 20 & 20 & 20 & 20 \\
\hline Media & 6,1 & 5,25 & 5,15 & 4,45 \\
\hline Mediana & 6,0 & 5,0 & 5,0 & 4,0 \\
\hline Moda & 6,0 & 6,0 & 5,0 & 3,0 \\
\hline Varianza & 1,56842 & 1,77632 & 1,81842 & 1,62895 \\
\hline Desviación típica & 1,25237 & 1,33278 & 1,34849 & 1,2763 \\
\hline Mínimo & 4,0 & 3,0 & 3,0 & 3,0 \\
\hline Máximo & 8,0 & 8,0 & 7,0 & 7,0 \\
\hline Rango & 4,0 & 5,0 & 4,0 & 4,0 \\
\hline Primer cuartil & 5,5 & 4,0 & 4,0 & 3,0 \\
\hline Segundo cuartil & 7,0 & 6,0 & 6,0 & 5,5 \\
\hline Asimetría tipi. & $-0,378319$ & 0,152208 & $-0,288026$ & 0,6879 \\
\hline Curtosis típificada & $-0,469823$ & $-0,328053$ & $-0,871674$ & $-0,93287$ \\
\hline Coef. de variación & $20,5306 \%$ & $25,3864 \%$ & $26,1842 \%$ & $28,6809 \%$ \\
\hline
\end{tabular}


Esta tabla muestra resúmenes estadísticos para cada una de las variables seleccionadas. Incluye medidas de tendencia central, de variabilidad y de forma. De particular interés están la asimetría estandarizada y la curtosis estandarizada, las cuales pueden utilizarse para determinar si la muestra procede de una distribución normal. Valores de estos estadísticos fuera del rango de -2 a +2 indican una desviación significativa de la normalidad, que tendería a invalidar muchos de los procedimientos estadísticos aplicados habitualmente a estos datos.

Tabla 5.2. Comparaciones de las características estadísticas de ambas pruebas en la categoría facilidad

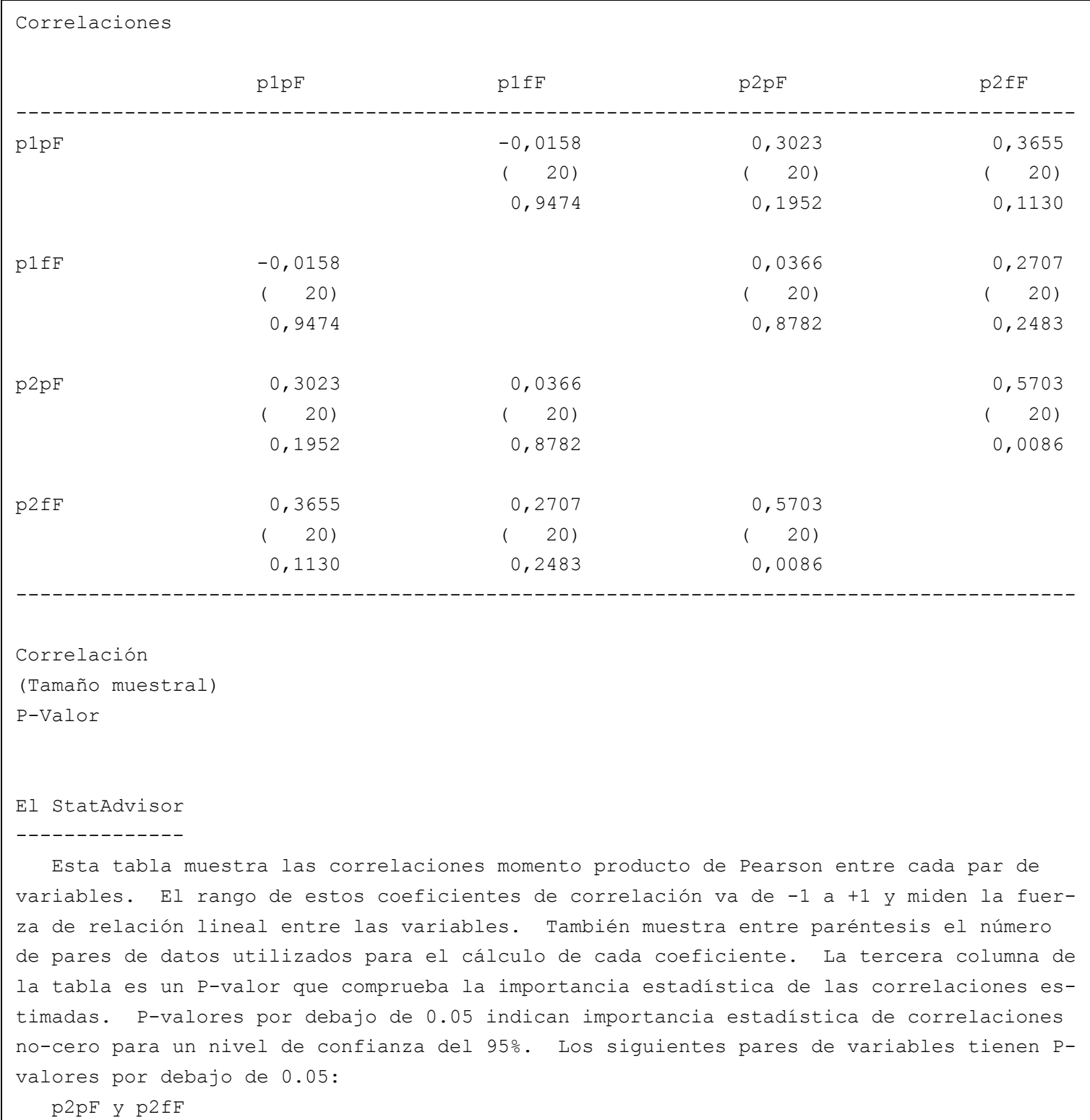

Tabla 5.3. Correlaciones de ambas pruebas en la categoría facilidad 
Por su parte, siguiendo en la tabla 5.2, la asimetría tipificada de las distribuciones viene a corroborar también la preferencia por las PPF: en ambos casos los resultados indican que las distribuciones de ambas PPF son asimétricas negativas, esto es, la mayoría de las calificaciones se agrupan al final y hay muy pocas calificaciones bajas; mientras que las distribuciones para las PF son asimétricas positivas, o sea, la mayoría de las calificaciones se agrupan al principio de la distribución y hay muy pocas calificaciones altas. Además, como explica el programa Statgraphics en la misma tabla, el hecho de que la asimetría tipificada y la curtosis tipificada (en el cuadro dice estandarizada, lo que es equivalente) estén entre -2 y 2 va a permitir tratar estas variables como que siguen distribuciones normales, lo que justificará la utilización de los contrastes de igualdad de varianzas y de igualdad de medias que se verán en los dos apartados siguientes.

Y por último y no menos importante, las correlaciones de la tabla 5.3 nos indican la escasa asociación que hay entre los pares de pruebas por lo general (los valores de estas correlaciones, por lo general, están más bien próximos a cero, lo que indica una escasa relación entre el par de variables correspondiente), salvo una ligera relación existente entre la PPF y la PF del segundo teorema. El p-valor corrobora la inexistencia de relaciones significativas, salvando este último par. Esto es, no hay relación entre las puntuaciones dadas por los alumnos en las distintas demostraciones.

\subsubsection{Comparaciones de los resultados de la facilidad (F) para la primera demostración}

Atendiendo a Pérez (2002, p. 312), vamos a realizar una serie de contrastes que corroboren la desigualdad de las distribuciones entre ambas demostraciones.

Todas las tablas y gráficos que aparecen a continuación vienen a confirmarnos que para el primer teorema es preferida la demostración PPF a la PF. Dada la igualdad de varianzas (de desviaciones típicas concretamente en el contraste) que se comprueba en la tabla 5.6 (en virtud de la normalidad comprobada con los coeficientes de asimetría y curtosis tipificados en la tabla 5.2), se puede realizar el contraste de 
igualdad de medias (tabla 5.5) donde se rechaza la hipótesis nula, por lo que no se puede admitir que las medias sean iguales entre ambas variables. Ya resulta definitivo el contraste de Kolmogorov-Smirnov (tabla 5.7) según el cual tampoco se puede aceptar la igualdad de las distribuciones, como queríamos comprobar.

Los gráficos ponen de manifiesto estas mismas diferencias:

1) Gráfico 5.2: comparación de histogramas, donde se observa cómo el histograma de arriba (correspondiente a la PPF) tiene las frecuencias mayores en la segunda mitad, mientras que el de abajo (correspondiente a la PF) los tiene en la primera.

2) Gráfico 5.3: comparación de densidades, donde la densidad de PPF está más a la derecha que la PF (dicha densidad se deduce a partir del histograma anterior).

3) Gráfico 5.4: con los dos diagramas de cajas y bigotes, en el que se observa cómo todo en el gráfico superior (que representa la PPF) está desplazado a la derecha respecto al inferior (que representa la PF).

4) Gráfico 5.5: con la representación de los cuantiles, donde la distribución de la PF está a la izquierda (y por lo tanto toma menores valores) que la PPF.

Por lo tanto la prueba PPF es claramente preferida a la PF en este caso.

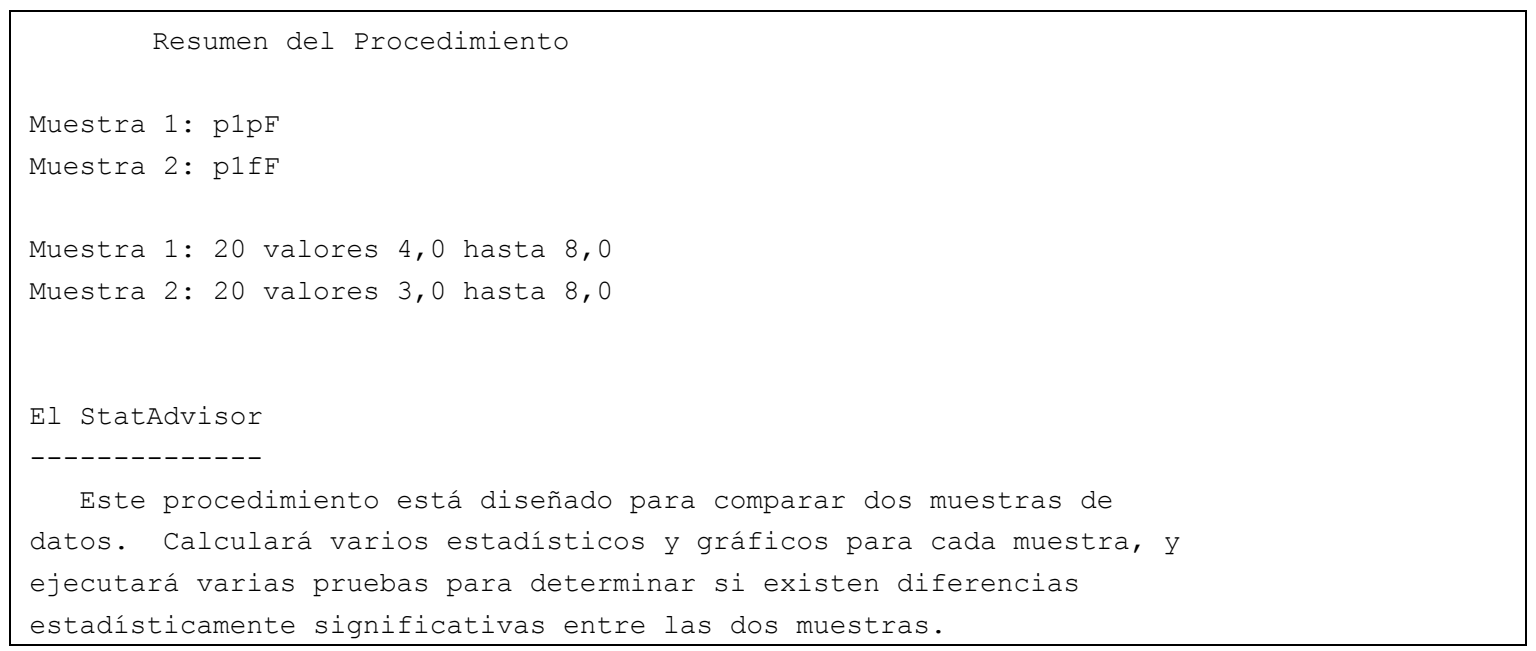

Tabla 5.4. Comparación de muestras p1pF-p1fF 


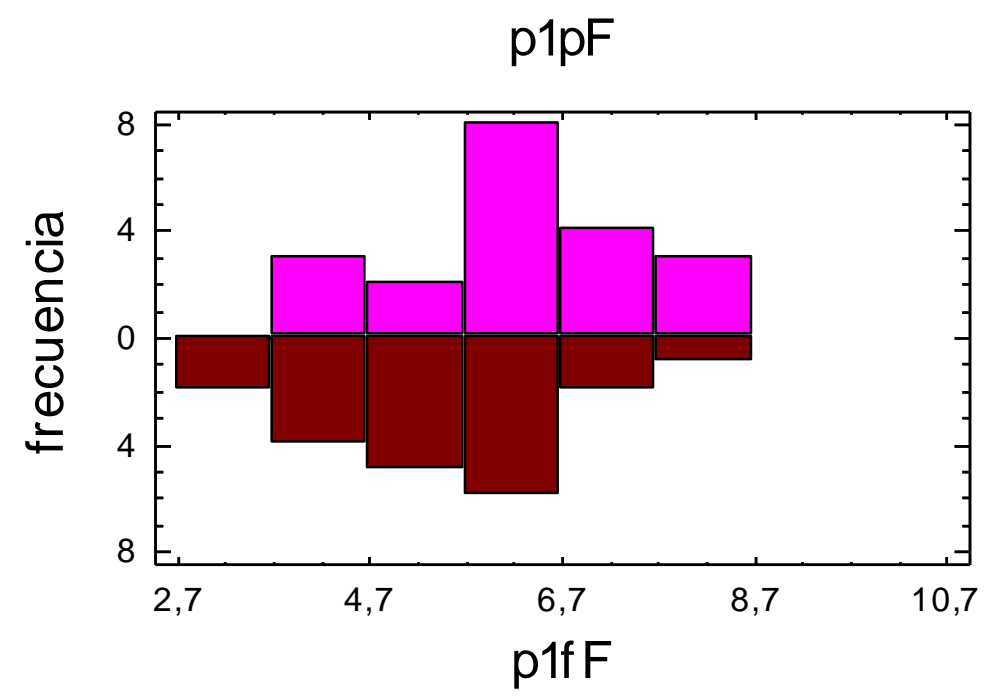

Gráfico 5.2. Comparación de histogramas p1pF-p1fF

Comparación de Medias

95, 0\% intervalo de confianza para la media de plpF: 6,1 +/- 0,586127

95,0\% intervalo de confianza para la media de p1fF: 5,25 +/- 0,623764

$[4,62624,5,87376]$

95, 0\% intervalos de confianza para la diferencia de medias:

suponiendo varianzas iguales: $0,85+/-0,82787 \quad[0,0221304,1,67787]$

contrastes t de comparación de medias

Hipótesis nula: medial $=$ media2

Hipótesis alt.: medial <> media2

suponiendo varianzas iguales: $t=2,07851 \quad$ P-Valor $=0,0444655$

El StatAdvisor

Esta opción ejecuta el t-test para comparar las medias de las dos muestras. También establece los intervalos de confianza o los límites para cada media y para la diferencia entre las medias. De particular interés está el intervalo de confianza para la diferencia entre las medias, el cual se extiende desde 0,0221304 hasta 1,67787. Dado que el intervalo no contiene el valor 0.0 , existe diferencia

estadísticamente significativa entre las medias de las dos muestras para un nivel de confianza del 95,0\%.

También puede aplicarse un t-test para probar una hipótesis específica sobre la diferencia entre las medias de las poblaciones de las que proceden las dos muestras. En este caso, el test se ha realizado para determinar si la diferencia entre las dos medias es igual a 0,0 frente a la hipótesis alternativa en la que la diferencia no es igual 0,0. Puesto que el p-valor calculado es inferior a 0,05, podemos rechazar la hipótesis nula en favor de la alternativa. 
NOTA: estos resultados asumen la igualdad de varianzas en las dos muestras. En este caso, esa asunción parece ser razonable teniendo en cuenta los resultados del F-test para comparar las desviaciones típicas. Puede ver los resultados de este test seleccionando Comparación de Desviaciones Típicas del menú Opciones Tabulares.

Tabla 5.5. Comparación de medias p1pF-p1fF

Density Traces

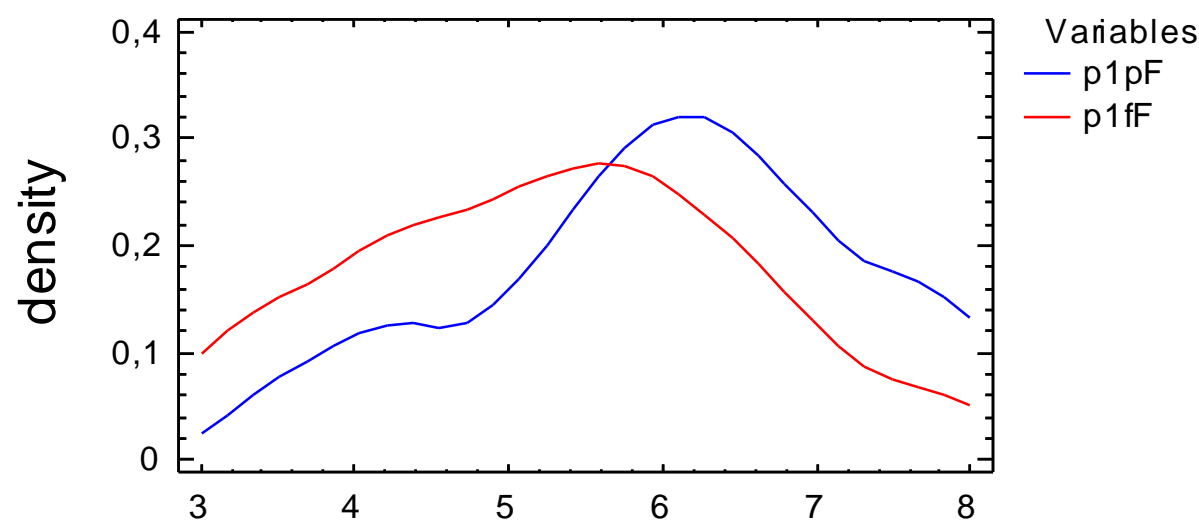

Gráfico 5.3. Comparación de densidades p1pF-p1fF

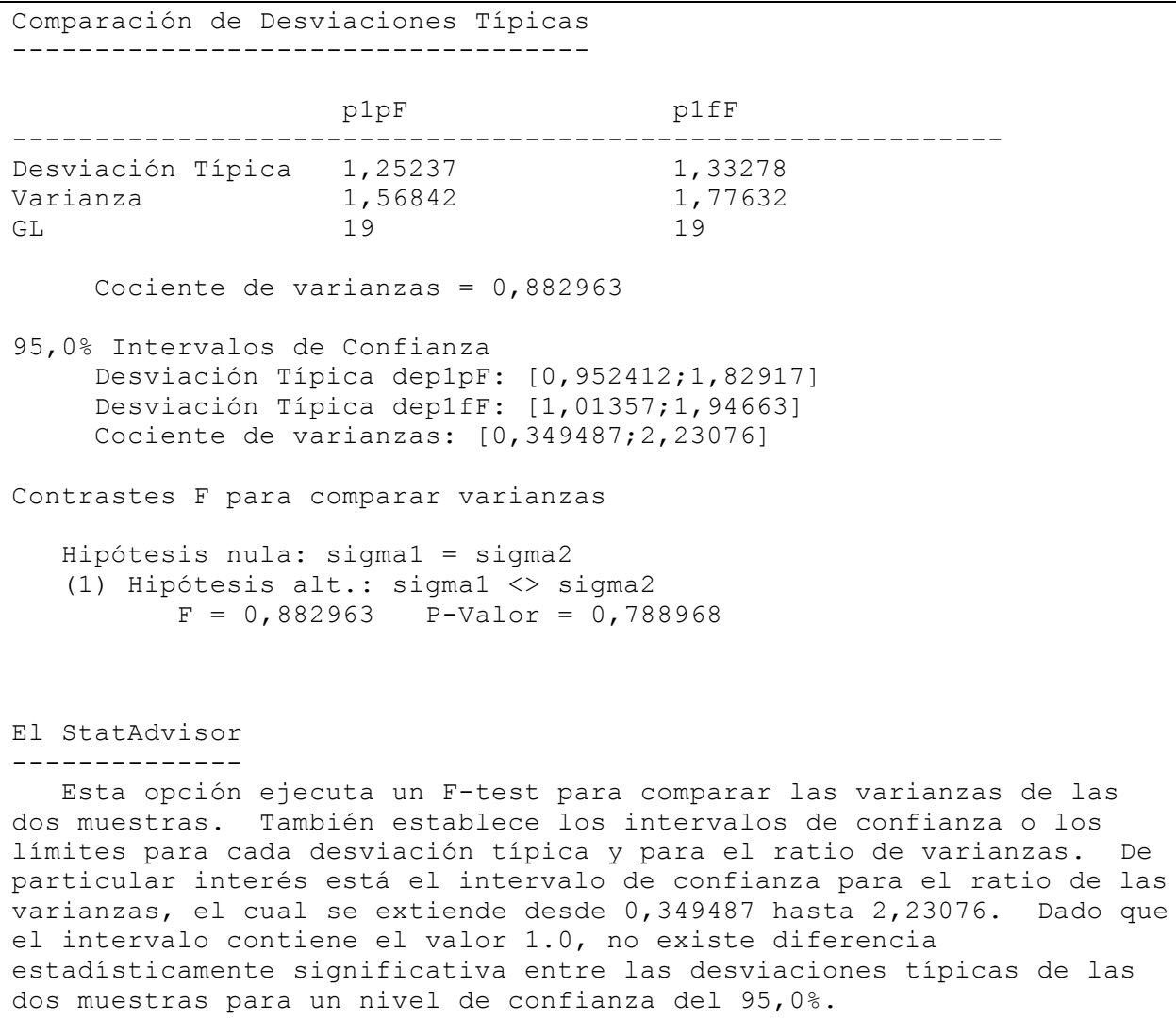


También puede utilizarse un F-test para probar una hipótesis específica sobre las desviaciones típicas de las poblaciones de las que proceden las dos muestras. En este caso, el test se ha realizado para determinar si el ratio de las desviaciones típicas son iguales 1,0 frente a la hipótesis alternativa en la que el ratio no es igual 1,0. Puesto que el p-valor calculado no es inferior a 0,05, no podemos rechazar la hipótesis nula.

NOTA IMPORTANTE: los F-test y los intervalos de confianza mostrados dependen de que las muestras procedan de distribuciones normales.

Para comprobar esta asunción, seleccione Resumen Estadístico de la lista de Opciones Tabulares y observe los valores de asimetría estandarizada y curtosis estandarizada.

Tabla 5.6. Comparación de desviaciones típicas p1pF-p1fF

\section{Box-and-Whisker Plot}

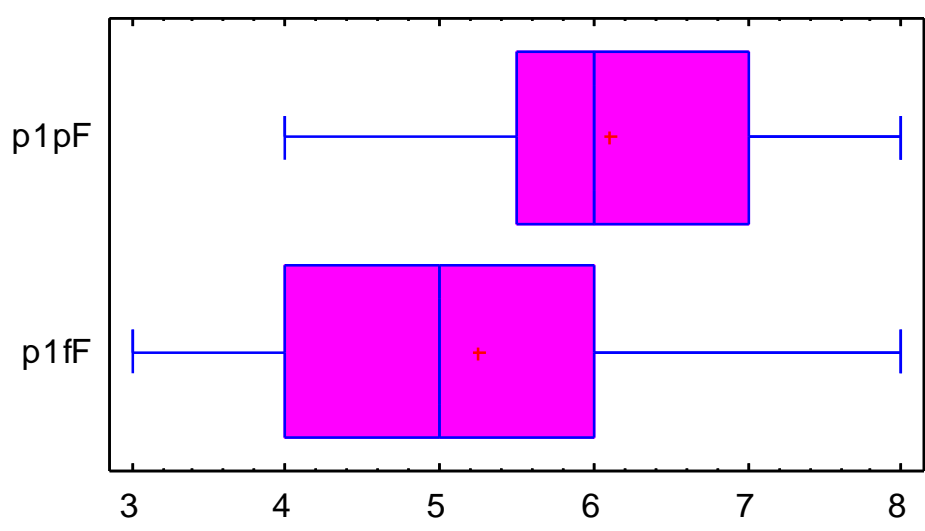

Gráfico 5.4. Comparación de diagramas de cajas y bigotes

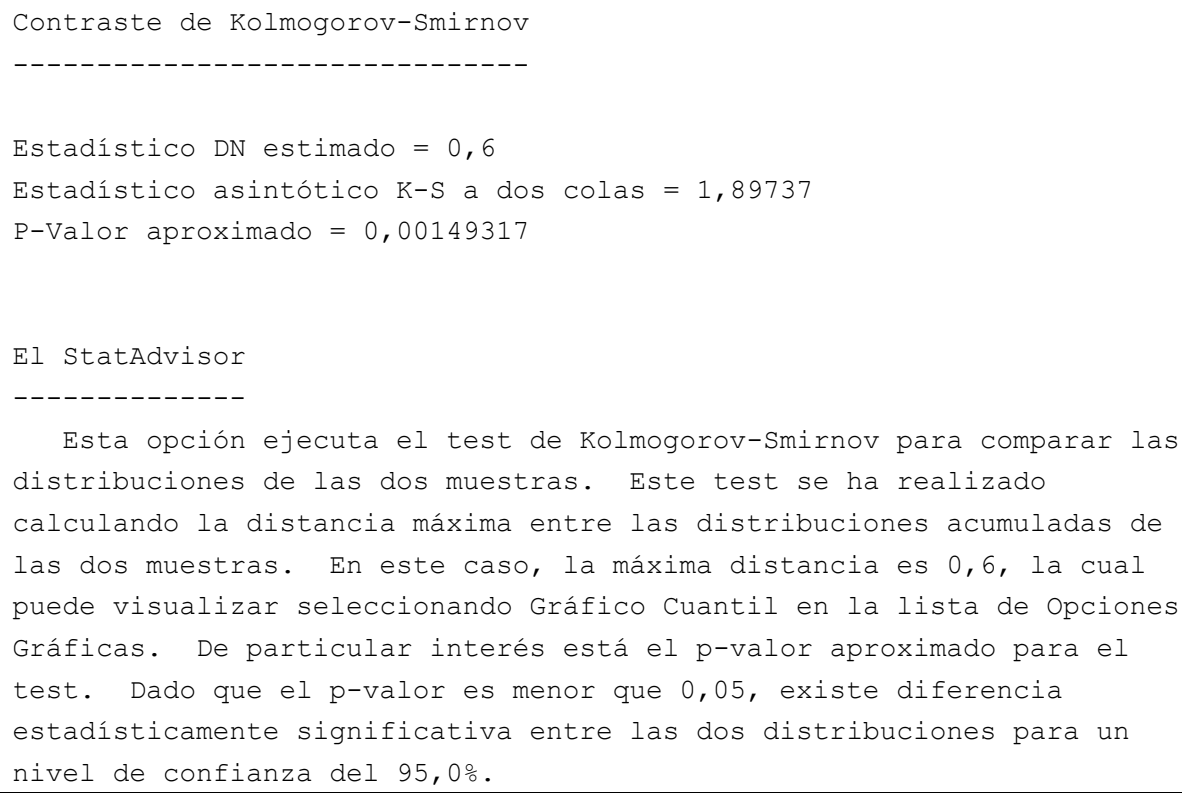

Tabla 5.7. Contraste de Kolmogorov-Smirnov, facilidad 1 


\section{Quantile Plot}

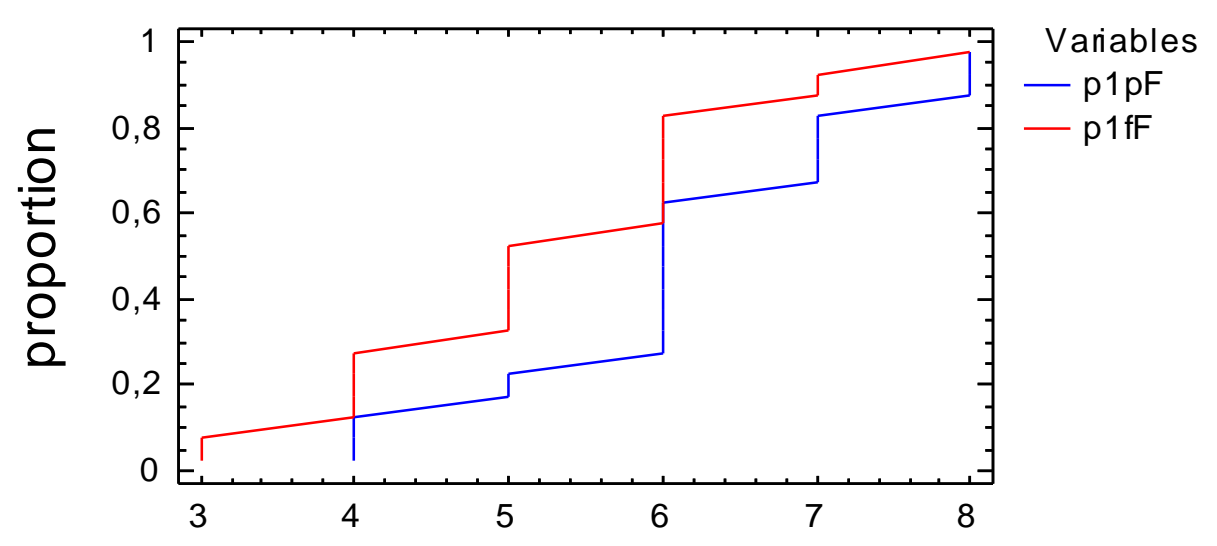

Gráfico 5.5. Representación de cuantiles p1pF-p1fF

\subsubsection{Comparaciones de los resultados de la facilidad (F) para la segunda demostración}

Los resultados no son tan contundentes en el caso del segundo teorema. Dada la igualdad de desviaciones típicas que se comprueba en la tabla 5.10 (en virtud de la normalidad comprobada con los coeficientes de asimetría y curtosis tipificados en la tabla 5.2), se puede realizar el contraste de igualdad de medias, resultando que no se puede rechazar que las medias sean distintas (tabla 5.9), pero al menos el contraste de Kolmogorov-Smirnov (tabla 5.11) nos permite rechazar que sean iguales las distribuciones, como queríamos demostrar.

Por su parte, los gráficos 5.6 a 5.9 corroboran la desigualdad de las distribuciones.

5) Gráfico 5.6: comparación de histogramas, donde se aprecia cómo el histograma de arriba (correspondiente a la PPF) tiene las frecuencias mayores en la segunda mitad, mientras que el de abajo (correspondiente a la PF) los tiene en la primera, destacando un claro decrecimiento de las frecuencias desde las calificaciones menores (mayoritarias) hacia el resto. 
6) Gráfico 5.7: comparación de densidades, donde la densidad de PPF está más a la derecha que la PF (dicha densidad se deduce a partir del histograma anterior, y se aprecia el mismo decrecimiento para la PF).

7) Gráfico 5.8: con los dos diagramas de cajas y bigotes, en el que se observa cómo todo en el gráfico superior (que representa la PPF) está desplazado a la derecha respecto al inferior (que representa la PF). Mientras que el de arriba muestra una situación de práctica simetría, el de abajo es claramente asimétrico positivo, acumulándose casi todos los valores en las calificaciones más bajas.

8) Gráfico 5.9: con la representación de los cuantiles, donde la distribución de la PF está a la izquierda (y por lo tanto toma menores valores) que la PPF.

Por tanto en esta segunda demostración también resulta preferida la PPF a la PF.

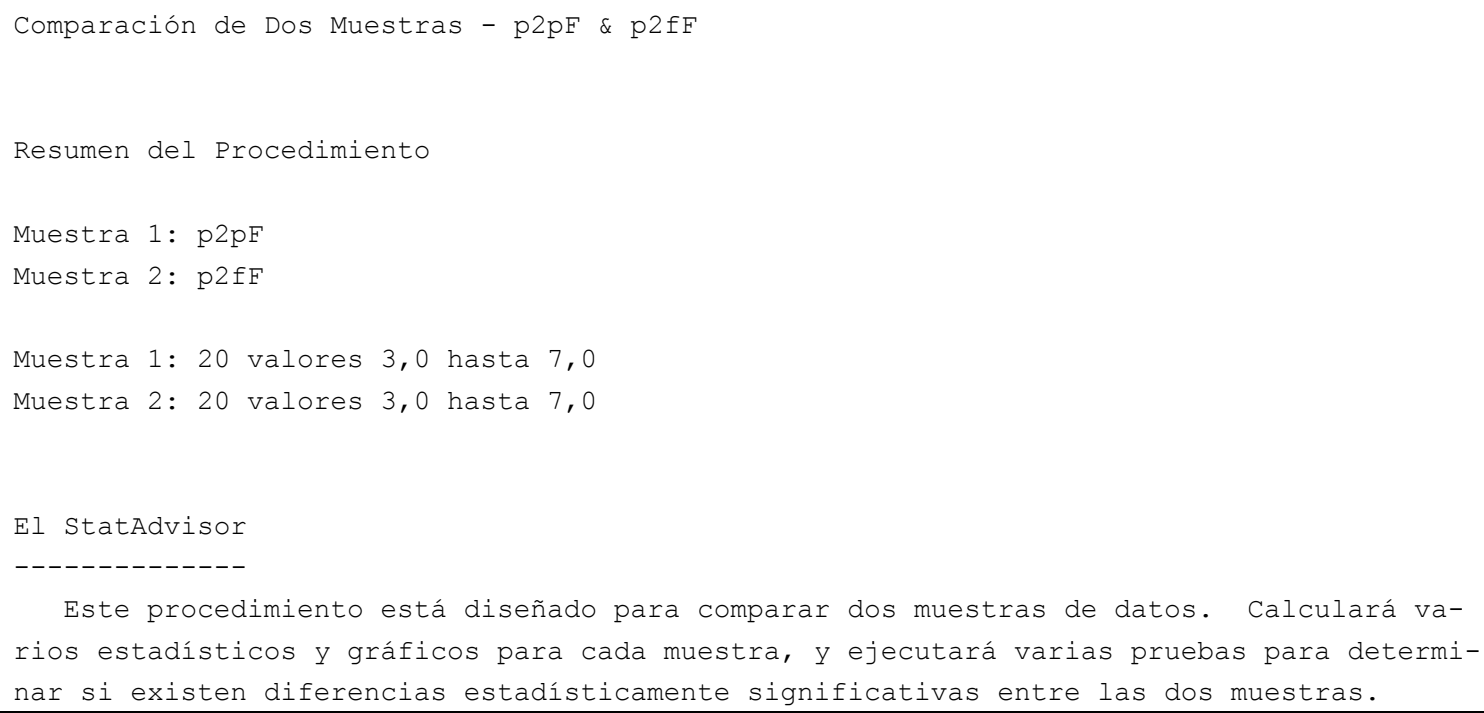

Tabla 5.8. Comparación de muestras p2pF-p2fF 


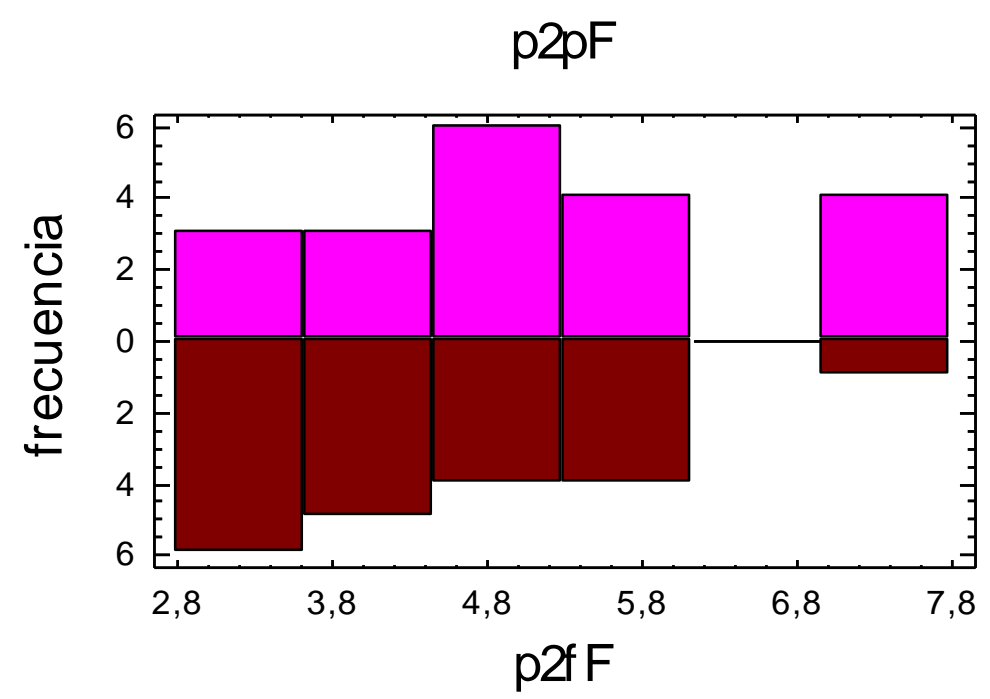

Gráfico 5.6. Comparación de histogramas p2pF-p2fF

Comparación de Medias

95, 0\% intervalo de confianza para la media de p2pF: 5,15 +/- 0,631113

$[4,51889,5,78111]$

95, 0\% intervalo de confianza para la media de p2ff: 4,45 +/- 0,597329

$[3,85267,5,04733]$

95, 0\% intervalos de confianza para la diferencia de medias:

suponiendo varianzas iguales: $0,7+/-0,840475 \quad[-0,140475,1,54048]$

contrastes t de comparación de medias

Hipótesis nula: medial = media2

Hipótesis alt.: medial <> media2

suponiendo varianzas iguales: $t=1,68605 \quad$ P-Valor $=0,0999824$

El StatAdvisor

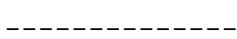

Esta opción ejecuta el t-test para comparar las medias de las dos muestras. También establece los intervalos de confianza o los límites para cada media y para la diferencia entre las medias. De particular interés está el intervalo de confianza para la diferencia entre las medias, el cual se extiende desde $-0,140475$ hasta 1,54048. Dado que el intervalo contiene el valor 0.0, no existe diferencia estadísticamente significativa entre las medias de las dos muestras para un nivel de confianza del 95,0\%.

También puede aplicarse un t-test para probar una hipótesis específica sobre la diferencia entre las medias de las poblaciones de las que proceden las dos muestras. En este caso, el test se ha realizado para determinar si la diferencia entre las dos medias es igual a 0,0 frente a la hipótesis alternativa en la que la diferencia no es igual 0,0. Puesto que el p-valor calculado no es inferior a 0,05, no podemos rechazar la hipótesis nula. 
NOTA: estos resultados asumen la igualdad de varianzas en las dos

muestras. En este caso, esa asunción parece ser razonable teniendo en

cuenta los resultados del F-test para comparar las desviaciones

típicas. Puede ver los resultados de este test seleccionando

Comparación de Desviaciones Típicas del menú Opciones Tabulares.

Tabla 5.9. Comparación de medias p2pF-p2ff

Density Traces

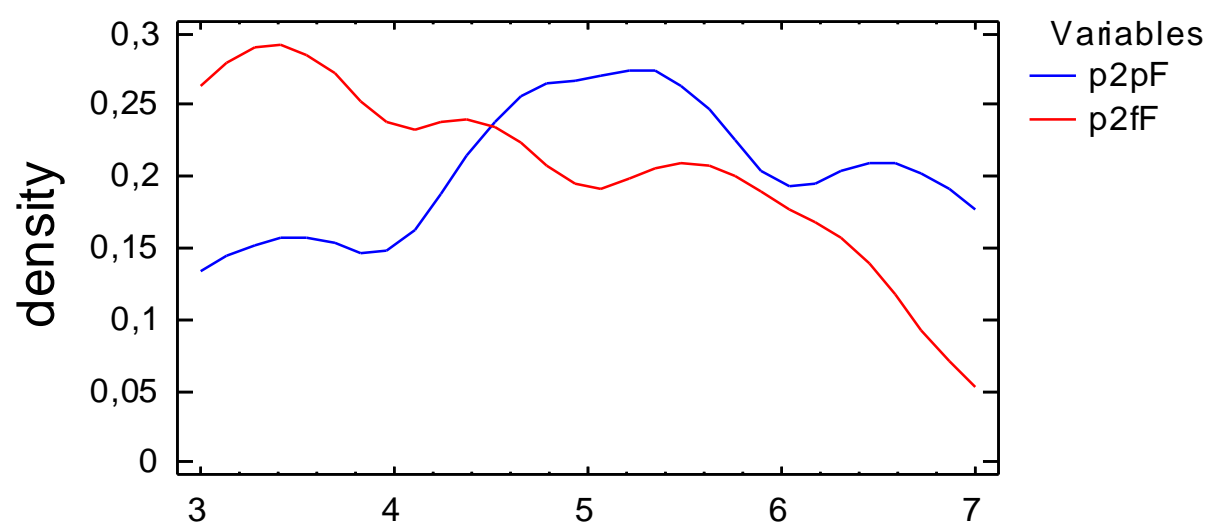

Gráfico 5.7. Comparación de densidades p2pF-p2fF

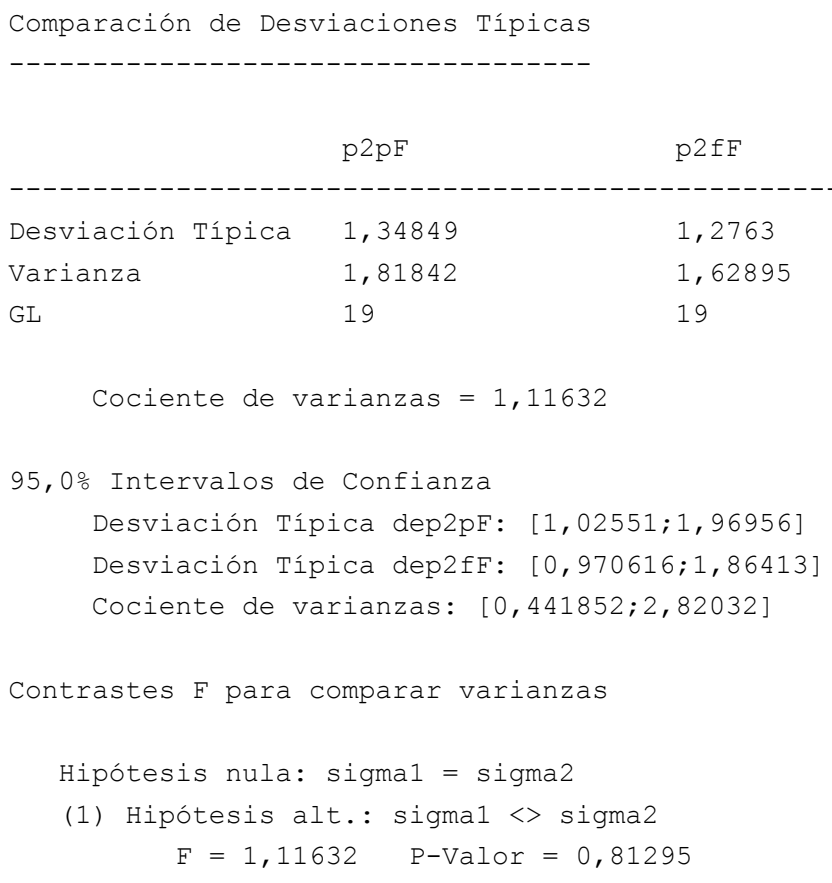


El StatAdvisor

\section{$------------1$}

Esta opción ejecuta un F-test para comparar las varianzas de las dos muestras. También establece los intervalos de confianza o los límites para cada desviación típica y para el ratio de varianzas. De particular interés está el intervalo de confianza para el ratio de las varianzas, el cual se extiende desde 0,441852 hasta 2,82032. Dado que el intervalo contiene el valor 1.0, no existe diferencia estadísticamente significativa entre las desviaciones típicas de las dos muestras para un nivel de confianza del 95,0\%.

También puede utilizarse un F-test para probar una hipótesis específica sobre las desviaciones típicas de las poblaciones de las que proceden las dos muestras. En este caso, el test se ha realizado para determinar si el ratio de las desviaciones típicas son iguales 1,0 frente a la hipótesis alternativa en la que el ratio no es igual

1,0. Puesto que el p-valor calculado no es inferior a 0,05, no podemos rechazar la hipótesis nula.

NOTA IMPORTANTE: los F-test y los intervalos de confianza mostrados

dependen de que las muestras procedan de distribuciones normales. Para comprobar esta asunción, seleccione Resumen Estadístico de la lista de Opciones Tabulares y observe los valores de asimetría estandarizada y curtosis estandarizada.

\section{Tabla 5.10. Comparación de desviaciones típicas p2pF-p2fF}

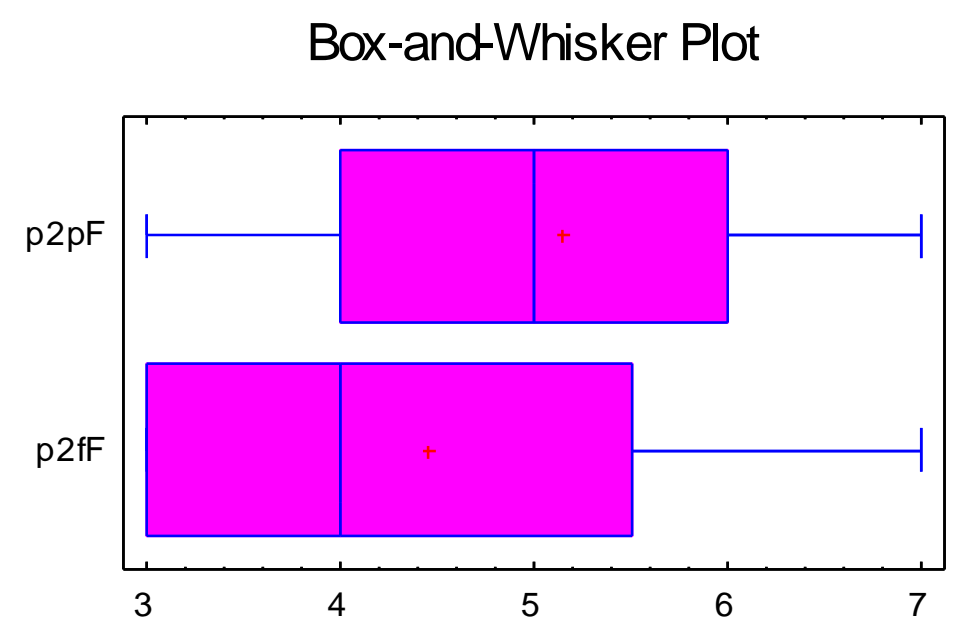

Gráfico 5.8. Comparación de diagramas de cajas y bigotes

Contraste de Kolmogorov-Smirnov

Estadístico DN estimado $=0,45$

Estadístico asintótico $\mathrm{K}-\mathrm{S}$ a dos colas $=1,42302$

$\mathrm{P}-\mathrm{Val}$ r aproximado $=0,0348448$ 


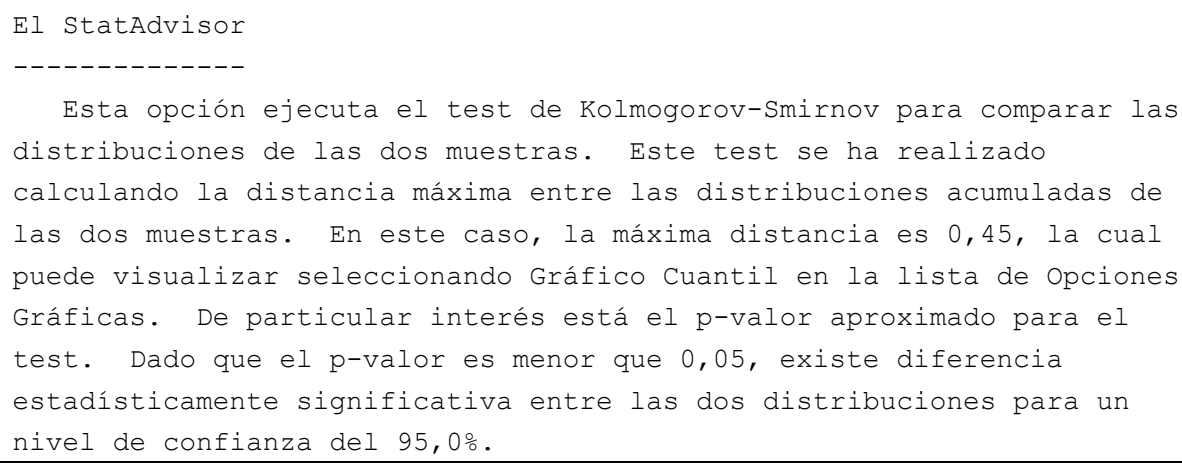

Tabla 5.11. Contraste de Kolmogorov-Smirnov, facilidad 2

Quantile Plot

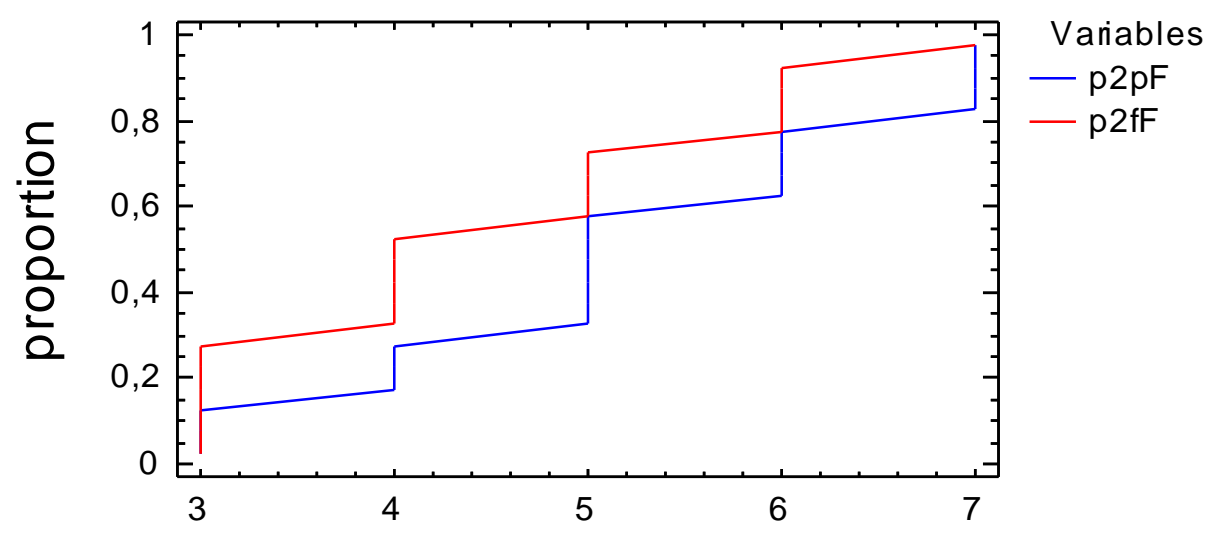

Gráfico 5.9. Representación de los cuantiles p2pF-p2fF

\subsection{Comparaciones de los resultados del gusto (G) de cada una de las demostraciones}

La tabla 5.12 recoge las frecuencias de cada una de las calificaciones asignadas por los alumnos en la categoría gusto en cada una de las cuatro demostraciones. 


\begin{tabular}{rrrrr}
\hline & $\mathbf{p 1 p G}$ & $\mathbf{p 1 f G}$ & $\mathbf{p 2 p G}$ & \multicolumn{2}{c}{$\mathbf{p 2 f G}$} \\
\hline $\mathbf{1}$ & 0 & 0 & 0 & 0 \\
$\mathbf{2}$ & 0 & 1 & 1 & 1 \\
$\mathbf{3}$ & 0 & 1 & 2 & 3 \\
$\mathbf{4}$ & 3 & 5 & 4 & 4 \\
$\mathbf{5}$ & 6 & 3 & 1 & 7 \\
$\mathbf{6}$ & 6 & 3 & 8 & 4 \\
$\mathbf{7}$ & 3 & 4 & 3 & 1 \\
$\mathbf{8}$ & 2 & 3 & 0 & 0 \\
$\mathbf{9}$ & 0 & 0 & 1 & 0 \\
$\mathbf{1 0}$ & 0 & 0 & 0 & 0 \\
\hline Total & 20 & 20 & 20 & 20 \\
\hline
\end{tabular}

Tabla 5.12. Frecuencias en la categoría gusto en cada una de las pruebas y de los teoremas

Asimismo, estos datos quedan reflejados en los gráficos 5.10 y 5.10 bis siguientes.

\section{Gráfico 5.10}

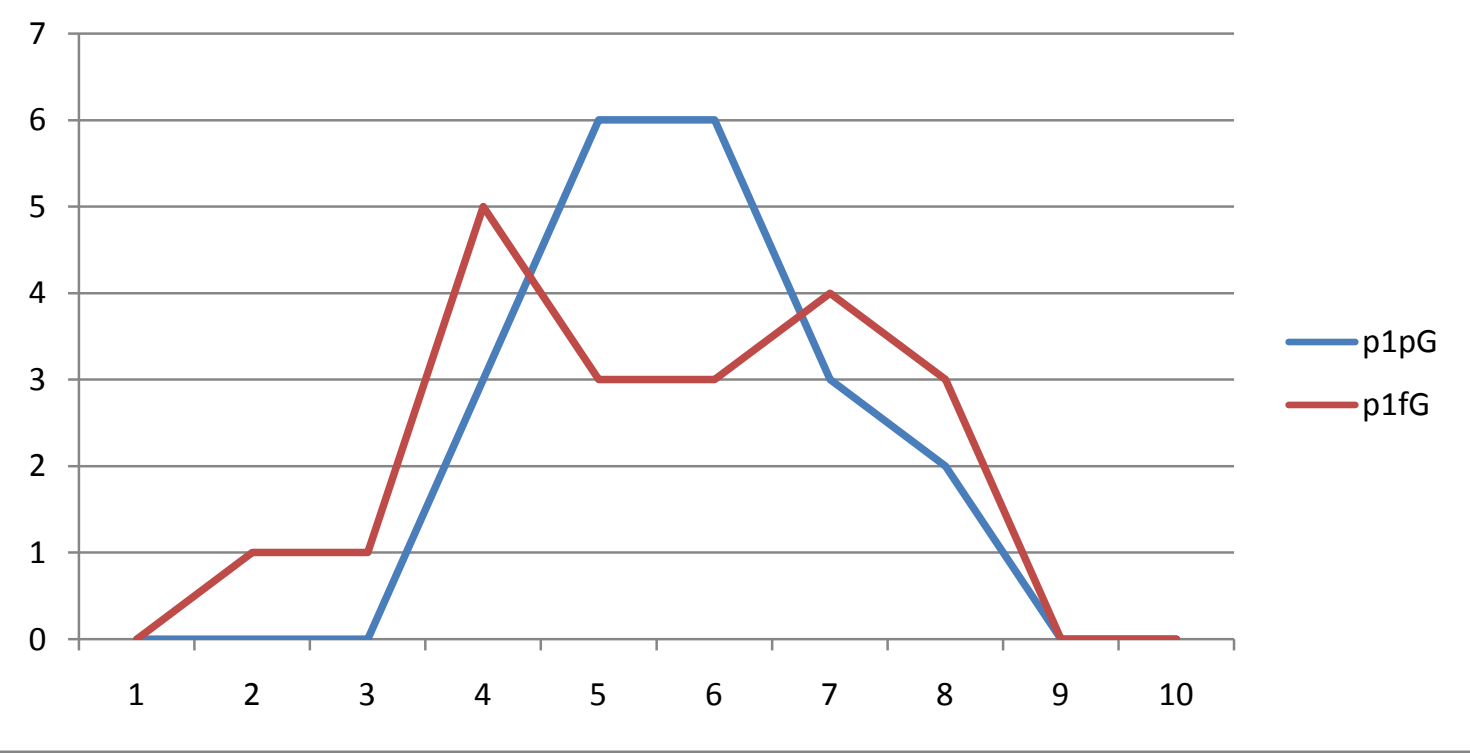




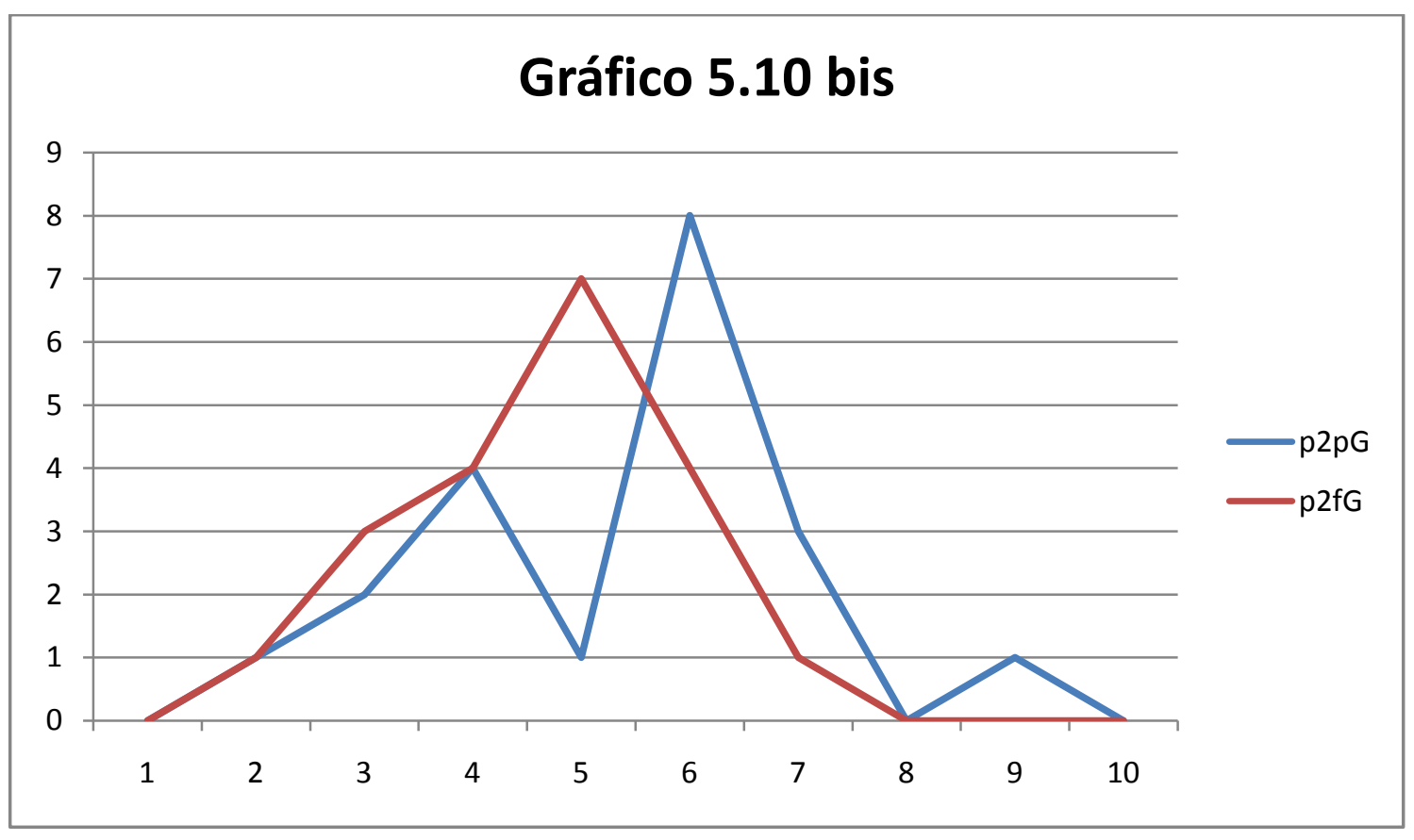

Tales distribuciones representan sólo en parte para el caso del gusto lo que queremos mostrar, que las pruebas PPF son mejor valoradas que las PF; para el primer teorema veremos cómo no se puede decir que haya grandes diferencias, aunque en el gráfico 5.10 la PPF (en azul) presenta una moda más a la derecha que la PF (en rojo), si bien luego presenta un segundo máximo local; sin embargo, para el segundo teorema claramente la PPF (en azul) está a la derecha de la PF (en rojo).

Las tablas 5.13 y 5.14 recogen las comparaciones de las características estadísticas de estas cuatro variables, que vienen a confirmarnos la preferencia por PPF. Así en la tabla 5.13, observamos que las características de tendencia central son superiores en las PPF ( $1^{\underline{a}}$ y $3^{\underline{a}}$ columna) respecto a las PF ( $2^{\underline{a}}$ y $4^{\underline{a}}$ columna) en media, mediana y moda en la primera y segunda prueba, con unas diferencias en los resultados de aproximadamente un punto a favor de las PPF, llegando a caer por debajo del 5 sólo en la media de la PF de la segunda prueba. Se observa lo mismo para mínimo, máximo y cuartiles, salvo en el segundo cuartil para la primera prueba.

Por lo que se refiere a la dispersión, tomando el coeficiente de variación se aprecia cómo va creciendo de PPF a PF en la primera demostración, lo que significaría una 
mayor unanimidad en las votaciones para aquellas pruebas con un coeficiente de variación menor. En la segunda sin embargo es la PF la que presenta una menor dispersión sobre la PPF, aunque las diferencias no son tan amplias como en el caso anterior.

Por su parte, siguiendo en la tabla 5.13, la asimetría tipificada de las distribuciones no viene a corroborar la preferencia por las PPF: en ambos casos los resultados indican que las distribuciones de ambas PF son asimétricas negativas, esto es, la mayoría de las calificaciones se agrupan al final y hay muy pocas calificaciones bajas; mientras que la distribuciones para la PPF del primer experimento es asimétrica positiva, o sea, la mayoría de las calificaciones se agrupan al principio de la distribución y hay muy pocas calificaciones altas; en el caso del segundo experimento estaría dentro de las negativas, pero algo menos que la PF. Además, el hecho de que la asimetría tipificada y la curtosis tipificada estén entre -2 y 2 va a permitir tratar estas variables como que siguen distribuciones normales, lo que justificará la utilización de los contrastes de igualdad de varianzas y de igualdad de medias que se verán en los dos apartados siguientes.

Las correlaciones de la tabla 5.14 nos indican la escasa asociación que hay entre los pares de calificaciones por lo general (los valores de estas correlaciones, por lo general, están más bien próximos a cero, lo que indica una escasa relación entre el par de variables correspondiente). El p-valor corrobora la inexistencia de relaciones significativas. Esto es, no hay relación entre las puntuaciones respecto del gusto dadas por los alumnos en las distintas demostraciones.

\begin{tabular}{|c|c|c|c|c|}
\hline \multicolumn{5}{|c|}{ Análisis Multivariable } \\
\hline \multicolumn{5}{|c|}{ Resumen Estadístico } \\
\hline & $\mathrm{p} 1 \mathrm{pg}$ & $\mathrm{p} 1 \mathrm{fG}$ & $\mathrm{p} 2 \mathrm{pG}$ & $\mathrm{p} 2 \mathrm{fG}$ \\
\hline Frecuencia & 20 & 20 & 20 & 20 \\
\hline Media & 5,75 & 5,5 & 5,35 & 4,65 \\
\hline Mediana & 6,0 & 5,5 & 6,0 & 5,0 \\
\hline Moda & & 4,0 & 6,0 & 5,0 \\
\hline Varianza & 1,46053 & 3,10526 & 2,87105 & 1,60789 \\
\hline Desviación típica & 1,20852 & 1,76218 & 1,69442 & 1,26803 \\
\hline
\end{tabular}




\begin{tabular}{|c|c|c|c|}
\hline Mínimo & 2,0 & 2,0 & 2,0 \\
\hline Máximo & 8,0 & 9,0 & 7,0 \\
\hline Rango & 6,0 & 7,0 & 5,0 \\
\hline Primer cuartil & 4,0 & 4,0 & 4,0 \\
\hline Segundo cuartil & 7,0 & 6,0 & 5,5 \\
\hline Asimetría tipi. & $-0,292676$ & $-0,200489$ & $-0,527073$ \\
\hline Curtosis típificad $-0,49055$ & $-0,821841$ & $-0,0196062$ & $-0,268029$ \\
\hline Coef. de variación 21,0178\% & $32,0396 \%$ & $31,6714 \%$ & $27,2694 \%$ \\
\hline \multicolumn{4}{|l|}{ El StatAdvisor } \\
\hline-------------- & & & \\
\hline \multicolumn{4}{|c|}{$\begin{array}{l}\text { Esta tabla muestra resúmenes estadísticos para cada una de las variables selecciona- } \\
\text { das. Incluye medidas de tendencia central, de variabilidad y de forma. De particular } \\
\text { interés están la asimetría estandarizada y la curtosis estandarizada, las cuales pueden } \\
\text { utilizarse para determinar si la muestra procede de una distribución } \\
\text { normal. Valores de estos estadísticos fuera del rango de }-2 \text { a +2 indican una desviación } \\
\text { significativa de la normalidad, que tendería a invalidar muchos de los procedimientos } \\
\text { estadísticos aplicados habitualmente a estos datos. En este caso, las siguientes varia- } \\
\text { bles muestran valores de asimetría estandarizada fuera del rango esperado: } \\
\text { <ninguna> }\end{array}$} \\
\hline \multicolumn{4}{|c|}{$\begin{array}{l}\text { Las siguientes variables muestran valores de curtosis estandarizada fuera del rango es- } \\
\text { perado: } \\
\quad<\text { ninquna> }\end{array}$} \\
\hline
\end{tabular}

Tabla 5.13. Comparaciones de las características estadísticas de ambas pruebas en la categoría gusto

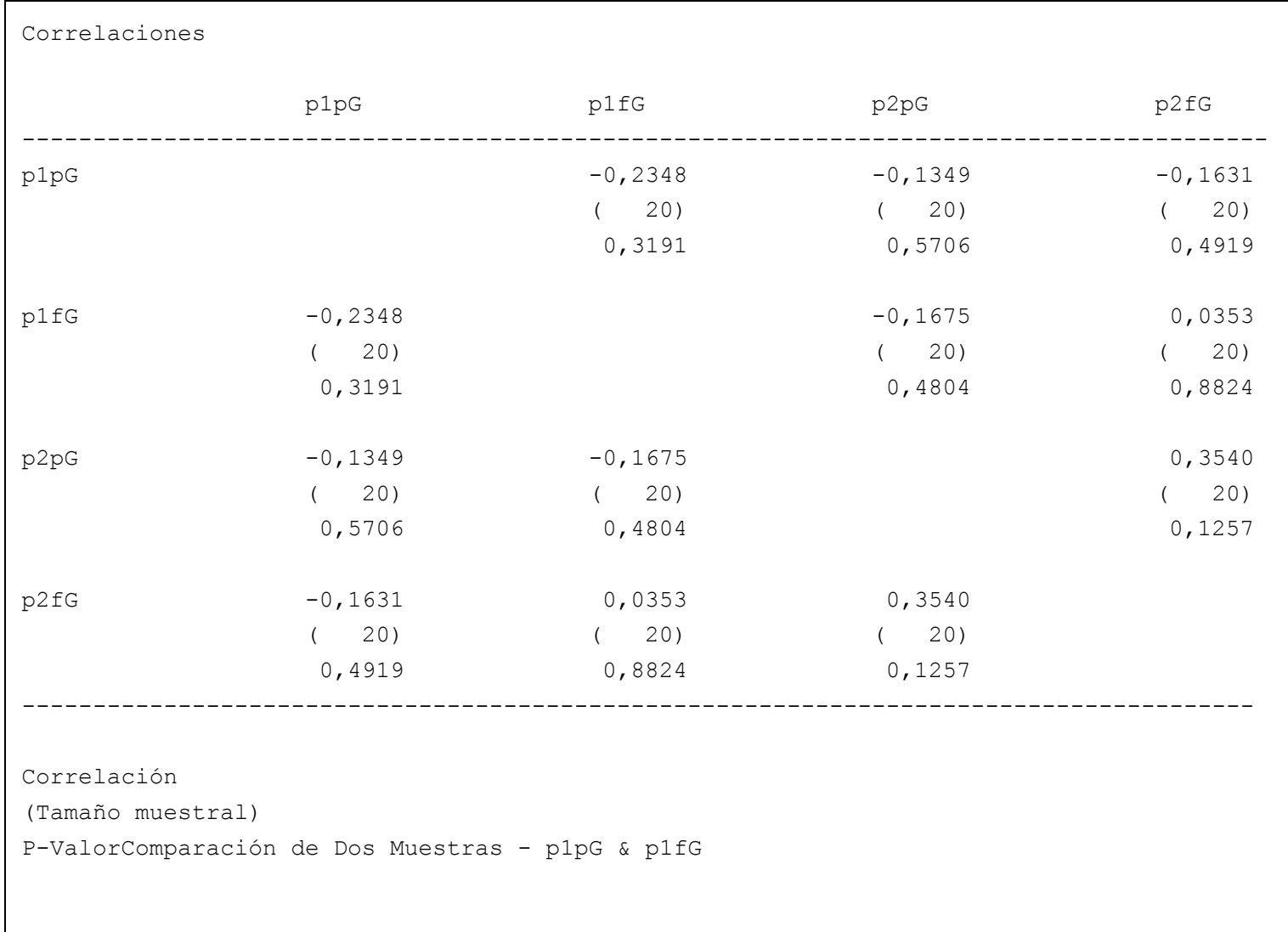




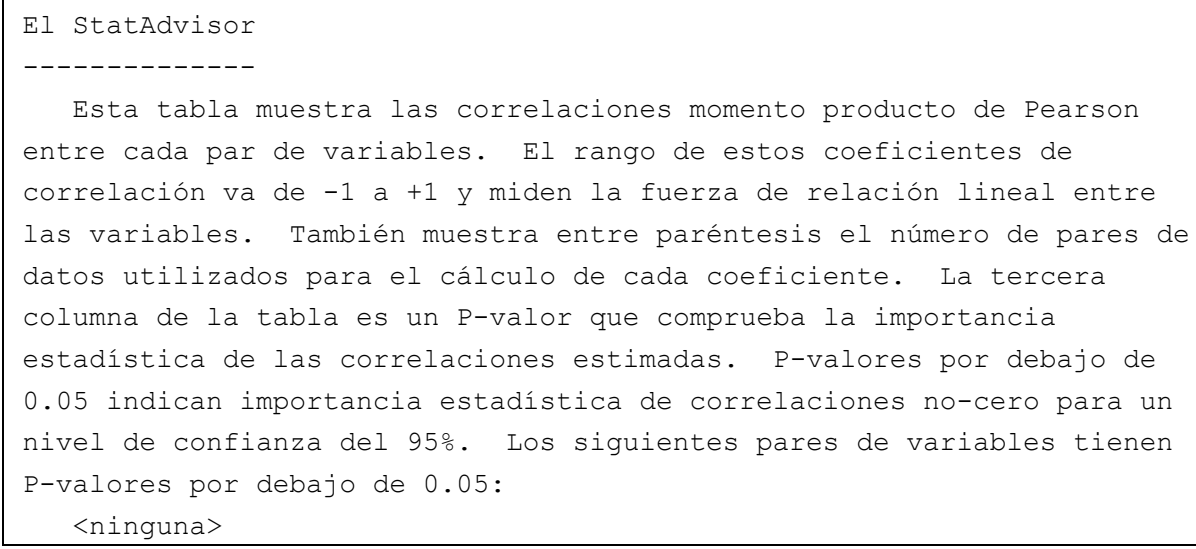

Tabla 5.14. Correlaciones de ambas pruebas en la categoría gusto

\subsubsection{Comparaciones de los resultados de gusto (G) para la primera demostración}

Partiendo de la normalidad comprobada con los coeficientes de asimetría y curtosis tipificados en la tabla 5.13, en la tabla 5.17 comprobamos que no se puede rechazar la igualdad de desviaciones típicas; a su vez esto nos permite realizar el contraste de igualdad de medias que aparece en la tabla 5.16, resultando que tampoco podemos rechazar la hipótesis nula, de modo que se puede admitir que las medias sean iguales entre ambas variables. El resultado del contraste de Kolmogorov-Smirnov (tabla 5.18) indica en la misma dirección, ya que tampoco se puede rechazar la igualdad de las distribuciones.

Los gráficos ponen de manifiesto sólo ciertas diferencias:

9) Gráfico 5.11: comparación de histogramas, donde se observa cómo el histograma de arriba (correspondiente a la PPF) tiene una mayor continuidad que el de abajo (correspondiente a la PF) con un máximo y un máximo local.

10) Gráfico 5.12: comparación de densidades, con formas similares a la del histograma anterior.

11) Gráfico 5.13: con los dos diagramas de cajas y bigotes, en el que se observa cómo todo en el gráfico superior (que representa la PPF) está 
desplazado a la derecha y más reducido que el inferior (que representa la PF).

12) Gráfico 5.14: con la representación de los cuantiles, donde la distribución de la PF se cruza con la PPF.

Por tanto no podemos decir que en este caso el gusto suponga una diferencia sustancial entre ambas pruebas.

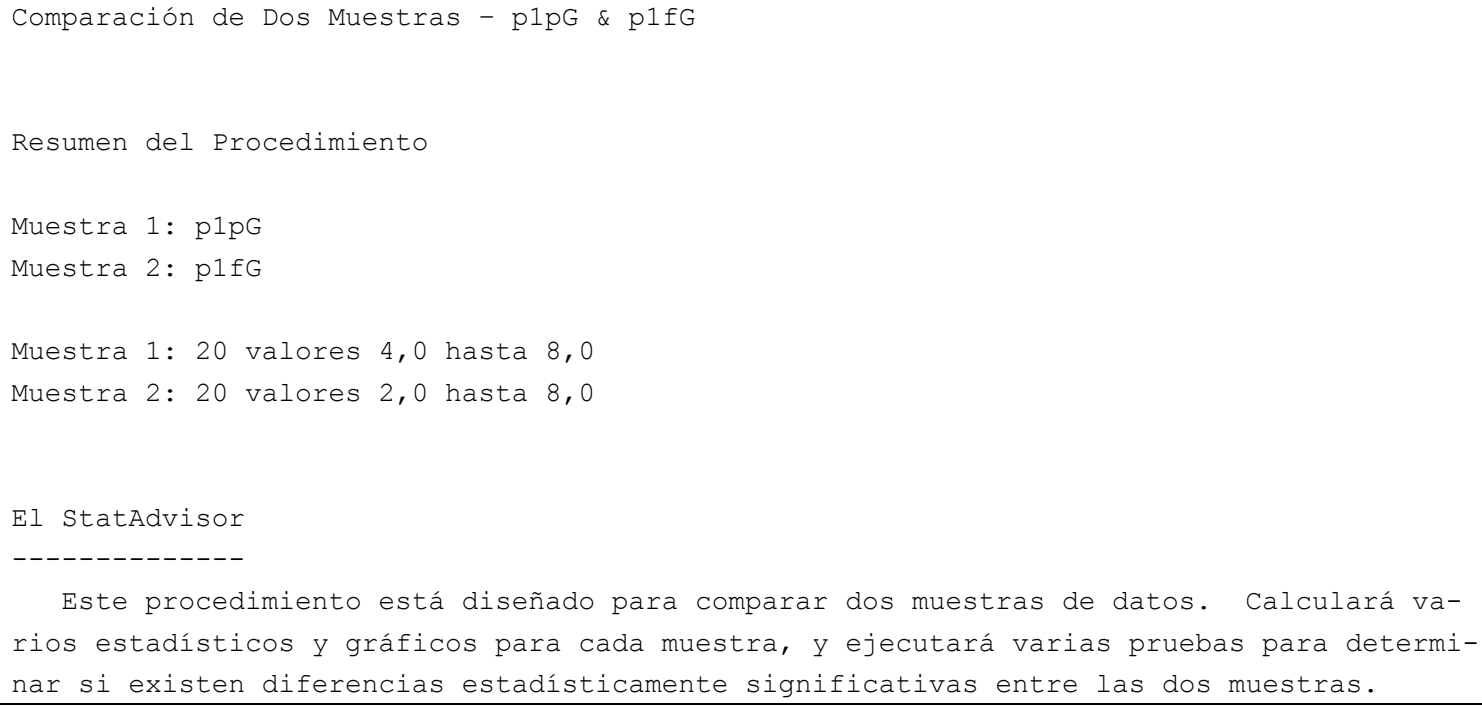

Tabla 5.15. Comparación de muestras p1pG-p1fG

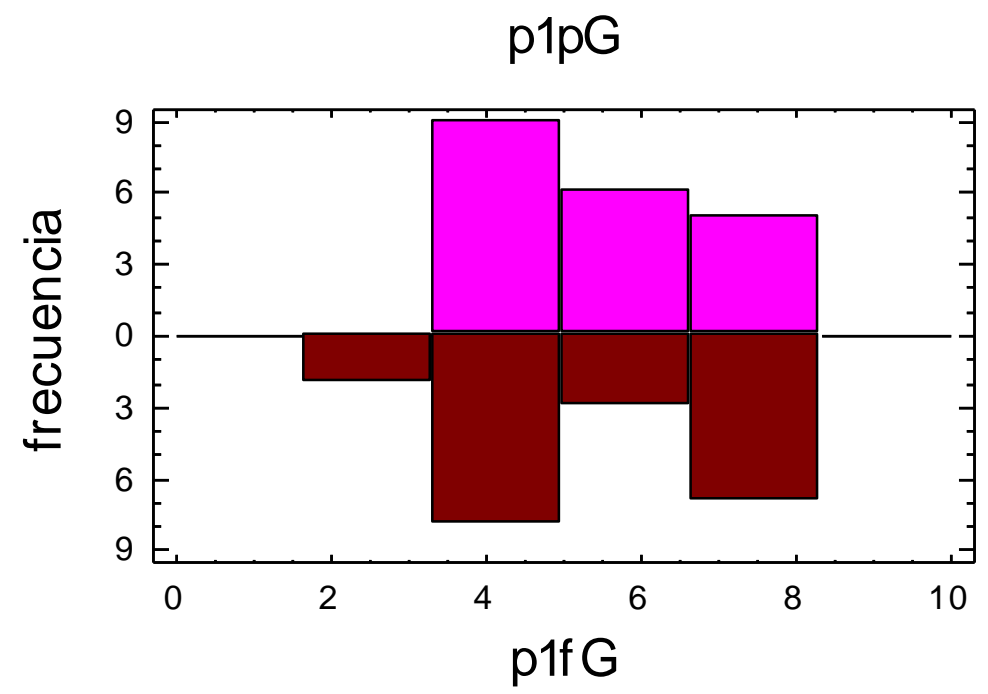

Gráfico 5.11. Comparación de histogramas p1pG-p1fG 


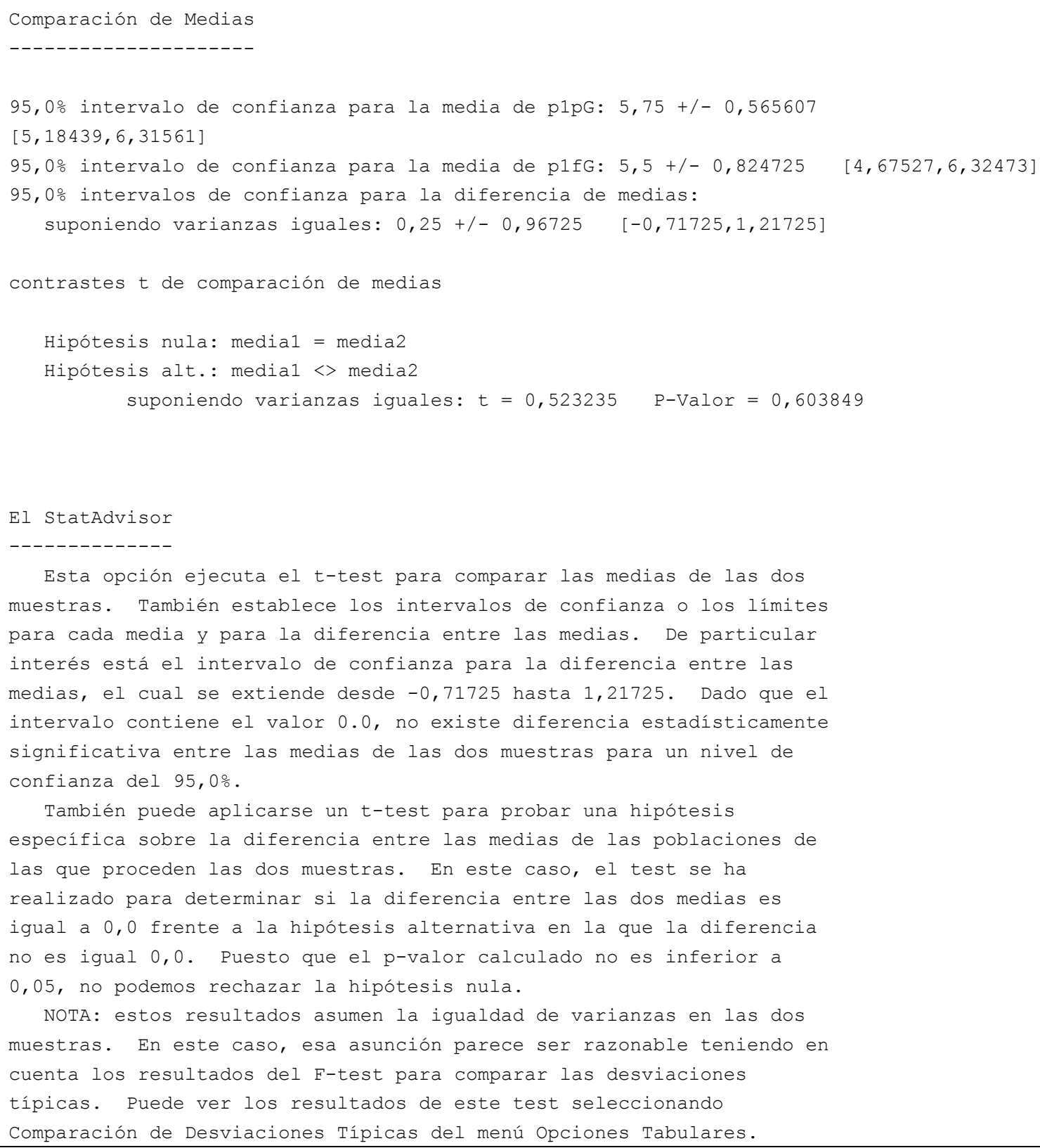

Tabla 5.16. Comparación de medias p1pG-p1fG 


\section{Density Traces}

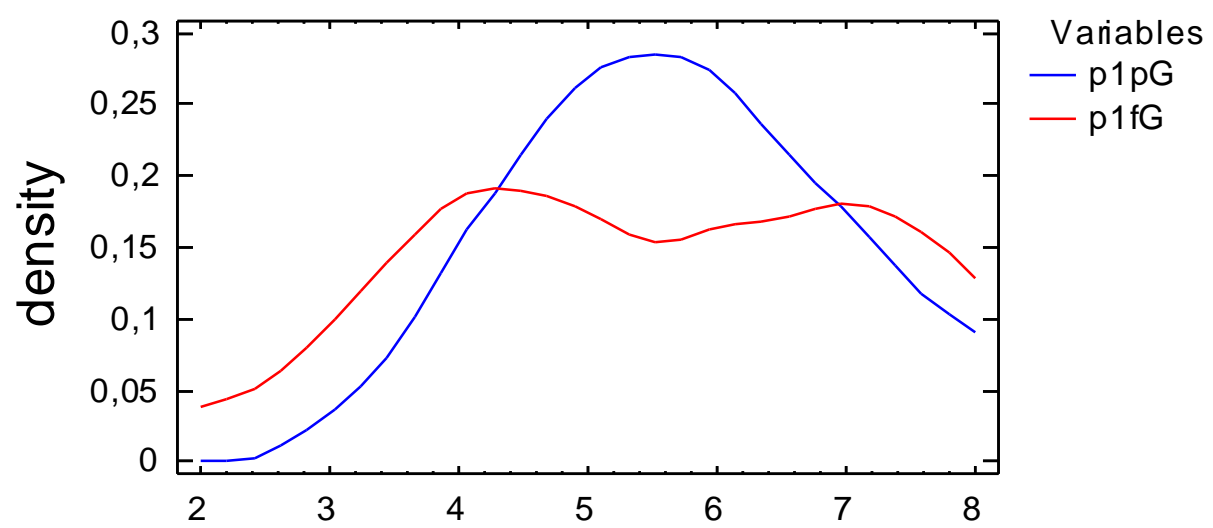

Gráfico 5.12. Comparación de densidades p1pG-p1fG

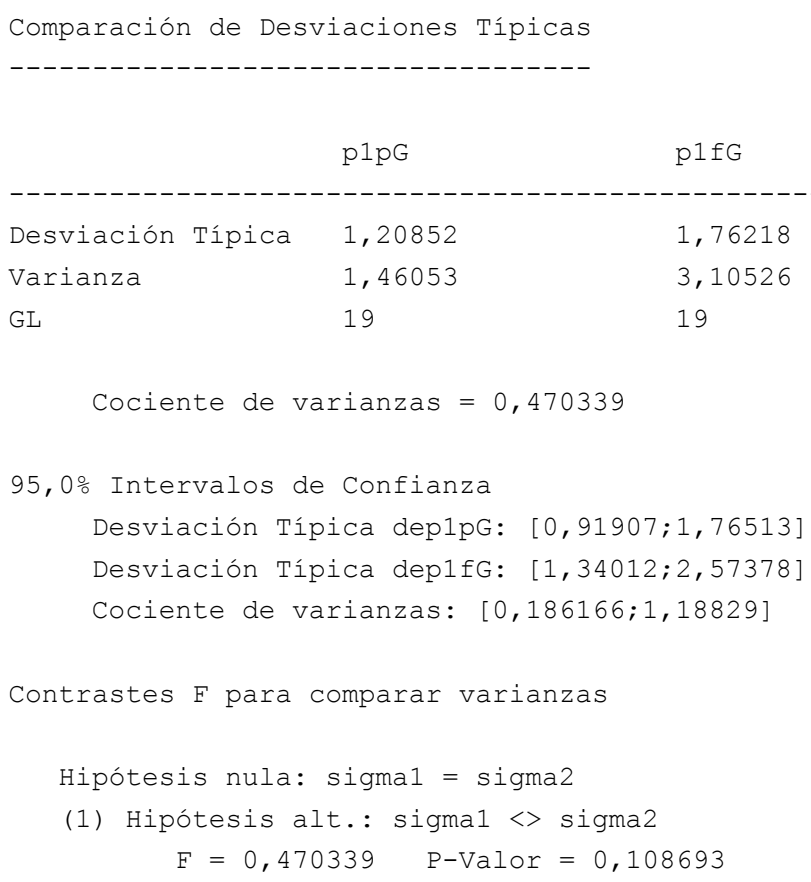

El StatAdvisor

Esta opción ejecuta un F-test para comparar las varianzas de las dos muestras. También establece los intervalos de confianza o los límites para cada desviación típica y para el ratio de varianzas. De particular interés está el intervalo de confianza para el ratio de las varianzas, el cual se extiende desde 0,186166 hasta 1,18829. Dado que el intervalo contiene el valor 1.0, no existe diferencia estadísticamente significativa entre las desviaciones típicas de las 
dos muestras para un nivel de confianza del 95,0\%.

También puede utilizarse un F-test para probar una hipótesis

específica sobre las desviaciones típicas de las poblaciones de las

que proceden las dos muestras. En este caso, el test se ha realizado

para determinar si el ratio de las desviaciones típicas son iguales

1,0 frente a la hipótesis alternativa en la que el ratio no es igual

1,0. Puesto que el p-valor calculado no es inferior a 0,05, no

podemos rechazar la hipótesis nula.

NOTA IMPORTANTE: los F-test y los intervalos de confianza mostrados

dependen de que las muestras procedan de distribuciones normales.

Para comprobar esta asunción, seleccione Resumen Estadístico de la

lista de Opciones Tabulares y observe los valores de asimetría

estandarizada y curtosis estandarizada.

Tabla 5.17. Comparación de desviaciones típicas p1pG-p1fG

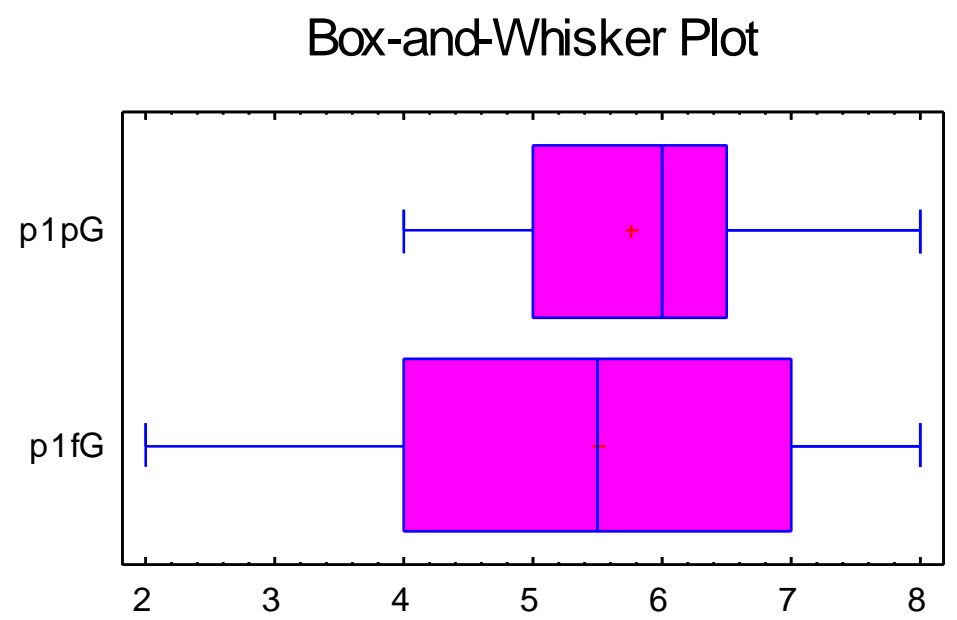

Gráfico 5.13. Comparación de diagramas de cajas y bigotes p1pG-p1fG

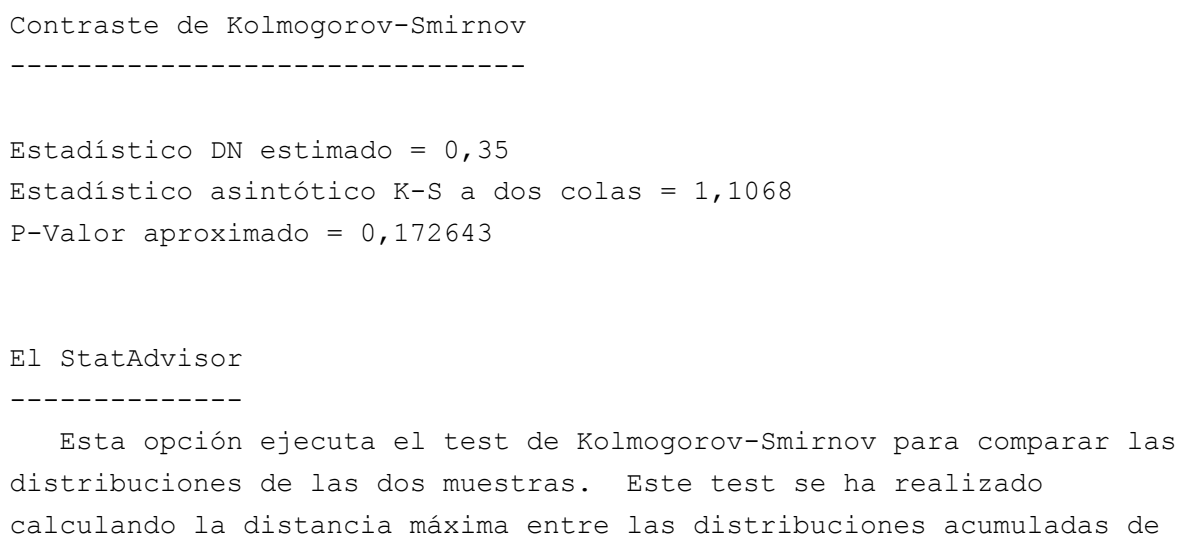


las dos muestras. En este caso, la máxima distancia es 0,35, la cual

puede visualizar seleccionando Gráfico Cuantil en la lista de Opciones

Gráficas. De particular interés está el p-valor aproximado para el

test. Dado que el p-valor es mayor o igual a 0,05, no existe

diferencia estadísticamente significativa entre las dos distribuciones

para un nivel de confianza del 95,0\%.

Tabla 5.18. Contraste de Kolmogorov-Smirnov, gusto 1

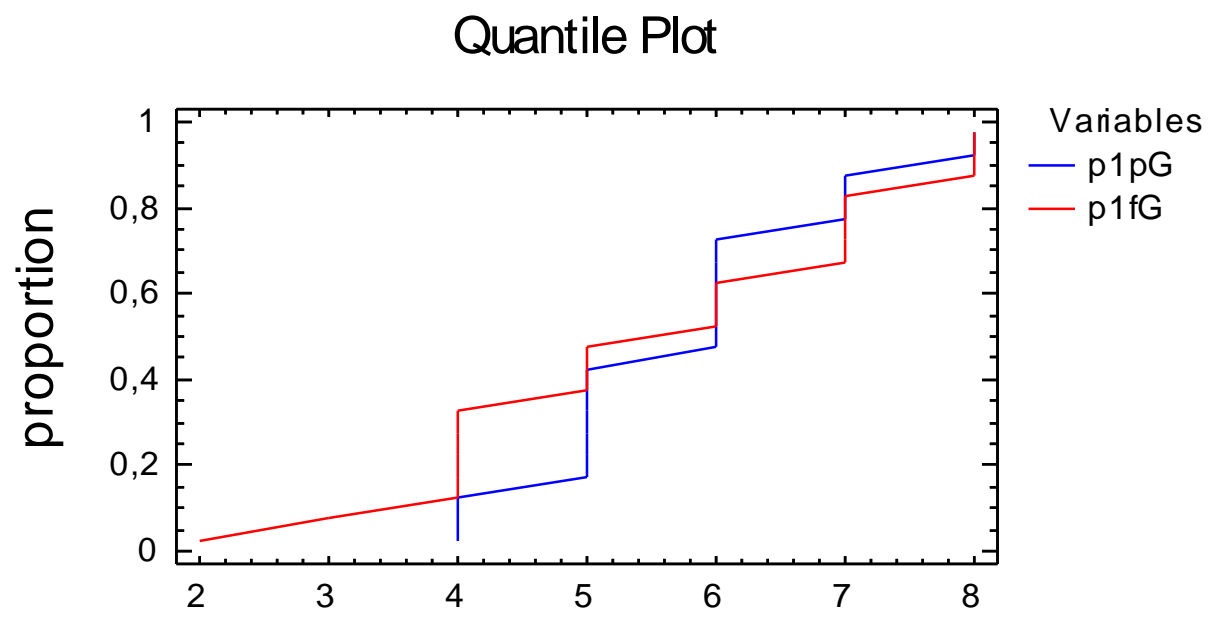

Gráfico 5.14. Representación de cuantiles p1pG-p1fG

\subsubsection{Comparaciones de los resultados de gusto (G) para la segunda demostración}

Partiendo de la normalidad comprobada con los coeficientes de asimetría y curtosis tipificados en la tabla 5.13, en la tabla 5.21 comprobamos que no se puede rechazar la igualdad de desviaciones típicas; tal conclusión nos permite realizar el contraste de igualdad de medias que aparece en la tabla 5.20, resultando que tampoco podemos rechazar la hipótesis nula, de modo que se puede admitir que las medias sean iguales entre ambas variables. El contraste de Kolmogorov-Smirnov (tabla 5.22) sin embargo sí que permite rechazar la igualdad de las distribuciones.

Los gráficos permiten advertir lo siguiente: 
13) Gráfico 5.15: comparación de histogramas, donde se observa cómo el histograma de arriba (correspondiente a la PPF) está desplazado hacia la derecha respecto al de abajo (correspondiente a la PF).

14) Gráfico 5.16: comparación de densidades, donde se aprecia ese mismo desplazamiento a la derecha.

15) Gráfico 5.17: con los dos diagramas de cajas y bigotes, en el que se observa cómo a partir del primer cuartil (el lado izquierdo de la caja) todo aparece antes en el de abajo (que representa la PF) que en el de arriba (que representa la PPF).

16) Gráfico 5.18: con la representación de los cuantiles, donde la distribución de la PF se separa cada vez más de la distribución de la PPF, que queda más a la derecha.

Por tanto en este caso el gusto sí que supone una diferencia sustancial entre ambas demostraciones, con clara preferencia por la PPF.

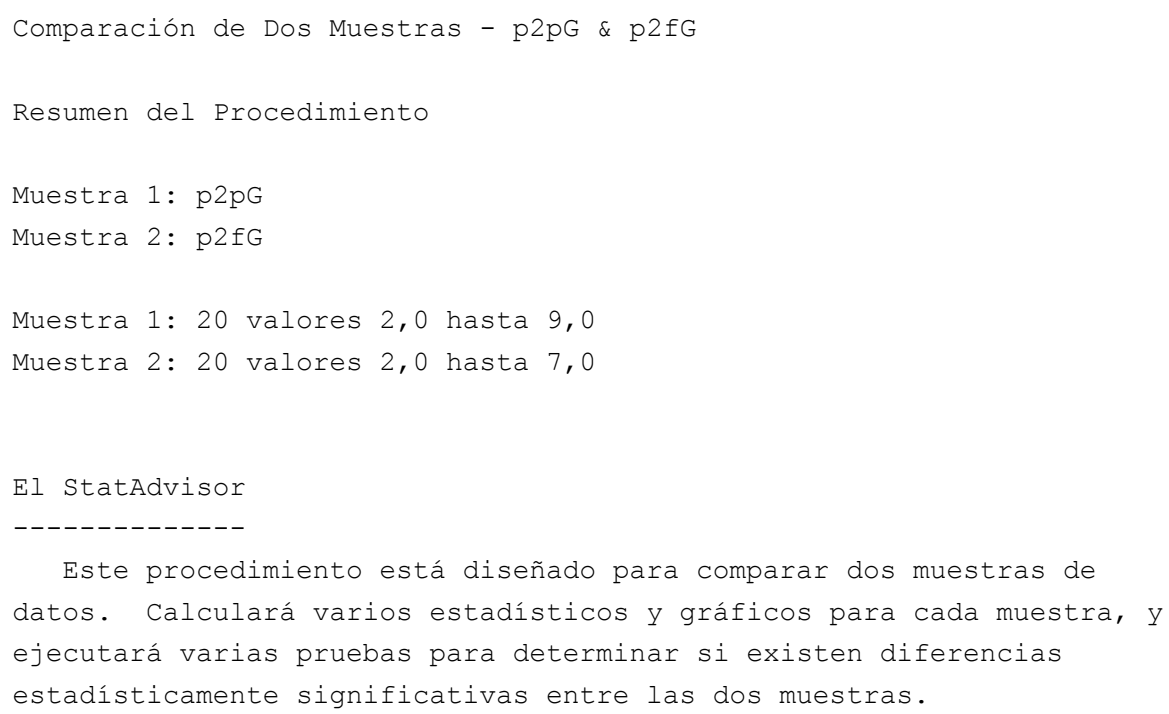

Tabla 5.19. Comparación de muestras p2pG-p2fG 


\section{p2pG}

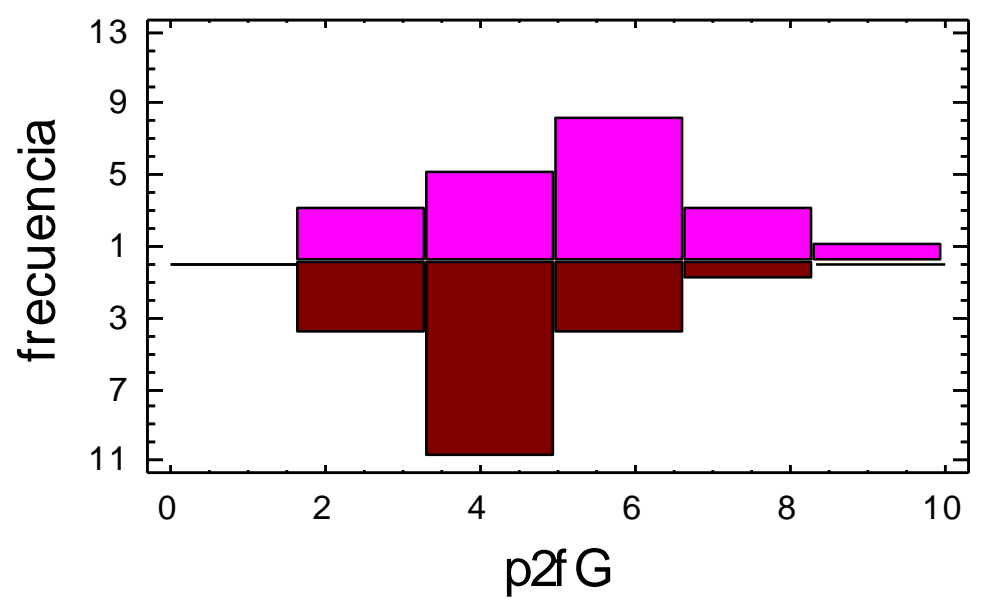

Gráfico 5.15. Comparación de histogramas p2pG-p2fG

Comparación de Medias

95, 0\% intervalo de confianza para la media de p2pG: 5,35 +/- 0,793014

$[4,55699,6,14301]$

95, 0\% intervalo de confianza para la media de p2fG: 4,65 +/- 0,593457

$[4,05654,5,24346]$

95, 0\% intervalos de confianza para la diferencia de medias:

suponiendo varianzas iguales: $0,7+/-0,958008 \quad[-0,258008,1,65801]$

contrastes $t$ de comparación de medias

Hipótesis nula: medial $=$ media2

Hipótesis alt.: medial <> media2

suponiendo varianzas iguales: $t=1,47919 \quad$ P-Valor $=0,147331$

El StatAdvisor

------------

Esta opción ejecuta el t-test para comparar las medias de las dos muestras. También establece los intervalos de confianza o los límites para cada media y para la diferencia entre las medias. De particular interés está el intervalo de confianza para la diferencia entre las medias, el cual se extiende desde $-0,258008$ hasta 1,65801. Dado que el intervalo contiene el valor 0.0 , no existe diferencia estadísticamente significativa entre las medias de las dos muestras para un nivel de confianza del 95, 0\%.

También puede aplicarse un t-test para probar una hipótesis específica sobre la diferencia entre las medias de las poblaciones de las que proceden las dos muestras. En este caso, el test se ha realizado para determinar si la diferencia entre las dos medias es igual a 0,0 frente a la hipótesis alternativa en la que la diferencia no es igual 0,0. Puesto que el p-valor calculado no es inferior a 0,05, no podemos rechazar la hipótesis nula. 
NOTA: estos resultados asumen la igualdad de varianzas en las dos

muestras. En este caso, esa asunción parece ser razonable teniendo en

cuenta los resultados del F-test para comparar las desviaciones

típicas. Puede ver los resultados de este test seleccionando

Comparación de Desviaciones Típicas del menú Opciones Tabulares.

Tabla 5.20. Comparación de medias p2pG-p2fG

Density Traces

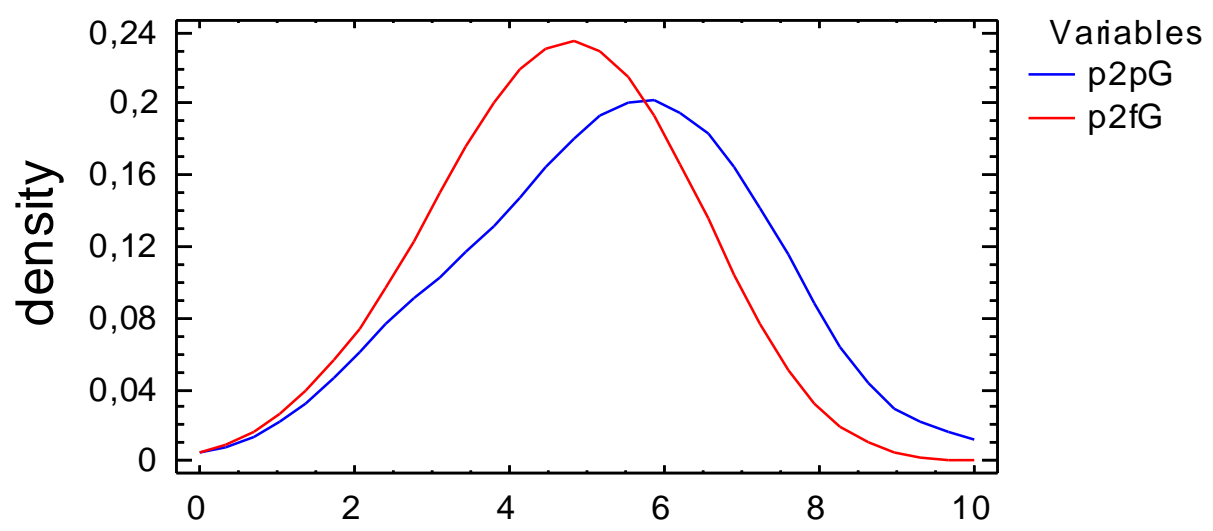

Gráfico 5.16. Comparación de densidades p2pG-p2fG

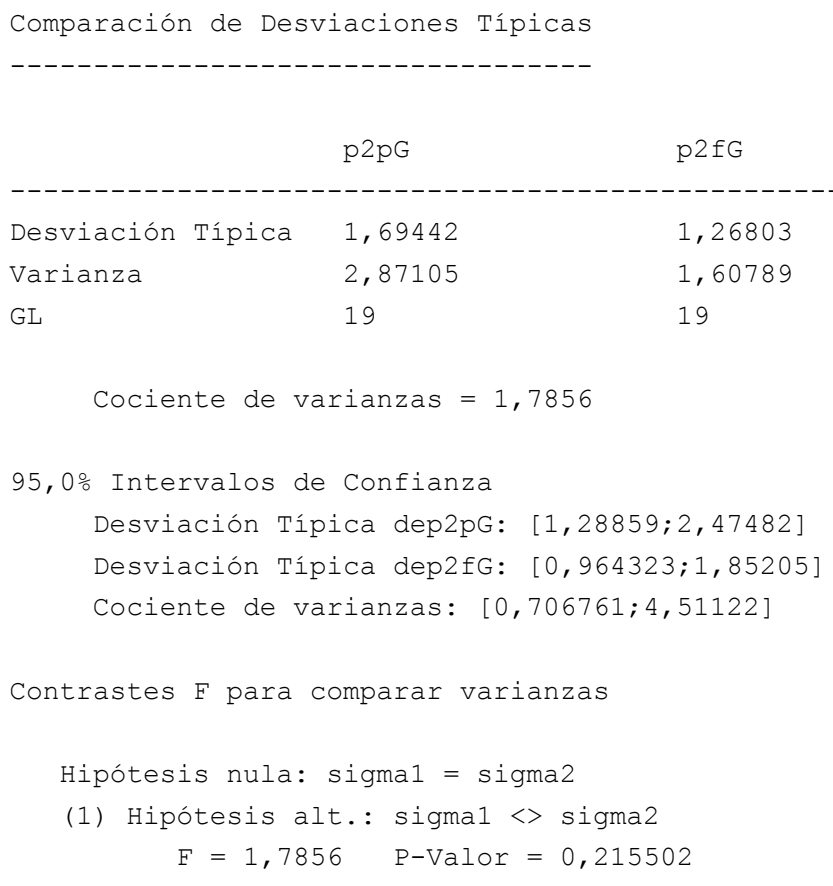


El StatAdvisor

Esta opción ejecuta un F-test para comparar las varianzas de las dos muestras. También establece los intervalos de confianza o los límites para cada desviación típica y para el ratio de varianzas. De particular interés está el intervalo de confianza para el ratio de las varianzas, el cual se extiende desde 0,706761 hasta 4,51122. Dado que el intervalo contiene el valor 1.0 , no existe diferencia estadísticamente significativa entre las desviaciones típicas de las dos muestras para un nivel de confianza del 95,0\%.

También puede utilizarse un F-test para probar una hipótesis específica sobre las desviaciones típicas de las poblaciones de las que proceden las dos muestras. En este caso, el test se ha realizado para determinar si el ratio de las desviaciones típicas son iguales 1,0 frente a la hipótesis alternativa en la que el ratio no es igual 1,0. Puesto que el p-valor calculado no es inferior a 0,05, no podemos rechazar la hipótesis nula.

NOTA IMPORTANTE: los F-test y los intervalos de confianza mostrados dependen de que las muestras procedan de distribuciones normales.

Para comprobar esta asunción, seleccione Resumen Estadístico de la lista de Opciones Tabulares y observe los valores de asimetría estandarizada y curtosis estandarizada.

Tabla 5.21. Comparación de desviaciones típicas p2pG-p2fG

\section{Box-and-Whisker Plot}

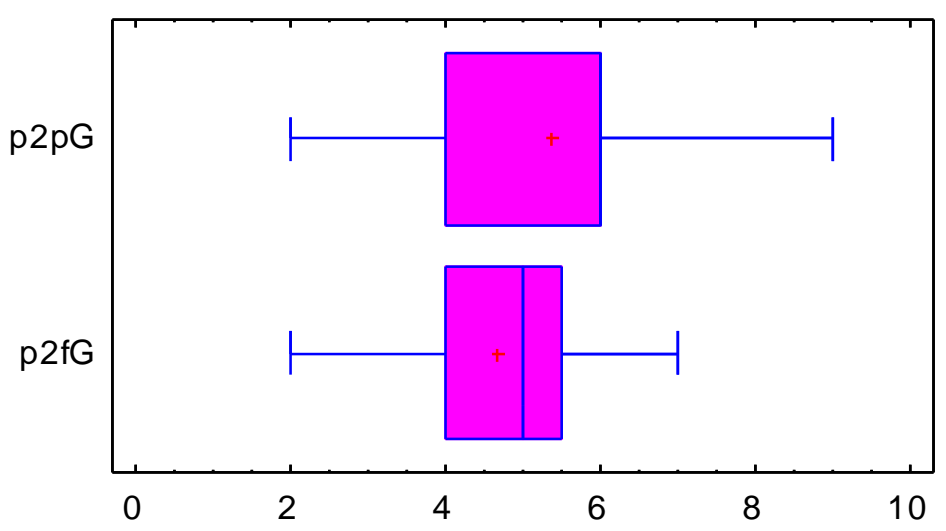

Gráfico 5.17. Comparación de diagramas de cajas y bigotes p2pG-p2fG

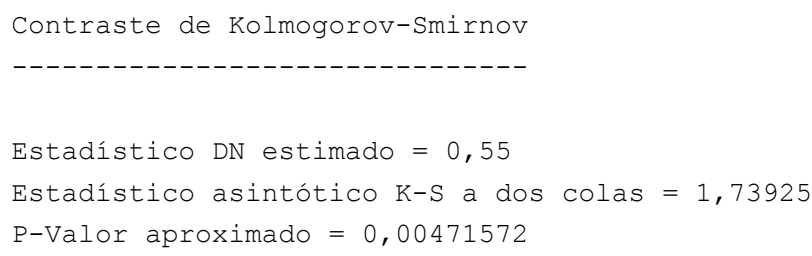




\section{El StatAdvisor}

Esta opción ejecuta el test de Kolmogorov-Smirnov para comparar las distribuciones de las dos muestras. Este test se ha realizado calculando la distancia máxima entre las distribuciones acumuladas de las dos muestras. En este caso, la máxima distancia es 0,55, la cual puede visualizar seleccionando Gráfico Cuantil en la lista de Opciones Gráficas. De particular interés está el p-valor aproximado para el

test. Dado que el p-valor es menor que 0,05, existe diferencia estadísticamente significativa entre las dos distribuciones para un nivel de confianza del 95, 0\%.

Tabla 5.22. Contraste de Kolmogorov-Smirnov, gusto 2

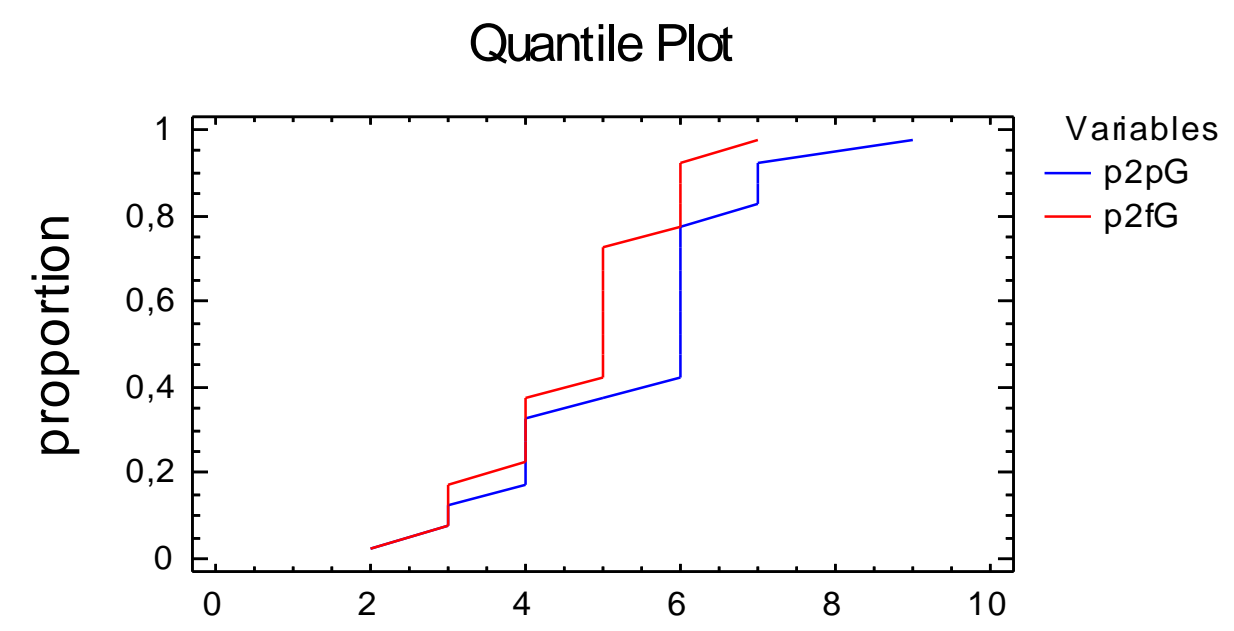

Gráfico 5.18. Representación de cuantiles p2pG-p2fG

\subsection{Comparaciones de los resultados de claridad (C) de cada una de las demostraciones}

La tabla 5.23 recoge las frecuencias de cada una de las calificaciones asignadas por los alumnos en la categoría claridad en cada una de las cuatro demostraciones. 


\begin{tabular}{rrrrrr} 
& $\mathbf{p 1 p C}$ & $\mathbf{p 1 f C}$ & \multicolumn{1}{c}{$\mathbf{p} \mathbf{p C}$} & \multicolumn{2}{c}{$\mathbf{p 2 f C}$} \\
\hline $\mathbf{1}$ & 0 & 0 & 0 & 0 \\
$\mathbf{2}$ & 0 & 1 & 1 & 0 \\
$\mathbf{3}$ & 0 & 1 & 1 & 3 \\
$\mathbf{4}$ & 0 & 1 & 1 & 2 \\
$\mathbf{5}$ & 7 & 5 & 8 & 7 \\
$\mathbf{6}$ & 8 & 3 & 3 & 5 \\
$\mathbf{7}$ & 3 & 5 & 4 & 2 \\
$\mathbf{8}$ & 2 & 4 & 2 & 1 \\
$\mathbf{9}$ & 0 & 0 & 0 & 0 \\
$\mathbf{1 0}$ & 0 & 0 & 0 & 0 \\
\hline Total & 20 & 20 & 20 & 20 \\
\hline
\end{tabular}

Tabla 5.23. Frecuencias en la categoría claridad en cada una de las pruebas y de los teoremas

Asimismo, estos datos quedan reflejados en los gráficos 5.19 y 5.19 bis siguientes.

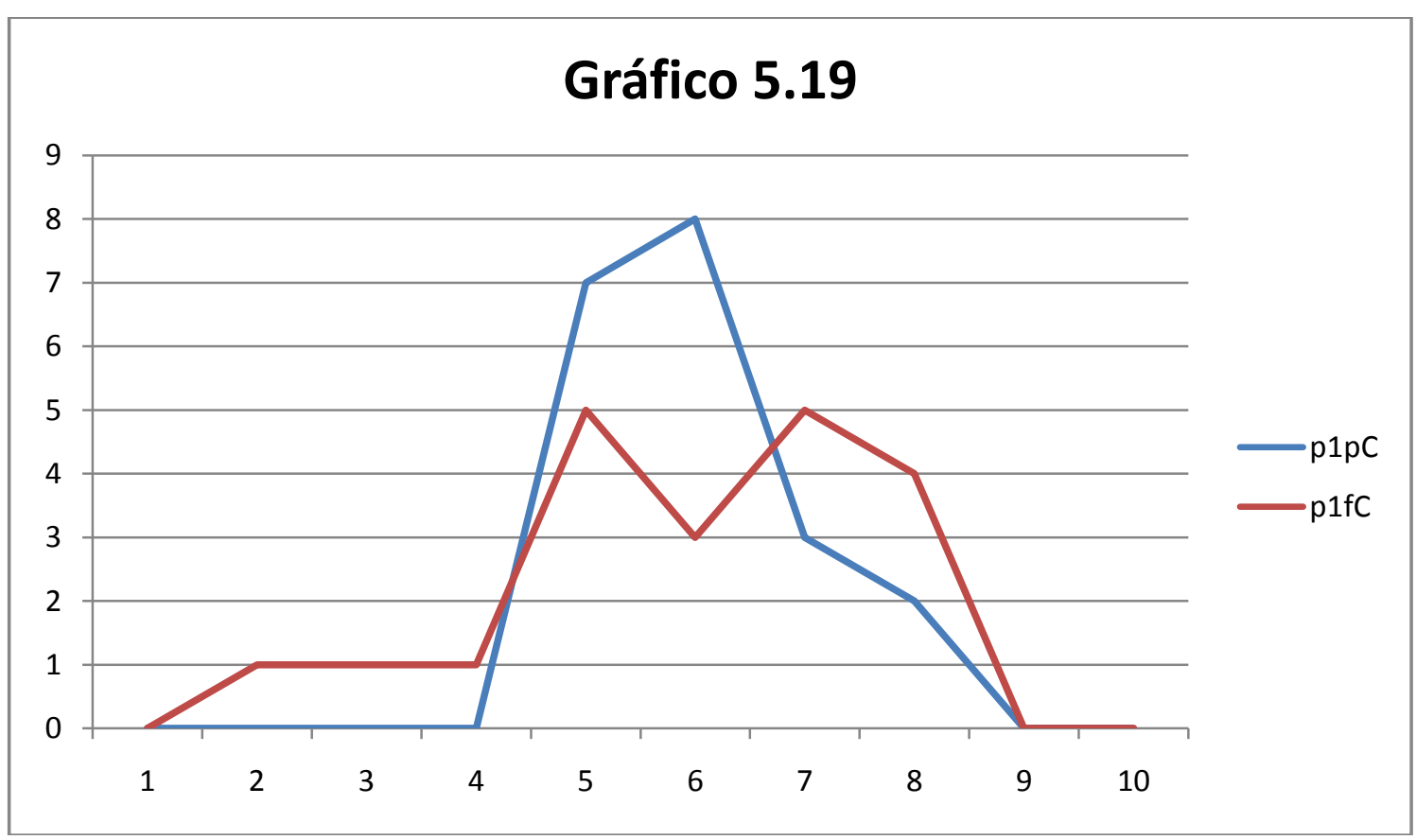




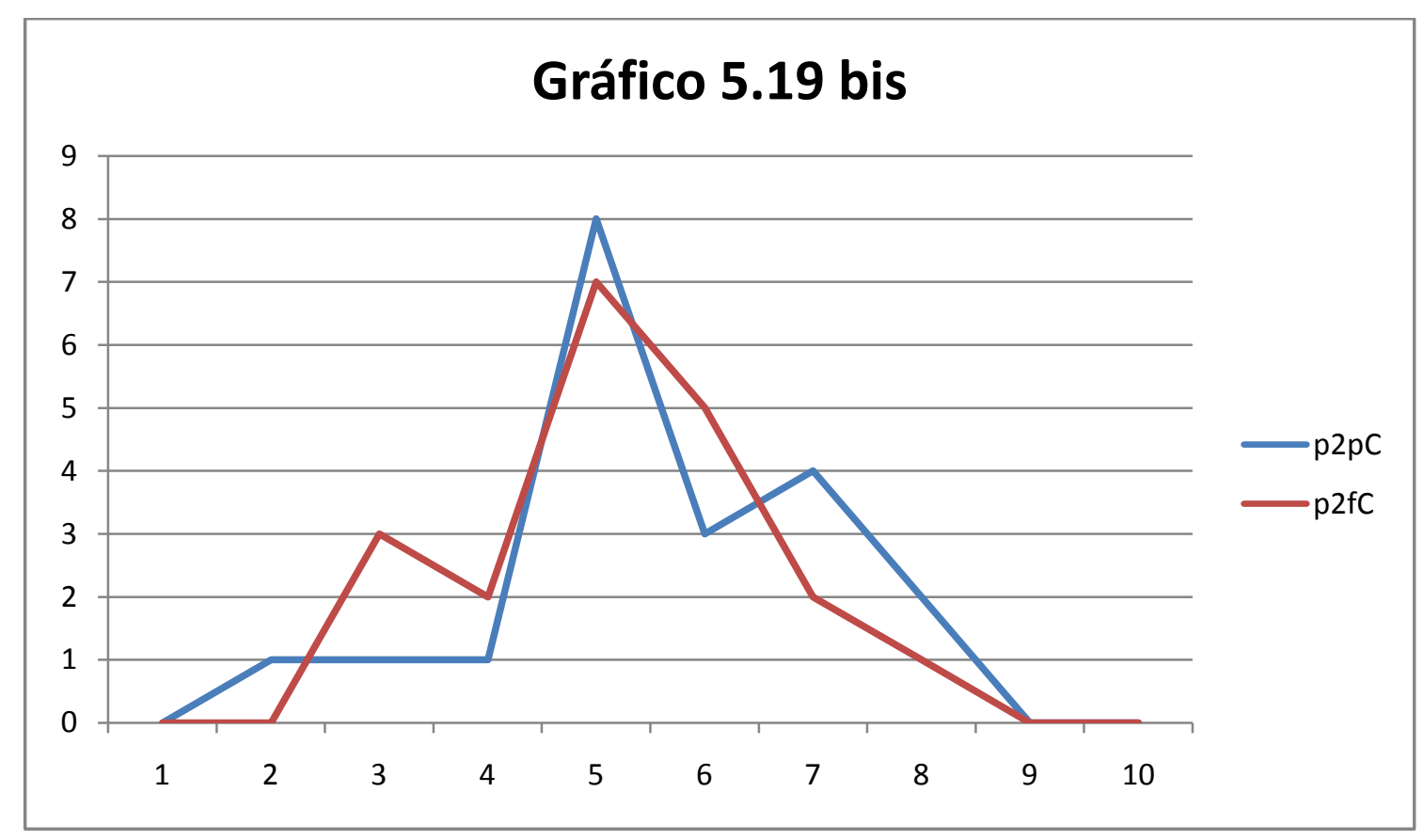

Estas distribuciones representan menos evidentemente para el caso de la claridad que las demostraciones PPF son mejor valoradas que las PF; para el primer teorema la distribución de la PPF (en azul) en general está más a la derecha que la PF (en rojo), y lo mismo ocurre para el segundo teorema (PPF en azul y PF en rojo).

Las tablas 5.24 y 5.25 recogen las comparaciones de las características estadísticas de estas cuatro variables, que vienen a confirmarnos una escasa preferencia por la PPF. Así en la tabla 5.24, observamos que las características de tendencia central son muy poco superiores en las PPF ( $1^{\underline{a}}$ y $3^{\underline{a}}$ columna) respecto a las $P F\left(2^{\underline{a}}\right.$ y $4^{\underline{a}}$ columna) en media, mediana y moda en la primera y segunda prueba. Se observa lo mismo para mínimo, máximo y cuartiles, salvo en el segundo cuartil para la primera prueba y para el mínimo de la segunda.

Por lo que se refiere a la dispersión, tomando el coeficiente de variación se aprecia cómo va creciendo de PPF a PF en la primera demostración, lo que significaría una mayor unanimidad en las votaciones para aquellas pruebas con un coeficiente de variación menor. En la segunda sin embargo es la PF la que presenta una menor 
dispersión sobre la PPF, aunque las diferencias no son tan amplias como en el caso anterior, y en todos los casos muy distantes de la menor dispersión de la PPF primera.

Por su parte, siguiendo en la tabla 5.24, la asimetría tipificada de las distribuciones no viene a corroborar en todos los casos la preferencia por las PPF: en el primer caso la PF es asimétrica negativa frente a una PPF asimétrica positiva, o sea, en la PPF la mayoría de las calificaciones se agrupan al principio de la distribución y hay menos calificaciones altas, lo que nos indica por tanto una preferencia por la PF; sin embargo, en el segundo caso la PPF es asimétrica negativa frente a una PF asimétrica positiva, o sea, en la PF la mayoría de las calificaciones se agrupan al principio de la distribución y hay menos calificaciones altas, lo que nos indica por tanto una preferencia por la PPF.

Además, el hecho de que la asimetría tipificada y la curtosis tipificada estén entre -2 y 2 va a permitir tratar estas variables como que siguen distribuciones normales, lo que justificará la utilización de los contrastes de igualdad de varianzas y de igualdad de medias que se verán en los dos apartados siguientes.

Las correlaciones de la tabla 5.25 nos indican la escasa asociación que hay mayoritariamente entre los pares de calificaciones (los valores de estas correlaciones, por lo general, están más bien próximos a cero, lo que indica una escasa relación entre el par de variables correspondiente). El p-valor corrobora la inexistencia de relaciones significativas salvo para la PPF y PF del segundo teorema. Esto es, mayoritariamente no hay relación entre las puntuaciones respecto de la claridad dadas por los alumnos en las distintas demostraciones.

\begin{tabular}{|c|c|c|c|c|}
\hline \multicolumn{5}{|c|}{ Resumen Estadístico } \\
\hline & $\mathrm{p} 1 \mathrm{pC}$ & $\mathrm{p} 1 \mathrm{fC}$ & $\mathrm{p} 2 \mathrm{pc}$ & $\mathrm{p} 2 \mathrm{fC}$ \\
\hline Frecuencia & 20 & 20 & 20 & 20 \\
\hline Media & 6,0 & 5,95 & 5,55 & 5,2 \\
\hline Mediana & 6,0 & 6,0 & 5,0 & 5,0 \\
\hline Moda & 6,0 & & 5,0 & 5,0 \\
\hline
\end{tabular}




\begin{tabular}{|c|c|c|c|c|}
\hline Varianza & 0,947368 & 2,89211 & 2,36579 & 1,85263 \\
\hline Desviación típica & 0,973329 & 1,70062 & 1,53811 & 1,36111 \\
\hline Mínimo & 5,0 & 2,0 & 2,0 & 3,0 \\
\hline Máximo & 8,0 & 8,0 & 8,0 & 8,0 \\
\hline Rango & 3,0 & 6,0 & 6,0 & 5,0 \\
\hline Primer cuartil & 5,0 & 5,0 & 5,0 & 4,5 \\
\hline Segundo cuartil & 6,5 & 7,0 & 7,0 & 6,0 \\
\hline Asimetría tipi. & 1,38946 & $-1,27546$ & $-0,718715$ & 0,030485 \\
\hline Curtosis típificada & $-0,144853$ & 0,0365431 & 0,274099 & $-0,17698$ \\
\hline Coef. de variación & $16,2221 \%$ & $28,5818 \%$ & $27,7137 \%$ & $26,1753 \%$ \\
\hline \multicolumn{5}{|l|}{----------------- } \\
\hline \multicolumn{5}{|l|}{ El StatAdvisor } \\
\hline \multicolumn{5}{|c|}{$\begin{array}{l}\text { Esta tabla muestra resúmenes estadísticos para cada una de las variables selecciona- } \\
\text { das. Incluye medidas de tendencia central, de variabilidad y de forma. De particular } \\
\text { interés están la asimetría estandarizada y la curtosis estandarizada, las cuales pueden } \\
\text { utilizarse para determinar si la muestra procede de una distribución normal. Valores de } \\
\text { estos estadísticos fuera del rango de -2 a t2 indican una desviación significativa de la } \\
\text { normalidad, que tendería a invalidar muchos de los procedimientos estadísticos aplicados } \\
\text { habitualmente a estos datos. En este caso, las siguientes variables muestran valores de } \\
\text { asimetría estandarizada fuera del rango esperado: } \\
\text { <ninguna> }\end{array}$} \\
\hline \multicolumn{5}{|c|}{$\begin{array}{l}\text { Las siguientes variables muestran valores de curtosis estandarizada fuera del rango es- } \\
\text { perado: } \\
\quad \text { <ninguna> }\end{array}$} \\
\hline
\end{tabular}

Tabla 5.24. Comparaciones de las características estadísticas de ambas pruebas en la categoría claridad

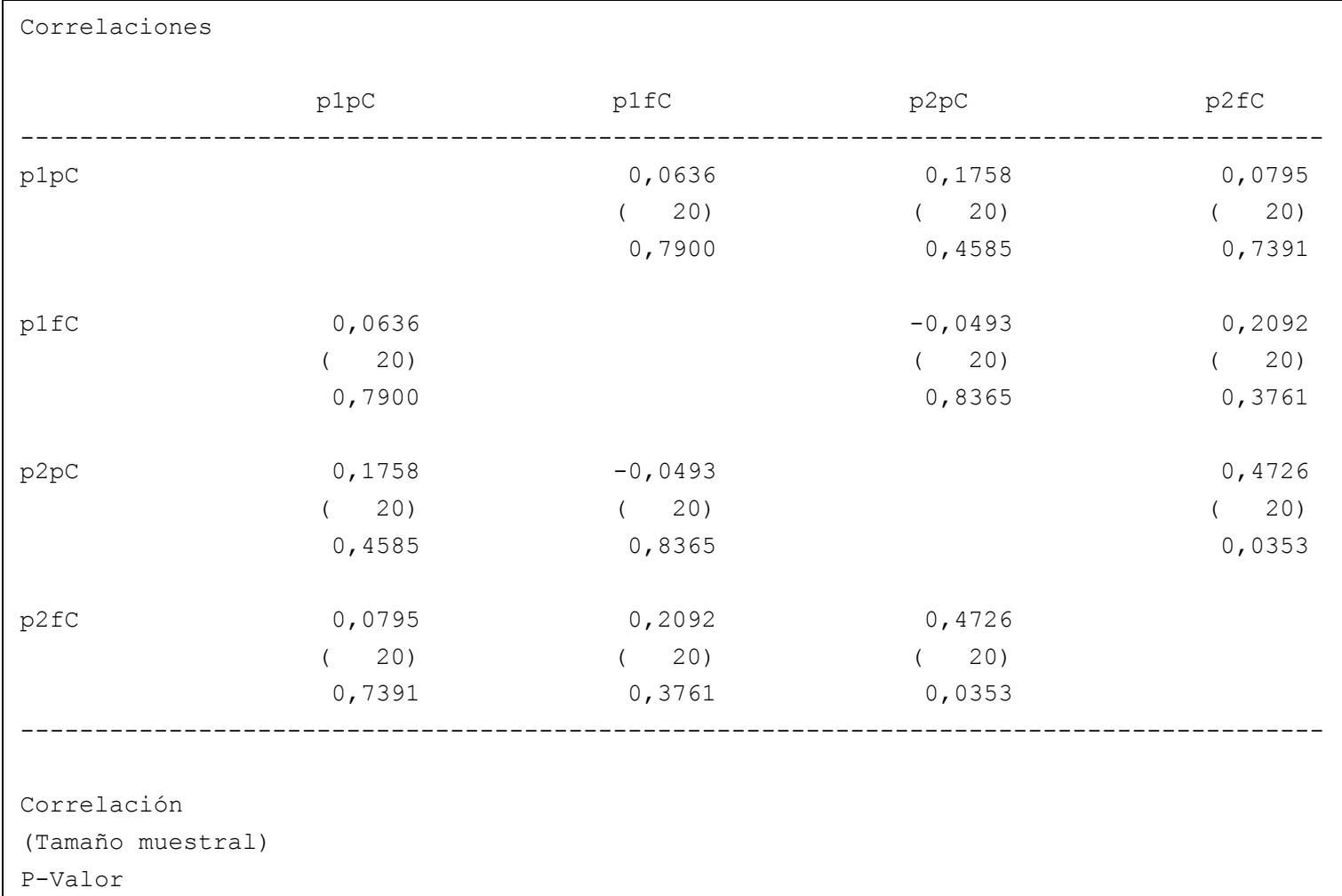




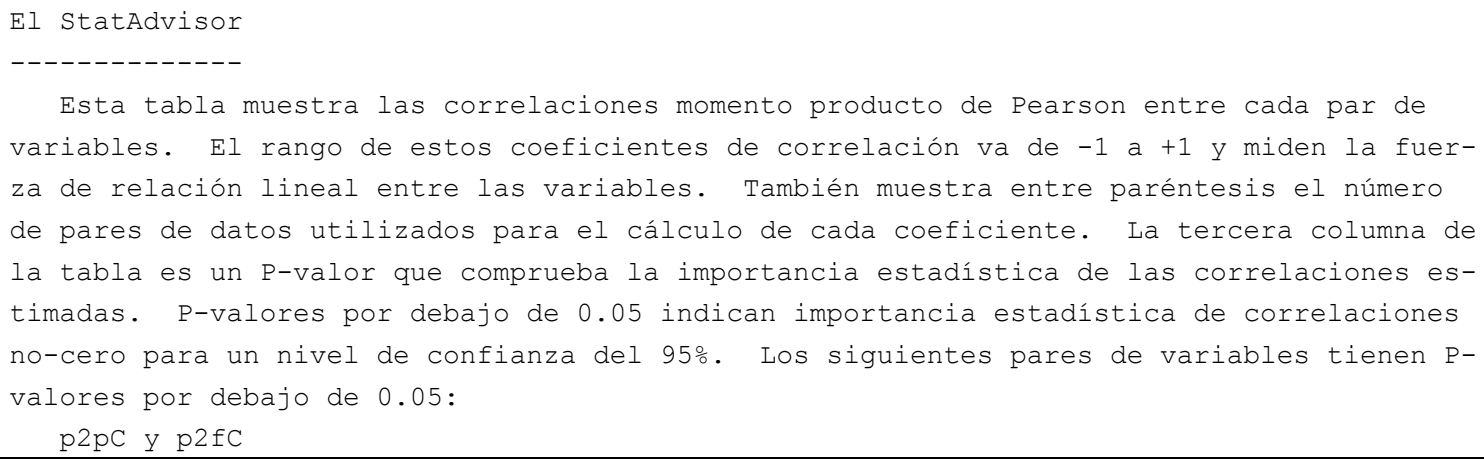

Tabla 5.25. Correlaciones de ambas pruebas en la categoría claridad

\subsubsection{Comparaciones de los resultados de claridad (C) para la primera demostración}

Partiendo de la normalidad comprobada con los coeficientes de asimetría y curtosis tipificados en la tabla 5.24, en la tabla 5.28 comprobamos que no se puede rechazar la igualdad de desviaciones típicas; a su vez esto nos permite realizar el contraste de igualdad de medias que aparece en la tabla 5.27, resultando que tampoco podemos rechazar la hipótesis nula, de modo que se puede admitir que las medias sean iguales entre ambas variables. El resultado del contraste de Kolmogorov-Smirnov (tabla 5.29) indica en el mismo sentido, ya que tampoco se puede rechazar la igualdad de las distribuciones.

Los gráficos ponen de manifiesto sólo ciertas diferencias:

17) Gráfico 5.20: comparación de histogramas, donde se observa cómo el histograma de arriba (correspondiente a la PPF) presenta por lo general mayores valores que el de abajo (correspondiente a la PF) que sólo destaca en ese último intervalo su alta frecuencia.

18) Gráfico 5.21: comparación de densidades, con formas similares a la del histograma anterior y una distribución bastante plana para la PF en comparación con la de la PPF.

19) Gráfico 5.22: con los dos diagramas de cajas y bigotes, en el que se observa cómo todo en el gráfico superior (que representa la PPF) está más concentrado a partir de la mediana (el trazo central de la caja) lo que supondría una mayor preferencia a partir de ese punto por la PF. 
20) Gráfico 5.23: con la representación de los cuantiles, donde la distribución de la PF se cruza con la PPF, con lo que no podríamos afirmar nada sobre cuál es preferida.

Por tanto no podemos decir que en este caso la claridad suponga una diferencia sustancial entre ambas pruebas.

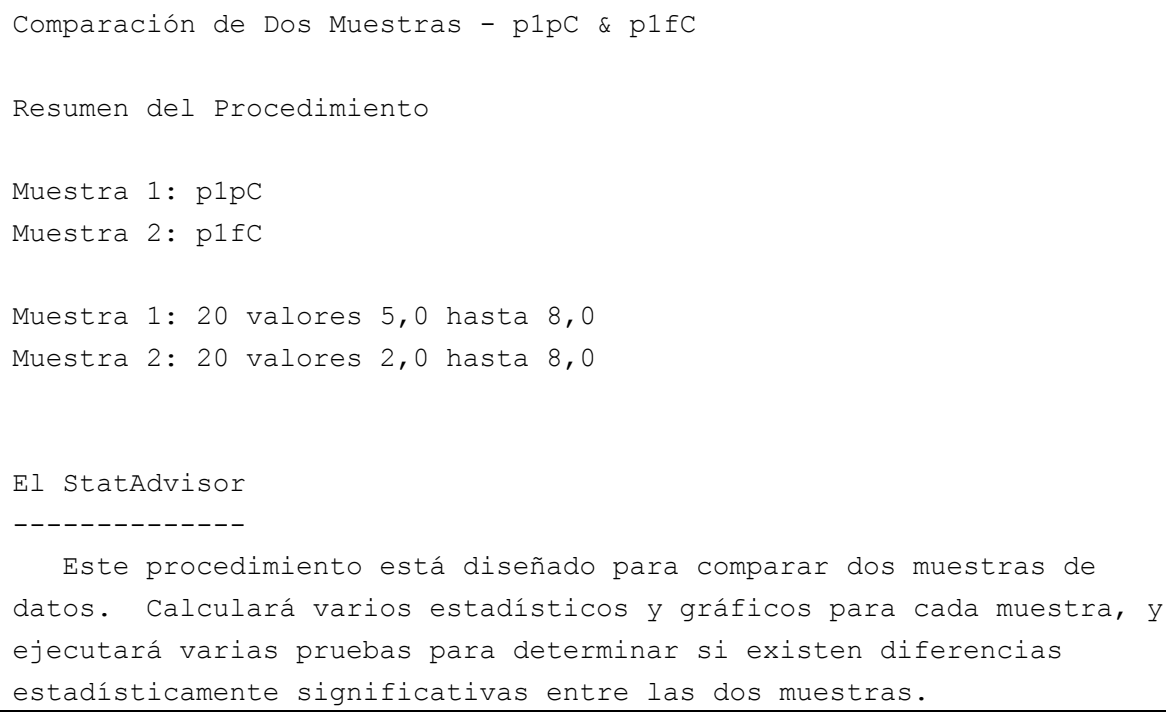

Tabla 5.26. Comparación de muestras p1pC-p1fC

$$
\mathrm{p} 1 \mathrm{pC}
$$

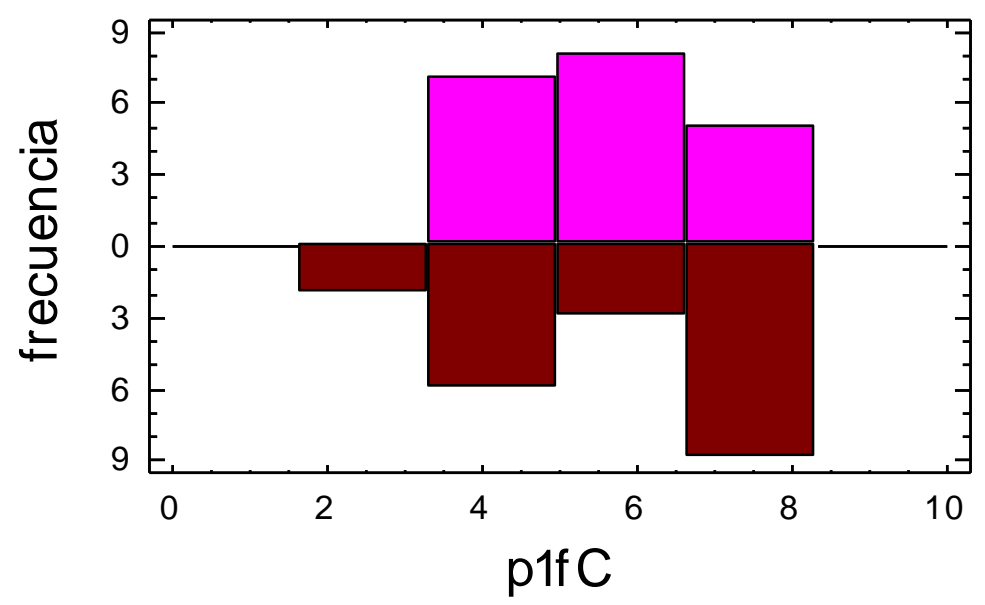

Gráfico 5.20. Comparación de histogramas p1pC-p1fC 


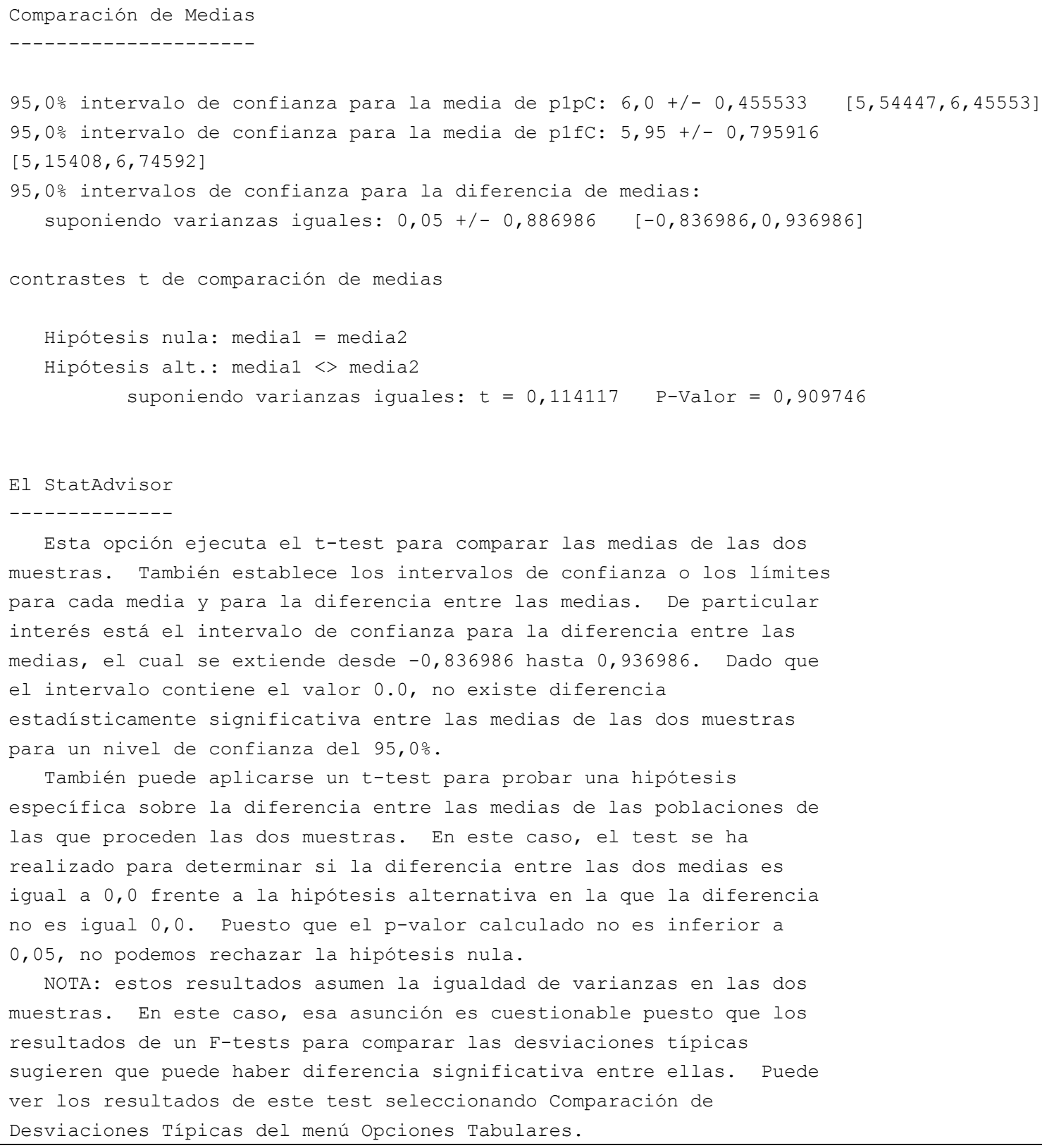

Tabla 5.27. Comparación de medias p1pC-p1fC 


\section{Density Traces}

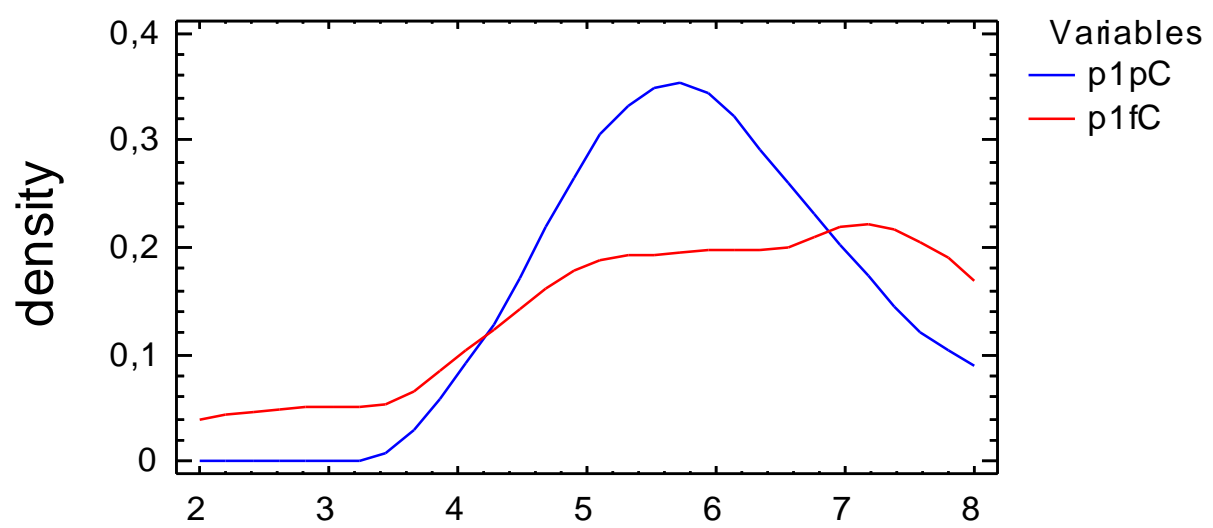

Gráfico 5.21 Comparación de densidades p1pC-p1fC

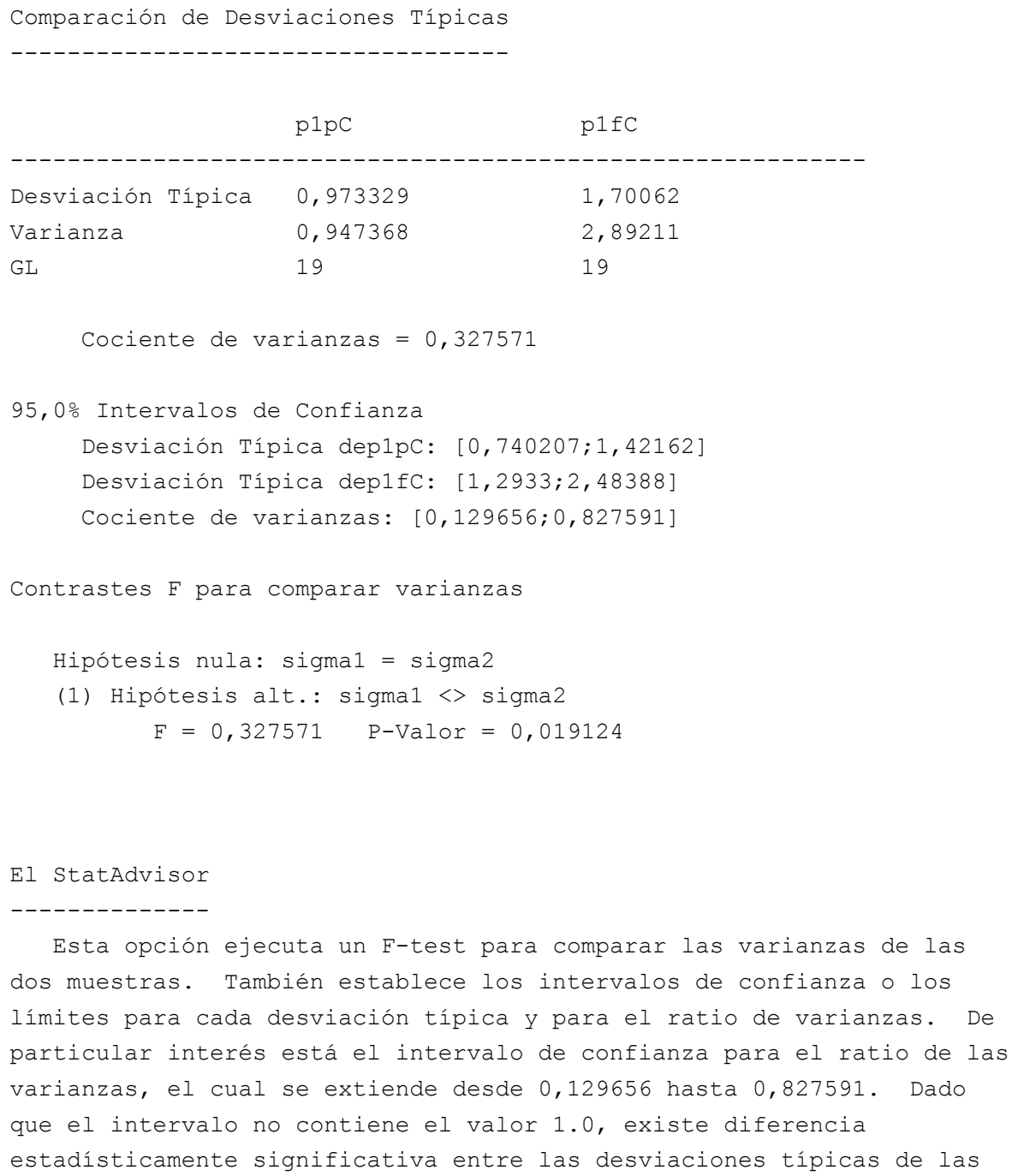


dos muestras para un nivel de confianza del 95,0\%.

También puede utilizarse un F-test para probar una hipótesis específica sobre las desviaciones típicas de las poblaciones de las que proceden las dos muestras. En este caso, el test se ha realizado para determinar si el ratio de las desviaciones típicas son iguales 1,0 frente a la hipótesis alternativa en la que el ratio no es igual 1,0. Puesto que el p-valor calculado es inferior a 0,05, podemos rechazar la hipótesis nula en favor de la alternativa.

NOTA IMPORTANTE: los F-test y los intervalos de confianza mostrados dependen de que las muestras procedan de distribuciones normales.

Para comprobar esta asunción, seleccione Resumen Estadístico de la lista de Opciones Tabulares y observe los valores de asimetría estandarizada y curtosis estandarizada.

Tabla 5.28. Comparación de desviaciones típicas p1pC-p1fC

\section{Box-and-Whisker Plot}

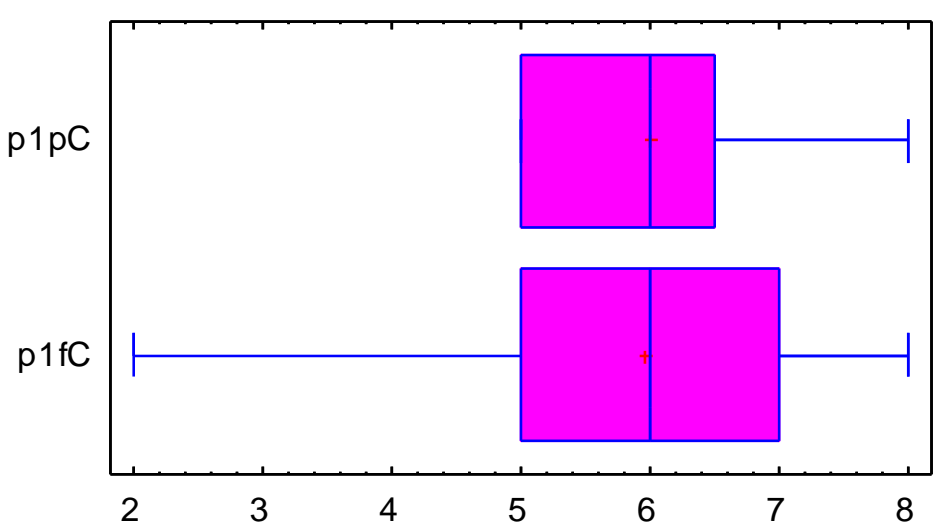

Gráfico 5.22. Comparación de diagramas de cajas y bigotes p1pC-p1fC

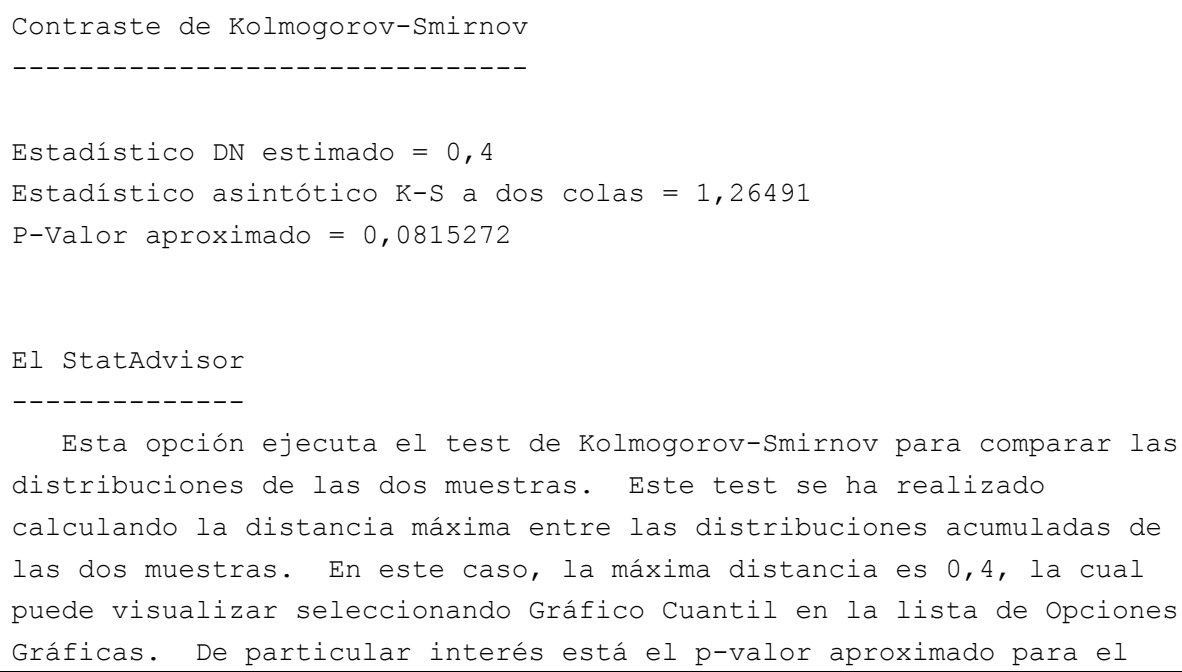


test. Dado que el p-valor es mayor o igual a 0,05, no existe

diferencia estadísticamente significativa entre las dos distribuciones

para un nivel de confianza del 95,0\%.

Tabla 5.29. Contraste de Kolmogorov-Smirnov claridad 1

\section{Quantile Plot}

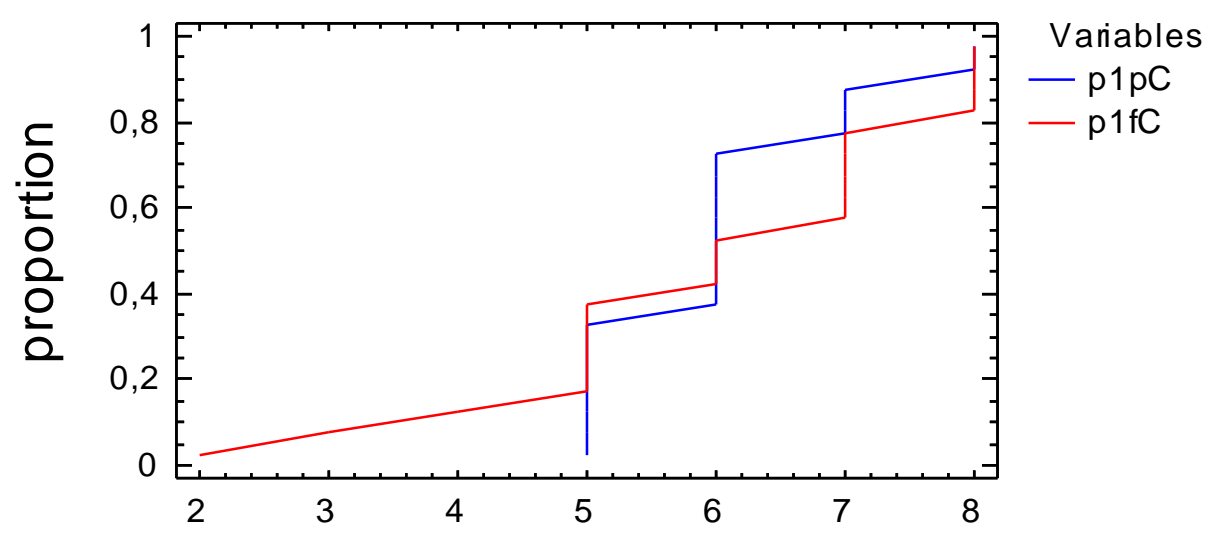

Gráfico 5.23. Representación de cuantiles p1pC-p1fC

\subsubsection{Comparaciones de los resultados de claridad (C) para la segunda demostración}

Partiendo de la normalidad comprobada con los coeficientes de asimetría y curtosis tipificados en la tabla 5.24, en la tabla 5.32 comprobamos que no se puede rechazar la igualdad de desviaciones típicas; a su vez esto nos permite realizar el contraste de igualdad de medias que aparece en la tabla 5.31, resultando que tampoco podemos rechazar la hipótesis nula, de modo que se puede admitir que las medias sean iguales entre ambas variables. El resultado del contraste de Kolmogorov-Smirnov (tabla 5.33) indica sin embargo que se puede rechazar la igualdad de las distribuciones.

Los gráficos ponen de manifiesto sólo ciertas diferencias:

21) Gráfico 5.24: comparación de histogramas, donde se observa cómo el histograma de arriba (correspondiente a la PPF) es menos continuo que 
el de abajo (correspondiente a la PF), destacando en el de arriba la alta frecuencia del último intervalo.

22) Gráfico 5.25: comparación de densidades, donde vemos cómo la distribución de la PF (en rojo) está a la izquierda de la de la PPF (en azul), lo que indicaría cierta preferencia por esta última.

23) Gráfico 5.26: con los dos diagramas de cajas y bigotes, en el que se observa cómo en el gráfico superior (que representa la PPF) a partir del primer cuartil (lado izquierdo de la caja) todo está más a la derecha que en el de abajo, lo que supondría una mayor preferencia a partir de ese punto por la PPF.

24) Gráfico 5.27: con la representación de los cuantiles, donde la distribución de la PF (en rojo) está a la izquierda siempre de la distribución de la PPF, de donde deducimos también que es preferida la PPF.

Por tanto, en este caso la claridad sí que supone una preferencia por la PPF.

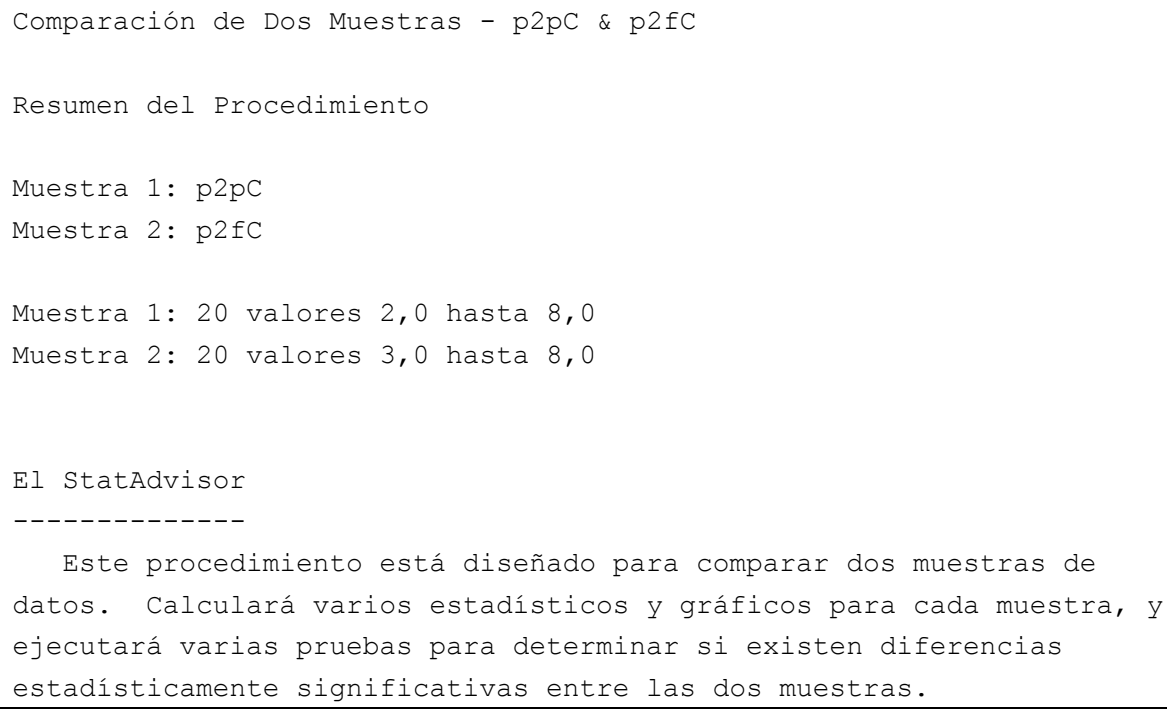

Tabla 5.30. Comparación de muestras p2pC-p2fC 


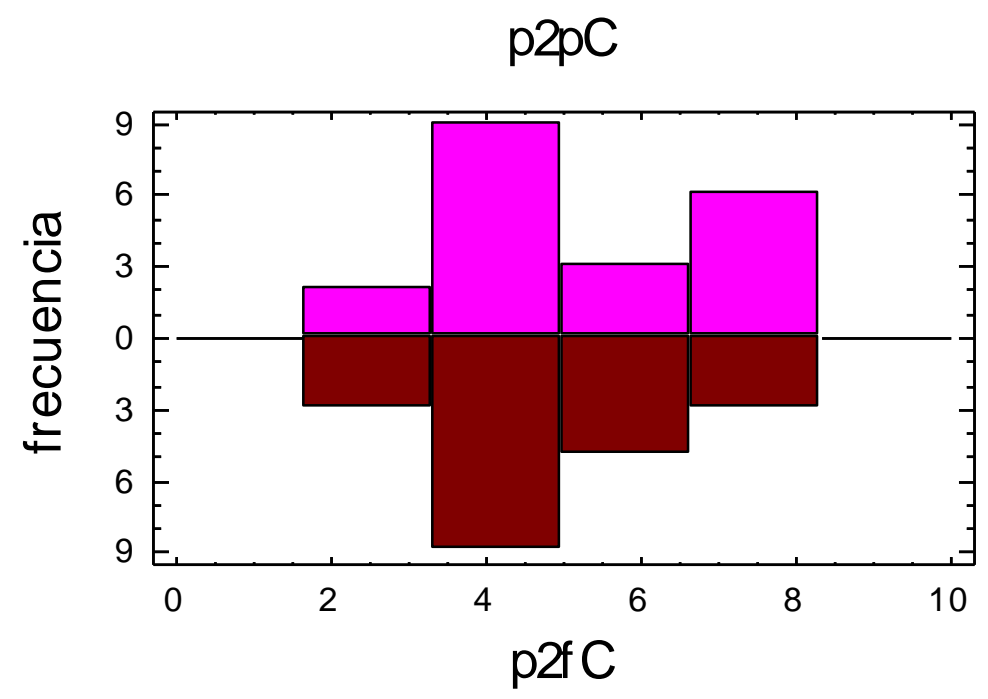

Gráfico 5.24. Comparación de histogramas p2pC-p2fC

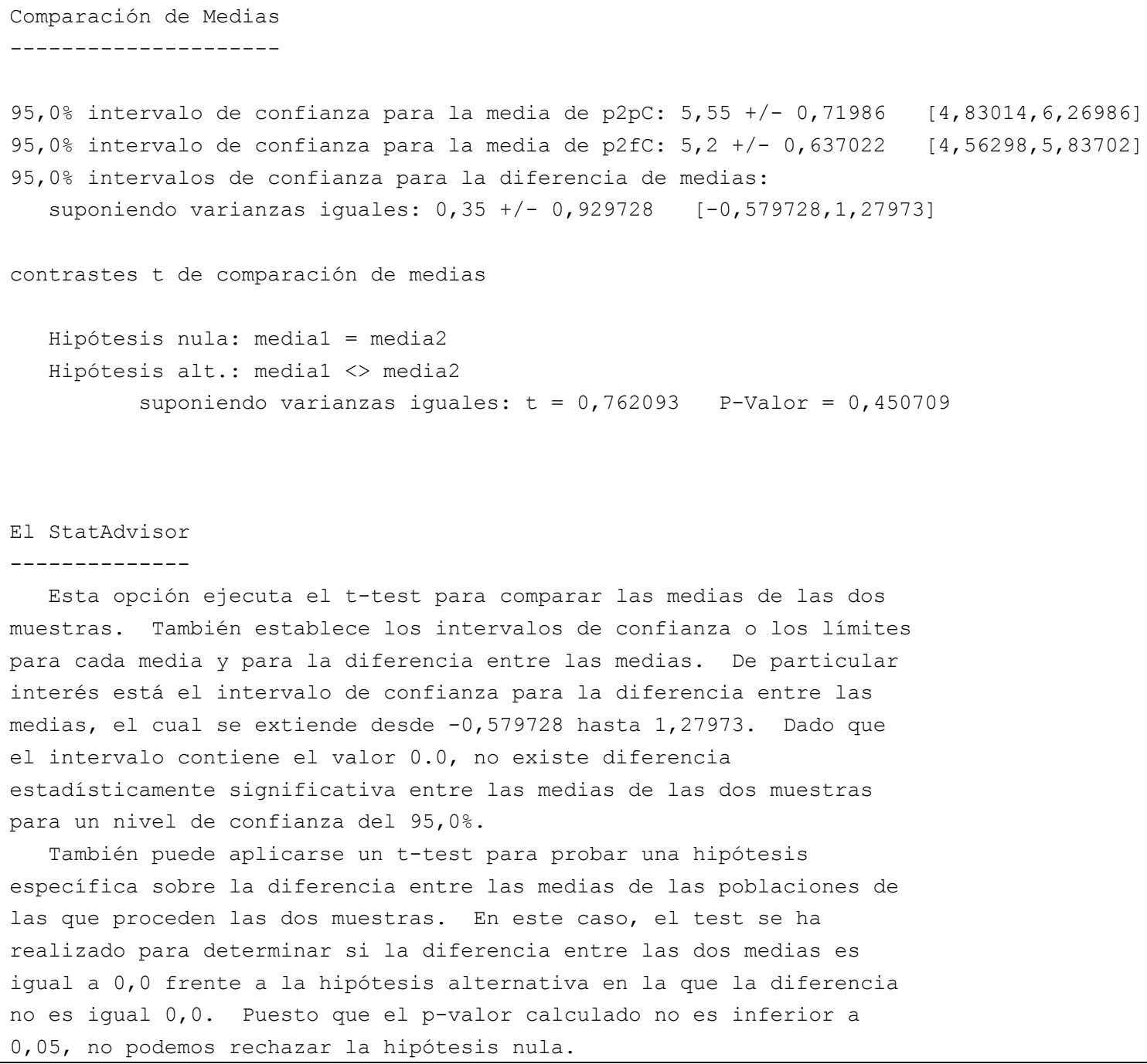


NOTA: estos resultados asumen la igualdad de varianzas en las dos

muestras. En este caso, esa asunción parece ser razonable teniendo en

cuenta los resultados del F-test para comparar las desviaciones

típicas. Puede ver los resultados de este test seleccionando

Comparación de Desviaciones Típicas del menú Opciones Tabulares.

Tabla 5.31. Comparación de medias p2pC-p2fC

Density Traces

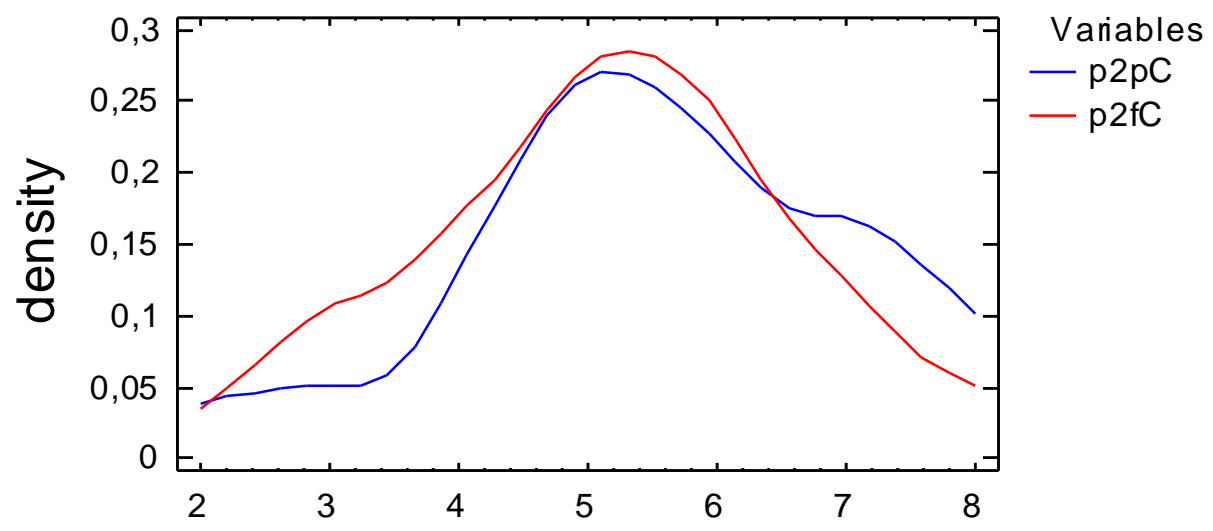

Gráfico 5.25. Comparación de densidades p2pC-p2fC

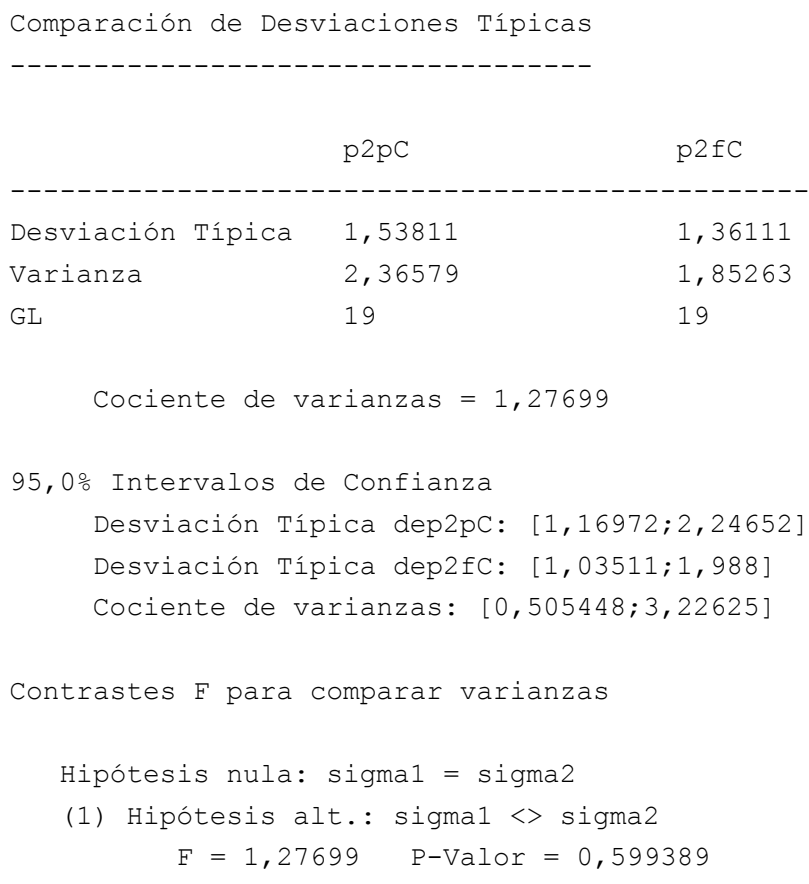


El StatAdvisor

------------

Esta opción ejecuta un F-test para comparar las varianzas de las dos muestras. También establece los intervalos de confianza o los límites para cada desviación típica y para el ratio de varianzas. De particular interés está el intervalo de confianza para el ratio de las varianzas, el cual se extiende desde 0,505448 hasta 3,22625. Dado que el intervalo contiene el valor 1.0, no existe diferencia estadísticamente significativa entre las desviaciones típicas de las dos muestras para un nivel de confianza del 95,0\%.

También puede utilizarse un F-test para probar una hipótesis específica sobre las desviaciones típicas de las poblaciones de las que proceden las dos muestras. En este caso, el test se ha realizado para determinar si el ratio de las desviaciones típicas son iguales 1,0 frente a la hipótesis alternativa en la que el ratio no es igual 1,0. Puesto que el p-valor calculado no es inferior a 0,05, no podemos rechazar la hipótesis nula.

NOTA IMPORTANTE: los F-test y los intervalos de confianza mostrados dependen de que las muestras procedan de distribuciones normales.

Para comprobar esta asunción, seleccione Resumen Estadístico de la lista de Opciones Tabulares y observe los valores de asimetría estandarizada y curtosis estandarizada.

Tabla 5.32. Comparación de desviaciones típicas p2pC-p2fC

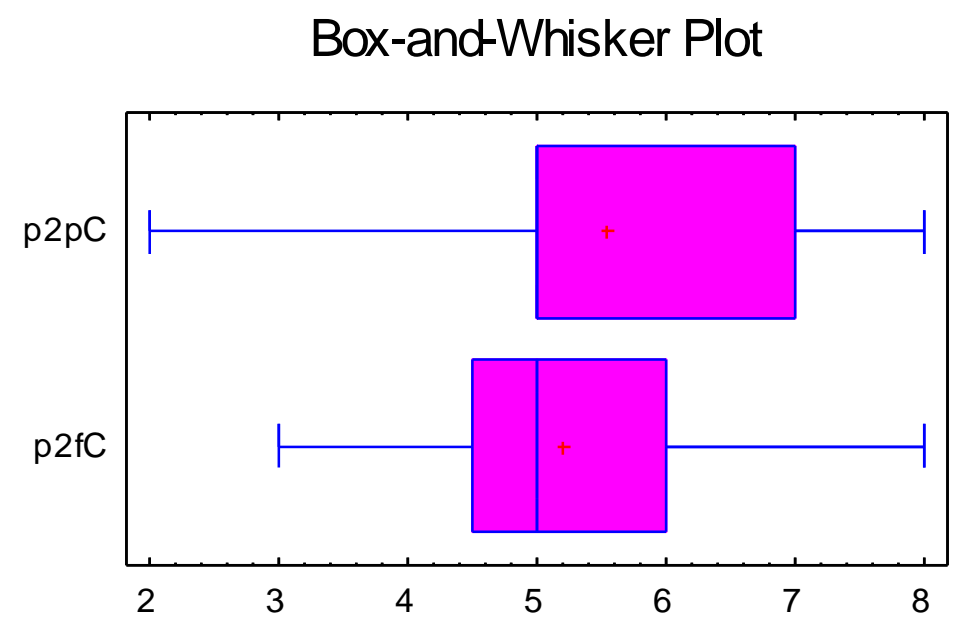

Gráfico 5.26. Comparación de diagramas de cajas y bigotes p2pC-p2fC

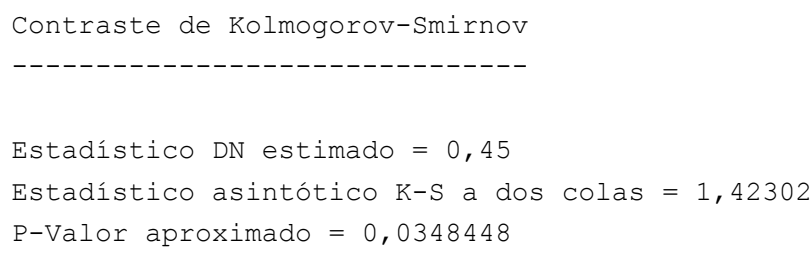




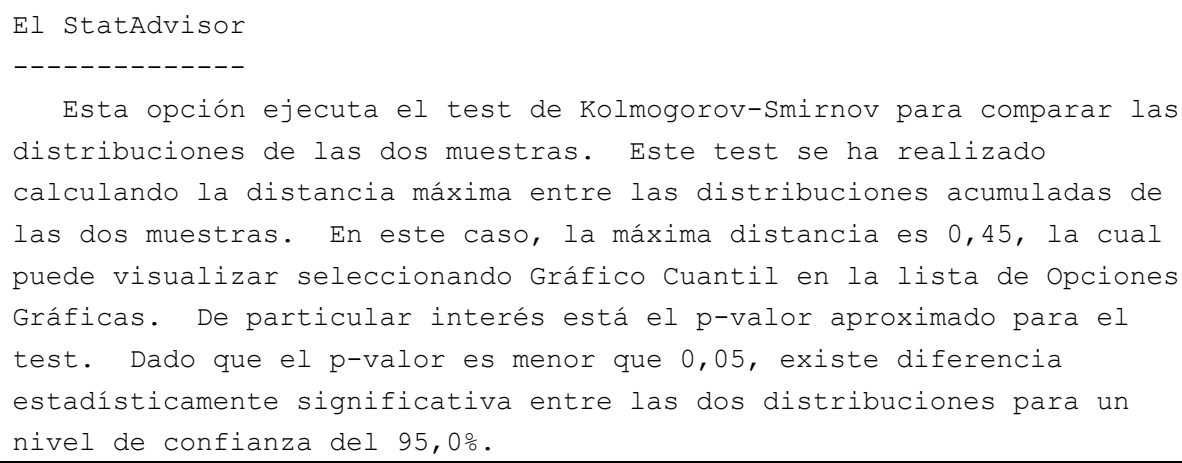

Tabla 5.33. Contraste de Kolmogorov-Smirnov, claridad 2

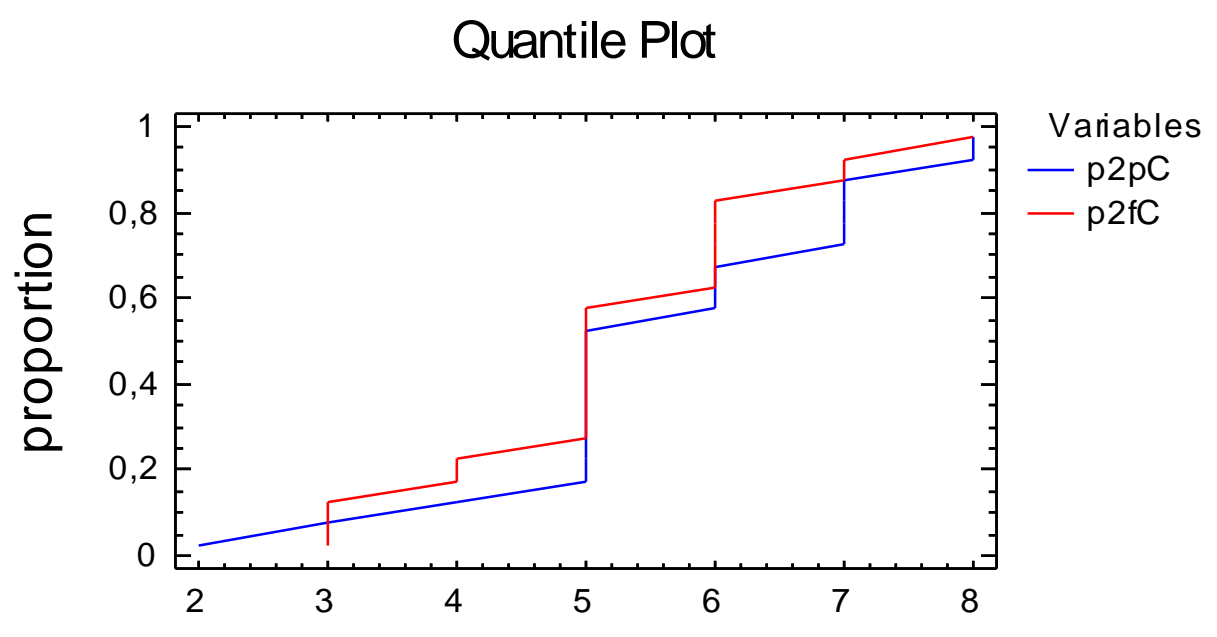

Gráfico 5.27. Representación de cuantiles p2pC-p2fC

\subsection{Comparaciones de los resultados de aprendizaje (A) de cada una de las demostraciones}

La tabla 5.34 recoge las frecuencias de cada una de las calificaciones asignadas por los alumnos en la categoría aprendizaje en cada una de las cuatro demostraciones. 


\begin{tabular}{rrrrr}
\hline & $\mathbf{p 1 p A}$ & $\mathbf{p 1 f A}$ & $\mathbf{p 2 p A}$ & $\mathbf{p 2 f A}$ \\
\hline $\mathbf{1}$ & 0 & 0 & 0 & 0 \\
$\mathbf{2}$ & 0 & 1 & 0 & 0 \\
$\mathbf{3}$ & 0 & 2 & 1 & 2 \\
$\mathbf{4}$ & 2 & 1 & 3 & 7 \\
$\mathbf{5}$ & 5 & 2 & 5 & 4 \\
$\mathbf{6}$ & 5 & 4 & 7 & 3 \\
$\mathbf{7}$ & 4 & 6 & 1 & 0 \\
$\mathbf{8}$ & 2 & 4 & 3 & 4 \\
$\mathbf{9}$ & 2 & 0 & 0 & 0 \\
$\mathbf{1 0}$ & 0 & 0 & 0 & 0 \\
\hline Total & 20 & 20 & 20 & 20 \\
\hline
\end{tabular}

Tabla 5.34. Frecuencias en la categoría aprendizaje en cada una de las pruebas y de los teoremas

Asimismo, estos datos quedan reflejados en los gráficos 5.28 y 5.28 bis siguientes.

\section{Gráfico 5.28}

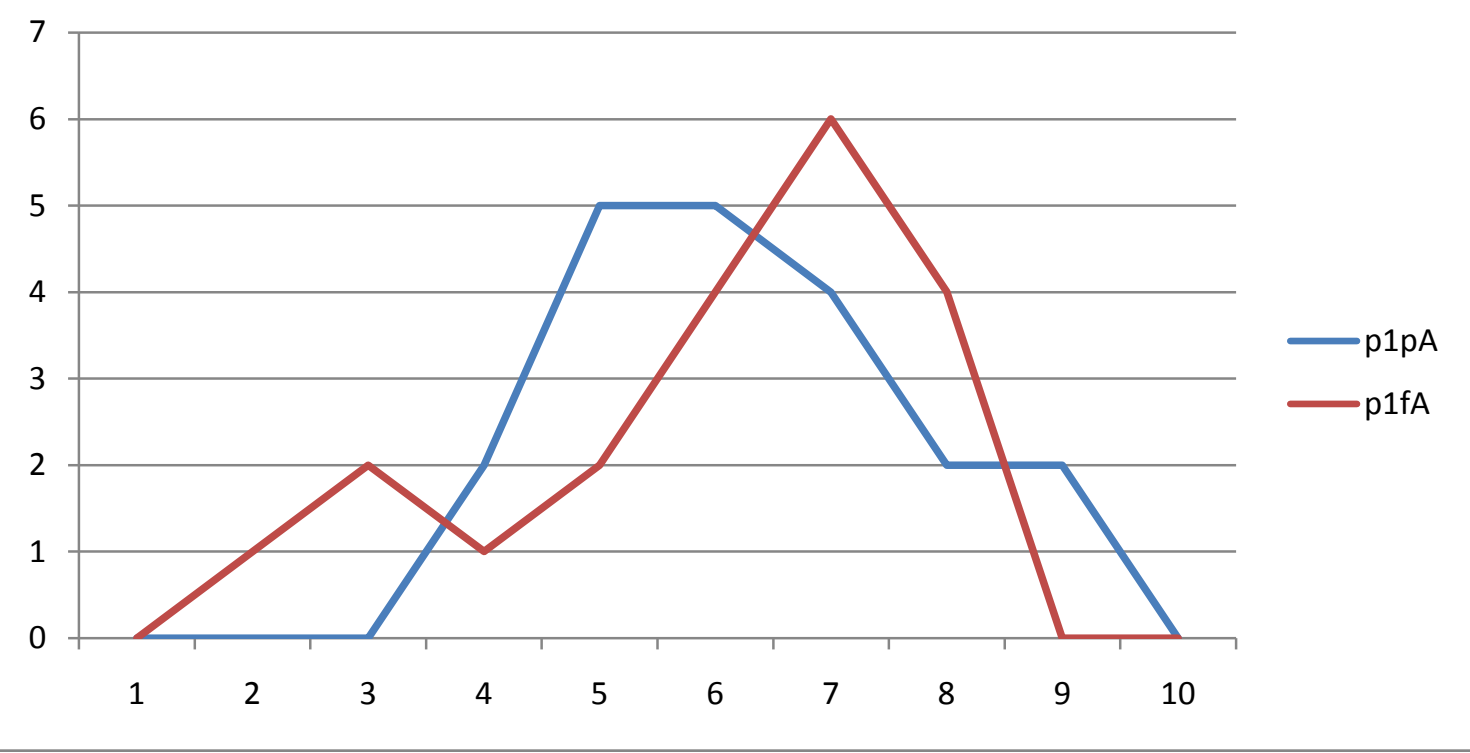




\section{Gráfico 5.28 bis}

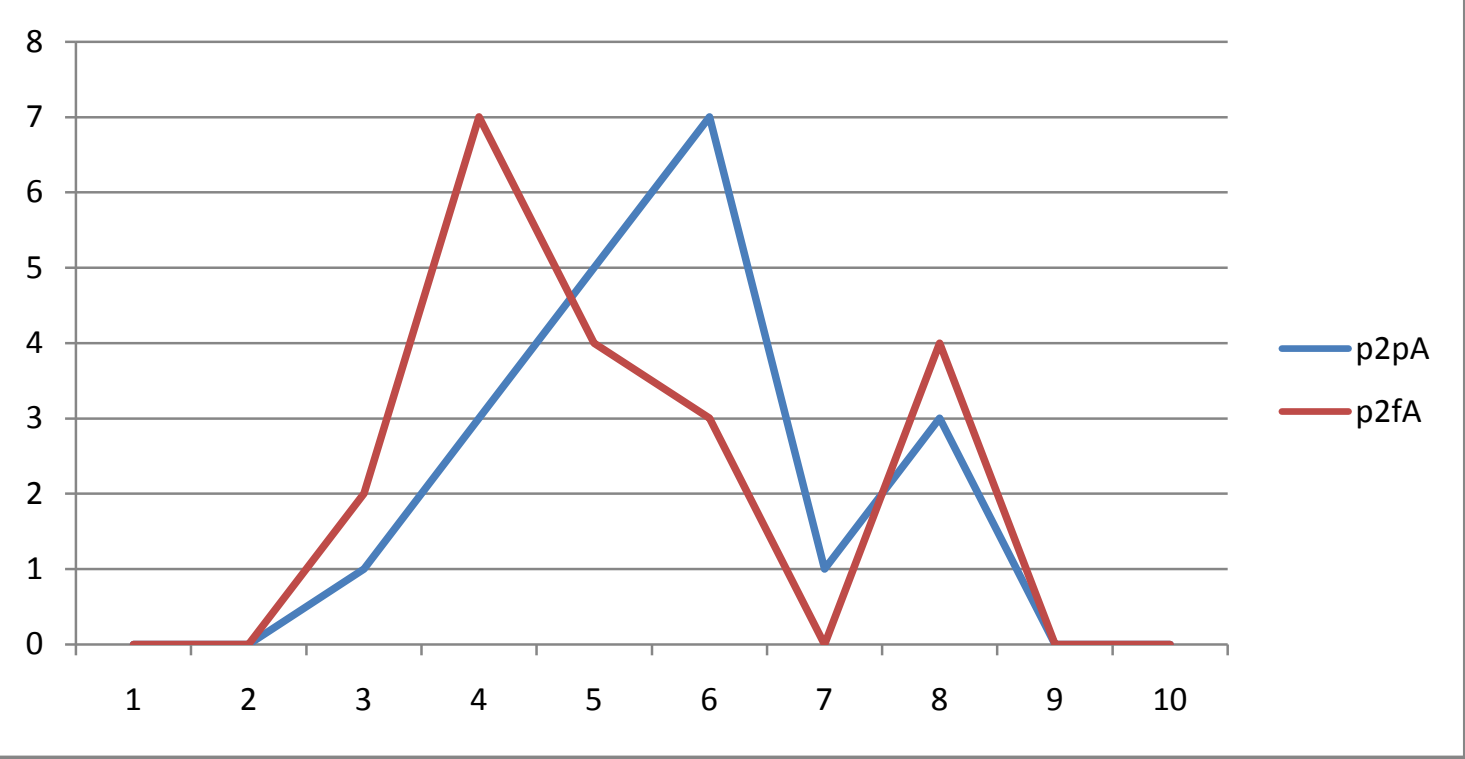

Estas distribuciones representan menos evidentemente para el caso del aprendizaje que las demostraciones PPF son mejor valoradas que las PF; para el primer teorema la distribución de la PPF (en azul) en general está más a la izquierda que la PF (en rojo), mientras que para el segundo teorema la preferencia es más clara por la PPF (en azul) respecto la PF (en rojo).

Las tablas 5.35 y 5.36 recogen las comparaciones de las características estadísticas de estas cuatro variables, que vienen a confirmarnos una preferencia por la PPF en la segunda demostración, mientras que no lo es tan evidente en el caso de la primera. Así en la tabla 5.35, observamos que las características de tendencia central para la segunda demostración son superiores en la PPF ( $3^{\text {a }}$ columna) respecto a la PF ( $4^{\text {a }}$ columna) en media, mediana y moda mientras que en la primera la media es mayor en la PPF y la mediana y la moda en la PF. Se observa sin embargo unos mayores valores en las dos demostraciones para la PPF para mínimo, máximo y cuartiles. 
Por lo que se refiere a la dispersión, tomando el coeficiente de variación se aprecia cómo va creciendo de PF a PPF en ambas demostraciones, aunque las diferencias no son demasiado amplias.

Por su parte, siguiendo en la tabla 5.35, la asimetría tipificada de las distribuciones no viene a corroborar en todos los casos la preferencia por las PPF: en el primer caso la PF es asimétrica negativa frente a una PPF asimétrica positiva, o sea, en la PPF la mayoría de las calificaciones se agrupan al principio de la distribución y hay menos calificaciones altas, lo que nos indica por tanto una preferencia por la PF; sin embargo, en el segundo caso las dos pruebas son asimétricas positivas, aunque lo es mucho más la PF, o sea, en la PF se nota más que la mayoría de las calificaciones se agrupan al principio de la distribución y hay menos calificaciones altas, lo que nos indica por tanto una preferencia por la PPF.

Además, el hecho de que la asimetría tipificada y la curtosis tipificada estén entre -2 y 2 va a permitir tratar estas variables como que siguen distribuciones normales, lo que justificará la utilización de los contrastes de igualdad de varianzas y de igualdad de medias que se verán en los dos apartados siguientes.

Las correlaciones de la tabla 5.36 nos indican la escasa asociación que hay entre todos los pares de calificaciones (los valores de estas correlaciones, por lo general, están más bien próximos a cero, lo que indica una escasa relación entre el par de variables correspondiente). El p-valor corrobora la inexistencia de relaciones significativas. Esto es, en todos los casos podemos decir que no hay relación entre las puntuaciones respecto del aprendizaje dadas por los alumnos en las distintas demostraciones. 
Análisis Multivariable

Resumen Estadístico

$\begin{array}{lllll} & \mathrm{p} 1 \mathrm{pA} & \mathrm{p} 1 \mathrm{fA} & \mathrm{p} 2 \mathrm{pA} & \mathrm{p} 2 \mathrm{fA} \\ \text { Frecuencia } & 20 & 20 & 20 & 20 \\ \text { Media } & 6,25 & 6,0 & 5,65 & 5,2 \\ \text { Mediana } & 6,0 & 6,5 & 6,0 & 5,0 \\ \text { Moda } & & 7,0 & 6,0 & 4,0 \\ \text { Varianza } & 2,19737 & 3,26316 & 1,92368 & 2,8 \\ \text { Desviación típica } & 1,48235 & 1,80642 & 1,38697 & 1,67332 \\ \text { Mínimo } & 4,0 & 2,0 & 3,0 & 3,0 \\ \text { Máximo } & 9,0 & 8,0 & 8,0 & 8,0 \\ \text { Rango } & 5,0 & 6,0 & 5,0 & 5,0 \\ \text { Primer cuartil } & 5,0 & 5,0 & 5,0 & 4,0 \\ \text { Segundo cuartil } & 7,0 & 7,0 & 6,0 & 6,0 \\ \text { Asimetría tipi. } & 0,70064 & -1,63015 & 0,329539 & 1,2743 \\ \text { Curtosis típificada } & -0,528114 & -0,0971431 & -0,248352 & -0,63623 \\ \text { Coef. de variación } & 23,7176 \% & 30,107 \% & 24,5481 \% & 32,1792 \% \\ \text { M. } & & \end{array}$

\section{El StatAdvisor}

Esta tabla muestra resúmenes estadísticos para cada una de las variables seleccionadas. Incluye medidas de tendencia central, de variabilidad y de forma. De particular interés están la asimetría estandarizada y la curtosis estandarizada, las cuales pueden utilizarse para determinar si la muestra procede de una distribución normal. Valores de estos estadísticos fuera del rango de -2 a +2 indican una desviación significativa de la normalidad, que tendería a invalidar muchos de los procedimientos estadísticos aplicados habitualmente a estos datos. En este caso, las siguientes variables muestran valores de asimetría estandarizada fuera del rango esperado:

$<$ ninguna $>$

Las siguientes variables muestran valores de curtosis estandarizada fuera del rango esperado:

$<$ ninguna $>$

Tabla 5.35. Comparaciones de las características estadísticas de ambas pruebas en la categoría aprendizaje

\begin{tabular}{|c|c|c|c|c|}
\hline \multicolumn{5}{|c|}{ Correlaciones } \\
\hline & $\mathrm{p} 1 \mathrm{pA}$ & $\mathrm{p} 1 \mathrm{fA}$ & $\mathrm{p} 2 \mathrm{pA}$ & $\mathrm{p} 2 \mathrm{fA}$ \\
\hline \multirow[t]{3}{*}{$\mathrm{p} 1 \mathrm{pA}$} & & 0,2555 & $-0,2112$ & 0,4244 \\
\hline & & $(\quad 20)$ & $(20)$ & $(20)$ \\
\hline & & 0,2769 & 0,3714 & 0,0622 \\
\hline \multirow[t]{3}{*}{$\mathrm{p} 1 \mathrm{fA}$} & 0,2555 & & $-0,0840$ & 0,0871 \\
\hline & $(20)$ & & $(20)$ & $(20)$ \\
\hline & 0,2769 & & 0,7247 & 0,7151 \\
\hline
\end{tabular}




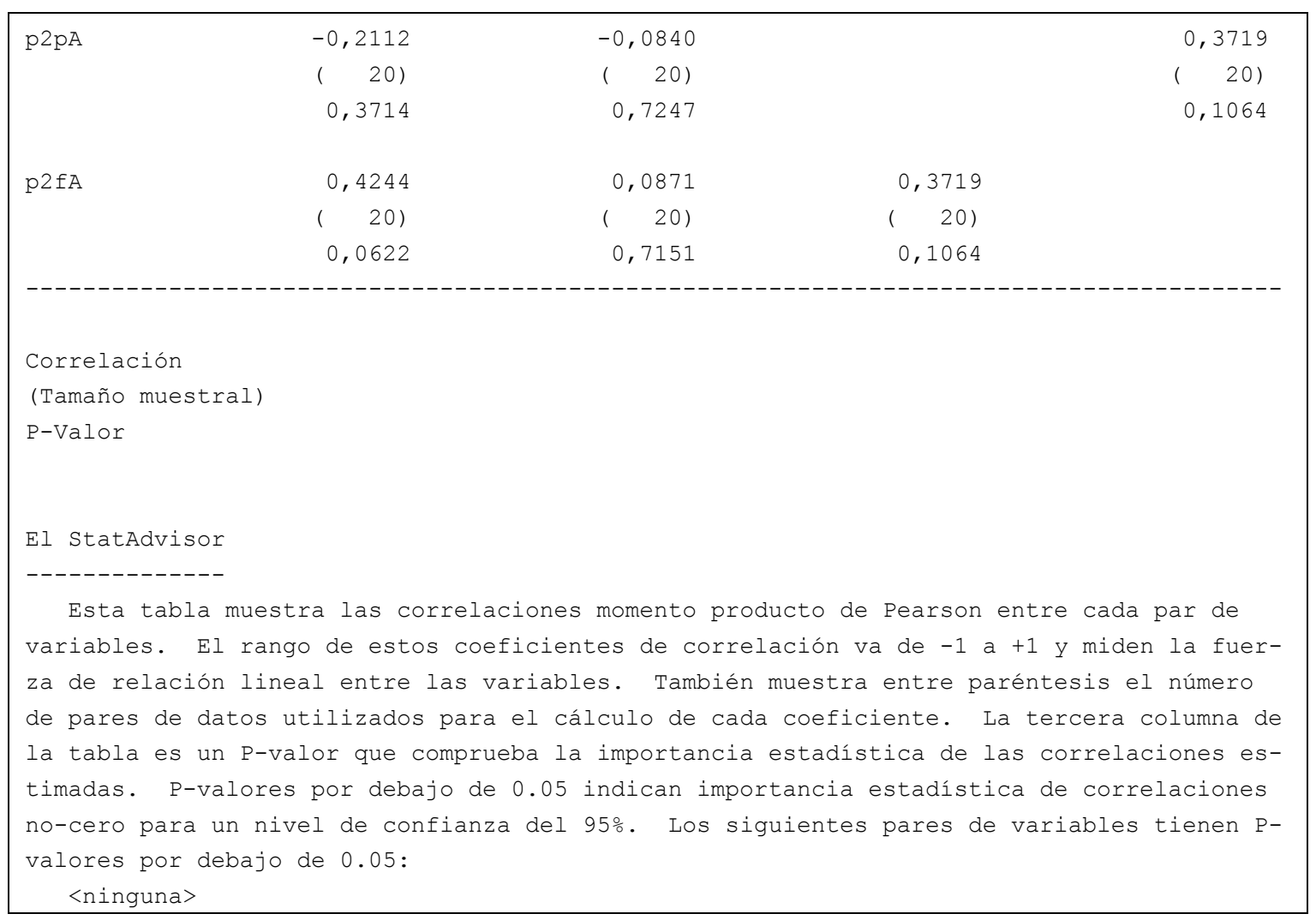

Tabla 5.36. Correlaciones de ambas pruebas en la categoría aprendizaje

\subsubsection{Comparaciones de los resultados de aprendizaje (A) para la primera demostración}

Partiendo de la normalidad comprobada con los coeficientes de asimetría y curtosis tipificados en la tabla 5.35, en la tabla 5.39 comprobamos que no se puede rechazar la igualdad de desviaciones típicas; a su vez esto nos permite realizar el contraste de igualdad de medias que aparece en la tabla 5.38, resultando que tampoco podemos rechazar la hipótesis nula, de modo que se puede admitir que las medias sean iguales entre ambas variables. El resultado del contraste de Kolmogorov-Smirnov (tabla 5.40) indica en el mismo sentido, ya que tampoco se puede rechazar la igualdad de las distribuciones.

Los gráficos ponen de manifiesto sólo ciertas diferencias:

25) Gráfico 5.29: comparación de histogramas, donde se observa cómo el histograma de arriba (correspondiente a la PPF) presenta por lo gene- 
ral mayores valores que el de abajo (correspondiente a la PF) que sólo destaca en ese último intervalo su alta frecuencia.

26) Gráfico 5.30: comparación de densidades, con una distribución algo más a la derecha para la PF en los valores más altos de la variable, aunque se cruzan por varias veces.

27) Gráfico 5.31: con los dos diagramas de cajas y bigotes, en el que se observa cómo las cajas de ambos gráficos están en los mismos valores, variando sólo los bigotes, que representan mayores valores en la PPF y menores en la PF.

28) Gráfico 5.32: con la representación de los cuantiles, donde la distribución de la PF se cruza con la PPF, con lo que no podríamos afirmar nada sobre cuál es preferida.

Por tanto no podemos decir que en este caso el aprendizaje suponga una diferencia sustancial entre ambas demostraciones.

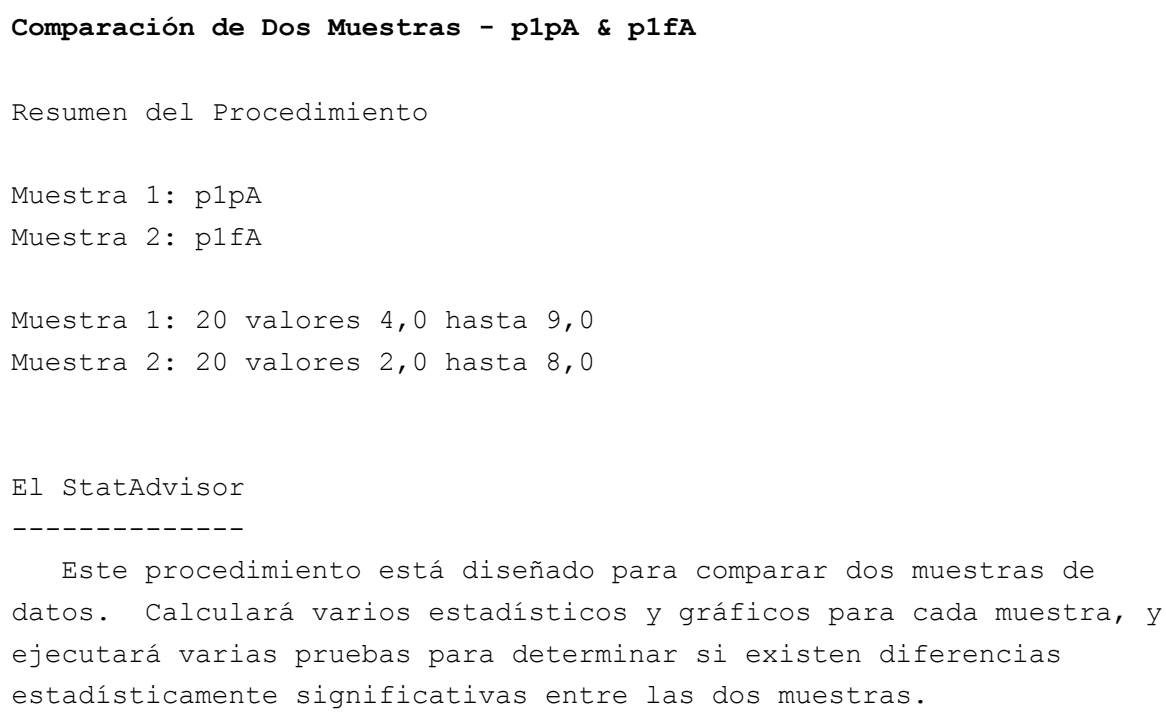

Tabla 5.37. Comparación de muestras p1pA-p1fA 


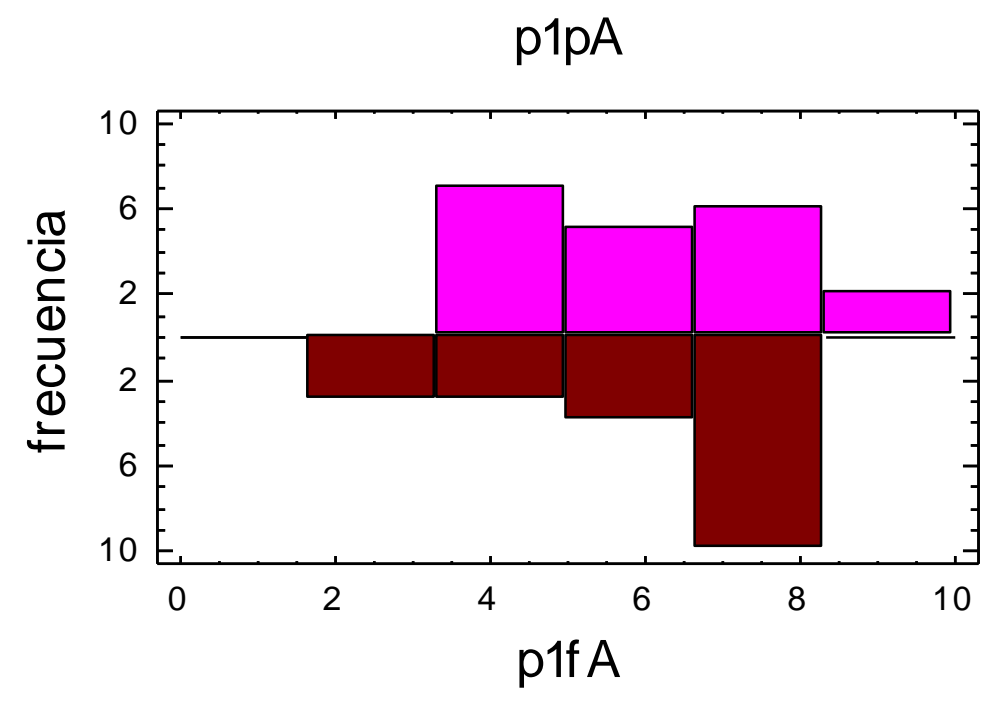

Gráfico 5.29. Comparación de histogramas p1pA-p1fA

Comparación de Medias

95, 0\% intervalo de confianza para la media de p1pA: 6,25 +/- 0,693764

$[5,55624,6,94376]$

95,0\% intervalo de confianza para la media de p1fA: 6,0 +/- 0,845433 [5, 15457,6,84543]

95, 0\% intervalos de confianza para la diferencia de medias:

suponiendo varianzas iguales: $0,25+/-1,05779 \quad[-0,807787,1,30779]$

contrastes t de comparación de medias

Hipótesis nula: medial = media2

Hipótesis alt.: medial <> media2

suponiendo varianzas iguales: $t=0,478451 \quad$ P-Valor $=0,63507$

El StatAdvisor

Esta opción ejecuta el t-test para comparar las medias de las dos muestras. También establece los intervalos de confianza o los límites para cada media y para la diferencia entre las medias. De particular interés está el intervalo de confianza para la diferencia entre las medias, el cual se extiende desde $-0,807787$ hasta 1,30779. Dado que el intervalo contiene el valor 0.0 , no existe diferencia estadísticamente significativa entre las medias de las dos muestras para un nivel de confianza del 95,0\%.

También puede aplicarse un t-test para probar una hipótesis específica sobre la diferencia entre las medias de las poblaciones de las que proceden las dos muestras. En este caso, el test se ha realizado para determinar si la diferencia entre las dos medias es igual a 0,0 frente a la hipótesis alternativa en la que la diferencia no es igual 0,0. Puesto que el p-valor calculado no es inferior a 0,05, no podemos rechazar la hipótesis nula. 
NOTA: estos resultados asumen la igualdad de varianzas en las dos

muestras. En este caso, esa asunción parece ser razonable teniendo en

cuenta los resultados del F-test para comparar las desviaciones

típicas. Puede ver los resultados de este test seleccionando

Comparación de Desviaciones Típicas del menú Opciones Tabulares.

Tabla 5.38. Comparación de medias p1pA-p1fA

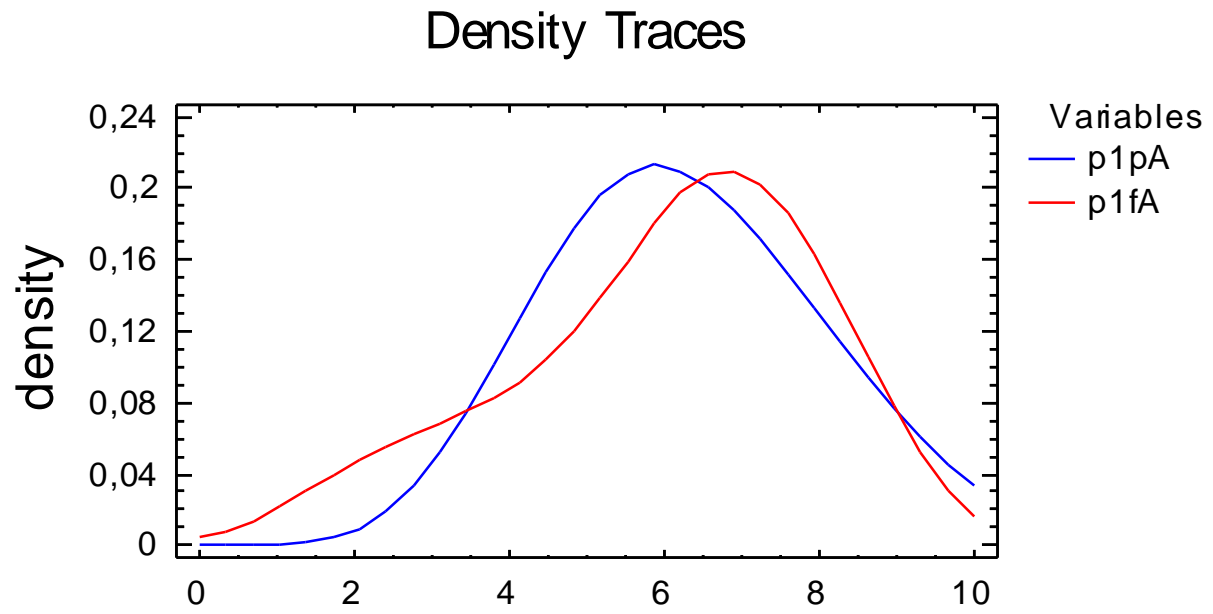

Gráfico 5.30. Comparación de densidades p1pA-p1fA

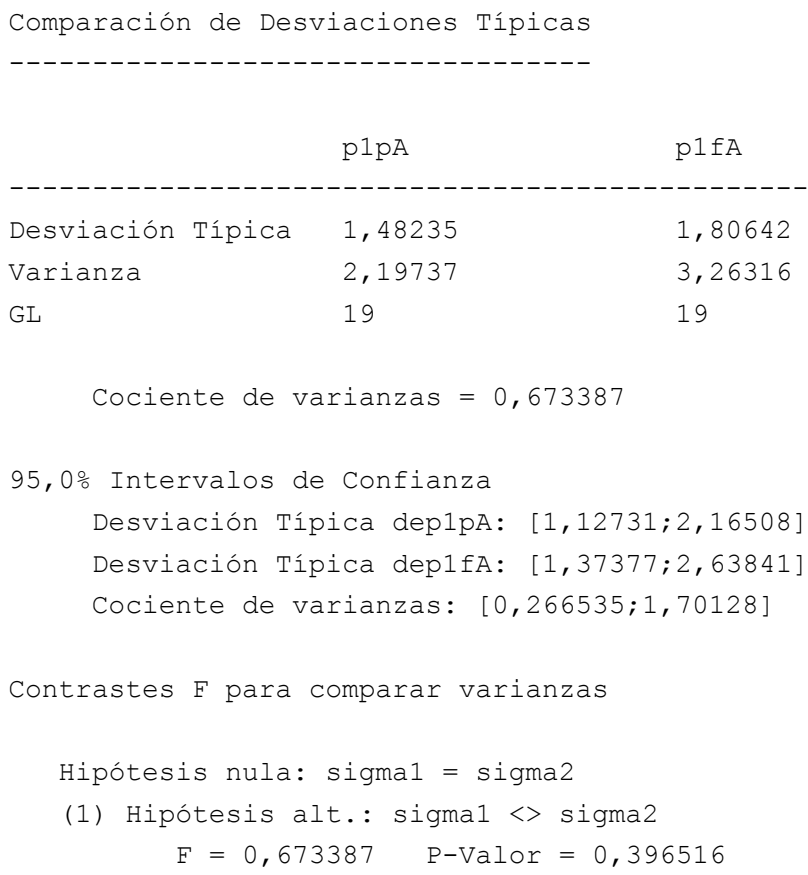


El StatAdvisor

-------------

Esta opción ejecuta un F-test para comparar las varianzas de las dos muestras. También establece los intervalos de confianza o los límites para cada desviación típica y para el ratio de varianzas. De particular interés está el intervalo de confianza para el ratio de las varianzas, el cual se extiende desde 0,266535 hasta 1,70128. Dado que el intervalo contiene el valor 1.0, no existe diferencia estadísticamente significativa entre las desviaciones típicas de las dos muestras para un nivel de confianza del 95,0\%.

También puede utilizarse un F-test para probar una hipótesis específica sobre las desviaciones típicas de las poblaciones de las que proceden las dos muestras. En este caso, el test se ha realizado para determinar si el ratio de las desviaciones típicas son iguales 1,0 frente a la hipótesis alternativa en la que el ratio no es igual 1,0. Puesto que el p-valor calculado no es inferior a 0,05, no podemos rechazar la hipótesis nula.

NOTA IMPORTANTE: los F-test y los intervalos de confianza mostrados dependen de que las muestras procedan de distribuciones normales.

Para comprobar esta asunción, seleccione Resumen Estadístico de la lista de Opciones Tabulares y observe los valores de asimetría estandarizada y curtosis estandarizada.

Tabla 5.39. Comparación de desviaciones típicas p1pA-p1fA

Box-and-Whisker Plot

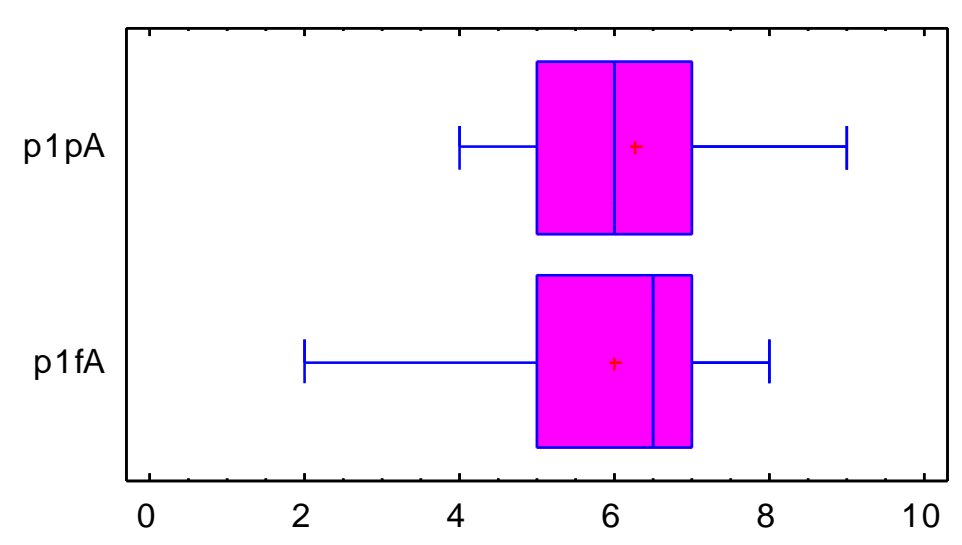

Gráfico 5.31. Comparación de diagramas de cajas y bigotes p1pA-p1fA

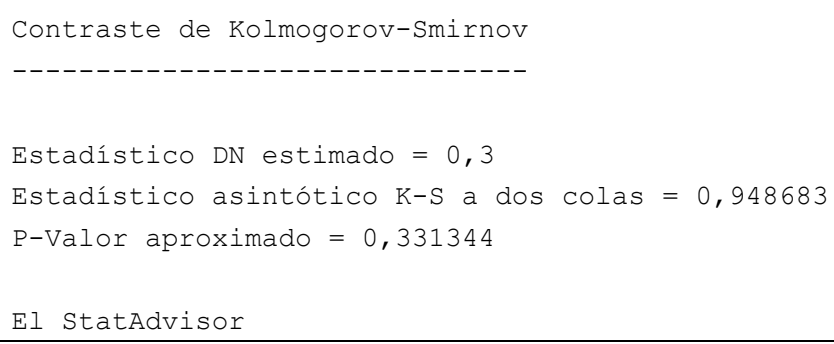


Esta opción ejecuta el test de Kolmogorov-Smirnov para comparar las distribuciones de las dos muestras. Este test se ha realizado

calculando la distancia máxima entre las distribuciones acumuladas de

las dos muestras. En este caso, la máxima distancia es 0,3, la cual

puede visualizar seleccionando Gráfico Cuantil en la lista de Opciones

Gráficas. De particular interés está el p-valor aproximado para el

test. Dado que el p-valor es mayor o igual a 0,05, no existe

diferencia estadísticamente significativa entre las dos distribuciones

para un nivel de confianza del 95,0\%.

Tabla 5.40. Contraste de Kolmogorov-Smirnov p1pA-p1fA

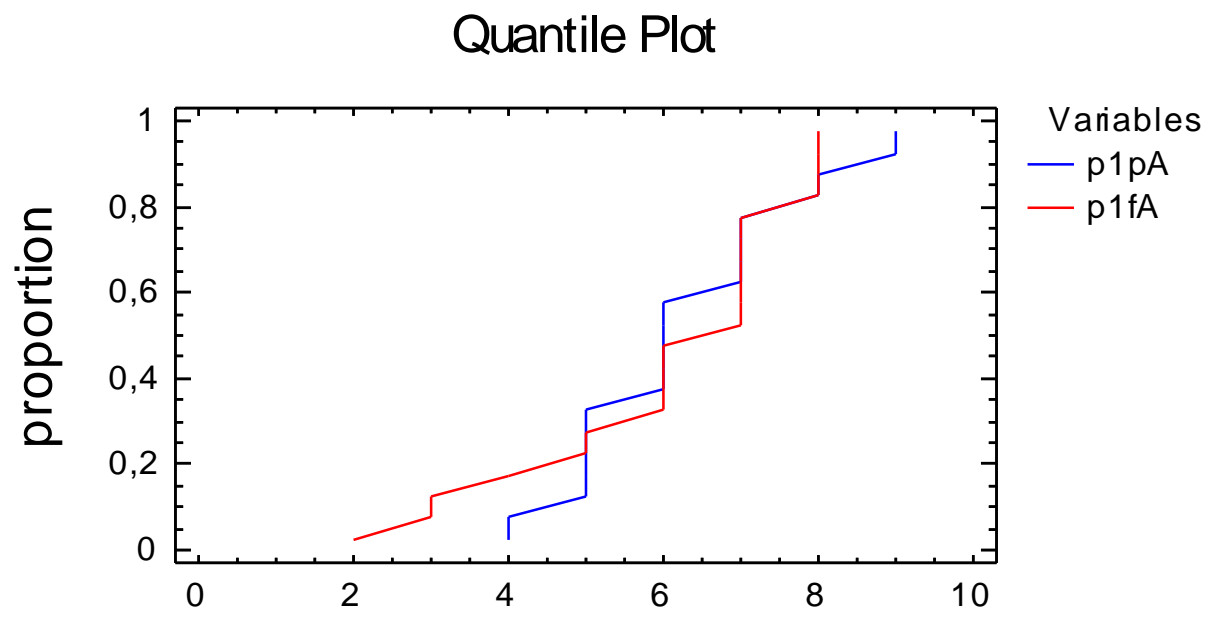

Gráfico 5.32. Representación de cuantiles p1pA-p1fA

\subsubsection{Comparaciones de los resultados de aprendizaje (A) para la segunda demostración}

Partiendo de la normalidad comprobada con los coeficientes de asimetría y curtosis tipificados en la tabla 5.35, en la tabla 5.43 comprobamos que no se puede rechazar la igualdad de desviaciones típicas; a su vez esto nos permite realizar el contraste de igualdad de medias que aparece en la tabla 5.42, resultando que tampoco podemos rechazar la hipótesis nula, de modo que se puede admitir que las medias sean iguales entre ambas variables. Sin embargo, el resultado del contraste de Kolmogorov-Smirnov (tabla 5.44) indica que se puede rechazar la igualdad de las distribuciones. 
Los gráficos ponen de manifiesto sólo ciertas diferencias:

29) Gráfico 5.33: comparación de histogramas, donde se observa cómo el histograma de arriba (correspondiente a la PPF) presenta mayores frecuencias para los valores más altos que el de abajo (correspondiente a la PF).

30) Gráfico 5.34: comparación de densidades, con una distribución de la PF claramente a la izquierda de la distribución de la PPF.

31) Gráfico 5.35: con los dos diagramas de cajas y bigotes, en el que se observa cómo todo en el gráfico superior (que representa a la PPF) está más concentrado que el inferior (caja y bigotes más reducidos) y que existen valores atípicos en el de arriba.

32) Gráfico 5.36: con la representación de los cuantiles, donde la distribución de la PPF está a la derecha de la distribución de la PF, salvo al final que se cruzan.

Por tanto en este caso el aprendizaje presenta una clara diferencia a favor de la PPF.

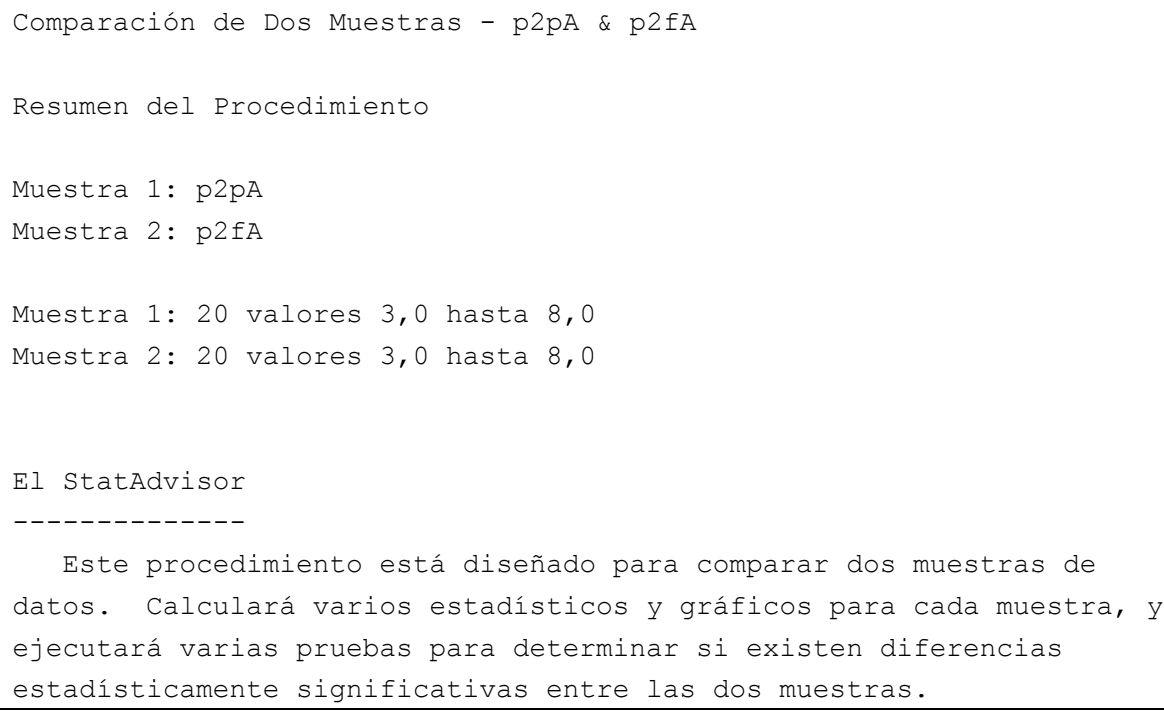

Tabla 5.41. Comparación de muestras p2pA-p2fA 


\section{p2pA}

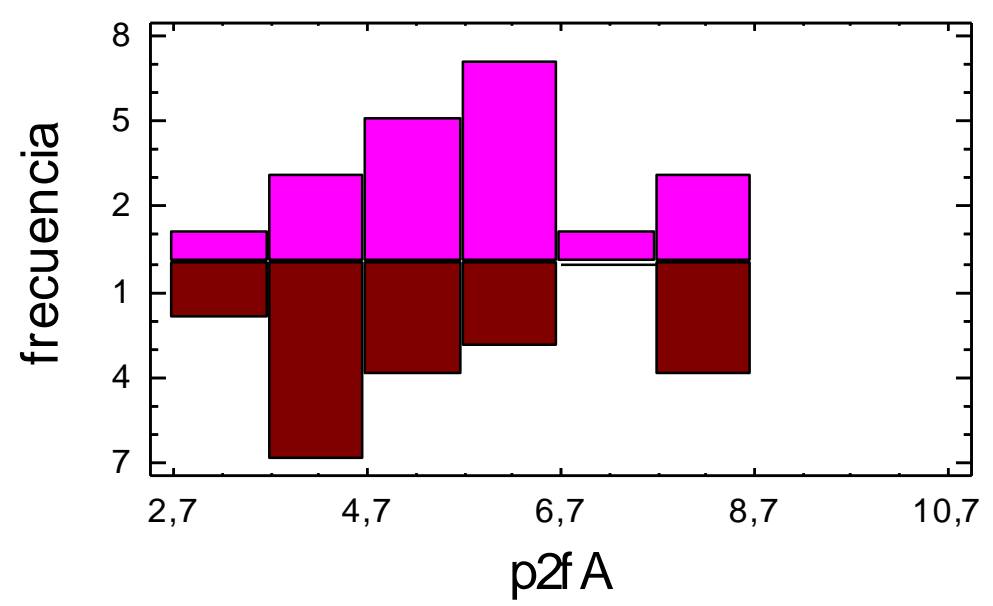

Gráfico 5.33. Comparación de histogramas p2pA-p2fA

Comparación de Medias

95, 0\% intervalo de confianza para la media de p2pA: 5,65 +/- 0,649123

$[5,00088,6,29912]$

95,0\% intervalo de confianza para la media de p2fA: 5,2 +/- 0,78314 [4,41686,5,98314]

95,0\% intervalos de confianza para la diferencia de medias:

suponiendo varianzas iguales: $0,45+/-0,983833 \quad[-0,533833,1,43383]$

contrastes $t$ de comparación de medias

Hipótesis nula: medial = media2

Hipótesis alt.: medial $<>$ media2

suponiendo varianzas iguales: $t=0,925949 \quad$ P-Valor $=0,360316$

El StatAdvisor

Esta opción ejecuta el t-test para comparar las medias de las dos muestras. También establece los intervalos de confianza o los límites para cada media y para la diferencia entre las medias. De particular interés está el intervalo de confianza para la diferencia entre las medias, el cual se extiende desde -0,533833 hasta 1,43383. Dado que el intervalo contiene el valor 0.0, no existe diferencia estadísticamente significativa entre las medias de las dos muestras para un nivel de confianza del 95,0\%.

También puede aplicarse un t-test para probar una hipótesis específica sobre la diferencia entre las medias de las poblaciones de las que proceden las dos muestras. En este caso, el test se ha realizado para determinar si la diferencia entre las dos medias es igual a 0,0 frente a la hipótesis alternativa en la que la diferencia no es igual 0,0. Puesto que el p-valor calculado no es inferior a 0,05, no podemos rechazar la hipótesis nula. 
NOTA: estos resultados asumen la igualdad de varianzas en las dos

muestras. En este caso, esa asunción parece ser razonable teniendo en

cuenta los resultados del F-test para comparar las desviaciones

típicas. Puede ver los resultados de este test seleccionando

Comparación de Desviaciones Típicas del menú Opciones Tabulares.

Tabla 5.42. Comparación de medias p2pA-p2fA

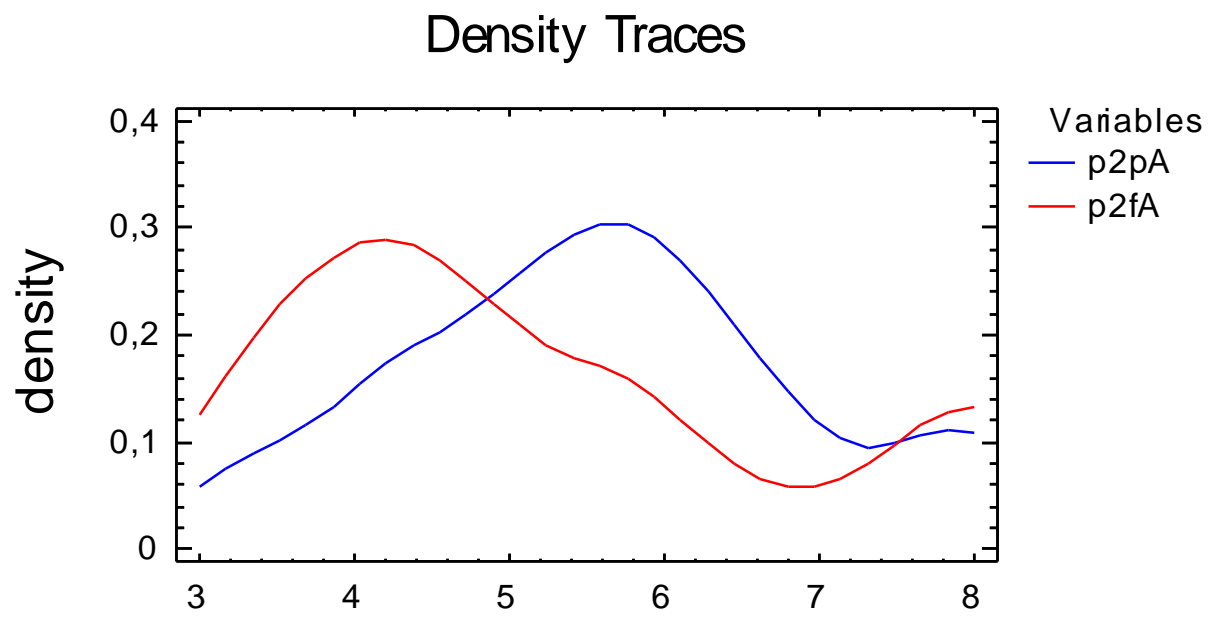

Gráfico 5.34. Comparación de densidades p2pA-p2fA

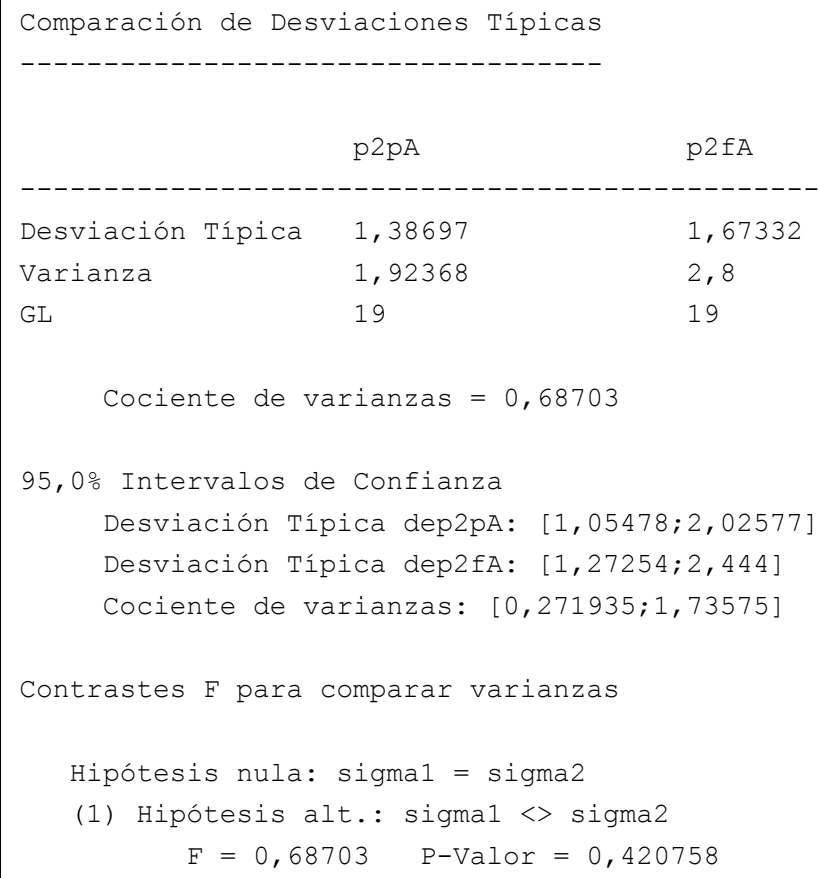


El StatAdvisor

-------------

Esta opción ejecuta un F-test para comparar las varianzas de las dos muestras. También establece los intervalos de confianza o los límites para cada desviación típica y para el ratio de varianzas. De particular interés está el intervalo de confianza para el ratio de las varianzas, el cual se extiende desde 0,271935 hasta 1,73575. Dado que el intervalo contiene el valor 1.0, no existe diferencia estadísticamente significativa entre las desviaciones típicas de las dos muestras para un nivel de confianza del 95,0\%.

También puede utilizarse un F-test para probar una hipótesis específica sobre las desviaciones típicas de las poblaciones de las que proceden las dos muestras. En este caso, el test se ha realizado para determinar si el ratio de las desviaciones típicas son iguales 1,0 frente a la hipótesis alternativa en la que el ratio no es igual 1,0. Puesto que el p-valor calculado no es inferior a 0,05, no podemos rechazar la hipótesis nula.

NOTA IMPORTANTE: los F-test y los intervalos de confianza mostrados dependen de que las muestras procedan de distribuciones normales.

Para comprobar esta asunción, seleccione Resumen Estadístico de la lista de Opciones Tabulares y observe los valores de asimetría estandarizada y curtosis estandarizada.

Tabla 5.43. Comparación de desviaciones típicas p2pA-p2fA.

\section{Box-and-Whisker Plot}

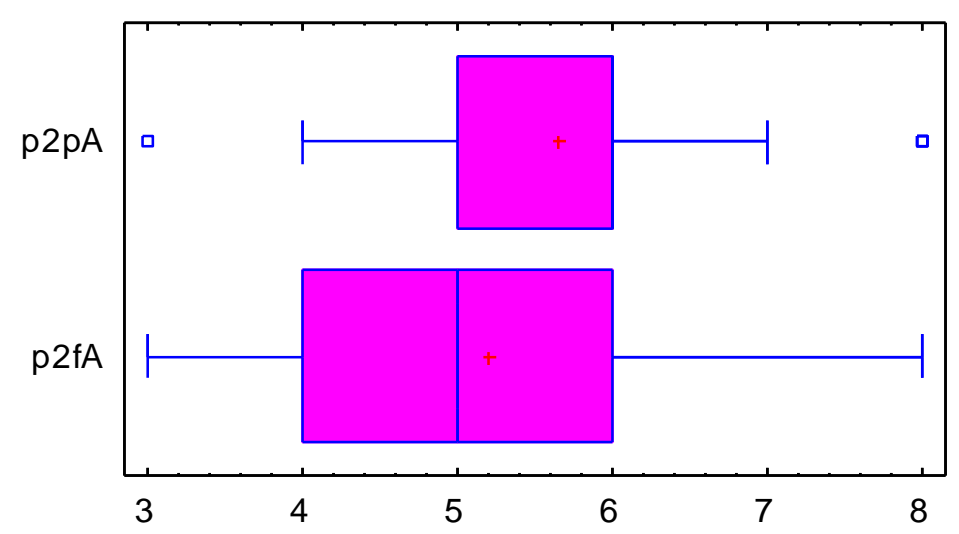

Gráfico 5.35. Comparación de diagramas de cajas y bigotes p2pA-p2fA

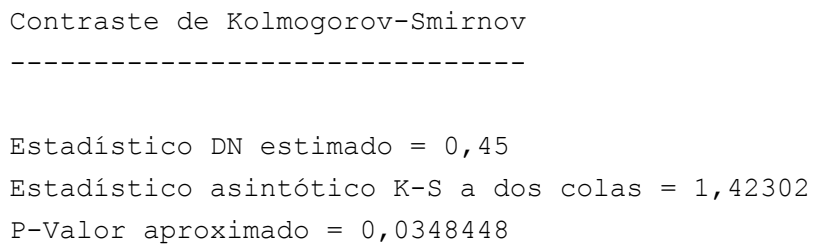




\section{El StatAdvisor}

Esta opción ejecuta el test de Kolmogorov-Smirnov para comparar las

distribuciones de las dos muestras. Este test se ha realizado

calculando la distancia máxima entre las distribuciones acumuladas de

las dos muestras. En este caso, la máxima distancia es 0,45, la cual

puede visualizar seleccionando Gráfico Cuantil en la lista de Opciones

Gráficas. De particular interés está el p-valor aproximado para el

test. Dado que el p-valor es menor que 0,05, existe diferencia

estadísticamente significativa entre las dos distribuciones para un

nivel de confianza del 95, 0\%.

Tabla 5.44. Contraste de Kolmogorov-Smirnov p2pA-p2fA

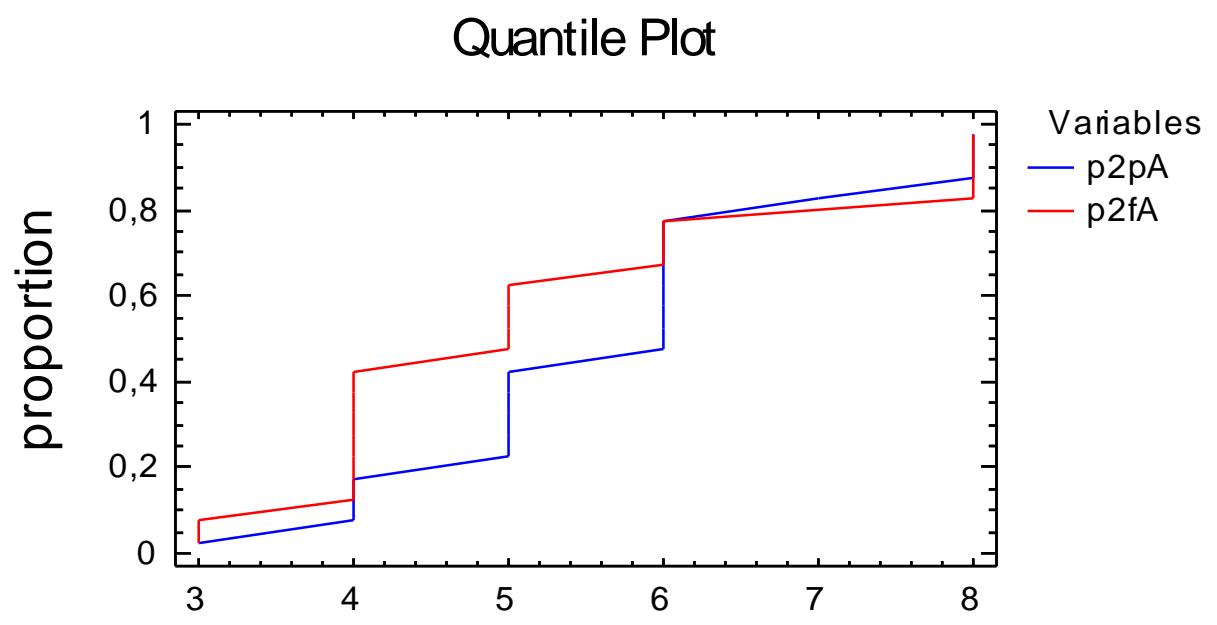

Gráfico 5.36. Representación de cuantiles p2pA-p2fA

\subsection{Comparaciones de los resultados medios para el conjunto de las demostraciones}

La tabla 5.45 recoge las frecuencias de cada una de las calificaciones medias (Me) de las cuatro categorías anteriores asignadas por los alumnos en cada una de las cuatro demostraciones. 


\begin{tabular}{rrrrr} 
& p1pMe & p1fMe & p2pMe & \multicolumn{2}{c}{ p2fMe } \\
\hline $\mathbf{1 - 2}$ & 0 & 0 & 0 & 0 \\
$\mathbf{2}-\mathbf{3}$ & 0 & 1 & 1 & 1 \\
$\mathbf{3 - 4}$ & 0 & 3 & 1 & 4 \\
$\mathbf{4 - 5}$ & 3 & 4 & 6 & 5 \\
$\mathbf{5 - 6}$ & 9 & 2 & 5 & 8 \\
$\mathbf{6 - 7}$ & 4 & 8 & 6 & 2 \\
$\mathbf{7 - 8}$ & 4 & 2 & 1 & 0 \\
\hline $\mathbf{8 - 9}$ & 0 & 0 & 0 & 0 \\
$\mathbf{9 - 1 0}$ & 0 & 0 & 0 & 0 \\
\hline Total & 20 & 20 & 20 & 20 \\
\hline
\end{tabular}

Tabla 5.45. Frecuencias de las calificaciones medias de las cuatro categorías anteriores en cada una de las cuatro demostraciones p1pMe-p1fMe; p2pMep2fMe

Asimismo, estos datos quedan reflejados en los gráficos 5.37 y 5.37 bis siguientes.

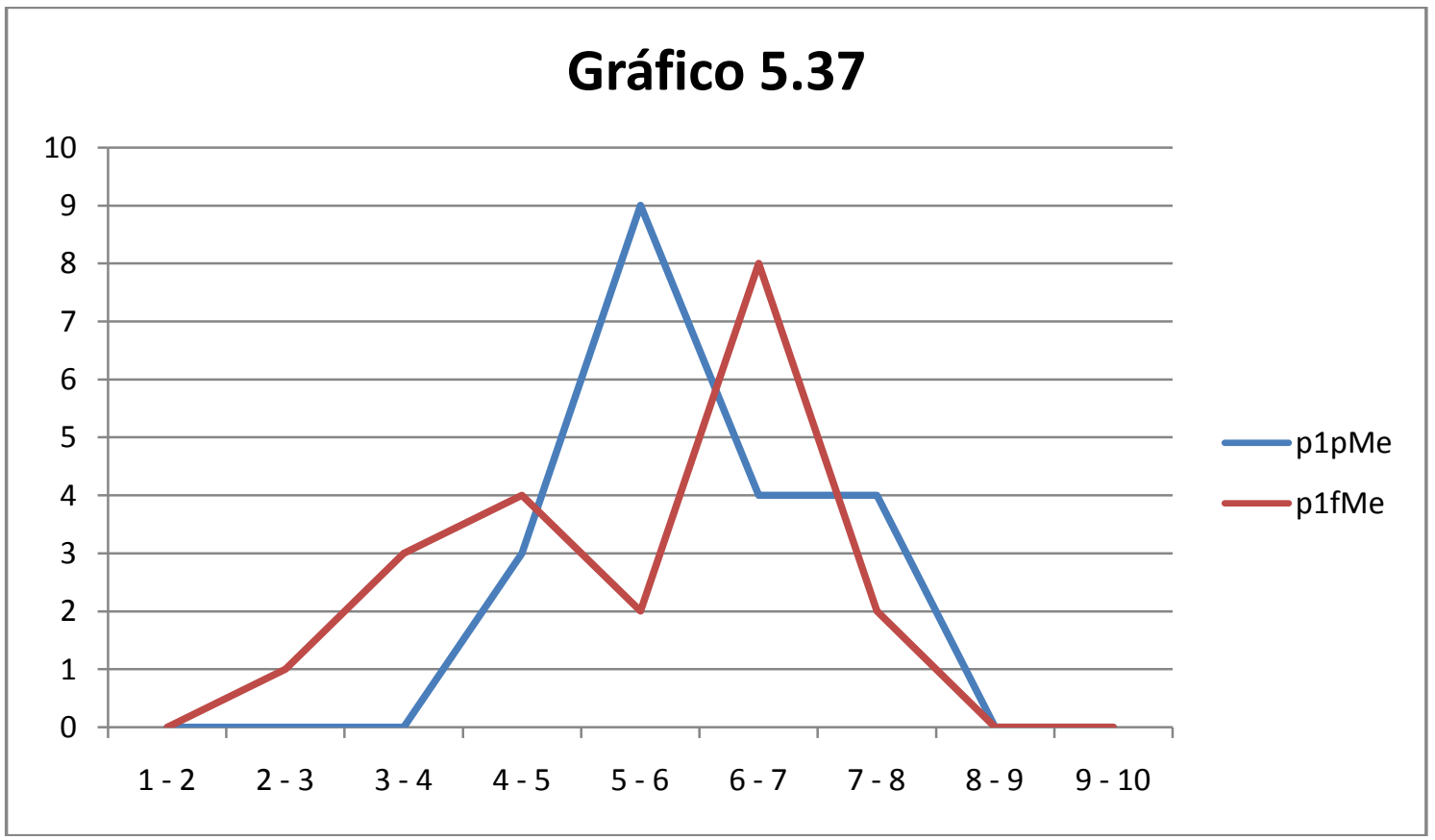

Gráfico 5.37. Representación de resultados medios para el conjunto de la primera demostración (teorema 1) 


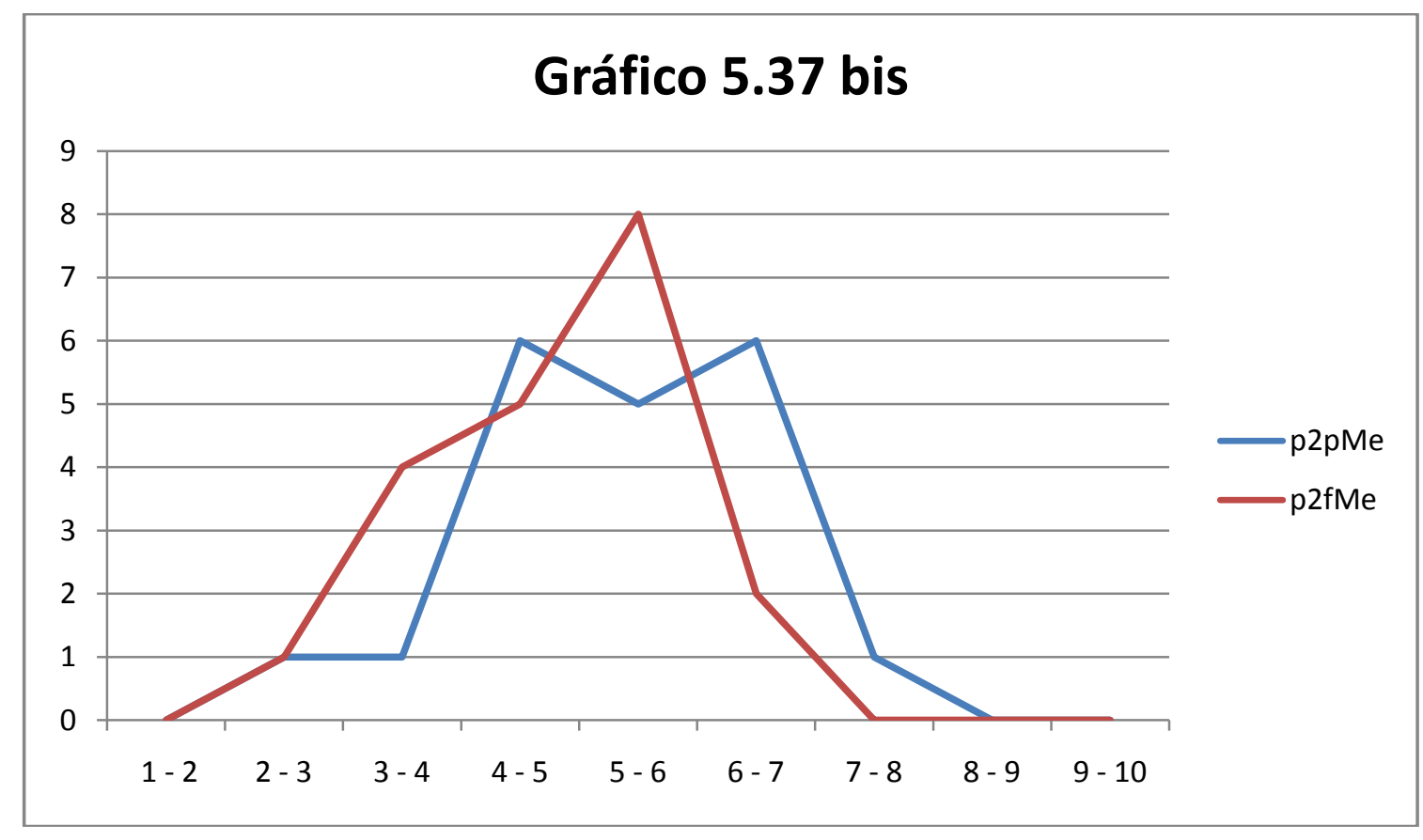

\section{Gráfico 5.37 bis. Representación de resultados medios para el conjunto de la segunda demostración (teorema 2)}

Estas distribuciones no representan claramente que las demostraciones PPF son mejor valoradas que las PF; para el primer teorema la distribución de la PPF (en azul) en general está más a la izquierda que la PF (en rojo), mientras que para el segundo teorema la preferencia es más clara por la PPF (en azul, más a la derecha a pesar de tener dos máximos) respecto la PF (en rojo).

Las tablas 5.46 y 5.47 recogen las comparaciones de las características estadísticas de estas cuatro variables, que vienen a confirmarnos por lo general una preferencia por PPF en ambas demostraciones. Así en la tabla 5.46, observamos que las características de tendencia central son superiores en la PPF (1aㅡ y $3^{\underline{a}}$ columna) respecto a la PF (2 ${ }^{a}$ y $4^{\underline{a}}$ columna) en la media. En la mediana esto sólo se cumple para la segunda demostración, mientras que los valores son mayores en la PF que en la PPF de la primera; y la moda no se calcula por tratarse de una continua en alguna de las variables. Se observan sin embargo por lo general unos mayores valores en las dos demostraciones para la PPF para mínimo, máximo y cuartiles. 
Por lo que se refiere a la dispersión, tomando el coeficiente de variación se aprecia cómo va creciendo de PPF a PF en la primera demostración, mientras que la relación es la opuesta entre ambas en la segunda, aunque en esta última las diferencias no son demasiado amplias.

Por su parte, siguiendo en la tabla 5.46, la asimetría tipificada de las distribuciones no viene a corroborar en todos los casos la preferencia por las PPF: en el primer caso la PF es asimétrica negativa frente a una PPF asimétrica positiva, o sea, en la PPF la mayoría de las calificaciones se agrupan al principio de la distribución y hay menos calificaciones altas, lo que nos indica por tanto una preferencia por la PF; sin embargo, en el segundo caso las dos pruebas son asimétricas negativas, aunque lo es un poco más la PPF, o sea, en la PPF se nota más que la mayoría de las calificaciones se agrupan al final de la distribución y hay menos calificaciones bajas, lo que nos indica por tanto una preferencia por la PPF.

Además, el hecho de que la asimetría tipificada y la curtosis tipificada estén entre -2 y 2 va a permitir tratar estas variables como que siguen distribuciones normales, lo que justificará la utilización de los contrastes de igualdad de varianzas y de igualdad de medias que se verán en los dos apartados siguientes.

Las correlaciones de la tabla 5.47 nos indican la escasa asociación que hay entre la mayoría de los pares de calificaciones (los valores de estas correlaciones, por lo general, están más bien próximos a cero, lo que indica una escasa relación entre el par de variables correspondiente). El p-valor corrobora la inexistencia de relaciones significativas salvo para la PPF y PF del segundo teorema. Esto es, en la mayoría de los casos podemos decir que no hay relación entre las puntuaciones medias dadas por los alumnos en las distintas demostraciones. 


\begin{tabular}{|c|c|c|c|c|}
\hline \multicolumn{5}{|l|}{ Resumen Estadístico } \\
\hline & plpMe & $\mathrm{p} 1 \mathrm{fMe}$ & $\mathrm{p} 2 \mathrm{pMe}$ & $\mathrm{p} 2 \mathrm{fMe}$ \\
\hline Frecuencia & 20 & 20 & 20 & 20 \\
\hline Media & 6,025 & 5,675 & 5,425 & 4,875 \\
\hline Mediana & 5,75 & 6,0 & 5,5 & 5,125 \\
\hline Moda & 5,5 & & & 5,25 \\
\hline Varianza & 1,13092 & 2,19803 & 1,55987 & 1,09539 \\
\hline Desviación típica & 1,06345 & 1,48257 & 1,24895 & 1,04661 \\
\hline Mínimo & 4,25 & 2,75 & 2,5 & 3,0 \\
\hline Máximo & 8,0 & 8,0 & 8,0 & 6,5 \\
\hline Rango & 3,75 & 5,25 & 5,5 & 3,5 \\
\hline Primer cuartil & 5,25 & 4,625 & 4,75 & 4,125 \\
\hline Segundo cuartil & 6,75 & 6,875 & 6,25 & 5,75 \\
\hline Asimetría tipi. & 0,567111 & $-0,859066$ & $-0,594862$ & $-0,52385$ \\
\hline Curtosis típificada & $-0,740658$ & $-0,751574$ & 0,647565 & $-0,86989$ \\
\hline Coef. de variación & $17,6506 \%$ & $26,1247 \%$ & $23,0221 \%$ & $21,4689 \%$ \\
\hline
\end{tabular}

\section{El StatAdvisor}

Esta tabla muestra resúmenes estadísticos para cada una de las variables seleccionadas. Incluye medidas de tendencia central, de variabilidad y de forma. De particular interés están la asimetría estandarizada y la curtosis estandarizada, las cuales pueden utilizarse para determinar si la muestra procede de una distribución normal. Valores de estos estadísticos fuera del rango de -2 a +2 indican una desviación significativa de la normalidad, que tendería a invalidar muchos de los procedimientos estadísticos aplicados habitualmente a estos datos. En este caso, las siguientes variables muestran valores de asimetría estandarizada fuera del rango esperado:

$$
<\text { ninguna }>
$$

Las siguientes variables muestran valores de curtosis estandarizada fuera del rango esperado:

$<$ ninguna $>$

Tabla 5.46. Comparaciones de las características estadísticas de medias de ambas pruebas en estas cuatro variables p1pMe-p1fMe; p2pMe-p2fMe

\begin{tabular}{|c|c|c|c|c|}
\hline & plpMe & plfme & $\mathrm{p} 2 \mathrm{pMe}$ & $\mathrm{p} 2 \mathrm{fme}$ \\
\hline \multirow[t]{3}{*}{$\mathrm{p} 1 \mathrm{pMe}$} & & $-0,0008$ & 0,0535 & 0,2867 \\
\hline & & $(20)$ & 20) & 20) \\
\hline & & 0,9972 & 0,8228 & 0,2204 \\
\hline \multirow[t]{3}{*}{$\mathrm{p} 1 \mathrm{fMe}$} & $-0,0008$ & & $-0,0974$ & 0,1887 \\
\hline & $(20)$ & & $(20)$ & $(20)$ \\
\hline & 0,9972 & & 0,6830 & 0,4257 \\
\hline
\end{tabular}




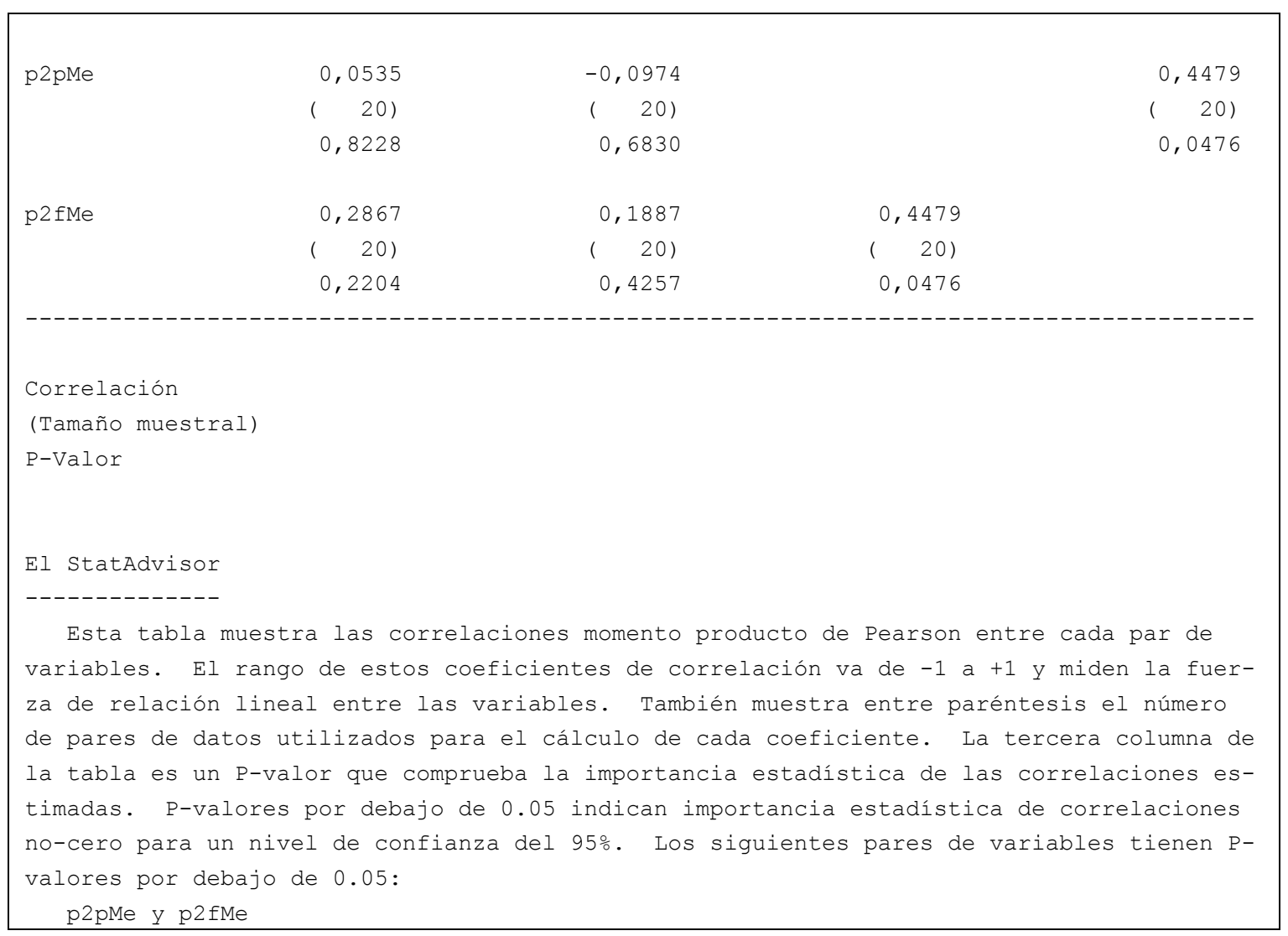

Tabla 5.47. Correlaciones p1pMe-p1fMe; p2pMe-p2fMe

\subsubsection{Comparaciones de los resultados medios para la primera demostración}

Partiendo de la normalidad comprobada con los coeficientes de asimetría y curtosis tipificados en la tabla 5.46, en la tabla 5.50 comprobamos que no se puede rechazar la igualdad de desviaciones típicas; a su vez esto nos permite realizar el contraste de igualdad de medias que aparece en la tabla 5.49, resultando que tampoco podemos rechazar la hipótesis nula, de modo que se puede admitir que las medias sean iguales entre ambas variables. El resultado del contraste de Kolmogorov-Smirnov (tabla 5.51) indica en el mismo sentido, ya que tampoco se puede rechazar la igualdad de las distribuciones.

Los gráficos ponen de manifiesto sólo ciertas diferencias:

33) Gráfico 5.38: comparación de histogramas, donde se observa cómo el histograma de arriba (correspondiente a la PPF) presenta por lo gene- 
ral mayores valores que el de abajo (correspondiente a la PF) que sólo destaca en ese penúltimo intervalo su alta frecuencia.

34) Gráfico 5.39: comparación de densidades, con una distribución algo más a la derecha para la PF en los valores más altos de la variable, aunque se cruzan por varias veces.

35) Gráfico 5.40: con los dos diagramas de cajas y bigotes, en el que se observa cómo las cajas del gráfico superior están a la derecha del inferior, lo mismo que el primer bigote, representando al menos una mayor unanimidad en las valoraciones medias de la PPF.

36) Gráfico 5.41: con la representación de los cuantiles, donde la distribución de la PF se cruza con la PPF, con lo que no podríamos afirmar nada sobre cuál es preferida.

Por tanto no podemos decir que para la media de las cuatro características analizadas para la primera demostración exista una diferencia sustancial entre ambas pruebas.

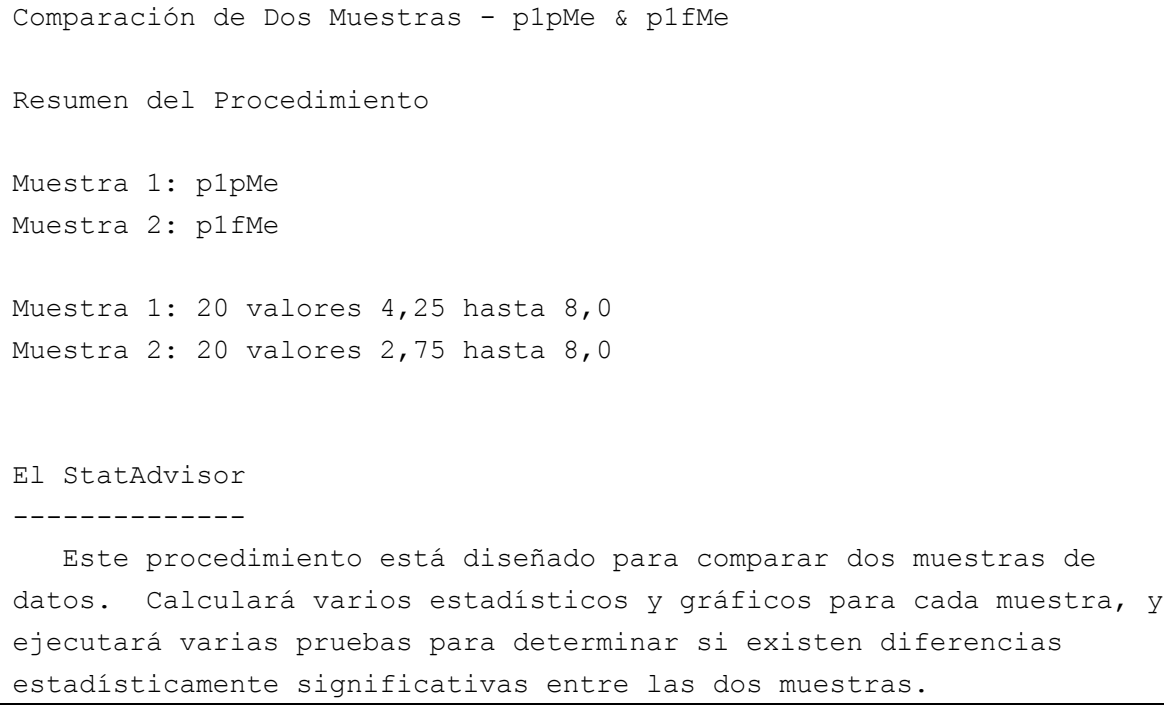

Tabla 5.48. Comparación de muestras p1pMe-p1fMe 


\section{p1pMe}

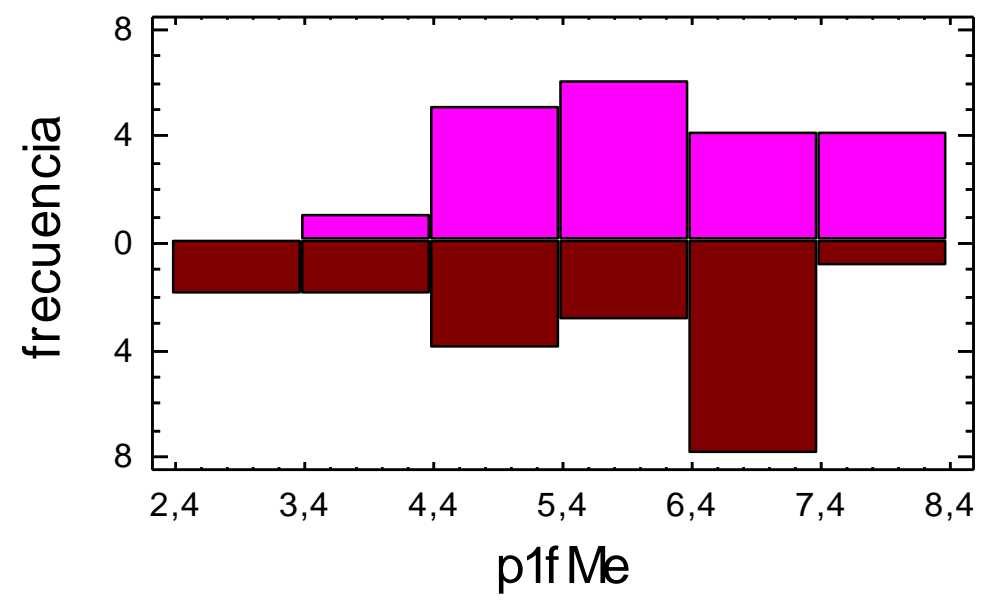

Gráfico 5.38. Comparación de histogramas p1pMe-p1fMe

Comparación de Medias

95, 0\% intervalo de confianza para la media de plpMe: 6,025 +/- 0,49771

$[5,52729,6,52271]$

95,0\% intervalo de confianza para la media de plfMe: 5,675 +/- 0,693868

$[4,98113,6,36887]$

95, 0\% intervalos de confianza para la diferencia de medias:

suponiendo varianzas iguales: 0,35 +/- 0,825913 [-0,475913,1,17591]

contrastes $t$ de comparación de medias

Hipótesis nula: medial $=$ media2

Hipótesis alt.: medial <> media2

suponiendo varianzas iguales: $t=0,857886 \quad$ P-Valor $=0,396334$

El StatAdvisor

Esta opción ejecuta el t-test para comparar las medias de las dos muestras. También establece los intervalos de confianza o los límites para cada media y para la diferencia entre las medias. De particular interés está el intervalo de confianza para la diferencia entre las medias, el cual se extiende desde $-0,475913$ hasta 1,17591. Dado que el intervalo contiene el valor 0.0 , no existe diferencia estadísticamente significativa entre las medias de las dos muestras para un nivel de confianza del 95,0\%.

También puede aplicarse un t-test para probar una hipótesis específica sobre la diferencia entre las medias de las poblaciones de las que proceden las dos muestras. En este caso, el test se ha realizado para determinar si la diferencia entre las dos medias es igual a 0,0 frente a la hipótesis alternativa en la que la diferencia no es igual 0,0. Puesto que el p-valor calculado no es inferior a 0,05 , no podemos rechazar la hipótesis nula. 
NOTA: estos resultados asumen la igualdad de varianzas en las dos

muestras. En este caso, esa asunción parece ser razonable teniendo en

cuenta los resultados del F-test para comparar las desviaciones

típicas. Puede ver los resultados de este test seleccionando

Comparación de Desviaciones Típicas del menú Opciones Tabulares.

Tabla 5.49. Comparación de medias p1pMe-p1fMe

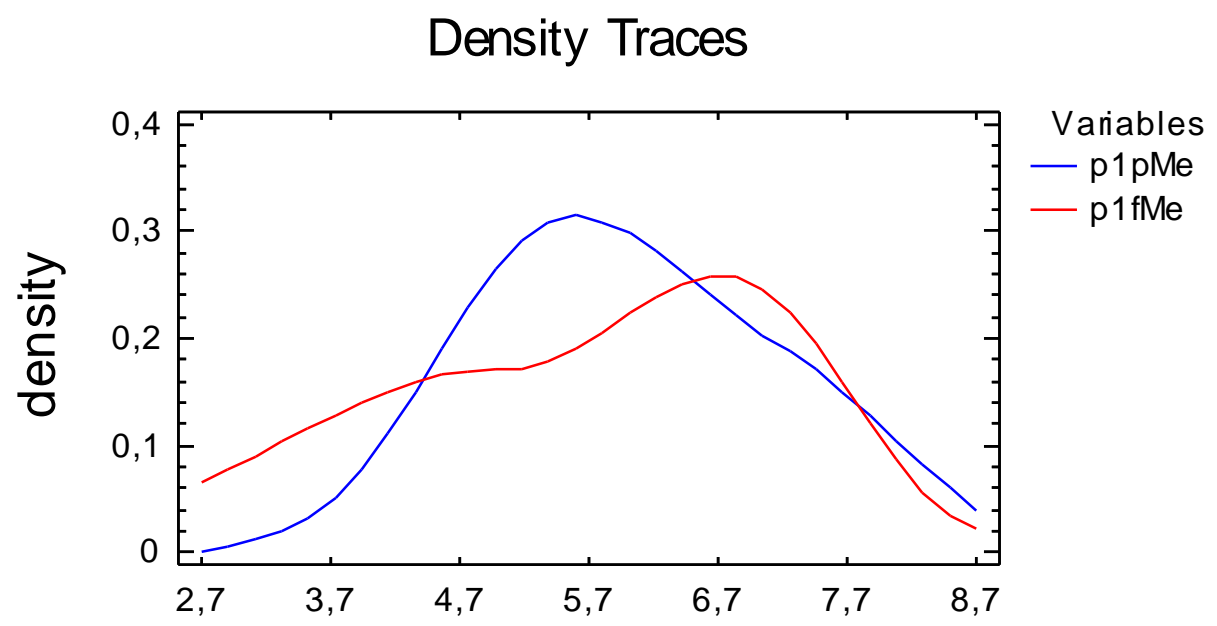

Gráfico 5.39. Comparación de densidades p1pMe-p1fMe

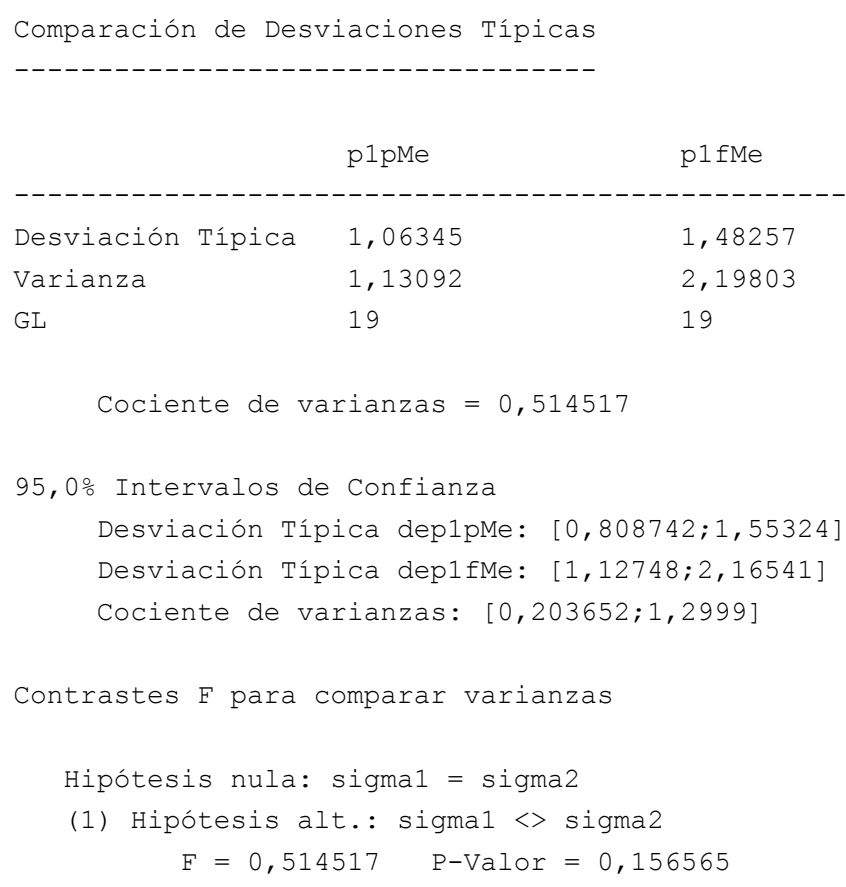


El StatAdvisor

-------------

Esta opción ejecuta un F-test para comparar las varianzas de las dos muestras. También establece los intervalos de confianza o los límites para cada desviación típica y para el ratio de varianzas. De particular interés está el intervalo de confianza para el ratio de las varianzas, el cual se extiende desde 0,203652 hasta 1,2999. Dado que el intervalo contiene el valor 1.0, no existe diferencia estadísticamente significativa entre las desviaciones típicas de las dos muestras para un nivel de confianza del 95,0\%.

También puede utilizarse un F-test para probar una hipótesis específica sobre las desviaciones típicas de las poblaciones de las que proceden las dos muestras. En este caso, el test se ha realizado para determinar si el ratio de las desviaciones típicas son iguales 1,0 frente a la hipótesis alternativa en la que el ratio no es igual 1,0. Puesto que el p-valor calculado no es inferior a 0,05, no podemos rechazar la hipótesis nula.

NOTA IMPORTANTE: los F-test y los intervalos de confianza mostrados dependen de que las muestras procedan de distribuciones normales.

Para comprobar esta asunción, seleccione Resumen Estadístico de la lista de Opciones Tabulares y observe los valores de asimetría estandarizada y curtosis estandarizada.

Tabla 5.50. Comparación de desviaciones típicas p1pMe-p1fMe.

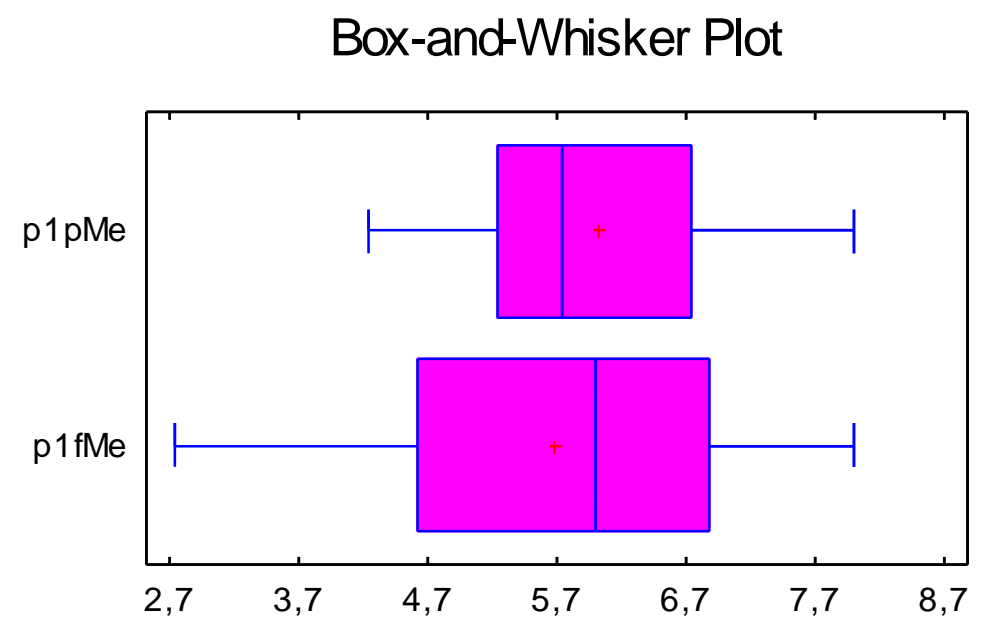

Gráfico 5.40. Comparación de diagramas de cajas y bigotes p1pMe-p1fMe

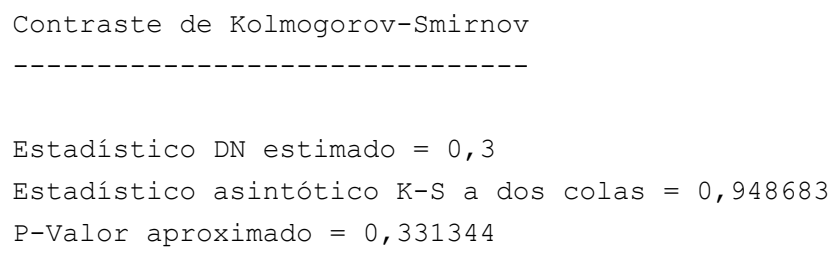




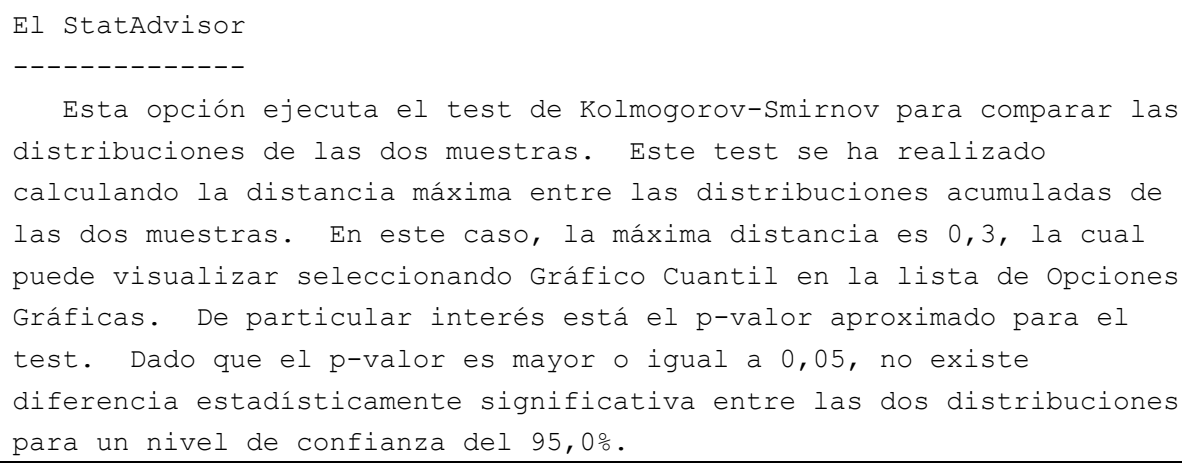

Tabla 5.51. Contraste de Kolmogorov-Smirnov p1pMe-p1fMe

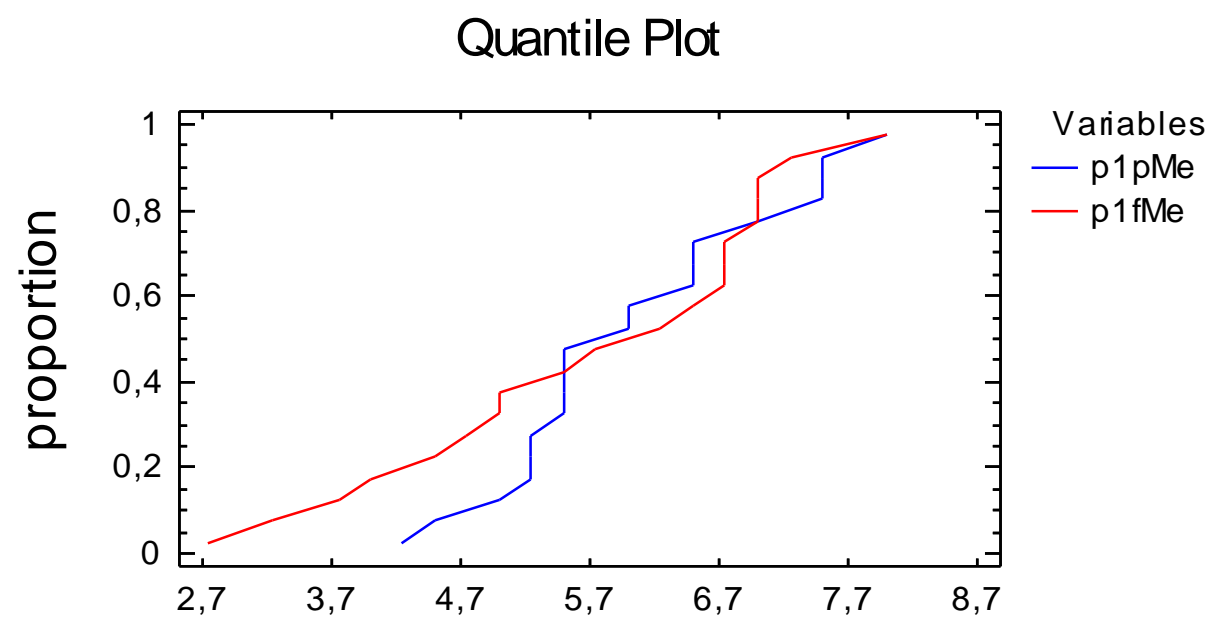

Gráfico 5.41. Representación de cuantiles p1pMe-p1fMe

\subsubsection{Comparaciones de los resultados medios para la segunda demostración}

Partiendo de la normalidad comprobada con los coeficientes de asimetría y curtosis tipificados en la tabla 5.46, en la tabla 5.54 comprobamos que no se puede rechazar la igualdad de desviaciones típicas; a su vez esto nos permite realizar el contraste de igualdad de medias que aparece en la tabla 5.53, resultando que tampoco podemos rechazar la hipótesis nula, de modo que se puede admitir que las medias sean iguales entre ambas variables. El resultado del contraste de Kolmogorov-Smirnov (tabla 5.55) indica en el mismo sentido, ya que tampoco se puede rechazar la igualdad de las distribuciones. 
Los gráficos ponen de manifiesto sólo ciertas diferencias:

37) Gráfico 5.42: comparación de histogramas, donde se observa cómo el histograma de arriba (correspondiente a la PPF) presenta por lo general mayores frecuencias en valores más altos que el de abajo (correspondiente a la PF) que sólo destaca en ese penúltimo intervalo su alta frecuencia.

38) Gráfico 5.43: comparación de densidades, con una distribución algo más a la derecha para la PPF en los valores más altos de la variable.

39) Gráfico 5.44: con los dos diagramas de cajas y bigotes, en el que se observa cómo las cajas del gráfico superior están a la derecha del inferior, si bien los bigotes del superior son mucho más amplios.

40) Gráfico 5.45: con la representación de los cuantiles, donde la distribución de la PPF está a la derecha de la distribución de la PF, con lo que podríamos afirmar cierta preferencia a favor de la PPF.

Por tanto no podemos decir que en este caso exista una diferencia sustancial entre ambas demostraciones.

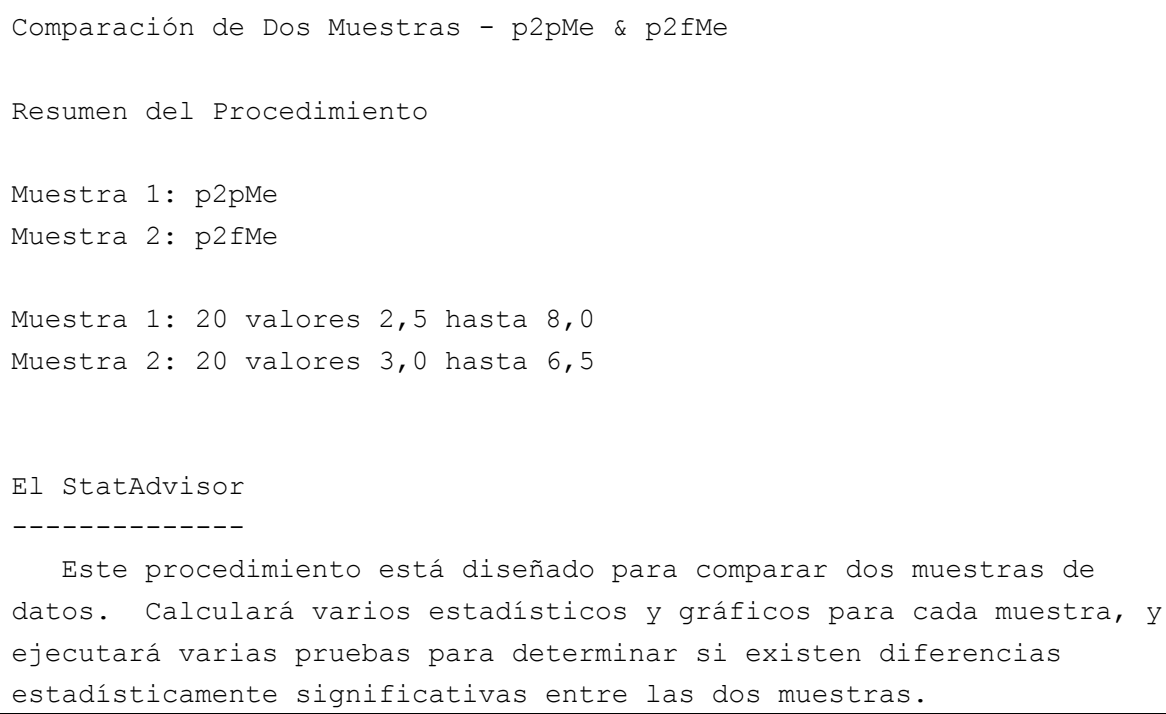

Tabla 5.52. Comparación de muestras p2pMe-p2fMe 


\section{p2pMe}

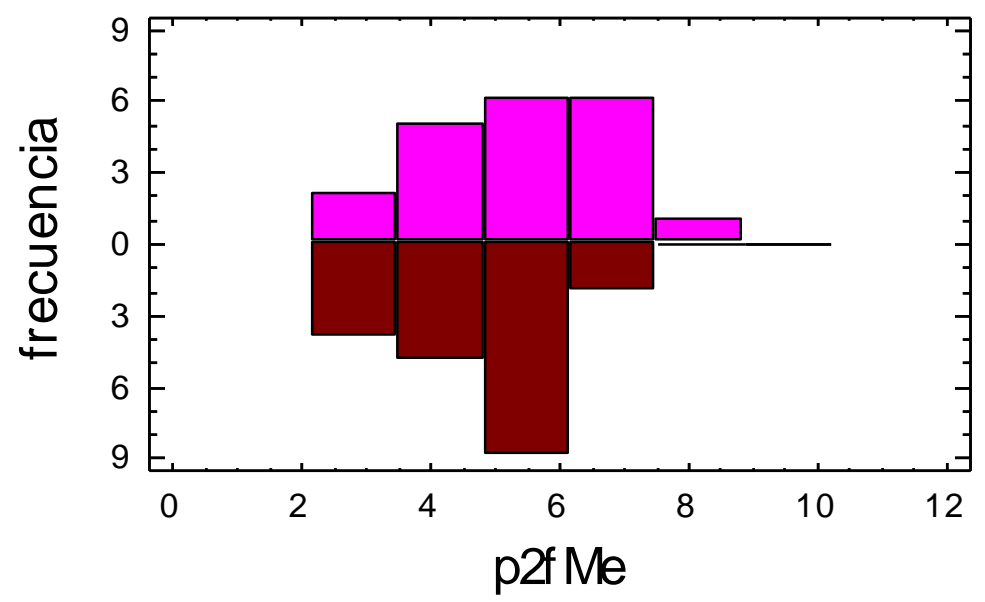

Gráfico 5.42. Representación de histogramas p2pMe-p2fMe

Comparación de Medias

95, 0\% intervalo de confianza para la media de p2pMe: 5,425 +/- 0,584526

$[4,84047,6,00953]$

95,0\% intervalo de confianza para la media de p2fMe: 4,875 +/- 0,48983

$[4,38517,5,36483]$

95, 0\% intervalos de confianza para la diferencia de medias:

suponiendo varianzas iguales: $0,55+/-0,737623 \quad[-0,187623,1,28762]$

contrastes t de comparación de medias

Hipótesis nula: medial = media2

Hipótesis alt.: medial <> media2

suponiendo varianzas iguales: $t=1,50947 \quad$ P-Valor $=0,13945$

El StatAdvisor

Esta opción ejecuta el t-test para comparar las medias de las dos muestras. También establece los intervalos de confianza o los límites para cada media y para la diferencia entre las medias. De particular interés está el intervalo de confianza para la diferencia entre las medias, el cual se extiende desde $-0,187623$ hasta 1,28762. Dado que el intervalo contiene el valor 0.0, no existe diferencia

estadísticamente significativa entre las medias de las dos muestras para un nivel de confianza del 95,0\%.

También puede aplicarse un t-test para probar una hipótesis específica sobre la diferencia entre las medias de las poblaciones de las que proceden las dos muestras. En este caso, el test se ha realizado para determinar si la diferencia entre las dos medias es igual a 0,0 frente a la hipótesis alternativa en la que la diferencia 
no es igual 0,0. Puesto que el p-valor calculado no es inferior a

0,05, no podemos rechazar la hipótesis nula.

NOTA: estos resultados asumen la igualdad de varianzas en las dos

muestras. En este caso, esa asunción parece ser razonable teniendo en

cuenta los resultados del F-test para comparar las desviaciones

típicas. Puede ver los resultados de este test seleccionando

Comparación de Desviaciones Típicas del menú Opciones Tabulares.

Tabla 5.53. Comparación de medias p2pMe-p2fMe

Density Traces

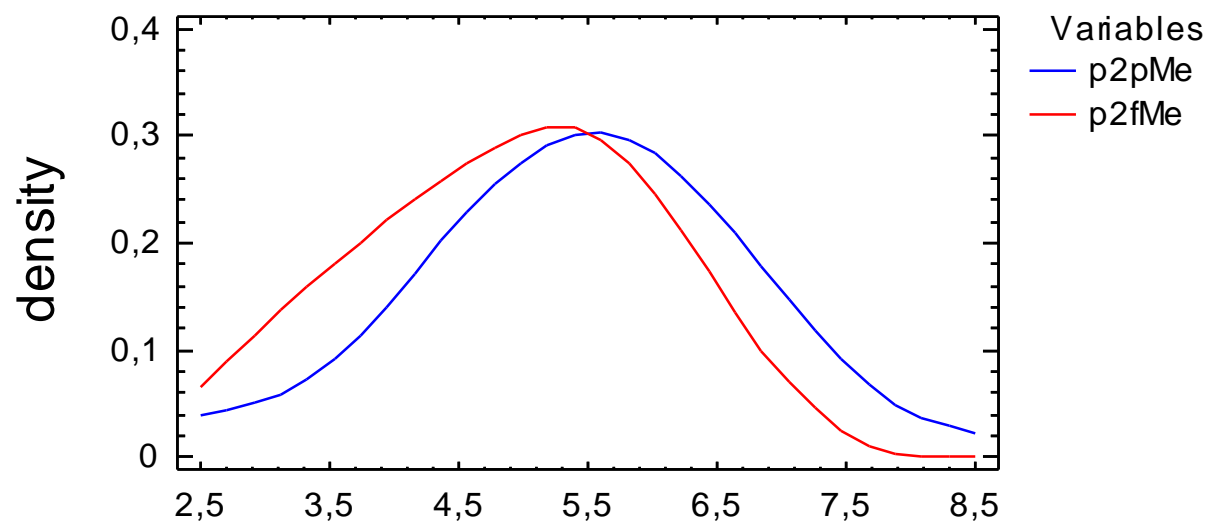

Gráfico 5.43. Comparación de densidades p2pMe-p2fMe

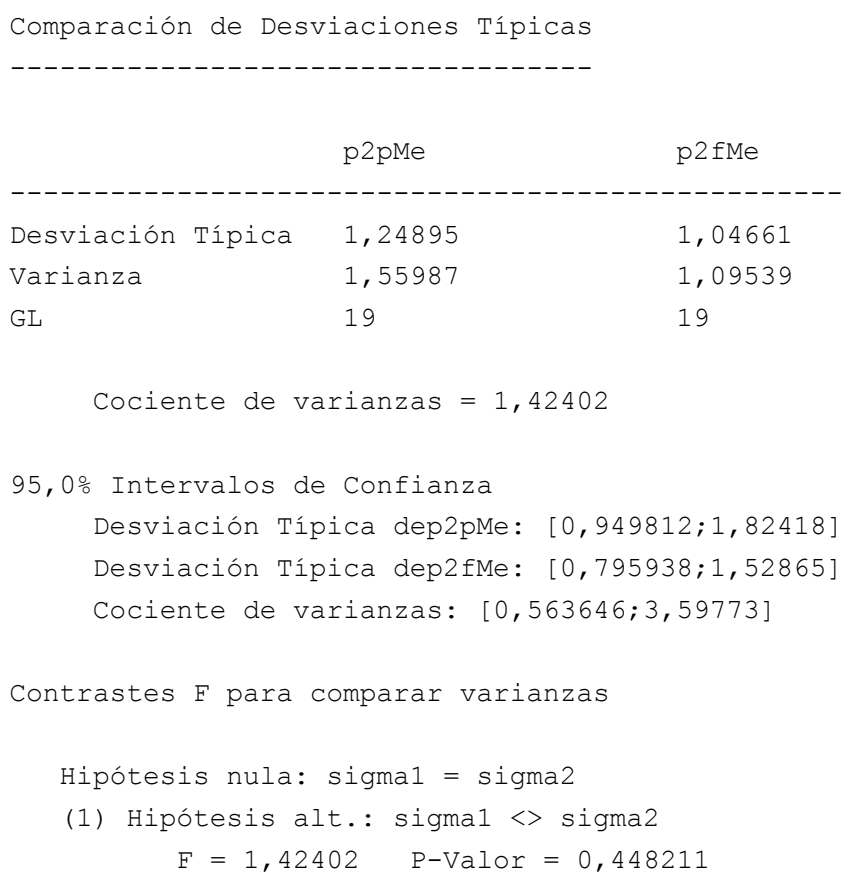


El StatAdvisor

------------

Esta opción ejecuta un F-test para comparar las varianzas de las dos muestras. También establece los intervalos de confianza o los límites para cada desviación típica y para el ratio de varianzas. De particular interés está el intervalo de confianza para el ratio de las varianzas, el cual se extiende desde 0,563646 hasta 3,59773. Dado que el intervalo contiene el valor 1.0, no existe diferencia estadísticamente significativa entre las desviaciones típicas de las dos muestras para un nivel de confianza del 95,0\%.

También puede utilizarse un F-test para probar una hipótesis específica sobre las desviaciones típicas de las poblaciones de las que proceden las dos muestras. En este caso, el test se ha realizado para determinar si el ratio de las desviaciones típicas son iguales 1,0 frente a la hipótesis alternativa en la que el ratio no es igual 1,0. Puesto que el p-valor calculado no es inferior a 0,05, no podemos rechazar la hipótesis nula.

NOTA IMPORTANTE: los F-test y los intervalos de confianza mostrados dependen de que las muestras procedan de distribuciones normales.

Para comprobar esta asunción, seleccione Resumen Estadístico de la lista de Opciones Tabulares y observe los valores de asimetría estandarizada y curtosis estandarizada.

Tabla 5.54. Comparación de desviaciones típicas p2pMe-p2fMe

\section{Box-and-Whisker Plot}

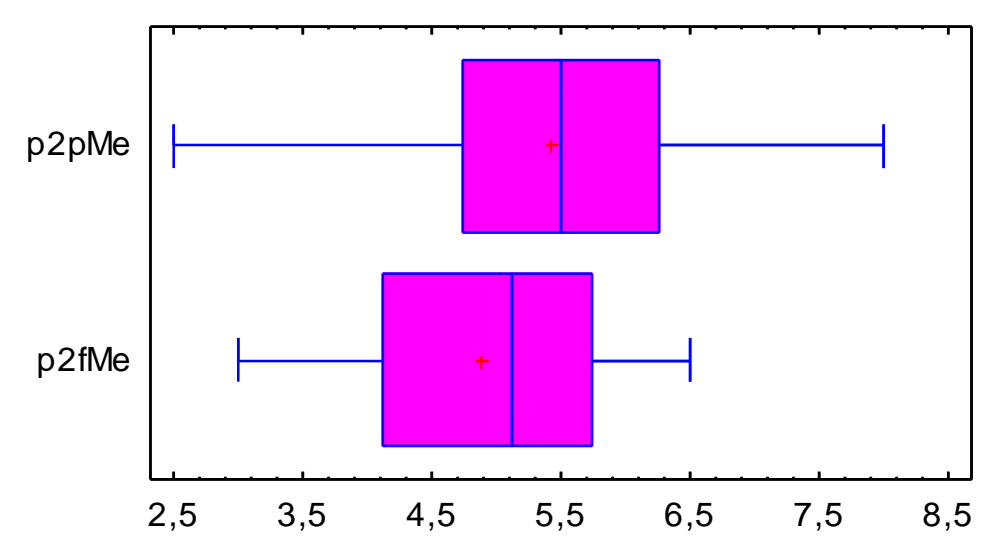

Gráfico 5.44. Comparación de diagramas y cajas de bigotes p2pMe-p2fMe

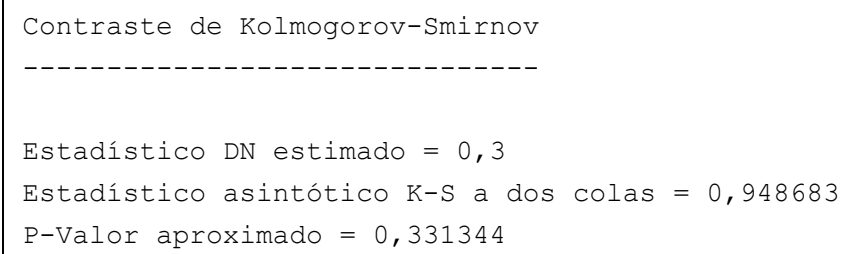




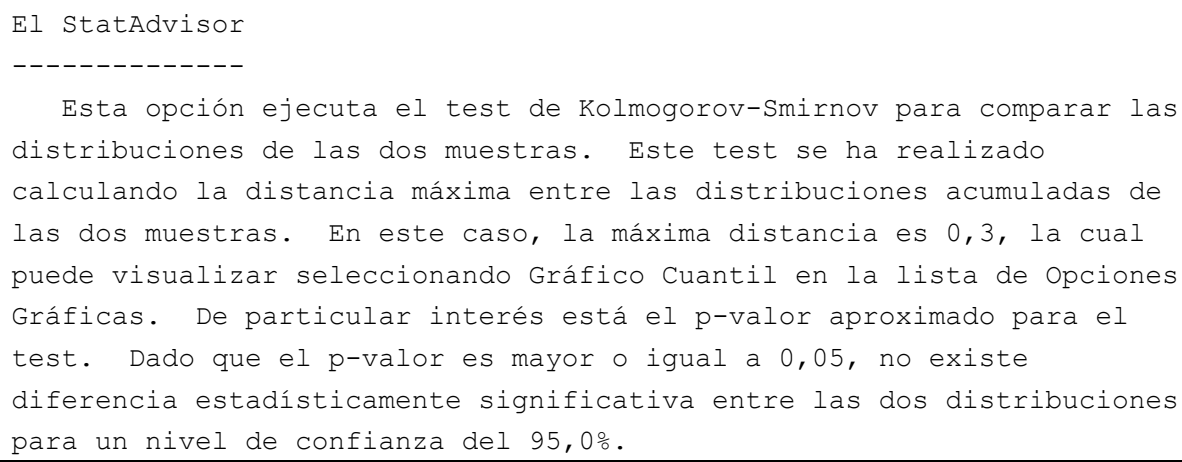

Tabla 5.55. Contraste de Kolmogorov-Smirnov p2pMe-p2fMe

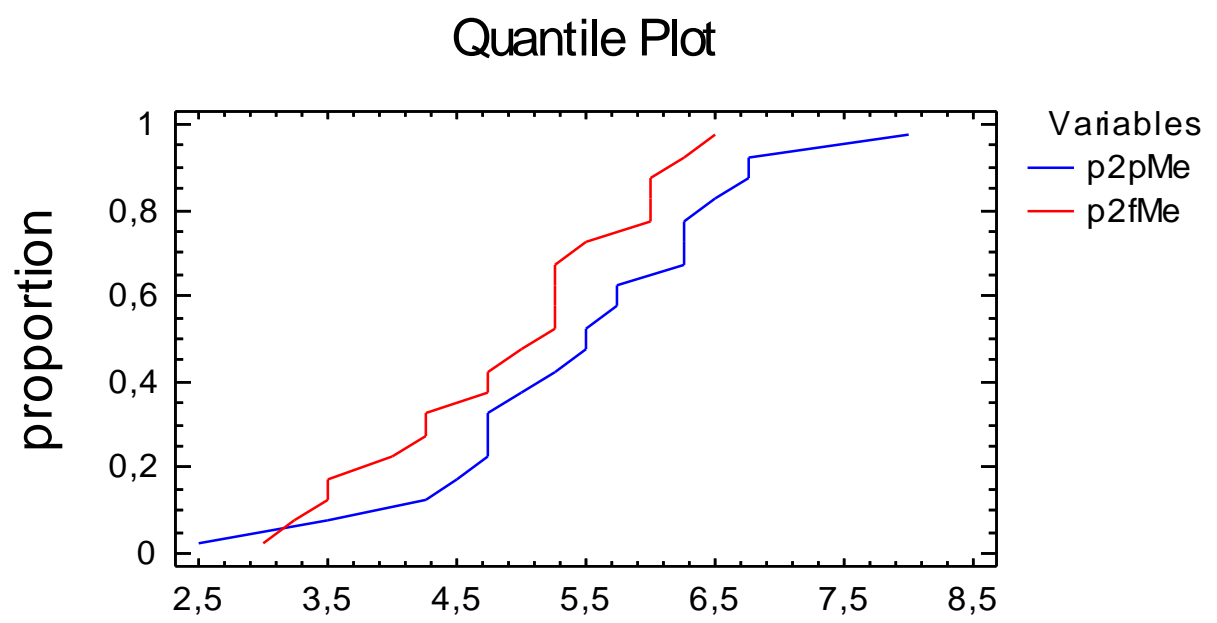

Gráfico 5.45. Representación de cuantiles p2pMe-p2fMe

\subsection{Reflexiones derivadas del análisis cuantitativo}

Con los datos del experimento realizado en 2009 con las opiniones de 20 alumnos no hay una gran evidencia sobre si son preferidas las pruebas PPF sobre las PF.

En las calificaciones sobre la facilidad (sección 5.1) hay evidencias sobre la preferencia por las PPF en ambas demostraciones. Sin embargo, en las valoraciones sobre el gusto, la claridad y el aprendizaje sólo se aprecia una preferencia por la PPF en la segunda demostración, no habiendo grandes diferencias entre ambas pruebas en la primera. Y si analizamos la puntuación media de las cuatro variables, no 
se aprecian grandes diferencias entre ambas pruebas en ninguna de las dos demostraciones. Lo que sin embargo resulta obvio es que en ningún caso una prueba PF resulta preferida sobre la PPF.

Se corresponden estos resultados con los del análisis cualitativo, en los que también se constatan estas apreciaciones (cf. 4.3.5.1, tabla 4.27, p. 127, y gráfico 4.5, p. 128, y motivos invocados en las tablas 4.24 y 4.25, p. 121). Los estadísticos descriptivos en los casos que no permiten suponer diferencia sustancial, estadísticamente significativa entre las distribuciones, sí aportan ciertas diferencias a favor siempre de la prueba preformal, diferencias que se muestran más acusadas en el análisis cualitativo, e incluso pueden observarse también en la mayoría de los gráficos del mismo análisis cuantitativo. Cuando es analizado el total de los alumnos de los tres ciclos, estas diferencias quedan integradas a la vez que más diluidas en todo el conjunto (cf. capítulo 6). Ya hemos señalado que el orden de presentación de las demostraciones ha podido influir en la posterior valoración de los alumnos; en este ciclo el orden fue: en el primer teorema, primera demostración, la prueba formal en primer lugar, y la prueba preformal en segundo lugar; en el segundo teorema, segunda demostración, fue presentada primeramente la prueba preformal, y después de ella la prueba formal. En cuanto a los motivos de las valoraciones, recordemos que es constante en los tres ciclos otorgar a la demostración formal mayor generalización, abstracción, ayuda a memorizar, considerarla más teórica, compleja, difícil; y mayor sencillez, concreción, facilidad, ayuda al mejor entendimiento de los teoremas y más útil para ilustrar sus aplicaciones, a la demostración preformal. 



\section{CAPÍTULO 6}

\section{ANÁLISIS ESTADÍSTICO GLOBAL}

A partir de las respuestas de los alumnos obtenidas en las encuestas que se les pasaron a continuación de cada una de las pruebas, vamos a analizar si alguna de las dos pruebas tiene mejores resultados o si por el contrario los alumnos son indiferentes ante la aplicación de ambas metodologías.

Para ello, analizamos cuantitativamente los datos numéricos relativos a cada modalidad de prueba (preformal y formal) comparando los resultados mediante técnicas de contraste. Se ha considerado como muestra la totalidad de los alumnos que han participado en los tres ciclos de investigación-acción.

Los resultados en la mayoría de los casos apuntan una preferencia por las demostraciones preformales sobre las formales, o en todo caso una indiferencia entre ambas demostraciones. Las tablas que aparecen a continuación plantean un análisis descriptivo donde ya se ve unas mejores calificaciones de las pruebas preformales (p1p) sobre las formales (p1f); en todos los casos a partir de los estadísticos de asimetría y curtosis comprobamos que se encuentran en el intervalo $(-2,2)$ con lo que podemos aceptar que cada una de estas muestras sigue una distribución normal (cf. Harvey (1993), p. 45). A continuación vemos que en todos los casos se puede aceptar, basados en esa normalidad, una igualdad de medias y de desviaciones típicas; pero con el test de Kolmogorov comprobamos que las dos variables no 
tienen una misma distribución, y en los gráficos de la distribución apreciamos que la de las pruebas preformales siempre está desplazada a la derecha respecto de las formales, lo que significa unas mejores puntuaciones de las preformales sobre las formales.

\subsection{Comparaciones sobre la facilidad (F) de las pruebas}

Resultados conjuntos de los tres ciclos en la categoría Facilidad en ambas pruebas, preformal y formal, en cada uno de los dos teoremas (p1, p2) en que se han utilizado. La facilidad de la prueba preformal la denotamos con $\mathrm{p} 1 \mathrm{pF}$ y $\mathrm{p} 2 \mathrm{pF}$, y la de la prueba formal p1fF y $\mathrm{p} 2 \mathrm{fF}$.

\begin{tabular}{|c|c|c|c|c|}
\hline Facilidad & $\mathbf{p 1 p F}$ & $\mathbf{p 1 f F}$ & $\mathbf{p 2 p F}$ & $\mathbf{p 2 f F}$ \\
\hline $\mathbf{1}$ & 0 & 1 & 1 & 2 \\
\hline $\mathbf{2}$ & 6 & 1 & 2 & 5 \\
\hline $\mathbf{3}$ & 2 & 9 & 9 & 12 \\
\hline $\mathbf{4}$ & 12 & 13 & 7 & 13 \\
\hline $\mathbf{5}$ & 10 & 17 & 13 & 15 \\
\hline $\mathbf{6}$ & 13 & 11 & 17 & 12 \\
\hline $\mathbf{7}$ & 14 & 10 & 13 & 6 \\
\hline $\mathbf{8}$ & 10 & 5 & 4 & 2 \\
\hline $\mathbf{9}$ & 0 & 0 & 1 & 0 \\
\hline $\mathbf{1 0}$ & 0 & 0 & 0 & 0 \\
\hline Total & 67 & 67 & 67 & 67 \\
\hline
\end{tabular}

Tabla 6.1. Resultados conjuntos de los tres ciclos en la categoría facilidad en ambas pruebas en cada uno de los dos teoremas. 


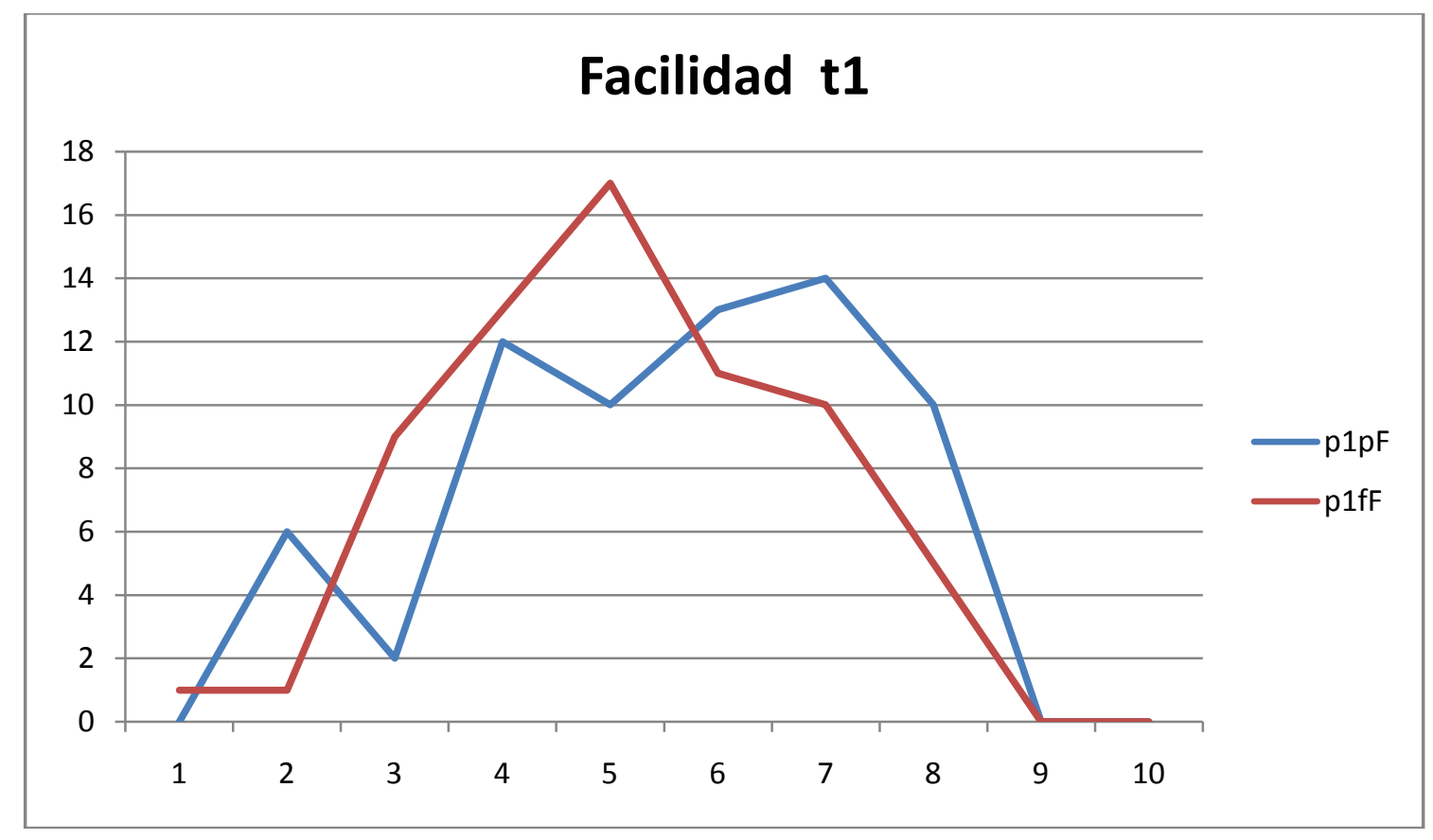

Gráfico 6.1. Frecuencias de los tres ciclos en ambas pruebas en la categoría facilidad, teorema 1

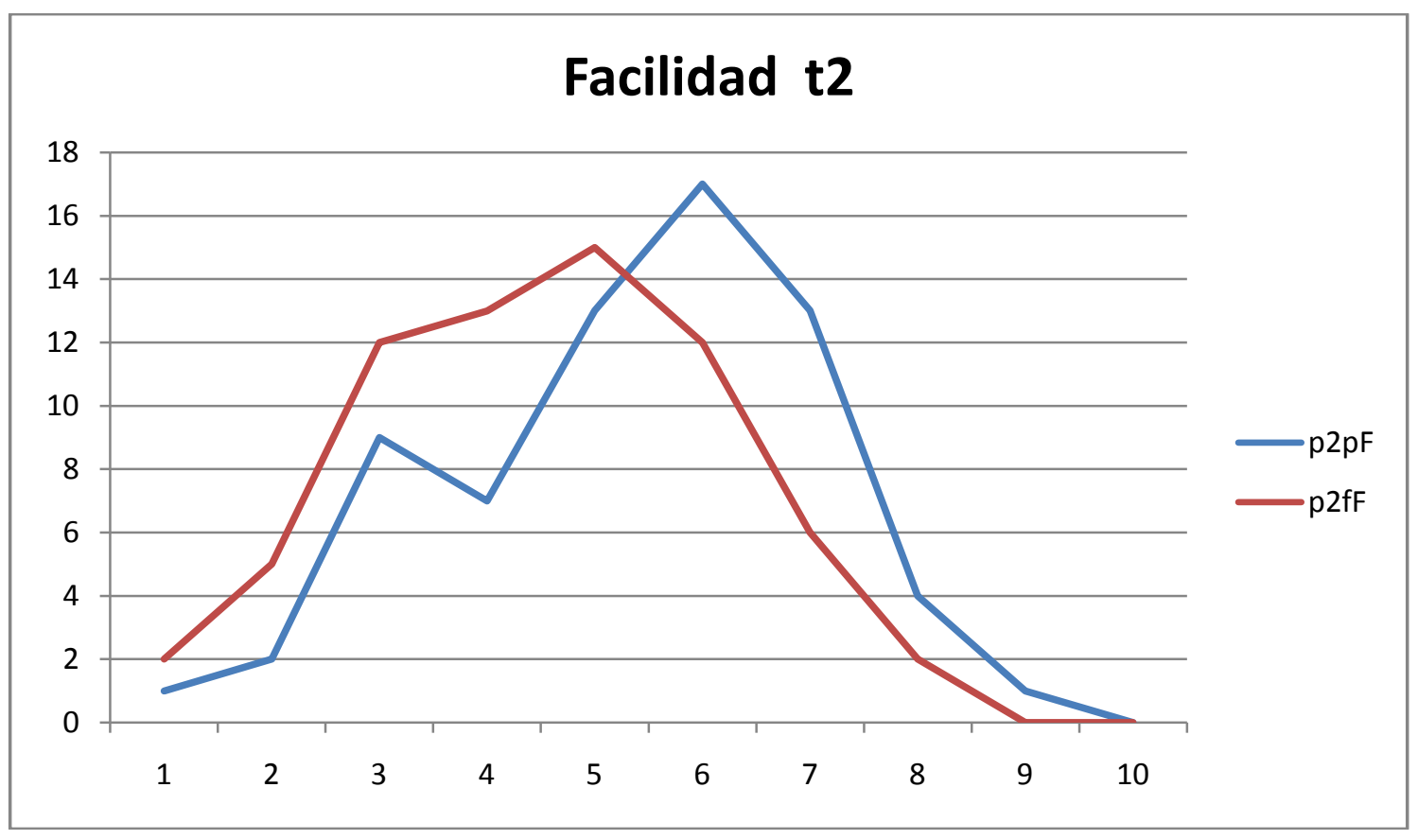

Gráfico 6.1 bis. Frecuencias de los tres ciclos en ambas pruebas en la categoría facilidad, teorema 2 
En la tabla 6.2 se recogen los estadísticos descriptivos, donde se aprecian unas mayores valoraciones de la prueba preformal siendo algo superior la media, la moda, la mediana y el segundo cuartil (cuartil superior). Por la asimetría y curtosis comprobamos que se encuentran en el intervalo $(-2,2)$ con lo que podemos aceptar que cada una de estas muestras sigue una distribución normal.

\section{TEOREMA 1}

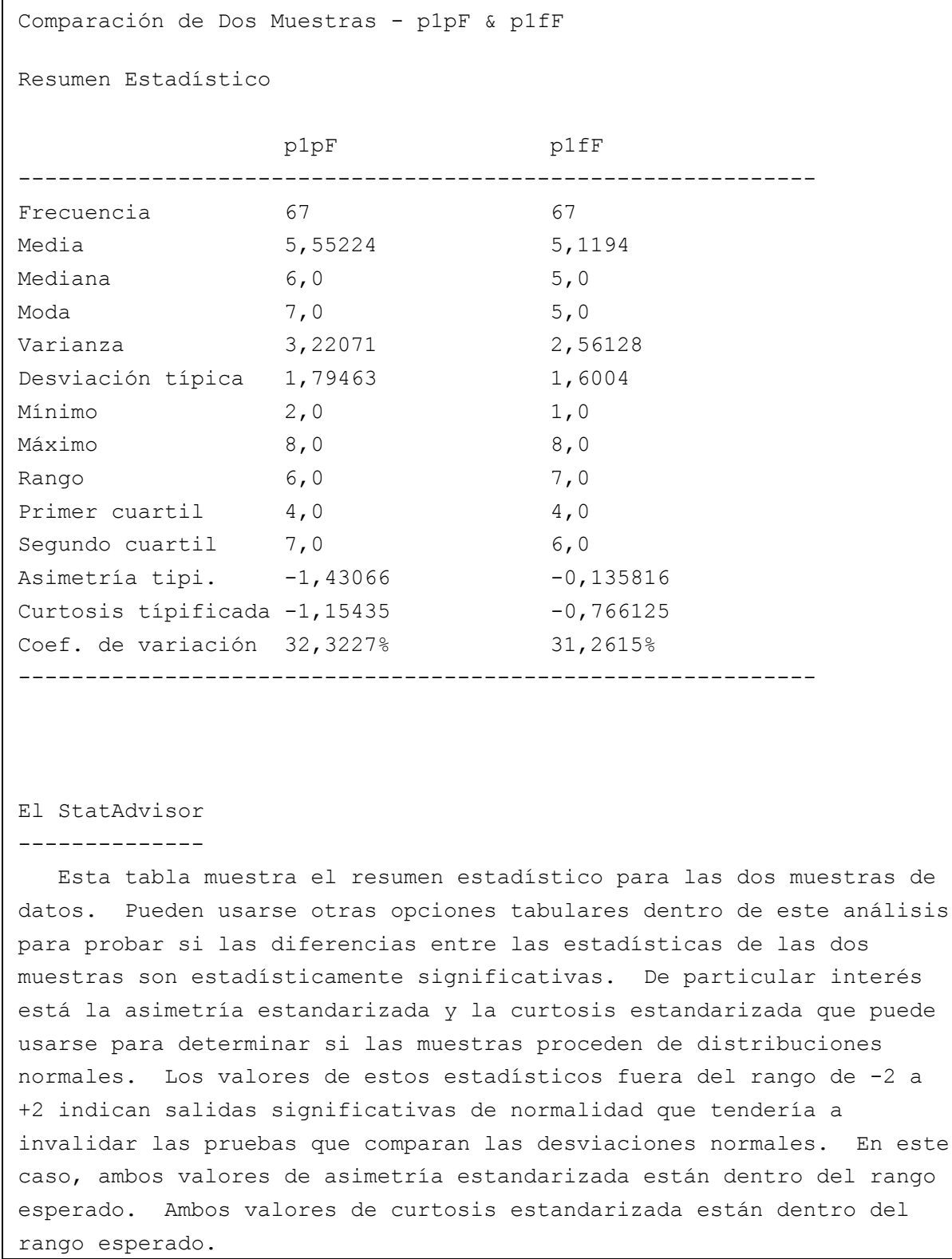

Tabla 6.2. Comparación de muestras p1pF-p1fF 


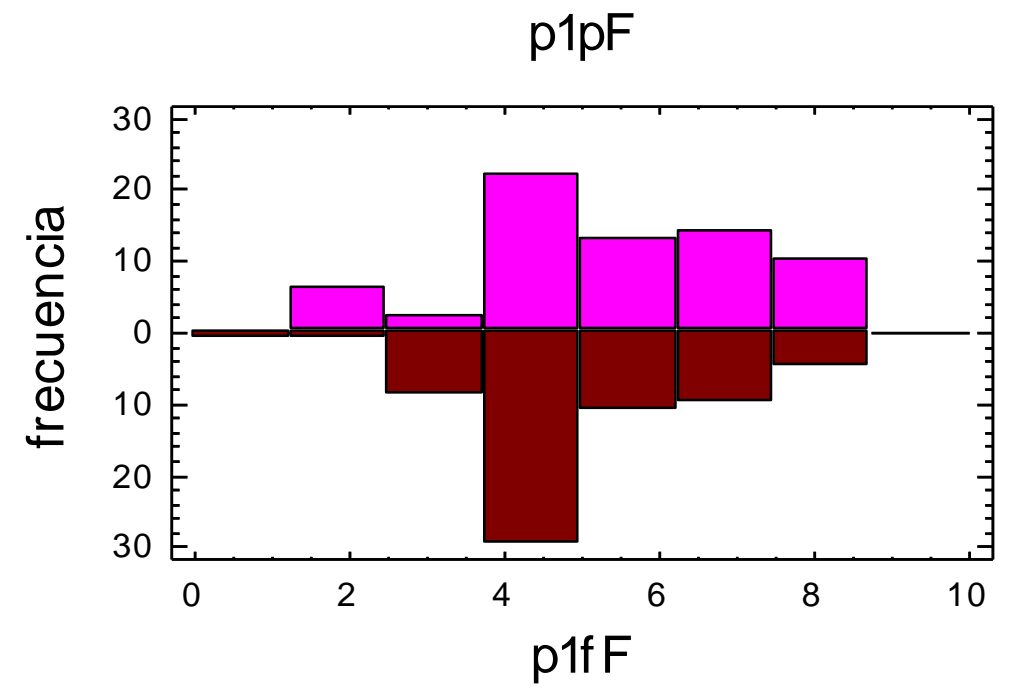

Gráfico 6.2. Comparación de histogramas p1pF-p1fF

Comparación de Medias

95,0\% intervalo de confianza para la media de plpF: 5,55224 +/- 0,437747

$[5,11449,5,98999]$

95, 0\% intervalo de confianza para la media de plfF: 5,1194 +/- 0,390369

$[4,72903,5,50977]$

95, 0\% intervalos de confianza para la diferencia de medias:

suponiendo varianzas iguales: 0,432836 +/- 0,581099 [-0,148264,1,01394]

contrastes $t$ de comparación de medias

Hipótesis nula: medial = media2

Hipótesis alt.: medial <> media2

suponiendo varianzas iguales: $t=1,4734 \quad$ P-Valor $=0,143023$

El StatAdvisor

Esta opción ejecuta el t-test para comparar las medias de las dos muestras. También establece los intervalos de confianza o los límites para cada media y para la diferencia entre las medias. De particular interés está el intervalo de confianza para la diferencia entre las medias, el cual se extiende desde $-0,148264$ hasta 1,01394. Dado que el intervalo contiene el valor 0.0, no existe diferencia estadísticamente significativa entre las medias de las dos muestras para un nivel de confianza del 95,0\%.

También puede aplicarse un t-test para probar una hipótesis

específica sobre la diferencia entre las medias de las poblaciones de 
las que proceden las dos muestras. En este caso, el test se ha

realizado para determinar si la diferencia entre las dos medias es

igual a 0,0 frente a la hipótesis alternativa en la que la diferencia

no es igual 0,0. Puesto que el p-valor calculado no es inferior a

0,05, no podemos rechazar la hipótesis nula.

NOTA: estos resultados asumen la igualdad de varianzas en las dos

muestras. En este caso, esa asunción parece ser razonable teniendo en

cuenta los resultados del F-test para comparar las desviaciones

típicas. Puede ver los resultados de este test seleccionando

Comparación de Desviaciones Típicas del menú Opciones Tabulares.

Tabla 6.3. Comparación de medias p1pF-p1ff

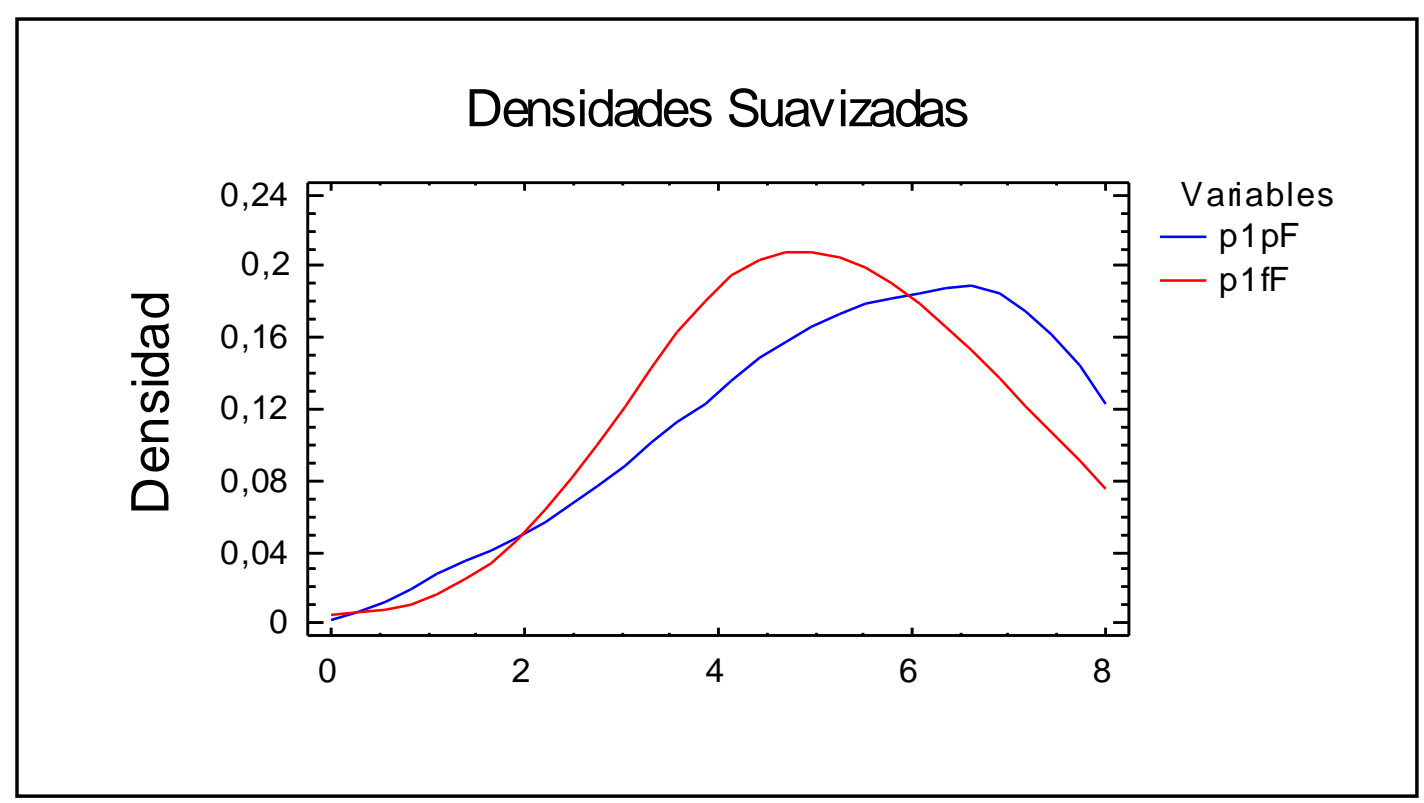

Gráfico 6.3. Comparación de densidades suavizadas p1pF-p1fF

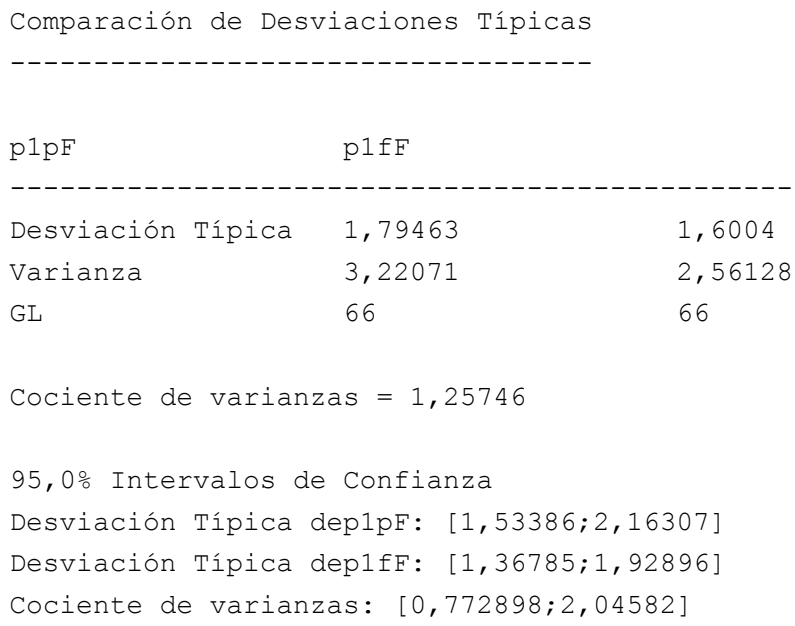




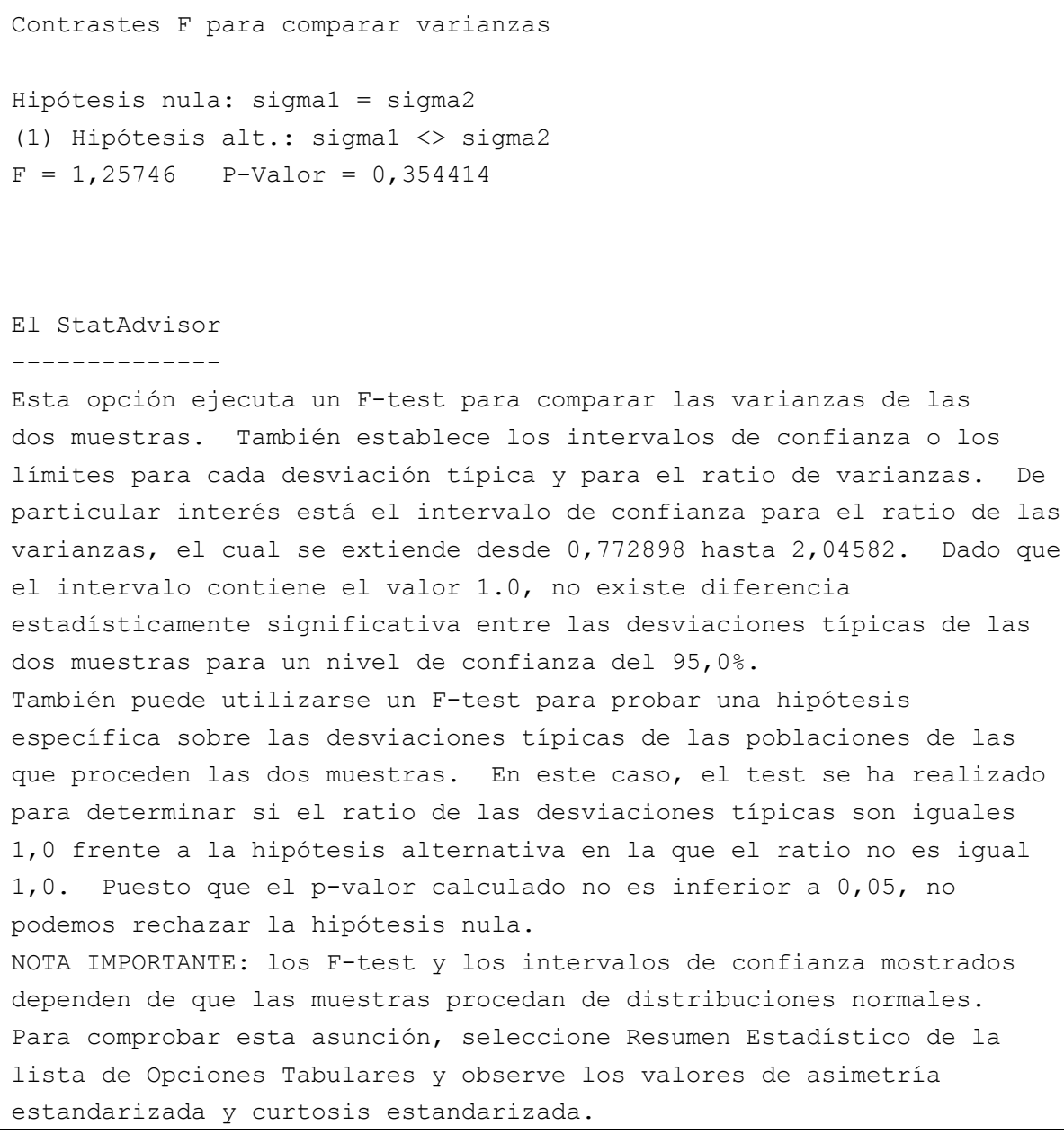

Tabla 6.4. Comparación de desviaciones típicas p1pF-p1fF

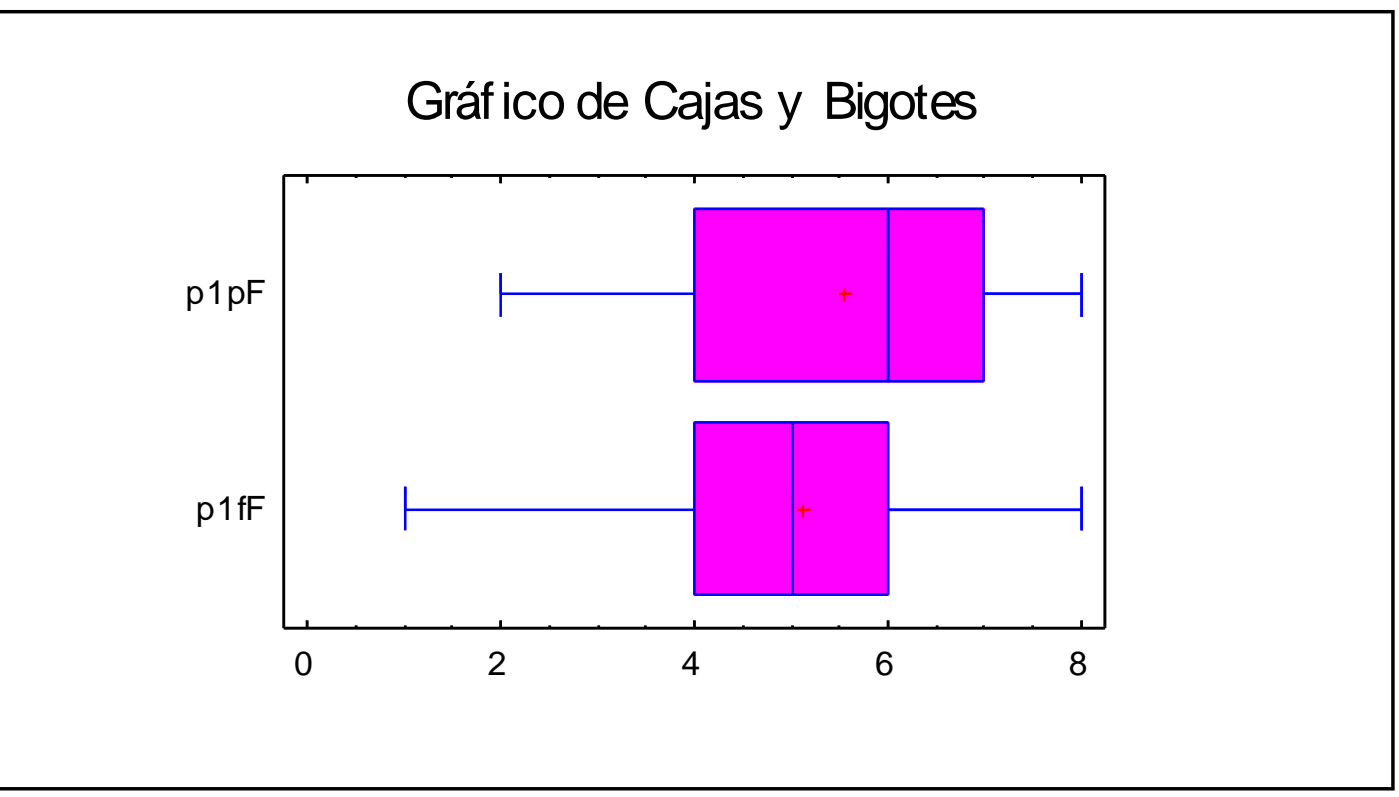

Gráfico 6.4. Comparación de diagramas de cajas y bigotes p1pF-p1fF 


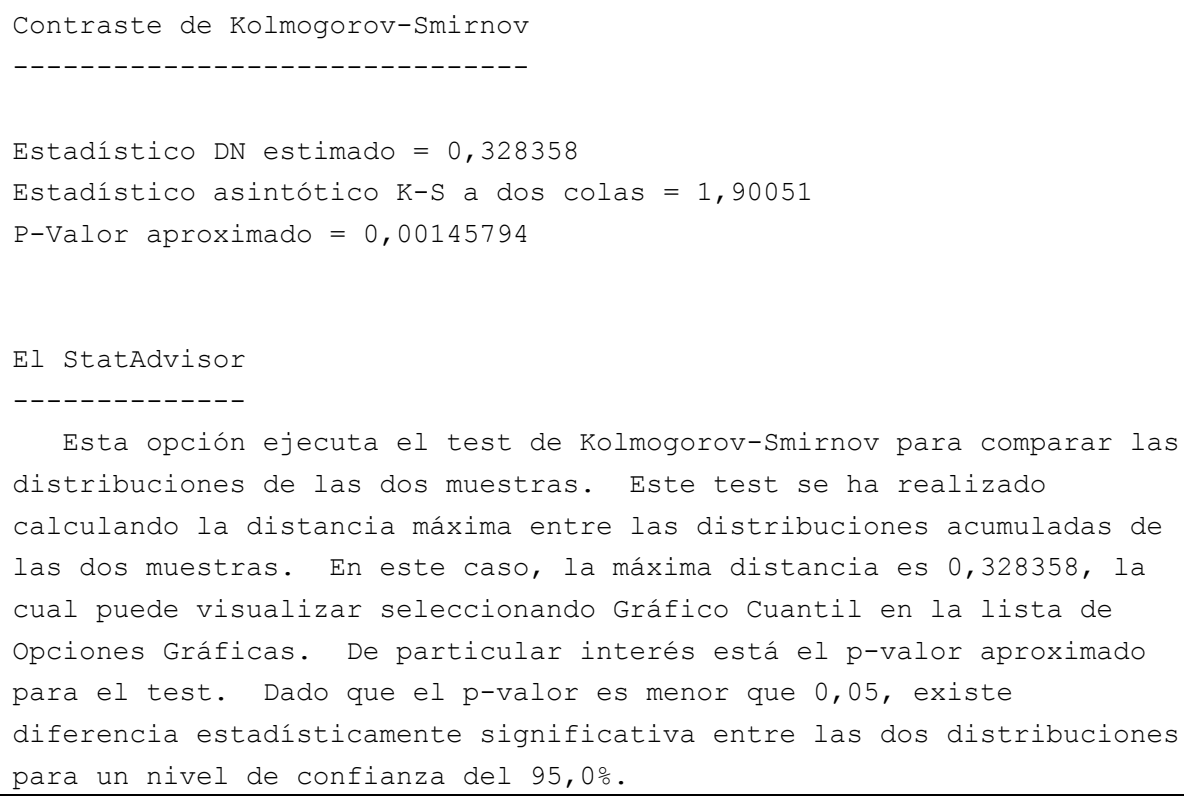

Tabla 6.5. Contraste de Kolmogorov-Smirnov p1pF-p1fF

\section{Función de distribución}

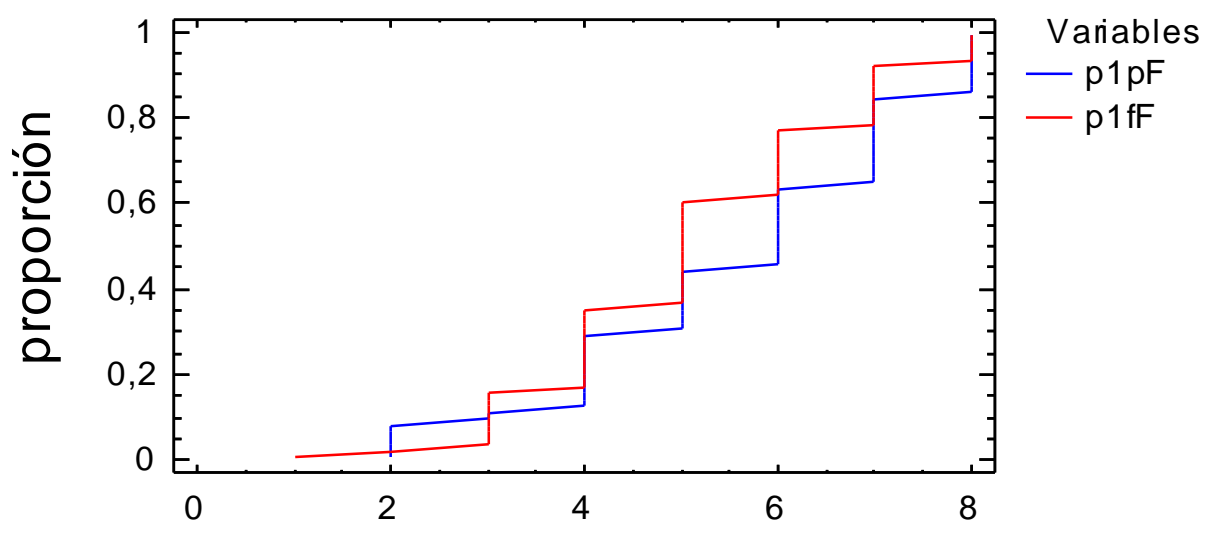

\section{Gráfico 6.5. Comparación de funciones de distribución p1pF-p1fF}

Los contrastes de igualdad de medias y de desviaciones típicas, basados en la normalidad de las distribuciones, no permiten rechazar la hipótesis nula, de modo que aceptaríamos que ambas medidas son iguales para las dos muestras de datos. Sin embargo el test de Kolmogorov-Smirnov nos permite rechazar la igualdad de distribuciones, y tanto en el gráfico de densidades suavizadas como en el de cajas y 
bigotes y en la función de distribución se observa que la distribución para la prueba preformal está más a la derecha que la formal, situándose en valores mayores para las puntuaciones otorgadas a esta prueba.

\section{TEOREMA 2}

\begin{tabular}{|c|c|c|}
\hline \multirow[t]{2}{*}{ Resumen Estadístico } & & \\
\hline & $\mathrm{p} 2 \mathrm{pF}$ & $\mathrm{p} 2 \mathrm{fF}$ \\
\hline Frecuencia & 67 & 67 \\
\hline Media & 5,35821 & 4,55224 \\
\hline Mediana & 6,0 & 5,0 \\
\hline Moda & 6,0 & 5,0 \\
\hline Varianza & 2,86974 & 2,70556 \\
\hline Desviación típica & 1,69403 & 1,64486 \\
\hline Mínimo & 1,0 & 1,0 \\
\hline Máximo & 9,0 & 8,0 \\
\hline Rango & 8,0 & 7,0 \\
\hline Primer cuartil & 4,0 & 3,0 \\
\hline Segundo cuartil & 7,0 & 6,0 \\
\hline Asimetría tipi. & $-1,18751$ & $-0,142121$ \\
\hline Curtosis típificada & $-0,583962$ & $-0,882333$ \\
\hline Coef. de variación & $31,6156 \%$ & $36,133 \%$ \\
\hline
\end{tabular}

\section{El StatAdvisor}

Esta tabla muestra el resumen estadístico para las dos muestras de datos. Pueden usarse otras opciones tabulares dentro de este análisis para probar si las diferencias entre las estadísticas de las dos muestras son estadísticamente significativas. De particular interés está la asimetría estandarizada y la curtosis estandarizada que puede usarse para determinar si las muestras proceden de distribuciones normales. Los valores de estos estadísticos fuera del rango de -2 a +2 indican salidas significativas de normalidad que tendería a invalidar las pruebas que comparan las desviaciones normales. En este caso, ambos valores de asimetría estandarizada están dentro del rango esperado. Ambos valores de curtosis estandarizada están dentro del rango esperado.

Tabla 6.6. Comparación de muestras p2pF-p2fF 


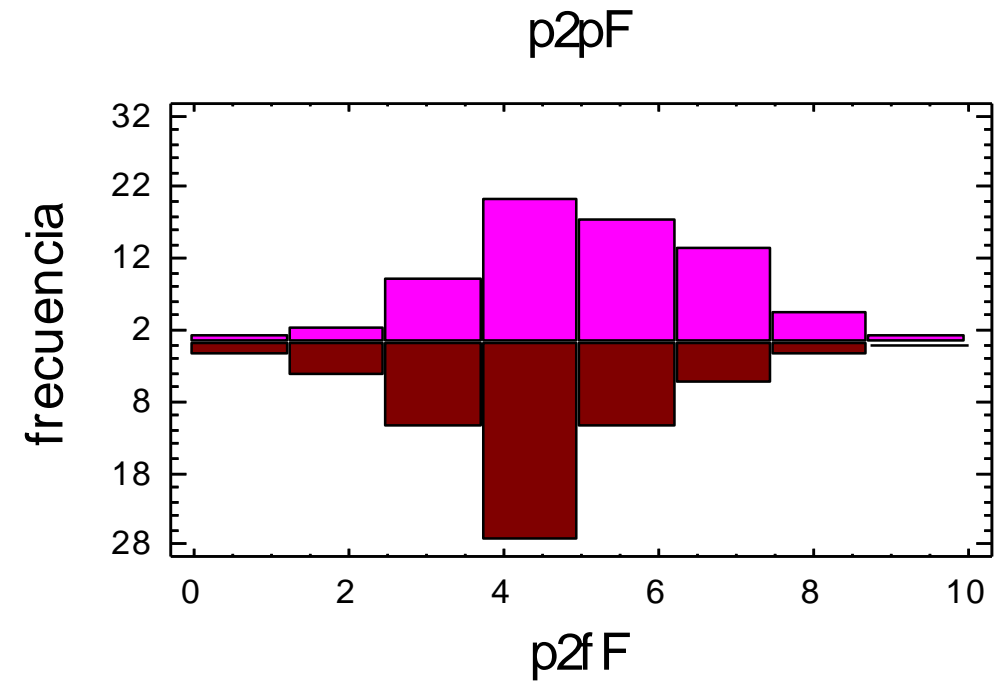

Gráfico 6.6. Comparación de histogramas p2pF-p2fF

Comparación de Medias

95, 0\% intervalo de confianza para la media de p2pF: 5,35821 +/- 0,413208

$[4,945,5,77142]$

95, 0\% intervalo de confianza para la media de p2fF: 4,55224 +/- 0,401214

$[4,15103,4,95345]$

95, 0\% intervalos de confianza para la diferencia de medias:

suponiendo varianzas iguales: 0,80597 +/- 0,570618 [0,235352,1,37659]

contrastes $t$ de comparación de medias

Hipótesis nula: medial = media2

Hipótesis alt.: medial <> media2

suponiendo varianzas iguales: $t=2,79397 \quad \mathrm{P}$-Valor $=0,00598228$

El StatAdvisor

$--------------$

Esta opción ejecuta el t-test para comparar las medias de las dos muestras. También establece los intervalos de confianza o los límites para cada media y para la diferencia entre las medias. De particular interés está el intervalo de confianza para la diferencia entre las medias, el cual se extiende desde 0,235352 hasta 1,37659. Dado que el intervalo no contiene el valor 0.0, existe diferencia estadísticamente significativa entre las medias de las dos muestras para un nivel de confianza del 95,0\%.

También puede aplicarse un t-test para probar una hipótesis específica sobre la diferencia entre las medias de las poblaciones de las que proceden las dos muestras. En este caso, el test se ha realizado para determinar si la diferencia entre las dos medias es 
igual a 0,0 frente a la hipótesis alternativa en la que la diferencia

no es igual 0,0. Puesto que el p-valor calculado es inferior a 0,05,

podemos rechazar la hipótesis nula en favor de la alternativa.

NOTA: estos resultados asumen la igualdad de varianzas en las dos

muestras. En este caso, esa asunción parece ser razonable teniendo en

cuenta los resultados del F-test para comparar las desviaciones

típicas. Puede ver los resultados de este test seleccionando

Comparación de Desviaciones Típicas del menú Opciones Tabulares.

Tabla 6.7. Comparación de medias p2pF-p2ff

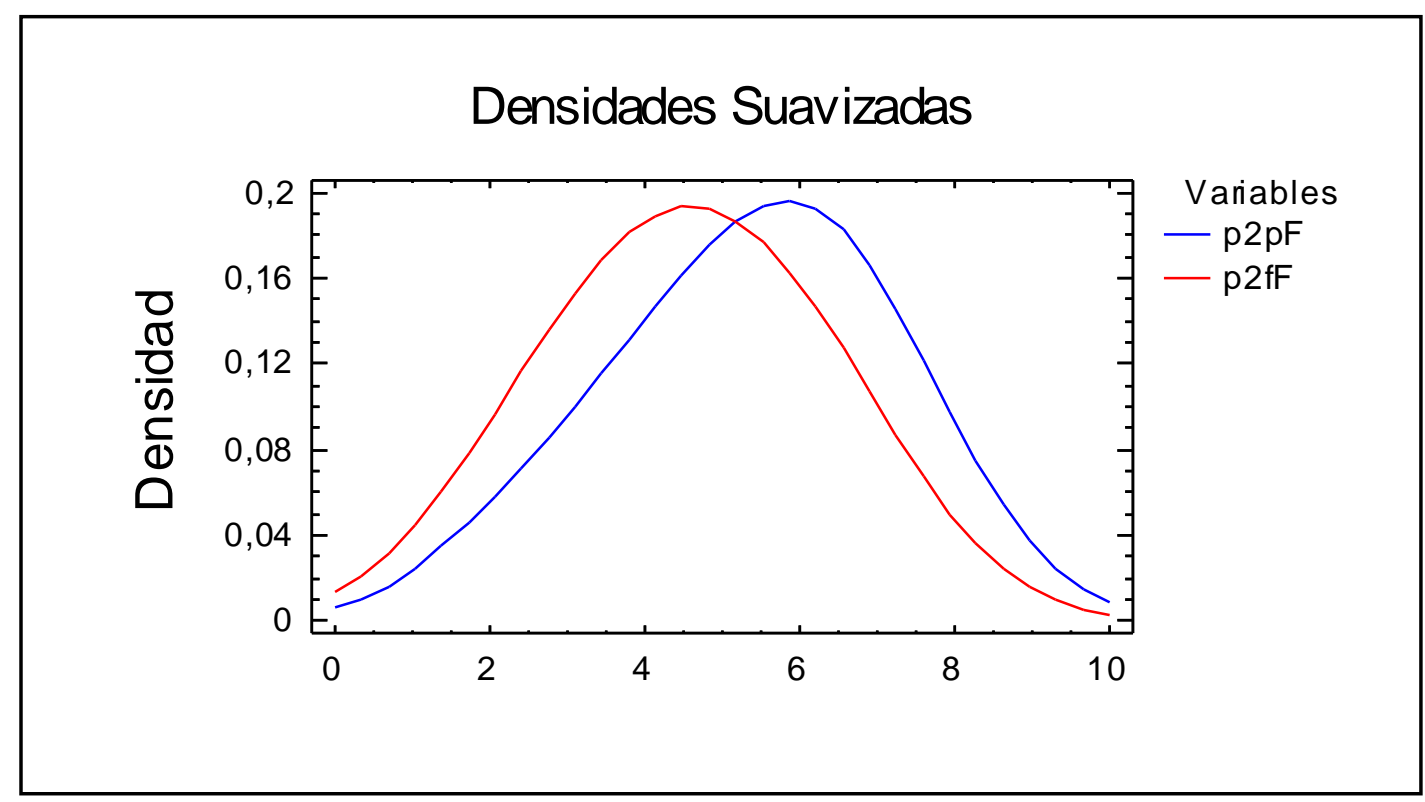

Gráfico 6.7. Comparación de densidades suavizadas p2pF-p2fF

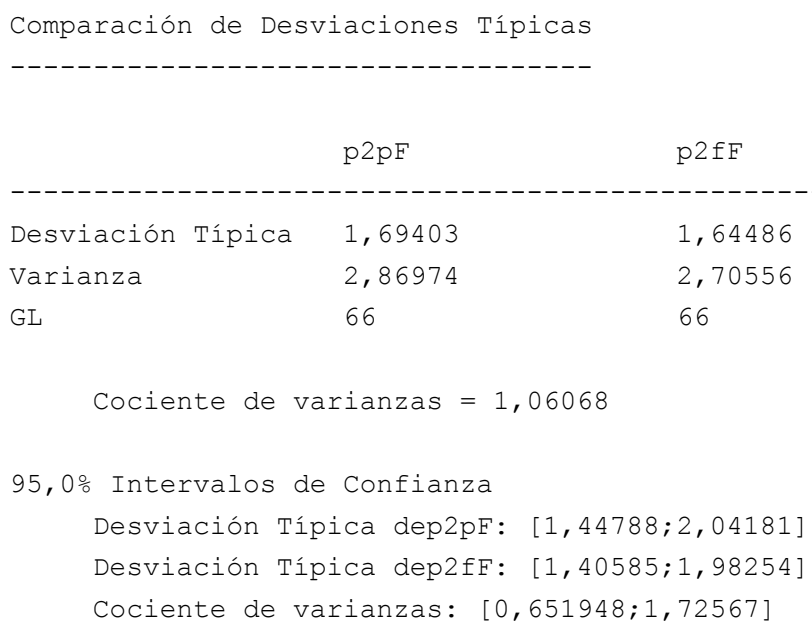


Contrastes F para comparar varianzas

Hipótesis nula: sigmal = sigma2

(1) Hipótesis alt.: sigmal <> sigma2

$$
\mathrm{F}=1,06068 \quad \mathrm{P} \text {-Valor }=0,811586
$$

El StatAdvisor

\section{El statidvisor}

Esta opción ejecuta un F-test para comparar las varianzas de las dos muestras. También establece los intervalos de confianza o los límites para cada desviación típica y para el ratio de varianzas. De particular interés está el intervalo de confianza para el ratio de las varianzas, el cual se extiende desde 0,651948 hasta 1,72567. Dado que el intervalo contiene el valor 1.0, no existe diferencia estadísticamente significativa entre las desviaciones típicas de las dos muestras para un nivel de confianza del 95,0\%.

También puede utilizarse un F-test para probar una hipótesis específica sobre las desviaciones típicas de las poblaciones de las que proceden las dos muestras. En este caso, el test se ha realizado para determinar si el ratio de las desviaciones típicas son iguales 1,0 frente a la hipótesis alternativa en la que el ratio no es igual 1,0. Puesto que el p-valor calculado no es inferior a 0,05, no podemos rechazar la hipótesis nula.

NOTA IMPORTANTE: los F-test y los intervalos de confianza mostrados dependen de que las muestras procedan de distribuciones normales. Para comprobar esta asunción, seleccione Resumen Estadístico de la lista de Opciones Tabulares y observe los valores de asimetría estandarizada y curtosis estandarizada.

Tabla 6.8. Comparación de desviaciones típicas p2pF-p2fF

\section{Gráf ico de Cajas y Bigotes}

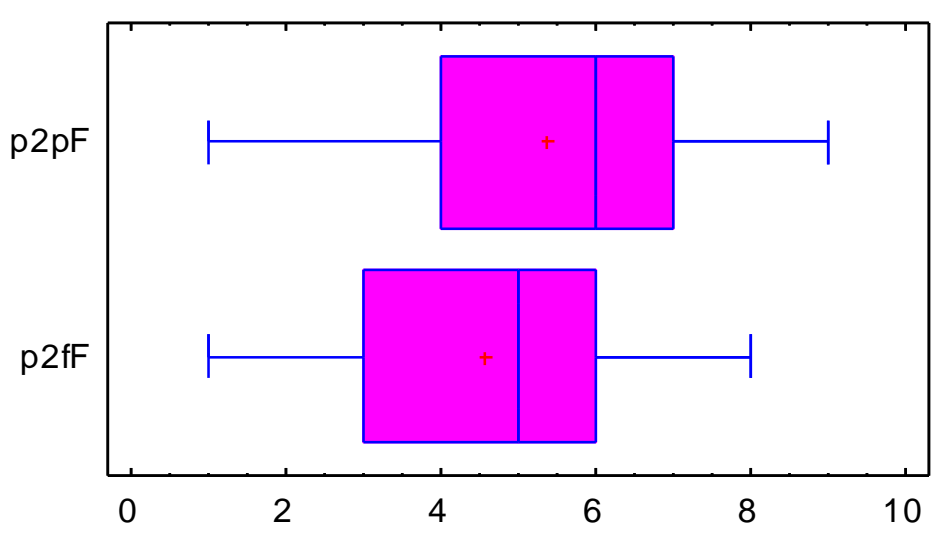

Gráfico 6.8. Comparación de diagramas de cajas y bigotes p2pF-p2fF 
Contraste de Kolmogorov-Smirnov

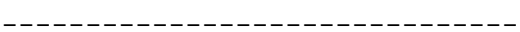

Estadístico DN estimado $=0,41791$

Estadístico asintótico $\mathrm{K}-\mathrm{S}$ a dos colas $=2,41883$

P-Valor aproximado $=0,0000165629$

El StatAdvisor

Esta opción ejecuta el test de Kolmogorov-Smirnov para comparar las distribuciones de las dos muestras. Este test se ha realizado

calculando la distancia máxima entre las distribuciones acumuladas de

las dos muestras. En este caso, la máxima distancia es 0,41791, la

cual puede visualizar seleccionando Gráfico Cuantil en la lista de

opciones Gráficas. De particular interés está el p-valor aproximado

para el test. Dado que el p-valor es menor que 0,05, existe

diferencia estadísticamente significativa entre las dos distribuciones

para un nivel de confianza del 95,0\%.

Tabla 6.9. Contraste de Kolmogorov-Smirnov p2pF-p2fF

Función de distribución

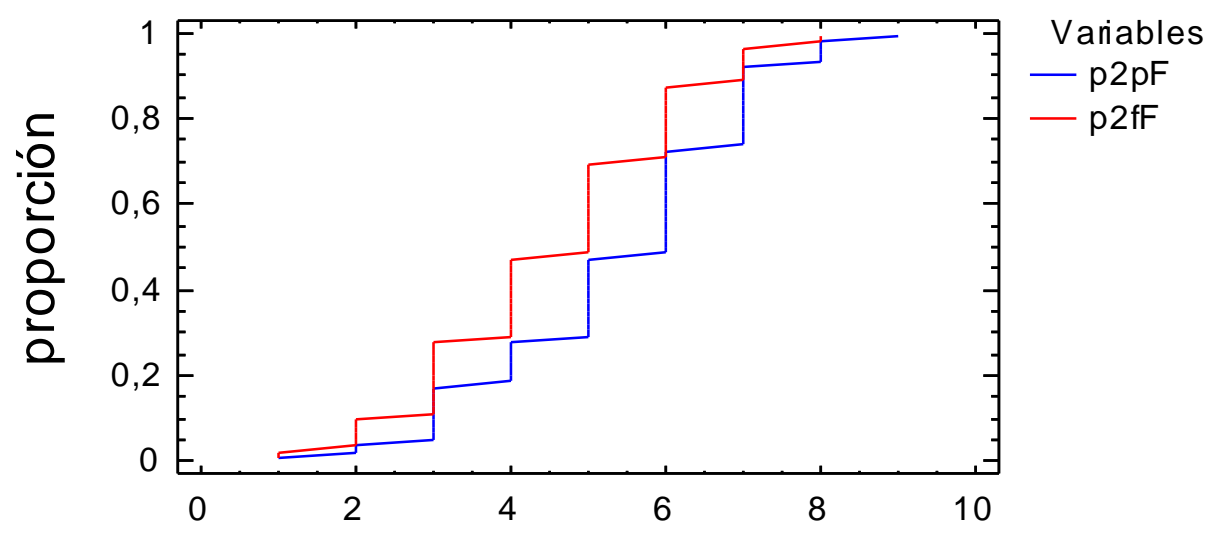

Gráfico 6.9. Comparación de funciones de distribución p2pF-p2fF 


\subsection{Comparaciones sobre el gusto (G) de las pruebas}

Resultados conjuntos de los tres ciclos en la categoría Gusto en ambas pruebas, preformal y formal, en cada uno de los dos teoremas $(\mathbf{p 1}, \mathbf{p 2})$ en que se han utilizado. El gusto de la prueba preformal lo denotamos con p1pG y p2pG, y el de la prueba formal p1fG y p2fG.

\begin{tabular}{|c|c|c|c|c|}
\hline Gusto & p1pG & p1fG & p2pG & p2fG \\
\hline $\mathbf{1}$ & 3 & 2 & 2 & 3 \\
\hline $\mathbf{2}$ & 4 & 6 & 4 & 4 \\
\hline $\mathbf{3}$ & 2 & 3 & 6 & 12 \\
\hline $\mathbf{4}$ & 7 & 11 & 8 & 9 \\
\hline $\mathbf{5}$ & 16 & 13 & 14 & 15 \\
\hline $\mathbf{6}$ & 15 & 13 & 15 & 14 \\
\hline $\mathbf{7}$ & 12 & 10 & 14 & 9 \\
\hline $\mathbf{8}$ & 7 & 8 & 3 & 1 \\
\hline $\mathbf{9}$ & 1 & 1 & 1 & 0 \\
\hline $\mathbf{1 0}$ & 0 & 0 & 0 & 0 \\
\hline Total & 67 & 67 & 67 & 67 \\
\hline
\end{tabular}

Tabla 6.10. Resultados conjuntos de los tres ciclos en la categoría gusto en ambas pruebas en cada uno de los dos teoremas

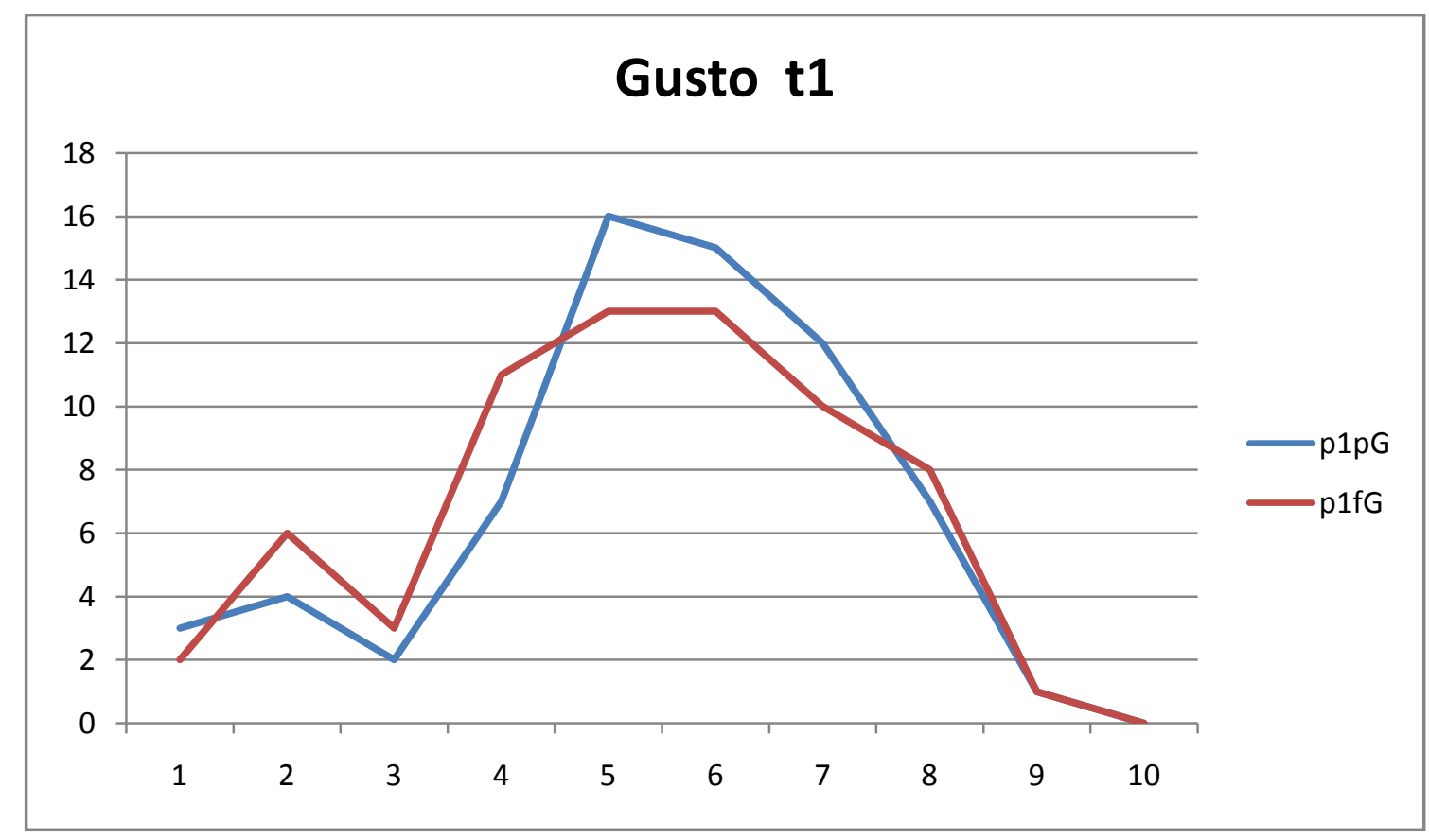

Gráfico 6.10. Frecuencias de los tres ciclos en ambas pruebas en la categoría gusto, teorema 1 


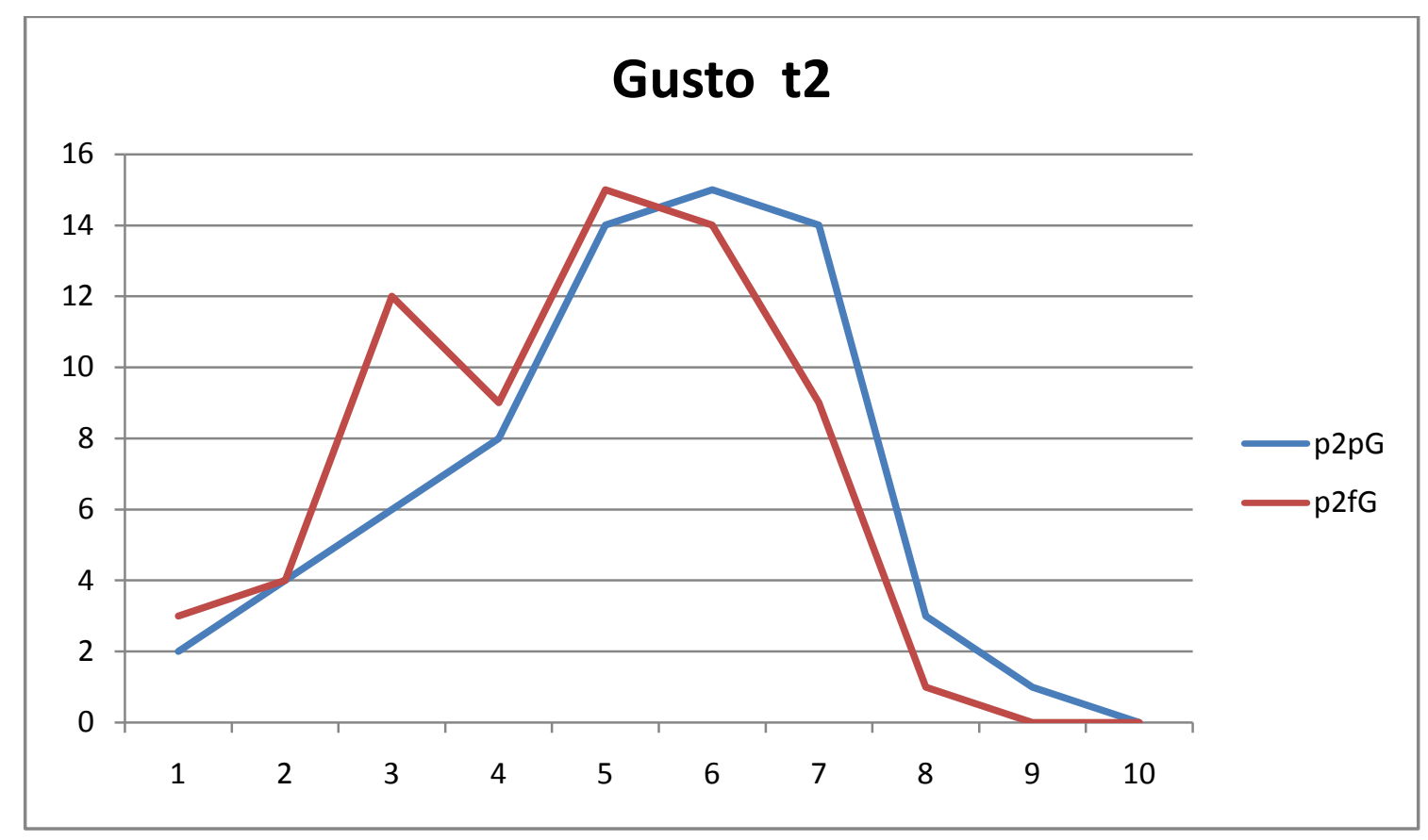

\section{Gráfico 6.10 bis. Frecuencias de los tres ciclos en ambas pruebas en la categoría gusto, teorema 2}

En la tabla 6.11 se recogen los estadísticos descriptivos, donde se aprecian unas mayores valoraciones de la prueba preformal siendo algo superior la media, la moda, la mediana y el primer cuartil (cuartil inferior). Por la asimetría y curtosis comprobamos que se encuentran en el intervalo $(-2,2)$ con lo que podemos aceptar que cada una de estas muestras sigue una distribución normal.

\section{TEOREMA 1}

\begin{tabular}{|c|c|c|}
\hline \multicolumn{3}{|l|}{ Resumen Estadístico } \\
\hline & $\mathrm{plpG}$ & $\mathrm{p} 1 \mathrm{fG}$ \\
\hline Frecuencia & 67 & 67 \\
\hline Media & 5,43284 & 5,26866 \\
\hline Mediana & 6,0 & 5,0 \\
\hline Moda & 5,0 & \\
\hline Varianza & 3,46133 & 3,71461 \\
\hline Desviación típica & 1,86046 & 1,92733 \\
\hline Mínimo & 1,0 & 1,0 \\
\hline Máximo & 9,0 & 9,0 \\
\hline
\end{tabular}




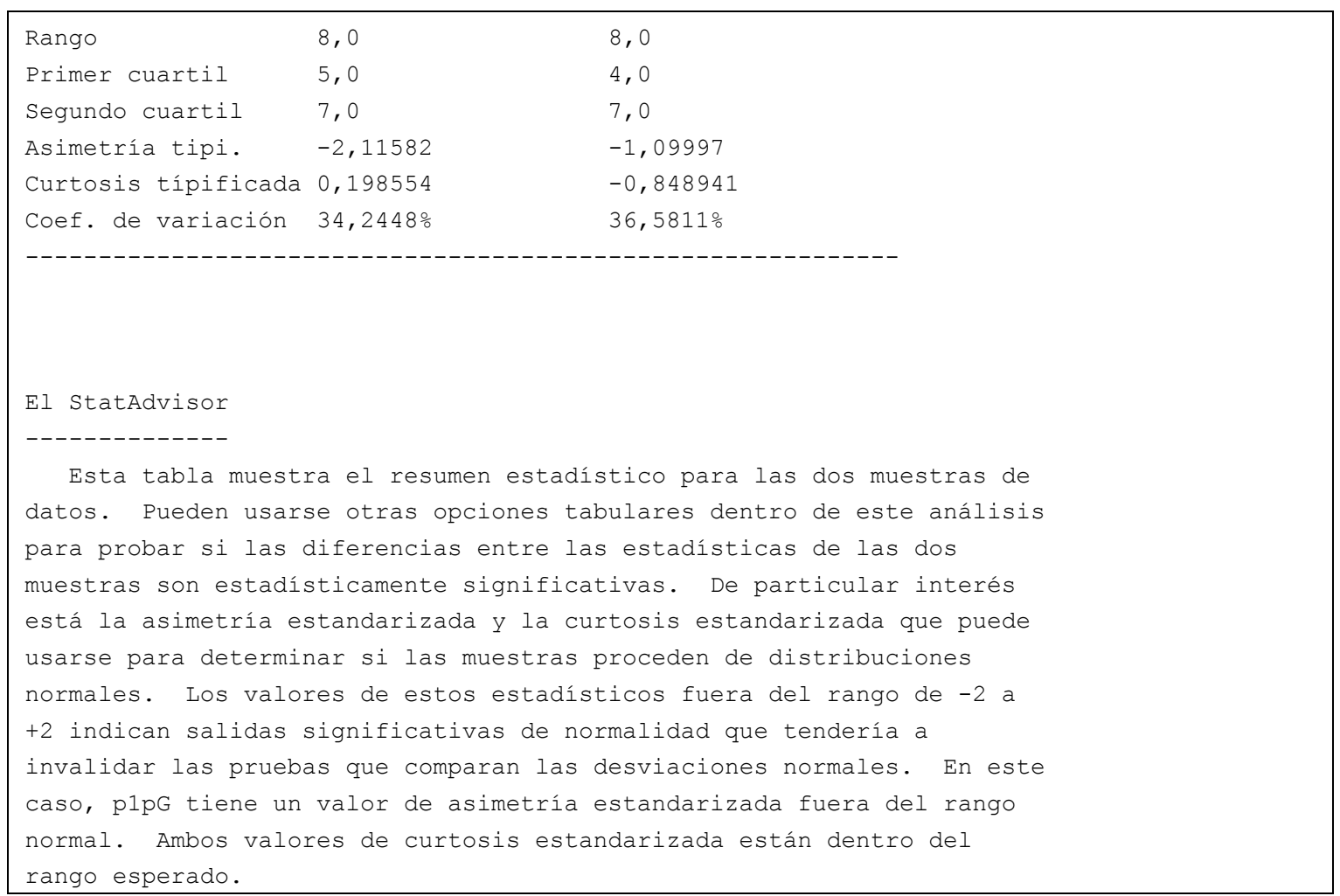

Tabla 6.11. Comparación de muestras p1pG-p1fG

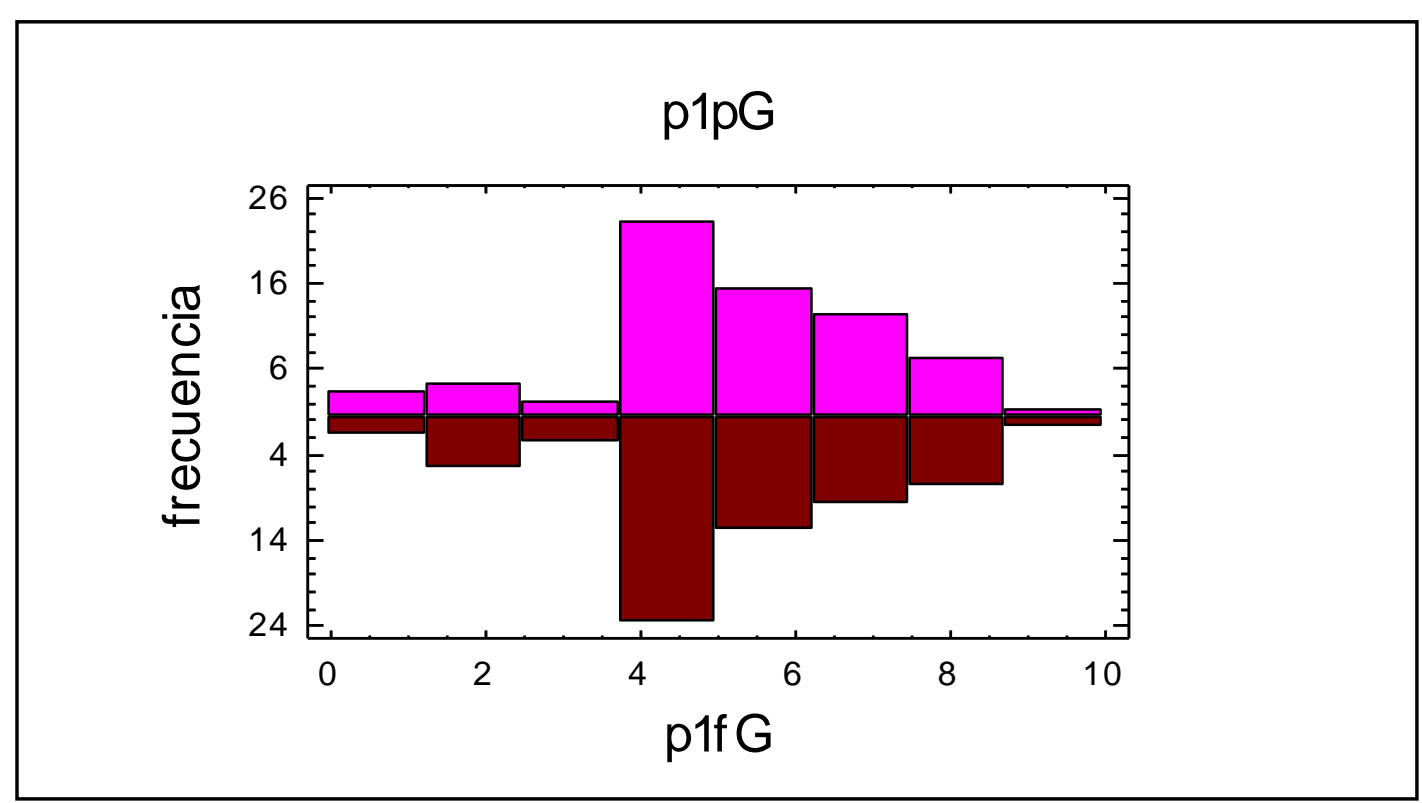

Gráfico 6.11. Comparación de histogramas p1pG-p1fG 


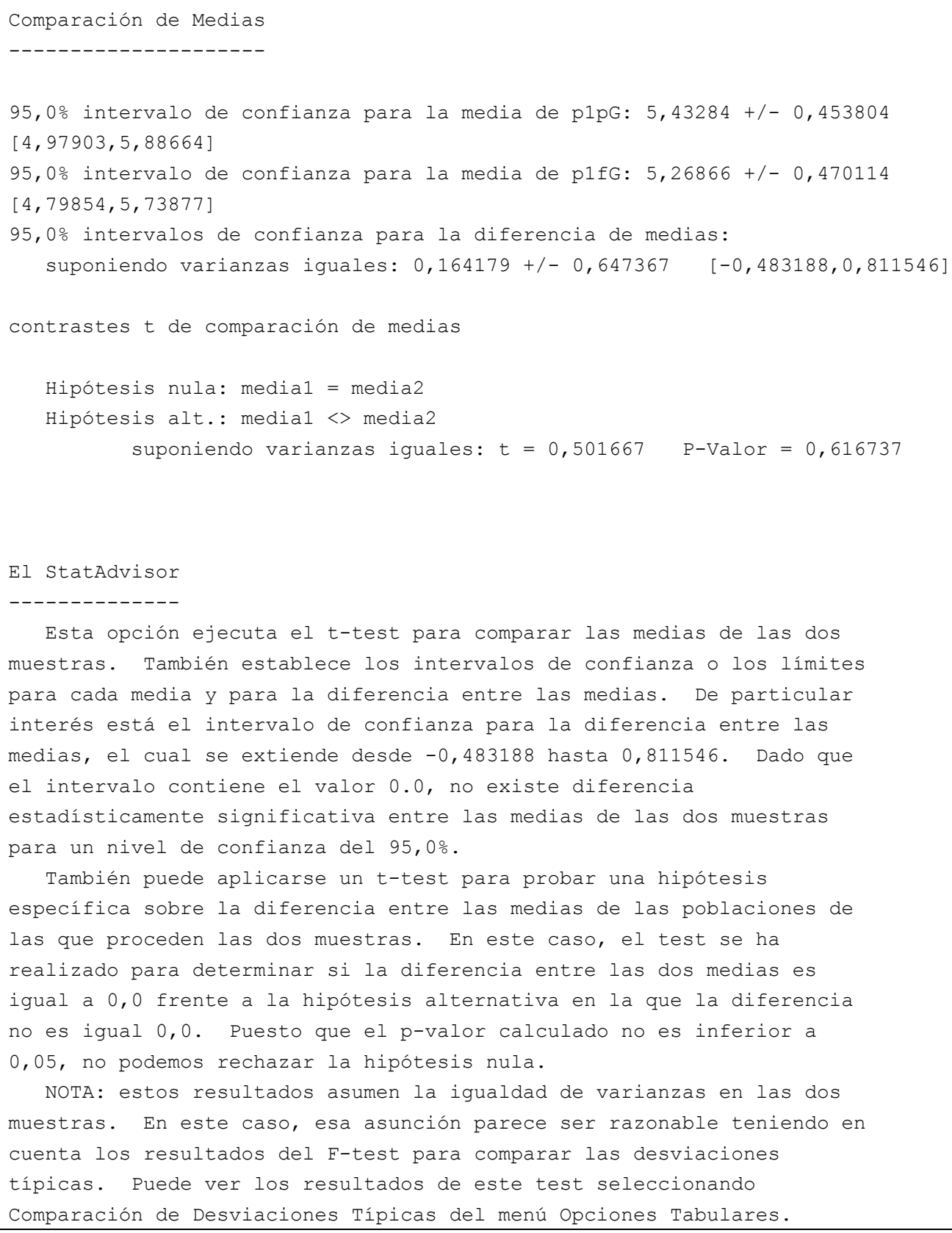

Tabla 6.12. Comparación de medias p1pG-p1fG 


\section{Densidades Suavizadas}

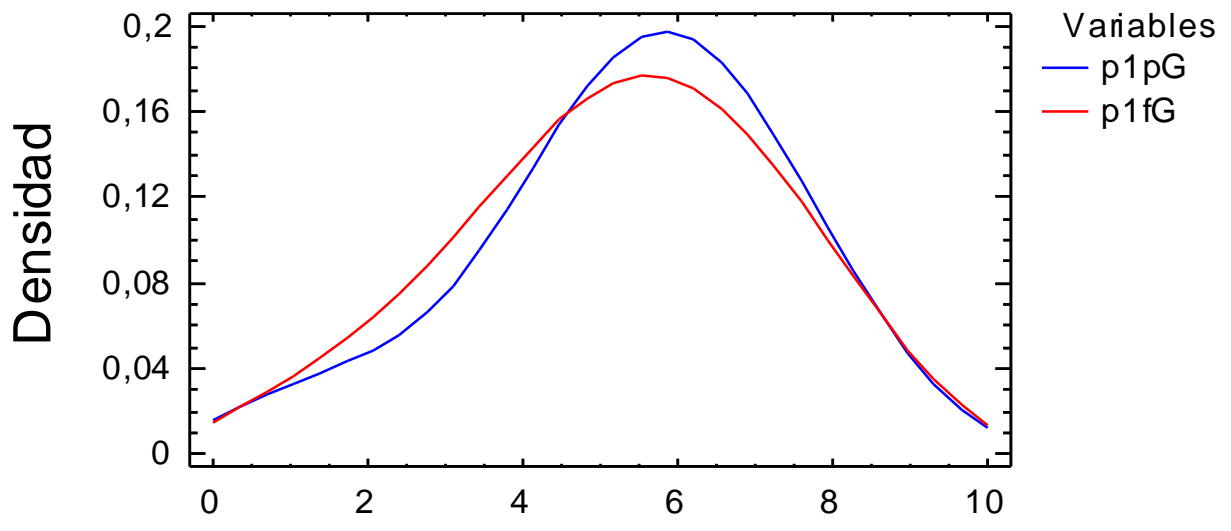

Gráfico 6.12. Comparación de densidades suavizadas p1pG-p1fG

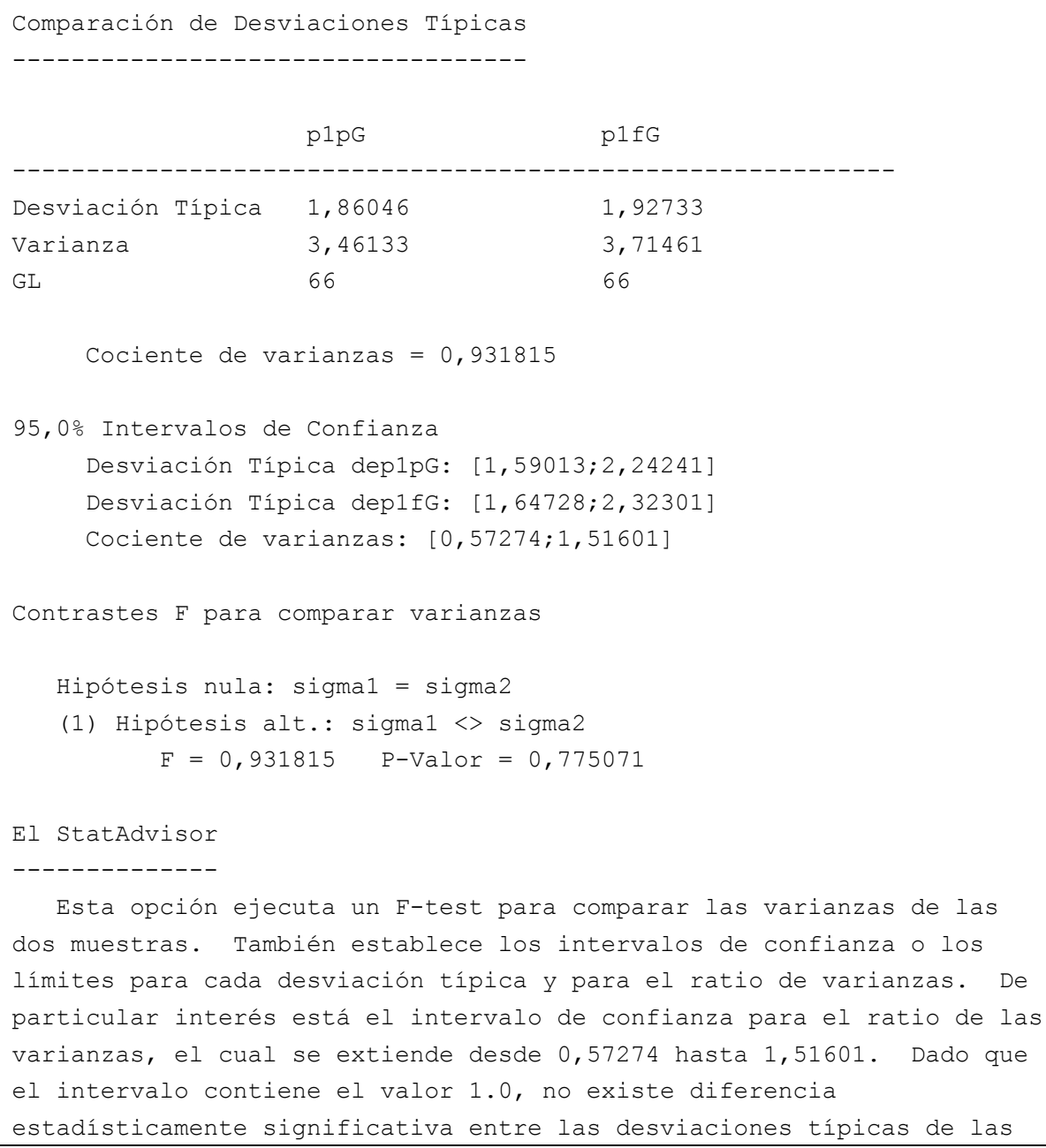

El StatAdvisor

Esta opción ejecuta un F-test para comparar las varianzas de las dos muestras. También establece los intervalos de confianza o los límites para cada desviación típica y para el ratio de varianzas. De particular interés está el intervalo de confianza para el ratio de las varianzas, el cual se extiende desde 0,57274 hasta 1,51601. Dado que el intervalo contiene el valor 1.0, no existe diferencia estadísticamente significativa entre las desviaciones típicas de las 
dos muestras para un nivel de confianza del 95,0\%.

También puede utilizarse un F-test para probar una hipótesis

específica sobre las desviaciones típicas de las poblaciones de las

que proceden las dos muestras. En este caso, el test se ha realizado

para determinar si el ratio de las desviaciones típicas son iguales

1,0 frente a la hipótesis alternativa en la que el ratio no es igual

1,0. Puesto que el p-valor calculado no es inferior a 0,05, no

podemos rechazar la hipótesis nula.

NOTA IMPORTANTE: los F-test y los intervalos de confianza mostrados

dependen de que las muestras procedan de distribuciones normales.

Para comprobar esta asunción, seleccione Resumen Estadístico de la

lista de Opciones Tabulares y observe los valores de asimetría

estandarizada y curtosis estandarizada.

Tabla 6.13. Comparación de desviaciones típicas p1pG-p1fG

\section{Gráf ico de Cajas y Bigotes}

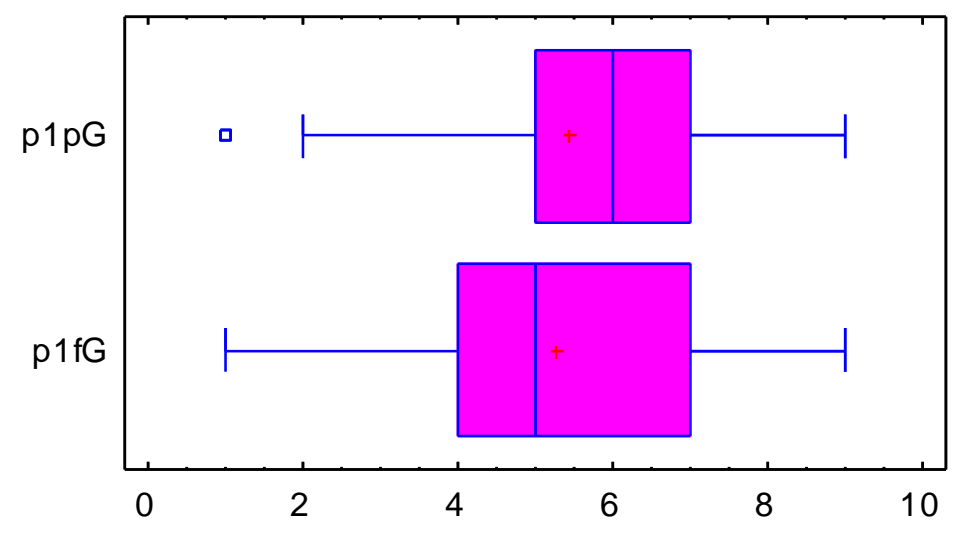

Gráfico 6.13. Comparación de diagramas de cajas y bigotes p1pG-p1fG

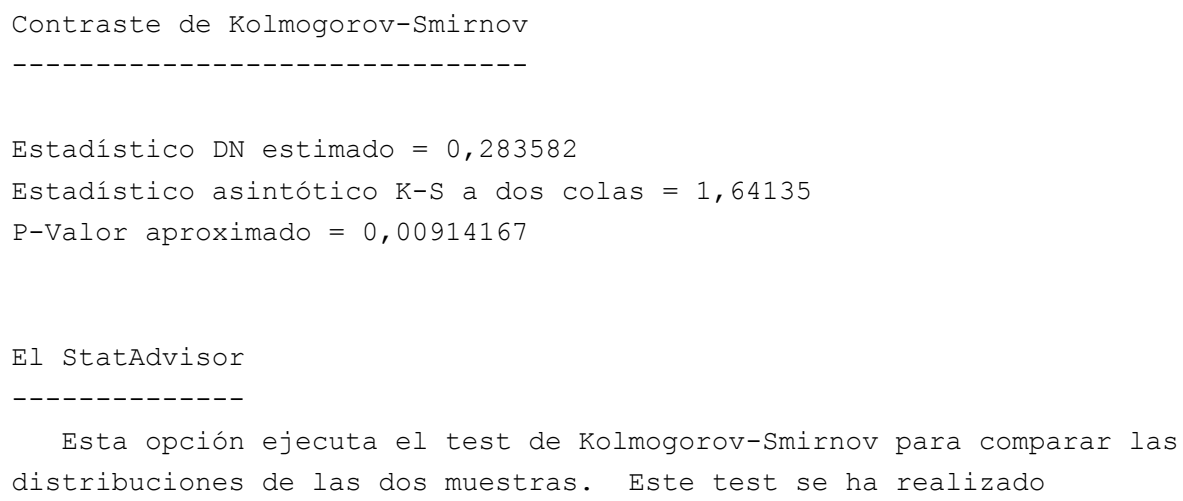

El StatAdvisor

Esta opción ejecuta el test de Kolmogorov-Smirnov para comparar las distribuciones de las dos muestras. Este test se ha realizado 
calculando la distancia máxima entre las distribuciones acumuladas de

las dos muestras. En este caso, la máxima distancia es 0,283582, la

cual puede visualizar seleccionando Gráfico Cuantil en la lista de

Opciones Gráficas. De particular interés está el p-valor aproximado

para el test. Dado que el p-valor es menor que 0,05, existe

diferencia estadísticamente significativa entre las dos distribuciones

para un nivel de confianza del 95,0\%.

Tabla 6.14. Contraste de Kolmogorov-Smirnov p1pG-p1fG

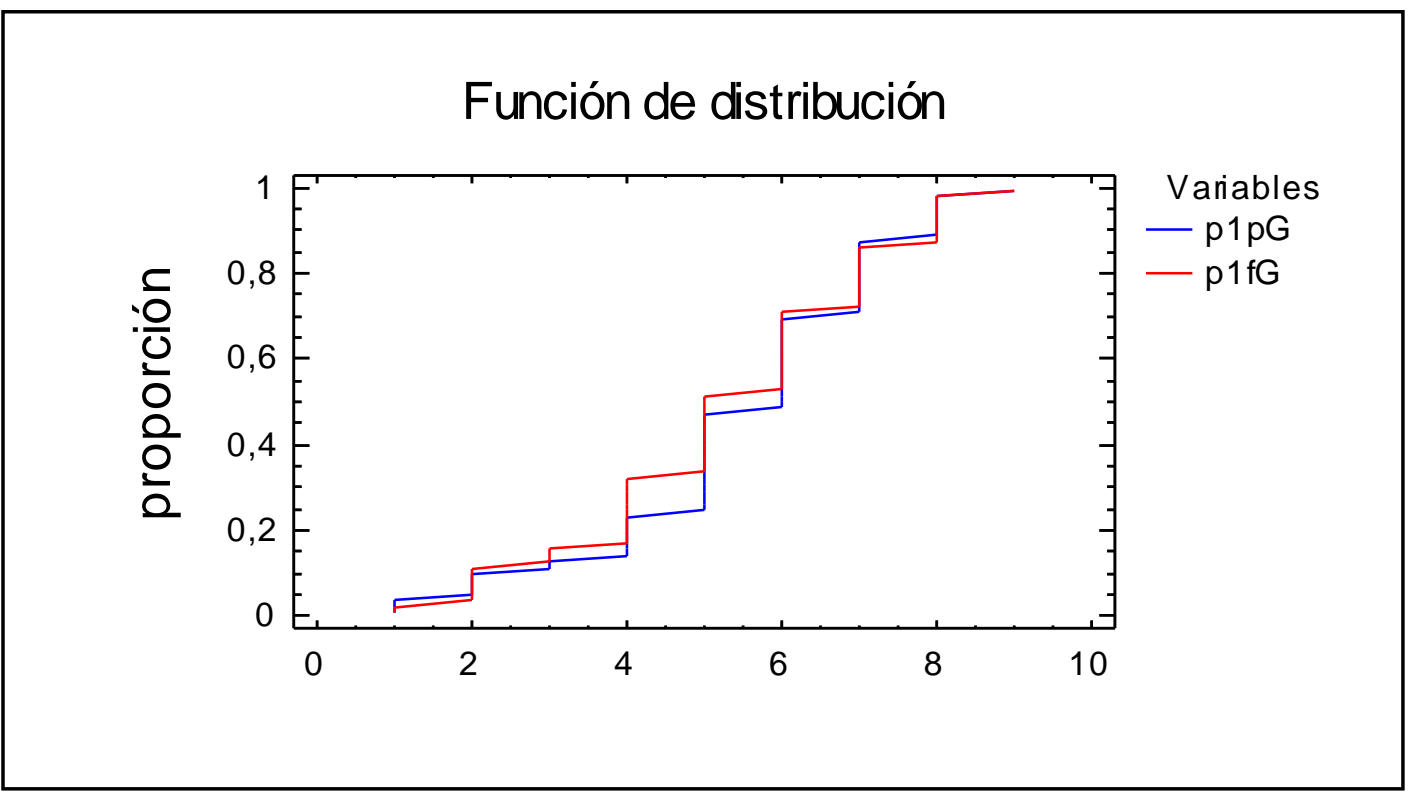

Gráfico 6.14. Comparación de funciones de distribución p1pG-p1fG

Los contrastes de igualdad de medias y de desviaciones típicas, basados en la normalidad de las distribuciones, no permiten rechazar la hipótesis nula, de modo que aceptaríamos que ambas medidas son iguales para las dos muestras de datos. Sin embargo el test de Kolmogorov-Smirnov nos permite rechazar la igualdad de distribuciones, y tanto en el gráfico de densidades suavizadas como en el de cajas y bigotes y en la función de distribución se observa que la distribución para la prueba preformal está más a la derecha que la formal, situándose en valores mayores para las puntuaciones otorgadas a esta prueba. 


\section{TEOREMA 2}

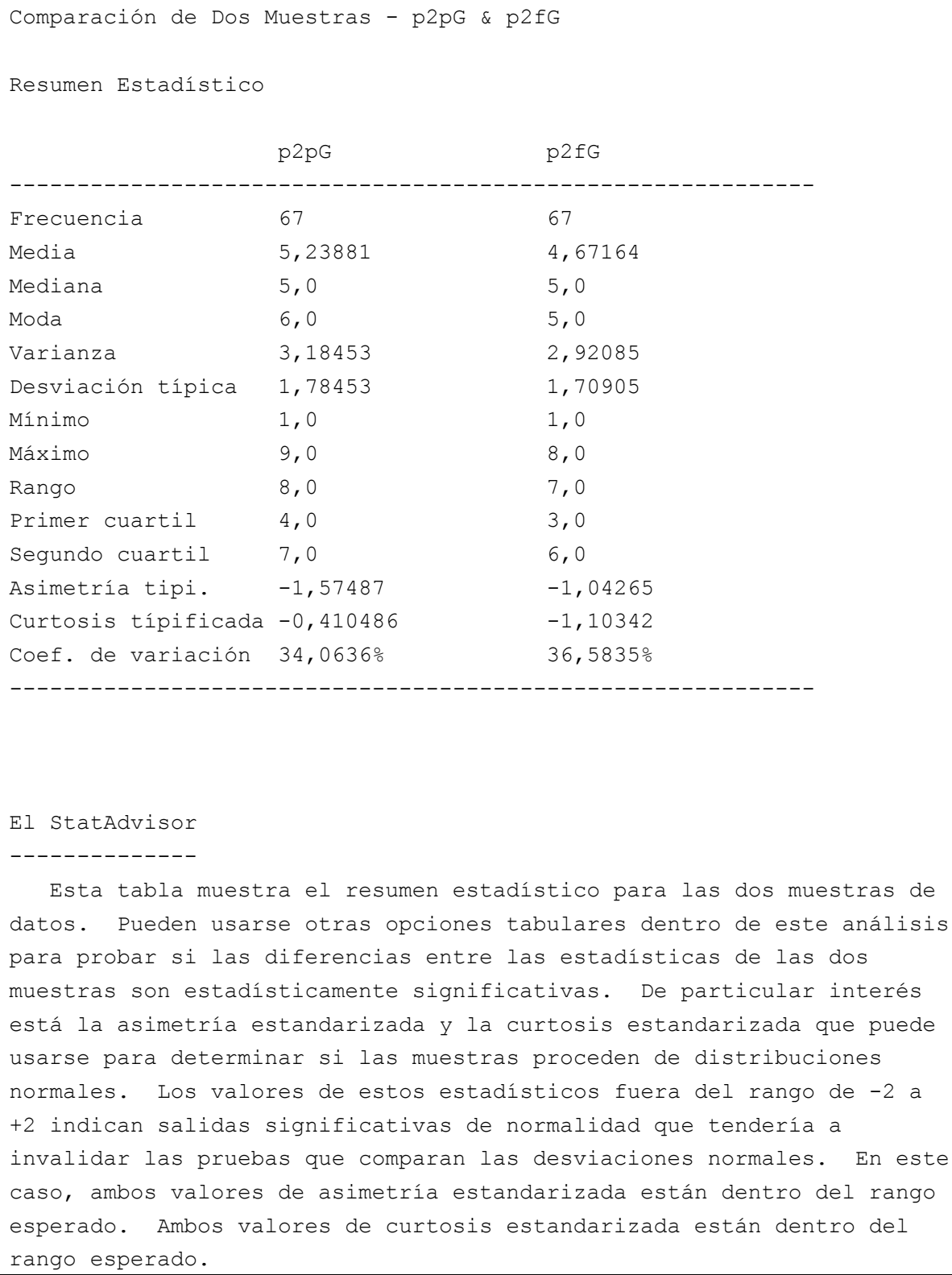

Tabla 6.15. Comparación de muestras p2pG-p2fG 


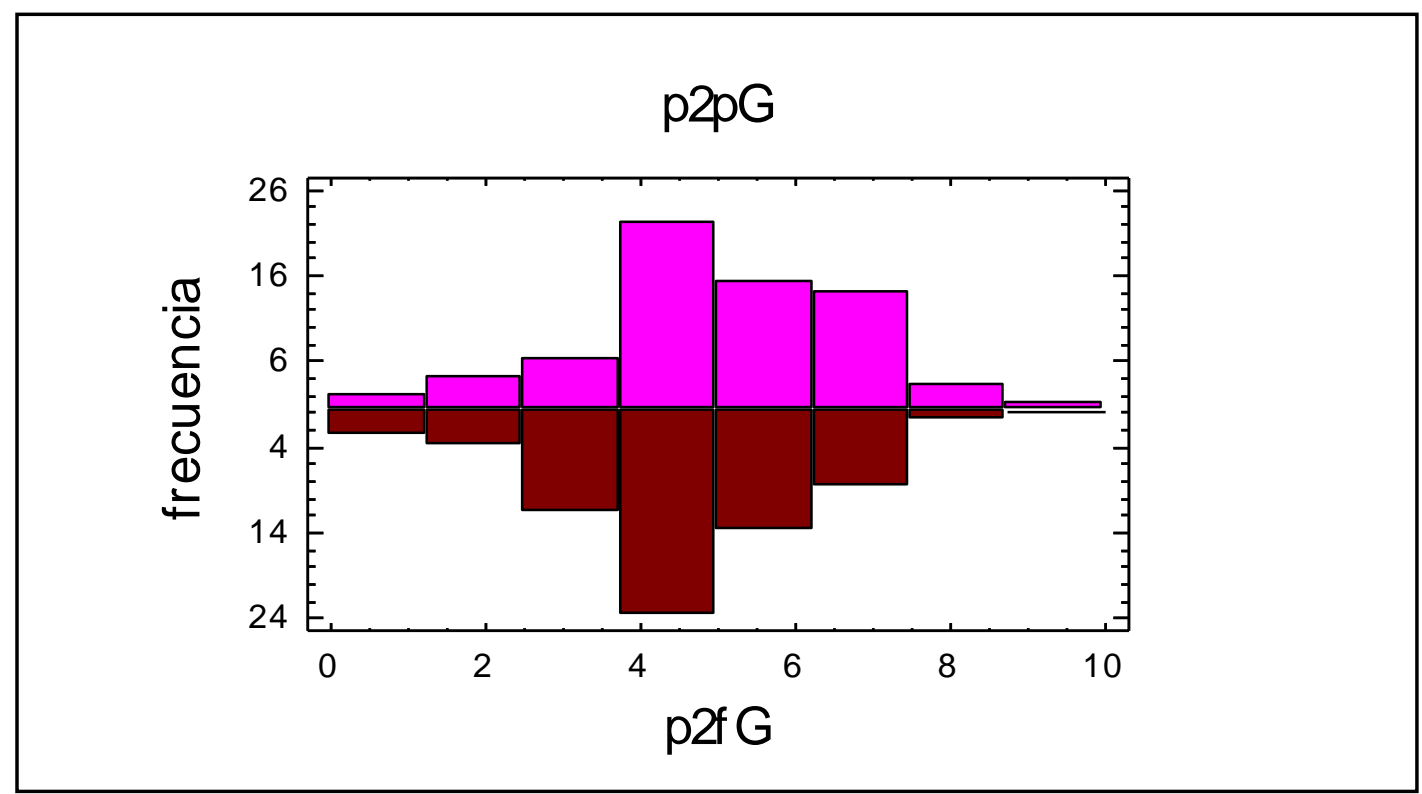

Gráfico 6.15. Comparación de histogramas p2pG-p2fG

Comparación de Medias

95,0\% intervalo de confianza para la media de p2pG: 5,23881 +/- 0,435281

$[4,80353,5,67409]$

95,0\% intervalo de confianza para la media de p2fG: 4,67164 +/- 0,416871

$[4,25477,5,08851]$

95, 0\% intervalos de confianza para la diferencia de medias:

suponiendo varianzas iguales: 0,567164 +/- 0,597129 [-0,0299644,1,16429]

contrastes $t$ de comparación de medias

Hipótesis nula: medial = media2

Hipótesis alt.: medial $<>$ media2

suponiendo varianzas iguales: $t=1,87884 \quad$ P-Valor $=0,0624716$

El StatAdvisor

Esta opción ejecuta el t-test para comparar las medias de las dos muestras. También establece los intervalos de confianza o los límites para cada media y para la diferencia entre las medias. De particular interés está el intervalo de confianza para la diferencia entre las medias, el cual se extiende desde -0,0299644 hasta 1,16429. Dado que el intervalo contiene el valor 0.0, no existe diferencia

estadísticamente significativa entre las medias de las dos muestras para un nivel de confianza del 95,0\%.

También puede aplicarse un t-test para probar una hipótesis específica sobre la diferencia entre las medias de las poblaciones de las que proceden las dos muestras. En este caso, el test se ha realizado para determinar si la diferencia entre las dos medias es 
igual a 0,0 frente a la hipótesis alternativa en la que la diferencia

no es igual 0,0 . Puesto que el p-valor calculado no es inferior a

0,05, no podemos rechazar la hipótesis nula.

NOTA: estos resultados asumen la igualdad de varianzas en las dos

muestras. En este caso, esa asunción parece ser razonable teniendo en

cuenta los resultados del F-test para comparar las desviaciones

típicas. Puede ver los resultados de este test seleccionando

Comparación de Desviaciones Típicas del menú Opciones Tabulares.

Tabla 6.16. Comparación de medias p2pG-p2fG

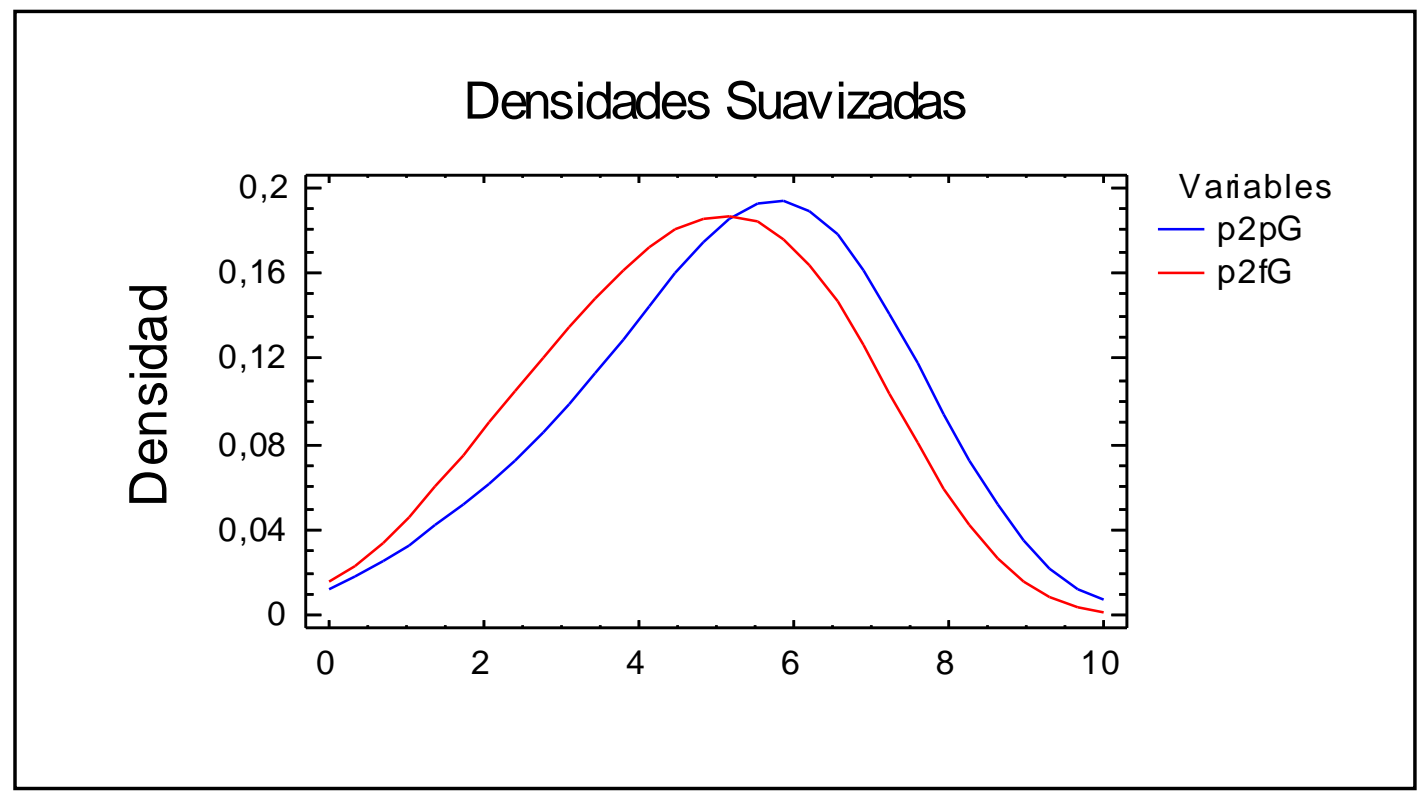

Gráfico 6.16. Comparación de densidades suavizadas p2pG-p2fG

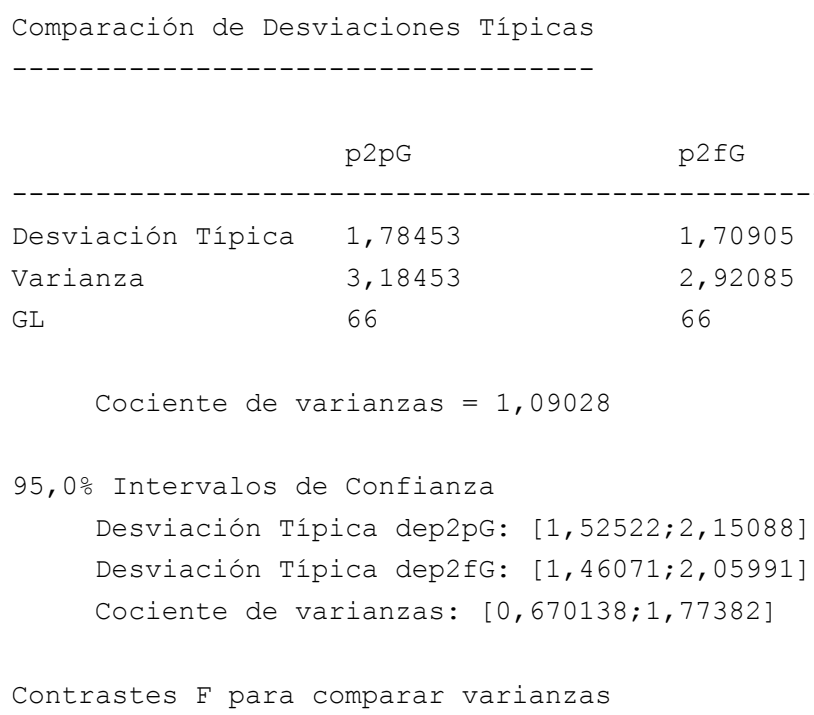




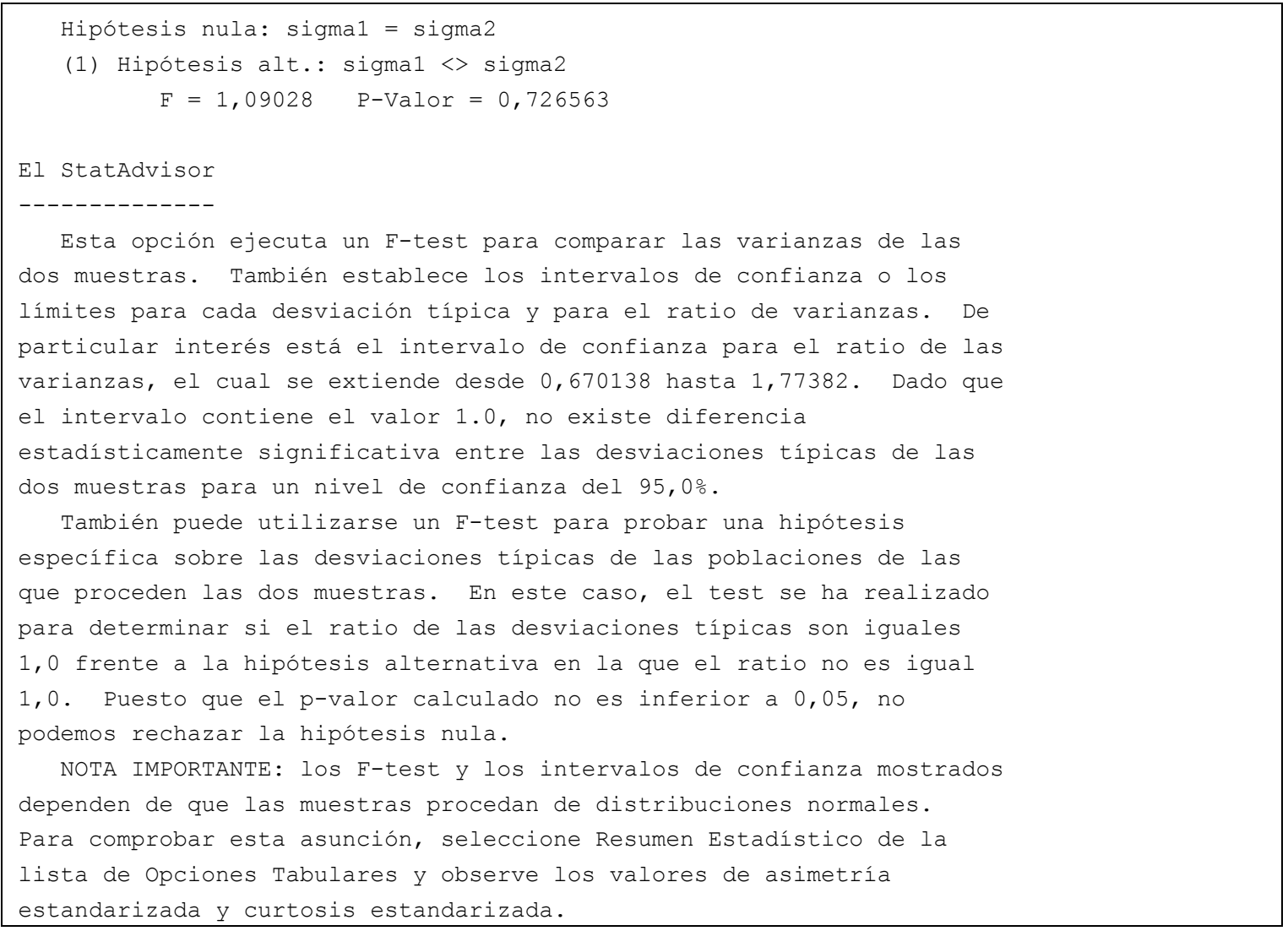

Tabla 6.17. Comparación de desviaciones típicas p2pG-p2fG

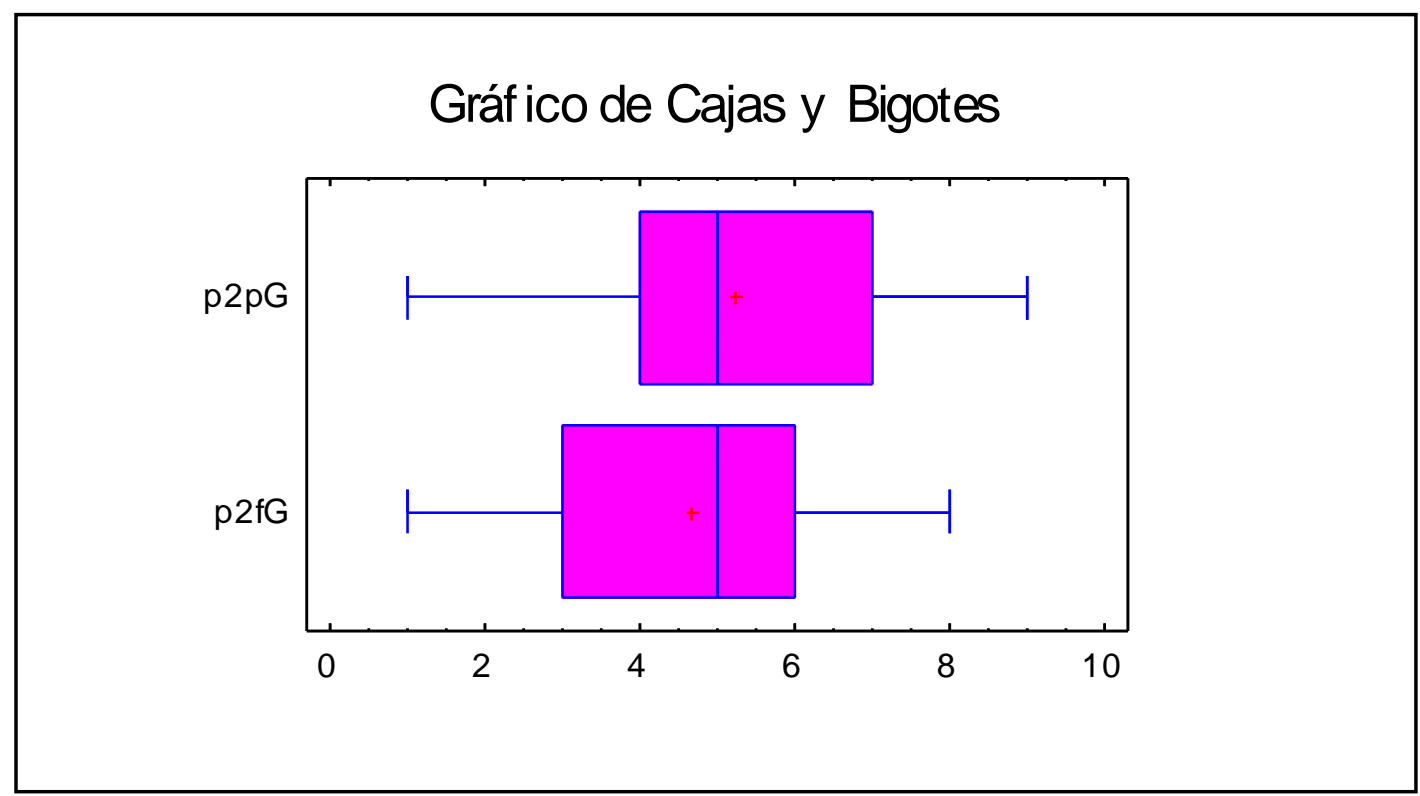

Gráfico 6.17. Comparación de diagramas de cajas y bigotes p2pG-p2fG 


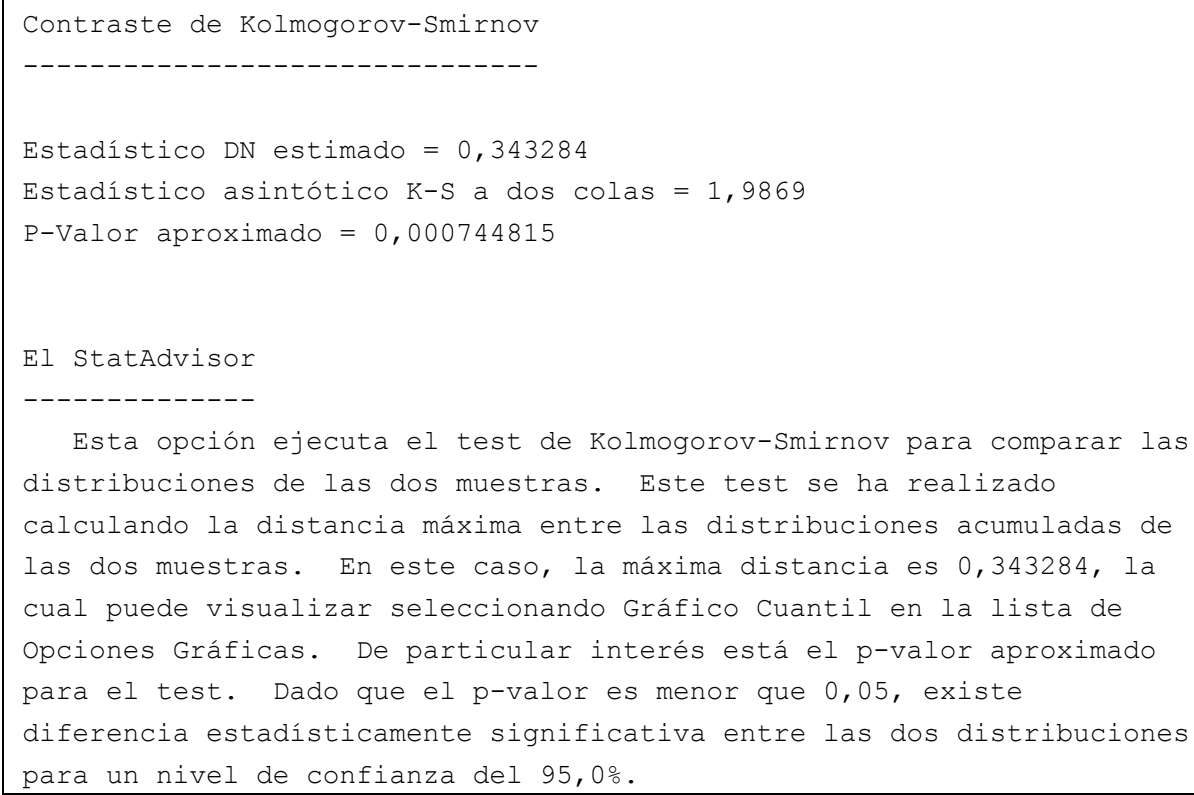

Tabla 6.18. Contraste de Kolmogorov-Smirnov p2pG-p2fG

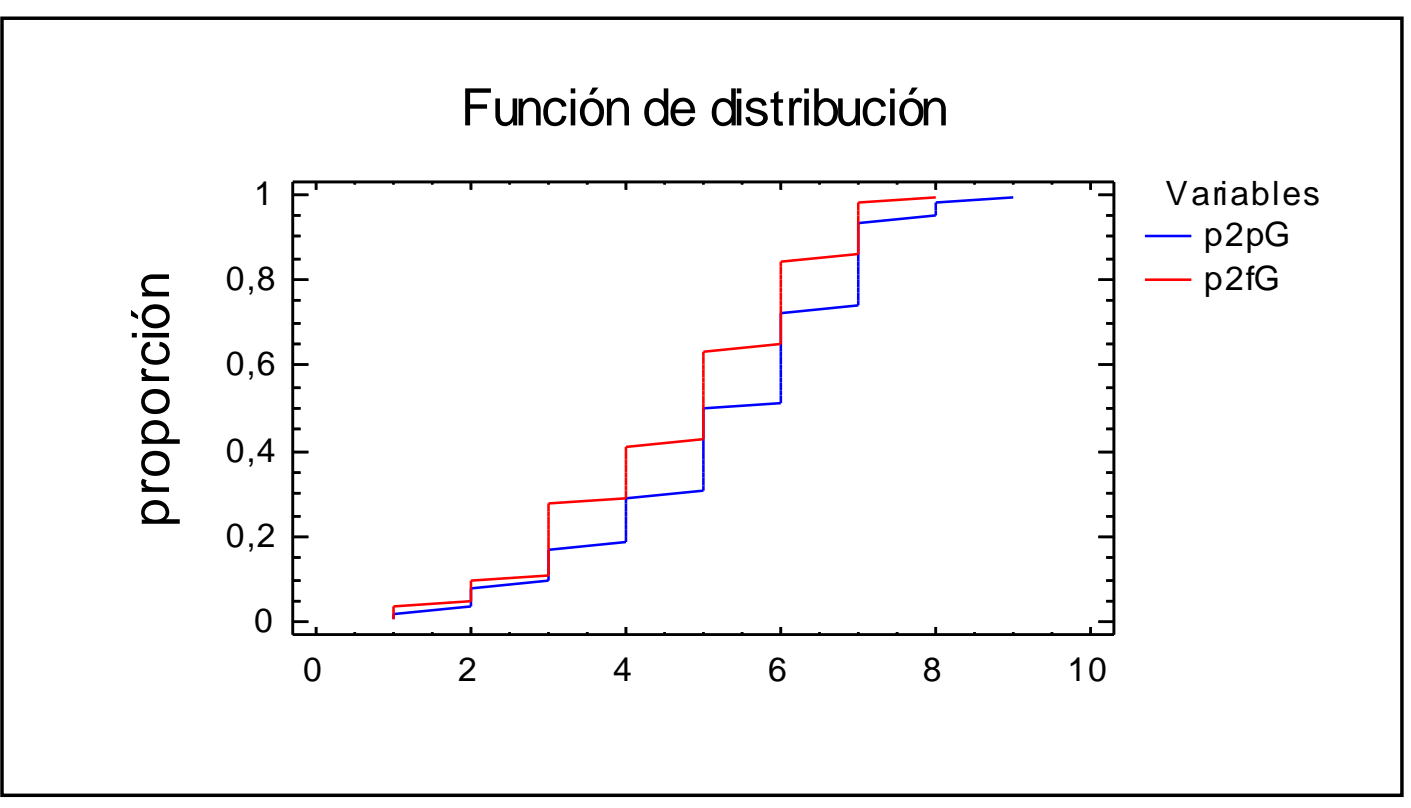

Gráfico 6.18. Comparación de funciones de distribución p2pG-p2fG

\subsection{Comparaciones sobre claridad (C) de las pruebas}

Resultados conjuntos de los tres ciclos en la categoría Claridad en ambas pruebas, preformal y formal, en cada uno de los dos teoremas $(\mathbf{p 1}, \mathbf{p 2})$ en que se han utili- 
zado. La claridad de la prueba preformal la denotamos con $\mathrm{p} 1 \mathrm{pC}$ y $\mathrm{p} 2 \mathrm{pC}$, y la de la prueba formal p1fC y p2fC.

\begin{tabular}{|c|c|c|c|c|}
\hline Claridad & p1pC & p1fC & p2pC & p2fC \\
\hline $\mathbf{1}$ & 2 & 1 & 1 & 2 \\
\hline $\mathbf{2}$ & 1 & 2 & 1 & 4 \\
\hline $\mathbf{3}$ & 7 & 7 & 7 & 9 \\
\hline $\mathbf{4}$ & 4 & 5 & 7 & 12 \\
\hline $\mathbf{5}$ & 13 & 12 & 17 & 13 \\
\hline $\mathbf{6}$ & 19 & 10 & 14 & 13 \\
\hline $\mathbf{7}$ & 11 & 16 & 10 & 8 \\
\hline $\mathbf{8}$ & 7 & 13 & 8 & 6 \\
\hline $\mathbf{9}$ & 2 & 1 & 1 & 0 \\
\hline $\mathbf{1 0}$ & 1 & 0 & 1 & 0 \\
\hline Total & 67 & 67 & 67 & 67 \\
\hline
\end{tabular}

Tabla 6.19. Resultados conjuntos de los tres ciclos en la categoría claridad en ambas pruebas en cada uno de los dos teoremas

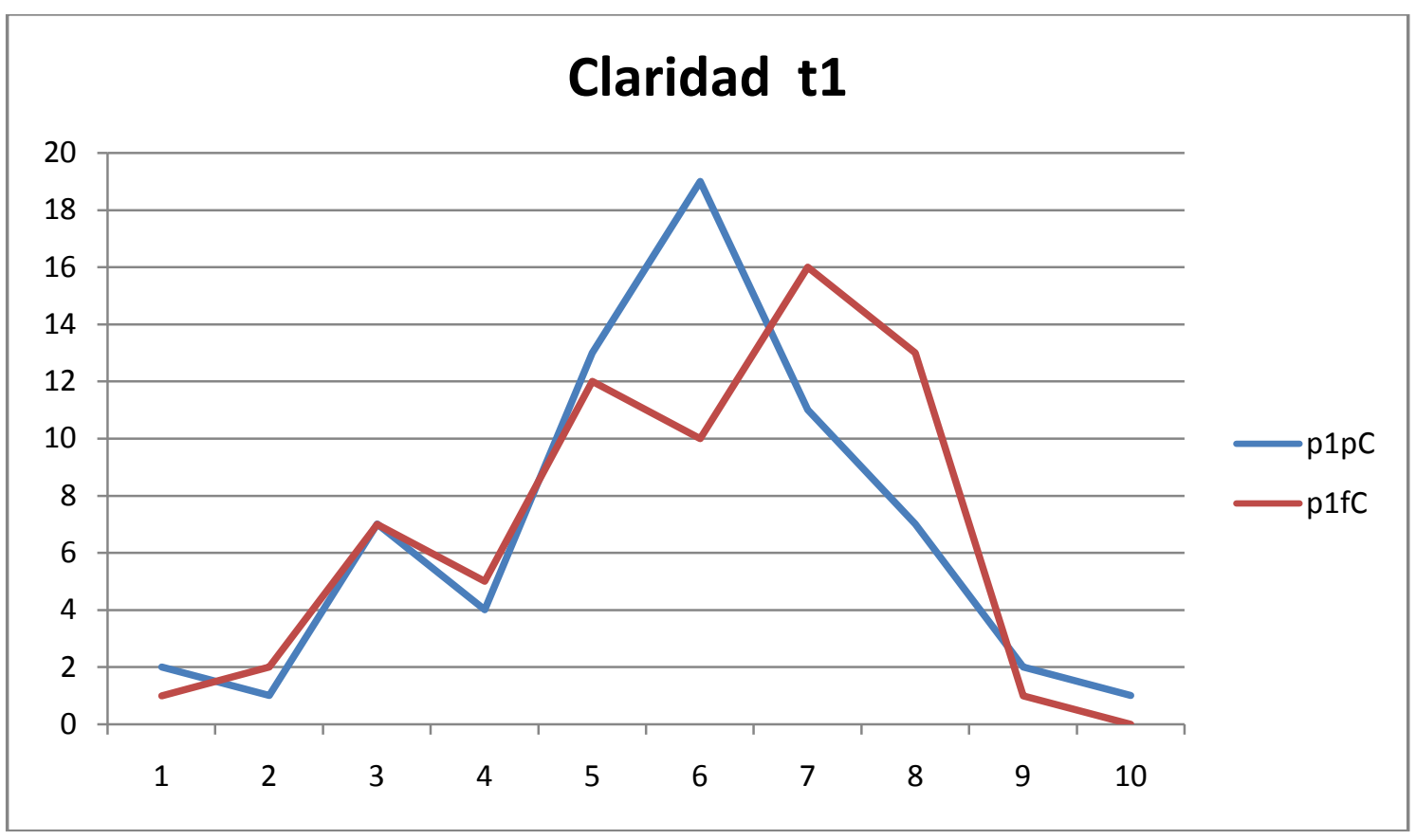

Gráfico 6.19. Frecuencias de los tres ciclos en ambas pruebas en la categoría claridad, teorema 1 


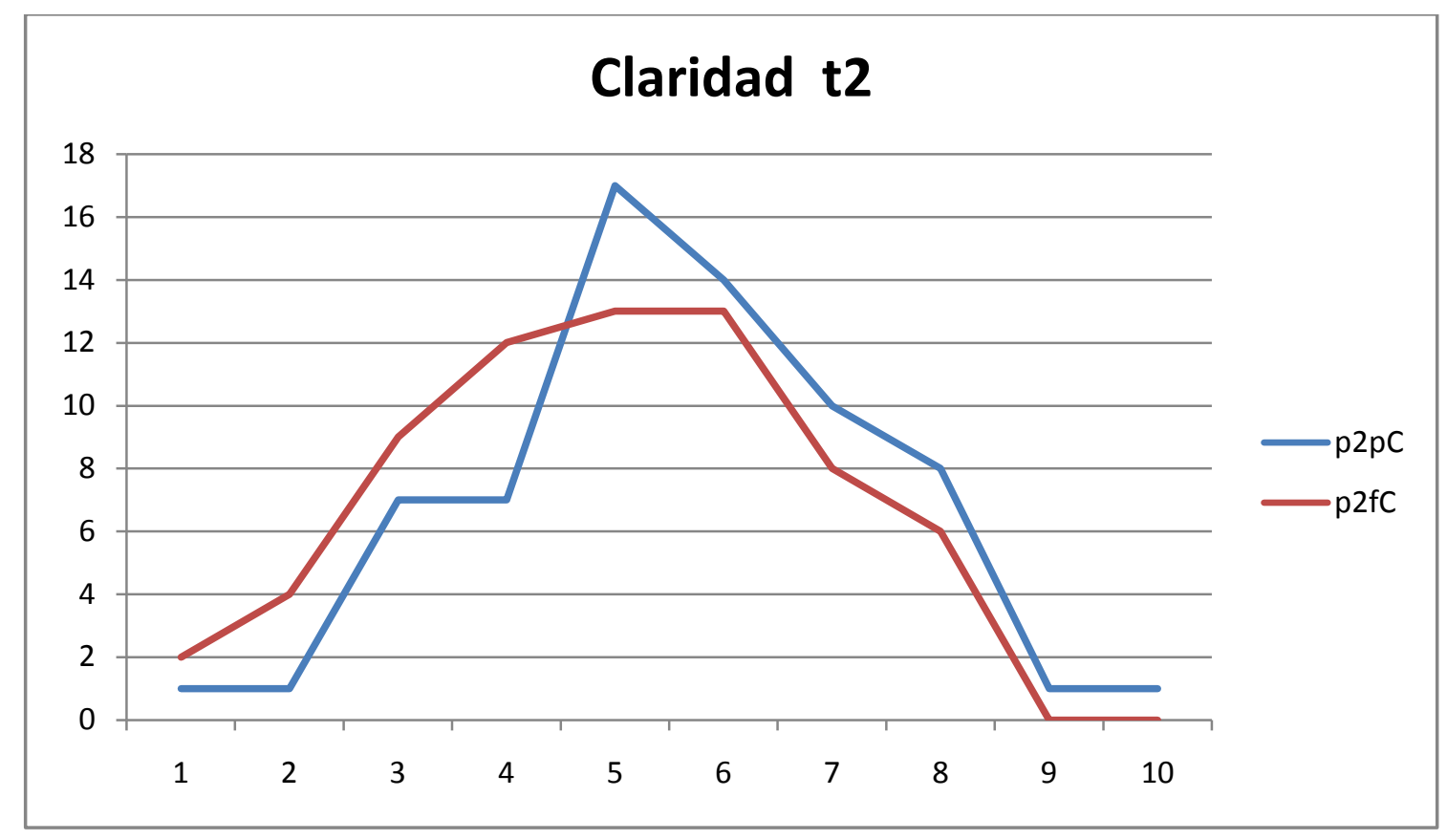

\section{Gráfico 6.19 bis. Frecuencias de los tres ciclos en ambas pruebas en la categoría claridad, teorema 2}

En la tabla 6.20 se recogen los estadísticos descriptivos, donde se aprecian unas valoraciones muy similares en ambas pruebas siendo algo superior la media de la prueba formal y su moda, mientras que el resto de estadísticos son iguales. Por la asimetría y curtosis comprobamos que se encuentran en el intervalo $(-2,2)$ con lo que podemos aceptar que cada una de estas muestras sigue una distribución normal.

\section{TEOREMA 1}

\begin{tabular}{|c|c|c|}
\hline \multicolumn{3}{|l|}{ Resumen Estadístico } \\
\hline & $\mathrm{plpC}$ & $\mathrm{p} 1 \mathrm{fC}$ \\
\hline Frecuencia & 67 & 67 \\
\hline Media & 5,68657 & 5,83582 \\
\hline Mediana & 6,0 & 6,0 \\
\hline Moda & 6,0 & 7,0 \\
\hline Varianza & 3,40027 & 3,44233 \\
\hline Desviación típica & 1,84398 & 1,85535 \\
\hline
\end{tabular}




\begin{tabular}{lll}
\hline Mínimo & 1,0 & 1,0 \\
Máximo & 10,0 & 9,0 \\
Rango & 9,0 & 8,0 \\
Primer cuartil & 5,0 & 5,0 \\
Segundo cuartil & 7,0 & 7,0 \\
Asimetría tipi. & $-1,24784$ & $-1,91868$ \\
Curtosis típificada & 0,434915 & $-0,779676$ \\
Coef. de variación & $32,427 \%$ & $31,7925 \%$
\end{tabular}

El StatAdvisor

E-1 Statidvisor

Esta tabla muestra el resumen estadístico para las dos muestras de datos. Pueden usarse otras opciones tabulares dentro de este análisis para probar si las diferencias entre las estadísticas de las dos muestras son estadísticamente significativas. De particular interés está la asimetría estandarizada y la curtosis estandarizada que puede usarse para determinar si las muestras proceden de distribuciones normales. Los valores de estos estadísticos fuera del rango de -2 a +2 indican salidas significativas de normalidad que tendería a invalidar las pruebas que comparan las desviaciones normales. En este caso, ambos valores de asimetría estandarizada están dentro del rango esperado. Ambos valores de curtosis estandarizada están dentro del rango esperado.

Tabla 6.20. Comparación de muestras p1pC-p1fC

\section{p1pC}

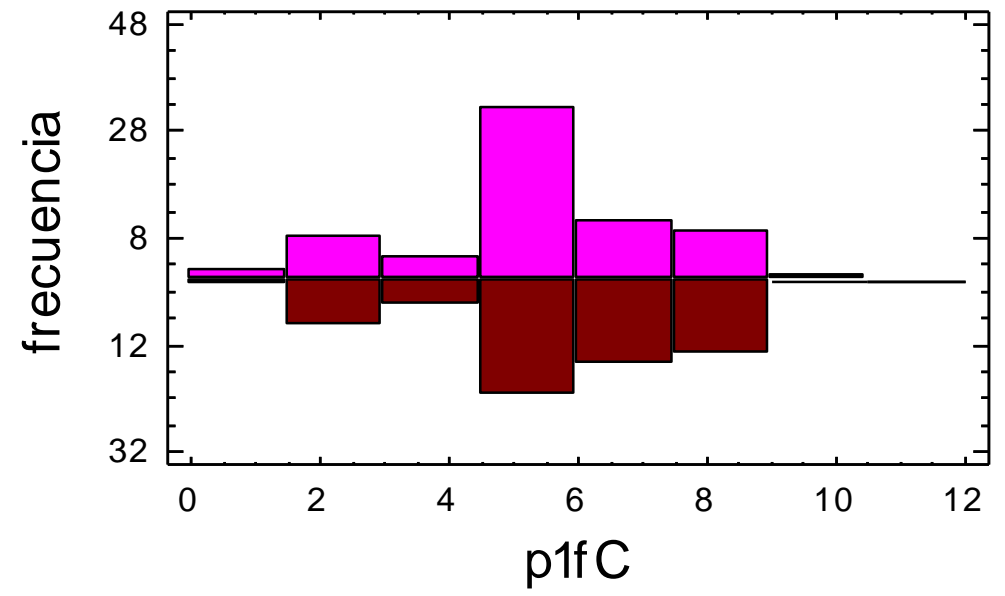

Gráfico 6.20. Comparación de histogramas p1pC-p1fC 


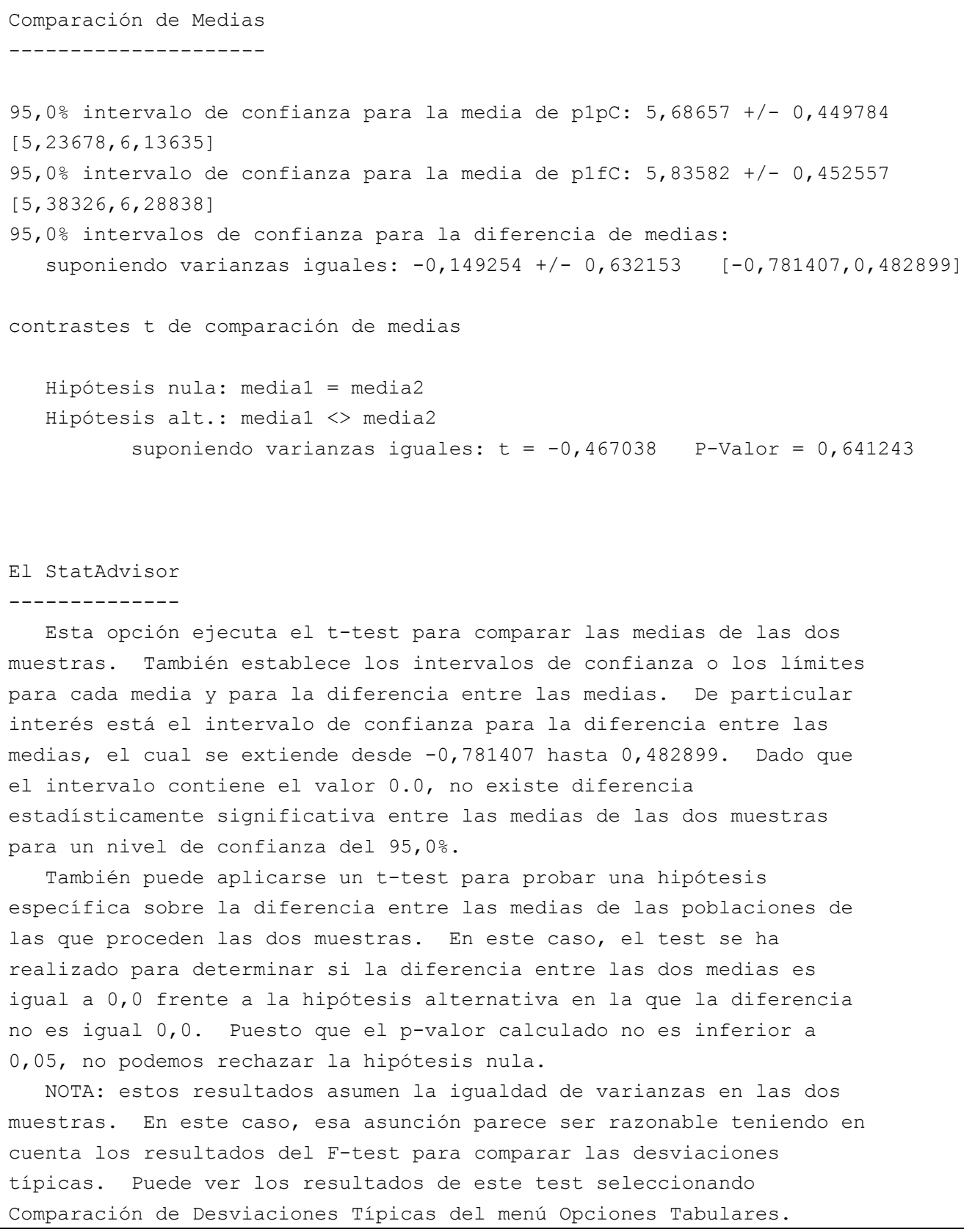

Tabla 6.21. Comparación de medias p1pC-p1fC 


\section{Densidades Suavizadas}

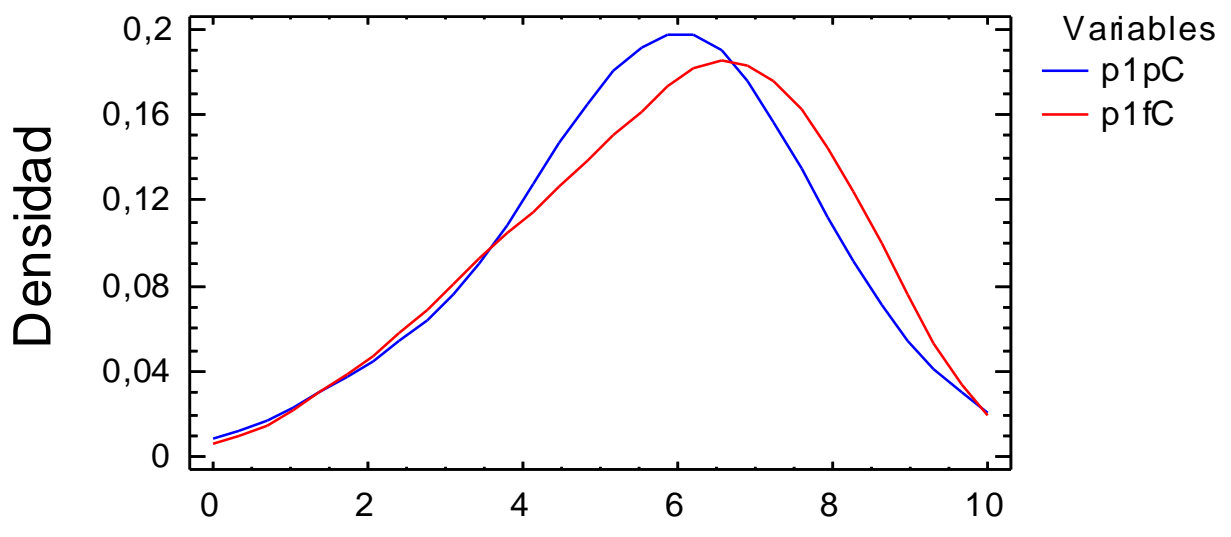

Gráfico 6.21. Comparación de densidades suavizadas p1pC-p1fC

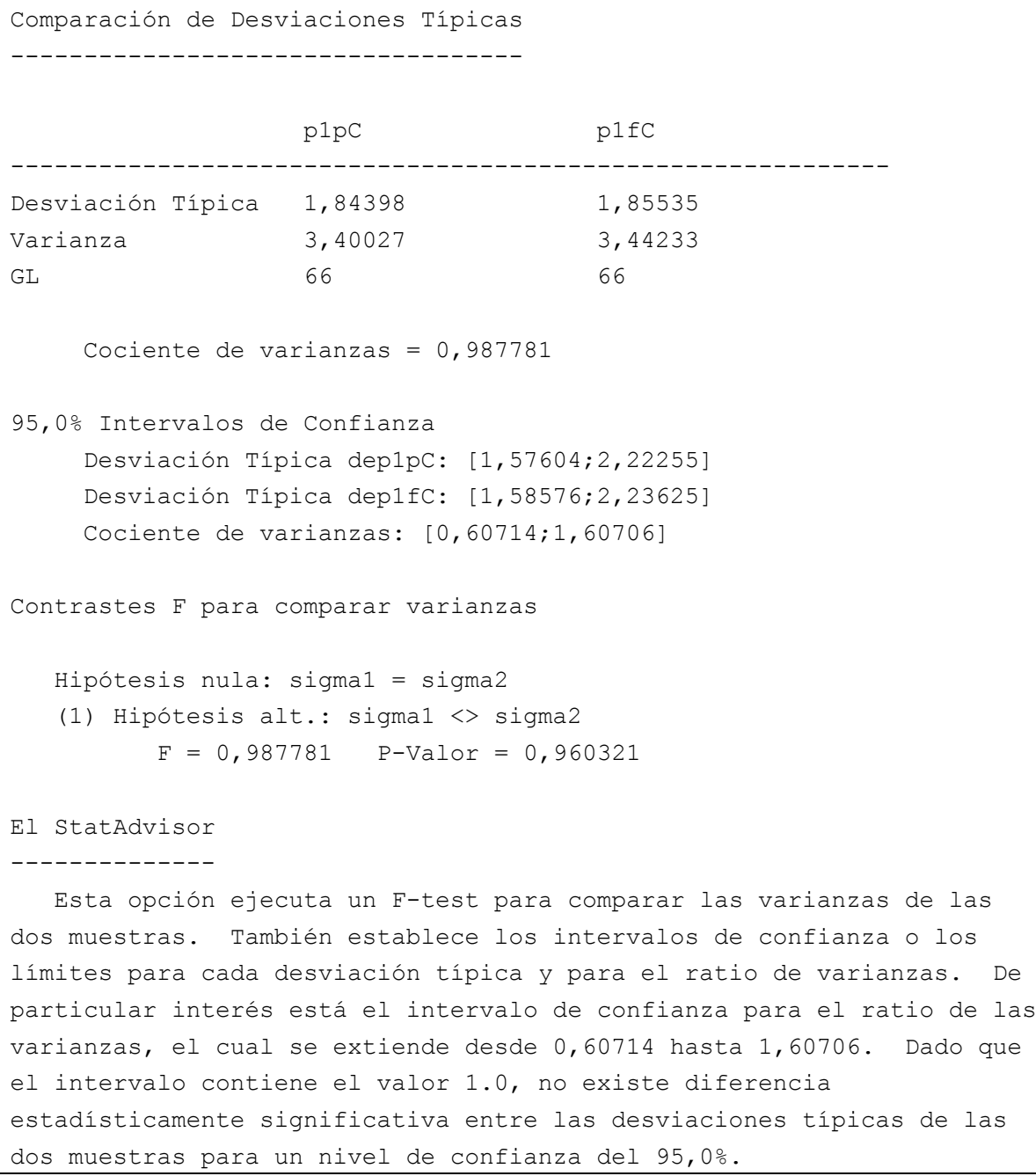


También puede utilizarse un F-test para probar una hipótesis

específica sobre las desviaciones típicas de las poblaciones de las

que proceden las dos muestras. En este caso, el test se ha realizado

para determinar si el ratio de las desviaciones típicas son iguales

1,0 frente a la hipótesis alternativa en la que el ratio no es igual

1,0. Puesto que el p-valor calculado no es inferior a 0,05, no

podemos rechazar la hipótesis nula.

NOTA IMPORTANTE: los F-test y los intervalos de confianza mostrados

dependen de que las muestras procedan de distribuciones normales.

Para comprobar esta asunción, seleccione Resumen Estadístico de la

lista de Opciones Tabulares y observe los valores de asimetría

estandarizada y curtosis estandarizada.

Tabla 6.22. Comparación de desviaciones típicas p1pC-p1fC

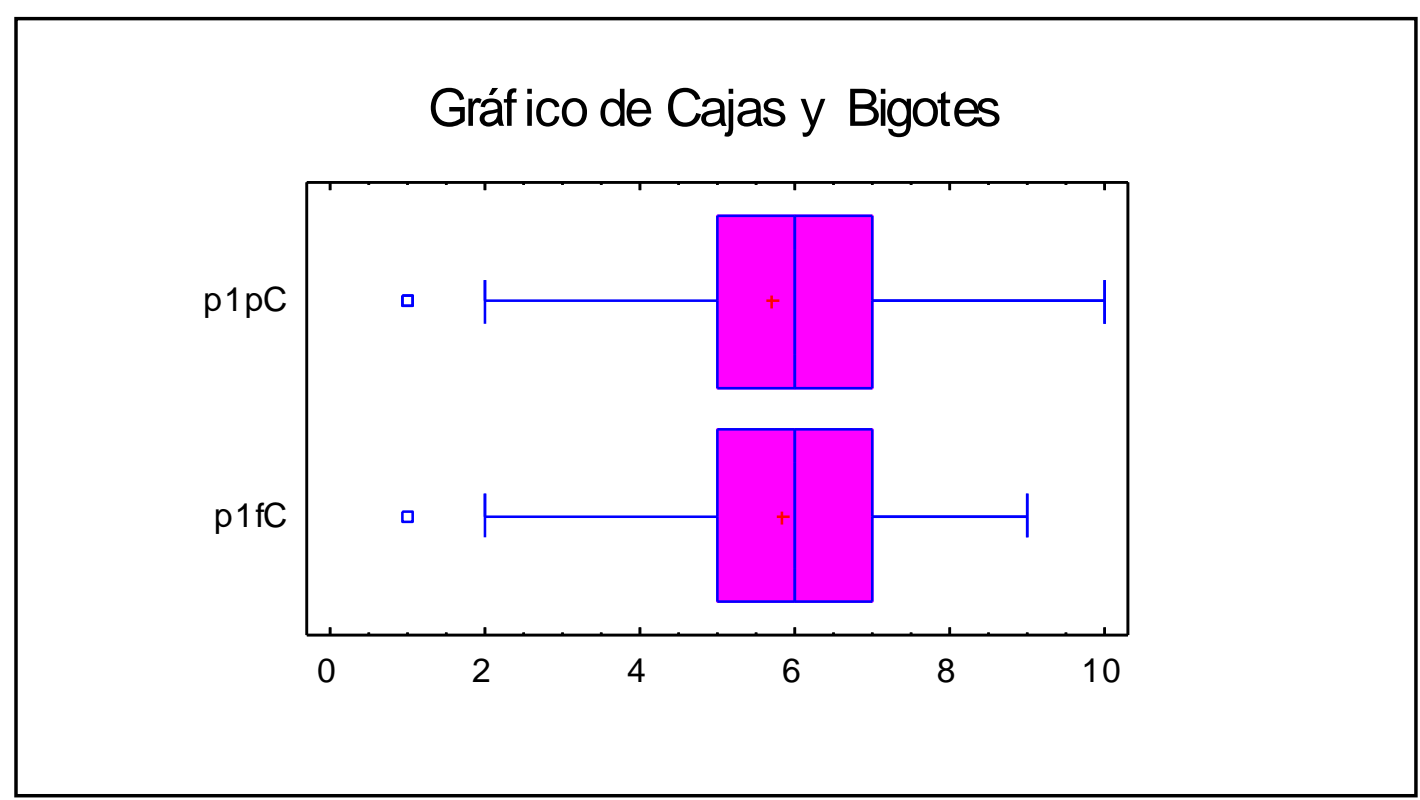

Gráfico 6.22. Comparación de diagramas de cajas y bigotes p1pC-p1fC

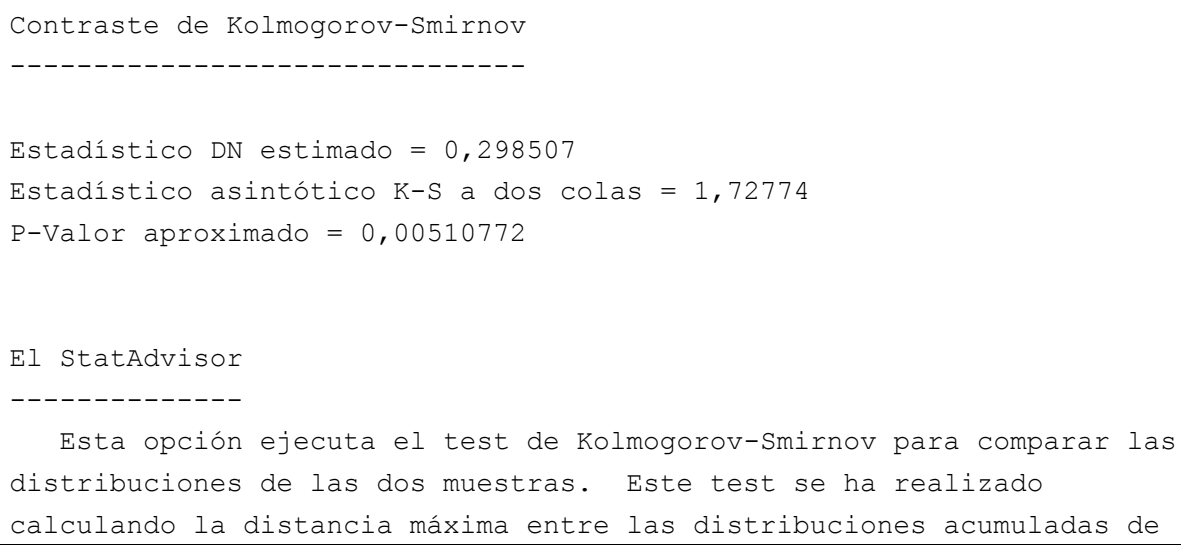


las dos muestras. En este caso, la máxima distancia es 0,298507, la

cual puede visualizar seleccionando Gráfico Cuantil en la lista de

Opciones Gráficas. De particular interés está el p-valor aproximado

para el test. Dado que el p-valor es menor que 0,05, existe

diferencia estadísticamente significativa entre las dos distribuciones

para un nivel de confianza del 95,0\%.

Tabla 6.23. Contraste de Kolmogorov-Smirnov p1pC-p1fC

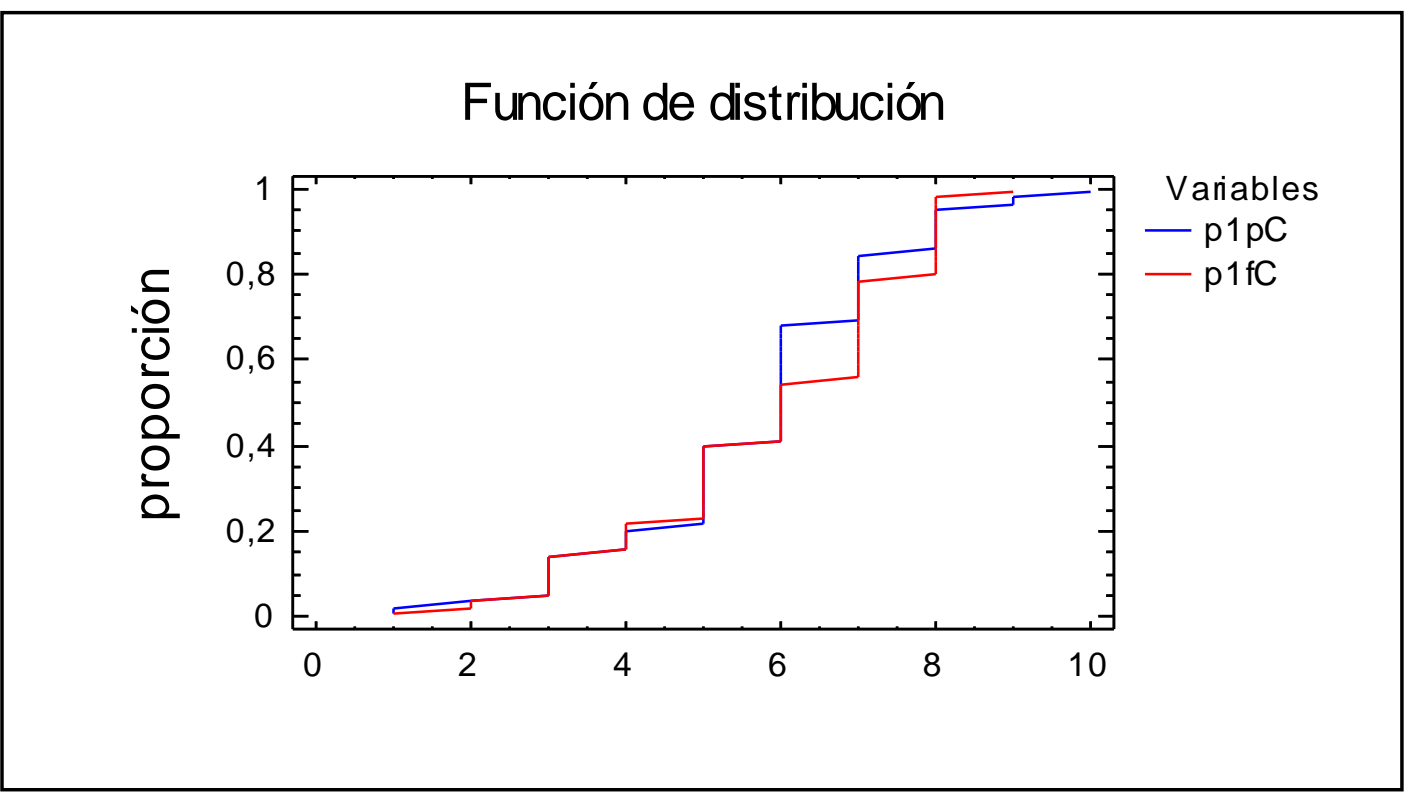

Gráfico 6.23. Comparación de funciones de distribución p1pC-p1fC

Los contrastes de igualdad de medias y de desviaciones típicas, basados en la normalidad de las distribuciones, no permiten rechazar la hipótesis nula, de modo que aceptaríamos que ambas medidas son iguales para las dos muestras de datos. Sin embargo el test de Kolmogorov-Smirnov nos permite rechazar la igualdad de distribuciones, y tanto en el gráfico de densidades suavizadas como en el de cajas y bigotes y en la función de distribución se observa que la distribución para la prueba formal está más a la derecha que la preformal, situándose en valores mayores para las puntuaciones otorgadas a esta prueba. 


\section{TEOREMA 2}

Comparación de Dos Muestras - p2pC \& p2fC

Resumen Estadístico

$\mathrm{p} 2 \mathrm{pC} \quad \mathrm{p} 2 \mathrm{fC}$

Frecuencia $\quad 67 \quad 67$

Media $\quad 5,58209 \quad 4,95522$

Mediana $6,0 \quad 5,0$

Moda 5, 0

$\begin{array}{lll}\text { Varianza } & 3,09543 & 3,25554\end{array}$

Desviación típica 1,75938 1,80431

Mínimo $1,0 \quad 1,0$

Máximo $10,0 \quad 8,0$

Rango $9,0 \quad 7,0$

Primer cuartil 5,0 4,0

Segundo cuartil $7,0 \quad 6,0$

Asimetría tipi. $\quad-0,239327 \quad-0,463174$

Curtosis típificada $-0,0572034 \quad-1,04459$

Coef. de variación 31,5184\% 36,4123\%

El StatAdvisor

-----------

Esta tabla muestra el resumen estadístico para las dos muestras de datos. Pueden usarse otras opciones tabulares dentro de este análisis para probar si las diferencias entre las estadísticas de las dos muestras son estadísticamente significativas. De particular interés está la asimetría estandarizada y la curtosis estandarizada que puede usarse para determinar si las muestras proceden de distribuciones normales. Los valores de estos estadísticos fuera del rango de -2 a +2 indican salidas significativas de normalidad que tendería a invalidar las pruebas que comparan las desviaciones normales. En este caso, ambos valores de asimetría estandarizada están dentro del rango esperado. Ambos valores de curtosis estandarizada están dentro del rango esperado.

Tabla 6.24. Comparación de muestras p2pC-p2fC 


\section{p2pC}

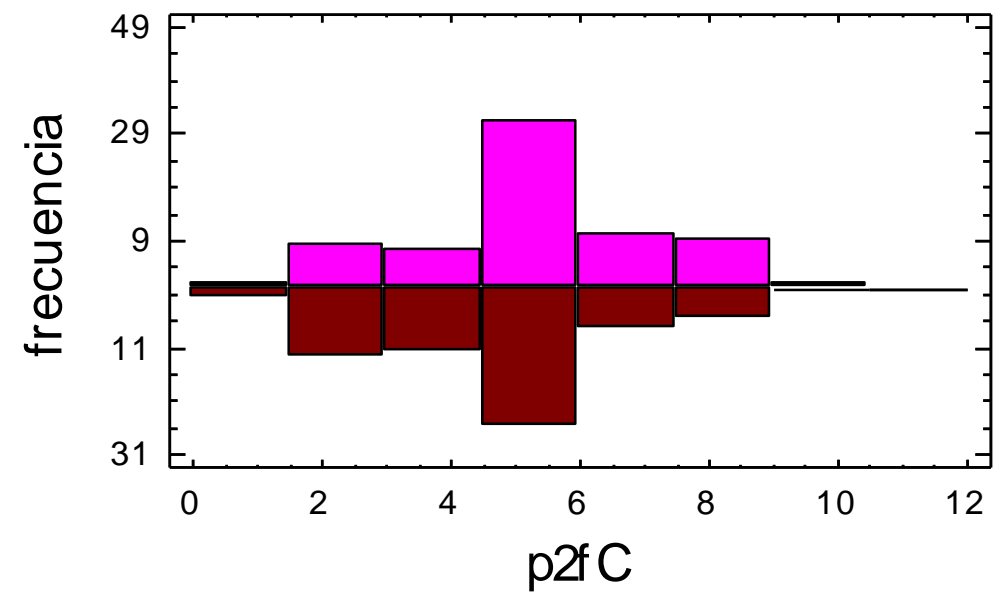

Gráfico 6.24. Comparación de histogramas p2pC-p2fC

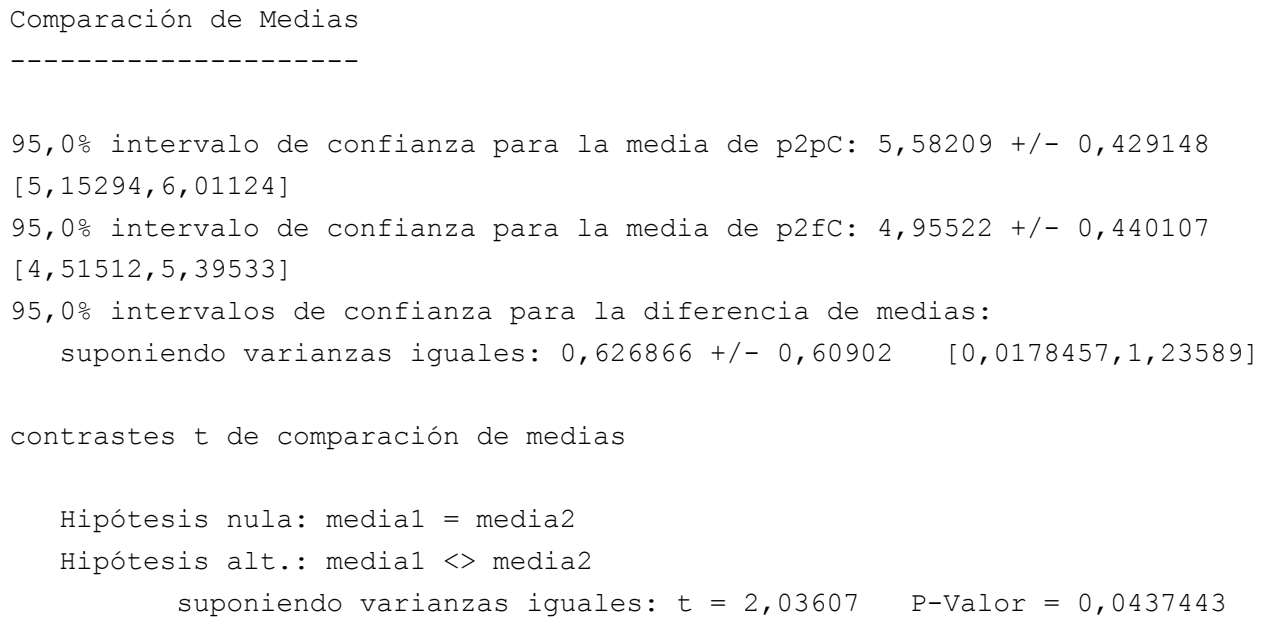


realizado para determinar si la diferencia entre las dos medias es

igual a 0,0 frente a la hipótesis alternativa en la que la diferencia

no es igual 0,0. Puesto que el p-valor calculado es inferior a 0,05,

podemos rechazar la hipótesis nula en favor de la alternativa.

NOTA: estos resultados asumen la igualdad de varianzas en las dos

muestras. En este caso, esa asunción parece ser razonable teniendo en

cuenta los resultados del F-test para comparar las desviaciones

típicas. Puede ver los resultados de este test seleccionando

Comparación de Desviaciones Típicas del menú Opciones Tabulares.

Tabla 6.25. Comparación de medias p2pC-p2fC

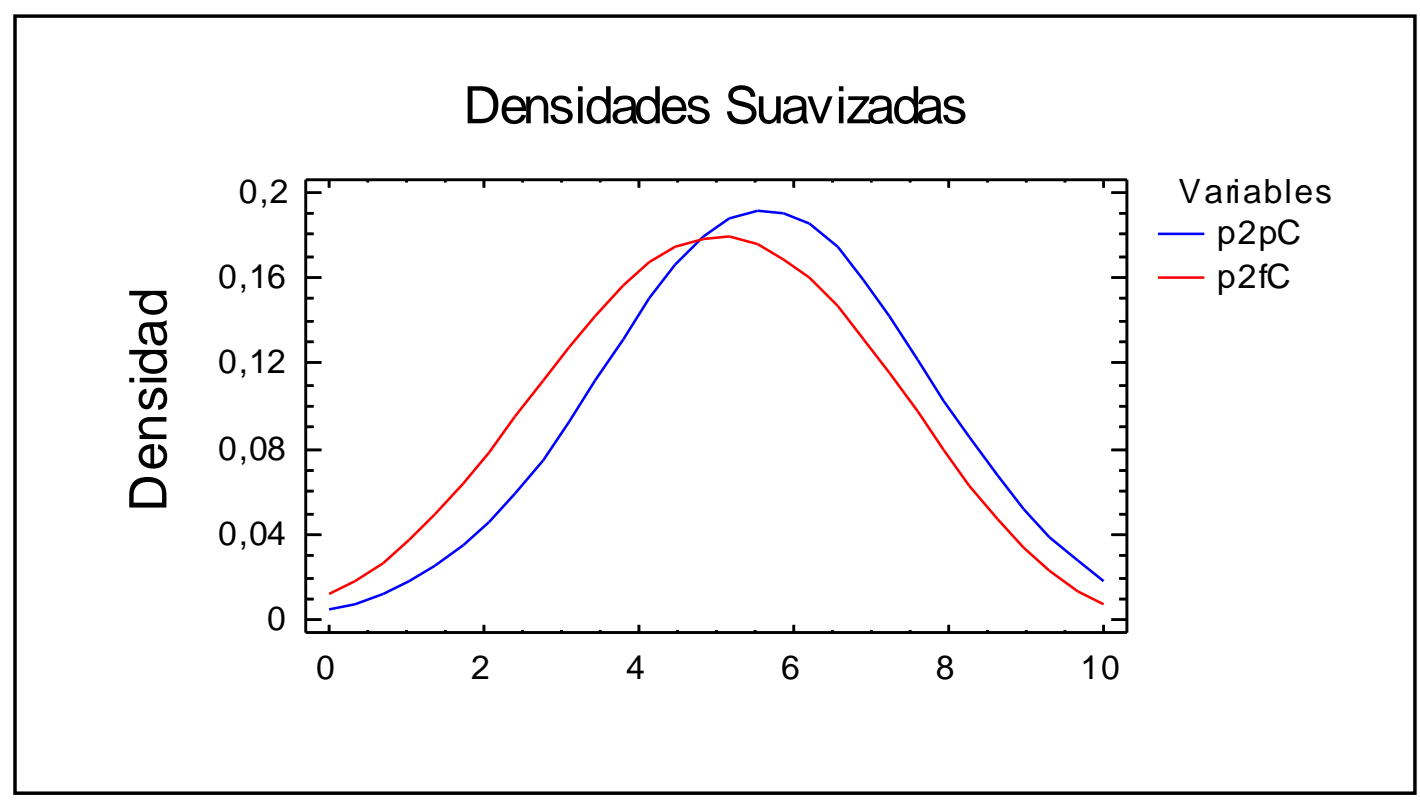

Gráfico 6.25. Comparación de densidades suavizadas p2pC-p2fC

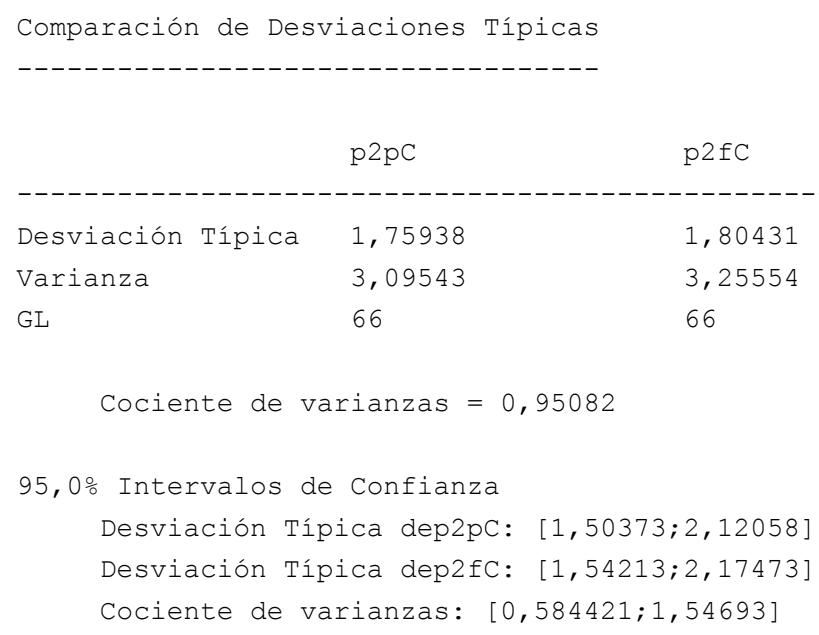


Contrastes F para comparar varianzas

Hipótesis nula: sigmal = sigma2

(1) Hipótesis alt.: sigmal <> sigma2

$$
\mathrm{F}=0,95082 \quad \text { P-Valor }=0,838302
$$

El StatAdvisor

\section{El statidvisor}

Esta opción ejecuta un F-test para comparar las varianzas de las dos muestras. También establece los intervalos de confianza o los límites para cada desviación típica y para el ratio de varianzas. De particular interés está el intervalo de confianza para el ratio de las varianzas, el cual se extiende desde 0,584421 hasta 1,54693. Dado que el intervalo contiene el valor 1.0, no existe diferencia estadísticamente significativa entre las desviaciones típicas de las dos muestras para un nivel de confianza del 95,0\%.

También puede utilizarse un F-test para probar una hipótesis específica sobre las desviaciones típicas de las poblaciones de las que proceden las dos muestras. En este caso, el test se ha realizado para determinar si el ratio de las desviaciones típicas son iguales 1,0 frente a la hipótesis alternativa en la que el ratio no es igual 1,0. Puesto que el p-valor calculado no es inferior a 0,05, no podemos rechazar la hipótesis nula.

NOTA IMPORTANTE: los F-test y los intervalos de confianza mostrados dependen de que las muestras procedan de distribuciones normales. Para comprobar esta asunción, seleccione Resumen Estadístico de la lista de Opciones Tabulares y observe los valores de asimetría estandarizada y curtosis estandarizada.

Tabla 6.26. Comparación de desviaciones típicas p2pC-p2fC

\section{Gráf ico de Cajas y Bigotes}

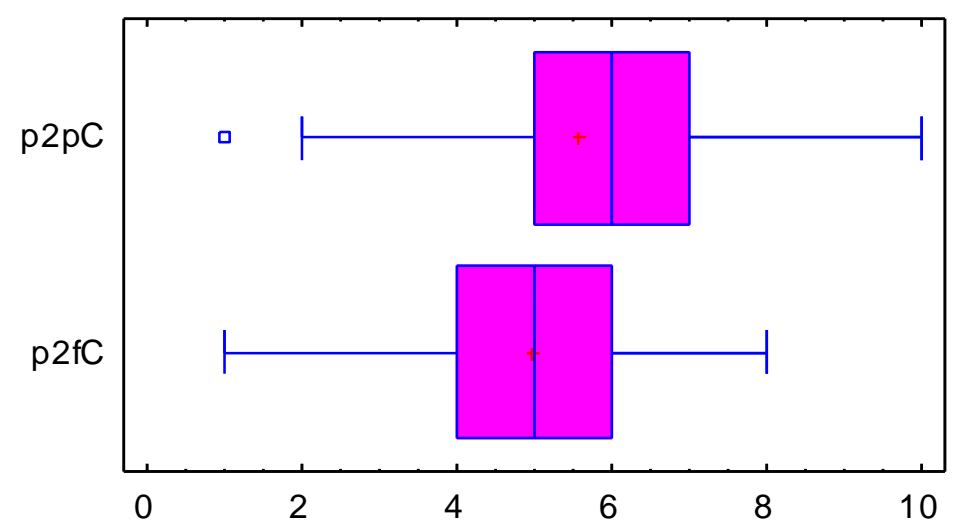

Gráfico 6.26. Comparación de diagramas de cajas y bigotes p2pC-p2fC 


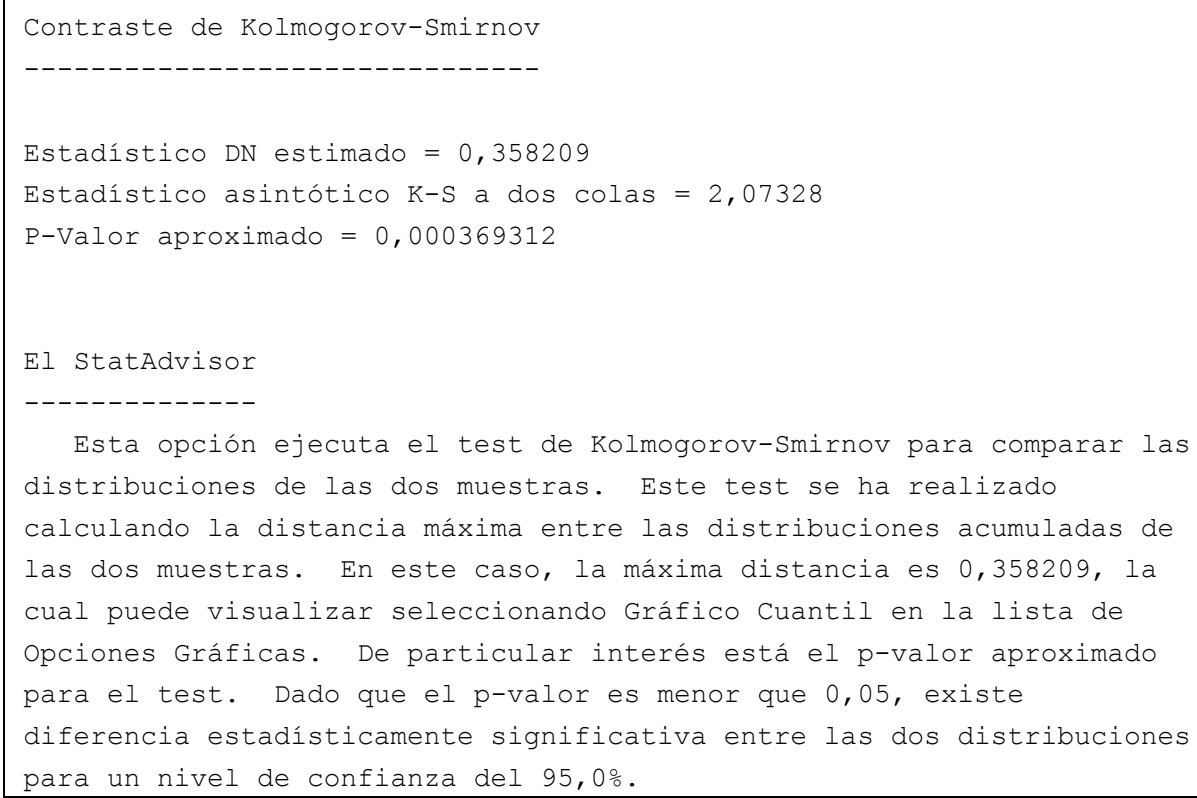

Tabla 6.27. Contraste de Kolmogorov-Smirnov p2pC-p2fC

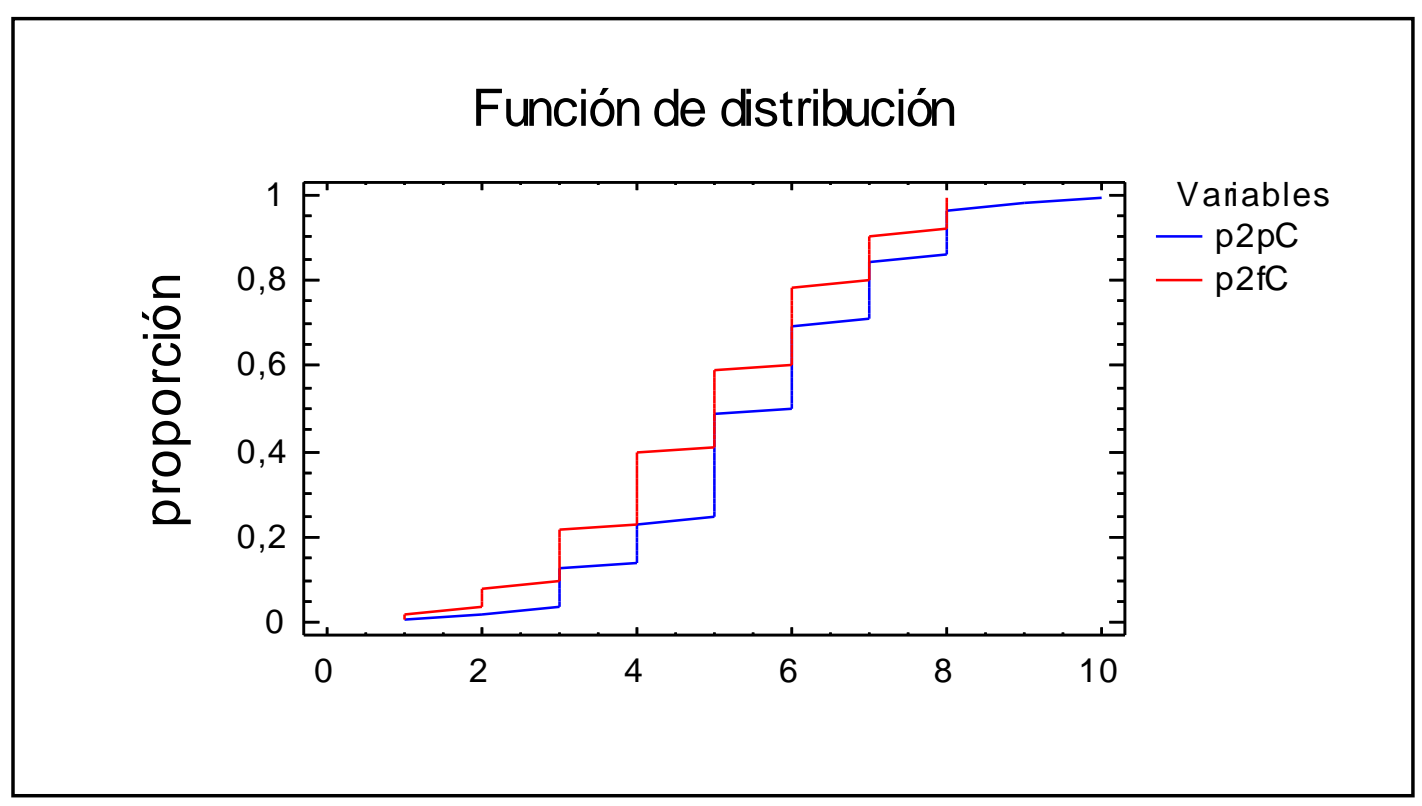

Gráfico 6.27. Comparación de funciones de distribución p2pC-p2fC

\subsection{Comparaciones sobre el aprendizaje (A) de las pruebas}

Resultados conjuntos de los tres ciclos en la categoría Aprendizaje en ambas pruebas, preformal y formal, en cada uno de los dos teoremas $(\mathbf{p} 1, \mathbf{p} 2)$ en que se han 
utilizado. El aprendizaje de la prueba preformal lo denotamos con p1pA y p2pA, y el de la prueba formal p1fA y p2fA.

\begin{tabular}{|c|c|c|c|c|}
\hline Aprendizaje & p1pA & p1fA & p2pA & p2fA \\
\hline $\mathbf{1}$ & 1 & 1 & 1 & 1 \\
\hline $\mathbf{2}$ & 3 & 2 & 0 & 4 \\
\hline $\mathbf{3}$ & 4 & 4 & 5 & 10 \\
\hline $\mathbf{4}$ & 5 & 9 & 7 & 15 \\
\hline $\mathbf{5}$ & 13 & 9 & 17 & 15 \\
\hline $\mathbf{6}$ & 11 & 11 & 18 & 9 \\
\hline $\mathbf{7}$ & 17 & 16 & 8 & 5 \\
\hline $\mathbf{8}$ & 9 & 11 & 11 & 7 \\
\hline $\mathbf{9}$ & 4 & 4 & 0 & 1 \\
\hline $\mathbf{1 0}$ & 0 & 0 & 0 & 0 \\
\hline Total & 67 & 67 & 67 & 67 \\
\hline
\end{tabular}

Tabla 6.28. Resultados conjuntos de los tres ciclos en la categoría aprendizaje en ambas pruebas en cada uno de los dos teoremas

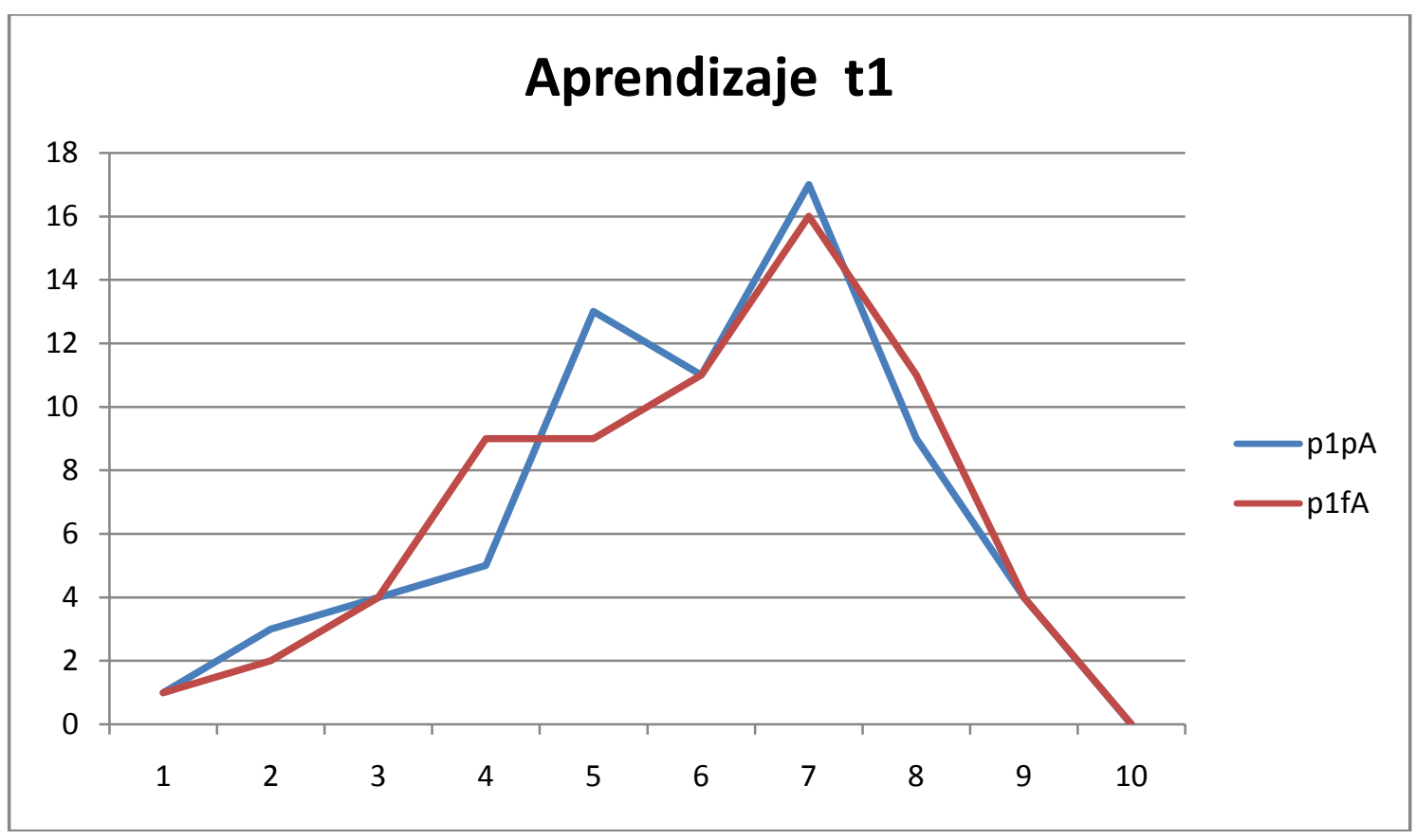

Gráfico 6.28. Frecuencias de los tres ciclos en ambas pruebas en la categoría aprendizaje, teorema 1 


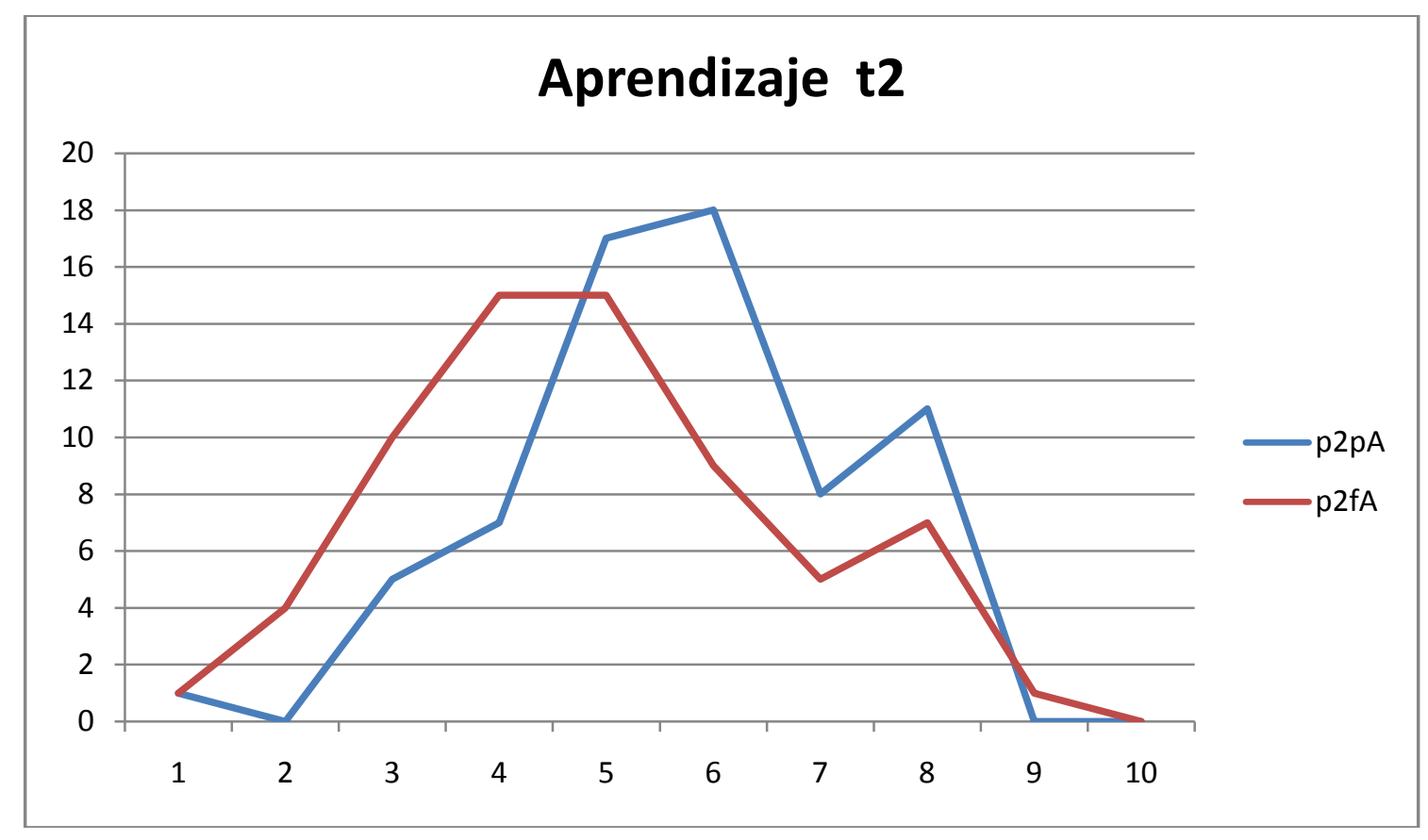

\section{Gráfico 6.28 bis. Frecuencias de los tres ciclos en ambas pruebas en la categoría aprendizaje, teorema 2}

En la tabla 6.29 se recogen los estadísticos descriptivos, donde se aprecian unas valoraciones muy similares en ambas pruebas siendo algo superior la media de la prueba formal, mientras que el resto de estadísticos son iguales. Por la asimetría y curtosis comprobamos que se encuentran en el intervalo $(-2,2)$ con lo que podemos aceptar que cada una de estas muestras sigue una distribución normal.

\section{TEOREMA 1}

\begin{tabular}{|c|c|c|}
\hline \multicolumn{3}{|l|}{ Resumen Estadístico } \\
\hline & $\mathrm{p} 1 \mathrm{pA}$ & $\mathrm{p} 1 \mathrm{fA}$ \\
\hline Frecuencia & 67 & 67 \\
\hline Media & 5,92537 & 5,97015 \\
\hline Mediana & 6,0 & 6,0 \\
\hline Moda & 7,0 & 7,0 \\
\hline Varianza & 3,49435 & 3,54455 \\
\hline Desviación típica & 1,86932 & 1,8827 \\
\hline Mínimo & 1,0 & 1,0 \\
\hline Máximo & 9,0 & 9,0 \\
\hline
\end{tabular}




\begin{tabular}{lll}
\hline Rango & 8,0 & 8,0 \\
Primer cuartil & 5,0 & 5,0 \\
Segundo cuartil & 7,0 & 7,0 \\
Asimetría tipi. & $-1,83235$ & $-1,63487$ \\
Curtosis típificada & $-0,210504$ & $-0,606084$ \\
Coef. de variación & $31,5477 \%$ & $31,5352 \%$
\end{tabular}

El StatAdvisor

$-----------1$

Esta tabla muestra el resumen estadístico para las dos muestras de datos. Pueden usarse otras opciones tabulares dentro de este análisis para probar si las diferencias entre las estadísticas de las dos muestras son estadísticamente significativas. De particular interés está la asimetría estandarizada y la curtosis estandarizada que puede usarse para determinar si las muestras proceden de distribuciones normales. Los valores de estos estadísticos fuera del rango de -2 a +2 indican salidas significativas de normalidad que tendería a invalidar las pruebas que comparan las desviaciones normales. En este caso, ambos valores de asimetría estandarizada están dentro del rango esperado. Ambos valores de curtosis estandarizada están dentro del rango esperado.

Tabla 6.29. Comparación de muestras p1pA-p1fA

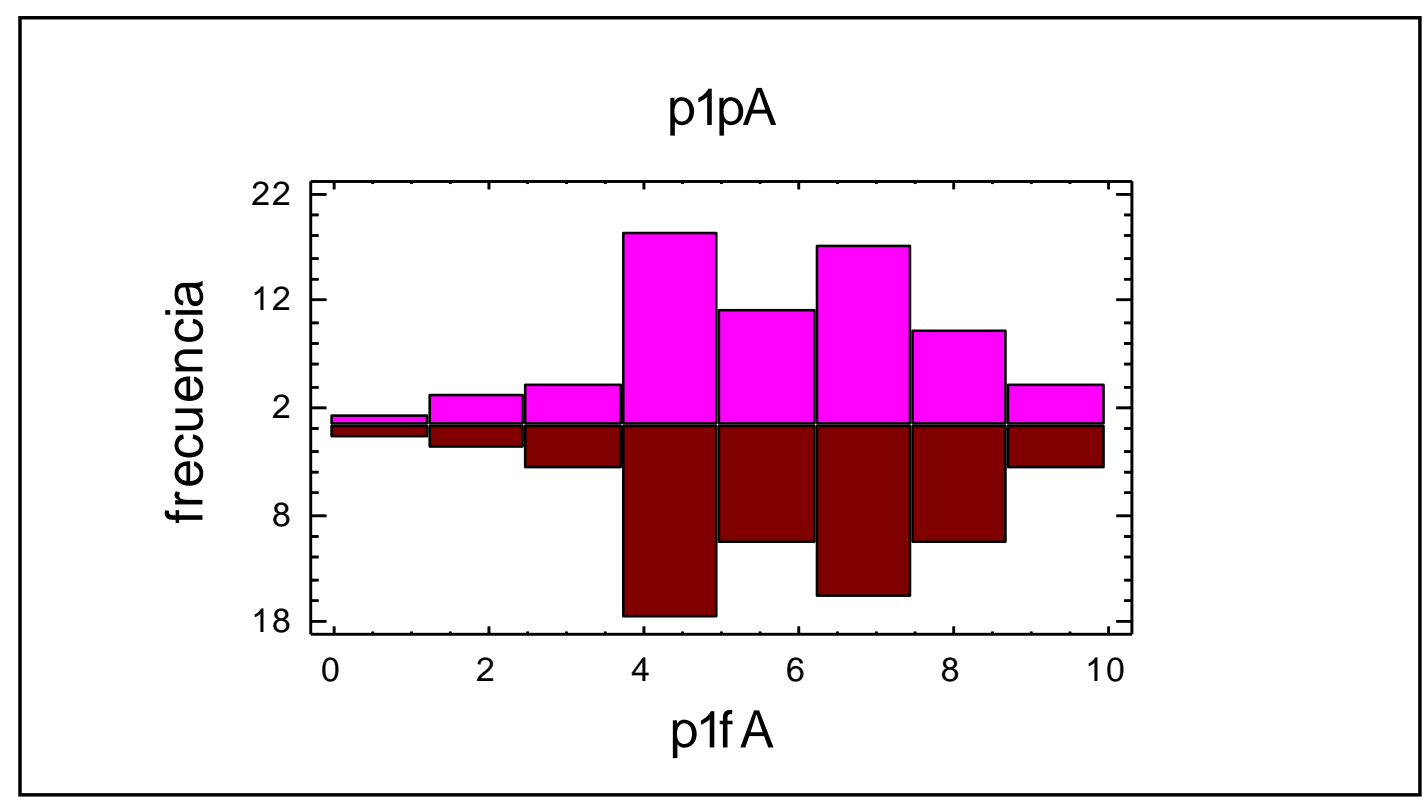

Gráfico 6.29. Comparación de histogramas p1pA-p1fA 


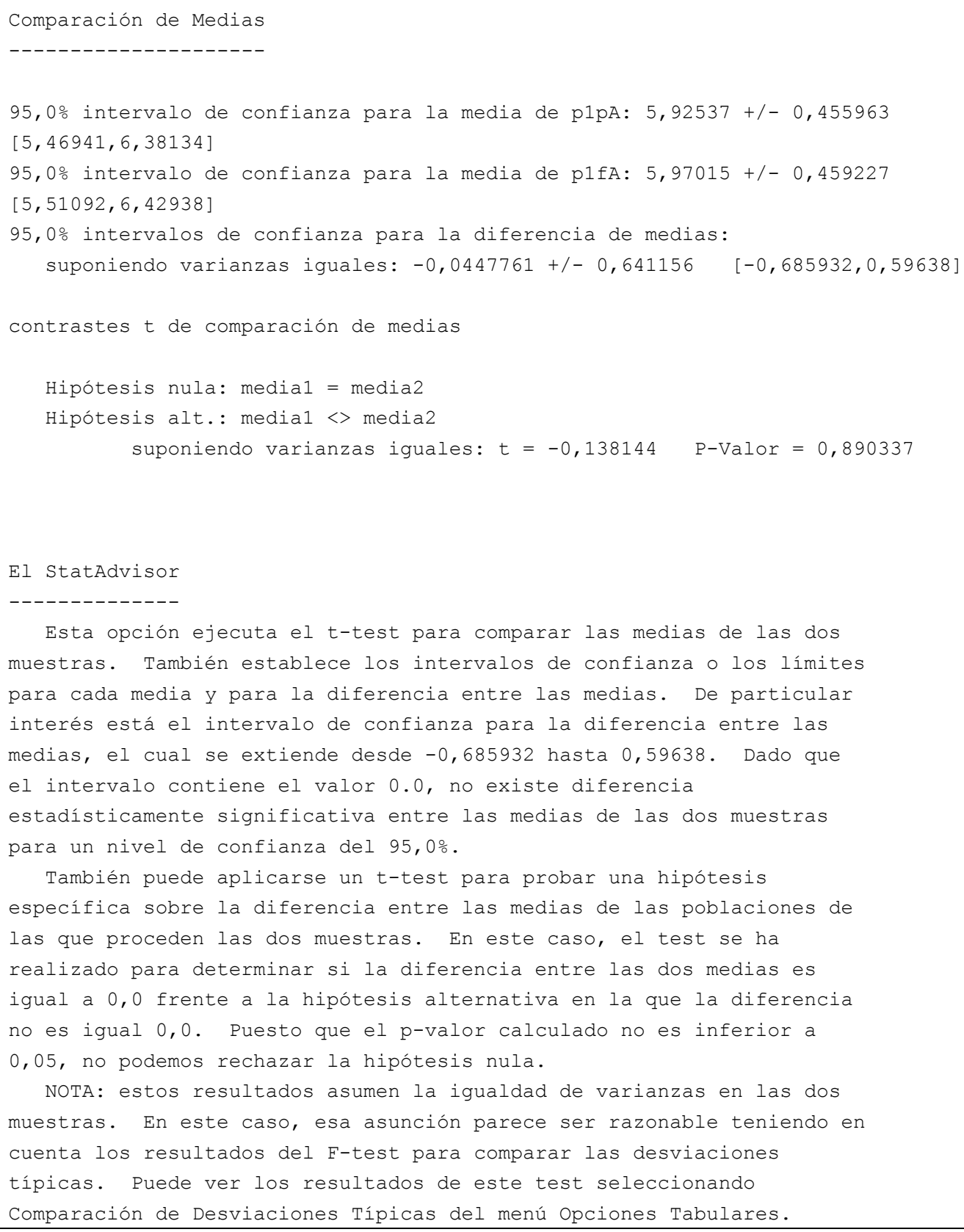

Tabla 6.30. Comparación de medias p1pA-p1fA 


\section{Densidades Suavizadas}

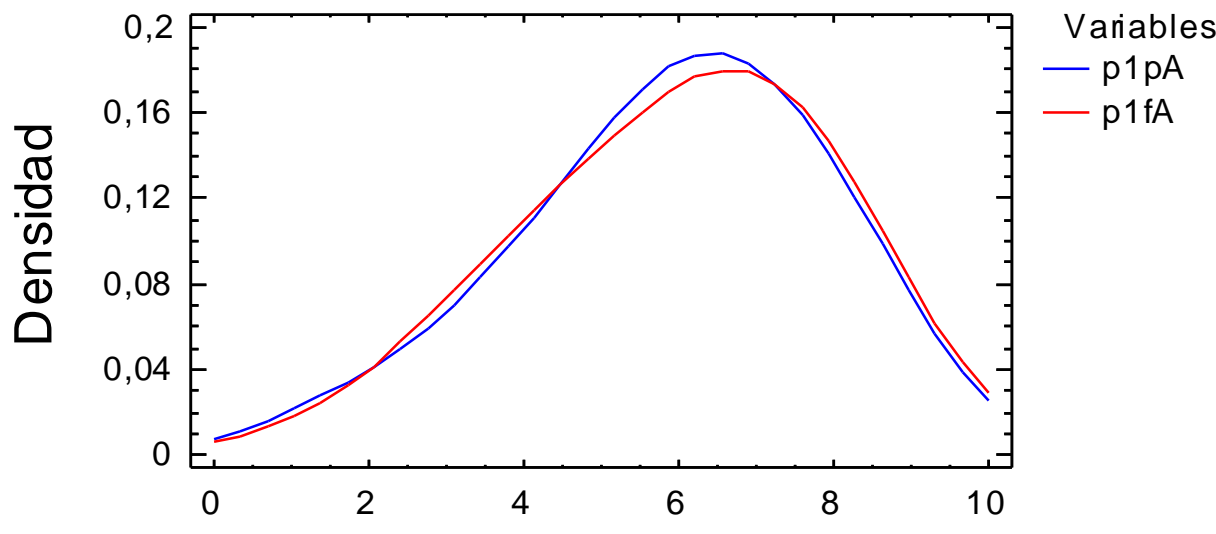

Gráfico 6.30. Comparación de densidades suavizadas p1pA-p1fA

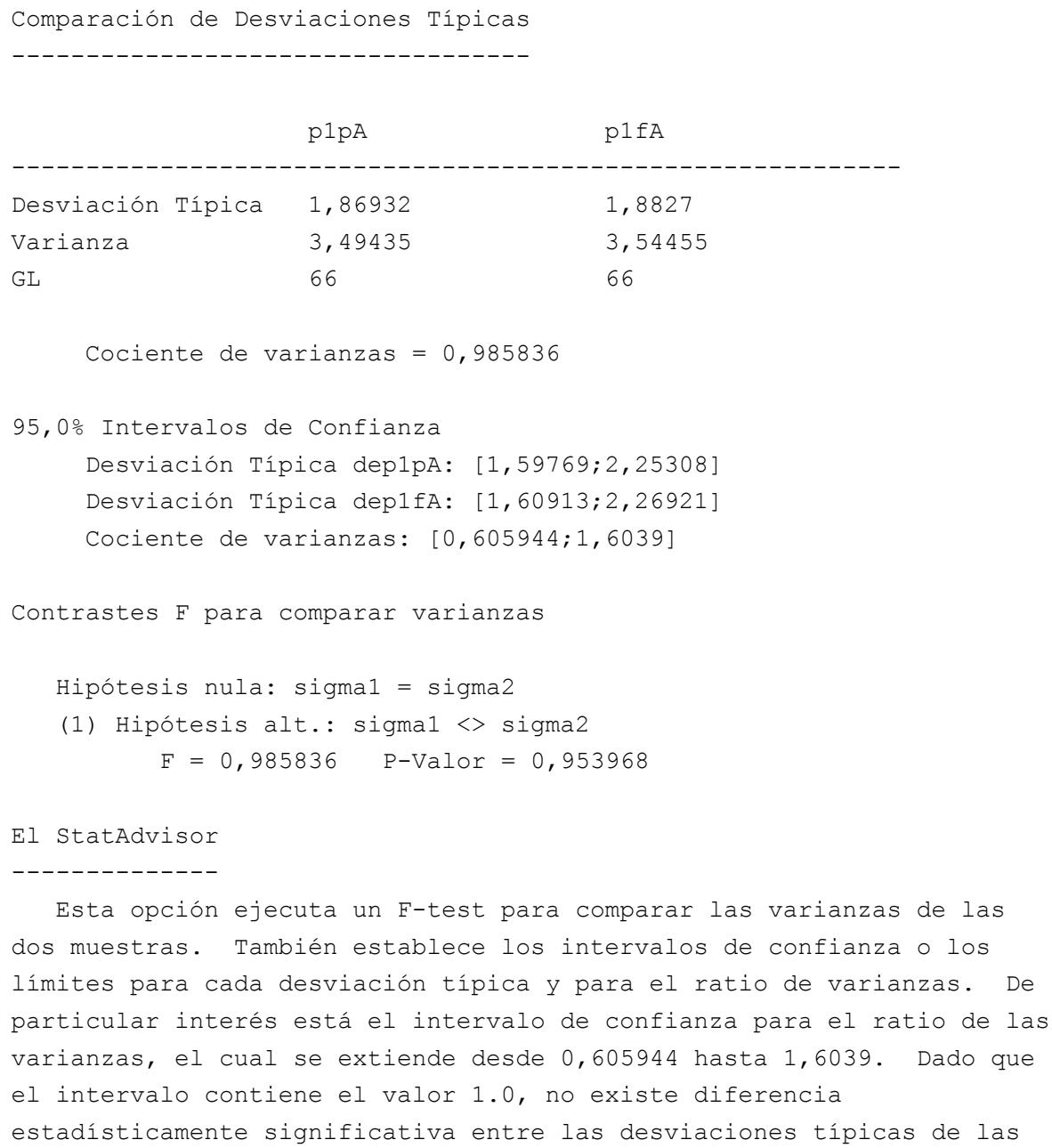


dos muestras para un nivel de confianza del 95,0\%.

También puede utilizarse un F-test para probar una hipótesis específica sobre las desviaciones típicas de las poblaciones de las que proceden las dos muestras. En este caso, el test se ha realizado para determinar si el ratio de las desviaciones típicas son iguales 1,0 frente a la hipótesis alternativa en la que el ratio no es igual 1,0. Puesto que el p-valor calculado no es inferior a 0,05, no podemos rechazar la hipótesis nula.

NOTA IMPORTANTE: los F-test y los intervalos de confianza mostrados dependen de que las muestras procedan de distribuciones normales.

Para comprobar esta asunción, seleccione Resumen Estadístico de la lista de Opciones Tabulares y observe los valores de asimetría estandarizada y curtosis estandarizada.

Tabla 6.31. Comparación de desviaciones típicas p1pA-p1fA

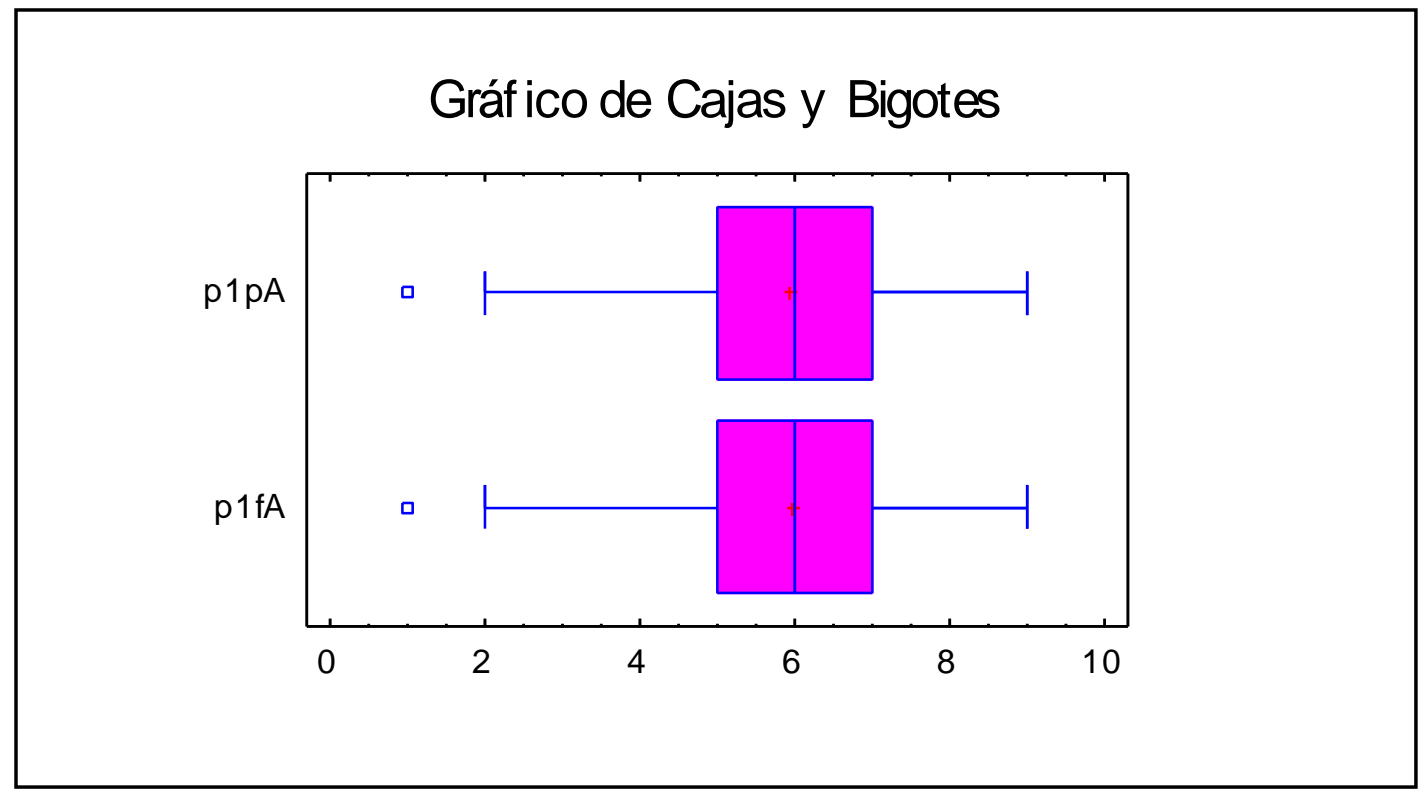

Gráfico 6.31. Comparación de diagramas de cajas y bigotes p1pA-p1fA

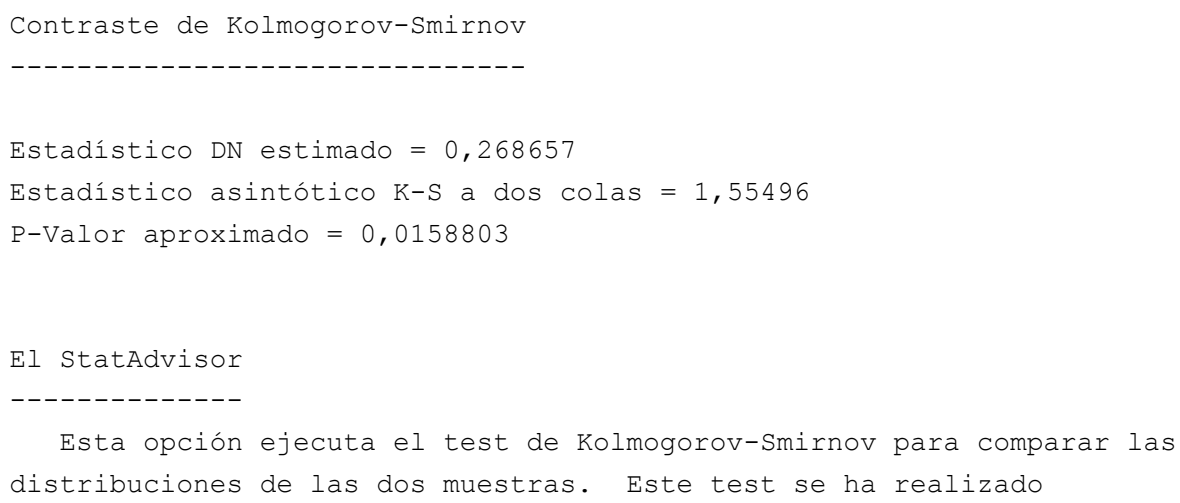


calculando la distancia máxima entre las distribuciones acumuladas de

las dos muestras. En este caso, la máxima distancia es 0,268657, la

cual puede visualizar seleccionando Gráfico Cuantil en la lista de

Opciones Gráficas. De particular interés está el p-valor aproximado

para el test. Dado que el p-valor es menor que 0,05, existe

diferencia estadísticamente significativa entre las dos distribuciones

para un nivel de confianza del 95,0\%.

Tabla 6.32. Contraste de Kolmogorov-Smirnov p1pA-p1fA

Los contrastes de igualdad de medias y de desviaciones típicas, basados en la normalidad de las distribuciones, no permiten rechazar la hipótesis nula, de modo que aceptaríamos que ambas medidas son iguales para las dos muestras de datos. Sin embargo el test de Kolmogorov-Smirnov nos permite rechazar la igualdad de distribuciones, y sobre todo en el gráfico de densidades suavizadas se observa que la distribución para la prueba formal está un poco más a la derecha que la preformal, situándose en valores mayores para las puntuaciones otorgadas a esta prueba.

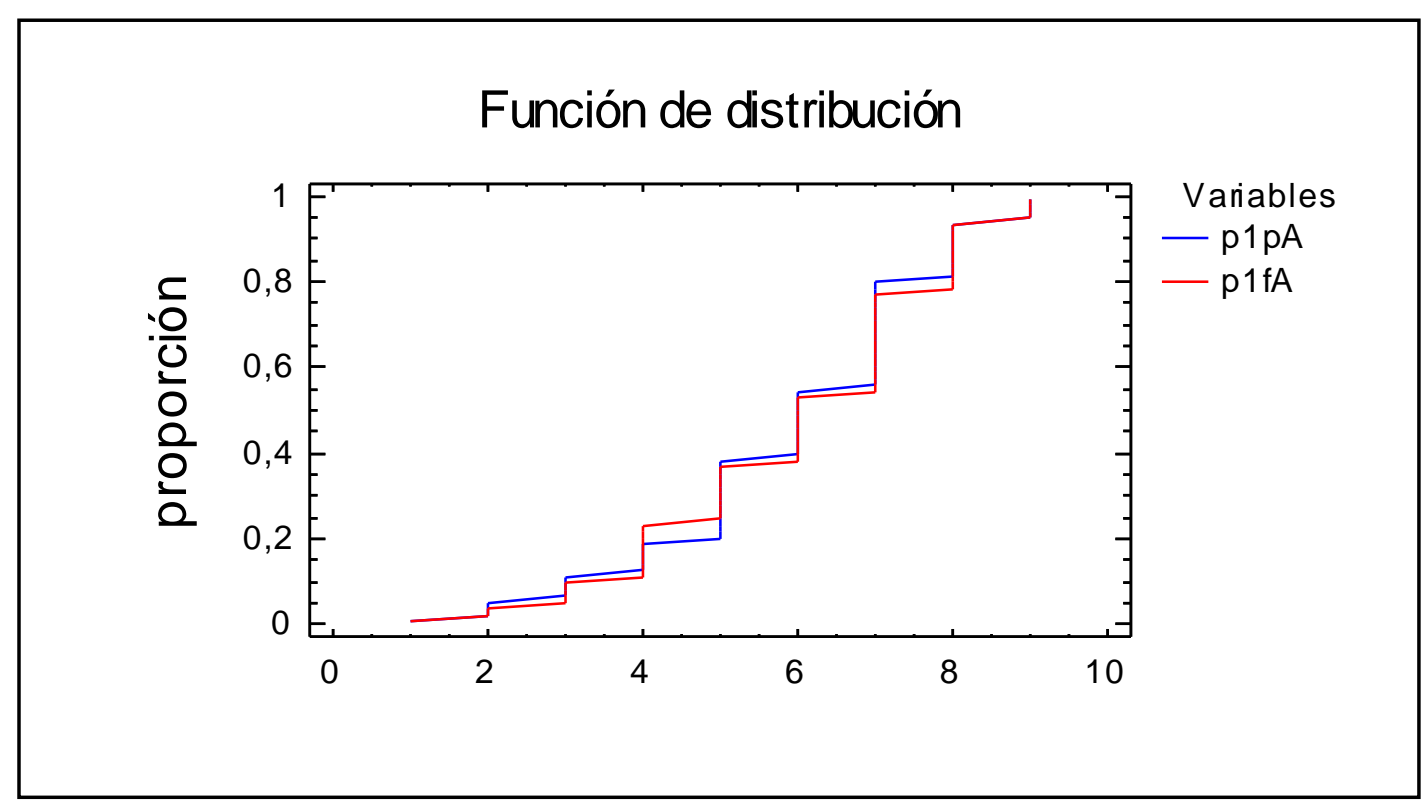

Gráfico 6.32. Comparación de funciones de distribución p1pA-p1fA 


\section{TEOREMA 2}

\begin{tabular}{|c|c|c|}
\hline \multicolumn{3}{|l|}{ Resumen Estadístico } \\
\hline & $\mathrm{p} 2 \mathrm{pA}$ & $\mathrm{p} 2 \mathrm{fA}$ \\
\hline Frecuencia & 67 & 67 \\
\hline Media & 5,68657 & 4,89552 \\
\hline Mediana & 6,0 & 5,0 \\
\hline Moda & 6,0 & \\
\hline Varianza & 2,43057 & 3,24649 \\
\hline Desviación típica & 1,55903 & 1,8018 \\
\hline Mínimo & 1,0 & 1,0 \\
\hline Máximo & 8,0 & 9,0 \\
\hline Rango & 7,0 & 8,0 \\
\hline Primer cuartil & 5,0 & 4,0 \\
\hline Segundo cuartil & 7,0 & 6,0 \\
\hline Asimetría tipi. & $-1,15822$ & 0,912132 \\
\hline Curtosis típificada & 0,112579 & $-0,788045$ \\
\hline Coef. de variación & $27,416 \%$ & $36,8051 \%$ \\
\hline
\end{tabular}

El StatAdvisor

Esta tabla muestra el resumen estadístico para las dos muestras de datos. Pueden usarse otras opciones tabulares dentro de este análisis para probar si las diferencias entre las estadísticas de las dos muestras son estadísticamente significativas. De particular interés está la asimetría estandarizada y la curtosis estandarizada que puede usarse para determinar si las muestras proceden de distribuciones normales. Los valores de estos estadísticos fuera del rango de -2 a +2 indican salidas significativas de normalidad que tendería a invalidar las pruebas que comparan las desviaciones normales. En este caso, ambos valores de asimetría estandarizada están dentro del rango esperado. Ambos valores de curtosis estandarizada están dentro del rango esperado.

Tabla 6.33. Comparación de muestras p2pA-p2fA 


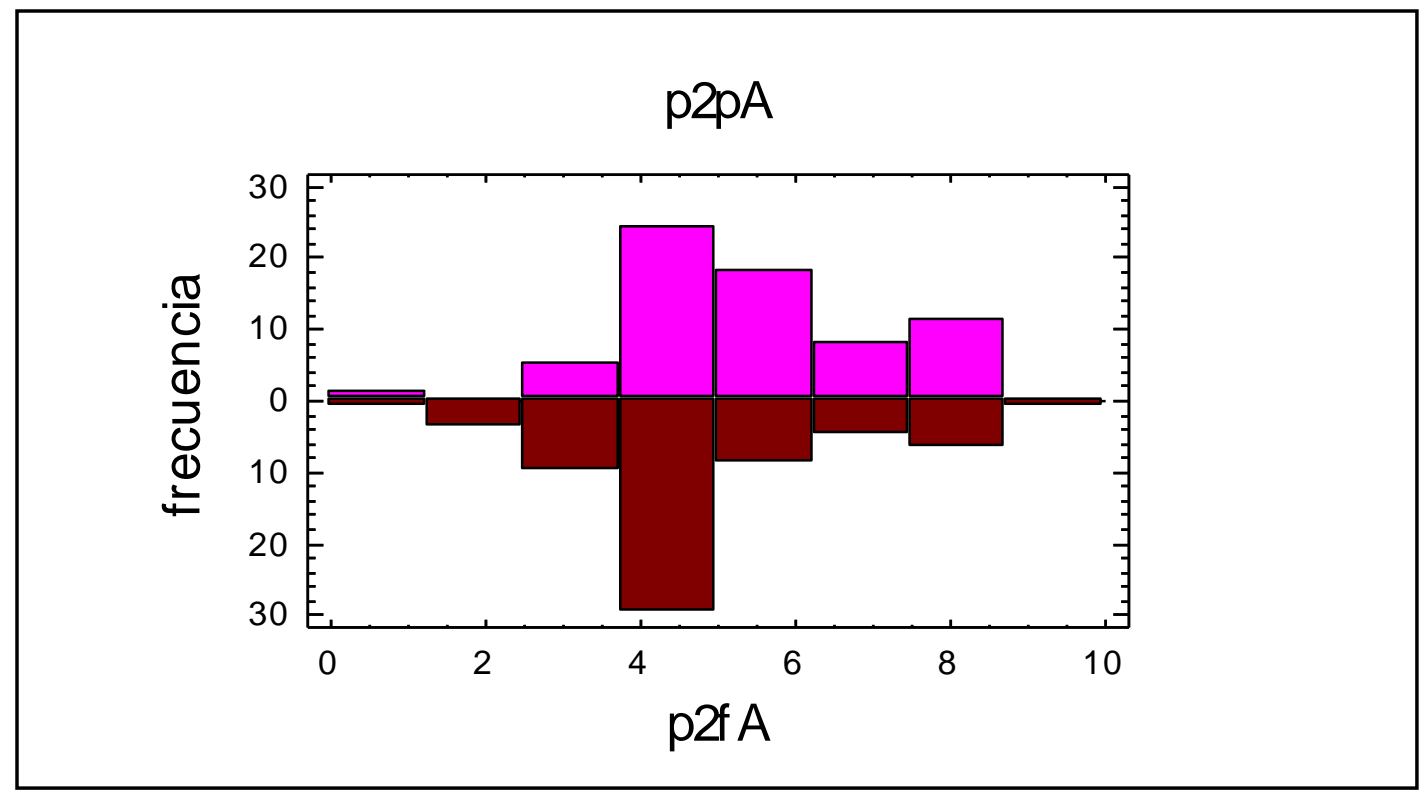

\section{Gráfico 6.33. Comparación de histogramas p2pA-p2fA}

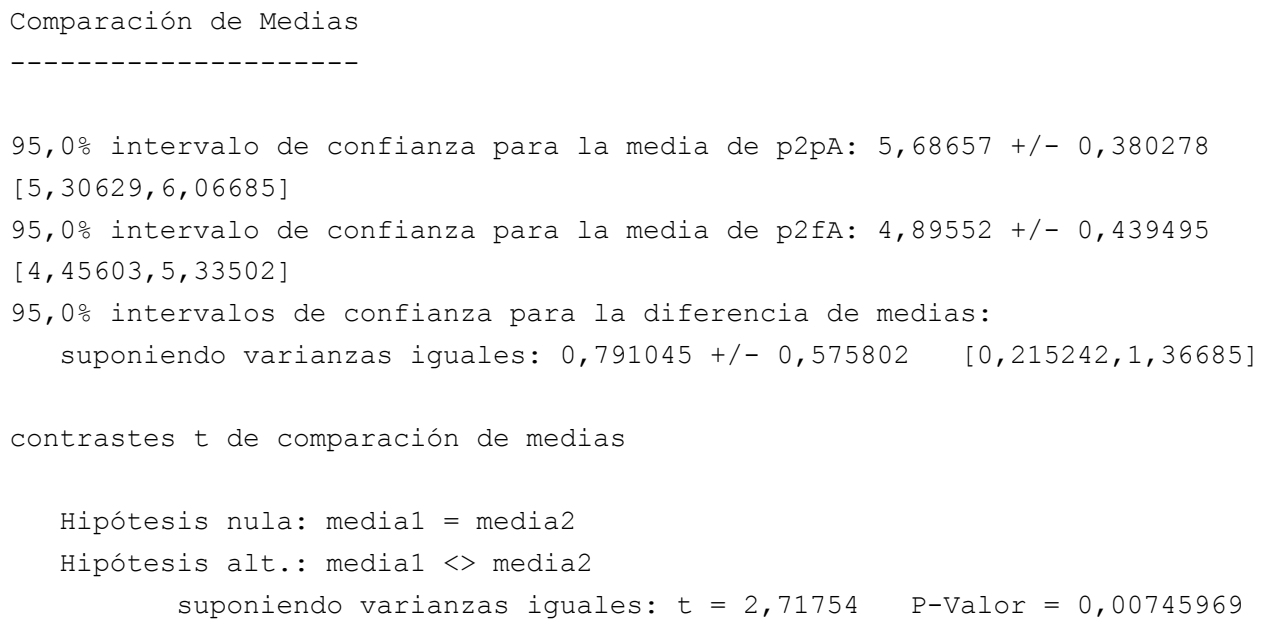


realizado para determinar si la diferencia entre las dos medias es

igual a 0,0 frente a la hipótesis alternativa en la que la diferencia

no es igual 0,0. Puesto que el p-valor calculado es inferior a 0,05,

podemos rechazar la hipótesis nula en favor de la alternativa.

NOTA: estos resultados asumen la igualdad de varianzas en las dos

muestras. En este caso, esa asunción parece ser razonable teniendo en

cuenta los resultados del F-test para comparar las desviaciones

típicas. Puede ver los resultados de este test seleccionando

Comparación de Desviaciones Típicas del menú Opciones Tabulares.

Tabla 6.34. Comparación de medias p2pA-p2fA

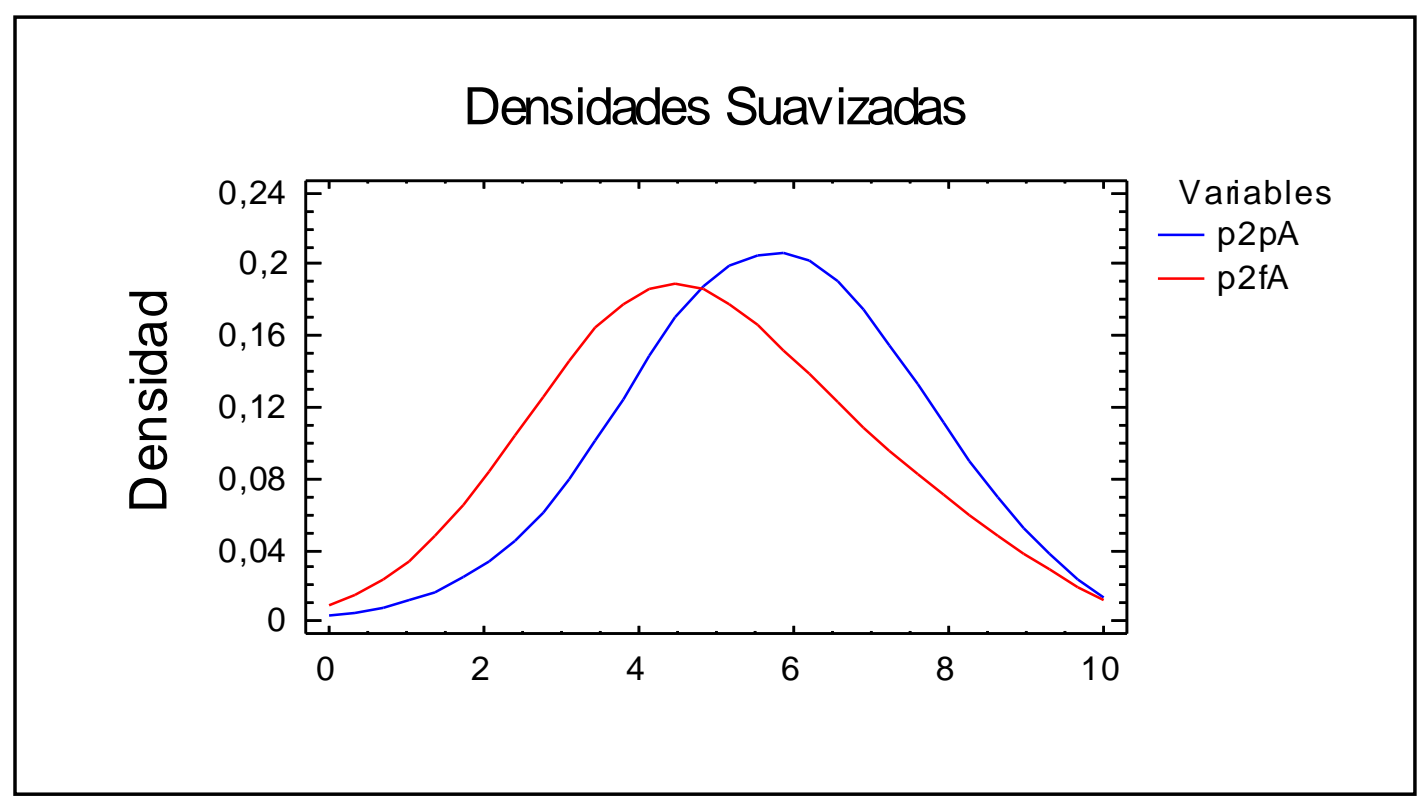

Gráfico 6.34. Comparación de densidades suavizadas p2pA-p2fA

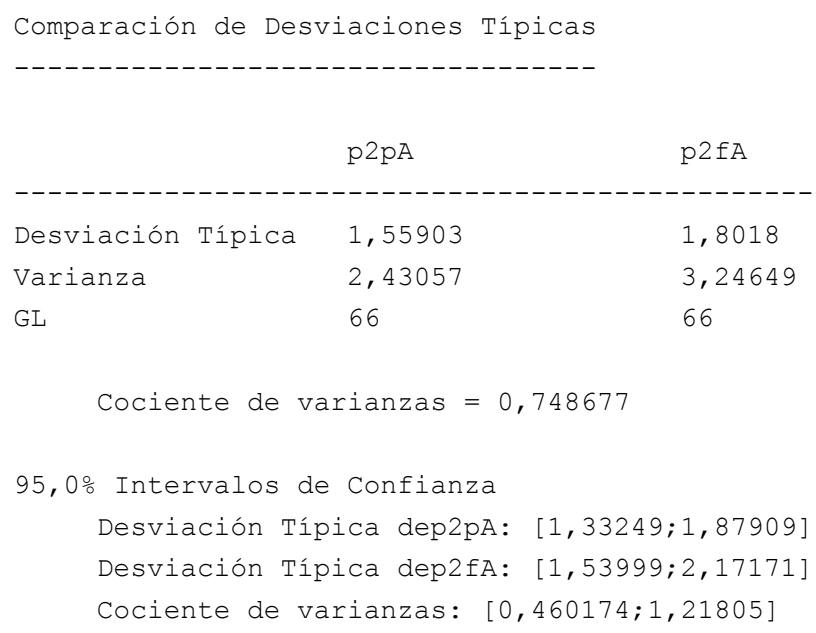


Contrastes F para comparar varianzas

Hipótesis nula: sigmal = sigma2

(1) Hipótesis alt.: sigmal <> sigma2

$$
\mathrm{F}=0,748677 \quad \mathrm{P} \text {-Valor }=0,242294
$$

El StatAdvisor

\section{El statidvisor}

Esta opción ejecuta un F-test para comparar las varianzas de las dos muestras. También establece los intervalos de confianza o los límites para cada desviación típica y para el ratio de varianzas. De particular interés está el intervalo de confianza para el ratio de las varianzas, el cual se extiende desde 0,460174 hasta 1,21805. Dado que el intervalo contiene el valor 1.0, no existe diferencia estadísticamente significativa entre las desviaciones típicas de las dos muestras para un nivel de confianza del 95,0\%.

También puede utilizarse un F-test para probar una hipótesis específica sobre las desviaciones típicas de las poblaciones de las que proceden las dos muestras. En este caso, el test se ha realizado para determinar si el ratio de las desviaciones típicas son iguales 1,0 frente a la hipótesis alternativa en la que el ratio no es igual 1,0. Puesto que el p-valor calculado no es inferior a 0,05, no podemos rechazar la hipótesis nula.

NOTA IMPORTANTE: los F-test y los intervalos de confianza mostrados dependen de que las muestras procedan de distribuciones normales. Para comprobar esta asunción, seleccione Resumen Estadístico de la lista de Opciones Tabulares y observe los valores de asimetría estandarizada y curtosis estandarizada.

Tabla 6.35. Comparación de desviaciones típicas p2pA-p2fA

\section{Gráf ico de Cajas y Bigotes}

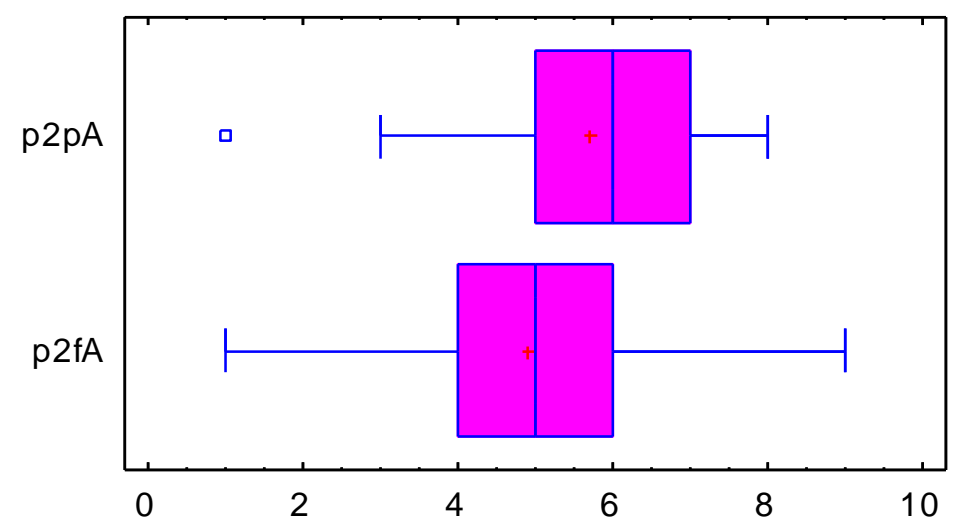

Gráfico 6.35. Comparación de diagramas de cajas y bigotes p2pA-p2fA 
Contraste de Kolmogorov-Smirnov

Contraste de Kolmogorov-Smirnov

Estadístico DN estimado $=0,477612$

Estadístico asintótico $\mathrm{K}-\mathrm{S}$ a dos $\mathrm{colas}=2,76438$

$\mathrm{P}-$ Valor aproximado $=4,60739 \mathrm{E}-7$

El StatAdvisor

Esta opción ejecuta el test de Kolmogorov-Smirnov para comparar las distribuciones de las dos muestras. Este test se ha realizado

calculando la distancia máxima entre las distribuciones acumuladas de

las dos muestras. En este caso, la máxima distancia es 0,477612, la

cual puede visualizar seleccionando Gráfico Cuantil en la lista de

opciones Gráficas. De particular interés está el p-valor aproximado

para el test. Dado que el p-valor es menor que 0,05, existe

diferencia estadísticamente significativa entre las dos distribuciones

para un nivel de confianza del 95,0\%.

Tabla 6.36. Contraste de Kolmogorov-Smirnov p2pA-p2fA

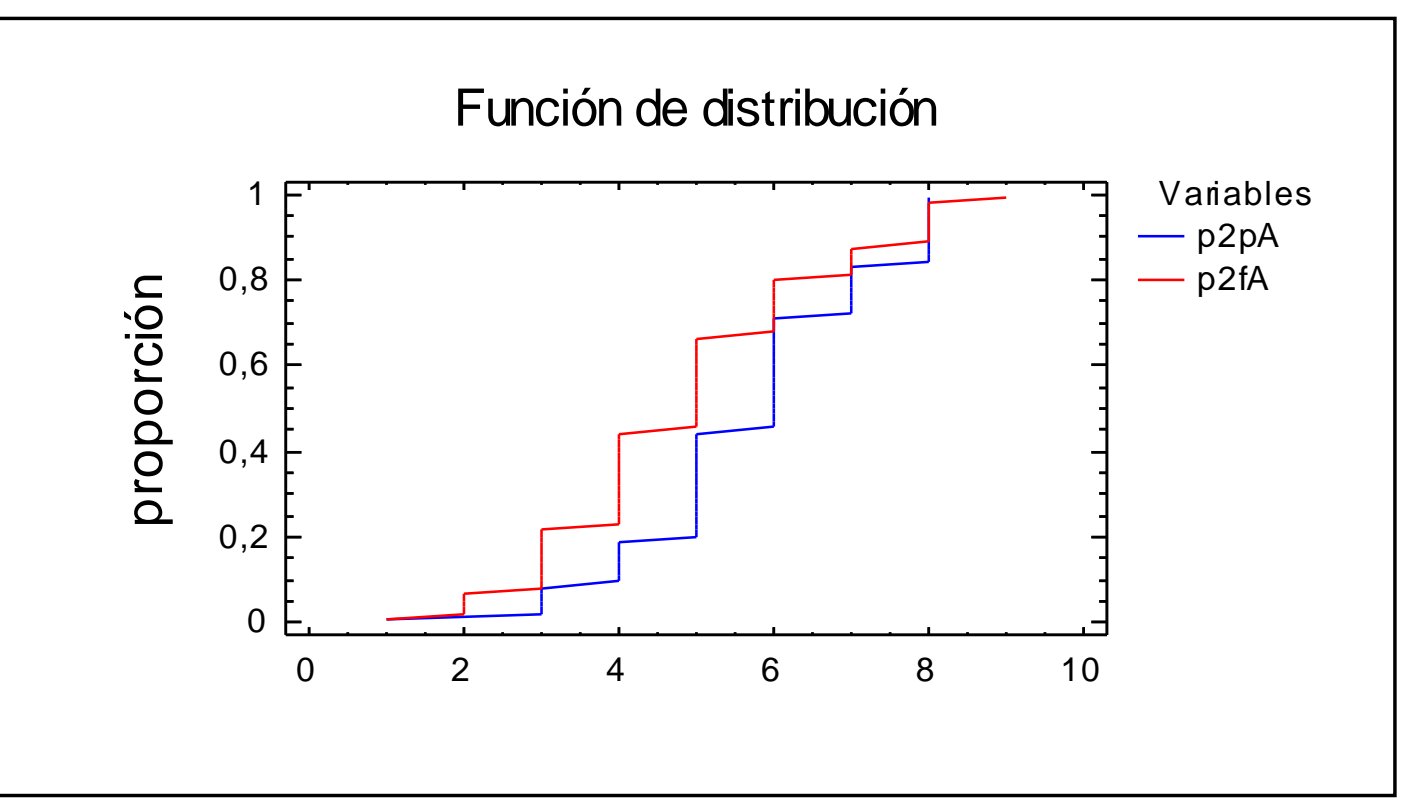

Gráfico 6.36. Comparación de funciones de distribución p2pA-p2fA 
Presentamos ahora los resultados conjuntos de los tres ciclos en las categorías Facilidad, Gusto, Claridad y Aprendizaje de cada una de las dos pruebas, preformal y formal, en ambos teoremas.

\begin{tabular}{|c|c|c|c|c|c|}
\hline \multicolumn{3}{|c|}{ Facilidad } & \multicolumn{3}{c|}{ Gusto } \\
\hline & Fp1,2 & Ff1,2 & & Gp1,2 & Gf1,2 \\
\hline $\mathbf{1}$ & 1 & 3 & $\mathbf{1}$ & 5 & 5 \\
\hline $\mathbf{2}$ & 8 & 6 & $\mathbf{2}$ & 8 & 10 \\
\hline $\mathbf{3}$ & 11 & 21 & $\mathbf{3}$ & 8 & 15 \\
\hline $\mathbf{4}$ & 19 & 26 & $\mathbf{4}$ & 15 & 20 \\
\hline $\mathbf{5}$ & 23 & 32 & $\mathbf{5}$ & 30 & 28 \\
\hline $\mathbf{6}$ & 30 & 23 & $\mathbf{6}$ & 30 & 27 \\
\hline $\mathbf{7}$ & 27 & 16 & $\mathbf{7}$ & 26 & 19 \\
\hline $\mathbf{8}$ & 14 & 7 & $\mathbf{8}$ & 10 & 9 \\
\hline $\mathbf{9}$ & 1 & 0 & $\mathbf{9}$ & 2 & 1 \\
\hline $\mathbf{1 0}$ & 0 & 0 & $\mathbf{1 0}$ & 0 & 0 \\
\hline Total & 134 & 134 & Total & 134 & 134 \\
\hline
\end{tabular}

Tabla 6.37. Conjunto ambas pruebas, tres ciclos, facilidad-gusto en cada teorema ( 1 y 2)

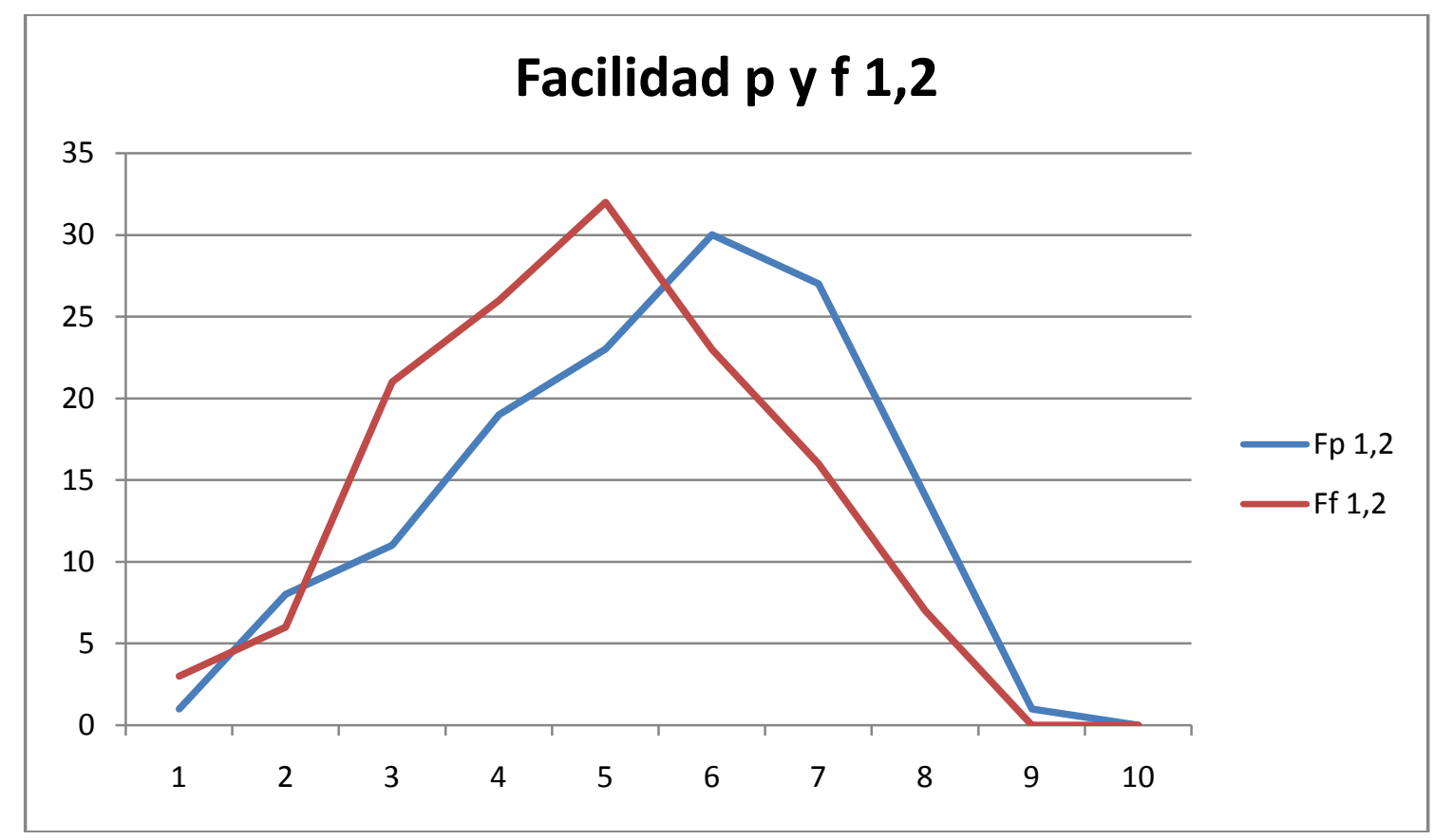

Gráfico 6.37. Conjunto ambas pruebas, tres ciclos, facilidad en cada teorema (1 y 2) 


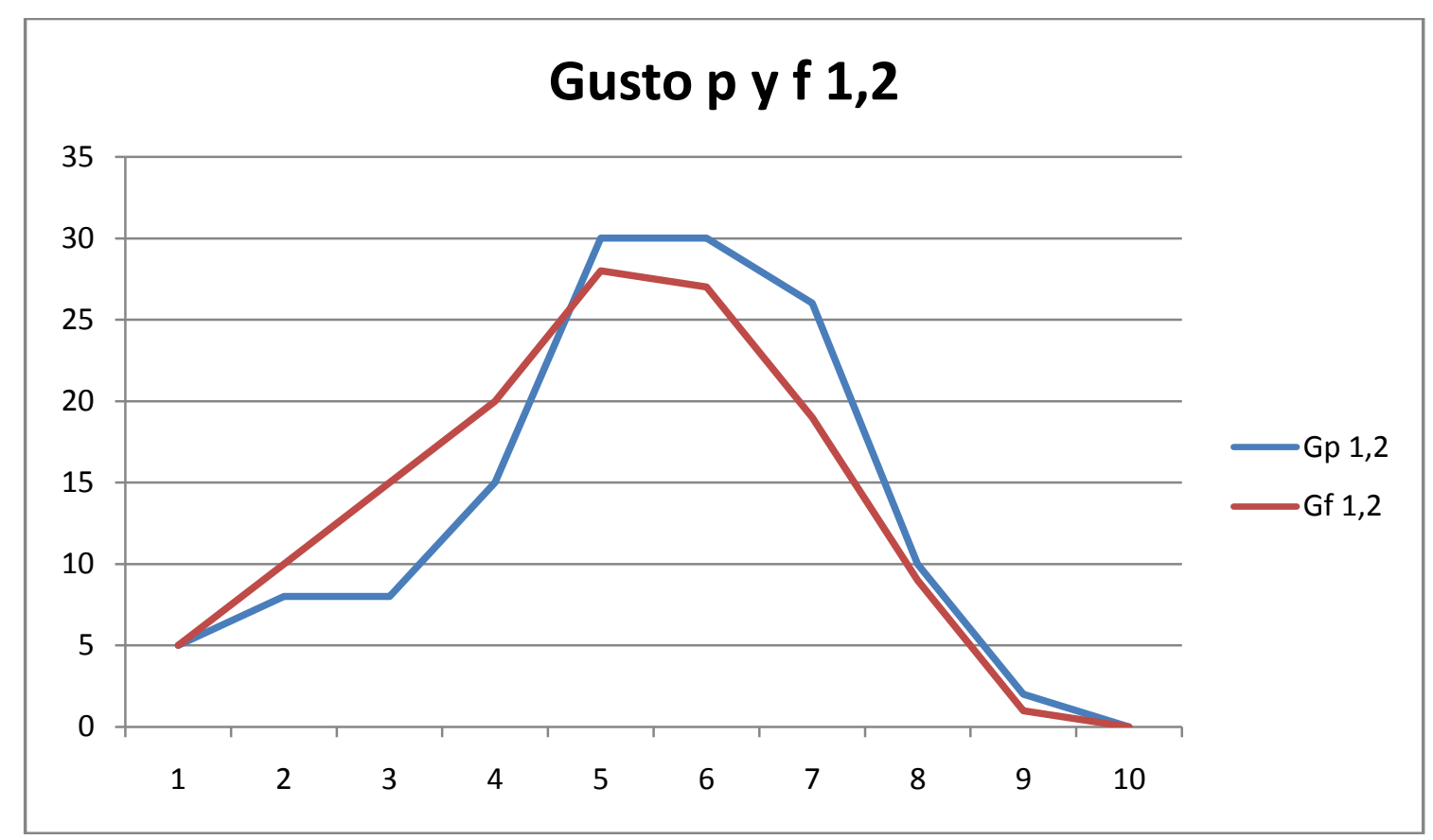

Gráfico 6.37 bis. Conjunto ambas pruebas, tres ciclos, gusto en cada teorema (1 y 2)

\begin{tabular}{|c|c|c|c|c|c|}
\hline \multicolumn{3}{|c|}{ Claridad } & \multicolumn{3}{c|}{ Aprendizaje } \\
\hline & Cp1,2 & Cf1,2 & & Ap1,2 & Af1,2 \\
\hline $\mathbf{1}$ & 3 & 3 & $\mathbf{1}$ & 2 & 2 \\
\hline $\mathbf{2}$ & 2 & 6 & $\mathbf{2}$ & 3 & 6 \\
\hline $\mathbf{3}$ & 14 & 16 & $\mathbf{3}$ & 9 & 14 \\
\hline $\mathbf{4}$ & 11 & 17 & $\mathbf{4}$ & 12 & 24 \\
\hline $\mathbf{5}$ & 30 & 25 & $\mathbf{5}$ & 30 & 24 \\
\hline $\mathbf{6}$ & 33 & 23 & $\mathbf{6}$ & 29 & 20 \\
\hline $\mathbf{7}$ & 21 & 24 & $\mathbf{7}$ & 25 & 21 \\
\hline $\mathbf{8}$ & 15 & 19 & $\mathbf{8}$ & 20 & 18 \\
\hline $\mathbf{9}$ & 3 & 1 & $\mathbf{9}$ & 4 & 5 \\
\hline $\mathbf{1 0}$ & 2 & 0 & $\mathbf{1 0}$ & 0 & 0 \\
\hline Total & 134 & 134 & Total & 134 & 134 \\
\hline
\end{tabular}

Tabla 6.38. Conjunto ambas pruebas, tres ciclos, claridad-aprendizaje en cada teorema ( 1 y 2 ) 


\section{Claridad p y f 1,2}

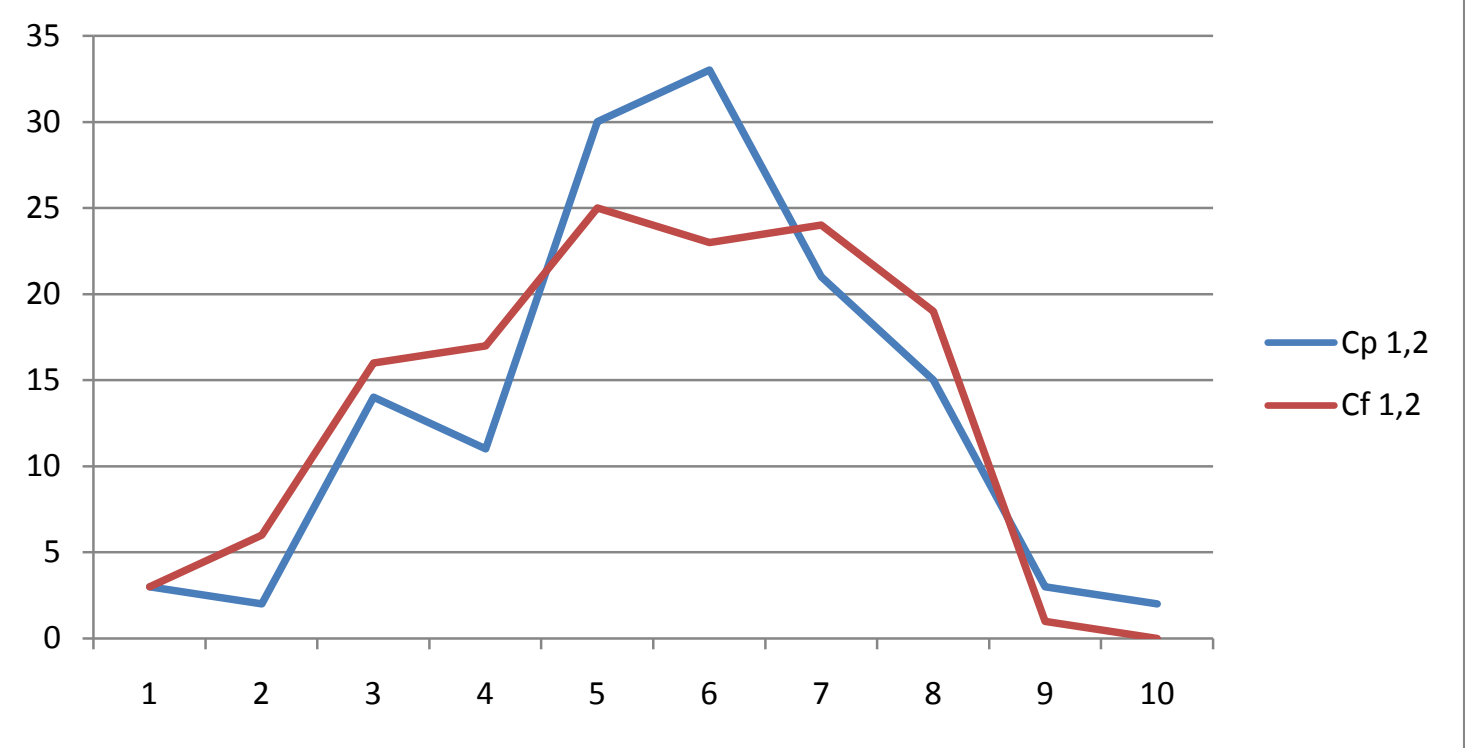

Gráfico 6.38. Conjunto ambas pruebas, tres ciclos, claridad en cada teorema (1 y 2)

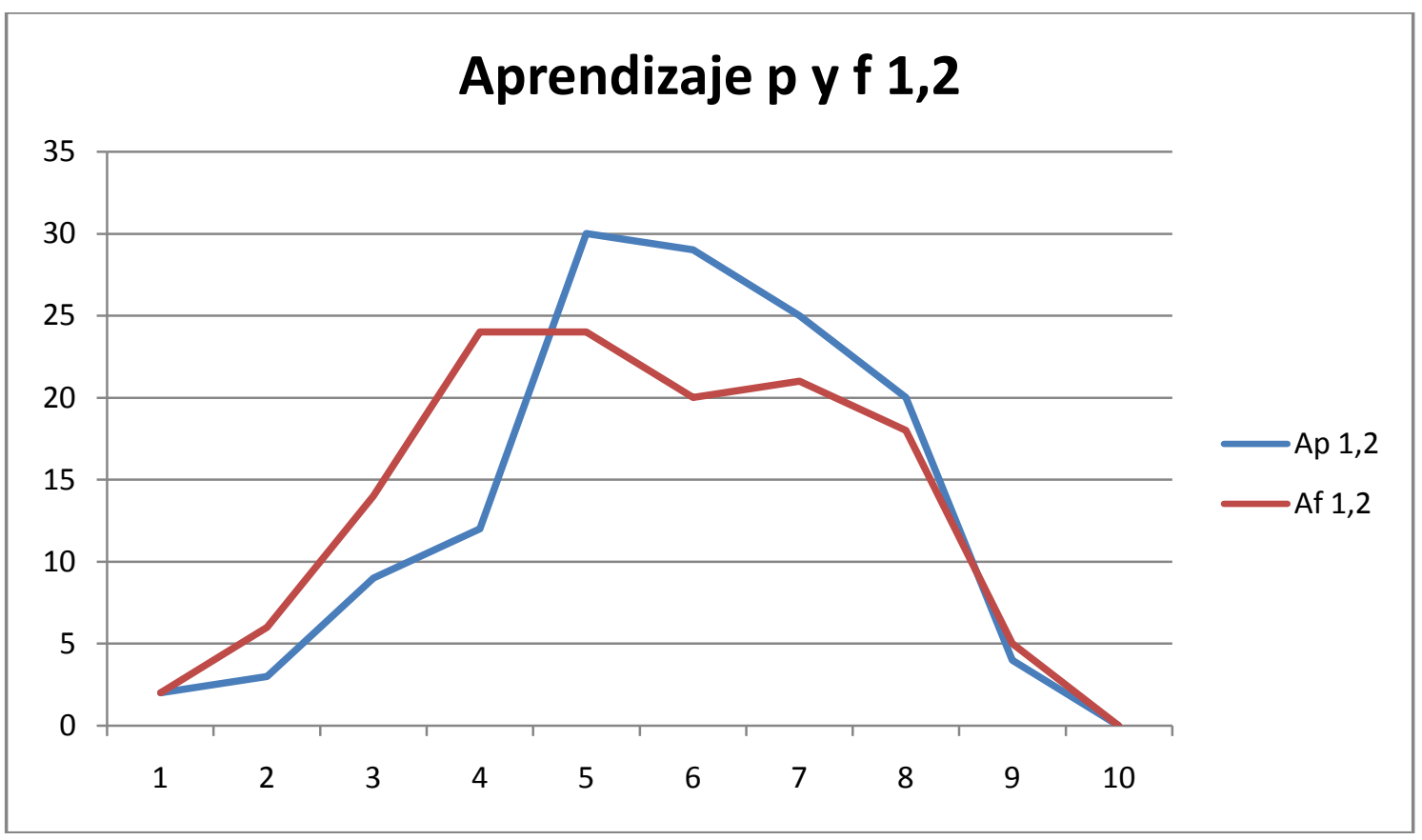

Gráfico 6.38 bis. Conjunto ambas pruebas, tres ciclos, aprendizaje en cada teorema (1 y 2) 
Finalmente, ofrecemos en una doble tabla los datos globales de las valoraciones otorgadas por los alumnos en el conjunto de los tres ciclos a las dos pruebas, preformal y formal, conjuntamente a las cuatro categorías facilidad, gusto, claridad y aprendizaje, y en ambos teoremas. La tabla de la izquierda se refiere a la prueba preformal (PPF), la de la derecha a la formal (PF).

\begin{tabular}{|c|c|c|c|c|c|c|c|c|c|}
\hline PPF & 2007 & 2008 & 2009 & TOTAL & PF & 2007 & 2008 & 2009 & TOTAL \\
\hline 1 & 7 & 4 & 0 & 11 & 1 & 8 & 5 & 0 & 13 \\
\hline 2 & 4 & 15 & 2 & 21 & 2 & 13 & 11 & 4 & 28 \\
\hline 3 & 19 & 16 & 7 & 42 & 3 & 22 & 24 & 20 & 66 \\
\hline 4 & 26 & 12 & 19 & 57 & 4 & 33 & 25 & 29 & 87 \\
\hline 5 & 37 & 36 & 40 & 113 & 5 & 42 & 30 & 37 & 109 \\
\hline 6 & 43 & 30 & 49 & 122 & 6 & 39 & 22 & 32 & 93 \\
\hline 7 & 45 & 28 & 26 & 99 & 7 & 38 & 21 & 21 & 80 \\
\hline 8 & 36 & 9 & 14 & 59 & 8 & 25 & 11 & 17 & 53 \\
\hline 9 & 5 & 2 & 3 & 10 & 9 & 4 & 3 & 0 & 7 \\
\hline 10 & 2 & 0 & 0 & 2 & 10 & 0 & 0 & 0 & 0 \\
\hline
\end{tabular}

Tabla 6.39. Resultados globales, de los tres ciclos en ambos teoremas unidos, en el conjunto de las cuatro categorías

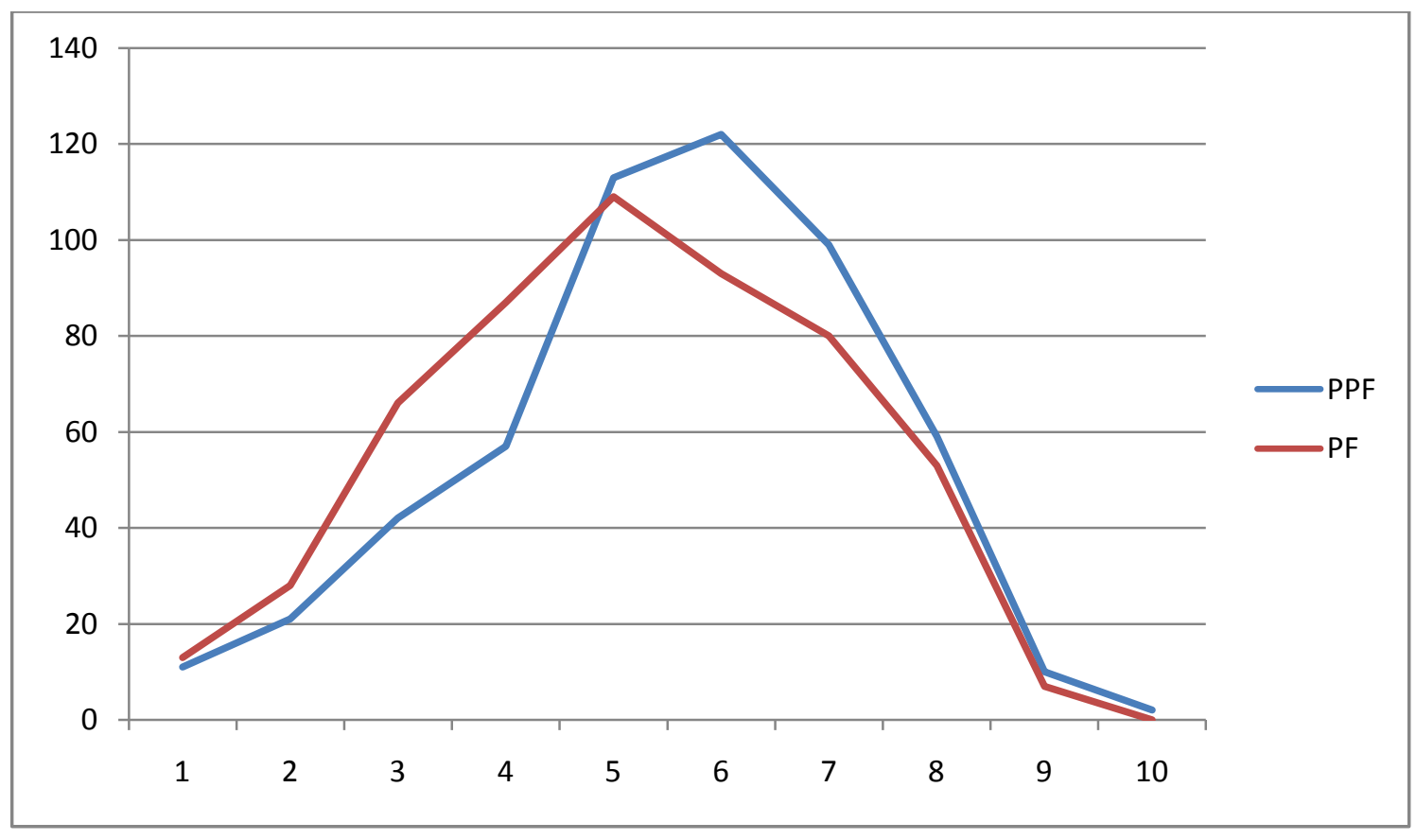

Gráfico 6.39. Resultados globales, de los tres ciclos en ambos teoremas unidos, en el conjunto de las cuatro categorías 
En las tablas 6.37 y 6.38 y los gráficos $6.37,6.37$ bis, 6.38 y 6.38 bis, en las valoraciones otorgadas por los alumnos, aparece la preferencia por la prueba preformal en las cuatro categorías, con bastante claridad siempre en el teorema 2; en el teorema 1 y en las categorías gusto, claridad y aprendizaje esta diferencia no aparece tan patente. En la tabla 6.39 y el gráfico 6.39 esta preferencia aparece de nuevo en las cuatro categorías tanto en las valoraciones altas, 5-10, como sobre todo en las valoraciones bajas, 1-4: la línea azul en el gráfico (prueba preformal) aparece a la derecha de la roja (prueba formal), es decir, favorable en ambas valoraciones (altas y bajas) a la prueba preformal.

\subsection{Comparaciones sobre la valoración global de las pruebas}

Como ejercicio final pedí a los alumnos de cada uno de los tres grupos que han realizado esta experiencia que valorasen el grado de influencia de cada prueba, preformal y formal, en una aplicación concreta del teorema del valor medio (Lagrange) a la función seno.

Utilizando ahora la valoración general que los mismos alumnos otorgaron a las dos modalidades de prueba hechas para el teorema del valor medio podemos comprobar de nuevo la preferencia por la prueba preformal. La valoración de la prueba preformal la denotamos con PPF, y la de la prueba formal con PF. 


\begin{tabular}{|c|c|c|}
\hline & PPF & PF \\
\hline 1 & 2 & 5 \\
\hline 2 & 1 & 2 \\
\hline 3 & 5 & 2 \\
\hline 4 & 2 & 6 \\
\hline 5 & 3 & 9 \\
\hline 6 & 12 & 9 \\
\hline 7 & 9 & 14 \\
\hline 8 & 13 & 7 \\
\hline 9 & 11 & 2 \\
\hline 10 & 0 & 1 \\
\hline Sin nota & & 1 \\
\hline Total general & 58 & 58 \\
\hline
\end{tabular}

Tabla 6.40. Grado de influencia de cada prueba, PPF-PF, al aplicar el teorema 1 a la función seno

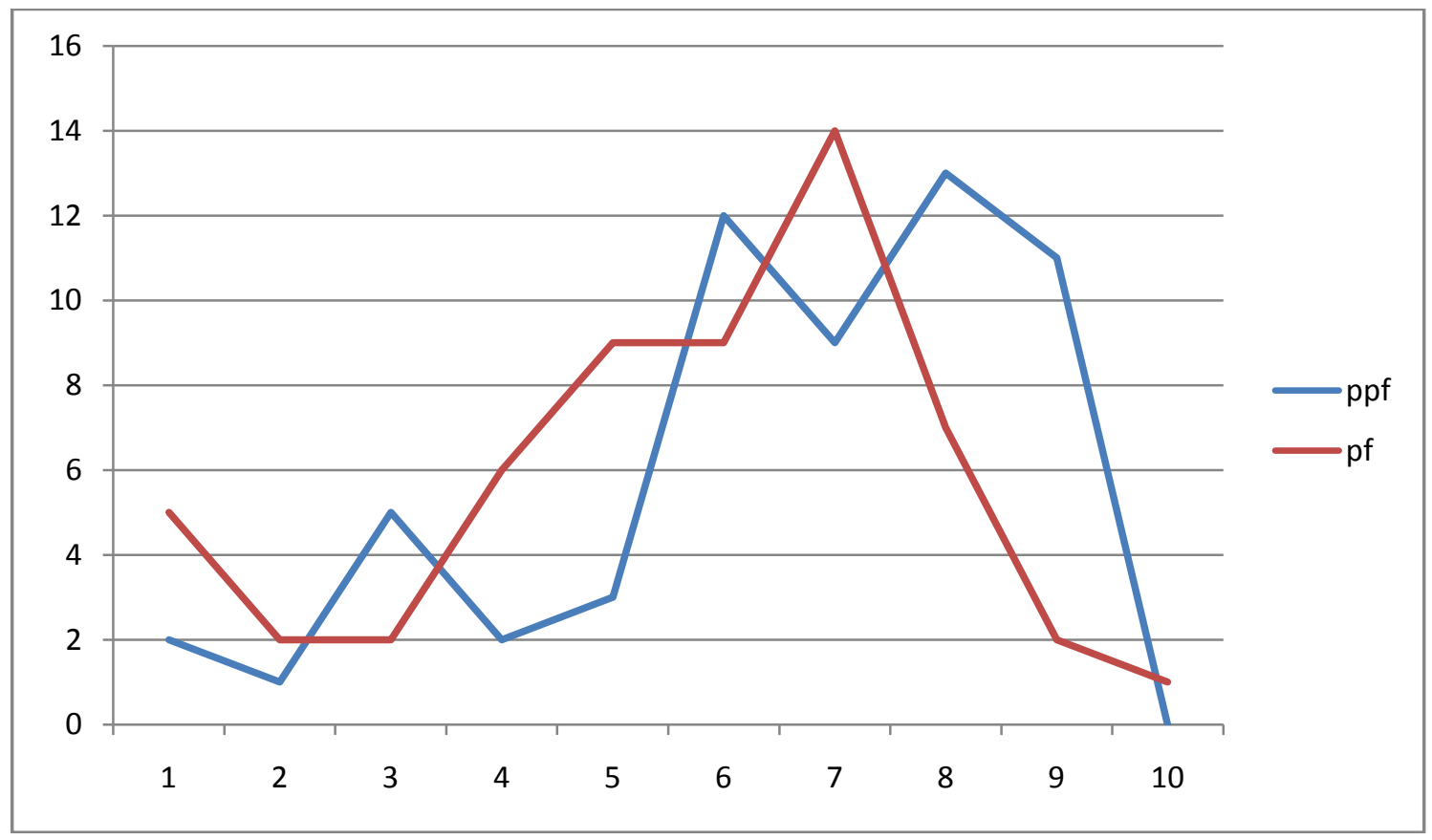

Gráfico 6.40. Refleja el grado de influencia de cada prueba, PPF-PF, al aplicar el teorema 1 a la función seno

En la tabla 6.41 se recogen los estadísticos descriptivos, donde se aprecian unas mayores valoraciones de la prueba preformal siendo algo superior la media, la moda, la mediana y en ambos cuartiles. 
Por la asimetría y curtosis comprobamos que no se encuentran en el intervalo $(-2,2)$ con lo que no podemos aceptar que cada una de estas muestras sigue una distribución normal. Esto significa que los contrastes de igualdad de medias y de desviaciones típicas, basados en la normalidad de las distribuciones, no tienen sentido en este caso.

Sin embargo el test de Kolmogorov-Smirnov de igualdad de distribuciones (no se restringe a normalidad) nos permite rechazar la igualdad de distribuciones, y tanto en el gráfico de densidades suavizadas como en el de cajas y bigotes y en la función de distribución se observa que la distribución para la prueba preformal está más a la derecha que la formal, situándose en valores mayores para las puntuaciones otorgadas a esta prueba.

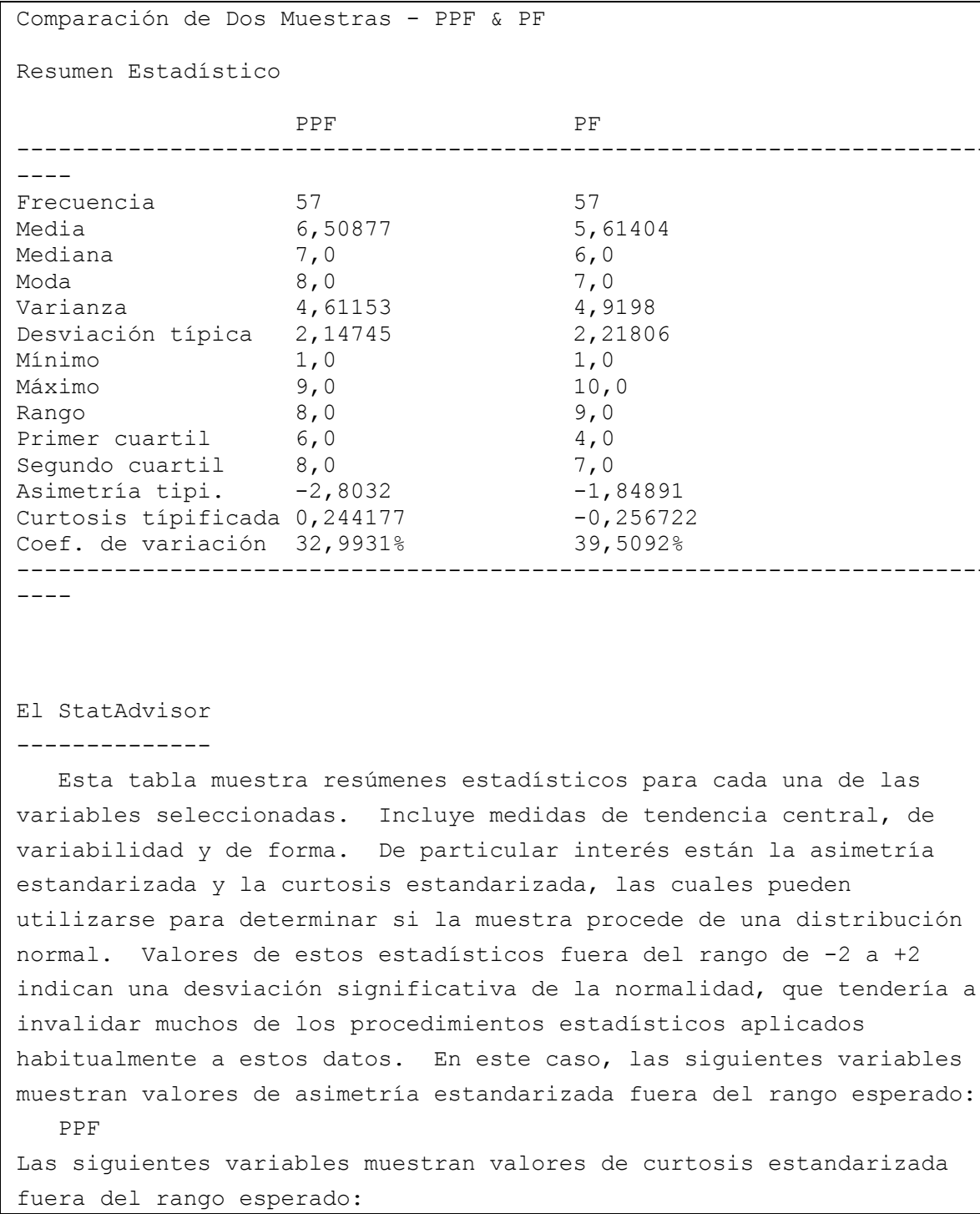




\section{$<$ ninguna $>$}

Para hacer las variables más normales, podría intentar una

transformación como LOG(Y), SQRT(Y), O 1/Y.

\section{Tabla 6.41. Comparación de muestras PPF-PF}

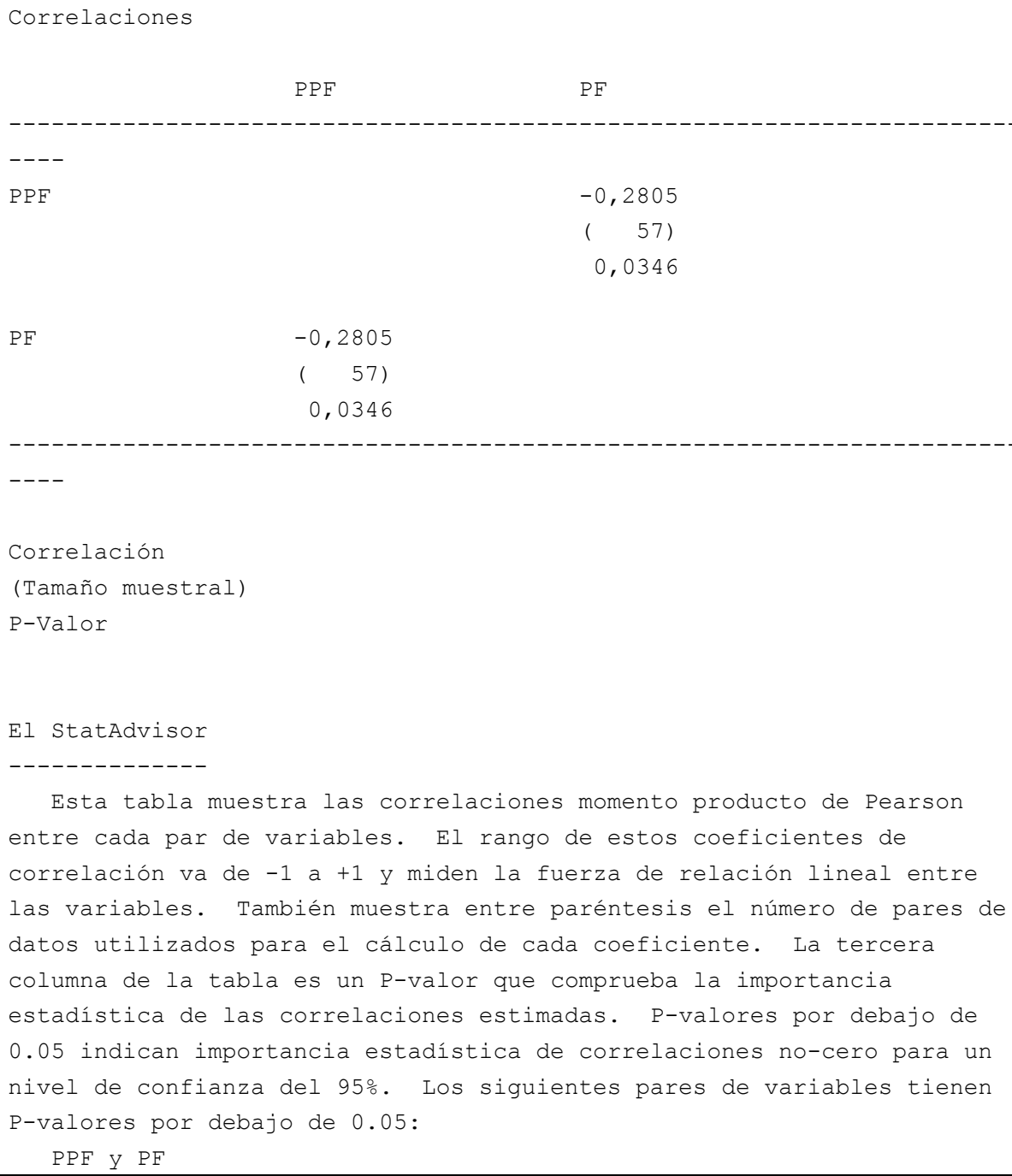

Tabla 6.42. Correlaciones PPF-PF, momento producto de Pearson

$\begin{array}{lc}\text { Correlaciones por Rangos de Spearman } & \\ & \text { PPF } \\ & \text { PF } \\ --- & -0,2563 \\ \text { PPF } & (57) \\ & 0,0551\end{array}$




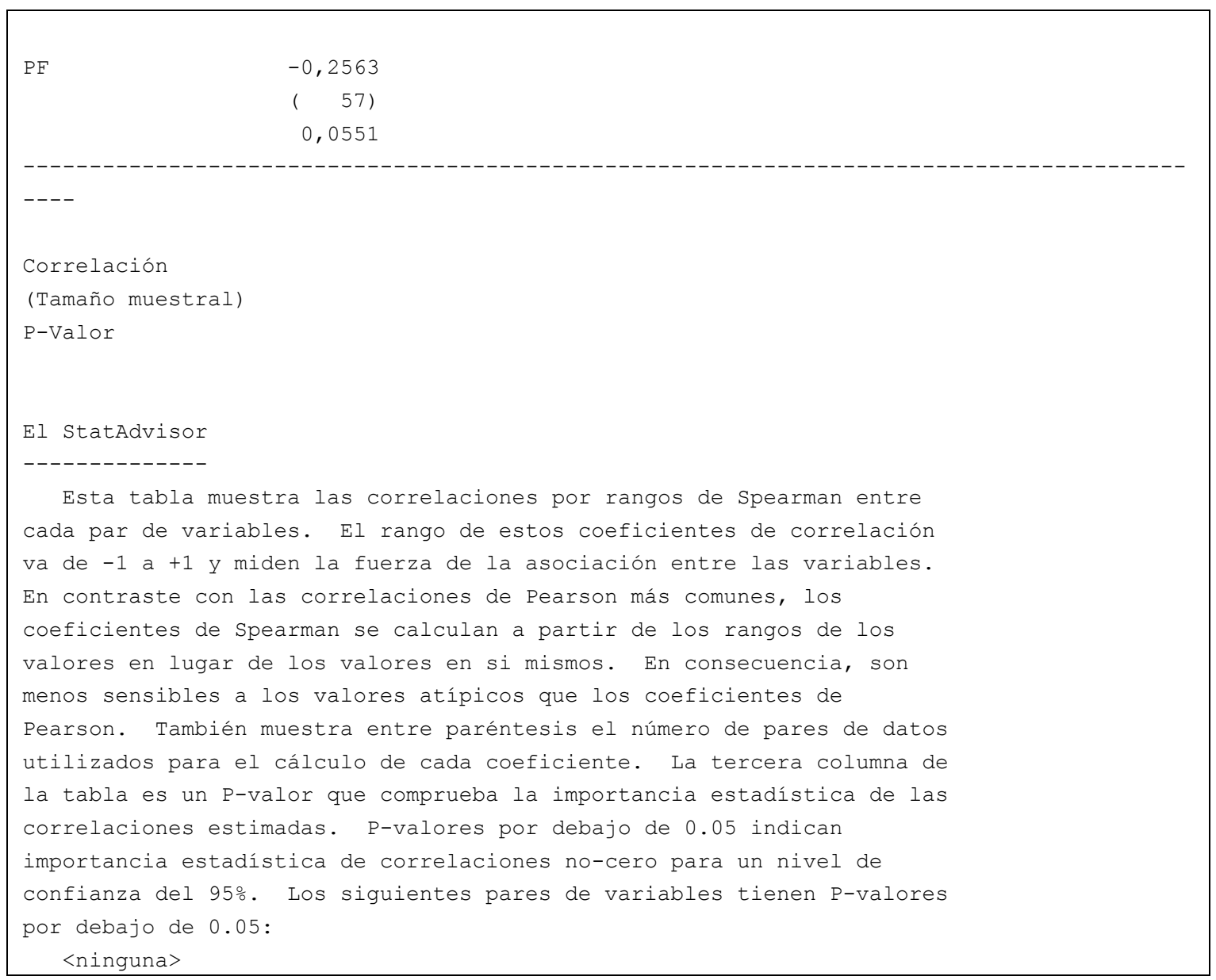

Tabla 6.43. Correlaciones por rangos de Spearman PPF-PF.

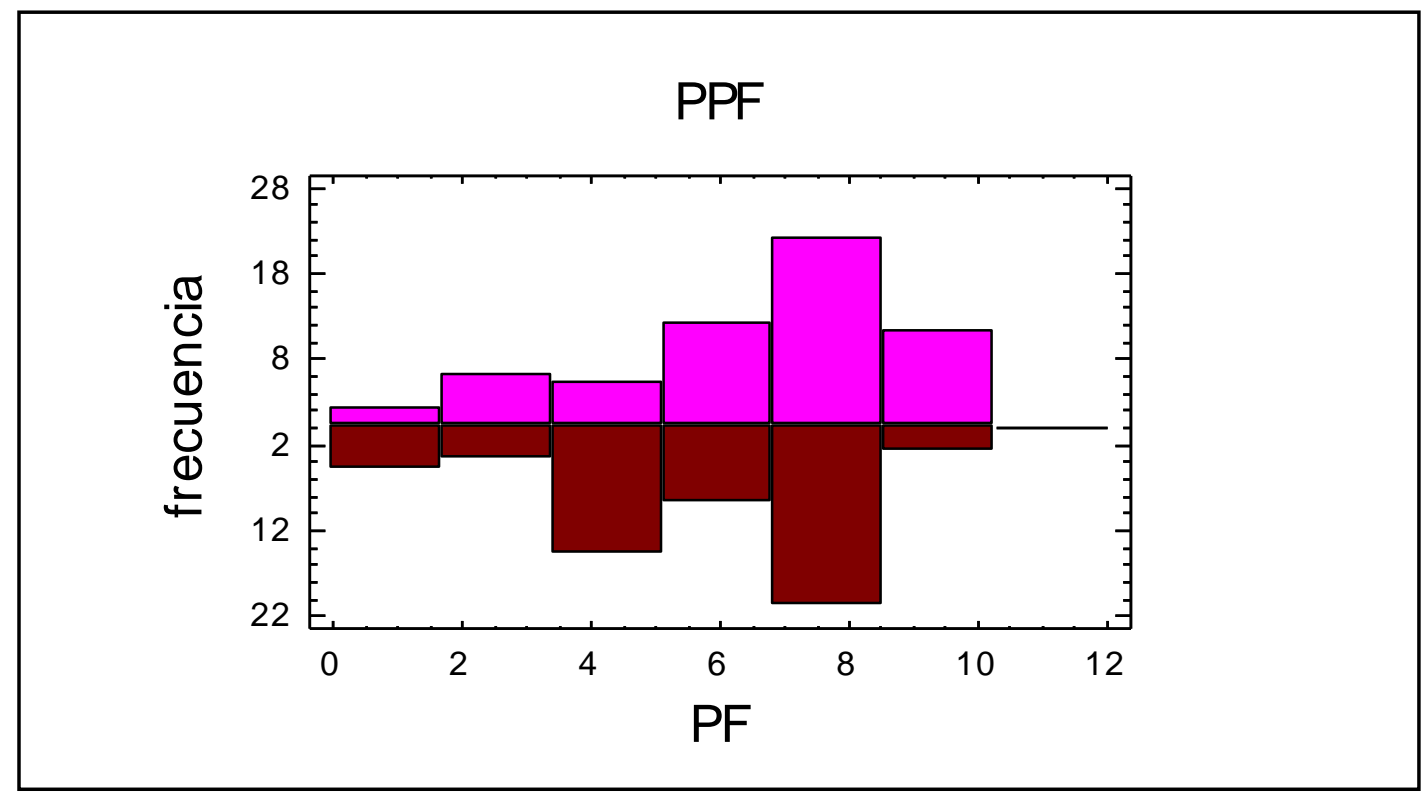

Gráfico 6.41.Comparación de histogramas PPF-PF 


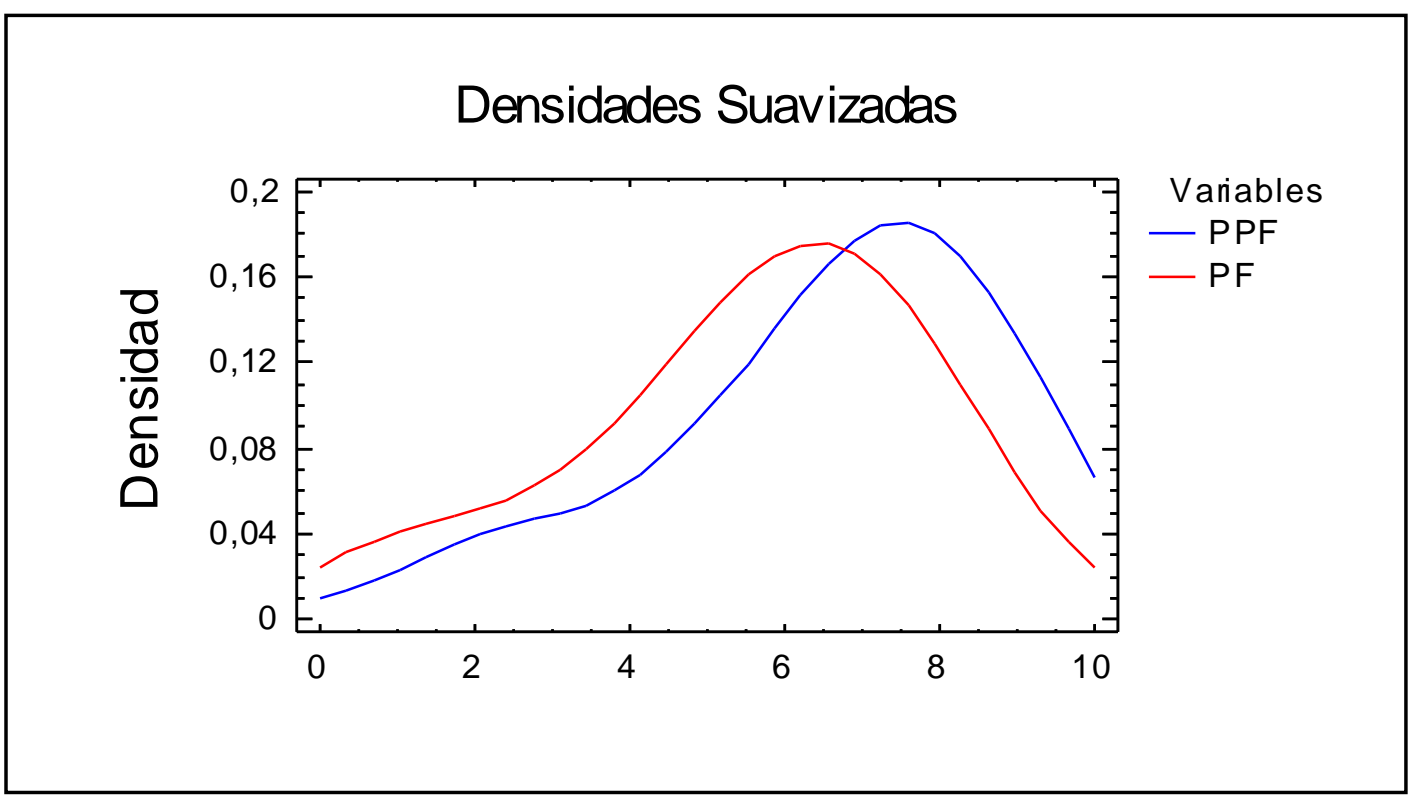

Gráfico 6.42. Comparación de densidades suavizadas PPF-PF

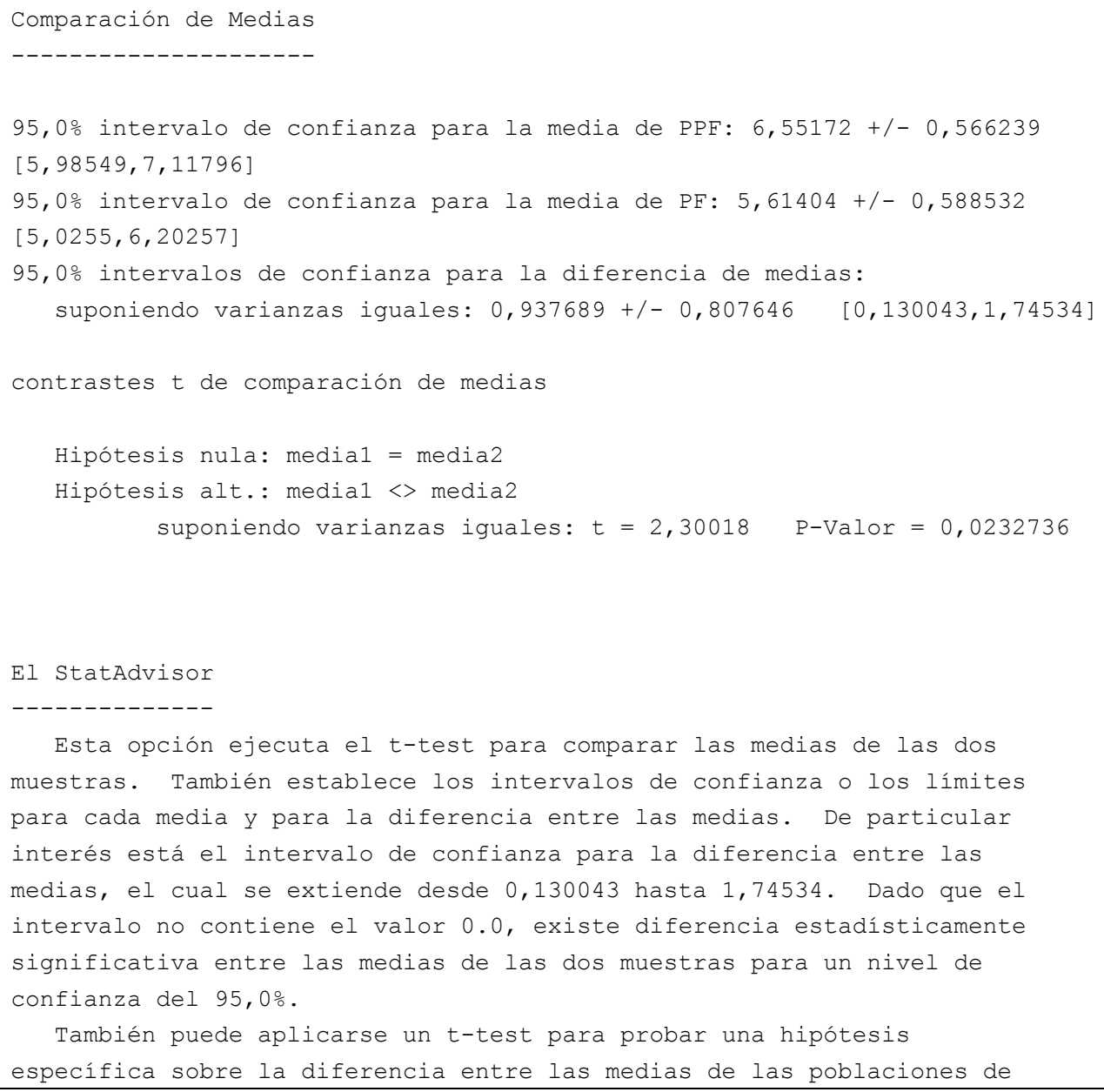


las que proceden las dos muestras. En este caso, el test se ha

realizado para determinar si la diferencia entre las dos medias es

igual a 0,0 frente a la hipótesis alternativa en la que la diferencia

no es igual 0,0. Puesto que el p-valor calculado es inferior a 0,05,

podemos rechazar la hipótesis nula en favor de la alternativa.

NOTA: estos resultados asumen la igualdad de varianzas en las dos

muestras. En este caso, esa asunción parece ser razonable teniendo en

cuenta los resultados del F-test para comparar las desviaciones

típicas. Puede ver los resultados de este test seleccionando

Comparación de Desviaciones Típicas del menú Opciones Tabulares.

Tabla 6.44. Comparación de medias PPF-PF

\section{Gráf ico de Cajas y Bigotes}

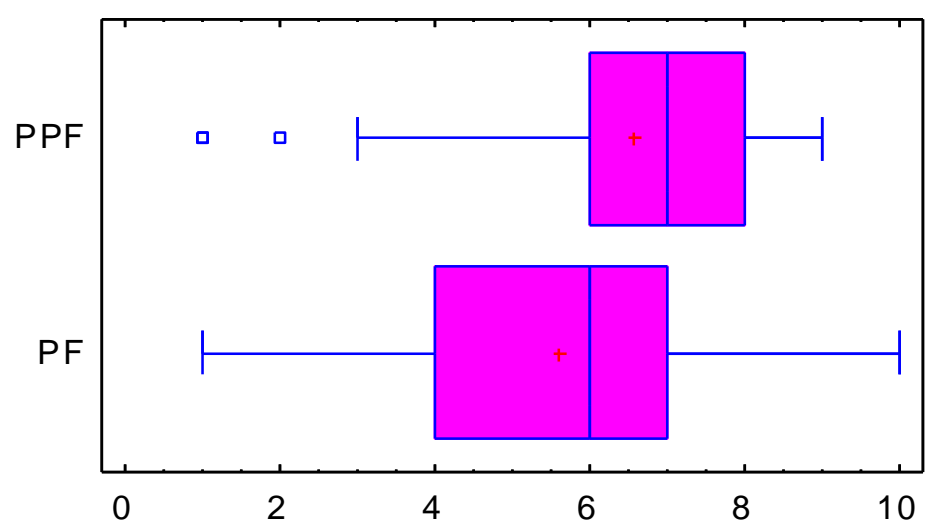

Gráfico 6.43. Comparación de diagramas de cajas y bigotes PPF-PF

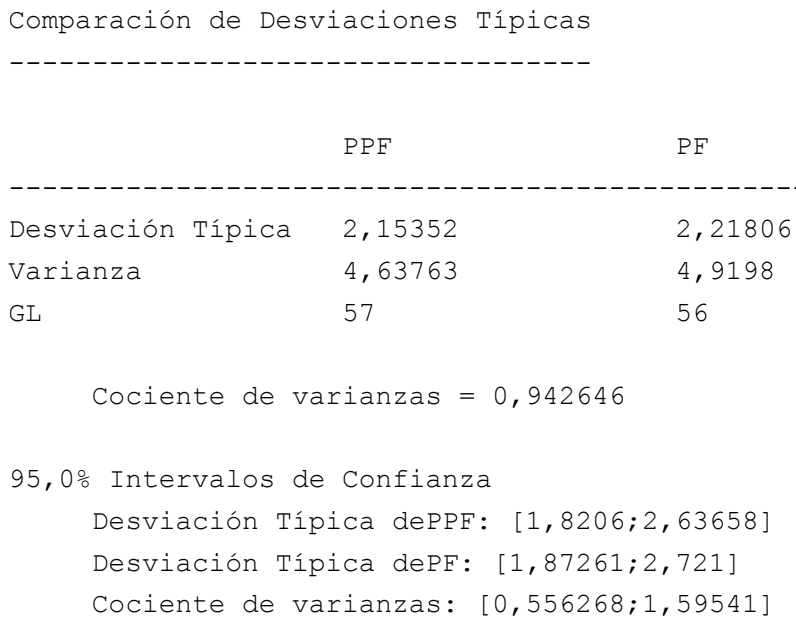


Contrastes $F$ para comparar varianzas

Hipótesis nula: sigmal = sigma2

(1) Hipótesis alt.: sigmal <> sigma2

$$
\mathrm{F}=0,942646 \quad \text { P-Valor }=0,824481
$$

El StatAdvisor

\section{----------}

Esta opción ejecuta un F-test para comparar las varianzas de las dos muestras. También establece los intervalos de confianza o los límites para cada desviación típica y para el ratio de varianzas. De particular interés está el intervalo de confianza para el ratio de las varianzas, el cual se extiende desde 0,556268 hasta 1,59541. Dado que el intervalo contiene el valor 1.0, no existe diferencia estadisticamente significativa entre las desviaciones típicas de las dos muestras para un nivel de confianza del 95,0\%.

También puede utilizarse un F-test para probar una hipótesis específica sobre las desviaciones típicas de las poblaciones de las que proceden las dos muestras. En este caso, el test se ha realizado para determinar si el ratio de las desviaciones típicas son iguales 1,0 frente a la hipótesis alternativa en la que el ratio no es igual 1,0. Puesto que el p-valor calculado no es inferior a 0,05, no podemos rechazar la hipótesis nula.

NOTA IMPORTANTE: los F-test y los intervalos de confianza mostrados dependen de que las muestras procedan de distribuciones normales. Para comprobar esta asunción, seleccione Resumen Estadístico de la lista de Opciones Tabulares y observe los valores de asimetría estandarizada y curtosis estandarizada.

Tabla 6.45. Comparación de desviaciones típicas PPF-PF

\section{Función de distribución}

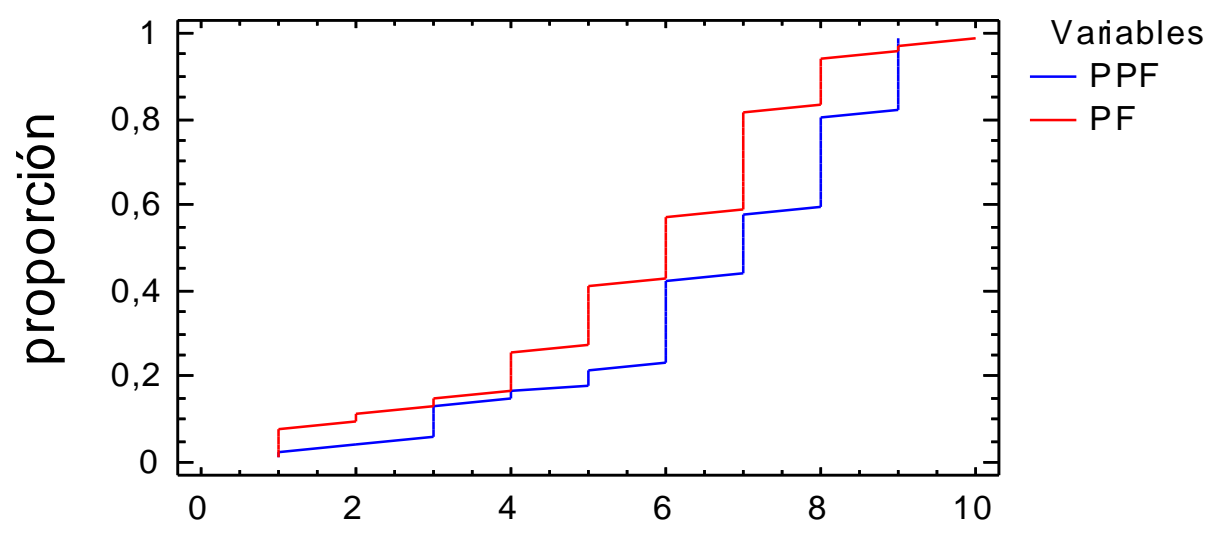

Gráfico 6.44. Comparación de funciones de distribución PPF-PF 


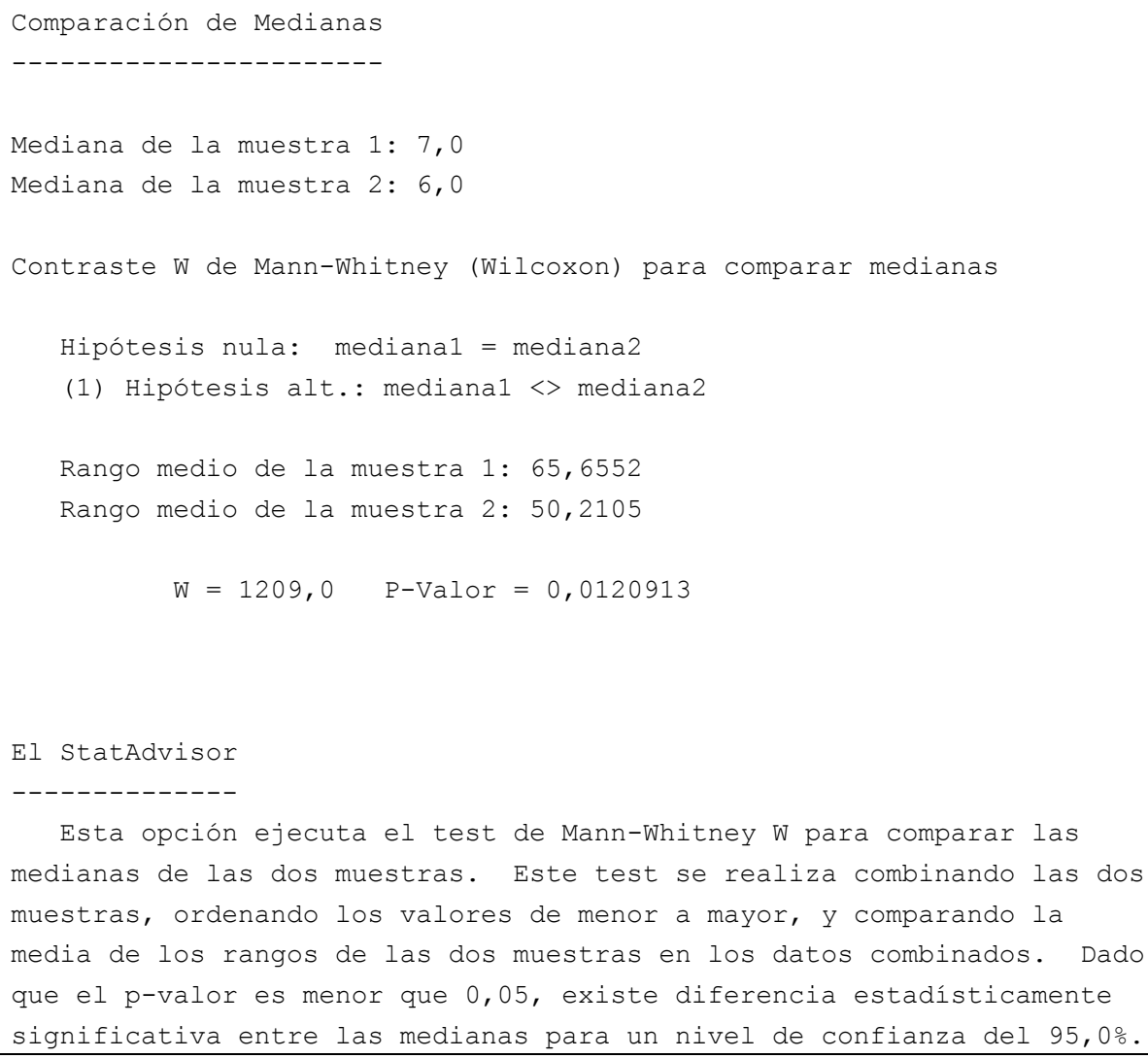

Tabla 6.46. Comparación de medianas PPF-PF

\section{Gráf ico Cuantil-Cuantil}

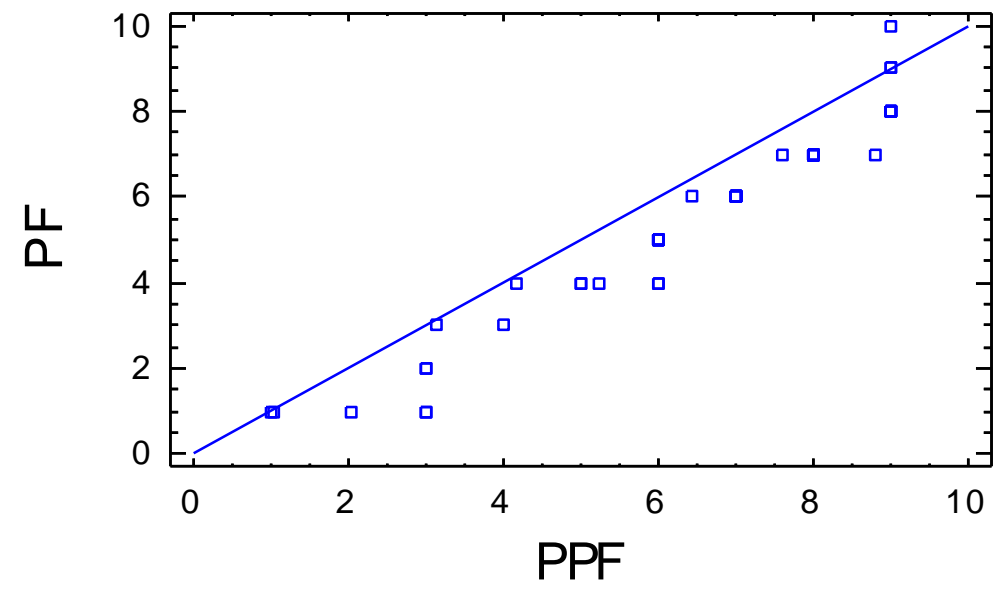

Gráfico 6.45. Representación de cuantiles PPF-PF 


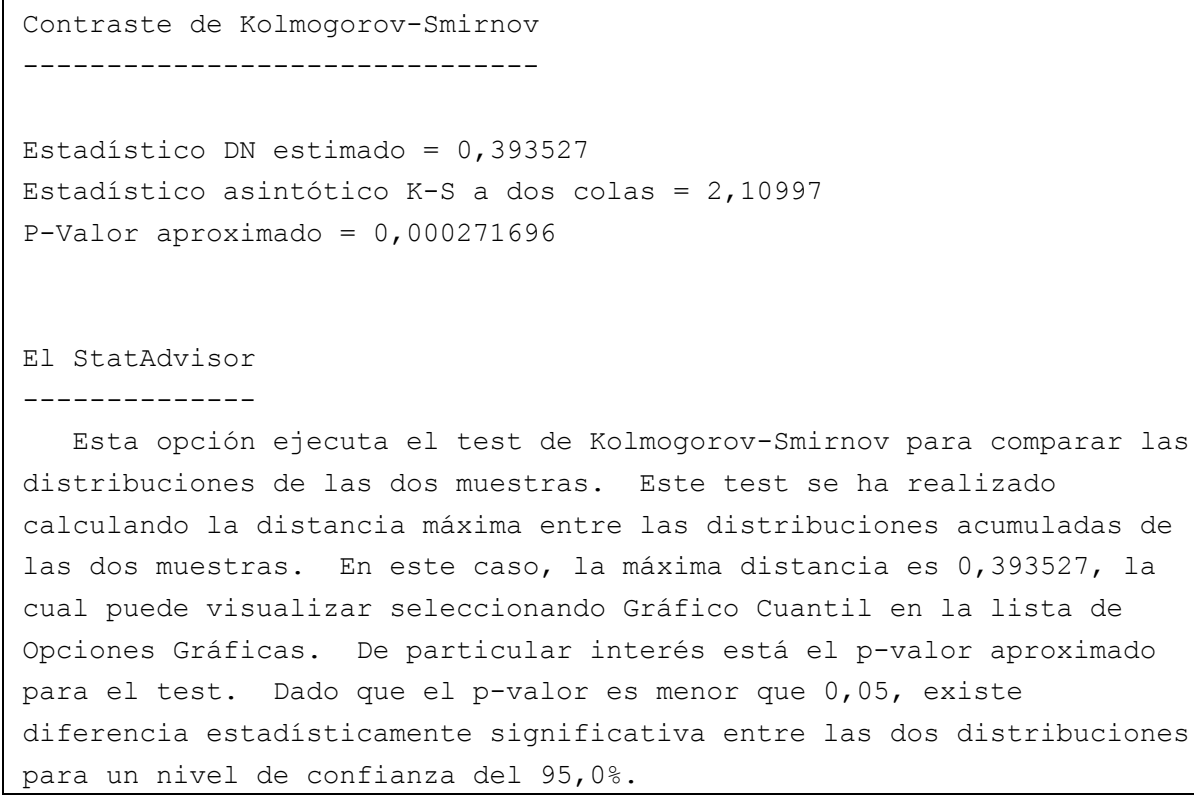

\section{Tabla 6.47. Contraste de Kolmogorov-Smirnov PPF-PF}

Como no existe normalidad los test de igualdad de medias y de varianzas no serían válidos. En cualquier caso el de Kolmogorov sí que prueba que las distribuciones son desiguales, suponiendo unas mayores valoraciones la PPF.

\subsection{Reflexiones que se desprenden de este análisis}

Todo este capítulo es un análisis estadístico de las respuestas del conjunto de los alumnos de los tres ciclos de investigación-acción realizados en esta experiencia. Las respuestas analizadas son las otorgadas a cada una de las pruebas formal y preformal en la demostración de dos teoremas: teorema del valor medio (Lagrange) (teorema 1) y teorema de la unicidad del límite (teorema 2). Los alumnos responden sobre las categorías de facilidad, gusto, claridad e influjo en el aprendizaje de cada una de estas pruebas y en cada una de las dos demostraciones.

Al principio del análisis de cada una de las cuatro categorías señaladas se muestran dos gráficos, que describen las respuestas referidas a cada prueba. Sigue a continuación el estudio estadístico correspondiente a cada una de las dos demostracio- 
nes. Las muestras siguen una distribución normal. En todas ellas, son mayores las valoraciones asignadas a la prueba preformal. En los contrastes de desviaciones típicas y de igualdad de medias no aparece con claridad la desigualdad de las distribuciones para la prueba formal y preformal. El test de Kolmogorov-Smirnov sí permite rechazar su igualdad. Así se muestra en los gráficos de densidades suavizadas, cajas y bigotes y la función de distribución, en los que se sitúan siempre a la derecha las mayores valoraciones otorgadas a la prueba preformal (excepto en $\mathrm{p} 1 \mathrm{fC}$ y $\mathrm{p} 1 \mathrm{fA}$ ). Estas dos excepciones a favor de la prueba formal corresponden a las categorías de claridad e influjo en el aprendizaje, ambas en la primera demostración (teorema del valor medio); en los datos detallados por ciclos, corresponden a los ciclos II y III en los que la prueba formal fue explicada en primer lugar, antes que la preformal. Por otra parte, varios alumnos tienden a considerar, erróneamente, el aprendizaje de las fórmulas matemáticas como una facilidad que aclara, complica menos, el aprendizaje de los teoremas, lo que les induce a valorar lo formal como más útil al aprendizaje matemático. Sin duda estas particularidades han podido motivar estas únicas excepciones que, en pequeña proporción ciertamente, apenas atenúan la recurrente preferencia general de estos alumnos por la demostración preformal.

Termina el capítulo con el análisis estadístico, similar a los precedentes, de la valoración que hacen los propios alumnos sobre el grado de influencia de cada prueba, formal y preformal, en su aplicación concreta del teorema 1 (valor medio) a la función seno. De nuevo el test de Kolmogorov-Smirnov permite rechazar la igualdad de distribuciones, y también los gráficos de densidades suavizadas, cajas y bigotes, y función de distribución muestran los valores mayores para las puntuaciones otorgadas a la prueba preformal situados más a la derecha que los de las valoraciones otorgadas a la prueba formal.

Los análisis cuantitativos confirman los cualitativos, y con los matices que se van señalando en los comentarios en el texto, podemos afirmar la preferencia general de estos alumnos por el estilo de demostración preformal, por su concreción, facilidad, sencillez, utilidad, ayuda, significación y atractivo. 


\section{CAPÍTULO 7}

\section{CONCLUSIONES}

\subsection{Introducción}

La investigación que se ha recogido en este trabajo ha consistido en analizar si las pruebas preformales son más significativas que las pruebas formales para los alumnos en las demostraciones matemáticas.

Con este propósito, después de una experiencia previa (curso 2005-2006) la investigación se ha realizado a través del empleo comparativo de pruebas formales y pruebas preformales. Se ha llevado a efecto con los tres grupos de alumnos, cuya docencia estaba a cargo del PI, en cada uno de tres cursos sucesivos, que han cursado la asignatura Cálculo en primero de Ingeniería Técnica en Informática de Gestión de la Universidad Europea Miguel de Cervantes de Valladolid.

Como ya indicamos (capítulo 2), por las características específicas del tema, consideramos más apropiado utilizar una metodología de integración cualitativocuantitativa, integrando por una parte docencia, praxis e investigación y, por otra, la validez de las pruebas estadísticas y de los resultados.

En este capítulo, final de nuestro trabajo de tesis, resumimos las aportaciones que se han producido en el desarrollo de todo el proceso investigador y las conclusiones del mismo. Señalamos también sus fortalezas y algunas de sus limitaciones. 
Sugerimos posibles cuestiones abiertas o perspectivas de posteriores investigaciones y hacemos una propuesta de desarrollo curricular sobre las demostraciones, ofreciendo varios ejemplos de pruebas preformales.

\subsection{Aportaciones}

\subsubsection{Las pruebas preformales}

En este trabajo se ofrece una variedad de ejemplos de pruebas preformales, según la definición y características de van Asch (1993), comparándolas con las pruebas formales (cf. ANEXO I).

Ya hemos señalado la experiencia previa (sección 3.2, p. 38) realizada en el curso 2005-2006, que supuso al PI remodelar sus materiales de Cálculo Diferencial utilizando estas pruebas (preformales) en la docencia, experiencia que permitió establecer la conjetura de que este tipo de pruebas son más beneficiosas que las demostraciones formales para el aprendizaje de los alumnos.

En cada uno de los tres ciclos de investigación-acción, los alumnos han cursado los contenidos propios del análisis local de funciones explícitas y, concretamente se han realizado demostraciones preformales de los siguientes teoremas: desigualdad triangular, propiedad arquimediana, unicidad del límite, Bolzano, Weierstrass, "derivabilidad implica continuidad", extremo interior (o de singularidad), Rolle, valor medio (Lagrange), fundamental del Cálculo Integral (cf. ANEXO I).

Además, en el desarrollo de la investigación se ha dedicado una especial atención A los siguientes teoremas sobre los que se han basado los cuestionarios de los alumnos: unicidad del límite y valor medio de Lagrange, empleando ambos tipos de prueba: la formal y la preformal. La finalidad era la comparación y valoración por los propios alumnos de ambos procedimientos de demostración con respecto a las cuatro importantes "categorías" de facilidad, claridad, gusto e influjo en el aprendizaje, y con respecto a la significación de la demostración (Hanna, 1989): 
capacidad de convicción, ayuda a la comprensión, ayuda a la memorización, y ayuda a ilustrar cómo se aplica un teorema.

Aportamos, pues, las valoraciones y preferencias de un grupo de alumnos de primer curso universitario por estos dos procedimientos de demostración. La actividad ha supuesto también la iniciación y cierta familiaridad por parte de dichos alumnos en el aprendizaje de estas pruebas, la preformal especialmente, novedosa para ellos, acostumbrados a las demostraciones formales tan habituales en los textos al uso.

\subsubsection{La integración de las metodologías cualitativa y cuantitativa}

En el capítulo 2 señalábamos como más apropiada para nuestra investigación una metodología de integración cualitativo-cuantitativa. La cualitativa, integrando dos marcos metodológicos, investigación-acción y metodología de diseño; y la cuantitativa, aportando datos estadísticos y pruebas de validez de los resultados.

En la investigación-acción hemos seguido las indicaciones fundamentales establecidas por Pérez Serrano (1994) resumidas por Ibañes y Ortega (2005, pp. 30-32; cf. sección 2.2, pp. 21-23). En la metodología del diseño hemos procurado ajustarnos a las características señaladas por Brown (1992), Collins (2004) adaptadas al ámbito de la Didáctica de la Matemática, de los constructivistas Kelly (2003), Confrey et al. (2004) y la aplicación a experimentos dirigidos por una conjetura según Confrey y Lachance (2000) y, sobre todo, Molina (2006) que enuncia una serie de características propias de esta metodología (integración de docencia, praxis e investigación, ciclos, desarrollo en el aula, coparticipación de profesores y alumnos, elaboración de un diario, refinamiento del cuestionario mediante el cambio de orden, desarrollo de entrevistas,...). Finalmente, la unión de las metodologías cuantitativa y cualitativa aporta, sin duda, validez y garantía a las conclusiones y a los resultados obtenidos. 


\subsubsection{El protocolo de observación del $\mathrm{OE}$}

En los experimentos de enseñanza es importante la participación de un observador que colabore con el investigador-docente y el alumno o alumnos protagonistas de los episodios del experimento (Steffe y Thompson, 2000).

En nuestro trabajo ha llevado a cabo esta colaboración una profesora de matemáticas del mismo Centro Académico en que se ha realizado la experiencia, con su presencia en la sesión principal de los ciclos II y III, y con sus notas y respuestas a un "protocolo de observación" que hemos descrito en la planificación del ciclo II (cf. 4.2.1), y sobre el que ya hemos reflexionado (cf. 4.2.3.3 y 4.3.3.3). De esta manera, la profesora observadora ha participado imparcial y objetivamente en la investigación.

Esta actividad constituye una relevante aportación al proceso de la experiencia investigadora. De hecho, han sido tomadas en consideración, y aceptadas, sus observaciones sobre los tiempos dados a los alumnos para responder el cuestionario de la sesión principal, las sugerencias para motivarles más en la atención a la exposición del teorema abordado en segundo lugar, y para fomentar las interacciones profesor-alumnos, en dicha sesión, puntos en los que la OE insiste en sus respuestas al protocolo. La valoración general que hace la OE es de estimación de la experiencia, en lo que ella ha participado, como aceptable y positiva (cf. las observaciones recogidas en 4.2.3.1 y 4.3.3.1): se explican las tareas, se aclaran dudas, incluso individuales con claridad y rigor; los alumnos preguntan con confianza, sus intervenciones son de poca duración. El número de alumnos de los grupos ha resultado adecuado para las actividades y ha contribuido a la ausencia de dificultades, incidencias y disfunciones especiales, como afirma la OE.

\subsubsection{El cuestionario y protocolo de entrevistas}

El cuestionario es la base principal para la aportación de datos en nuestra investigación, por la amplitud de las cuestiones que afronta y por extenderse a todos los alumnos de los tres ciclos a quienes se les ha entregado para responderlo. 
El núcleo del mismo está orientado a la comparación de los dos sistemas de prueba, formal y preformal. El cuestionario quedó fijado después de una experiencia previa realizada en el curso 2005-2006. Se encuentra en el ANEXO II. Después de manifestar cada alumno su impresión general sobre la comprensión de los dos teoremas utilizados en esta experiencia con ambas modalidades de prueba, formal y preformal (cuestión 1), se pide su criterio de preferencia por una u otra en relación con las principales categorías (cuestión 2) y significación (cuestión 3) de dichas demostraciones. El cuestionario pretende valorar también las ventajas y grado de influencia de una y otra modalidad con una aplicación concreta.

Se ha elaborado un protocolo para realizar entrevistas semiestructuradas a los alumnos, protocolo que se ha utilizado en los tres ciclos de experimentación. Las entrevistas de los ciclos II y III se han grabado, y se han tomado las notas correspondientes de las del ciclo I. En el ANEXO V se reproduce el protocolo de las entrevistas. En los ANEXOS VI, VII y VIII, aparecen las transcripciones de las entrevistas de cada ciclo y las respuestas de los alumnos a la cuestión tercera del cuestionario.

\subsubsection{Los datos recogidos durante tres años}

Nuestro estudio ha sido principalmente exploratorio y descriptivo. Hemos pretendido indagar la preferencia por demostraciones preformales sobre las formales, en relación con el aprendizaje de lo demostrado, proponiendo a los alumnos que han realizado la experiencia, en tres cursos consecutivos, la comparación entre las principales categorías y significación de ambas pruebas. Aportamos así datos y experiencia que pueda colaborar a una mayor eficacia en la práctica de la enseñanzaaprendizaje de la demostración matemática en el ámbito del aula.

Ante el hecho de la utilización general de pruebas formales, investigamos la posibilidad (eficacia, resultado) del uso de otras pruebas, las preformales según definición de van Asch, así como el grado de aceptación y estima de este tipo de demostración por parte de los alumnos protagonistas en esta experiencia. 
Estos alumnos, 67 en total, de la asignatura Cálculo de primero de Ingeniería Técnica en Informática de Gestión, de la Universidad Europea Miguel de Cervantes de Valladolid, estaban distribuidos en tres grupos, uno en cada curso durante los años 2006-2007, 2007-2008, 2008-2009. Son los alumnos que acceden en la actualidad a la Universidad en España conforme a los planes de estudio vigentes, en los que, en general, se prima más la cantidad que la calidad en la enseñanza. La actitud de estos alumnos ha sido positiva, tenían interés y han colaborado en todo el desarrollo de la actividad.

Los trabajos se han desarrollado como queda descrito en la planificación, calendario y acción de los tres ciclos (cf. capítulo 4). La actividad principal se ha prolongado dos horas en el ciclo I, y dos horas y media en los ciclos II y III. En estos dos últimos ciclos se ha contado con la presencia de una Observadora Externa. Se han realizado también dos entrevistas en cada uno de los ciclos a sendas parejas de alumnos (cf. ANEXOS VI, VII y VIII).

Los datos son cuantiosos. Tomados conjuntamente en un resumen-síntesis generalizado tenemos que confirmar la preferencia de este colectivo de alumnos por las demostraciones preformales:

- En la comprensión de los teoremas: la prueba preformal convence más, se entiende mejor, es más fácil, más sencilla; la prueba formal es más difícil, compleja, general (tablas 4.2 y 4.3 , pp. 54-55; tablas 4.13 y 4.14, p. 90; tablas 4.24 y 4.25 , p. 121).

- En las categorías facilidad, gusto, claridad, influjo en el aprendizaje: en los gráficos (6.1 y 6.1bis, p. 227; 6.10 y 6.10bis, pp. 238-239; 6.19 y 6.19bis, pp. 250-251; 6.28 y 6.28bis, pp. 262-263) que describen las valoraciones de los alumnos, las correspondientes a la prueba formal se presentan a la izquierda en las apreciaciones más bajas, y en las más altas son las atribuidas a la prueba preformal las que se mantienen a la derecha.

- En las funciones de significación capacidad de convicción, gusto, memorización y utilidad para ilustrar su aplicación también prefieren los alumnos la 
demostración preformal, concediendo ventaja a la formal en la función de ayuda a memorizar (cf. 4.3.8, tabla 4.34, p. 144; gráfico 4.7, pág. 145).

- $\quad$ En el grado de influencia de cada prueba en una aplicación concreta (cf. sección 6.5), de nuevo las mayores valoraciones se otorgan a la demostración preformal.

\subsection{Conclusiones}

Nos propusimos investigar que las pruebas preformales son más significativas para los alumnos que las pruebas formales. Establecimos seis hipótesis (H1-H6) y seis objetivos (01-06) correlativos según queda señalado en el capítulo 3, sección 3.3. Aquí, para facilitar la lectura se repiten las seis hipótesis y a continuación de cada una de ellas se presentan y se discuten las conclusiones correspondientes:

H1. El aprendizaje en relación con la demostración matemática es más activo, más participativo y los aprendizajes son más significativos a través de las pruebas preformales.

H2. Las pruebas preformales son un complemento de las pruebas formales y como tales cumplen una función de explicación.

H3. No es difícil que los alumnos aprendan a usar pruebas preformales con precisión y que las distingan de las pruebas formales.

H4. El proceso de aprendizaje de las demostraciones matemáticas mediante pruebas preformales resulta más significativo para los alumnos, son más motivadoras (gusto) y consideran que las entienden mejor, que son más fáciles, más claras.

H5. Los alumnos prefieren este tipo de pruebas, las comprenden mejor y perduran más en la memoria.

H6. Las pruebas preformales son más convincentes para estos alumnos y son más fáciles de aplicar. 
Aparecen conclusiones que están relacionadas con varias hipótesis y, por esta razón, en la discusión que sigue, tras enunciar la conclusión, entre paréntesis, se escriben las hipótesis y objetivos relacionados.

7.3.1. Con las pruebas preformales el aprendizaje en relación con la demostración matemática es más activo, más participativo, más significativo. (01, 06, H1, H4).

El aprendizaje significativo, según Ausubel (cf. capítulo 1) implica una apropiación de conocimiento que se realiza encajando unas piezas nuevas con otras ya existentes en un todo coherente. Es "un proceso de relación del contenido o material a aprender con el bagaje o almacenamiento de conocimientos ya poseído por el alumno que aprende y la estructura mental de ese bagaje". Encajar el concepto que se desea transmitir y acogerlo, aprehenderlo, encajándose en el conjunto de los conocimientos de quien lo recibe. Esta transmisión no se consigue en el aprendizaje por pura "memorización", sino en el aprendizaje por "comprensión" (Katona, 1940; Wertheimer, 1959). En consecuencia, las pruebas que faciliten más esta transmisión, comprensión, ajuste y posterior aplicación de lo así aprendido (aprehendido) harán el aprendizaje más significativo.

Los alumnos que han practicado esta experiencia manifiestan en sus respuestas, como queda reflejado en los análisis que de ellas hemos realizado, lo siguiente:

- La "comprensión" de los teoremas es decisiva para el aprendizaje, especialmente significativo, de los alumnos. Éstos, en este punto, se decantan notablemente a favor de la modalidad de prueba preformal: "es más sencilla, más clara, más útil, más fácil, ayuda a entender los pasos de la demostración", "convence más", con esta prueba "se entiende mejor" (tablas 4.2 y 4.3, pp. 54-55; tablas 4.13 y 4.14 , p. 90 ; tablas 4.24 y 4.25 , p. 121).

- En relación con la pregunta sobre el influjo de cada una de las modalidades de prueba (formal y preformal) en el aprendizaje, también se inclinan los alumnos en sus respuestas por la preformal, como puede apreciarse en la tabla 6.38 (p. 275), el gráfico 6.38bis (p. 276) y el gráfico resumen 6.39 (p. 277). El gráfico 6.39 (que reproducimos más abajo, gráfico 7.1) muestra la 
respuesta global de estos alumnos sobre cuatro categorías referentes a la demostración de gran relevancia en el aprendizaje: facilidad, gusto, claridad e influjo en el aprendizaje, y el gráfico 6.38bis muestra la respuesta a la categoría aprendizaje. Las líneas que en el gráfico 7.1 manifiestan las apreciaciones de los alumnos, cuando éstas son más bajas (1 a 4) son favorables a la prueba formal, (línea roja), y cuando son más altas (5 a 10) son favorables, en conjunto, a la prueba preformal (línea azul).

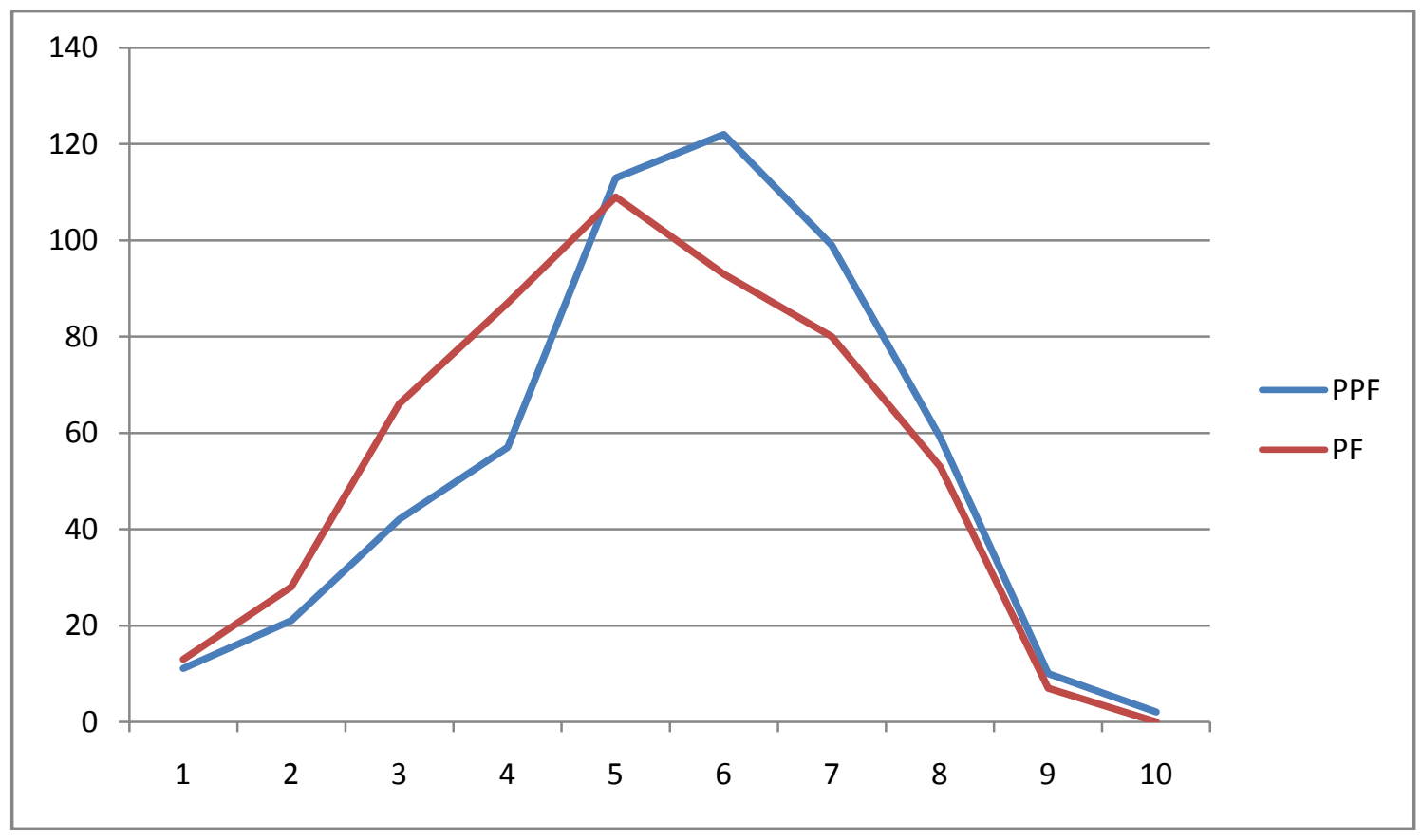

\section{Gráfico 7.1. Resultados globales, de los tres ciclos en ambos teoremas unidos, en el conjunto de las cuatro categorías}

- Otras características del aprendizaje significativo son las funciones de las pruebas, importantes en la demostración según Hanna (1989), Bell (1976), de Villiers (1993), Ibañes y Ortega (2005), y sobre las que se ha preguntado también a los alumnos. Se han considerado cuatro de estas funciones en cada una de las dos modalidades de prueba: su capacidad para convencer, su ayuda a comprender, a memorizar y su utilidad para ilustrar la aplicación. En las respuestas a esta cuestión (cf. 4.3.8, tabla 4.34, p. 144 y gráfico 4.7, p. 145) destaca la preferencia por la prueba preformal (61 vs. 10) en la fun- 
ción de ilustrar la aplicación de lo aprendido, lo que, una vez más, estima muy positivamente lo significativo del aprendizaje adquirido con esta modalidad de prueba. Ya se ha considerado anteriormente la preferencia de estos alumnos por la prueba preformal en su ayuda para convencer de la verdad y para entender el significado de los teoremas. Solamente es valorada más positivamente la prueba formal (47 vs. 26, tabla 4.34, pág. 144) en la función de ayudar a memorizar el teorema. Repetimos aquí la consideración que, a este respecto, se hace al comentar esta valoración: los alumnos suelen interpretar las fórmulas como más fáciles de retener, pero debe distinguirse muy bien "memorizar una fórmula", comprendida y entendida perfectamente, de un mero aprendizaje mecanicista de esa fórmula. Los psicólogos de la Gestalt, Katona (1940) y Wertheimer (1959), compararon aprendizajes por memorización y por comprensión, y demostraron que sólo este último transfiere aprendizaje a nuevas situaciones (cf. capítulo 1, p. 11).

7.3.2. Los alumnos aprenden con facilidad el concepto y uso de la prueba preformal y su distinción de la prueba formal. (02, H3).

Estos alumnos han acogido desde el principio la demostración preformal con interés y satisfacción. Rápidamente han aceptado esta prueba, como se ha señalado en los varios análisis realizados sobre sus respuestas, porque para ellos es más fácil, más clara y más sencilla, incluso en mayor grado que la prueba formal. No conocían esta modalidad de demostración matemática; alguno ha evolucionado en el concepto de la misma desde verla como un ejemplo hasta considerarla como una auténtica prueba. En sus valoraciones y comparaciones con la prueba formal, en esta característica de facilidad habitualmente la conceden las apreciaciones más altas.

También han sabido captar la distinción entre ambas modalidades de demostración, como muestran en las distintas características y singularidades que detectan en cada una de ellas. Recordemos los gráficos 6.1 y 6.1bis (p. 227), y la tabla 6.37 
con el gráfico 6.37 (p. 274) sobre las respuestas de los tres ciclos al valorar esta característica de facilidad en cada una de las pruebas.

7.3.3. Los alumnos prefieren las demostraciones preformales, al comparar las características y funciones con las pruebas formales. $(03, \mathrm{H} 4, \mathrm{H} 5)$.

La consideración conjunta de los diversos análisis (y estudio estadístico) realizados sobre características y significación de ambas pruebas, formal y preformal, conduce a que estos alumnos se decantan en sus valoraciones a favor de la prueba preformal. Esta es la conclusión general de conjunto, como ha quedado patente en los numerosos gráficos y tablas que reflejan los resultados de dichos análisis a lo largo del texto de este trabajo.

Al concurrir varias y diversas variables en esta cuestión (pruebas, teoremas, características, funciones, ciclos), adquiere esta opción por la prueba preformal una mayor significación. Al mismo tiempo tiene también interés señalar, como así hemos hecho en su lugar respectivo, algunos datos concretos del comportamiento de estas variables.

En las características analizadas, facilidad-gusto-claridad-aprendizaje, los valores más altos en las valoraciones bajas, 1 a 5, corresponden a la prueba formal (izquierda en los gráficos 6.37 y 6.37bis, 6.38 y 6.38bis, pp. 274-276, resumen de los anteriores 6.1 y 6.1bis, 6.19 y 6.19bis, 6.28 y 6.28bis, línea roja) y los más altos en las valoraciones altas, 6 a 10, se aplican a la prueba preformal (derecha del gráfico, línea azul): así ocurre en las categorías facilidad, gusto, aprendizaje; aparecen más equilibrados sus valores en la categoría claridad, teorema 1.

Se concluye que es la preformal la prueba que supone menos esfuerzo en su comprensión por su sencillez, facilidad, intuición, práctica, concreción. Algunos alumnos que la han considerado un ejemplo han terminado convencidos de que se trata de una prueba verdadera. Queda, pues, integrada sin dificultad en su esquema cog- 
noscitivo. El gusto por dicha prueba se desprende lógicamente de esta facilidad de comprensión.

La prueba formal supone más esfuerzo asimilarla e integrarla por la utilización de letras (mayor abstracción); es más difícil, general, compleja...

Respecto a las funciones (significación) ayuda a convencer de la veracidad, a entender el significado del teorema, a memorizar, a ilustrar la aplicación del teorema, las valoraciones se decantan así (cf. tabla 4.34, p. 144 y gráfico 4.7, p. 145): una casi unanimidad a favor de la prueba preformal en la función de ilustrar la aplicación del teorema, lo que es muy importante para estos alumnos de carrera técnica. Consecuentes con sus valoraciones de las características de las pruebas, también conceden una destacada preferencia a la prueba preformal en la función de ayudar a entender el significado de los teoremas $\mathrm{y}$, aunque en menor porcentaje, en la ayuda a convencer de su veracidad. En la función ayuda a memorizar se cambia la preferencia a favor de la prueba formal: los alumnos ven esta prueba como una "fórmula" de fácil retención extensible a la generalidad de casos posibles. ¿Podría ser una secuela del aprendizaje habitual en los textos al uso?

Creemos confirmado con estas valoraciones lo que ya apuntábamos en la introducción a este trabajo (p. 4): que las pruebas preformales permiten amortiguar o incluso resolver las dificultades en el grado de comprensión, de significación y utilidad de las demostraciones matemáticas.

7.3.4. Ambas demostraciones, preformal y formal, son complementarias, las primeras cumplen una función de explicación de las segundas. $(04, \mathrm{H} 2)$.

Son varios los alumnos que afirman la necesidad o conveniencia de la utilización de ambas pruebas, preformal y formal, en las demostraciones matemáticas. Después de manifestar su preferencia por una u otra, estiman y valoran también la prueba no preferida y señalan, incluso, el "orden" de su uso en conformidad con las características de su estructura mental y hábito de aprendizaje. Se apoyan en las condiciones, muy recurrentes, como hemos visto en toda esta experiencia, de la "genera- 
lización" que aplican a la prueba formal y en la mayor facilidad, claridad, sencillez, concreción y utilidad de aplicación que atribuyen a la prueba preformal. Condiciones que, sin rebajar en nada la propiedad probatoria de la preformal, la hacen además, por su concreción, cumplir una función de explicación de la prueba formal.

El PI estima que, en este punto, las respuestas y explicaciones de los alumnos pueden elevarse a integrar las conclusiones de esta experiencia realizada, con el deseo de que en los libros al uso se utilicen mucho más las pruebas preformales.

7.3.5. Las pruebas preformales son mejor valoradas y más fáciles y convincentes al aplicar un teorema a algún ejercicio práctico matemático. $(05, \mathrm{H6})$.

Como ejercicio para calcular la valoración de las dos pruebas, formal y preformal, se pidió a los alumnos que manifestaran (con apreciaciones del 1 al 10) el grado de influencia experimentado en la aplicación del teorema 1 , del valor medio, a la función seno (cf. ANEXO II).

Las valoraciones, en los tres ciclos de la experiencia, son favorables a la prueba preformal, como aparece en las tablas y gráficos de la sección 6.5. Podemos comprobar de nuevo, decíamos como conclusión, la preferencia por la prueba preformal.

\subsection{Debilidades y fortalezas}

\subsubsection{La falta de bibliografía al respecto}

Es abundante el material existente sobre las metodologías que se han practicado y sobre temas relacionados con el aprendizaje en general, el aprendizaje significativo, la educación y las demostraciones en matemáticas. Investigadores y autores en sus publicaciones y revistas, ofrecen sobre estas cuestiones en sus artículos orien- 
taciones, criterios y experiencias prácticas (éstas de manera predominante en ámbitos de enseñanza primaria y secundaria).

Varios de estos medios han servido de apoyo y orientación en este trabajo. Señalamos entre los españoles por más cercanos, a los profesores Ibañes, Ortega y a Pérez Serrano (citados en las referencias). También se ha podido disponer de algunas tesis doctorales, relacionadas con temas aludidos en el curso de las actividades realizadas, y presentadas en los Departamentos de Didáctica de la Matemática de las Universidades de Valladolid y de Granada ${ }^{1}$.

Sin embargo, no es tan abundante el material sobre demostraciones "prácticas" y sobre ofertas de demostraciones preformales, sobre todo destinado a ámbitos docentes de estudios superiores, universitarios. En este sentido, nuestras conclusiones podrían resultar un tanto subjetivas si no pudieran constatarse suficientemente con más trabajos y otros posibles criterios, investigaciones, análisis y resultados similares y referidos a estos temas.

\subsubsection{Las deficiencias matemáticas del alumnado}

En todo aprendizaje son importantes las condiciones del sujeto que aprende. El aprendizaje es un "cambio relativamente estable en el conocimiento de alguien como consecuencia de la experiencia de esa persona" (Mayer, 2004). Depende, pues, de la experiencia del aprendiz, de lo que ha hecho y de cómo ha interpretado lo ocurrido.

Según Ausubel, el aprendizaje significativo es un proceso de relación, no mecánica, al pie de la letra, sino sustancial, integrador del contenido a aprender con el bagaje de lo ya adquirido y de la estructura mental de ese bagaje. Lo que exige en el aprendiz disposición y capacidad para encajar lo nuevo que accede. El resultado, pues, variará según los antecedentes, la edad, el grado de madurez, los factores socioculturales, afectivos, etc.... del aprendiz y de su entorno (cf. capítulo 1, p. 11).

\footnotetext{
${ }^{1}$ Las tesis de Maㅡ Asunción García Olivares y de Marta Molina González, citadas en las referencias.
} 
¿Cómo son los alumnos que han realizado esta experiencia? ¿Cómo es su entorno? Una nueva sociedad en el mundo y un nuevo "aprendiz" (alumno) en las aulas está emergiendo entre nosotros. Los avances sin pausa de la tecnología, la informática y sus nuevos lenguajes están influyendo en las personas y en las formas (procedimientos, práctica, modos) del aprendizaje. Estos avances, con logros y ventajas, presentan también riesgos y servidumbres: es posible un mayor desarrollo de la rapidez, intuición, agilidad, observación, fantasía de la mente, pero además, puede apoderarse de ella el pragmatismo, la comodidad, la primacía de lo fácil, concreto, inmediato, y perturbarse el esfuerzo, la atención a lo abstracto y hasta los esquemas intelectuales.

Estos alumnos no son ajenos a este entorno. Son resultado de las leyes y planes oficiales de enseñanza españoles de la última década. Pertenecen a un Centro Académico Universitario privado. Utilizan los medios modernos a su alcance de información, consulta, comunicación, cálculo... sin evidenciar adición a ellos. Están habituados a las demostraciones formales y han conocido y practicado la demostración preformal por primera vez en el desarrollo de esta experiencia: se ha dedicado poco más de un mes en cada ciclo, (como consta en los respectivos calendarios 4.1.2, $4.2 .2,4.3 .2)$ a familiarizarles con este procedimiento de prueba. El resultado ha demostrado ser positivo en el ejercicio de valoración realizado por los alumnos al final de cada ciclo, calculando la influencia, para ellos, de cada prueba en una aplicación del teorema del valor medio a la función seno (cf. sección 6.5).

Las limitaciones de los alumnos pueden haber sido los antecedentes académicos de los periodos anteriormente cursados en ESO y Bachillerato (cambios en leyes y planes), y esa especie de predisposición habitual, ambigua y tácitamente aceptada en amplios sectores de la sociedad, poco reflexivos, de cierta y equívoca aversión a temas y disciplinas relacionados con las matemáticas. Pero, en general, este alumnado se ha comportado responsablemente y en todo momento ha ofrecido su colaboración con interés a las actividades realizadas. 


\subsubsection{La experimentación. Combinación de metodologías}

En el capítulo 2 tratamos la planificación de esta experimentación y la metodología a emplear en su realización. Se estudió la combinación de dos metodologías juzgadas complementarias, cuantitativa y cualitativa y, dentro de ésta, la integración de dos marcos metodológicos, la investigación-acción y el diseño. Consideradas sus características, objetivos, ventajas y limitaciones, se decidió como línea conductora de la investigación la basada en el diseño y en la integración de éste en la metodología de investigación-acción. Con el objetivo de presentar las pruebas preformales como adecuadas (como otra opción) para facilitar un aprendizaje significativo de las demostraciones matemáticas.

En todo el proceso del trabajo llevado a cabo se ha procurado fidelidad a las características y rasgos de estas metodologías, particularmente de la investigaciónacción, establecidos por iniciadores como Lewin, Kemmis y McTaggart (1988) y enriquecidos por continuadores como Pérez Serrano (1994), Ibañes y Ortega (2005).

La experimentación parte de una situación real, con perspectiva ecológica en el aula: las demostraciones demasiado abstractas, formalistas, en matemáticas. Problema que implica a todos los responsables, con el afán de mejorarlo. El profesor es a la vez investigador, próximo a la situación y su entorno. Se ha seguido la espiral de tres ciclos, integrando docencia, praxis e investigación; se han realizado entrevistas y análisis cuantitativos y cualitativos, cuyos resultados se han analizado y reflexionado. Todo ello ha conducido a establecer, modestamente, las conclusiones que aportamos $y$, humildemente ofrecemos.

La combinación de metodologías llevada a efecto ha sido una experiencia positiva así como la figura del profesor investigador, tan cercano a las cuestiones planteadas, y la participación de los alumnos. Estos han expresado su voluntad de ser más activos en el aprendizaje. No aceptan actitudes pasivas, quieren un aprendizaje que conexione los contenidos aprendidos con la realidad y con el bagaje mental de sus conocimientos ya adquiridos, un aprendizaje en verdad significativo. Y las pruebas 
preformales en las demostraciones matemáticas, les parecen, en general, más conformes con este tipo de aprendizaje (cf. sección 7.3).

\subsection{Problemas abiertos}

Una demostración es valorada, según Hanna, cuando saca a relucir relaciones matemáticas esenciales, más que cuando simplemente demuestra la exactitud de un resultado. Deben presentarse a los estudiantes, siempre que sea posible, demostraciones que expliquen, en lugar de aquellas que simplemente prueben, que muestren el porqué es cierto un teorema (rationes essendi) en lugar de las que solamente prueban que un teorema es cierto (rationes cognoscendi). El objeto primario es que los estudiantes entiendan las matemáticas (cf. capítulo 1, p. 13).

Nuestro trabajo ha pretendido una humilde aportación al uso de un tipo concreto de demostración en matemáticas, prueba en sintonía con las afirmaciones de Hanna, razonamiento totalmente válido, distinto al utilizado habitualmente en los libros de texto al uso, el procedimiento preformal, según lo define van Asch (cf. capítulo 1, p. 14). Los alumnos que han realizado la experiencia, sin infravalorar los procedimientos de las pruebas formales, han manifestado que el procedimiento preformal es preferido por ellos por su sencillez, claridad, concreción (menos abstracto), facilidad, significación en el aprendizaje, mayor participación e interacción y ayuda a ilustrar la comunicación a otros, de los contenidos matemáticos. El propio van Asch se expresaba en este sentido al pedir que los matemáticos "deberían estar dispuestos a demostrar teoremas de forma menos abstracta a la que ellos probablemente preferirían".

Un desafío que puede plantearse a los matemáticos ante estas manifestaciones, es la presentación más frecuente de pruebas preformales en las aulas y en sus publicaciones de texto para los cursos de los planes académicos. En este sentido hacemos una propuesta de desarrollo curricular aportando algunas pruebas preformales incluidas en el ANEXO I de esta memoria. Sería bueno también llevar a cabo 
trabajos parecidos a éste con más alumnos y en otros ámbitos académicos y la intercomunicación de sus resultados. Siguiendo esta línea de investigación, en la que se han utilizado pruebas preformales de Análisis Matemático, se podrían plantear los siguientes problemas de investigación:

- Analizar el impacto de este tipo de pruebas en otras áreas de la matemática.

- Realizar estudios de contraste en otras áreas de la matemática.

- Investigar hasta qué punto estas pruebas contribuyen a que los alumnos comprendan los enunciados de los teoremas.

- Averiguar si los alumnos son capaces de reproducir y, por qué no, realizar pruebas preformales sencillas.

- Creación de equipos investigadores eficaces para la mejora de cuestiones prácticas detectadas en el aula.

- Integración de docencia, praxis e investigación en las metodologías de investigación-acción y diseño.

- Problemas y preocupaciones educativas que sienten como propios los enseñantes-educadores-profesores.

- Dimensión formativa de la investigación-acción que potencia el desarrollo personal y profesional.

- Estudio y comparación de contextos (ecológicos) diversos y su influjo en los problemas educativos.

- $\quad$ La ausencia de pruebas preformales en los libros de texto al uso.

- Demostraciones que prueban y demostraciones que explican, elementos que facilitan cada una de ellas. 


\section{REFERENCIAS}

ACKERMANN, E. (1995): "Construction and transference of meaning through form". En L. P. Steffe y J. Gale (Eds.), Constructivism in education, 341-354. Lawrence Erlbaum Associates. Hillsdale, New Jersey.

van ASCH, A. G. (1993): “To prove, why and how?”. International Journal of Mathematics Education, Science and Technology, 24(2), 301-313.

AUSUBEL, D. P. (1976): Psicología educativa. Un punto de vista cognoscitivo. Trillas. México.

AUSUBEL, D.; NOVAK, J.; HANESIAN, H. (1983): Psicología educativa. Un punto de vista cognoscitivo. $2^{\underline{a}}$ ed. Trillas. México.

BALACHEFF, N. (1987): "Processus de preuve et situations de validation". Educational Studies in Mathematics, 18, 147-176.

BARAB, S.; SQUIRE, K. (2004): "Design-Based Research: Putting a Stake in the Ground". The Journal of the Learning Sciences, 13(1), 1-14.

BELL, A. W. (1976): "A study of pupils' proof-explanations in mathematical situations". Educational Studies in Mathematics, 7, 23-40. 
BLUM, W.; KIRSCH, A. (1991): "mal proving: examples and reflections". Educational Studies in Mathematics, 22, 183-203.

BROWN, A. L. (1992): “Design experiments: Theoretical and methodological challenges in creating complex interventions in classroom settings". The Journal of the Learning Sciences, 2(2), 141-178.

COBB, P. et al. (2003): “Design Experiments in Educational Research". Educational Researcher, 32(1), 9-13.

COLLINS, A. (1992): “Toward a design science of education”. En E. Scanlon y T. O’Shea (Eds.), New directions in educational technology, 15-22. Springer. Berlin.

COLLINS, A. et al. (2004): "Design Research: Theoretical and Methodological Issues". The Journal of the Learning Sciences, 13(1), 15-42.

CONFREY, J. (2006): “The Evolution of Design Studies as Methodology”. En R. K. Sawyer (Ed.), The Cambridge Handbook of the Learning Sciences, 135-151. Cambridge University Press. New York.

CONFREY, J.; LACHANCE, A. (2000): "Transformative teaching experiments through conjecture-driven research design". En A. E. Kelly y R. A. Lesh (Eds.), Handbook of research design in mathematics and science education, 231-265. Lawrence Erlbaum Associates. Mahwah, New Jersey.

DEWEY, J. (1938): Logic: the theory of inquiry. Henry Holt and Co. New York.

DEWEY. J. (1981): The philosophy of John Dewey: Volume I - the structure of the experience, Volume II - the lived experience. University of Chicago Press. Chicago.

FISCHBEIN, E. (1982): “Intuition and Proof”. For the Learning of Mathematics, 3(2), 9-18. 
GARCÍA, Ma A. (2008): Educación matemática atendiendo a la diversidad: análisis de una metodología específica. Tesis doctoral. Universidad de Valladolid.

GEERTZ, C. (1983): "From the native's point of view: on the nature of anthropological understanding". En Local knowledge: further essays in interpretive anthropology, 55-70. Basic Books. New York.

HANNA, G. (1989): "Proofs that prove and proofs that explain". Proceedings of the $13^{\text {th }}$ International Conference on the Psychology of Mathematics Education, 45-51. París.

HANNA, G.; JAHNKE, H. N. (1996): “Proof and proving”. En A. J. Bishop, M. A. (Ken) Clements, Ch. Keitel, J. Kilpatrick, C. Laborde (Eds.), International Handbook of Mathematics Education, 877-908. Kluwer Academic Publishers. Dordrecht. The Netherlands.

HAREL, G.; SOWDER, L. (1998): “Students' Proof Schemes: Results from exploratory studies". En E. Dubinski, A. Schoenfeld y J. Kaput (Eds.), Research on Collegiate Mathematics Education, vol. III, 234-283. American Mathematical Society. Providence. USA.

HARVEY, A. C. (1993): Time series models. Second edition. Harvester Wheatsheaf. New York.

HERSH, R. (1993): "Proving is convincing and explaining". Educational Studies in Mathematics, 24, 389-399.

IBAÑES, M.; ORTEGA, T. (2002): "Reconocimiento de procesos matemáticos en alumnos de primer curso de Bachillerato". Enseñanza de las Ciencias, 21(1), 49-63.

IBAÑES, M.; ORTEGA, T. (2005): “Dimensiones de la demostración matemática en Bachillerato". Números, 61 (junio), 19-40. 
JUDD, C. H. (1908): "The relation of special training and general intelligence". Educational Review, 36, 28-42.

KATONA, G. (1940): Organizing and memorizing. Columbia University Press. New York.

KEGAN, R. (2000): “What 'form' transforms? A constructive-developmental approach to transformative learning". En J. Mezirow \& Associates (Eds.), Learning as transformation: critical perspectives on a theory in progress, 35-69. Jossey-Bass. San Francisco.

KELLY, A. E. (2003): “Research as design”. Educational researcher, 32(1), 3-4.

KEMMIS, S.; McTAGGART, R. (1988): Cómo planificar la investigación-acción. Laertes. Barcelona.

LAKATOS, I. (1978): Pruebas y refutaciones: la lógica del descubrimiento matemático. Alianza Editorial. Madrid.

MAYER, R. E. (2004): Psicología de la educación Vol. II. Enseñar para un aprendizaje significativo. Prentice Hall. Madrid.

MOLINA, M. (2006): Desarrollo de pensamiento relacional y comprensión del signo igual por alumnos de tercero de educación primaria. Tesis Doctoral. Universidad de Granada.

ORTEGA, T. (2004): “¿Se pueden crear matemáticas desde la Didáctica de la Matemática?" XVII RELME. Tuxtla Gutiérrez. México.

PECHARROMÁN, C.; ORTEGA, T. (2009): “Diseño de un marco de investigación. Aplicación al proceso de aprendizaje de las propiedades globales de las funciones". En M. J. González, M. T. González y J. Murillo (Eds.), Investigación en Educación Matemática XIII (págs. 367-378). SEIEM. Santander. 
PÉREZ, C. (2002): Estadística práctica con Statgraphics. Prentice Hall. Madrid.

PÉREZ SERRANO, G. (1994): Investigación cualitativa. Retos e interrogantes. La Muralla. Madrid.

REID, D. A. (1996): “The role of proving: students and mathematicians”. En M. De Villiers (Coord.), Proofs and proving: why, when and how? 185-199. The Association for Mathematics Education of South Africa (AMESA), PO Box 12833, Centrahil 6006. South Africa.

RENZ, P. (1981): "Mathematical proof: what it is and what it ought to be". The TwoYear College Mathematics Journal, 12(2), 83-103.

SHAVELSON, R. J.; TOWNE, L. (Eds.) (2002): Scientific Research in Education. National Academy Press. Washington, DC.

SOCAS, M. M. (2007): “Dificultades y errores en el aprendizaje de las matemáticas. Análisis desde el Enfoque Lógico Semiótico”. En M. C. Machín, P. F. Martínez y P. B. Catalán (Eds.), Investigación en Educación Matemática XI (págs. 19-52). SEIEM. Santander.

STAKE, R. E. (1995): The art of case study research. Sage Publications. Thousand Oaks, California.

STEFFE, L.; THOMPSON, P. W. (2000): “Teaching experiment methodology: underlying principles and essential elements". En A. E. Kelly y R. A. Lesh (Eds.), Handbook of research design in mathematics and science education, 267-306. Lawrence Erlbaum Associates. Mahwah, New Jersey.

THURSTON, W. P. (1995): “On proof and progress in mathematics”. For the Learning of Mathematics, 15(1), 29-37. 
VILLIERS, M. de (1993): "El papel y la función de la demostración en matemáticas". Épsilon, 26, 15-30. Original de 1990.

WERTHEIMER, M. (1959): Productive thinking. Harper \& Row. New York. 


\section{ANEXOS}

Anexo I: Las pruebas preformales

Anexo II: El cuestionario

Anexo III: El protocolo de observación

Anexo IV: Respuestas al protocolo de observación del ciclo III

Anexo V: El protocolo de las entrevistas

Anexo VI: Primer ciclo: Respuestas de los alumnos al cuestionario y transcripción de las entrevistas

Anexo VII: Segundo ciclo: Respuestas de los alumnos al cuestionario y transcripción de las entrevistas

Anexo VIII: Tercer ciclo: Respuestas de los alumnos al cuestionario y transcripción de las entrevistas. 



\section{ANEXO I}

En este anexo se describen las pruebas preformales que han servido de formación para el Profesor Investigador. Con ellas ha aprendido el fundamento de estas pruebas y adquirido la habilidad necesaria para desarrollar una docencia basada en las mismas. Algunas de estas pruebas se han utilizado en la investigación tanto en la fase de docencia (acción) como en la fase de recogida y análisis de datos.

\section{Teorema}

Si $x, y \in \boldsymbol{R}$, entonces $|x+y| \leq|x|+|y|$.

\section{Prueba preformal}

En lugar de $x$ e $y$ consideramos los números reales 3 y -5 . Puesto que $|3|=3$ y $|-5|=5$, se tiene

$$
-|3|<3=|3| \quad y \quad-|-5|=-5<|-5| .
$$

Sumando resulta

$$
-(|3|+|-5|)<3+(-5)<|3|+|-5|
$$

es decir

$$
|3+(-5)|<|3|+|-5| \text {. }
$$

Aquí termina la demostración, pero además aparece una verificación directa

$$
2<8
$$

\section{Observación}

Este proceso es completamente diferente de la verificación, ya que ésta consiste en ver que se cumple la propiedad en un caso concreto. Con los mismos números el proceso de verificación es el siguiente:

$$
2=|-2|=|3+(-5)|<|3|+|-5|=3+5=8 .
$$

\section{Teorema (Propiedad arquimediana)}

Si $a, b \in \boldsymbol{R}$ y $a>0$, entonces existe $n \in \boldsymbol{N}$ tal que $n a>b$. 


\section{Prueba preformal}

En lugar de $a$ y $b$ consideramos los números 0.01 y $10^{20}$. Sea

$$
A=\{n \cdot 0.01: n \in N\} .
$$

Si fuese $n \cdot 0.01 \leq 10^{20}$ para todo $n \in N$, el conjunto $A$ estaría acotado superiormente por $10^{20}$, de modo que por el axioma de completitud tendría un supremo $s \in \boldsymbol{R}$. En tal caso $s-0.01$ no sería cota superior de $A$, por lo que existiría $n^{*} \in \boldsymbol{N}$ tal que

$$
n^{*} \cdot 0.01>s-0.01 \text {. }
$$

Se tendría pues

$$
\left(n^{*}+1\right) \cdot 0.01 \in A \quad \text { y } \quad\left(n^{*}+1\right) \cdot 0.01>s,
$$

En contra de la elección de $s$. Por tanto se verifica la propiedad.

Además, el conocimiento de los números $a$ y $b$ permite hallar el número natural $n$. La igualdad $x \cdot 0.01=10^{20}$ permite obtener $x=10^{22}$, y por tanto, $n=10^{22}+1$.

\section{Observación}

Una regla corta puede medir distancias largas colocándola consecutivamente. Verificación: con $a=0.01$ y $b=10^{20}$ podemos elegir $n=10^{22}+1$ y se tiene

$$
\left(10^{22}+1\right) \cdot 0.01>10^{20} \text {. }
$$

\section{Teorema (Unicidad del límite)}

Si $f$ tiene límite en $a$, entonces $f$ tiene un solo límite en $a$.

\section{Prueba preformal}

Supongamos que $f$ tiene límite 1 y límite 3 en el punto 4 .

Como 1 es límite de $f$ en 4 , existe $\delta_{1}>0$ tal que para

$$
x \in \operatorname{Dom}(f) \cap\left(\left(4-\delta_{1}, 4+\delta_{1}\right)-\{4\}\right)
$$

es

$$
f(x) \in(0,2) .
$$

Como 3 es límite de $f$ en 4 , existe $\delta_{2}>0$ tal que para

$$
x \in \operatorname{Dom}(f) \cap\left(\left(4-\delta_{2}, 4+\delta_{2}\right)-\{4\}\right)
$$

es

$$
f(x) \in(2,4)
$$


Sea $\delta$ el menor de los números $\delta_{1}$ y $\delta_{2}$. Entonces, si

$$
x \in \operatorname{Dom}(f) \cap((4-\delta, 4+\delta)-\{4\})
$$

es

$$
f(x) \in(0,2) \quad \text { y } \quad f(x) \in(2,4),
$$

pero esto es imposible pues $(0,2) \cap(2,4)=\emptyset$.

Lo que hemos realizado para $L_{1}=1, L_{2}=3$ y $a=4$ se podía haber hecho para otros números cualesquiera. Por tanto, una función no puede tener dos límites diferentes en un punto.

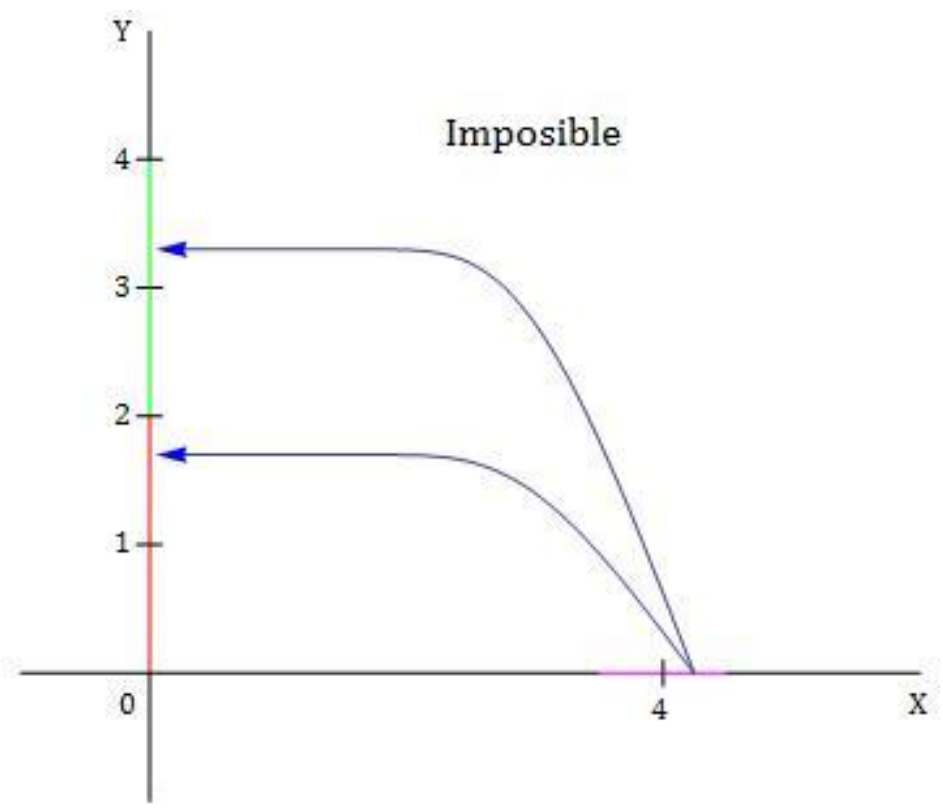

\section{Teorema de Bolzano}

Sea $f:[a, b] \rightarrow \boldsymbol{R}$ continua en $[a, b]$. Si $f(a)$ y $f(b)$ tienen signos distintos, entonces existe $c \in(a, b)$ tal que $f(c)=0$.

\section{Prueba preformal}

La función seno es continua en el intervalo $[2,4] \mathrm{y}$

$$
\operatorname{sen}(2)=0.909297 \ldots>0, \quad \operatorname{sen}(4)=-0.7568025 \ldots<0 \text {. }
$$


El punto que divide al intervalo [2, 4] en dos intervalos de la misma longitud es

$$
\frac{1}{2}(2+4)=3
$$

Como $\operatorname{sen}(3)=0.141120 \ldots>0$, se considera $a_{1}=3$ y $b_{1}=4$. Así se obtiene el intervalo $I_{1}=[3,4]$ en el que la función seno toma valores de diferente signo en sus extremos. Se tiene que

$$
\frac{1}{2}(3+4)=3.5
$$

y como $\operatorname{sen}(3.5)=-0.350783 \ldots<0$, se considera $a_{2}=3$ y $b_{2}=3.5$, obteniéndose el intervalo $I_{2}=[3,3.5]$ en el que la función seno toma valores de diferente signo en sus extremos. Prosiguiendo de esta manera, para $n \in \boldsymbol{N}$ se construye $I_{n}=\left[a_{n}, b_{n}\right]$ donde

$$
\operatorname{sen} a_{n}>0>\operatorname{sen} b_{n}
$$

$\mathrm{y}$

$$
a_{n} \leq a_{n+1}<b_{n+1} \leq b_{n}
$$

con

$$
b_{n+1}-a_{n+1}=\frac{1}{2}\left(b_{n}-a_{n}\right)
$$

La sucesión $\left(a_{n}\right)$ es monótona creciente y está acotada superiormente por 4 , luego es convergente. Sea $\operatorname{lím}_{n \rightarrow \infty} a_{n}=A$.

La sucesión $\left(b_{n}\right)$ es monótona decreciente y está acotada inferiormente por 2, luego es convergente. Sea $\operatorname{lím}_{n \rightarrow \infty} b_{n}=B$.

Por la continuidad de la función seno

$$
\lim _{n \rightarrow \infty} \operatorname{sen} a_{n}=\operatorname{sen}\left(\lim _{n \rightarrow \infty} a_{n}\right)=\operatorname{sen} A
$$

$\mathrm{y}$

$$
\lim _{n \rightarrow \infty} \operatorname{sen} b_{n}=\operatorname{sen}\left(\lim _{n \rightarrow \infty} b_{n}\right)=\operatorname{sen} B \text {. }
$$




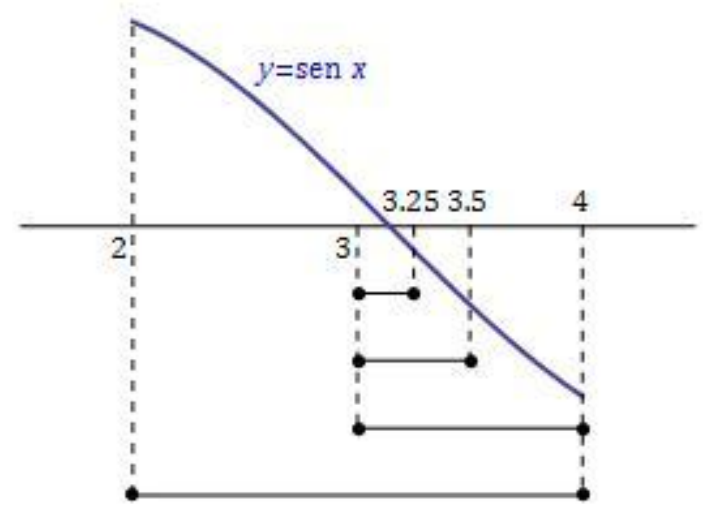

Como sen $a_{n}>0$ para todo $n \in N$, entonces

$$
\lim _{n \rightarrow \infty} \operatorname{sen} a_{n}=\operatorname{sen} A \geq 0 \text {; }
$$

Como sen $b_{n}<0$ para todo $n \in \boldsymbol{N}$, entonces

$$
\lim _{n \rightarrow \infty} \operatorname{sen} b_{n}=\operatorname{sen} B \leq 0 \text {. }
$$

Por otra parte, se tiene

$$
\begin{gathered}
B-A=\lim _{n \rightarrow \infty} b_{n}-\lim _{n \rightarrow \infty} a_{n}=\lim _{n \rightarrow \infty}\left(b_{n+1}-a_{n+1}\right)=\lim _{n \rightarrow \infty} \frac{1}{2}\left(b_{n}-a_{n}\right) \\
=\frac{1}{2}\left(\lim _{n \rightarrow \infty} b_{n}-\lim _{n \rightarrow \infty} a_{n}\right)=\frac{1}{2}(B-A)
\end{gathered}
$$

de donde

$$
A=B
$$

Así pues

$$
\operatorname{sen} A \geq 0 \geq \operatorname{sen} A \text {, }
$$

lo que implica $\operatorname{sen} A=0$.

Igual que se ha considerado la función seno y el intervalo $[2,4]$ se podría haber hecho idéntico desarrollo con cualquier otra función continua en cualquier otro intervalo cerrado y acotado tal que en sus extremos la función tome valores de signos distintos y, por tanto, el teorema es válido. 
Además de existir, por tratarse de una función particular se puede intentar obtener el punto en el que la función se anula. En este caso es claro que sen $c=0$ implica que $c=\pi$.

\section{Teorema de Weierstrass}

Toda función $f$ continua en un intervalo cerrado y acotado $I$ admite un máximo absoluto y un mínimo absoluto en dicho intervalo.

\section{Prueba preformal}

Se considera la función $f(x)=\cos x$ e $I=[\pi / 10, \pi / 4]$. La función coseno es continua en el intervalo $[\pi / 10, \pi / 4]$, luego está acotada. Sea $M$ el extremo superior del conjunto de valores de la función coseno en ese intervalo. Si existiera un $x^{*}$ del intervalo tal que $M-\cos x^{*}=0$ entonces $M$ sería el máximo absoluto en dicho intervalo y el teorema estaría demostrado. Si fuese $M-\cos x>0$ para todo $x \in[\pi / 10, \pi / 4]$, la función $1 /(M-\cos x)$ está definida en $[\pi / 10, \pi / 4]$, es continua y es positiva en dicho intervalo. Por tanto está acotada, es decir, existe un número real $K>0$ tal que

$$
\frac{1}{M-\cos x}<K
$$

de donde se deduce

$$
\frac{1}{K}<M-\cos x
$$

y finalmente,

$$
\cos x<M-\frac{1}{K}
$$

lo que significa que $M$ no es el extremo superior de los valores de la función coseno, en contra de la elección de $M$. Así pues, tiene que existir un $x^{*} \in[\pi / 10, \pi / 4]$ tal que $\cos x^{*}=M$ y por tanto, $M$ es el máximo absoluto en el intervalo.

Con un razonamiento análogo se prueba la existencia de mínimo absoluto $m$ en el intervalo. 
Igual que se ha considerado la función coseno y el intervalo $[\pi / 10, \pi / 4]$, se podría haber hecho idéntico desarrollo con cualquier otra función continua en cualquier otro intervalo cerrado y acotado y, por tanto, el teorema es válido.

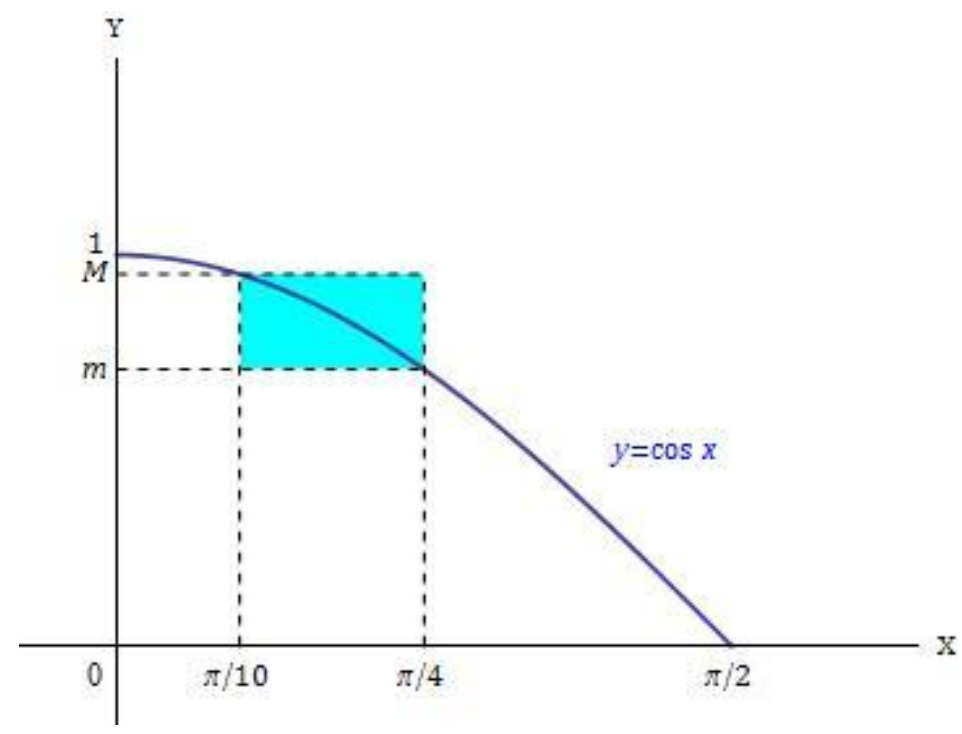

\section{Teorema (derivabilidad implica continuidad)}

Si $f$ es derivable en un punto $a$, entonces $f$ es continua en $a$.

\section{Prueba preformal}

Para cualquier $x \neq 1$, se tiene

$$
e^{x}=(x-1) \cdot \frac{e^{x}-e^{1}}{x-1}+e^{1}
$$

Tomando límites cuando $x \rightarrow 1$ en la expresión anterior

$$
\lim _{x \rightarrow 1} e^{x}=\lim _{x \rightarrow 1}\left[(x-1) \cdot \frac{e^{x}-e^{1}}{x-1}+e^{1}\right]=\lim _{x \rightarrow 1}(x-1) \cdot \lim _{x \rightarrow 1} \frac{e^{x}-e^{1}}{x-1}+\lim _{x \rightarrow 1} e^{1} \text {. }
$$

Como $f(x)=e^{x}$ es derivable en $x=1$,

$$
\lim _{x \rightarrow 1} \frac{e^{x}-e^{1}}{x-1}
$$

existe y es finito; además $\operatorname{lím}_{x \rightarrow 1}(x-1)=0$. Entonces

$$
\lim _{x \rightarrow 1} e^{x}=0 \cdot e^{1}+e^{1}=e^{1}=\left.e^{x}\right|_{x=1},
$$


y por consiguiente $f$ es continua en $x=1$.

\section{Teorema del extremo interior (o de singularidad)}

Sea $a$ un punto interior del intervalo $I$ en el que $f: I \rightarrow \boldsymbol{R}$ tiene un extremo relativo. Si $f$ es derivable en $a$, entonces $f^{\prime}(a)=0$.

\section{Prueba preformal}

La función coseno definida en el intervalo $[0,4]$ tiene un mínimo relativo en $x=\pi$. Existe un intervalo abierto centrado en $\pi$ tal que para los $x$ de ese intervalo es $\cos x \geq \cos \pi$. Así pues, en esos puntos

$$
\frac{\cos x-\cos \pi}{x-\pi}\left\{\begin{array}{l}
\leq 0 \text { si } x \text { está a la izquierda de } \pi \\
\geq 0 \text { si } x \text { está a la derecha de } \pi .
\end{array}\right.
$$

La función coseno es derivable en $x=\pi$. Por tanto, la derivada de la función coseno en $x=\pi$ debe coincidir con las derivadas laterales en dicho punto. Así pues

$$
\left.\frac{d \cos x}{d x}\right|_{x=\pi}=\lim _{x \rightarrow \pi^{-}} \frac{\cos x-\cos \pi}{x-\pi} \leq 0
$$

$\mathrm{y}$

$$
\left.\frac{d \cos x}{d x}\right|_{x=\pi}=\lim _{x \rightarrow \pi^{+}} \frac{\cos x-\cos \pi}{x-\pi} \geq 0,
$$

de donde

$$
\left.\frac{d \cos x}{d x}\right|_{x=\pi}=0
$$




\section{Teorema de Rolle}

Sea $f:[a, b] \rightarrow \boldsymbol{R}$ continua en $[a, b]$, derivable en $(a, b)$ y tal que $f(a)=f(b)$. Entonces existe $c \in(a, b)$ tal que $f^{\prime}(c)=0$.

\section{Prueba preformal}

La función seno es continua en $[0, \pi]$, derivable en $(0, \pi)$ y $\operatorname{sen} 0=0=\operatorname{sen} \pi$. Además no es constante, luego existe un punto $c \in(0, \pi)$ en el que la función seno tiene un extremo relativo. Por el teorema del extremo interior resulta

$$
\left.\frac{d \operatorname{sen} x}{d x}\right|_{x=c}=0
$$

Además de existir, puede encontrarse el punto que satisface el teorema: $\cos c=0$, de donde $c=\pi / 2$. En este punto la función tiene un máximo, cuyo valor es 1 .

\section{Teorema del valor medio (Lagrange)}

Sea $f:[a, b] \rightarrow \boldsymbol{R}$ continua en $[a, b]$ y derivable en $(a, b)$. Entonces existe $c \in(a, b)$ tal que

$$
f(b)-f(a)=f^{\prime}(c)(b-a)
$$

\section{Prueba preformal}

La función logaritmo neperiano es continua en $[2,7]$ y derivable en $(2,7)$.

La función $L:[2,7] \rightarrow \boldsymbol{R}$ cuya gráfica es el segmento que une los puntos $(2, \ln 2)$ y $(7, \ln 7)$ (secante a la gráfica de la función logarítmica) viene dada por

$$
L(x)=\frac{\ln 7-\ln 2}{7-2}(x-2)+\ln 2 .
$$

Sea $g:[2,7] \rightarrow \boldsymbol{R}$ dada por

$$
g(x)=\ln x-L(x)=\ln x-\frac{\ln 7-\ln 2}{7-2}(x-2)-\ln 2
$$


La función $g$ es continua en $[2,7]$, derivable en $(2,7)$ y $g(2)=0=g(7)$. Por el teorema de Rolle existe $c \in(2,7)$ tal que

$$
0=g^{\prime}(c)=\left.\frac{d}{d x}\left(\ln x-\frac{\ln 7-\ln 2}{7-2}(x-2)-\ln 2\right)\right|_{x=c},
$$

luego

$$
0=\left.\frac{d \ln x}{d x}\right|_{x=c}-\frac{\ln 7-\ln 2}{7-2}
$$

de donde

$$
\ln 7-\ln 2=\left.\frac{d \ln x}{d x}\right|_{x=c}(7-2)
$$

Además de existir, puede encontrarse el punto que satisface el teorema:

$$
\frac{1}{c}=\frac{\ln 7-\ln 2}{7-2}
$$

de donde

$$
c=\frac{7-2}{\ln 7-\ln 2}
$$

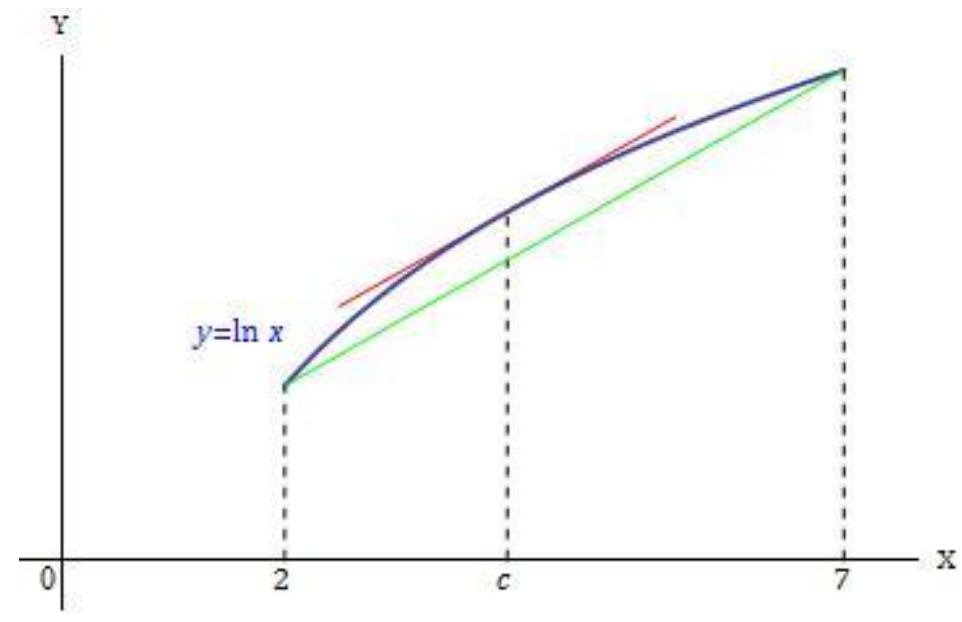




\section{Teorema fundamental del Cálculo Integral}

Si $f$ es integrable Riemann en $[a, b]$ y $F$ es una primitiva suya sobre este intervalo, entonces

$$
\int_{a}^{b} f(x) d x=F(b)-F(a)
$$

\section{Prueba preformal}

La función $f(x)=3 x^{2}$ es integrable Riemann en [1,2], y $F(x)=x^{3}$ es una primitiva suya en $[1,2]$.

Sea $P=\left\{x_{0}, x_{1}, x_{2}, x_{3}\right\}$ una partición de $[1,2]$. Aplicando el teorema del valor medio a la primitiva en cada subintervalo $\left[x_{k-1}, x_{k}\right]$ resulta que existe $t_{k} \in\left(x_{k-1}, x_{k}\right)$ tal que

$$
x_{k}^{3}-x_{k-1}^{3}=3 t_{k}^{2}\left(x_{k}-x_{k-1}\right) .
$$

Como

$$
x_{k-1}^{2}<t_{k}^{2}<x_{k}^{2}
$$

resulta que

$$
\begin{aligned}
\underline{S}(f, P)= & \sum_{k=1}^{3} 3 x_{k-1}^{2}\left(x_{k}-x_{k-1}\right)<\sum_{k=1}^{3} 3 t_{k}^{2}\left(x_{k}-x_{k-1}\right) \\
& <\sum_{k=1}^{3} 3 x_{k}^{2}\left(x_{k}-x_{k-1}\right)=\bar{S}(f, P) .
\end{aligned}
$$

Ahora bien

$$
\sum_{k=1}^{3} 3 t_{k}^{2}\left(x_{k}-x_{k-1}\right)=\sum_{k=1}^{3}\left(x_{k}^{3}-x_{k-1}^{3}\right)=x_{3}^{3}-x_{0}^{3}=2^{3}-1^{3},
$$

luego

$$
\underline{\int_{1}^{2}} 3 x^{2} d x \leq 2^{3}-1^{3} \leq \overline{\int_{1}^{2}} 3 x^{2} d x
$$

y como

$$
\underline{\int_{1}^{2}} 3 x^{2} d x=\overline{\int_{1}^{2}} 3 x^{2} d x=\int_{1}^{2} 3 x^{2} d x
$$

se concluye que 


$$
\int_{1}^{2} 3 x^{2} d x=2^{3}-1^{3}
$$




\section{ANEXO II}

En este anexo se presenta el cuestionario para recoger los datos que serán analizados:

1. Explica cómo has entendido cada uno de los teoremas con cada procedimiento. Indica en cada caso claramente el porqué.

- Teorema 1:

- Teorema 2:

2. Puntúa de 1 a 10 las cuatro pruebas que hemos realizado atendiendo a las siguientes categorías: facilidad (F), gusto (G), claridad (C), aprendizaje (A). Entiende que:

1 es muy difícil y 10 es muy fácil.

1 no me gusta y 10 me gusta mucho.

1 no es nada clara y 10 es muy clara.

1 no se aprende nada y 10 se aprende mucho.

- Preformal del teorema 1: $F_{ـ}, G_{-}, C_{\longrightarrow}, A_{-}$.

- Formal del teorema 1: $\mathrm{F}_{\_}, \mathrm{G}_{\_}, \mathrm{C}_{\_}, \mathrm{A}_{-}$.

- Preformal del teorema 2: $\mathrm{F}_{\_}, \mathrm{G}_{\_}, \mathrm{C}_{\_}, \mathrm{A}_{-}$.

- Formal del teorema 2: $F_{\_}, G_{\_}, C_{-}, A_{-}$. 
En la columna COMPRENSIÓN: se reescriben las puntuaciones de la cuestión 2. En la columna PREFERENCIA: para cada teorema, comparando las puntuaciones de cada modalidad de prueba en cada una de las cuatro categorías, se indica el número de veces en que "gana" (supera) la prueba preformal a la formal, y viceversa.

\begin{tabular}{|c|c|c|c|}
\hline TEOREMA & MODALIDAD & COMPRENSIÓN & PREFERENCIA \\
\hline \multirow{2}{*}{ Teorema 1} & Preformal & $\mathrm{F}_{-}, \mathrm{G}_{-}, \mathrm{C}_{-}, \mathrm{A}_{-}$ & \\
\hline & Formal & $F_{-}, G_{-}, C_{-}, A_{-}$ & \\
\hline \multirow{2}{*}{ Teorema 2} & Preformal & $F_{-}, G_{-}, C_{-}, A_{-}$ & \\
\hline & Formal & F_, $G_{-}, C_{2}, A_{-}$ & \\
\hline \multirow{2}{*}{ Total } & Preformal & & \\
\hline & Formal & & \\
\hline
\end{tabular}

3. Responde a las siguientes preguntas, señalando en cada caso claramente el porqué:

- ¿Cuál de las dos formas te convence más de la veracidad del teorema?

- ¿Cuál de las dos pruebas puede ayudar más a entender el significado del teorema?

- ¿Cuál de las dos pruebas puede ayudar más a memorizar el teorema?

- ¿Cuál de las dos pruebas es más útil para ilustrar cómo se aplica el teorema? 
Considera la función seno definida en el intervalo [0, $\pi / 3]$. Aplica el teorema del Valor Medio (Lagrange) para determinar $c$ con $0<c<\pi / 3$ y tal que la pendiente de la tangente a la curva $y=\operatorname{sen} x$ en el punto $(c, \operatorname{sen} c) \quad($ el valor de la derivada en $c$ ) coincida con la pendiente de la secante que corta a la curva en los puntos $(0, \operatorname{sen} 0)$ y $(\pi / 3, \operatorname{sen}(\pi / 3))$.

Valora de 1 a 10 el grado de influencia de cada una de las pruebas en esta aplicación.

De la prueba preformal: 1, 2, 3, 4, 5, 6, 7, 8, 9, 10.

De la prueba formal: $1,2,3,4,5,6,7,8,9,10$. 



\section{ANEXO III}

En este anexo se presenta el protocolo de observación utilizado por el observador externo en los ciclos II y III:

\section{PROTOCOLO DE OBSERVACIÓN}

\section{a) Tratamiento y metodología}

1. Finalidad/grado de adecuación del diseño:

- Objetivos

- Contenidos

- Metodología

2. ¿Se especifican adecuadamente los diferentes contenidos?

3. Viabilidad/utilidad práctica del tratamiento.

4. Tiempo: ¿Se ajusta el desarrollo al tiempo previsto? ¿Qué modificaciones son necesarias en tiempo?

\section{b) Profesor}

1. Comportamiento y papel durante el desarrollo. (Actitud, interés, atención,...).

2. Tipo de participación en el proceso docente.

3. Calidad y claridad de las intervenciones.

4. Tiempo de intervención.

\section{c) Alumnos}

1. Actividad central durante el desarrollo del tratamiento. ¿Qué hacen? 
2. Tipo de participación (Iniciativa, pasividad, silencio, hacen las tareas, siguen las explicaciones).

3. Tipo de preguntas que realizan.

4. Atención/asimilación:
a) ¿Atienden a las explicaciones?
b) ¿Parece que entienden las explicaciones?
c) ¿Qué síntomas se detectan?

5. Actitud general: ¿Positiva, neutra, negativa,...?

\section{d) Interacciones profesor-alumnos}

1. Frecuencia: Nunca, a menudo, constantemente. Duración de las intervenciones.

2. Tipo:

- Atención fluida con diálogo profesor-alumno.

- A instancias del alumno.

- El profesor crea situaciones de interacción.

- El profesor muestra una actitud que favorece el diálogo.

\section{e) Recursos y condiciones materiales}

1. Idoneidad de los recursos empleados en relación con el tratamiento.

2. Inconvenientes materiales observados.

3. Otras observaciones.

\section{f) Incidencias especiales}

1. Retrasos dignos de mención, puntualidad: alumnos y profesor.

2. Disfunciones o inconvenientes producidos. Posibles motivos.

3. Otras observaciones. (Orden en la clase, alboroto, atención, participación, estudio, trabajo). 


\section{ANEXO IV}

Aquí se reproducen las respuestas proporcionadas por el $\mathrm{OE}$ al protocolo de observación correspondiente al ciclo III.

\section{Tratamiento y metodología}

1 Finalidad/grado de adecuación del diseño:

- Objetivos

Analizar la enseñanza-aprendizaje de las demostraciones con pruebas formales y preformales, así como las preferencias de los alumnos sobre dichas pruebas.

- Contenidos

Teorema del valor medio.

Demostración formal del teorema del valor medio.

Demostración preformal del teorema del valor medio.

Teorema de unicidad del límite.

Demostración preformal del teorema de unicidad del límite.

Demostración formal del teorema de unicidad del límite.

- Metodología

Exposición por parte del profesor de los teoremas y sus respectivas demostraciones preformal y formal, en el orden cronológico que se indica en los contenidos. Además, explica cómo deben cumplimentarse las tareas que deben realizar los alumnos.

A continuación los alumnos realizan las tres tareas solicitadas por el profesor, relativas a los contenidos que se acaban de exponer.

Tanto en el periodo de exposición por parte del profesor como en el de realización de tareas, el profesor pregunta a los alumnos e informa que se pueden preguntar dudas en cualquier momento. 
2 ¿Se especifican adecuadamente los diferentes contenidos?

Sí, la exposición y especificación de cada uno de los contenidos se realiza de forma clara y detallada.

3 Viabilidad/utilidad práctica del tratamiento.

Enseñanza y aprendizaje de algunos teoremas de análisis utilizando pruebas preformales.

4 Tiempo: ¿Se ajusta el desarrollo al tiempo previsto? ¿Qué modificaciones son necesarias en el tiempo?

Sólo 4 alumnos han entregado los cuestionarios cumplimentados antes de finalizar el tiempo previsto, una hora y 45 minutos, por lo que parece aconsejable ampliar el tiempo aún más o realizar alguna de las tareas en otro momento.

\section{Profesor}

1 Comportamiento y papel durante el desarrollo. (Actitud, interés, atención,...)

El profesor muestra interés por el aprendizaje de los alumnos. Está pendiente en todo momento de las dudas del alumno, resolviendo éstas de forma individual y prestando especial interés por aquellos alumnos que tienen más dificultades. Además, realiza algunas preguntas para motivar la participación de los alumnos y observar la atención prestada. Muestra en todo momento una actitud positiva.

2 Tipo de participación en el proceso docente.

Durante la primera hora del proceso el profesor recuerda qué es una demostración preformal, a continuación expone los teoremas y sus demostraciones y explica la forma de cumplimentar los cuestionarios; deteniéndose en algunos momentos para resolver las dudas de los alumnos o preguntar él si existen dudas. 
En la segunda parte de la sesión, cuando los alumnos responden a las preguntas que se realizan en los cuestionarios, resuelve de forma individual las dudas que surgen a cada uno de los alumnos.

3 Calidad y claridad de las intervenciones.

Las intervenciones del profesor son claras, rigurosas y concisas en todo momento.

4 Tiempo de intervención.

Durante la primera hora interviene casi de forma continua, y en los 45 minutos que los alumnos dedican a realizar las tareas, de forma puntual resolviendo sus dudas.

\section{Alumnos}

1 Actividad central durante el desarrollo del tratamiento. ¿Qué hacen?

Durante el periodo de exposición del profesor, la gran mayoría de los alumnos prestan atención a la explicación y toman notas como apoyo en la resolución de las tareas propuestas.

Están expectantes ya que se encuentran en una clase distinta y especial.

2 Tipo de participación (Iniciativa, pasividad, silencio, hacen las tareas, siguen las explicaciones).

Siguen las explicaciones del profesor en silencio y tomando notas. En esta fase la participación es escasa, con una actitud de cierta pasividad en bastantes alumnos.

Durante la realización de tareas los alumnos muestran bastante interés en su desarrollo, preguntando al profesor cuando surgen dudas.

3 Tipo de preguntas que realizan.

Un alumno solicita al profesor que le recuerde la forma punto-pendiente de la ecuación de una recta. 
La mayoría de sus preguntas hacen referencia a cumplimentar adecuadamente las tareas propuestas.

4 Atención/asimilación.

a) ¿Atienden a las explicaciones?

En el periodo de exposición los alumnos han estado muy atentos en la parte correspondiente al teorema del valor medio; se observa menor atención durante el desarrollo correspondiente al teorema de unicidad del límite.

b) ¿Parece que entienden las explicaciones?

Se aprecia un entendimiento bastante claro de la exposición y demostración formal del teorema del valor medio, en menor medida cuando se realiza la demostración preformal al utilizar la función logaritmo neperiano. En el teorema de unicidad del límite se aprecia que algunos alumnos no entienden toda la explicación o que necesitarían dedicar más tiempo para que sea asimilada.

c) ¿Qué síntomas se detectan?

En el período relativo al teorema del valor medio se percibe interés por parte de la mayoría de los alumnos. En el teorema de unicidad del límite algunos alumnos dejan de tomar notas, hablan entre ellos, y dedican más tiempo a mirar y cumplimentar los cuestionarios que a atender al profesor.

5 Actitud general: ¿Positiva, neutra, negativa,...?

Positiva.

\section{Interacciones profesor-alumnos}

1 Frecuencia: Nunca, a menudo, constantemente.

Duración de las intervenciones.

Las intervenciones son pocas y de duración breve. 


\section{Tipo:}

Atención fluida con diálogo profesor-alumno.

(No responde).

A instancias del alumno.

Los alumnos intervienen principalmente para que el profesor aclare dudas sobre la forma de cumplimentar adecuadamente las tareas.

El profesor crea situaciones de interacción.

Sí.

El profesor muestra una actitud que favorece el diálogo.

Respondida en el apartado Profesor (1).

\section{Recursos y condiciones materiales}

1 Idoneidad de los recursos empleados en relación con el tratamiento. Se ha desarrollado en la misma aula teórica que se imparten las clases de la asignatura, cuyo tamaño es adecuado al número de alumnos, y se ha utilizado pizarra y tizas de diversos colores, considerando estos recursos suficientes para el tratamiento realizado.

2 Inconvenientes materiales observados.

Ninguno.

3 Otras observaciones.

(No responde). 


\section{Incidencias especiales}

1 Retrasos dignos de mención, puntualidad: alumnos y profesor.

Puntualidad tanto por parte del profesor como de la mayoría de los alumnos, sólo unos pocos alumnos llegaron unos minutos tarde.

2 Disfunciones o inconvenientes producidos. Posibles motivos. No se detectan.

3 Otras observaciones. (Orden en la clase, alboroto, atención, participación, estudio, trabajo).

(No responde). 


\section{ANEXO V}

En este anexo se presenta el protocolo utilizado en las entrevistas realizadas en los tres ciclos de la investigación.

1 ¿Tenías ya alguna convicción de estos teoremas antes de la demostración?

2 Si ya tenías algún grado de convicción anterior, ¿qué te han proporcionado las demostraciones realizadas?

3 Después del estudio de estas pruebas, ¿puedes afirmar que para ti es absoluta y rigurosamente completa la verdad de esos teoremas?

4 Facilidad de aplicación de la PF y de la PPF. Al estudiar estas pruebas has aprendido dos procedimientos, uno formal y otro preformal. A la hora de hacer ejercicios o trabajar en matemáticas ¿cuál de los dos procedimientos crees que te ayudará más?

5 ¿En cuál de ellos has aportado más de ti mismo, trabajo, ingenio, esfuerzo...? Expresa en breves palabras tu impresión general sobre toda esta actividad.

6 ¿Cuál de los dos procedimientos te aporta mayor ayuda a la hora de comunicar contenidos matemáticos?

7 ¿Cuál de los dos procedimientos explica mejor los pasos?

8 ¿En cuál de los dos procedimientos entiendes mejor los pasos?

9 ¿Crees que influye en la comprensión el orden de presentación de los dos procedimientos? 



\section{ANEXO VI (2007)}

Aquí se reproducen íntegramente las respuestas que emitieron los alumnos del grupo a la cuestión primera.

Cuestión 1: Explica cómo has entendido cada uno de los teoremas con cada procedimiento. Indica en cada caso claramente el porqué.

El objetivo de esta cuestión es analizar la comprensión de los alumnos de las demostraciones matemáticas sobre las pruebas preformales en contraste con las pruebas formales. Los números se refieren a los alumnos (28 en total). En adelante, PF designa prueba formal y PPF prueba preformal.

\section{Teorema 1. Valor Medio (Lagrange). Prueba Preformal (1º)}

1: Un ejemplo para ver la aplicación del teorema.

2: Este teorema es, por su naturaleza, más claro e intuitivo que el teorema 2, parte con ventaja. La demostración preformal ayuda más que la formal a entender su veracidad.

3: La he entendido como una aplicación a un caso concreto, en la que se dan por sabidas muchas cosas, por haber tomado una función que cumple con exactitud las condiciones de posible aplicación del teorema. Se ha procedido explicando la resolución de las ecuaciones planteadas "basándonos en el teorema formal"; el resultado carece de la generalización propia de un teorema.

4: Me ha resultado más sencillo al usar valores concretos en lugar de "letras".

5: (Sin aportación apreciable). 
6: Creo bueno hacer primero la prueba preformal por dar ejemplos concretos, funciones conocidas, pasos y operaciones más fáciles.

7: He entendido mejor la prueba preformal por partir de una función concreta, la función logarítmica; es más concisa y clara y ya está presente la idea esencial. Más fácil de entender.

8: Más intuitiva y mucho más fácil de entender.

9: Ilustra el uso; puede hacer pensar que sólo es aplicable a un caso determinado previamente ajustado.

10: Con esta prueba lo he comprendido peor, con la función logarítmica es más difícil de comprender.

11: No es "general". Ambas pruebas se apoyan en el teorema de Rolle.

12: No me agrada; el enunciado no me especifica con claridad lo que persigo; y no sé si los puntos $(\sqrt{2}, \ln \sqrt{2}),(6, \ln 6)$ vienen dados o son anteriormente calculados.

13: Con esta prueba se ve mucho mejor, clarifica mucho y ayuda a su comprensión.

14: No he entendido por qué se hacen las funciones ni el teorema de Rolle.

15: En esta prueba necesitas recurrir a la formal; no se muestran todas las fórmulas necesarias para resolverlo.

16: Esta prueba me resulta "tangible", algo clarificador; el resultado numérico ayuda a verlo mejor. 
17: Me resultó un poco complicada.

18: Me ha resultado más fácil entenderlo con la preformal por la ayuda del ejemplo numérico.

19: Me gusta más, se entiende mejor cómo llegar a la conclusión.

20: Me ha resultado un ejemplo. Lo entiendo igual con las dos pruebas.

21: Bien entendido de ambas maneras, preformal y formal; mejor en la preformal, al poner un ejemplo previo.

22: Es la comprobación del teorema en un caso particular y el procedimiento lo llevamos a un caso general; así resulta más sencilla la comprensión del teorema.

23: He llegado a la conclusión apoyándome en las dos pruebas, pero más en la preformal, en la que se muestra más el funcionamiento.

24: Algo lioso y complicado de ver lo que está realizado.

25: En esta prueba uso el teorema, no su explicación o demostración.

26: Las explicaciones son más claras con la prueba preformal.

27: Se entiende mejor con la prueba preformal. El teorema es sencillo y se comprende mejor el funcionamiento con casos concretos.

28: La que más me ha ayudado a entenderlo. 


\section{Teorema 1. Valor Medio (Lagrange). Prueba formal ( $2^{\circ}$ )}

1: Con la prueba formal lo he entendido mejor; se ven mejor los pasos a seguir para su uso. Pienso que es más eficiente la prueba formal.

2: Me ha resultado menos enrevesada la prueba formal por obviarse las derivadas.

3: Lo he entendido como demostración formal "de libro". Cabría esperar que fuera igual que la preformal, pero hay matices, sobre todo en la notación.

4: Es más sencillo pasar del método preformal al formal generalizando el procedimiento visto ya con valores concretos, teniendo ya la idea básica del teorema.

5: (Sin aportación apreciable).

6: Es necesaria, pues ayuda a generalizar el razonamiento seguido en la prueba preformal y facilita futuros ejercicios. Me parece confusa.

7: No se dan valores explícitos. Menos fácil de entender.

8: Es más completa, de cara a explicar la teoría; pero más compleja de entender por el alumno, según mi experiencia: me hallaba perdido por momentos.

9: Me quedo con la formal, me parece más general abarcando todos los casos posibles, también el contemplado en la preformal.

10: Es más sencilla por ser forma general, hay que aprenderla como se muestra. Lo más complejo es imaginarse la función. 
11: Al ser "general" vale para todas las funciones que verifiquen las hipótesis iniciales del teorema.

12: Es la mejor opción: más generalizada, sé hasta dónde tengo que llegar en el análisis del problema.

13: Con esta prueba no está claro, pero es aplicable a otros datos, da la perspectiva mejor sobre el teorema.

14: La he entendido un poco más por la función $L(x)$.

15: Me parece mejor, se muestran todas las fórmulas necesarias para resolverlo. Al terminar la demostración y aplicar Rolle se ve mucho más clara la resolución que en la preformal.

16: Es la prueba con la que realmente he comprendido el teorema.

17: Me resultó más claro por este método.

18: El desarrollo formal es una forma más precisa y más aplicable a múltiples casos.

19: (No opina).

20: Lo entiendo igual con las dos pruebas, me resulta más claro con la prueba formal.

21: Bien entendido de ambas maneras, preformal y formal, peor en la formal, en mi caso. 
22: Más difícil.

23: He llegado a la conclusión apoyándome en las dos pruebas; en la formal, menos.

24: Más sencilla, aunque la función de la prueba es casi imposible obtenerla.

25: Con esta prueba lo he entendido mejor.

26: Menos claras las explicaciones que en la preformal.

27: Se entiende peor que con la preformal.

28: Lo mejor: dar primero la explicación formal y luego la preformal para acabar con un ejemplo.

\section{Teorema 2. Unicidad del Límite. Prueba formal (1ํㅜㄱ}

1: La prueba formal al ser más estricta y utilizar muchas variables hace difícil su comprensión, por eso el ejemplo ha sido más fácil de entender.

2: Es una dificultad añadida tratar de demostrar este teorema mediante la reducción al absurdo. Aunque igualmente queda clara su veracidad.

3: Demostración formal "de libro"; más fácil de entender que el teorema 1, por suponer menos y más sencillos conocimientos.

4: Me gusta menos el orden primero formal y luego preformal porque se pasa de lo más abstracto a lo más sencillo. 
5: (Sin aportación apreciable).

6: Me parece confusa.

7: Me ha resultado también bastante clara.

8: Más difícil de entender el teorema en la prueba formal.

9: Esta prueba es poco intuitiva.

10: Con esta prueba es más difícil comprender el teorema, aunque no es mal método al ser general.

11: Me satisface mucho más, se extiende a más casos.

12: Con la prueba formal me quedaría sin palabras ante mis múltiples dudas.

13: Prefiero la preformal aunque no sea la forma "genérica" que ha de memorizarse.

14: No he entendido nada.

15: Me quedo con la prueba formal, me ha ayudado mejor a entender los intervalos de $f(x)$ para los distintos límites 1 y 3 ; la veo más sencilla y útil.

16: Una vez vista la prueba preformal, volviendo a la formal sí pude entenderlo y generalizarlo.

17: Esta prueba me ha resultado peor por las letras. 
18: Con la prueba formal ya me ha quedado bastante claro.

19: Esta prueba es más difícil de entender porque no tomamos números concretos.

20: Es más difícil de entender.

21: Me quedaría con la demostración formal, pues la preformal no sería suficiente para entender de dónde salen los resultados.

22: El teorema se demuestra en la prueba formal; la preformal lo aplica a un caso particular.

23: Lo entiendo mejor con la prueba formal.

24: Con esta prueba se ve mejor lo que está realizado.

25: También lo he entendido con esta prueba.

26: Es menos sencilla que la prueba preformal.

27: Prefiero esta prueba: es más técnica, la reducción al absurdo parece lo más apropiado.

28: Lo mejor: dar primero la explicación formal y luego la preformal, para acabar con un ejemplo. 


\section{Teorema 2. Unicidad del Límite. Prueba preformal (2º)}

1: Me ha parecido más fácil la prueba preformal, la identifica con un ejemplo; ni una ni otra me han ayudado a entender del todo el teorema.

2: El ejemplo de la demostración preformal es más claro. Supongo que la reducción al absurdo es posiblemente el camino más fácil para resolver teoremas sobre unicidad.

3: Este teorema lo he entendido mejor que el teorema 1, por ser más sencillo y por seguir el orden de explicación habitual (explicación y después aplicación). Hay matices que aportan mayor precisión a la prueba formal.

4: Me decanto por el método preformal, porque primero se hace la aplicación de forma muy visual y luego se pasa al método formal que sirva para aprenderlo en conceptos generales.

5: Con la prueba preformal he entendido el teorema (que una función en un punto sólo puede tener un límite en un intervalo abierto).

6: La prueba preformal ha sido de más ayuda por dar ejemplos concretos, con funciones conocidas, pasos y operaciones más fáciles.

7: He entendido algo mejor con la prueba preformal, por dar valores concretos. Me ha resultado bastante clara. Me decanto a favor de la preformal.

8: Más fácil de intuir el teorema en la prueba preformal. 
9: Esta prueba es más ilustrativa: dados los intervalos $(0,2)$ y $(2,4)$ se ve que la imagen de la función no puede estar a la vez en los dos, pues no tienen ningún punto común.

10: Es más sencilla. El teorema se entiende mejor con esta prueba.

11: Sólo demuestra que la función no puede tener límites 1 y 3 en el punto 4; pero no demuestra nada para el resto de puntos.

12: Este teorema es más complejo que el anterior. La única forma de entenderlo es con un ejemplo.

13: Ver el teorema de una forma más concreta me ayudó a comprenderlo mejor. Prefiero la prueba preformal.

14: No entiendo por qué utiliza el $\varepsilon$.

15: Me ayudó en la última parte de la demostración formal donde se comprueba que en los intervalos no hay puntos comunes, se ve más claro con los puntos.

16: La prueba preformal me ha ayudado más. La representación gráfica ayuda bastante a ver que es imposible que en un punto tengamos dos límites distintos.

17: Con la prueba preformal me ha quedado más claro.

18: El uso de la forma preformal no le resta dificultad al teorema.

19: Con esta prueba es más fácil de entender, porque tomamos valores concretos en vez de $L_{1}$ y $L_{2}$. 
20: He entendido mejor con la prueba preformal.

21: Sólo con esta prueba no sería suficiente para entender de dónde salen los resultados. Ahora, pienso que una prueba preformal puede ayudar siempre a entender cualquier teorema.

22: Esta prueba me sirve para aplicar la demostración formal a un caso particular: así se verá mejor si no se ha entendido algún paso del caso formal.

23: La prueba preformal ayuda bastante a seguir el razonamiento.

24: Me resulta algo más lioso y complicado de entender.

25: Lo he entendido mejor con esta prueba con un ejemplo con números.

26: Mejor que la prueba formal: es más sencilla de ejecución y ofrece una visión de conjunto bastante mejor.

27: No la prefiero en este caso.

28: Esta prueba preformal es la que más me ha ayudado a entenderlo. 
Las respuestas a la tercera cuestión fueron las siguientes:

\section{CUÁL DE LAS DOS FORMAS TE CONVENCE MÁS DE LA VERACIDAD DEL TEOREMA}

\begin{tabular}{|c|c|}
\hline 1 & La PF, porque la PPF la veo más como un ejemplo práctico. \\
\hline 2 & $\begin{array}{l}\text { La PPF: aunque matemáticamente no tenga la misma validez que la PF, se suele en- } \\
\text { tender mejor la vida con casos prácticos, aunque un caso aislado no sirva como de- } \\
\text { mostración. }\end{array}$ \\
\hline 3 & $\begin{array}{l}\text { La PF: la PPF no daría veracidad por sí sola, tendríamos que probar todos los casos,... } \\
\text { no sé si la PPF se puede considerar demostración. }\end{array}$ \\
\hline 4 & $\begin{array}{l}\text { La PPF por demostrarse cierto en un caso, lo que con la PF me resulta imposible al no } \\
\text { tener valores concretos, no puedo comprobar su veracidad con constantes... }\end{array}$ \\
\hline 5 & Las pruebas PF me parecen más veraces. \\
\hline 6 & $\begin{array}{l}\text { La PF porque parte de un caso general. En la PPF puede quedar la duda de si se cum- } \\
\text { plirá para cualquier función o solo la del ejemplo. }\end{array}$ \\
\hline 7 & La PPF porque es más concisa, se apoya en ejemplos para explicar los teoremas. \\
\hline 8 & La PF por lógica, dado que abarca todos los casos convence más. \\
\hline 9 & $\begin{array}{l}\text { La PF es más concreta, pero la PPF puede ayudar a hacerse una idea rápida sin ser } \\
\text { muy específico. }\end{array}$ \\
\hline 10 & La PF ya que es el teorema en sí y es la forma general. \\
\hline 11 & La PF porque demuestra los teoremas para todos los casos. \\
\hline 12 & $\begin{array}{l}\text { La PF enuncia el teorema de forma más técnica y precisa, aunque la PPF te demuestra } \\
\text { la veracidad del teorema con un ejemplo. }\end{array}$ \\
\hline 13 & La PPF porque con un ejemplo se ve todo mejor. \\
\hline 14 & (Respuesta irrelevante). \\
\hline 15 & $\begin{array}{l}\text { La PF, ya que al ser un caso general se entiende mejor y se puede aplicar a cualquier } \\
\text { caso. }\end{array}$ \\
\hline 16 & $\begin{array}{l}\text { La PF ya que es general, aplicable a cualquier función que cumpla las condiciones. La } \\
\text { PPF no da esa sensación por ser un caso concreto. }\end{array}$ \\
\hline 17 & $\ldots$ \\
\hline 18 & La PF porque es mucho más factible. \\
\hline 19 & La PPF porque tiene más claridad para llegar a la misma conclusión. \\
\hline 20 & La PF me resulta más veraz en ambos casos, pero más difícil de entender. \\
\hline 21 & La PF porque es general para todos; la PPF nos habla de un ejemplo. \\
\hline 22 & La PF por ser general para cualquier valor de las variables. \\
\hline 23 & $\begin{array}{l}\text { La PPF al realizarse con números, la idea ya está presente en la forma que más me } \\
\text { convence de la veracidad. }\end{array}$ \\
\hline 24 & La PF (no indica motivo). \\
\hline 25 & La PF (no indica motivo). \\
\hline 26 & La PPF, ya que se demuestra a base de números y claridad. \\
\hline 27 & La PPF, sobre todo en el teorema de Lagrange (no señala motivos). \\
\hline 28 & La PPF (no aporta motivos). \\
\hline
\end{tabular}




\section{CUÁL DE LAS DOS PRUEBAS PUEDE AYUDAR MÁS A ENTENDER EL SIGNIFICADO DEL TEOREMA}

\begin{tabular}{|c|c|}
\hline 1 & La PPF porque es más fácil asimilarlo al verlo en un caso práctico y su aplicación. \\
\hline 2 & $\begin{array}{l}\text { La PPF: los ejemplos numéricos clarifican el entendimiento; las múltiples variables y } \\
\text { las letras griegas de la PF complican bastante la comprensión. }\end{array}$ \\
\hline 3 & $\begin{array}{l}\text { La PF; la PPF tendría que formalizarla. Me ayuda más ambas pruebas en este orden: } \\
\text { PF + PPF, demostración más aplicación. }\end{array}$ \\
\hline 4 & $\begin{array}{l}\text { La PPF por ser directa y visual, teniendo en cuenta que ya posee la idea central del } \\
\text { teorema. }\end{array}$ \\
\hline 5 & La PPF: es más fácil y se ve mejor. \\
\hline 6 & La PPF, más fácil de entender. \\
\hline 7 & La PPF, a mi parecer (no indica motivo). \\
\hline 8 & Mucho más intuitivo para mí la PPF. \\
\hline 9 & $\begin{array}{l}\text { La PPF es bastante intuitiva, pero al cumplirse en un caso determinado, la PF es más } \\
\text { clara al ser extrapolable a todos los casos posibles. }\end{array}$ \\
\hline 10 & La PPF: un ejemplo aplicado es más fácil de comprender. \\
\hline 11 & Las dos PPF - PF: se complementan y ayudan a su comprensión. \\
\hline 12 & $\begin{array}{l}\text { Para entender con mayor precisión e intensidad el teorema, la mejor manera un } \\
\text { ejemplo, la PPF; }\end{array}$ \\
\hline 13 & La PF, pues se ve el verdadero significado del teorema. \\
\hline 14 & (Respuesta irrelevante). \\
\hline 15 & La PPF me ayudó más, ya que con un ejemplo se puede entender mejor. \\
\hline 16 & $\begin{array}{l}\text { La PF ya que no se ciñe a un caso concreto y me da una visión más amplia del teore- } \\
\text { ma. }\end{array}$ \\
\hline 17 & ( \\
\hline 18 & A mí la PPF me sirve para verlo antes, pero también es necesaria la PF. \\
\hline 19 & La PPF porque usa valores reales más fáciles de entender por el alumno. \\
\hline 20 & $\begin{array}{l}\text { La PF es más técnica, pero la PPF resulta mucho más visual, todos los teoremas de- } \\
\text { berían apoyarse en ejemplos parecidos. }\end{array}$ \\
\hline 21 & $\begin{array}{l}\text { La PPF, siempre con un ejemplo (al menos en mi caso) se entiende mejor el concepto } \\
\text { matemático. }\end{array}$ \\
\hline 22 & La PPF, se ve mejor con un ejemplo concreto y práctico. \\
\hline 23 & La PF, el concepto lo veo mejor en la forma generalizada del teorema. \\
\hline 24 & La PF (no indica motivo). \\
\hline 25 & La PF (no indica motivo). \\
\hline 26 & La PF, es más estructurada y tiene más que ver con el teorema. \\
\hline 27 & La PPF, sobre todo en el teorema de Lagrange (no señala motivos). \\
\hline 28 & La PPF al ser un determinado caso. \\
\hline & $\begin{array}{c}\text { Tabla VI/2. Respuestas de los alumnos sobre la ayuda al entendimiento del } \\
\text { significado del teorema }\end{array}$ \\
\hline
\end{tabular}




\section{CUÁL DE LAS DOS PRUEBAS PUEDE AYUDAR MÁS A MEMORIZAR EL TEOREMA}

\begin{tabular}{|c|c|}
\hline 1 & $\begin{array}{l}\text { Las dos son necesarias, ya que es necesario para ello una prueba estricta y un ejem- } \\
\text { plo práctico. }\end{array}$ \\
\hline 2 & $\begin{array}{l}\text { En el teorema } 1 \text { la PF y en el teorema } 2 \text { la PPF: se memorizan mejor las cosas que se } \\
\text { entienden, pero también las más cortas. }\end{array}$ \\
\hline 3 & $\begin{array}{l}\text { En esta vida se valora la aplicación; si dispones de una aplicación directa de un teo- } \\
\text { rema, la PPF, tiendes a memorizar cómo se aplica, pero de poco sirve si no tienes una } \\
\text { idea genérica, la PF, aunque podemos llegar a generalizar sustituyendo números por } \\
\text { letras. En resumen, la PPF ayuda más a memorizar. }\end{array}$ \\
\hline 4 & La PF más fácil de recordar de manera general y aplicar valores según se necesite. \\
\hline 5 & La PF (no señala motivo). \\
\hline 6 & La PF, que al ser general te vale para cualquier ejercicio. \\
\hline 7 & La PF por ser general vale para todas las situaciones. \\
\hline 8 & $\begin{array}{l}\text { La PPF al ser mucho más intuitiva, la entiendes antes, acordándote de un ejemplo } \\
\text { puedes memorizarlo antes mejor que la PF que quizá no llegas a entender. }\end{array}$ \\
\hline 9 & La PF es la que más juego puede dar ya que se puede ajustar a más casos. \\
\hline 10 & La PPF: los números te ayudan a memorizar el procedimiento. \\
\hline 11 & La PF por ser más clara. \\
\hline 12 & $\begin{array}{l}\text { La PF: una explicación técnica me parece más útil que con un ejemplo, que aunque } \\
\text { éste sea fácil de memorizar, puedes cometer más errores que si memorizas el teore- } \\
\text { ma de forma general. }\end{array}$ \\
\hline 13 & La PF, pues es la que se aplicará en los distintos casos posibles. \\
\hline 14 & (Respuesta irrelevante). \\
\hline 15 & La PF ya que es un caso general. \\
\hline 16 & La PF es más impactante, pero la PPF ayuda a tenerlo más claramente. \\
\hline 17 & $\ldots$ \\
\hline 18 & Quizás la PF. \\
\hline 19 & La PF porque es más corto que la PPF. \\
\hline 20 & La PF (no aporta motivo). \\
\hline 21 & La PF al no tratarse de un caso concreto. \\
\hline 22 & La PPF y la PF ambas por igual. \\
\hline 23 & La PF: es más genérica, la "máquina" en la que meter los "ingredientes". \\
\hline 24 & La PF (no indica motivo). \\
\hline 25 & La PF (no indica motivo). \\
\hline 26 & La PF es más común y más mecánica. \\
\hline 27 & La PF puedes ver en mayor medida el desarrollo teórico. \\
\hline 28 & La PPF aunque se complementan. \\
\hline
\end{tabular}




\section{CUÁL DE LAS DOS PRUEBAS ES MÁS ÚTIL PARA ILUSTRAR CÓMO SE APLICA EL TEOREMA}

\begin{tabular}{|c|c|}
\hline 1 & La PPF, se ve mejor la utilidad. \\
\hline 2 & $\begin{array}{l}\text { La PPF. Cuando se da un ejemplo numérico, se está aplicando en cierta forma el teo- } \\
\text { rema, es más difícil visualizar una variable o punto que no se conoce. }\end{array}$ \\
\hline 3 & $\begin{array}{l}\text { La PPF: siempre se ilustrará mejor con números que con letras, poco útiles para ilus- } \\
\text { trar la aplicación de un teorema. }\end{array}$ \\
\hline 4 & $\begin{array}{l}\text { La PPF porque esa es su función, ilustrar una aplicación concreta del teorema y no } \\
\text { tanto su visión general, aunque a partir de ella se puede generalizar fácilmente. }\end{array}$ \\
\hline 5 & La PPF, es más clara y se ve mejor. \\
\hline 6 & $\begin{array}{l}\text { La PPF para demostrar cómo se aplica. Pero para entenderlo a la perfección, hacer } \\
\text { ambas demostraciones sería lo mejor. }\end{array}$ \\
\hline 7 & La PPF te ayuda a ver cómo se aplica el teorema con un ejemplo concreto. \\
\hline 8 & La PPF es la mejor manera para ilustrarlo, con un ejemplo concreto. \\
\hline 9 & La PPF ya que es un caso práctico de la aplicación formal con datos concretos. \\
\hline 10 & La PPF ya que es un ejemplo "aplicado" al teorema. \\
\hline 11 & $\begin{array}{l}\text { La PPF aplica para un caso el teorema, por lo que se ve mejor, pero siempre ayudán- } \\
\text { dola con la PF. }\end{array}$ \\
\hline 12 & $\begin{array}{l}\text { La PPF sin lugar a dudas porque con ejemplos te guía hasta concluir con lo que el teo- } \\
\text { rema persigue. }\end{array}$ \\
\hline 13 & La PPF sin duda porque se ve mejor con un caso concreto. \\
\hline 14 & (Respuesta irrelevante). \\
\hline 15 & $\begin{array}{l}\text { La PF lo explica de un modo general, aunque creo que es necesaria la PPF para de- } \\
\text { mostrarlo a modo de ejemplo. }\end{array}$ \\
\hline 16 & $\begin{array}{l}\text { La PPF ya que tratamos de un caso concreto en el que la idea esencial está presente y } \\
\text { su aplicación es mucho más obvia. }\end{array}$ \\
\hline 17 & $\ldots$ \\
\hline 18 & La PPF (no aporta motivo). \\
\hline 19 & La PPF, la gente lo entiende mejor con unos valores definidos que sin ellos. \\
\hline 20 & La PPF da una idea mejor de lo que es el teorema y lo que se quiere indicar con él. \\
\hline 21 & La PPF te ayudará más a entender la idea de la aplicación. \\
\hline 22 & La PPF porque se realiza con números concretos. \\
\hline 23 & $\begin{array}{l}\text { La PPF: representa de forma más gráfica la aplicación del teorema, ya que a la hora de } \\
\text { ver el funcionamiento es mejor verlo con datos reales y no "letras". }\end{array}$ \\
\hline 24 & La PF (no indica motivo). \\
\hline 25 & La PPF (no indica motivo). \\
\hline 26 & La PPF porque es la forma más sencilla gráficamente. \\
\hline 27 & $\begin{array}{l}\text { La PPF evidentemente la mejor, al utilizarse pruebas con situaciones más concretas } \\
\text { se ve mejor cómo se aplica el teorema en situaciones más prácticas. }\end{array}$ \\
\hline 28 & Cualquiera de las dos, aunque la PPF es más de mi gusto. \\
\hline \multicolumn{2}{|r|}{$\begin{array}{c}\text { Tabla VI/4. Respuestas de los alumnos sobre la utilidad para ilustrar la aplicación } \\
\text { del teorema }\end{array}$} \\
\hline
\end{tabular}




\title{
TRANSCRIPCIÓN DE LA ENTREVISTA I
}

\author{
José Luis Ramos Jiménez (JLR). Prefiere la PF \\ Eduardo Cristóbal Enríquez (ECE). Prefiere la PPF
}

\section{1 ¿Tenías ya alguna convicción de estos teoremas antes de la demostración?}

- No; no los había visto nunca demostrados; los resultados los recordaba sólo vagamente (JLR).

- Sí; en Bachillerato los había visto²; el de unicidad del límite sin demostración (ECE).

2 Si ya tenías algún grado de convicción anterior, ¿qué te han proporcionado las demostraciones realizadas?

- PPF: es un ejemplo ${ }^{3}$ (JLR).

- PPF: razonamiento completo 4 que me ayudó a aplicar el resultado (ECE).

- PF: es la prueba completa (ECE).

- PF: es un razonamiento abstracto que conduce al resultado (JLR).

3 Después del estudio de estas pruebas, ¿puedes afirmar que para ti es absoluta y rigurosamente completa la verdad de esos teoremas?

- Sí. Ahora que he visto demostraciones me convenzo de la veracidad (JLR).

- Sí. Al ver las demostraciones te convences de que no puede aparecer ningún caso extraño que lo incumpla (ECE).

¿No habrá algún caso que lo incumple?

- $\quad$ No (JLR).

- $\quad$ No (ECE).

4 Facilidad de aplicación de la PF y de la PPF. Al estudiar estas pruebas has aprendido dos procedimientos, uno formal y otro preformal. A la hora de

\footnotetext{
${ }^{2}$ A efectos de la instrucción relativa a las pruebas se puede considerar que el punto de partida de ambos es similar, pero en el caso de JLR, quizás sí que pudieran haber realizado en clase las demostraciones correspondientes.

${ }^{3}$ El alumno está equivocado.

${ }^{4}$ Este alumno se fija en la característica de la PPF, el razonamiento, a diferencia del anterior que, equivocadamente, piensa que es un ejemplo.
} 


\section{hacer ejercicios o trabajar en matemáticas ¿cuál de los dos procedimientos crees que te ayudará más?}

- El procedimiento formal porque es el que cubre todos los casos. No obstante, reconozco que el preformal puede proporcionar alguna ayuda en las aplicaciones $^{5}$ (JLR).

- En las aplicaciones, el procedimiento preformal. También el formal, pero es con la PPF con la que me quedo por ser más intuitiva, más cercana ${ }^{6}$ (ECE).

5 ¿En cuál de ellos has aportado más de ti mismo, trabajo, ingenio, esfuerzo...? Expresa en breves palabras tu impresión general sobre toda esta actividad.

- El procedimiento preformal es el que más trabajo y esfuerzo me ha supuesto, lo encontré más lioso.

- Impresión general: la actividad me ha ayudado a pensar y a razonar y a tener un conocimiento más profundo de los teoremas ${ }^{7}$ (JLR).

- Más esfuerzo en el procedimiento formal, por la abstracción.

- Impresión general: la actividad me ha ayudado a profundizar en los teoremas; especialmente positivos han resultado los procedimientos preformales para aplicar los resultados ${ }^{8}$ (ECE).

\section{6 ¿Cuál de los dos procedimientos te aporta mayor ayuda a la hora de comu- nicar contenidos matemáticos?}

- $\quad$ El procedimiento formal por su rigor y por ser el que mejor entendí (JLR).

- Utilizaría el procedimiento preformal, aunque, naturalmente, ofrecería también el formal (ECE).

\footnotetext{
5 Señala la "generalización" como razón de su preferencia por la PF. Es una observación válida. Asigna también a la PPF alguna ayuda.

${ }^{6}$ Aduce la condición de "más intuitiva" para preferir la PPF. Efectivamente, así es.

${ }^{7}$ Quizá su preferencia por la PF le hace manifestarse así, pues no es "más lioso" el procedimiento de la PPF. La respuesta a su impresión general muestra el interés del alumno y el resultado positivo para él de esta experiencia.

${ }^{8}$ Lógica la respuesta a tenor de su preferencia por la PPF y en razón del motivo que señala, la "abstracción" de la PF. En la impresión general manifiesta su interés en la experiencia y los efectos positivos que ha supuesto para él.
} 


\title{
7 ¿Cuál de los dos procedimientos explica mejor los pasos?
}

- El procedimiento formal. Cualquier situación queda contemplada en el razonamiento (JLR).

- El procedimiento preformal; ahora bien, la PF es la que explica de forma definitiva cubriendo todos los $\operatorname{casos}^{9}$ (ECE).

\section{8 ¿En cuál de los dos procedimientos entiendes mejor los pasos?}

- En el procedimiento formal (JLR).

- Sin duda alguna en el procedimiento preformal. Lo veo más manejable, más fácil (ECE).

\section{TRANSCRIPCIÓN DE LA ENTREVISTA II}

\author{
Roberto García López (RGL). Prefiere la PF
}

Fernando Hernández Esguevillas (FHE). Prefiere la PPF

\section{1 ¿Tenías ya alguna convicción de estos teoremas antes de la demostración?}

- Sí; más convicción respecto del teorema del valor medio (RGL).

- Sí, de ambos; aunque no había visto ninguna demostración del teorema de unicidad del límite (FHE). ${ }^{10}$

2 Si ya tenías algún grado de convicción anterior, ¿qué te han proporcionado las demostraciones realizadas?

- PPF: la veo como una situación concreta, como un ejemplo; me ayuda a recor$\operatorname{dar}^{11}(R G L)$.

- PPF: lo veo como un caso concreto, como un ejemplo que esclarece lo visto en la PF y que me ayuda a recordar ${ }^{12}$ (FHE).

\footnotetext{
${ }^{9}$ Respuesta sensata, acorde con su preferencia, pero sin rehusar el procedimiento formal, que califica como definitivo.

10 También en esta segunda pareja es muy similar el punto de partida de sus conocimientos previos sobre los dos teoremas de la experiencia. Coinciden ambos incluso en una mayor convicción respecto al teorema del valor medio.

${ }^{11}$ Se confunde al considerar la PPF como un mero ejemplo.

12 Tampoco está acertado en su apreciación de la PPF solamente como un ejemplo.
} 
- PF: es la prueba definitiva, la que permite establecer el resultado (FHE).

- PF: la veo como la justificación propiamente dicha del teorema, el "teorema en sí" (RGL).

3 Después del estudio de estas pruebas, ¿puedes afirmar que para ti es absoluta y rigurosamente completa la verdad de esos teoremas?

- Sí. La prueba que me convenció completamente es la PF (RGL).

- Sí. Ambas pruebas clarifican, especialmente la PPF por su concreción (FHE). ${ }^{13}$

¿No habrá algún caso que lo incumple?

- $\quad$ No $(R G L)$.

- $\quad$ No (FHE).

4 Facilidad de aplicación de la PF y de la PPF. Al estudiar estas pruebas has aprendido dos procedimientos, uno formal y otro preformal. A la hora de hacer ejercicios o trabajar en matemáticas ¿cuál de los dos procedimientos crees que te ayudará más?

- A la hora de aplicar y trabajar de forma práctica, el procedimiento preformal es el que más me ayuda (RGL).

- -Para las aplicaciones ayuda más el procedimiento preformal siempre y cuando se sepa extraer la idea esencial y no se vea inflexiblemente con los objetos concretos que en él aparezcan (FHE). ${ }^{14}$

5 ¿En cuál de ellos has aportado más de ti mismo, trabajo, ingenio, esfuerzo...? Expresa en breves palabras tu impresión general sobre toda esta actividad.

- En el procedimiento formal, por su grado de abstracción, pero el esfuerzo queda compensado.

\footnotetext{
${ }^{13}$ Cada uno de los alumnos que integran esta pareja apoya la veracidad de los teoremas en la prueba de su preferencia, pero los dos afirman también que es el conjunto de ambas pruebas lo que justifica la absoluta verdad de los mismos.

${ }^{14}$ Los dos alumnos coinciden en que la prueba que más les ayuda en las aplicaciones es la PPF. FHE advierte, ahora sí acertadamente, corrigiendo la imprecisión de la respuesta a la cuestión 2, que para las aplicaciones debe evitarse considerar la PPF limitándola exclusivamente a un solo caso concreto.
} 
- Impresión general: la actividad me ha ayudado a profundizar en los teoremas $(R G L) \cdot{ }^{15}$

- En el procedimiento formal para el teorema de unicidad del límite (por el asunto del $\varepsilon$ ), este teorema se ve mejor con la PPF; la PPF me ayudó mucho a entender la PF.

- Impresión general: muy positiva: son los teoremas que mejor he asimilado porque la actividad me ha impulsado a trabajarlos con más profundidad (FHE).16

\section{6 ¿Cuál de los dos procedimientos te aporta mayor ayuda a la hora de comu- nicar contenidos matemáticos?}

- Utilizaría los dos procedimientos. Haría PF en primer lugar y después PPF $(R G L)$.

- El teorema del valor medio le comunicaría directamente con la PF. Para el de unicidad del límite haría en primer lugar PPF y después PF (FHE).

\section{7 ¿Cuál de los dos procedimientos explica mejor los pasos?}

- En el procedimiento formal se ven mejor explicados los pasos. Como es completamente general, se ven todos los casos de una vez (RGL).

- $\quad$ La PF es más completa, da la visión global del teorema (FHE).

\section{8 ¿En cuál de los dos procedimientos entiendes mejor los pasos?}

- Los pasos se entienden mejor en el procedimiento preformal, pero es la conjunción de los dos procedimientos la que realmente ayuda a entender mejor $(R G L)$.

- Los pasos se entienden mejor en la PPF porque todo es más cercano, más manejable. No obstante la PPF no me resulta tan necesaria en el caso del teorema del valor medio como en el caso del teorema de unicidad del límite. Para el teorema de unicidad del límite, la PPF me resulta imprescindible (FHE). ${ }^{17}$

\footnotetext{
15 Respuesta que supone el interés prestado y la satisfacción lograda.

${ }^{16}$ Expresa, coincidiendo con todos los demás, la satisfacción al realizar esta experiencia.

${ }^{17}$ Ambos alumnos coinciden en que los pasos de estas demostraciones se explican mejor en el procedimiento de la PF, pero se entienden mejor en el de la PPF.
} 
Nota: Entre estos dos alumnos se estableció un pequeño debate sobre el orden en que debían hacerse las pruebas. Ambos opinaban que la conjunción de los dos procedimientos es la que realmente proporciona el aprendizaje completo del teorema, pero mientras que Roberto prefería en primer lugar la PF y después la PPF (de abstracto a concreto), Fernando prefería primero la PPF y luego la PF (de concreto a abstracto). ${ }^{18}$

18 Tiene interés el debate sobre el orden de presentación de estas dos pruebas: RGL se inclina por la deducción y FHE por la inducción, preferencias que pueden responder a la configuración personal de su capacidad de aprendizaje. En las respuestas de estos dos alumnos subyacen también las características concreción y abstracción o generalización, de la pareja I, aplicadas a la PPF (concreción) y a la PF (abstracción). 



\section{ANEXO VII (2008)}

Aquí se reproducen íntegramente las respuestas que emitieron los alumnos del grupo a la cuestión primera.

Cuestión 1: Explica cómo has entendido cada uno de los teoremas con cada procedimiento. Indica en cada caso claramente el porqué.

El objetivo de esta cuestión es analizar la comprensión de los alumnos de las demostraciones matemáticas sobre las pruebas preformales en contraste con las pruebas formales. Los números se refieren a los alumnos (19 en total). En adelante, PF designa prueba formal y PPF prueba preformal.

\section{Teorema 1. Valor Medio (Lagrange). Prueba Formal (1º)}

1: He entendido la PF bastante bien, aunque no utiliza número como ejemplo. Es muy útil al desarrollar un problema de este tipo.

2: Lo he entendido perfectamente tanto en la PF como en la PPF: el proceso a realizar para obtener el resultado.

3: Fácil, excepto la fórmula de la función diferencia, un poco compleja. El problema se visualiza fácilmente.

4: Bastante sencilla de entender. Prefiero la PF por ser general, lo cual veo útil para poder aplicarlo a cualquier caso concreto. El orden ideal: $1^{\circ}$ la PF y $2^{\underline{o}}$ la PPF, porque primero se capta la idea general y después se concreta.

5: Es menos intuitiva, aunque viendo la resolución, no es muy diferente de la PPF. 
6: Mejor 1ำ la PF y luego la PPF: primero una visión más amplia de conceptos teóricos y a continuación (con la PPF) refuerzas los conceptos adquiridos.

7: Más clara la PF: las operaciones fáciles y la conclusión salía inmediata.

8: (Describe lo realizado, sin señalar preferencia ni motivos).

9: Con la PF he conseguido saber cuáles son las variables que he de cambiar y dónde colocarlas.

10: Con la PF he comprendido mejor, aunque me ha gustado más la PPF. Me ha costado comprender la parte en que se aplica el teorema de Rolle.

11: La PF: es general para cualquier caso y se entiende perfectamente.

12: Con la PF al ir expresándolo gráficamente se pueden sacar conclusiones a partir de estos cálculos.

13: Lo he entendido bien con las dos pruebas; quizá un poco mejor con la PF, porque se ve mejor de dónde sale cada dato.

14: Entendí mejor con la PF ya que solo operamos con derivadas y en la PPF se mezclan derivadas, logaritmos... Es verdad que ha sido la primera explicada y siempre parece más sencillo y estás más atento.

15: El punto más conflictivo es aplicar Rolle. Una vez esto, simplemente es derivar y despejar. Es bastante entendible.

16: La PF me ha parecido demasiado teórica. 
17: La PF resulta básica para entender el teorema de forma más clara y útil; pero con la PPF después se alcanza la comprensión completa.

18: Con la PF me parece más sencillo el teorema.

19: La gráfica no ayuda demasiado. Me decanto por la PF: parece más clarificante y simple de entender.

\section{Teorema 1. Valor Medio (Lagrange). Prueba Preformal (2º)}

1: La PPF al estar explicada con un ejemplo concreto, se entiende algo peor; si quiero resolver un problema de este tipo con datos diferentes me cuesta más asociar cuál es cada término.

2: Me ha resultado un poco más compleja la PPF, debido a los logaritmos, que me lían un poco; pero lo he entendido perfectamente tanto en la PF como en la PPF.

3: La PPF me ha resultado un poco más complicada, ya que al introducir valores concretos, las derivadas hacen la demostración menos clara.

4: La PPF en segundo lugar me ha servido para ver una aplicación concreta. Me ha parecido más útil en la última parte encontrar el valor concreto de $c$. El orden $1^{\circ}$ la PF y $2^{\circ}$ la PPF es el ideal.

5: La PPF me agrada más: al manejar números y no letras me hago más a la idea de lo que se pretende.

6: Al explicar la PPF después de la PF refuerzas los conceptos adquiridos en la anterior prueba. 
7: Me pareció más clara la PF.

8: (No indica preferencia ni motivo).

9: Con la PPF he conseguido averiguar errores que hubiera cometido con la PF a la hora de sustituir.

10: Me ha gustado más la PPF.

11: La PPF es más concreta al utilizar números; cuando relacionamos con el teorema de Rolle me complico con los cálculos.

12: Con la PPF al ser ejemplo concreto se puede apreciar de forma menos abstracta.

13: Lo he entendido bien con las dos pruebas.

14: En la PPF la mezcla de operaciones, derivadas, logaritmos... son más complejas.

15: Respecto a la PPF me parece más complejo utilizar logaritmos.

16: He entendido mejor la PPF: con valores concretos se ve mejor lo que se quiere demostrar. La PF es demasiado teórica.

17: Con la PPF a posteriori se alcanza la comprensión completa del teorema.

18: Con la PPF tanto la gráfica como el llegar al resultado me parece complicado. 
19: Quizás la función logarítmica no sea un ejemplo excesivamente fácil para ver los resultados.

\section{Teorema 2. Unicidad del Límite. Prueba Preformal (1º)}

1: Lo entiendo mejor con la PPF; está aplicada con un ejemplo concreto y es mucho más fácil de ver y de aplicar.

2: Ninguna prueba me ha resultado complicada. Ambas son sencillas. A la hora de representación gráfica me resulta más clara la PPF, debido a la aplicación de números concretos.

3: La PPF es fácil de comprender, gracias a que en el caso particular es más fácil visualizar el problema.

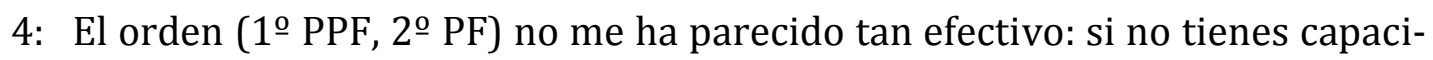
dad de abstracción se te pasan muchos detalles fijándote en los datos concretos. Pienso que también influye el orden.

5: Con la PPF los números no hacen mucho más visible la finalidad de la explicación del teorema.

6: Al explicar primero la PPF creo que se le pueden escapar algunos conceptos al alumno al no ver primero la PF. Considero que la PPF es más entendible y la PF más completa.

7: Me pareció bastante más fácil la PPF: visualmente se llegaba de una manera más clara y concisa a la solución, incluso antes de acabar la demostración.

8: (No señala preferencias ni motivos). 
9: Con la PPF se ve más claro que con la PF: numéricamente se ve por anticipado que un mismo punto no puede tener varios límites.

10: Prefiero la PPF: se ha explicado mejor. Creo que el orden más idóneo es primero la PPF y segundo la PF.

11: Con la PPF se me hace más sencillo porque al realizar cálculos se ve mucho más fácil la conclusión, que hemos partido de algo erróneo.

12: En ambas formas se puede deducir rápidamente el resultado.

13: El teorema 2 no le he entendido hasta llegar al final de la PPF; este teorema es más fácil de entender que con la PF.

14: La PPF: las operaciones son más sencillas, y se realiza en primer lugar.

15: La demostración de este teorema me parece más abstracta que el anterior (aunque con los esquemas y los colores ayudan bastante). La PPF me parece más sencilla, ya que utilizamos números concretos y el resultado satisface el teorema.

16: He entendido mejor la PPF por ser más concreta.

17: En este caso se hace más necesaria una explicación preformal.

18: La PPF me parece más entendible (no sé si porque se ha explicado más).

19: La PPF es algo más concreta y conclusa. La gráfica se hace imprescindible para ambas pruebas. 


\section{Teorema 2. Unicidad del Límite. Prueba Formal $\left(2^{\circ}\right)$}

1: La PF se entiende algo peor.

2: La PPF y la PF son sencillas. Ninguna me ha resultado complicada.

3: La PF se hace un poco más complicada al introducir más parámetros, pero reflexionando y pensando un poco no resulta demasiado difícil.

4: La PF me ha parecido efectiva, aunque algo más complicada que en el teorema 1. También influye el orden de las pruebas.

5: La PF parece ser la que me ha dejado entender el objetivo del teorema.

6: Considero que la PF es más completa y la PPF más entendible.

7: (No aporta nada sobre la PF en este teorema).

8: (No señala preferencia ni motivos).

9: Lo entendí con la PF y con un ejemplo práctico lo acabé de ver.

10: Creo que el orden más idóneo es $1^{\circ}$ la PPF y 2oa la PF.

11: No se ve tan claro como en la PPF con números concretos.

12: En ambas formas se puede deducir rápidamente el resultado.

13: En este teorema la PF no es tan fácil de entender como la PPF.

14: En la PF las operaciones y las explicaciones que hay que dar son complejas.

15: Me parece más sencilla de seguir la PPF que la PF. 
16: He entendido mejor la PPF por ser más concreta.

17: La explicación PF resulta más abstracta.

18: La PF menos entendible que la PPF.

19: La gráfica se hace imprescindible para ambas pruebas. La PPF es algo más concreta y conclusa.

Las respuestas a la tercera cuestión fueron las siguientes:

\section{CUÁL DE LAS DOS FORMAS TE CONVENCE MÁS DE LA VERACIDAD DEL TEOREMA}

\begin{tabular}{|c|c|}
\hline 1 & Las dos, PPF y PF, me convencen igual. \\
\hline 2 & Las dos, pero más la PPF por aplicar números concretos. \\
\hline 3 & "La PF, puesto que queda más claro que es válida para cualquier valor". \\
\hline 4 & $\begin{array}{l}\text { "La PPF porque al hacerlo con valores concretos conocidos ves de manera más clara } \\
\text { el resultado". }\end{array}$ \\
\hline 5 & La PPF en el teorema 1: los valores conocidos son un entorno más amigable. \\
\hline 6 & $\begin{array}{l}\text { "La PF porque considero que da una visión general de todos los casos posibles, aun- } \\
\text { que la conjunción de los dos métodos me parece lo ideal". }\end{array}$ \\
\hline 7 & "La PPF ya que la solución se ve de una manera más visual". \\
\hline 8 & La PF en el teorema 1, por más sencilla. La PPF en el teorema 2, por más fácil. \\
\hline 9 & La PPF si el teorema es complicado. La PF si el teorema es fácil de entender. \\
\hline 10 & La PPF: sustituimos las letras por valores; después es necesaria la PF. \\
\hline 11 & La PPF en el teorema 1: se ve más claro. La PF en el teorema 2: con números, mejor. \\
\hline 12 & "La PF porque al ser más general es aplicable a todos los casos". \\
\hline 13 & La PPF (no aporta motivo). \\
\hline 14 & Las dos, PPF y PF, si lo estudias bien. \\
\hline 15 & $\begin{array}{l}\text { "La PF es más genérica al no concretarse con un ejemplo; a mí particularmente me } \\
\text { parece que, a nuestro nivel, las pruebas preformales son más asequibles a nuestro ni- } \\
\text { vel". }\end{array}$ \\
\hline 16 & La PPF (no aporta motivo). \\
\hline 17 & La PF: sirve de base para su aplicación. \\
\hline 18 & La PF: me parece más sencilla. \\
\hline 19 & Ambas, PPF y PF (no aporta motivo). \\
\hline
\end{tabular}




\section{CUÁL DE LAS DOS PRUEBAS PUEDE AYUDAR MÁS A ENTENDER EL SIGNIFICADO DEL TEOREMA}

\begin{tabular}{|r|l|}
\hline $\mathbf{1}$ & Algo más la PPF. \\
\hline $\mathbf{2}$ & $\begin{array}{l}\text { "La PPF ya que en la representación gráfica se ve más claramente la interpretación } \\
\text { que hay que hacer". }\end{array}$ \\
\hline $\mathbf{3}$ & La PPF: ofrece un ejemplo más claro. \\
\hline $\mathbf{4}$ & $\begin{array}{l}\text { "La PF porque al explicarlo sin valores concretos no tienes que abstraerte de dichos } \\
\text { valores y puedes centrarte en el teorema como tal". }\end{array}$ \\
\hline $\mathbf{5}$ & $\begin{array}{l}\text { "Depende, ya que para mí en el teorema 1 prefiero la PF y en el 2 la PF ya que no me } \\
\text { distraigo tanto con los números y me ayuda a entenderlo mejor". }\end{array}$ \\
\hline $\mathbf{6}$ & La PPF: ayuda más con un ejemplo concreto y comprensible. \\
\hline $\mathbf{7}$ & La PF: se basa, dentro de lo que cabe, en el enunciado del teorema. \\
\hline $\mathbf{8}$ & $\begin{array}{l}\text { La PF en el teorema 1, por el dibujo de la gráfica. La PPF en el teorema 2, por la expli- } \\
\text { cación más sencilla. }\end{array}$ \\
\hline $\mathbf{9}$ & La PPF para el significado. \\
\hline $\mathbf{1 0}$ & La PPF: más acertada. \\
\hline $\mathbf{1 1}$ & "Para mí se entiende mejor con la PF en el primer teorema y la PPF en el 2". \\
\hline $\mathbf{1 2}$ & La PPF: al poner un caso concreto es más clara. \\
\hline $\mathbf{1 3}$ & La PF (no aporta motivo). \\
\hline $\mathbf{1 4}$ & "Para el entendimiento, aparte del estudio del teorema, por mi parte entiendo más la \\
\hline $\mathbf{1 5}$ & "La me parece la prueba más sencilla". \\
\hline $\mathbf{1 6}$ & La PPF (no aporta motivo). \\
\hline $\mathbf{1 7}$ & La PPF: resulta menos abstracta y más comprensible. \\
\hline $\mathbf{1 8}$ & La PF (no aporta motivo). \\
\hline $\mathbf{1 9}$ & La PPF (no aporta motivo). \\
\hline & Tabla VII/2. Respuestas de los alumnos sobre la ayuda al entendimiento del \\
& \\
\hline
\end{tabular}




\section{CUÁL DE LAS DOS PRUEBAS PUEDE AYUDAR MÁS A MEMORIZAR EL TEOREMA}

\begin{tabular}{|c|l|}
\hline $\mathbf{1}$ & La PPF en el teorema 2; la PF en el teorema 1 (no aporta motivo). \\
\hline $\mathbf{2}$ & "La PF porque está generalizada para cualquier aplicación". \\
\hline $\mathbf{3}$ & La PPF más fácil en el teorema 2; la PF más fácil en el teorema 1. \\
\hline $\mathbf{4}$ & La PF: al no utilizar valores concretos. \\
\hline $\mathbf{5}$ & $\begin{array}{l}\text { "La PF puede ser la más conveniente a la hora de memorizar ya que con variables } \\
\text { hace que se vea como más genérica, a forma de molde en que hay que introducir va- } \\
\text { lores y sale el resultado esperado". }\end{array}$ \\
\hline $\mathbf{6}$ & $\begin{array}{l}\text { "Creo que la PF es más fácil de memorizar al basarse en conceptos y no en números } \\
\text { concretos". }\end{array}$ \\
\hline $\mathbf{7}$ & $\begin{array}{l}\text { "La PPF ya que me resulta más fácil memorizar un teorema con aplicación numérica } \\
\text { que formal". }\end{array}$ \\
\hline $\mathbf{8}$ & $\begin{array}{l}\text { "La PPF en el teorema 2 por la explicación; la PF en el teorema 1 ya que la gráfica me } \\
\text { ha quedado más clara". }\end{array}$ \\
\hline $\mathbf{9}$ & La PPF: es lo que miro al estudiar. \\
\hline $\mathbf{1 0}$ & La PF: se puede generalizar. \\
\hline $\mathbf{1 1}$ & La PF: son casos genéricos. \\
\hline $\mathbf{1 2}$ & "La PPF si se usa un caso concreto llamativo". \\
\hline $\mathbf{1 3}$ & La PPF (no aporta motivo). \\
\hline $\mathbf{1 4}$ & La PF con su gráfica y puntos obtenidos. \\
\hline $\mathbf{1 5}$ & Creo que ninguna destaca para memorizar el teorema. \\
\hline $\mathbf{1 6}$ & La PPF (no aporta motivo). \\
\hline $\mathbf{1 7}$ & La PF: menos mecánica y no depende de un caso concreto. \\
\hline $\mathbf{1 8}$ & La PF: mucho más entendible. \\
\hline $\mathbf{1 9}$ & La PF (no aporta motivo). \\
\hline & Tabla VII/3. Respuestas de los alumnos sobre la ayuda a la memorización \\
& \\
\hline
\end{tabular}




\section{CUÁL DE LAS DOS PRUEBAS ES MÁS ÚTIL PARA ILUSTRAR CÓMO SE APLICA EL TEOREMA}

\begin{tabular}{|c|c|}
\hline 1 & La PPF en ambos teoremas (no aporta motivo). \\
\hline 2 & $\begin{array}{l}\text { "La PPF por la utilización de números concretos que hacen que me resulte más clara } \\
\text { la interpretación". }\end{array}$ \\
\hline 3 & La PPF: aclara más dónde se deben introducir los valores. \\
\hline 4 & $\begin{array}{l}\text { "La PPF por usar los valores concretos y una gráfica conocida, así hay más factores } \\
\text { que conoces de antemano". }\end{array}$ \\
\hline 5 & La PPF: con datos se ve mejor el molde (del punto anterior). \\
\hline 6 & $\begin{array}{l}\text { "En este sentido creo que es evidente que la PPF es la manera idónea de ilustrar cómo } \\
\text { se aplica el tema, ya que ves un ejemplo concreto, ves un caso en el plano, que resulta } \\
\text { real y entendible". }\end{array}$ \\
\hline 7 & La PPF: más fácil de mostrar la aplicación con números que con letras. \\
\hline 8 & $\begin{array}{l}\text { "Teorema } 1 \text { la PF (en la PPF partimos de logaritmos y pueden ser más difíciles las } \\
\text { operaciones); teorema } 2 \text { la PPF ya que partimos de valores inventados". }\end{array}$ \\
\hline 9 & La PPF (no aporta motivo). \\
\hline 10 & "Es más útil la PPF porque se comprende con un ejemplo". \\
\hline 11 & La PPF: manejamos números concretos. \\
\hline 12 & La PF (no aporta motivo). \\
\hline 13 & La PPF (no aporta motivo). \\
\hline 14 & $\begin{array}{l}\text { La PPF: obtienes todos los datos, puntos y demás información para la resolución del } \\
\text { ejercicio. }\end{array}$ \\
\hline 15 & La PPF; en el teorema 2 la PF no me ha quedado muy clara. \\
\hline 16 & La PPF (no aporta motivo). \\
\hline 17 & $\begin{array}{l}\text { La PPF; la PF resulta difícil de imaginar aplicarla. Ideal: las dos pruebas, primero la PF } \\
\text { como base, luego la PPF para ilustrar. }\end{array}$ \\
\hline 18 & "La PF me parece más ilustrativa". \\
\hline 19 & La PPF (no aporta motivo). \\
\hline
\end{tabular}




\section{TRANSCRIPCIÓN DE LA ENTREVISTA I}

\section{Pn: P, Profesor; $n, n^{\circ}$ de orden}

\section{A1: Cristina García Puente}

\section{A2: Luis Fernández Urbón}

P1: La primera pregunta es ¿teníais ya alguna convicción de estos teoremas antes de la demostración? Es decir, ¿los conocíais? ¿habíais tenido contacto con alguno de ellos? ¿los habíais practicado?

A2: El teorema de Lagrange sí, pero el de unicidad del límite no sabía nada de él.

A1: Yo lo mismo, el de Lagrange sí, pero el de unicidad del límite no sabía nada ${ }^{19}$.

P2: Entonces, para este teorema del que decís que ya teníais algún conocimiento de él, ¿qué os han proporcionado las demostraciones que hemos realizado?

A2: Me ha quedado más claro lo que dice, o sea, para lo que vale el teorema del valor medio, porque antes realmente lo utilizaba y ya está, pero no sabía realmente para qué valía. Y el de la unicidad del límite pues conocerle, que no le conocía.

A1: Yo el teorema del valor medio lo conocía superficialmente y bueno con demostraciones y viéndolo más en profundidad te queda más claro todo ${ }^{20}$.

P3: De acuerdo, muy bien. Entonces, después de estudiar estas pruebas ¿podéis afirmar que para vosotros es absoluta y rigurosamente completa la verdad de estos teoremas? o ¿pensáis, por el contrario, que puede haber algún caso por ahí extraño que lo incumpla?

\footnotetext{
19 Información interesante para analizar las respuestas de los alumnos.

${ }^{20}$ Ambos alumnos reconocen que la demostración de los teoremas ayuda a comprender el enunciado.
} 
A2: Yo creo que sí. Además, al ser teorema, se tiene que cumplir siempre ${ }^{21}$. A mí por lo menos... yo creo que sí que se cumple.

P4: Estás convencido entonces. ¿Tú también, Cristina?

A1: Yo creo que sí. Si realmente es un teorema y se puede demostrar ${ }^{22}$ pues me lo creo.

P5: Habéis visto la demostración por los dos procedimientos y entonces quedáis plenamente convencidos.

A2: Sí.

P6: Vale, muy bien. Ahora, en cuanto a facilidad en la aplicación de prueba formal y preformal, al estudiar estas pruebas hemos aprendido dos procedimientos ¿no?, el formal y el preformal. Ambos son completamente rigurosos, tanto el formal como el preformal, pero, a la hora de hacer ejercicios o trabajar de forma habitual de manera práctica en matemáticas ¿cuál de los dos procedimientos pensáis que os ayudaría más? ¿el formal o el preformal?

A2: El preformal porque yo con números me aclaro de lo que estoy viendo. Tanta letra..., luego no sé ni de lo que estoy hablando. Pero si veo números y veo las cosas que salen, sé lo que es. Si veo un neperiano o veo una raíz, sé lo que es, pero si veo $f(b), f(a) \ldots$, al final, me acabo liando y no sé lo que es ${ }^{23}$.

P7: Cristina.

A1: Yo la preformal también, porque con la formal me lío un poco más en la utilización de logaritmos..., no sé y todo eso. Entonces sí que lo veo más claro con la pre-

\footnotetext{
${ }^{21}$ Respuesta académica, invocando a la autoridad de la Matemática.

22 Además de la justificación relativa a la autoridad de la Matemática, invoca a la demostración. Ambos alumnos expresan una opinión subjetiva "yo creo", y no una razón universal.

23 Destaca la importancia de la representación numérica frente a la simbólica y, en consecuencia, da mayor validez a la PPF.
} 
formal, pero en el teorema de unicidad del límite las dos las entendí perfectamente, tanto la formal como la preformal 24 .

P8: Vale, de acuerdo, muy bien. ¿En cuál de los dos habéis aportado más de vosotros a la hora de entenderlo? Es decir, visto el desarrollo de cada una de las pruebas, ¿cuál os ha supuesto más trabajo, más esfuerzo, os ha movido más a la hora de entenderlo? De las dos pruebas, ¿cuál?

A2: ¿En cuanto a formal y preformal o en cuanto a unicidad del límite y valor medio?

P9: De los dos, en cuanto a prueba.

A2: Pues la unicidad del límite más que el de Lagrange y en los dos la prueba formal es la que más me ha costado ${ }^{25}$.

$\mathbf{P}^{10}$ : La que más te ha costado entender ¿no?

A1: La prueba preformal se ve más clara ${ }^{26}$.

P11: ¿Cuál es vuestra impresión general sobre toda esta actividad? Aquí decid todo lo que os parezca con total libertad. ¿Os resultó positiva? ¿Os resultó un rollo? ¿Os resultó pesada? ¿Os ha hecho profundizar más en los teoremas? En fin, lo que queráis decir.

A2: A mí me ha hecho ver de una manera distinta cómo un mismo problema se puede ver desde la base teórica y digamos con un ejemplo, y darme cuenta que con un ejemplo lo entiendo mucho mejor que con la base teórica ${ }^{27}$.

\footnotetext{
${ }^{24}$ Su declaración coincide con las respuestas del cuestionario.

${ }^{25}$ La comprensión del teorema de la unicidad del límite, totalmente nuevo para Luis, ha presentado más dificultad; además la PF, al ser general y abstracta, le ha exigido un esfuerzo superior.

${ }^{26}$ A Cristina también le ha resultado menos dificultosa la comprensión de la PPF en ambos teoremas.

${ }^{27}$ Luis parece confundir la PPF con un ejemplo; no obstante expresa la ventaja pedagógica que aporta una situación concreta a lo teórico.
} 
A1: A mí me resultó positiva, porque si realmente no hubiéramos hecho esto ${ }^{28}$, yo llego al examen y no tendría ni idea. 0 sea, me pones esto en el examen y si no lo hubiera visto en clase no... no sabría sacar nada ${ }^{29}$.

P12: Muy bien. Si vosotros tuvierais que comunicar esto a alguien ¿cuál de los dos procedimientos, el formal o el preformal, escogeríais? Es decir, ¿cuál aportaría mayor ayuda a la hora de comunicarle esto a otra persona a quien vosotros se lo fuerais a explicar?

A2: ¡Hombre!... yo le explicaría los $\operatorname{dos}^{30}$, porque tienes que saber la base teórica para hacer el ejercicio con números. Pero vamos, la prueba preformal es la que yo creo que entendería más la gente.

P13: ¿Harías una conjunción de las dos...?

A2: Yo creo que explicaría primero la prueba formal y luego haría la preformal. Haría las dos, pero primero la formal y luego la preformal ${ }^{31}$.

P14: De acuerdo. ¿Tú, Cristina?

A1: Yo haría las dos, pero me basaría más en la preformal, porque me resulta más fácil. Luego todo depende también de cada persona, cómo comprenda las cosas y cómo se te quede mejor ${ }^{32}$.

P15: Muy bien. ¿Cuál pensáis que explica mejor los pasos de los dos procedimientos?

A2: La preformal.

\footnotetext{
${ }^{28}$ Confianza en la elección procedimental del profesor.

${ }^{29}$ Se fija en lo beneficioso de este tipo de explicaciones de cara a los exámenes.

30 Expresa de manera inequívoca la complementariedad.

31 Señala con claridad el orden de las pruebas: primero formal, luego preformal.

32 También señala la complementariedad, pero enfatiza la facilidad de la PPF, lo que parece sugerir que haría en primer lugar la PPF. El orden que se establezca en la asignatura no debe ser estricto, sino flexible.
} 
A1: La preformal ${ }^{33}$.

P16: Bueno, se nota que sois partidarios de la preformal. Bien, lo habéis comentado un poquito ya... De los dos procedimientos ¿en cuál entendéis mejor los pasos, tanto de un teorema como del otro? A lo mejor podéis distinguir también... quizá en un teorema lo habéis entendido mejor con una prueba que con otra...

A1: Pero eso es lo que decía antes. Por ejemplo, en Lagrange entiendo mejor la preformal, pero en unicidad del límite me resultaban las dos muy fáciles, entendí las dos perfectamente.

A2: Yo también. En Lagrange, a mí muchísimo más fácil la preformal pero en unicidad del límite las dos las he llegado a entender porque no hay mucho cambio de una a otra 34 .

P17: De acuerdo, muy bien. Y por último, el orden en el que hicimos las pruebas, lo tenéis aquí escrito, para el teorema del valor medio primero se realizó la formal y después la preformal sin embargo, para unicidad del límite al revés, primero preformal y luego formal, ¿pensáis que puede influir el orden en el que se realicen las pruebas, primero formal y luego preformal o primero preformal y luego formal a la hora de entender mejor, de comprender mejor el teorema?

A2: Yo creo que sí, porque hay gente que le va a entrar mucho mejor si directamente se lo cuentas con la preformal que si se lo pones con la formal. A lo mejor con la formal no se empieza a enterar desde el principio y ya lo deja, pero con la preformal ve lo que está haciendo, o sea, va a ver el resultado real de lo que está haciendo en los dos $\operatorname{casos}^{35}$.

P18: ¿Tú cuál pondrías entonces primero?

\footnotetext{
33 Creen que los pasos de la prueba están mejor explicados en la PPF.

34 Ambos ven más fácil el proceso en la PPF y, en el teorema de Lagrange, también en la comprensión de los pasos de dicho proceso.

35 Cambio de opinión, quizás influenciado por la entrevista, pero que está en la misma línea de la respuesta a la $\mathrm{P}^{13}$, que él mismo emitió.
} 
A2: La preformal ${ }^{36 .}$

P19: La preformal y luego dirías: ahora el razonamiento que abarca la situación más general posible es la formal ¿no?

A2: (Asiente).

P20: ¿Tú, Cristina?

A1: Yo la preformal porque, como realmente yo lo comprendí mejor así, con la preformal, pues tendría una visión más clara de las cosas y luego, pues explicaría un poco más en general la formal ${ }^{37}$.

$\mathbf{P}^{21}$ : Es decir, en ese orden, primero preformal y después formal ¿no?

A1: (Asiente).

$\mathbf{P}^{22}$ : Muchas gracias.

\title{
TRANSCRIPCIÓN DE LA ENTREVISTA II
}

\author{
Pn: P, Profesor; n, no de orden
}

A1: Pablo Rey Núñez

\section{A2: Álvaro Lázaro Gallego}

P1: Comentad lo que queráis.

A1: Tomar los puntos $\sqrt{2}, \ln \sqrt{2}$, y hacerlo..., es más fácil a la vista lo de las funciones, lo que has visto toda la vida. Luego es aplicarlo aquí.

\footnotetext{
36 De lo más fácil o intuitivo, a lo menos fácil; se evitaría así algún posible desaliento.

37 Apela a su experiencia personal: ha visto mayor claridad en la PPF. Ambos establecen el mismo orden: primero la PPF y después la PF.
} 
$\mathbf{P}^{2}$ : Digamos que con unos puntos más sencillos..., eso es lo que me parece que querías decir.

A1: Yo no sé si con otros puntos quedaría más sencillo.

P3: Quizá sí, incluso se alargaba un poco más la demostración.

A1: Claro, también hay que encontrar unos puntos con los que quede simple la demostración.

A2: Esto es lo típico, que yo lo he usado en cursos anteriores. Cuando tienes un teorema que a lo mejor no entiendes muy bien en la prueba formal, pues luego te pones casos concretos y así lo visualizas mejor y creo que sí te ayuda.

P4: Quizá los números podían escogerse mejor ¿no?

A1: Yo creo que sí, porque aquí, en la prueba formal del teorema del valor medio es bastante... como es lo que se machaca en clase se reconoce nada más ver esta parte pero luego si te das cuenta aquí estamos haciendo lo mismo (se refiere a la prueba preformal). Yo simplemente digo que a lo mejor esto del resultado de que sea una razón irracional con logaritmo en el divisor y $\operatorname{tal}^{38} \ldots$

P5: Esa razón irracional y que puedes determinar exactamente el punto, ¿no?

A1: Para el otro caso (se refiere a la prueba formal del teorema del valor medio, donde no aparecen los "números raros" $\sqrt{2}, \ln \sqrt{2}$, ) sí que me parecía que era mucho más sencillo que tratar con cosas tan abstractas.

A2: Aquí (en el teorema de la unicidad del límite) en la prueba preformal se ve más claro que una función en un punto sólo puede tener, o sea, el límite siempre tiene el mismo valor. Se intuye antes de hacer la... el teorema ya se intuye, vamos.

\footnotetext{
38 Alude a la dificultad asociada a la tipología numérica.
} 
A1: Yo, si se lo enseñaras a los alumnos este año, yo lo dividiría en dos días ${ }^{39}$. Un día con esto, con el teorema de unicidad del límite, las dos pruebas, y otro día, con el intervalo de una semana o tal, porque en un día las cuatro... las dos primeras las puedes seguir pero ya las siguientes es muy...

P6: Pues muy bien la sugerencia, Pablo ${ }^{40}$. De acuerdo. Bueno, seguimos este cuestionario, ya. Muy bien todo lo que me habéis comentado. ¿Teníais ya alguna convicción de estos teoremas antes de la demostración? Es decir ¿los conocíais ya? ¿habíais visto sus demostraciones? ¿estabais convencidos de estos resultados antes de aquel día en el que hicimos esta experiencia?

A2: Yo sí que lo había estudiado, sobre todo cuando estuve en la Universidad de Valladolid, en la asignatura de Matemáticas III, creo que era. Estos teoremas sí que los tenía bastante claros, pero bueno, sí viene mejor repasarlos con estos méto$\operatorname{dos}^{41}$.

A1: Yo también, pero a lo que tú te refieres con prueba preformal... ellos hacen un ejemplo y ya está42.

P7: Entonces, como ya los conocíais, ¿estas demostraciones os han proporcionado algo nuevo? ¿Habéis profundizado en vuestro conocimiento de ellos? ¿Os habéis quedado igual?

A2: Sí, es una forma de tener el concepto bastante más claro y a la hora de recordarlo, sobre todo, es más fácil en casos concretos. Traes la imagen mental del teorema y a la hora de plasmarlo en papel te ayuda ${ }^{43}$.

\footnotetext{
${ }^{39}$ Organización de la actividad docente.

${ }^{40}$ Comienza esta entrevista con un diálogo de espontánea naturalidad en un clima de confianza entre profesor y entrevistados.

${ }^{41}$ Efectivamente las respuestas de este alumno fueron correctas.

${ }^{42}$ Confunde ejemplos de aplicación del teorema con prueba preformal.

${ }^{43}$ Ya conocía los teoremas; esta demostración le ha dado bastante más claridad y facilidad para recordar ("imagen mental"), en referencia sin duda a la PPF.
} 
A1: Yo sí que pienso que las pruebas preformales ayudan al alumno a entenderlo, porque es muy difícil de entender: épsilon ${ }^{44}$, o sea... muchas de las cosas... como no ves una cosa concreta... es muy enredado. Simplemente el ponerle una gráfica con puntos concretos ya te hace más que ver letras en los ejes ${ }^{45}$.

P8: Ese a que aparece ahí, ese $L_{1}, L_{2} \ldots$, ¡claro!

A2: El intervalo éste del épsilon...

$\mathbf{P}^{9}$ : Después del estudio de estas pruebas, ¿podéis afirmar que es absoluta y rigurosamente completa la verdad de estos teoremas? ¿No habrá algún caso que lo incumple?

A2: ¡No, hombre! Dado que se estudian los dos tipos de pruebas, la preformal y la formal, pues la formal te asegura que eso se cumple en todos los casos, la preformal siempre se te puede escapar algún caso, pues por ejemplo, los números negativos, puede que no se cumpla, pero vamos, la formal es completa ${ }^{46}$.

P10: La formal es una prueba que abarca todo tipo de situación ¿no? La preformal la veis entonces como algo concreto pero no sé si estáis convencidos de que es una prueba absolutamente rigurosa también, es decir, aunque se hace en una situación concreta, ¿eso os convence de que no puede haber ningún caso que lo incumpla?

A1: ¡Hombre!, si nos dices que es para todo, pues nos lo creemos ${ }^{47}$.

P11: Eso es lo que yo quería que precisarais un poco.

A1: Yo no tengo la certeza porque...

\footnotetext{
${ }^{44}$ Alude a la dificultad que entraña el simbolismo formal.

45 Señala la concreción de las pruebas preformales como ayuda para la comprensión de los teoremas.

46 Distinción entre razonamiento inductivo particular y universal deductivo.

47 Autoridad del profesor.
} 
P12: Se hace con unos números concretos, con una función concreta, pero el razonamiento... ¿qué pasa con él? Sigue los pasos del otro ¿no?

A1: No es lo mismo demostrar que existe un caso que para todo. Entonces, eso es la única... pero vamos, yo creo que para demostrar que para todo, está la prueba formal.

P13: Pero desde ese caso concreto que tú ahí ves, visualizas con números concretos, con funciones concretas ¿tú serías capaz de hacer una prueba formal que abarcase todos siguiendo esa misma línea que ahí se dice?

A1: ¡Hombre!, tomando valores sí. Simplemente sería sustituir esos...

P14: Efectivamente, ahí se ve en una situación concreta. Entonces eso no es una prueba que abarque todos los casos pero no quiere decir que no sea una prueba rigurosa, es decir, es una línea de razonamiento que luego se puede poner de manera completamente formal en una prueba que abarque todas las situaciones ¿de acuerdo?

A1: Diría yo que la prueba formal tiene unas ventajas, que puede ser que se ve fácilmente que es para todos los casos y tendrá la dificultad de entenderla. Y la prueba preformal tiene la ventaja de que es más fácil entenderla, pero que tiene la dificultad de si quiero demostrarlo para todos los casos tendré que transformar$\mathrm{la}^{48}$.

P15: Luego tienes tú que transformarla en una prueba formal. Bueno, en cuanto a la facilidad de aplicación. Habéis visto dos procedimientos, el formal y el preformal. A la hora de hacer ejercicios, de trabajar de manera práctica, ¿cuál de los dos procedimientos pensáis que os ayudaría más, el formal o el preformal?

\footnotetext{
48 Mantiene un diálogo interesante con el profesor sobre características de ambos tipos de prueba, que el mismo alumno resume indicando facilidades y dificultades de cada uno de ellos y aceptando que los dos tipos de prueba son absolutamente rigurosos.
} 
A1: El preformal, sin duda ${ }^{49}$.

A2: Yo también creo que a la hora de hacer un ejercicio, con la formal puedes tener dificultades a la hora de trasladarla a un ejercicio, y la preformal ya tienes un ejemplo que es casi como un ejercicio modelo, y sólo sería trasladarlo cambiando los valores ${ }^{50}$.

P16: Bien. ¿En cuál de los dos procedimientos habéis aportado más en cuanto a trabajo, en cuanto a esfuerzo que os haya supuesto, en cuanto a ingenio para desentrañar la demostración? ¿En cuál de ellos? Podéis distinguir también entre un teorema y otro.

A2: Pues la formal, si la estudias directamente requiere más atención y tomarlo con más cuidado, vamos, porque hay que hacerse la imagen de cada valor, de cada intervalo, de cada variable...

P17: ¿Supone más trabajo, en tu opinión?

A2: Supone más trabajo entenderlo.

A1: Yo es que creo... primero diferenciaría los teoremas porque el teorema del valor medio es algo que la gente tiene más conocido que el de unicidad del límite y en la prueba formal del teorema del valor medio yo creo que si vas a la Universidad de Valladolid, le pones esto a un alumno y sabe lo que es, a simple vista, más o menos, pero tanto si le das la formal como la preformal. Y como a mí el otro teorema me resulta un poco más distinto, la prueba formal me parece ya meterme... Si fuera otro teorema, un tercer teorema que no conociera, la prueba formal me sería mucho más difícil de asimilar ${ }^{51 .}$

\footnotetext{
${ }^{49}$ Consecuente con su respuesta anterior, expresa su total convicción a favor de la PPF a la hora de las aplicaciones.

${ }^{50}$ Asimismo se muestra partidario de la PPF a la hora de hacer ejercicios; ve en la PPF un ejemplo o ejercicio modelo al que referirse.

${ }^{51}$ Afirma, coincidiendo con Álvaro, que la PF es la que le supondría un mayor esfuerzo de seguimiento y asimilación.
} 


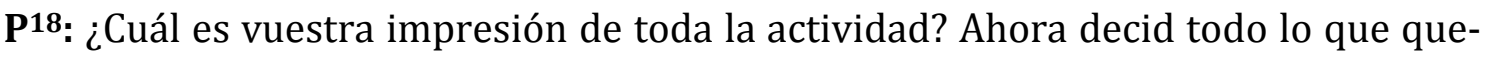
ráis.

A2: Yo creo que las dos pruebas se complementan, porque tú puedes tener el concepto claro o creer que tienes el concepto claro, y luego realizar la otra prueba y te das cuenta de que hay alguna cosa que no estaba tan clara. Entonces siempre viene bien hacer las dos. Puede llevar algo más de tiempo, pero los conceptos te quedan más claros y los teoremas se recuerdan mejor ${ }^{52}$.

P19: Tú crees que son complementarias en ese sentido y que conviene hacer las dos.

A1: Mi opinión. Creo que la asignatura... esto se lo das al... no te digo un porcentaje, pero más de la mitad de la clase le das cualquier teorema y cualquier prueba y si fuera sincero al cien por cien diría ¿qué es esto? ¿sabes? Porque digamos que las matemáticas a este nivel son algo difícil. Entonces la gente no sabe cómo afrontar esta asignatura y cuando no es el problema que sea formal o preformal yo intentaría explicar una demostración a nivel "subnormal".

P20: Muy elemental.

A1: Sí, porque me parece muy... o sea, cuando ya lo has trabajado y lo has estudiado puedes echar un vistazo y saber de qué va, pero cuando no has visto nada de eso o se te ha olvidado, pues no sabes por dónde cogerlo. Y hay gente que las matemáticas las toma por eso como decir, bueno, a ver si el día del examen hay suerte...

P21: Bueno, la gente, la gente... ¡Tú!

A1: Yo hablo con la gente cuando sale de clase y...

52 Decididamente apuesta por la complementariedad de los dos tipos de prueba, para lograr más claridad y recordar los resultados mejor. 
P22: Y esa es la impresión que tú recoges también.

A1: Muchas veces cuando sales de clase dices: ¡jobar!, es que por no preguntar qué es todo eso... Digamos que no es una cosa que sea formal o preformal. Está claro que es más fácil de entender la prueba preformal ${ }^{53}$, pero siempre que se haya estudiado. No puedes decirle: a ver, explícamelo. Yo tenía una profesora en la Universidad de Valladolid que llegaba, te hacía una demostración formal y te preguntaba ¿lo has entendido? Y llegaba el chico y decía: sí. Vale, pues sal a la pizarra y escríbelo.

$\mathbf{P}^{23}$ : Y a reproducirlo.

A1: Claro. Entonces el chico no sabía nada. Lo volvía a escribir. Le volvía a preguntar ¿lo has entendido? Sí. Sal a la pizarra. Así un día estuvo. Y el chico no aprendió nada.

P24: Bueno, muy bien. Si tuvierais que comunicar esto a alguien ¿cuál de los dos procedimientos utilizaríais? 0 ¿los utilizaríais combinadamente? 0 ¿utilizaríais para un teorema solamente uno y no el otro? En fin, ¿cómo lo haríais si tuvierais que comunicar esto a alguien?

A2: Yo creo que en estos teoremas concretos pues la verdad que el teorema de unicidad del límite es más fácil en la prueba preformal. Queda bastante más claro. Pero un teorema de un modo general depende de la dificultad del teorema y del nivel de matemáticas que tenga la persona ${ }^{54}$.

P25: A la que se lo quieras explicar.

\footnotetext{
${ }^{53}$ Actúa como portavoz de una sensación real o no y al final se decanta por la mayor facilidad de la prueba preformal.

${ }^{54} \mathrm{~A}$ la hora de comunicar los resultados a alguien, se inclina por la PPF en el teorema de la unicidad del límite; en general, considera los lógicos criterios de sopesar la dificultad del teorema y la capacidad del destinatario.
} 
A2: Si es una persona que ya ha estudiado matemáticas, le ponemos una prueba formal y lo puede entender rápidamente o más rápidamente que alguien que no lo sepa.

P26: Que está más iniciada digamos ¿no?

A2: Sólo... Hay muchos símbolos que mucha gente no sabe lo que significan. Por ejemplo, el pertenece o para todo o las intersecciones ${ }^{55}$.

A1: Yo, con alguien que tuviera conocimientos de matemáticas, lo que haría sería poner, por ejemplo: aquí teorema, la sección ésta, el teorema, explicaría la preformal, le explicaría que es para todos los casos y si quieren más información, que hagan la prueba formal. Pero digamos que el teorema y la prueba preformal, sí que lo pondría y luego ya... 0 si sobra tiempo, explicar la prueba formal. Pero digamos que pasar directamente... primero explicar el teorema y pasar a la prueba formal puede ser un poco de golpe. Entonces, por pasos ${ }^{56}$.

P27: Está claro. ¿Cuál de los dos procedimientos pensáis en general que explica mejor cada paso? ¿Cómo pasar de una situación a otra? ¿Cómo ir encadenando, entrelazando las piezas del puzle? ¿El formal o el preformal?

A1: Como en la prueba preformal son operaciones de sustituir o tal, se puede entender más fácil el paso de una operación a otra, simplemente explicando pues aquí he pasado esto dentro del divisor o he hecho la derivada. Con lo otro, prueba formal, a lo mejor si tuviera que hacer un caso real diría: bueno, ahora de aquí pasa a aquí y ahora ¿qué hago?, porque luego en la realidad es aplicarlo tú en un caso. Porque en el examen te toca demostrar la prueba formal y, bueno, será eso, pero luego en la realidad es utilizar números.

${ }^{55}$ Cree que el simbolismo es un obstáculo para seguir las pruebas formales.

${ }^{56}$ En este punto se muestra muy claro: primero la PPF; sería brusco empezar con la PF. 
A2: Lo que yo decía antes, que a la hora de hacer un ejercicio, la prueba preformal, como modelo para... es bastante más claro que el transformar la prueba formal a un ejercicio, va a ser más fácil con la prueba preformal ${ }^{57}$.

$\mathbf{P}^{28}$ : ¿En cuál de los dos procedimientos entiendes mejor los pasos?

A2: En general yo creo que con la preformal se entiende mejor, en general. Luego, en casos particulares... En general, la prueba preformal.

A1: Yo la preformal.

P29: Para ir terminando ya, el orden en el que hicimos las pruebas, recordad que en el teorema del valor medio primero hicimos la prueba formal y después la preformal y en el otro lo hicimos al revés, ¿creéis que puede influir a la hora de comprender mejor el resultado el orden en el que se hagan las pruebas?

A2: Yo creo, que como es más fácil de entender la prueba preformal, se debería hacer primero para tener una idea general del teorema. Luego, para abstraerla, pues llevarla ya a la prueba formal y acabar de comprender el teorema.

A1: Yo pienso lo mismo58.

P30: Coincidís. De acuerdo. Muchas gracias.

\footnotetext{
57 Facilidad de la PPF a la hora de entender cómo se aplica el teorema.

58 En estas últimas respuestas se muestran coherentes con sus intervenciones anteriores, y optan por la PPF; consideran que el orden adecuado es: en primer lugar PPF y después PF.
} 


\section{ANEXO VIII (2009)}

Aquí se reproducen íntegramente las respuestas que emitieron los alumnos del grupo a la cuestión primera.

Cuestión 1: Explica cómo has entendido cada uno de los teoremas con cada procedimiento. Indica en cada caso claramente el porqué.

El objetivo de esta cuestión es analizar la comprensión de los alumnos de las demostraciones matemáticas sobre las pruebas preformales en contraste con las pruebas formales. Los números se refieren a los alumnos (20 en total). En adelante, PF designa prueba formal y PPF prueba preformal.

\section{Teorema 1. Valor Medio (Lagrange). Prueba Formal (1ํㅜ}

1: La PF me ha parecido más claro que el PPF. En mi caso el dibujo me ha ayudado bastante.

2: Me ha sido muy útil el ver la PF antes que la PPF, pues me ha dado una gran base para entender mejor los pasos de la PPF.

3: Lo he entendido mejor con la PPF.

4: Me ha parecido bastante ilustrativo las gráficas para entender el teorema.

5: La PF requiere algo más tiempo para su entendimiento.

6: Me ha resultado más fácil de entender habiendo visto las demostraciones primero en la PF. 
7: Lo he entendido mejor con la PF porque lo ha demostrado para un caso genérico.

8: En la PF la comprensión de los conceptos se realiza de una forma más "abstracta".

9: Con la PF he entendido las cosas de una forma un poco abstracta.

10: Resulta muy abstracto.

11: Se ven peor los pasos a seguir.

12: Menos sencilla que la PPF.

13: No es mejor ni peor, simplemente prefiero la otra prueba (la PPF).

14: La PF me ha parecido más sencilla de entender, ya que llega a la solución de una manera más fácil.

15: Lo he entendido mejor con la PF, ya que es general para cualquier valor.

16: Lo he entendido de la misma forma con la PF que con la PPF; si tuviera que aplicarlo, recordaría la PF.

17: Ha quedado bastante claro al hacer primero la PF y después la PPF.

18: La idea del teorema se entiende bien por el ejemplo gráfico.

19: La PF toma valores genéricos, resulta menos fácil. 
20: La PF considero que es algo más sencilla a la hora de exponer los conceptos.

\section{Teorema 1. Valor Medio (Lagrange). Prueba Preformal (2º)}

1: El procedimiento ha sido más complicado, aunque el ejemplo utilizado del logaritmo neperiano es sencillo de aprender.

2: El orden de la PF primero me ha servido de base para entender mejor los pasos de la PPF.

3: El teorema le he comprendido mejor con la PPF, suelo entender mejor las cosas cuando se explican ejemplificadas.

4: Se ve muy bien la utilización de la derivada.

5: Este teorema se entiende mucho mejor con la PPF mediante la función logarítmica (logaritmo neperiano); es más clara y explícita.

6: Con la PPF, una vez visto primero la PF, la secuencia de los pasos seguidos era más evidente.

7: (No aporta nada en relación con la PPF).

8: PPF: queda ejemplificada la utilidad y resulta más claro.

9: Prefiero la PPF ya que se basa en un caso concreto, y a partir de ahí llegamos a la PF.

10: Hasta no hacer la PPF no lo he entendido, resulta muy abstracto. 
11: Me gusta la PPF, pues veo mejor los pasos a seguir para la resolución final del problema.

12: Me ha parecido más sencilla de entender la PPF al hacerlo con ejemplos prácticos, es decir, al conocer los números lo he visto más claro.

13: En este caso prefiero la PPF debido a que me resulta más sencilla.

14: (No aporta nada relativo a la PPF).

15: La PPF se ha realizado con la función logarítmica que no la entiendo bien.

16: El desarrollo se ve más claro con la PPF porque se hace con un caso concreto.

17: Con la PPF (después de la PF) se ha reforzado lo explicado anteriormente y se ha comprendido mucho mejor aún.

18: Con la PPF se entiende mejor ya que se cogen dos partes concretas y de manera algebraica se ve mejor que con la demostración formal (PF).

19: En la PPF se toman valores concretos, siendo así más fácil de comprender.

20: La PPF tiene la idea esencial, pero no me es suficiente para una comprensión bastante buena.

\section{Teorema 2. Unicidad del límite. Prueba Preformal (1º)}

1: Me ha resultado casi igual de complicada que la PF. 
2: Me ha costado seguir los pasos.

3: Siempre prefiero las explicaciones ejemplificadas.

4: Ha sido bastante sencillo. La utilización de los gráficos ayuda a entenderlo mejor.

5: (Nada aporta en relación a la PPF).

6: He entendido mejor, en este caso, la PPF.

7: En este caso prefiero la PPF, ya que al explicarlo particularmente lo he entendido mejor que de forma genérica (PF).

8: En este caso la explicación resulta abstracta tanto con la PF como con la PPF; sin embargo con la PPF, al tener casos concretos, se puede ver mejor la utilidad.

9: La PPF es más intuitiva y más fácil de ver. Me ha aclarado más la PPF.

10: Al ver primero la PPF me ha ayudado en la comprensión del teorema.

11: Me gusta la PPF por lo mismo que en el teorema 1 (veo mejor los pasos a seguir).

12: He entendido mejor la PPF; al hacerse primero, lo he entendido más rápidamente.

13: Prefiero la PPF por la misma razón (me resulta más sencilla). 
14: Me ha parecido más sencilla la PPF.

15: Lo he entendido mejor con la PPF, se ve más claro que cualquier función en un punto concreto no puede valer dos valores distintos.

16: Lo he entendido mucho mejor con la PPF, pues lo recuerdo mejor con un ejemplo que con su forma general.

17: Al empezar con la PPF, he llegado a entender algún concepto al continuar con la PF. Gráficamente me ha ayudado a entender ciertas cosas.

18: Con esta PPF no entendí el teorema.

19: No se decanta por ninguna preferencia de prueba.

20: Prefiero la PPF. He visto la explicación mucho más clara y creo que es fácil de comprender y recordarlo.

\section{Teorema 2. Unicidad del límite. Prueba Formal (2º)}

1: Me ha resultado complicada, ya que en el cálculo de límites puedes fallar en algún número.

2: Al final no me ha llegado a quedar nada claro.

3: Siempre prefiero las explicaciones ejemplificadas (la PPF).

4: La utilización de los gráficos es muy igual. Ayuda a entenderlo mejor.

5: Con la PF lo he visto como más práctico, más ligero para el entendimiento y algo más dinámico, aunque mínimamente. 
6: (Nada aporta en relación con esta prueba, PF).

7: (Nada aporta sobre la PF).

8: PF: es una demostración más técnica y que, a simple vista es difícil de captar la idea.

9: (Nada aporta sobre esta PF).

10: (Nada aporta sobre la PF).

11: (Nada aporta sobre la PF).

12: Al hacerse primero la PPF, la PF la he entendido más rápidamente.

13: (Nada dice de la PF).

14: (Nada aporta en relación con esta PF).

15: (Nada aporta sobre esta PF).

16: (Nada aporta sobre esta PF).

17: He llegado a entender algún concepto con la PF. Gráficamente me ha ayudado a entender ciertas cosas.

18: Gráficamente se entiende y visualiza mejor la PF.

19: No se decanta por ninguna preferencia de prueba. 
20: La PF me cuesta algo más (que la PPF).

Las respuestas a la tercera cuestión fueron las siguientes:

\section{CUÁL DE LAS DOS FORMAS TE CONVENCE MÁS DE LA VERACIDAD DEL TEOREMA}

\begin{tabular}{|c|c|}
\hline 1 & La PPF me convence más en los dos teoremas. \\
\hline 2 & "Verlo aplicado en la PPF me da una mejor idea de su veracidad". \\
\hline 3 & La PPF. \\
\hline 4 & "La PPF porque es más entendible que la PF". \\
\hline 5 & Dependiendo del teorema, me convence más una forma u otra. \\
\hline 6 & En el teorema 1 la PF, en el teorema 2 la PPF. \\
\hline 7 & "La PF puesto que es una demostración matemática seria para todos los casos". \\
\hline 8 & La PPF, se ve más clara su utilidad y veracidad. \\
\hline 9 & La PF, ya que la PPF no es general. \\
\hline 10 & La PPF, la aplicación resuelve dudas. \\
\hline 11 & La PPF, pues al ver que se cumple, puedes hacerlo para todos los casos. \\
\hline 12 & La PPF, al verlo aplicado en concreto. \\
\hline 13 & Ambas me convencen, pero escogería la PPF ya que me resulta más fácil de entender. \\
\hline 14 & Según el teorema, una u otra. \\
\hline 15 & La PF. (No aporta motivo). \\
\hline 16 & $\begin{array}{l}\text { "Me convence más la PPF, pues lo veo más claro, lo entiendo mejor y por tanto me lo } \\
\text { creo más". }\end{array}$ \\
\hline 17 & Para el teorema de Lagrange, la PF; para el de la unicidad, ambas. \\
\hline 18 & "La PF, aunque haya fórmulas "sin números", prefiero verlo gráficamente". \\
\hline 19 & La PPF, al verlo concreto, se entiende más claro. \\
\hline 20 & En el teorema 1 , la PF, en el teorema 2 , me han quedado más dudas. \\
\hline & $\begin{array}{c}\text { bla VIII/1. Respuestas de los alumnos sobre el convencimiento de la veracidad } \\
\text { del teorema }\end{array}$ \\
\hline
\end{tabular}




\section{CUÁL DE LAS DOS PRUEBAS PUEDE AYUDAR MÁS A ENTENDER EL SIGNIFICADO DEL TEOREMA}

\begin{tabular}{|c|l|}
\hline $\mathbf{1}$ & En el 1er. caso la PF; en el 2 o la PPF, al usar valores reales. \\
\hline $\mathbf{2}$ & "Creo que es importante ver las dos para entenderlo mejor". \\
\hline $\mathbf{3}$ & La PPF (no aporta motivo). \\
\hline $\mathbf{4}$ & "La PPF porque así va a ser cuando nos enfrentemos a un caso práctico". \\
\hline $\mathbf{5}$ & Una u otra dependiendo del teorema (no especifica más). \\
\hline $\mathbf{6}$ & En general, la PPF; al verlo en un caso práctico aclara la explicación. \\
\hline $\mathbf{7}$ & La PPF se entiende mejor a la hora de explicar el teorema. \\
\hline $\mathbf{8}$ & La PPF nos ayuda más que viéndolo en función de parámetros (PF). \\
\hline $\mathbf{9}$ & Sin duda la PPF, un ejemplo me ayuda más a asimilar el teorema. \\
\hline $\mathbf{1 0}$ & "La PPF: simplifica el proceso". \\
\hline $\mathbf{1 1}$ & La PPF, con un ejemplo te ayuda a entender el teorema. \\
\hline $\mathbf{1 2}$ & La PPF: al verlo aplicado en un caso concreto. \\
\hline $\mathbf{1 3}$ & La PPF (no aporta motivo). \\
\hline $\mathbf{1 4}$ & Ambas formas (no aporta motivo). \\
\hline $\mathbf{1 5}$ & En cada teorema una de las dos (sin mayor concreción). \\
\hline $\mathbf{1 6}$ & La PPF, pues al hacerse con un ejemplo concreto se entiende más claramente. \\
\hline $\mathbf{1 7}$ & "La PF: se asemeja más a la definición". \\
\hline $\mathbf{1 8}$ & La PPF ya que trata un caso concreto. \\
\hline $\mathbf{1 9}$ & "La PF da una visión más amplia, más genérica". \\
\hline $\mathbf{2 0}$ & La PF ya que expone todos los conceptos con la idea clara. \\
\hline \multicolumn{3}{|c|}{ Tabla VIII/2. Respuestas de los alumnos sobre la ayuda al entendimiento del } \\
\end{tabular}




\section{CUÁL DE LAS DOS PRUEBAS PUEDE AYUDAR MÁS A MEMORIZAR EL TEOREMA}

\begin{tabular}{|c|l|}
\hline $\mathbf{1}$ & En el teorema 1, la PPF: la gráfica es más fácil. En el teorema 2, difíciles las dos. \\
\hline $\mathbf{2}$ & La PF es más fácil de memorizar. \\
\hline $\mathbf{3}$ & La PPF (no aporta motivo). \\
\hline $\mathbf{4}$ & "La PPF, utilizándolo varias veces se te queda bien". \\
\hline $\mathbf{5}$ & Creo que la PPF (no aporta motivo). \\
\hline $\mathbf{6}$ & $\begin{array}{l}\text { "Creo que puede ayudar más la PF porque es una forma genérica para aplicar a cual- } \\
\text { quier teorema". }\end{array}$ \\
\hline $\mathbf{7}$ & La PF, ya que es una fórmula con unos pasos metódicos. \\
\hline $\mathbf{8}$ & Resulta más útil la PF, pues es genérica y no dará errores. \\
\hline $\mathbf{9}$ & La PPF, el ejemplo ayuda más a asimilar el teorema. \\
\hline $\mathbf{1 0}$ & La PPF: se aprende el método. \\
\hline $\mathbf{1 1}$ & "La PPF me ayuda a memorizar los pasos a seguir, pero la PF me ayuda a aprender las \\
& fórmulas utilizadas". \\
\hline $\mathbf{1 2}$ & La PF que es más memorizable para aplicarla a otros casos. \\
\hline $\mathbf{1 3}$ & La PF (no aporta motivo). \\
\hline $\mathbf{1 4}$ & La PF es más adecuada para memorizar los teoremas. \\
\hline $\mathbf{1 5}$ & La PF (sin aportar motivo). \\
\hline $\mathbf{1 6}$ & En el teorema del valor medio, la PF; en el de unicidad del límite, la PPF. \\
\hline $\mathbf{1 7}$ & "En ambos la PF porque resulta más genérica para aplicarla en cualquier caso". \\
\hline $\mathbf{1 8}$ & La PF: trata un método más general y vale para cualquier caso. \\
\hline $\mathbf{1 9}$ & La PPF porque creo más fácil de recordar un ejemplo concreto. \\
\hline $\mathbf{2 0}$ & "La PPF. Tiene menor cantidad de conceptos y para memorizar es mejor". \\
\hline \multicolumn{2}{|}{ Tabla VilI/3. Respuestas de los alumnos sobre la ayuda a la memorización } \\
& \\
\hline &
\end{tabular}




\section{CUÁL DE LAS DOS PRUEBAS ES MÁS ÚTIL PARA ILUSTRAR CÓMO SE APLICA EL TEOREMA}

\begin{tabular}{|c|c|}
\hline 1 & La PPF en el teorema $1^{\circ}$, el dibujo del logaritmo es más sencillo; en el $2^{\circ}$, las dos. \\
\hline 2 & La PPF (lo más útil la corrección de ejercicios). \\
\hline 3 & La PPF (no aporta motivo) \\
\hline 4 & 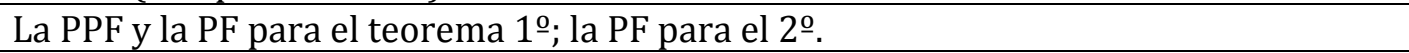 \\
\hline 5 & La PPF ilustra mejor la aplicación. \\
\hline 6 & Depende de los casos (no aclara más). \\
\hline 7 & $\begin{array}{l}\text { "La PPF es más útil porque ilustra con un ejemplo en particular, lo cual al llevarlo a la } \\
\text { práctica se aplica mejor". }\end{array}$ \\
\hline 8 & Para mostrar aplicaciones, lo mejor es una PPF, pues... el resultado es más claro. \\
\hline 9 & La PPF, los gráficos son más útiles para ilustrar el teorema. \\
\hline 10 & La PPF, nada mejor que un ejemplo. \\
\hline 11 & La PPF, pues se aprecia mejor cómo se aplica el teorema. \\
\hline 12 & La PPF (no aporta motivo). \\
\hline 13 & En mi opinión la PPF. Realmente ambas son igual de útiles. \\
\hline 14 & "La PPF es más ordenada para aplicar los teoremas". \\
\hline 15 & La PPF (sin aportar motivo). \\
\hline 16 & La PPF en ambos teoremas. \\
\hline 17 & A la hora de ilustrar, la PPF, pero habiendo explicado antes la PF. \\
\hline 18 & $\begin{array}{l}\text { "La PF, porque coges las partes que te parezca oportunas y dibujas la gráfica, siempre } \\
\text { que cumpla las condiciones lo comparas con el teorema". }\end{array}$ \\
\hline 19 & "La PPF es más útil por ver cómo se aplicaría en un caso concreto". \\
\hline 20 & "Prefiero la PPF, para enseñar cómo es de manera rápida" \\
\hline
\end{tabular}




\section{TRANSCRIPCIÓN DE LA ENTREVISTA I}

\section{Pn: P, Profesor; $n, n^{\circ}$ de orden}

\section{A1: David de la Calle Galindo}

\section{A2: Javier González Benavides}

P1: La primera pregunta es ¿teníais ya alguna convicción de estos teoremas antes de la demostración? Es decir, ¿los conocíais? ¿habíais tenido contacto con alguno de ellos?

A2: Uno de ellos sí que lo habíamos visto anteriormente en clase. Yo creo que nos resultó más fácil ver otra vez la demostración al haberla repetido, se te quedan mejor los conceptos, está claro.

$\mathbf{P}^{2}: ¿ Y$ en cursos anteriores?

A2: Yo es que hace mucho que empecé la carrera, entonces tengo mis conocimientos de matemáticas muy guardados dentro de la cabeza.

A1: Yo los conocía. El de unicidad del límite sabía lo que es, pero no sabía que había un teorema y el teorema de Lagrange también lo conocía ${ }^{59}$.

P3: Bueno, pues entonces las demostraciones que hemos hecho ¿os han proporcionado algo nuevo? ¿Qué os han proporcionado?

A2: Recordar, porque yo lo tengo muy olvidado todo. Por lo menos ver la demostración y el proceso completo te ayuda a recordarlo mejor que el que te pongan la demostración y hacer tú solo el ejercicio.

A1: Recordarlo de forma más clara.

\footnotetext{
${ }^{59}$ Ambos alumnos tienen alguna idea de los teoremas, pero sin claridad ni convicción.
} 
P4: Entonces, una vez que habéis visto estas demostraciones, las cuatro, las versiones formal y preformal, ¿podéis afirmar que es absoluta y rigurosamente completa la verdad de estos teoremas? ¿No habrá algún caso que lo incumple?

A2: Yo creo que sí. Tampoco tengo ciertos conocimientos matemáticos como para poder afirmar por mí mismo que son verdad, pero yo diría que sí.

A1: Yo también estoy de acuerdo 60.

P5: 0 sea, que no hay ningún caso extraño que incumpla estas cosas. En cuanto a la facilidad en la aplicación de la prueba formal y la preformal, al estudiar estas pruebas hemos aprendido dos procedimientos, el formal y el preformal. Ambos son completamente rigurosos, pero, a la hora de hacer ejercicios o trabajar de forma habitual de manera práctica en matemáticas ¿cuál de los dos procedimientos pensáis que os ayudaría más? ¿el formal o el preformal?

A2: A mí personalmente, el preformal, porque viendo antes un ejemplo de cómo es el desarrollo de un ejercicio, al ver luego la demostración, el porqué de la demostración, o viendo un contraejemplo del ejercicio también lo entiendes. Sin embargo, anteponiendo la demostración y luego estoy dando un ejercicio, yo creo que también se ve de manera clara, pero yo lo veo de manera menos clara. Primero prefiero ver un ejemplo y luego que me digan la demostración y luego, a partir de la demostración, si he entendido el ejemplo, me pongo a desarrollar el ejercicio sin problemas 61 .

A1: Yo lo que pienso es que la demostración formal está bien, pero está bien para indicar cómo es la forma de hacerlo y luego ya para explicarlo, pues el ejercicio, porque no puedes hacer un ejercicio sin saber lo que vas a utilizar. 0 sea, simple-

\footnotetext{
$60 \mathrm{~A}$ ambos alumnos la experiencia les ha dado claridad en los teoremas evocados.

${ }^{61}$ La opción espontánea de este alumno por la PPF queda algo confusa en la explicación que adjunta.
} 
mente, si utilizamos el teorema de Lagrange, se explica el teorema de Lagrange con la fórmula, y haces una aplicación. Yo así lo veo bien ${ }^{62}$.

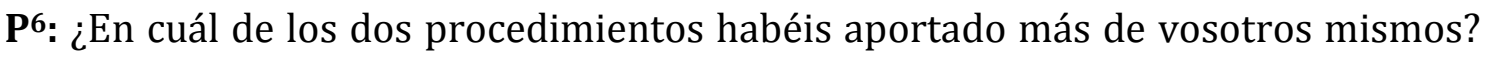
Es decir, visto el desarrollo de cada una de las pruebas, ¿cuál os ha supuesto más trabajo, más esfuerzo, más ingenio a la hora de entenderlo?

A2: La formal, porque partiendo de la fórmula o la demostración tienes que discurrir en cómo aplicarlo en tu caso en concreto. Yo creo que discurres más en la formal63.

A1: Si se supone que lo sabes ya, bueno, no sé... Es que si fuera otra cosa que no sabes, pues a lo mejor sí que me cuesta más la formal, pero si es algo que ya conoces, pues más o menos igual.

P7: Tú piensas que si es algo ya conocido...

A1: Algo ya conocido, la formal claramente la entiendes a la primera. Si es algo no conocido tienes que hacer el esfuerzo de intentar ver lo que te están explicando.

P8: Entonces, tú ves que es mejor introducirse primero por medio de la preformal si es algo más desconocido. ¿Es eso?

A1: Por ejemplo, lo de Riemann, la demostración así con la gráfica, sí que la entendía, porque también lo había dado, pero hacía mucho que no lo daba, y sí que, más o menos, me ha servido.

P9: Lo de las sumas de Riemann.

A1: Es que, además, me suena eso de los cuadrados. Por ejemplo, eso sí que se entiende bien, pero hay alguna demostración, cuando se utiliza mucho simbolito griego..., yo creo que la gente se pierde más. Yo sé que las matemáticas son riguro-

\footnotetext{
${ }^{62}$ Parece inclinarse por la PF, pero tampoco queda muy claro en la explicación que acompaña.
} ${ }^{63}$ Lo tiene claro, le supone mayor esfuerzo la PF. 
sas y a mí también me gusta, cuando sabes hacer las cosas, también te gusta ponerlas bien, pero primero hay que saber hacerlas, si no, no sabes lo que pones ${ }^{64}$.

P10: Primero uno se introduce, ¿no? Y luego uno pone el rigor. Bueno, expresad brevemente vuestra impresión general sobre toda la actividad. ¿Fue positiva? ¿Os ha hecho profundizar más en los teoremas? En fin, decid lo que queráis.

A2: Yo creo que es positivo hacer alguna prueba diferente, ver una demostración de dos maneras diferentes siempre es positivo.

A1: Yo también pienso lo mismo.

P11: A la hora de comunicar contenidos matemáticos. Pensad que tenéis que comunicaros uno a otro estos teoremas. ¿Cuál de los dos procedimientos, el formal o el preformal, escogeríais? Es decir, ¿cuál os aportaría mayor ayuda a la hora de comunicaros esto?

A2: Yo creo que el preformal. Siempre te vas a hacer un ejemplo de la aplicación del teorema en concreto. Entonces, siempre que yo voy a explicar algo a una persona que conozco o me tienen que explicar algo que yo desconozco, con un ejemplo siempre se ve mucho más fácil. Entonces yo creo que la preformal es una manera más fácil de hacerlo.

A1: Estoy de acuerdo. Normalmente, cuando te preguntan algo, te preguntan ejercicios: esto es así, así y así. Sabes hacer uno y, más o menos, sabes hacer todos.

P12: Entonces lo comunicarías en una situación concreta con números concretos.

A1: Pero claro, antes que explicar, hay que saber lo que hay que utilizar, porque en los teoremas que vimos del cociente y eso, de nada sirve que le diga cómo se hace

\footnotetext{
${ }^{64}$ Expresa algo elemental: es preciso saber qué se pretende hacer antes de realizarlo. En el diálogo acepta que, "aunque a mí también me gusta" el rigor matemático, le cuesta más trabajo el uso de símbolos (PF).
} 
si no sabe qué cosas hay que aplicar. Creo que a partir de un ejemplo desarrollado vuelves a lo mejor a la fórmula original.

P13: Pensáis los dos que una situación concreta ayuda...

A2: A conocer el desarrollo de una manera más clara.

A1: Así, por ejemplo, Juan Carlos, cuando vimos lo de las series convergentes y eso, me pareció la parte más fácil de entender, porque como era ver el teorema y ya decir ¡ah, vale!, esto se tiene que cumplir, ¿a que sí? Y aplicando la fórmula se entendía bastante bien. Sin embargo, no sé. Hay algunas cosas en los apuntes, como que hay muchas conclusiones de algunas cosas y no está nada claro qué es lo que realmente te va a hacer falta a ti para luego utilizarlo.

A2: Pierdes el rumbo del ejercicio. Sabes que estás haciendo algo pero llegas al momento que dices ¿por qué estaba haciendo yo esto? Pero bueno, yo creo que con la prueba preformal yo lo entiendo mejor65.

P14: ¿Cuál de los dos procedimientos explica mejor los pasos? Es decir, el encadenamiento de un paso a otro, que eso es una demostración ¿no? Es ir encadenando. Se parte de una hipótesis, se quiere llegar a una conclusión y hay unos pasos intermedios. Se van encadenando uno con otro hasta llegar. ¿Qué procedimiento los explica mejor?

A2: Yo creo que con un ejemplo una prueba preformal es más fácil, lo que pasa es que no siempre los números que uses te van a llevar a una conclusión cierta del teorema. Igual coges un ejemplo con algo que a lo mejor llegas a una conclusión que no es a la que tienes que llegar. Pero yo creo que con un ejemplo es más fácil.

P15: Ese ejemplo no cumpliría alguna de las hipótesis porque si se cumplen las hipótesis se llega necesariamente a la conclusión ¿no?

\footnotetext{
65 Ambos optan por la PPF, pero no terminan de distinguir con claridad "ejemplo" de "prueba".
} 
A2: Claro, si coges un ejemplo que cumpla las conclusiones o las directivas del teorema, pues yo creo que es más fácil entenderlo.

A1: Yo estoy de acuerdo también. Si se va a hacer un ejercicio, por ejemplo, se cumple esto, el teorema. En consecuencia, aplicamos esto y se hace el ejercicio. A mí es como me gusta hacerlo. Cuando hago las cosas es como... este teorema: se cumple esto, esto y ipum! Lo haces y se acabó.

P16: Bueno, y a la hora de entender el encadenamiento de un paso a otro ¿también la preformal?

A1 y A2: Yo creo que la preformal66.

P17: Finalmente, el orden en el que hicimos las pruebas, os lo recuerdo: para el teorema del valor medio primero se realizó la formal y después la preformal, sin embargo, para el teorema de unicidad del límite al revés, primero preformal y luego formal. ¿Creéis que puede influir el orden en el que se realicen las pruebas, a la hora de comprender mejor el teorema?

A2: Es que, como ya habíamos visto el teorema del valor medio en clase, pues al verlo por segunda vez y al hacer primero la prueba formal, igual en ese caso no. Pero yo creo que en el otro caso sí, en el caso del otro teorema de unicidad del límite.

P18: Ahí ¿influyó hacer primero la preformal?

A2: A mí me ayudó a comprenderla antes más la preformal, habiéndolo visto antes con un ejemplo. Al verlo con la demostración (se refiere a la formal) también veo ¡ah! pues es seguir estos pasos porque el teorema es cierto.

66 Afirmación unánime, pero confundiendo "prueba”, “ejemplo”, “ejercicio”. 
A1: Yo también pienso lo mismo: la preformal. Pero yo en mi elección me quedaría con una mezcla de las dos. La formal, como te he dicho, por explicar un poco el teorema y rápidamente, hacer un ejercicio práctico de lo que es.

A2: Pero intentar quizás explicarlo con menos...

A1: Con menos rigor...

A2: Matemático. Más palabras para que nosotros podamos entenderlo.

P19: Y luego ya vendría la demostración formal que abarca todos los casos. Pero utilizaríais las dos conjuntamente ¿no?

A2: Quizá buscar un lenguaje más cercano a nosotros, que lo podamos entender de alguna manera un poco más comprensible para nosotros, con una jerga matemática adecuada.

A1: Poner las cosas como siempre: para todo... no sé qué, pertenece a R. A mí me gusta ponerlo una vez que lo sabes hacer.

A2: Una vez que lo entiendes.

A2: Sabiendo lo que haces ¿sabes?

P20: Está claro.

A2: Yo creo que de nada sirve poner una demostración con todos los símbolos matemáticos si al final no se entiende lo que dices. Igual es más comprensible para nosotros explicarlo en un lenguaje coloquial, normal, y hacer la demostración y luego уа...

P21: Y luego ya vendrá el rigor, los símbolos y eso. 
A1: Para mí lo más importante es que quede claro cómo hay que desarrollar los ejercicios, o sea, este ejercicio se hace así, así y así y con el rigor que sea, pero lo más importante es que se entienda67.

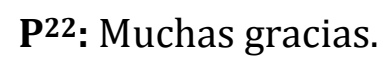

\section{TRANSCRIPCIÓN DE LA ENTREVISTA II}

Pn: P, Profesor; n, no de orden

A1: Julio Fernández Lasheras

\section{A2: Vicente Noriega Camarero}

P1: ¿Teníais ya alguna convicción de estos teoremas antes de la demostración? ¿Los conocíais? ¿Habíais trabajado con alguno de ellos?

A2: Con las dos demostraciones quedaron bastante más claras las cosas de cómo se aplicaban a los ejemplos los dos teoremas.

A1: Yo antes de estas dos demostraciones la verdad es que no tenía mucha idea de cómo iba a ser o no. Después de ver una demostración y otra, yo personalmente me quedo con la preformal, me resultó más clara, más simplificada para aplicar las posteriores, mejor ${ }^{68}$.

$\mathbf{P}^{2}:$ ¿Habíais visto en cursos anteriores estos teoremas?

A2: Yo la primera vez que los veía aquí contigo.

\footnotetext{
${ }^{67}$ Ambos, con una u otra expresión, aceptan como bueno el orden de la PPF en primer lugar.

${ }^{68}$ Muy espontáneamente, expresa desde el principio su convicción personal a favor de la PPF, que considera más clara y más sencilla.
} 
A1: Únicamente valor medio y un poco por encima, pasando rápidamente ${ }^{69}$.

P3: Para conocerlo simplemente. Entonces, estas demostraciones ¿qué os han proporcionado? Como novedad, digamos, para entender mejor estos teoremas.

A1: Pues, la verdad, ya sabemos básicamente de dónde sale el teorema, cómo se hace, y gracias a la prueba preformal y a la prueba formal, hemos podido comparar una y otra para poder escoger en un futuro cuál nos viene mejor o es más útil para aplicarla ${ }^{70}$.

A2: Yo estoy de acuerdo con él. Al hacer dos pruebas de cada teorema pues vemos claro de dónde han salido estos dos teoremas y, aplicándolo a los ejemplos de un examen o lo que sea, pues se puede entender mejor y hacer mejor el ejercicio.

P4: Entonces, una vez que habéis visto estas demostraciones, ¿podéis afirmar que es absoluta y rigurosamente completa la verdad de estos teoremas? ¿No habrá algún caso que lo incumple?

A2: Yo creo que sí se puede afirmar que para todos los casos que nos proponen los teoremas con esas condiciones de cada teorema, pues que no hay ningún caso excepcional en que no se cumpla el teorema, lo que afirma.

A1: Queda bastante claro cómo está una demostración, cómo está otra y se ve de dónde sale. Cuentas claras... Entonces creo que no hay ningún problema71.

P5: En cuanto a la facilidad en la aplicación de la prueba formal y la preformal, al estudiar estas pruebas hemos aprendido dos procedimientos, el formal y el preformal. Ambos son completamente rigurosos, pero, a la hora de hacer ejercicios

\footnotetext{
${ }^{69}$ No es muy diferente del compañero, $A_{2}$, su grado de conocimiento de estos teoremas.

${ }^{70} \mathrm{Han}$ comprobado, ambos, el fundamento de los teoremas, el proceso de las pruebas, la utilidad de cada una de ellas y pueden elegir, en consecuencia, la aplicación de la más adecuada; lo cual es laudable y valora la experiencia llevada a cabo.

${ }^{71}$ Han quedado convencidos de la verdad de los teoremas y han captado las diferencias de ambas pruebas.
} 
o trabajar de forma habitual de manera práctica en matemáticas ¿cuál de los dos procedimientos pensáis que os ayudaría más? ¿el formal o el preformal?

A2: Pues yo la preformal ya que aplicándolo con un ejemplo yo, a mi parecer, de mi forma de ser para realizar ejercicios, pues me ayuda más la preformal, aunque igual a otras personas les gusta más la otra prueba porque les gusta sólo hacer ejercicios más abstractos ${ }^{72}$.

A1: Yo también me tiro por la preformal. Aparte de que tiene la base de la formal, puesto que viene a ser lo mismo, pero una tiene más ejemplificación. Entonces, al ver ya cómo se aplica en un ejercicio práctico, ya puedes, básicamente, desde esa base, discurrir cómo sacar los demás ejercicios ${ }^{73}$.

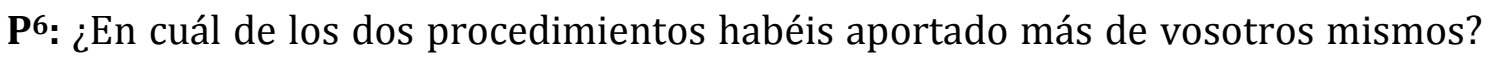
Es decir, visto el desarrollo de cada una de las pruebas, ¿cuál os ha supuesto más trabajo, más esfuerzo, más ingenio? ¿El preformal o el formal?

A1: A mí el formal me ha parecido bastante más abstracto, más... vamos, en vez de con números, con una aplicación práctica, es con valores un poco surrealistas. Entonces tienes que estar más pendiente de dónde salen los valores, de dónde salen las fórmulas y demás. Me ha parecido un poco más complicado ${ }^{74}$.

P7: Te ha supuesto más esfuerzo, por tanto.

A2: Yo también, porque la formal tienes que estar un poco más atento de dónde van saliendo las letras, porque luego igual yendo hacia atrás, lo puedes seguir un poco peor que la preformal 75 .

\footnotetext{
72 Vicente es sincero y respetuoso: a él le ayuda más la PPF; la característica de su aprendizaje parece ser la "inducción", prefiere lo concreto; pero es respetuoso con otra forma que prefiera lo abstracto, más deductivo.

${ }^{73} \mathrm{Su}$ razonamiento es que se trata de una auténtica prueba con más "ejemplificación", no es un simple ejemplo.

${ }^{74}$ Valores "surrealistas": indica que suponen más esfuerzo añadido a la PF.

75 También reconoce mayor esfuerzo ante la representación simbólica de la PF.
} 
P8: En breves palabras, vuestra impresión general sobre toda aquella actividad que hicimos aquel día. ¿Qué os pareció?

A1: Ha sido productiva. Ya te hemos visto en dos teoremas: uno que yo no conocía para nada; dos formas de aplicar, vamos, la prueba preformal y la prueba formal, tampoco las conocía hasta este curso y, bueno, un examen digamos que bien.

P9: ¿Os resultó interesante? ¿Os pareció un rollo? ¿Se podía haber hecho de otra forma?

A1: Bueno, además podíamos estar con el compañero medio debatiendo: ¿qué te parece uno?; ¿qué te parece la otra? Bastante positivo, interactivo y demás.

A2: Sí, estuvo muy bien. Además las tablas que había para poner las notas y eso, pues te da una mejor idea sobre las dos pruebas, de cuál te podía servir más para aplicarlo en un futuro ${ }^{76}$.

P11: ¿Os pareció claro el cuestionario, la distribución?

A2: Sí, estaba claro y ordenado.

A1: Luego, al final, además, tenías el ejercicio para demostrar cómo aplicar la prueba preformal o la prueba formal. Se podía ver por dónde ibas ${ }^{77}$.

P12: Muy bien. Imaginad que os tenéis que comunicar tú, Julio, a Vicente o tú, Vicente, a Julio, algo de esto. ¿Cuál de los dos procedimientos pensáis que os aportaría mayor ayuda?

A1: Yo creo que estando en nuestro nivel, así entre nosotros, un preformal sería más sencillo, más práctico, más asequible para entender. Pero con una persona que

\footnotetext{
${ }^{76}$ Ambos se expresan muy a favor de esta experiencia con manifestaciones que denotan sinceridad y convicción.

${ }_{77}$ Ambos alumnos se muestran satisfechos de una experiencia bastante positiva, que les ha enriquecido, "productiva" ha dicho este alumno (respuesta a la $\mathrm{P}^{8}$ ).
} 
tuviera un conocimiento más amplio en matemáticas una formal sería lo más conveniente para explicarle. En nuestro nivel, preformal, en el otro... ${ }^{78}$

A2: Además, de otros cursos, estamos más acostumbrados a ver ejemplos de preformal, mientras nos lo explican en $2^{\circ}$ de Bachillerato y otros cursos, que la formal. Entonces, a la hora de explicárselo a un compañero, hacer una preformal estaría mejor. Seríamos más capaces de explicárselo mejor que con una prueba formal ${ }^{79}$.

P13: ¿Cuál de los dos procedimientos explica mejor los pasos? Luego os preguntaré por en cuál entendéis mejor, pero es diferente ¿eh? Fijaos, una demostración es simplemente partir de una hipótesis, llegar a una conclusión y luego hay una serie de pasos intermedios que hay que ir enlazando, encadenando. ¿Cuál de los dos procedimientos lo explica mejor?

A1: Para mí, para explicar los pasos de cómo se tiene que ir realizando, yo creo que la formal a secas, puesto que la preformal ya lo da un poco por presupuesto, ya lo da como que sale así y no tiene que preocuparse de otra forma. Entonces, para ver los pasos que sigue el ejercicio, yo creo que uno formal ${ }^{80}$.

P14: Explica mejor los pasos la formal.

A2: Sí, yo también estoy de acuerdo que explica mejor los pasos uno formal porque esas letras o incógnitas que vayas poniendo van a valer para todos los casos. Entonces queda mejor explicado, aunque, bueno, depende de la gente, más claro o menos claro ${ }^{81 .}$

P15: Y ahora, a la hora de entender el encadenamiento de un paso a otro ¿por cuál os decantaríais?

\footnotetext{
${ }^{78}$ La "modestia" de Julio confirma la sencillez, facilidad y utilidad práctica de la PPF.

79 También el pudor de Vicente ratifica a la PPF como prueba fácil y sencilla.

${ }^{80}$ La respuesta supone considerar la PPF como prueba rigurosa y auténtica con ese matiz de lo "concreto" que no se detiene tanto en el "detalle" de sus pasos, y por ello se inclina hacia la PF en este aspecto.

${ }^{81}$ Distingue el ámbito objetivo (los enlaces lógicos) y el subjetivo (receptivo) de la demostración; en éste segundo será más o menos clara, según quién reciba la prueba.
} 
A1: La preformal para entender sí. Para seguir los pasos de cómo el ejercicio va creciendo una prueba formal, entonces ves las variables y demás, pero para entender, la preformal 82 .

A2: Yo también la preformal, pero también luego no está de más ver la formal para ver de dónde han salido esas demostraciones preformales ${ }^{83}$.

P16: O sea que de alguna manera, las dos de manera conjunta. Os inclináis más por la preformal pero hay una complementariedad ¿no?

A1 y A2: Sí, sí.

P17: Finalmente, el orden en el que hicimos las pruebas, os lo recuerdo: para el teorema del valor medio primero se realizó la formal y después la preformal; sin embargo, para el teorema de unicidad del límite, primero la preformal y luego la formal. ¿Pensáis que puede influir el orden en el que se presenten las pruebas, a la hora de la comprensión del teorema?

A1: En el primer teorema bueno, yo tenía un poco de idea de cómo iba, entonces, básicamente, una después de la otra: primero la formal y luego la preformal; no tuve problemas. Lo que pasa es que el siguiente teorema no tenía conocimiento previo de él. Entonces primero fue la preformal y luego la formal. Me costó más entenderle así, de primeras, con una preformal, sin saber de dónde salían previamente las cosas, pero...

P18: Pero porque lo desconocías totalmente.

A1: No lo conocía ni nada. Entonces al no saber de dónde salían las fórmulas ni na-

\footnotetext{
82 Interesante distinción: para "seguir, razonar, enlazar" los pasos, la PF; para "entender" los pasos, la PPF.

83 Vicente, que prefiere la PPF, no quiere prescindir tampoco de la PF, pues valora, también Julio al responder con Vicente a la siguiente pregunta, la complementariedad de ambas pruebas.
} 
da, me resultó un poco abstracto. Hubiera preferido la otra (el otro orden) ${ }^{84}$.

A2: Igual en los teoremas que no conoces de antes, quizá sea mejor hacer una demostración formal para ver en conjunto todo el teorema y luego ejemplificarlo con una demostración preformal. Igual queda más claro. Pero en otros teoremas que tengas conocimiento previo de ellos, con una preformal bastaría ${ }^{85}$.

P19: Como que te introduce mejor... Ya tienes la idea de eso y lo ves de esa manera concreta y digamos que te introduce mejor en el resultado. ¿Es eso lo que quieres decir?

A2: Sí.

P20: Muchas gracias.

84 Julio es partidario, en el orden de las pruebas, y para la asimilación personal por él mismo de los contenidos de los teoremas, la PF en primer lugar, sobre todo cuando no conoce nada de esos contenidos.

85 Un leve rebrote de la confusión "prueba-ejemplo"; prefiere la PPF, su aprendizaje va más por lo concreto. 